\title{
Influence of storage time and temperature on the activity of urease
}

\author{
B. Alev ${ }^{1 *}$, S. Tunali ${ }^{2}$, R. Yanardag ${ }^{2}$, A. Yarat ${ }^{1}$ \\ ${ }^{I}$ Marmara University, Faculty of Dentistry, Department of Basic Medical Sciences, Maltepe, Istanbul, Turkey \\ ${ }^{2}$ Istanbul University-Cerrahpasa, Faculty of Engineering, Department of Chemistry, Avcilar, Istanbul, Turkey
}

Received March 28, 2016; Accepted December 3, 2018

\begin{abstract}
Enzymes are made of protein, that is why they are sensitive molecules and are affected by storage conditions. A small change in enzyme activity during storage may cause a big error in analysis results. The aim of the study was to evaluate the effects of storage time and temperature on urease activity. Urease solutions were prepared at different activities (from 100 to $2000 \mathrm{U} / \mathrm{mL}$ ) and stored at room temperature, in the refrigerator $\left(4^{\circ} \mathrm{C}\right.$ ), and in the deep freezer $\left(-18^{\circ} \mathrm{C}\right.$ and $\left.-80^{\circ} \mathrm{C}\right)$. Activity measurements were made at regular intervals until 28 days by the modified Weatherburn method. The relative activities of $100-1000 \mathrm{U} / \mathrm{mL}$ urease solutions stored at room temperature, $4,-18$ or $-80^{\circ} \mathrm{C}$ were $75 \%$ and below after 4 days. Twenty-eight days later, for $2000 \mathrm{U} / \mathrm{mL}$ urease solutions, only at room temperature, the relative activity was reduced to $37 \%$, while at $4,-18$ or $-80^{\circ} \mathrm{C}$, the relative activities were above $80 \%$. Since urease can be maintained at $4^{\circ} \mathrm{C}$ for 28 days without significant loss of activity, it has practical importance. Low-activity urease solutions (such as $100-1000 \mathrm{U} / \mathrm{mL}$ ) should not be stored at -18 or $-80^{\circ} \mathrm{C}$ for short or long term storage, they should be stored at $4{ }^{\circ} \mathrm{C}$ only for one day.
\end{abstract}

Keywords: Urease activity, storage time, storage temperature

\section{INTRODUCTION}

Urease, (urea amidohydrolase, EC 3.5.1.5) is a multisubunit (homohexamer), nickel-dependent metalloenzyme synthesized by plants, some bacteria and fungi [1]. Urease plays a primary role in nitrogen metabolism in the nature. Its physiological role is to hydrolyze urea by forming ammonia and carbon dioxide. Because of released ammonia, nitrogen is produced during growth of organisms and the medium becomes alkaline [2].

Apart from its natural significance, there are many uses for urease. These include removal of urea from aqueous solutions, determination of urea concentration in aqueous solutions, precipitation of calcium carbonate in geoenvironmental applications, determination of heavy metal ions in aqueous solutions [3-10]. Urease is also used as an anticancer agent and a vaccine antigen, as well as for determining the concentration of urea in biological fluids (blood, urine, saliva) in medicine [11-15].

The storage of enzymes is a quite important issue from both practical and economical points of view. Enzymes undergo denaturation during production, storage, and application. Their activities may change in response to environmental factors such as temperature, $\mathrm{pH}$, chemical agents, autolysis or ionic strength [16]. Storage conditions may vary from enzyme to enzyme because of individual structural differences [17]. However,

\footnotetext{
* To whom all correspondence should be sent:
}

E-mail: burcinalew@yahoo.com.tr some basic rules for storage can be mentioned: Enzymes are more stable at low temperatures. They can be kept in a buffer in a $4^{\circ} \mathrm{C}$ refrigerator for short-term storage. There are different strategies for long-term storage, such as keeping them at $-20^{\circ} \mathrm{C}$ and $-80^{\circ} \mathrm{C}$ in the deep freezer, or under liquid nitrogen at $-196^{\circ} \mathrm{C}$, and storing in lyophilized or immobilized forms $[18,19]$.

In practice, enzymes that are not to be used immediately, are refrigerated or frozen. During storage, the enzyme stability can change according to its physical state such as being in liquid, lyophilized or immobilized form. Therefore, defining the appropriate storage conditions for each enzyme is very important to minimize preanalytical errors. The aim of our present study was to examine the effect of storage time and temperature on urease activity. This paper describes some recommendations for storage of urease enzyme.

\section{EXPERIMENTAL}

\section{Materials}

All chemicals used were of analytical grade from Merck (Darmstadt, Germany), Riedel-de Haën (Seelze, Germany) or Fluka (Buchs, Switzerland). Urease from Canavalia ensiformis was purchased from Merck Millipore (Massachusetts, USA). The lyophilized enzyme was stored at $-20{ }^{\circ} \mathrm{C}$ until use according to manufacturer's instructions. Ultra-pure water (Human Corporation Zeneer Power I) was 
used throughout the experiments. $\mathrm{pH}$ determinations were carried out on the HI 2211 pH/ORP-Meter (HANNA Instruments). The absorbance measurements were performed on a Shimadzu UVmini-1240 spectrophotometer.

\section{Methods}

Preparation of Urease Solutions and Storage Conditions. Lyophilized urease enzyme (10 kU) was reconstituted in $1 \mathrm{~mL}$ of $50 \mathrm{mM}$ phosphate buffer, pH 7.6 to obtain a stock urease solution. It was diluted with appropriate buffer to prepare 100 , 200, 300, 400, 500, 1000 and $2000 \mathrm{U} / \mathrm{mL}$ enzyme solutions. They were all divided into small volumes and were put into eppendorf tubes. To investigate the effect of storage temperature on urease activity, the tubes were stored at room temperature, in the refrigerator $\left(4^{\circ} \mathrm{C}\right)$, in the deep freezer $\left(-18,-80^{\circ} \mathrm{C}\right)$ separately. To determine the effect of storage time, urease activity was measured at $0,1,4,7,11,14$, 17, 21, 24, 28 days. Flow chart for all experiments is shown in Figure 1.

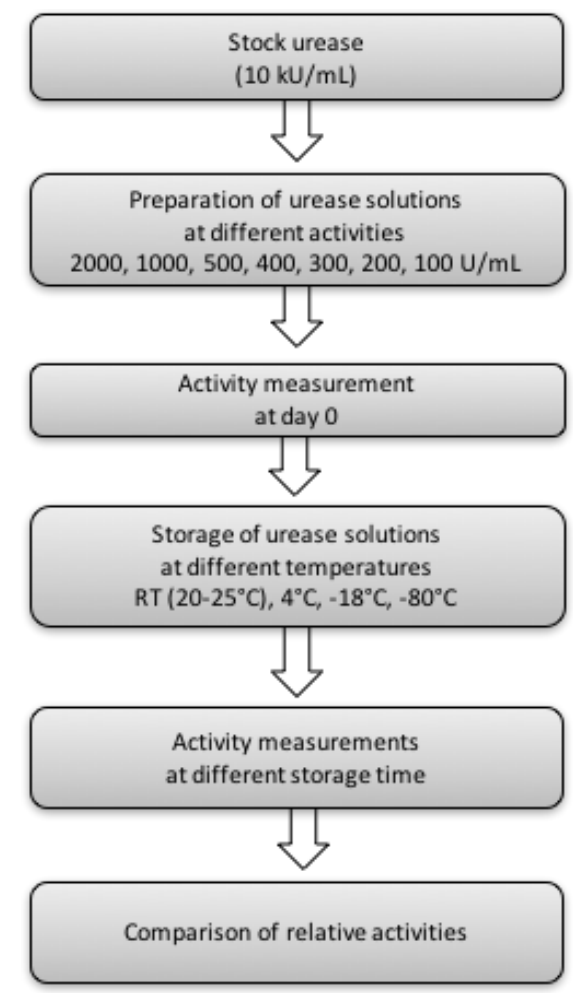

Fig. 1. Experimental flow chart (RT: Room temperature)

Measurement of Urease Activity. The urease activity was measured by a modified Weatherburn method [20]. The reaction was carried out in a tube containing $100 \mu \mathrm{L}$ of urease solution, $10 \mu \mathrm{L}$ of 7 $\mathrm{mM}$ urea and $1890 \mu \mathrm{L}$ of $50 \mathrm{mM}$ phosphate buffer,
$\mathrm{pH}$ 7.6. The reaction mixture was incubated at room temperature for $15 \mathrm{~min}$. $500 \mu \mathrm{L}$ of phenol reagent and $500 \mu \mathrm{L}$ of alkaline hypochlorite reagent were then added to the tube, and incubated at $60^{\circ} \mathrm{C}$ for $5 \mathrm{~min}$. After the tube was rapidly cooled under tap water to room temperature, absorbance was read at $630 \mathrm{~nm}$ against reagent blank. The amount of ammonia liberated was calculated using a standard curve obtained from ammonium sulfate. One unit urease activity was defined as the amount of enzyme required to hydrolyze $1 \mu \mathrm{mol}$ of urea per minute. All measurements were repeated six times. Results were reported as mean \pm standard deviation (SD). GraphPad Prism 5 version 5.0a (GraphPad Software, San Diego, CA, USA) was used to calculate means and SDs.

\section{RESULTS}

The urease activities of all solutions at zero time were accepted as $100 \%$ activity. The relative activities of solutions of $100-1000 \mathrm{U} / \mathrm{mL}$ were $75 \%$ and below after 4 days for all storage temperatures. Therefore the enzyme activities of urease solutions of $2000 \mathrm{U} / \mathrm{mL}$ were also measured.

After storage for 1 day at room temperature, the decrease in the relative activities of $100,200,300$, 400, 500 and $1000 \mathrm{U} / \mathrm{mL}$ urease solutions were 37, $34,38,37,37$ and $24 \%$, respectively; at $4^{\circ} \mathrm{C}$, they were $17,17,17,15,15$ and $6 \%$; at $-18^{\circ} \mathrm{C}$, they were $95,91,87,29,20$ and $18 \%$; at $-80^{\circ} \mathrm{C}$, they were 91 , $85,79,60,43$ and $22 \%$ (Figure 2A-F).

After storage for 4 days at room temperature, the decrease in the relative activities of 100, 200, 300, 400, 500 and $1000 \mathrm{U} / \mathrm{mL}$ urease solutions were 29, $28,25,30,28$ and $31 \%$, respectively; at $4^{\circ} \mathrm{C}$, they were $37,32,31,34,36$ and $29 \%$; at $-18^{\circ} \mathrm{C}$, they were $96,94,89,79,48$ and $37 \%$; at $-80^{\circ} \mathrm{C}$, they were 94, 88, 73, 62, 46 and $36 \%$ (Figure 2A-F).

As seen in Figure 2 there was unexpected increase in relative urease activities of 100-500 $\mathrm{U} / \mathrm{mL}$ urease solutions from day 1 to day 4 at room temperature.

At the end of the $14^{\text {th }}$ day, the relative activities of $2000 \mathrm{U} / \mathrm{mL}$ urease solutions, kept at all storage temperatures, were $84 \%$ and over (the decreases were $16 \%$ and below). After day 14 till day 28, only at room temperature, the relative activity was reduced to $37 \%$ (the decrease was $63 \%$ ), while at other storage temperatures, the relative activities were above $80 \%$ (the decreases were below 20\%) (Figure 3). 
B. Alev et al.: Influence of storage time and temperature on the activity of urease

A

$100 \mathrm{U} / \mathrm{mL}$

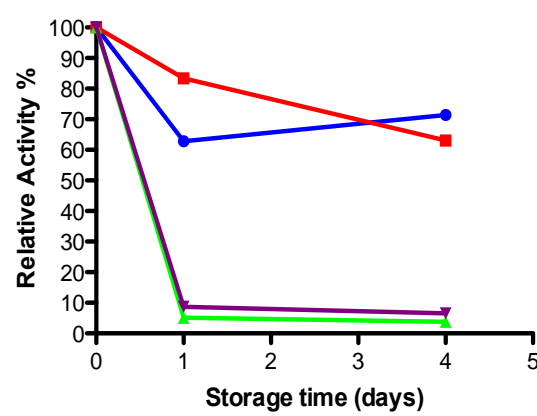

C

$300 \mathrm{U} / \mathrm{mL}$

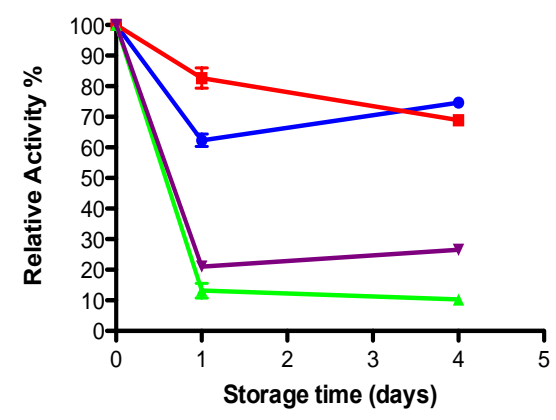

E

$500 \mathrm{U} / \mathrm{mL}$

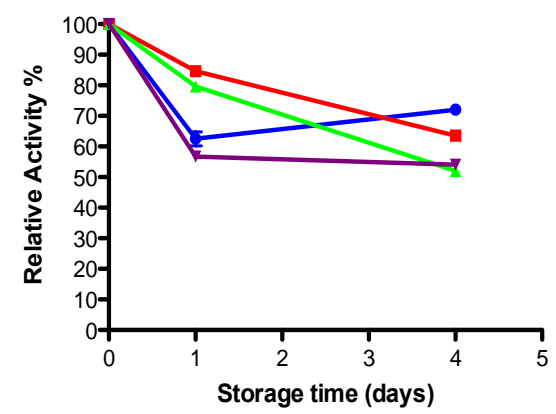

B

$200 \mathrm{U} / \mathrm{mL}$
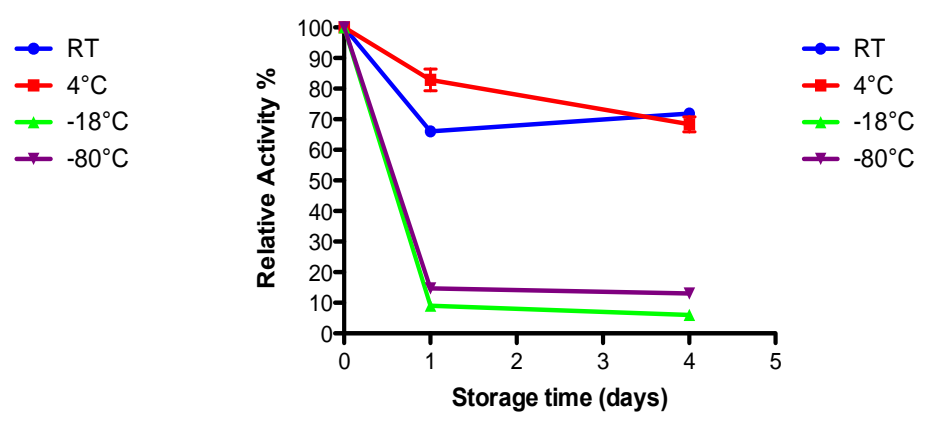

$\mathrm{D}$

$400 \mathrm{U} / \mathrm{mL}$

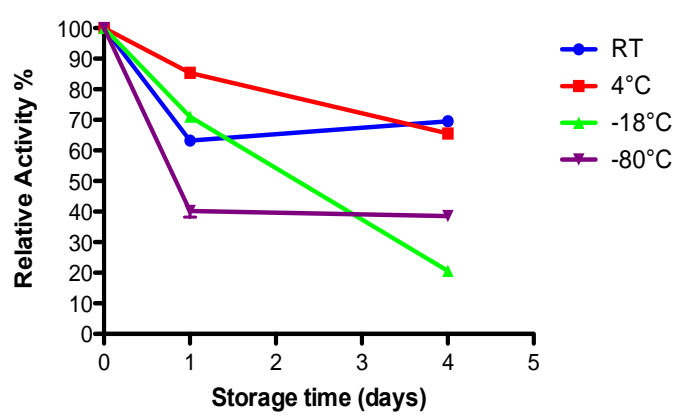

F

$1000 \mathrm{U} / \mathrm{mL}$

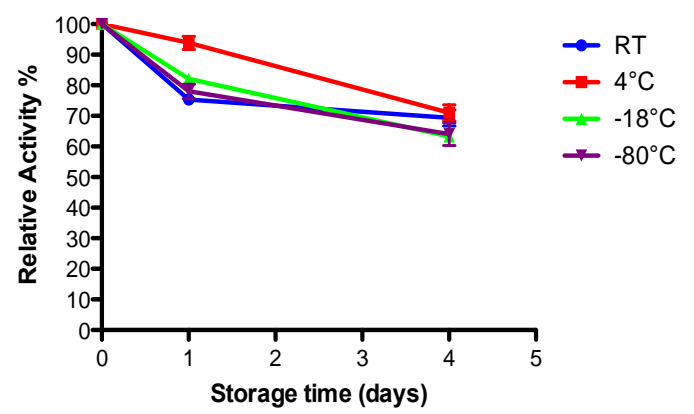

Fig. 2. Effects of storage time and temperature on urease activity. A: $100 \mathrm{U} / \mathrm{mL}, \mathrm{B}: 200 \mathrm{U} / \mathrm{mL}, \mathrm{C}: 300 \mathrm{U} / \mathrm{mL}, \mathrm{D}: 400$ $\mathrm{U} / \mathrm{mL}, \mathrm{E}: 500 \mathrm{U} / \mathrm{mL}, \mathrm{F}: 1000 \mathrm{U} / \mathrm{mL}$ urease solutions. RT: Room temperature

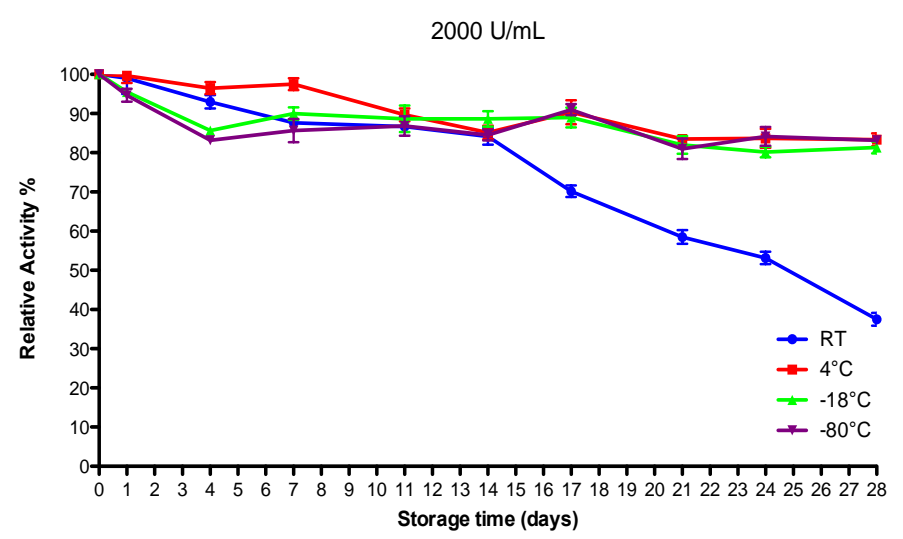

Fig. 3. Effects of storage time and temperature on $2000 \mathrm{U} / \mathrm{mL}$ urease solutions. RT: Room temperature 


\section{DISCUSSION}

Temperature, $\mathrm{pH}$, chemical agents, autolysis and ionic strength can affect enzyme structure and activity by causing unfolding, aggregation or covalent changes. For multimeric enzymes subunit dissociation is known to be an inactivation process [16]. Urease is a cysteine-rich enzyme and over time can aggregate through the formation of intermolecular disulfide bonds. Sumner et al. suggested that urease solution is inactivated on storage and the deactivation is caused by some oxidation reactions of sulfhydryl groups [21]. On the basis of previous investigations, urease aggregation and precipitation usually follows when it is stored in some conditions such as high protein concentrations, high temperature, absence of reducing agents, low $\mathrm{pH}$ or presence of salts. It is believed that exposed hydrophobic clusters of protein can cause aggregation [22].

It is known that denaturation of proteins during freezing is related to the physical and chemical changes of the local environment around the protein molecules during the process [23]. Follmer et al. showed that freezed sodium phosphate buffer induces crystallization of a component salt and a resulting shift of the local $\mathrm{pH}$ surrounding the protein. The acidification of medium, which leads to acidic residues' protonation, can decrease the electrostatic repulsion of the negative charges and thus could lead to aggregation of urease [22]. This may explain the drastic decrease in relative urease activity at low concentrations when stored at -18 and $-80^{\circ} \mathrm{C}$ in our study. In contrary, at a high enzyme concentration such as $2000 \mathrm{U} / \mathrm{mL}$, the decrease was not too big. This resistance of urease to damage during freeze-thawing might be due to high initial enzyme concentration [18].

An aqueous environment also introduces a significant risk of microbial contamination and proliferation, which can cause change in the enzyme activity in liquid products [24]. Urease is a microbial enzyme produced by some bacteria and fungi [25]. The unexpected increase in relative urease activities of $100-500 \mathrm{U} / \mathrm{mL}$ urease solutions from day 1 to day 4 at room temperature may be attributed to microbial contamination because of using non-sterilized eppendorf tubes and buffer solution [26].

The recommendation of the manufacturer for storage of urease that was used in this study is up to 2 months at $-20^{\circ} \mathrm{C}$ after reconstitution. However, there is a need for more descriptive information on the effect of enzyme concentration on its activity. Our results clearly showed that urease stability during storage is closely related to its concentration and urease is more stable at high than at low concentration.

In this study, it was observed that the highconcentration urease solution can be stored for a month at low temperatures such as $-18^{\circ} \mathrm{C}$ and $80^{\circ} \mathrm{C}$. This is an expected result but similar results were also obtained during storage at $4^{\circ} \mathrm{C}$. This stability of urease at $4^{\circ} \mathrm{C}$ is attractive for practical applications.

Jack bean (Canavalia ensiformis) urease was used in our study. In the literature, although urease used in another study was of bacterial origin, when it was stored at room temperature and $-70^{\circ} \mathrm{C}$, an activity decline, which was similar to the findings of our study, was detected [27]. Urease used in another study was also of fungal origin and when it was stored at -20 and $-80^{\circ} \mathrm{C}$, there was a similar decrease as our results in enzyme activity after 28 days [28].

\section{CONCLUSION}

The current study highlights the importance of defining the appropriate storage conditions for urease enzyme. Since urease enzyme with high activity can be maintained at $4^{\circ} \mathrm{C}$ for almost a month without significant loss of activity, it has practical importance. Low-activity urease solutions (such as $100-1000 \mathrm{U} / \mathrm{mL}$ ) should not be stored at 18 or $-80^{\circ} \mathrm{C}$ for short- or long-term storage; they should be stored at $4^{\circ} \mathrm{C}$ only for one day.

Acknowledgement: This study was supported by the Marmara University Scientific Research Projects Commission (SAG-D-070317-0097, 2017).

\section{REFERENCES}

1. A. Balasubramanian, K. Ponnuraj, J. Mol. Biol., 400, 274 (2010).

2. C. R. Carlini, R. Ligabue-Braun, Toxicon., 110, 90 (2016).

3. E. Urbańczyk, M. Sowa, W. Simka, J. Appl. Electrochem., 46, 1011 (2016).

4. R. Ramesh, P. Puhazhendi, J. Kumar, M. K. Gowthaman, S. F. D'Souza, N. R. Kamini, Mat. Sci. Eng. C-Bio. S., 49, 786 (2015).

5. A. Spiliotopoulou, K. M. S. Hansen, H. R. Andersen, in: Fifth International Conference Swimming Pool \& Spa - Abstract book, E. Ferretti, G. Fantuzzi, V. Romano Spica, S. Caroli, L. Bonadonna (eds.), Rome, Italy, 2013, p. 57.

6. M. Revilla, J. Alexander, P. M. Gilbert, Limnol. Oceanogr Methods, 3, 290 (2005).

7. M. Michalec, M. Granica, J. Bzura, R. Koncki, J. Matuszkiewicz-Rowińska, Ł. Tymecki, Sens. Actuators, B., 226, 563 (2016).

8. R. A. N. Dilrukshi, S. Kawasaki, J. Civil. Environ. Eng., 6, 207 (2016).

9. N. J. Nepomuscene, D. Daniel, A. Krastanov, Biotechnol. \& Biotechnol. Eq., 21, 377 (2007). 


\section{CONTENTS}

B. Alev, S. Tunali, R. Yanardag, A. Yarat, Influence of storage time and temperature on activity of urease.

M. Sinirkaya, Effect of ultrasound on the dissolution of Mardin-Mazıdağ (Turkey) phosphate ore in dilute $\mathrm{H}_{3} \mathrm{PO}_{4}$ solution.

G.S. Seth, R. Kumar, R. Tripathi, Thermo-дiffusion effects on magneto-hydrodynamic natural convection flow of a chemically reactive Brinkman type nanofluid in a porous medium.

M. Imran Khan, T. Hayat, M. Ijaz Khan, T. Yasmeen, Thermal properties and time dependent flow behavior of viscous fluid.

S. Hassanpour, M.Bayat, A.Chaichi Nosrati, M. Ghorannevis, S. Hashemi, The eliminatory effects of cold argon plasma jet on aflatoxin B1 produced by different isolates of Aspergillus section nigri.

B. Parvizi, A. Khanlarkhani, Y. Palizdar, A.Farshad, Comparison of ANFIS and ANN modeling for predicting the behavior of a catalytic methane reformer.

$\ddot{O}$. Özşen Batur, Ö. Atli, $\dot{I}$. Kiran, Biotransformation of oleic acid and antimicrobial and anticancer activities of its biotransformation extracts.

R. Rehman, T. Tanveer, Spectrophotometric determination of significant minerals in milk samples found in Lahore (Pakistan) for ensuring food safety.....

S. Sönmez, Ö. Özden, The influence of pigment proportions and calendering of coated paperboards on dot gain...

P.M. Rafailov, R. Todorov, V. Marinova, D.Z. Dimitrov, M. M. Gospodinov, Optical spectroscopic study of Ru- and Rh-DOPED $\mathrm{Bi}_{12} \mathrm{TiO}_{20}$ crystals....

S. Mohammadi Chalanchi, A. Ebrahimi , A. Nowroozi, Theoretical insight to intermolecular hydrogen bond interactions between methyl N-(2-pyridyl) carbamate and acetic acid: substituent effects, cooperativity and energy decomposition analysis...

V.A. Kadnor, S. N. Shelke, Synthesis and biological evaluation of novel carbazole chalcones

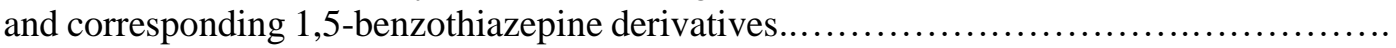

D.S. Bogdanović, D.H. Anđelković, I.S. Kostić, G.M. Kocić, T.D. Anđelković, The effects of temperature and ultrasound on the migration of di-(2-ethylhexyl) phthalate from plastic packaging into dairy products.

A.M. Vasileva, I.A. Iliev, V.S. Lozanov, M.B. Dimitrova, V.I. Mitev, I.P. Ivanov, In vitro study on the antitumor activity of Tanacetum vulgare L. extracts....

N.P. Gribacheva, G.M. Gecheva, V.M. Stefanova, Air pollution monitoring with mosses in Western Rhodopes, Bulgaria.

S.B. Seo, Y.M. Kim, Optimization of high ultrasound-assisted extraction (INEFU) of active components from natural materials by response HPLC-PDA analysis....

G. Karakus, Z. Akin Polat, M. Karahan, Design, synthesis, structural characterization and cell cytotoxicity of new derivative poly( maleic anhydride-co-vinyl acetate)/miltefosine polymer/drug conjugate.

N. Ebrahimi, A. Haghighi Asl, P. Mottahedin, Optimization of $\beta$-carotene solubility in pressurized hot water using a dynamic method and factorial methodology..................

E. Chorbadzhiyska, I. Bardarov, Y. Hubenova, M. Mitov, Modified graphite electrodes as potential cathodic electrocatalysts for microbial electrolysis cells........................ INSTRUCTIONS TO THE AUTHORS. 


\section{B. Alev et al.: Influence of storage time and temperature on the activity of urease}

10. X. Zeng, J. Liu, Z. Zhang, S. Kong, Int. J. Electrochem. Sci., 10, 8344 (2015).

11. W. Y. Wong, C. I. DeLuca, B. Tian, I. Wilson, S. Molund, N. Warriar, M.V. Govindan, D. Segal, H. Chao, J. Exp. Ther. Oncol., 5, 93 (2005).

12. M. Zeng, X. H. Mao, J. X. Li, W. D. Tong, B. Wang, Y. J. Zhang, G. Guo, Z. J. Zhao, L. Li, D. L. Wu, D. S. Lu, Z. M. Tan, H. Y. Liang, C. Wu, D. H. Li, P. Luo, H. Zeng, W. J. Zhang, J. Y. Zhang, B. T. Guo, F. C. Zhu, Q. M. Zou, Lancet, 386, 1457 (2015).

13. P. Lahiri, J. Chem. \& Chem. Sci., 5, 389 (2015).

14. G. K. Mishra, A. Sharma, K. Deshpande, S. Bhand, Appl. Biochem. Biotechnol., 174, 998 (2014).

15. T. J. Lasisi, Y. R. Raji, B. L. Salako, BMC Nephrol., 17, 10 (2016).

16. P. V. Iyer, L. Ananthanarayan, Process. Biochem., 43, 1019 (2008).

17. K. Johnson-Davis, G. A. McMillin, in: Clinical Chemistry: Principles, Techniques and Correlations, M. L. Bishop, E. P. Fody, L. E. Schoeff (eds.), Wolters Kluwer Health/Lippincott Williams \& Wilkins, 2010, p. 281.

18. J. F. Carpenter, M. C. Manning,T. W. Randolph,
Curr. Protoc. Protein Sci., 27, 4.6 .1 (2002).

19. R. A. Copeland, Methods for Protein Analysis: A Practical Guide for Laboratory Protocols, SpringerScience+Business Media B.V., Dordrecht, 1994, p. 17.

20. M. W. Weatherburn, Anal. Chem., 39, 971 (1967).

21. J. B. Sumner, N. Gralén, I. B. Eriksson-Quensel, J. Biol. Chem., 125, 37 (1938).

22. C. Follmer, F. V. Pereira, N. P. Silveira, C. R. Carlini, Biophys. Chem.,111,79 (2004).

23. E. Cao, Y. Chen, Z. Cui, P. R. Foster, Biotechnol. Bioeng., 82, 684 (2003).

24. M. A. El-Sherbiny, G. A. El-Chaghaby, J. Agrobiol., 28, 129 (2011).

25. B. Krajewska, J. Mol. Catal. B: Enzym., 59, 9 (2009).

26. M. R. Spier, F. B. Siepmann, L. Staack, P. Z. Souza, V. Kumar, A. B. P. Medeiros, C. R. Soccol, Prep. Biochem. Biotechnol., 46, 725 (2016).

27. H. L. T. Mobley, M. J. Cortesia, L. E. Rosenthal, B. D. Jones, J. Clin. Microbiol., 26, 831 (1988).

28. P. T. Smith, D. King Jr, N. Goodman, J. Gen. Microbiol., 139, 957 (1993). 


\title{
Effect of ultrasound on the dissolution of Mardin-Mazıdağı (Turkey) phosphate ore in dilute $\mathrm{H}_{3} \mathrm{PO}_{4}$ solutions
}

\author{
M. Sinirkaya \\ Department of Chemical Engineering, Atatürk University, 25240 Erzurum, Turkey
}

Received May 18, 2017; Accepted January 3, 2018

\begin{abstract}
The dissolution of phosphate ore in dilute phosphoric acid solutions was investigated in both the absence and presence of ultrasound. Acid concentration, reaction temperature and reaction time were chosen as parameters. While the conversion fractions decreased with increasing temperature in dilute phosphoric acid in absence of ultrasound, these rates increased with increasing temperature in the presence of ultrasound. In the experiments, the expected effects of ultrasound were observed, such as decrease in the thickness of the diffusion layer on the solid-liquid interfaces in the suspension, leading to increased surface area.
\end{abstract}

Key words: Phosphate rock, Phosphoric acid, Dissolution kinetics, Ultrasound, Cavitation

\section{INTRODUCTION}

Most of the phosphorus in the phosphate rock is found as fluorapatite $\mathrm{CaF}_{2}\left(\mathrm{PO}_{4}\right)_{6}$, which is a stable compound that can not be easily supplied as a plant nutrient. In order to obtain available phosphorus it is necessary to destroy the fluorapatite structure of the phosphate rock and to form soluble compounds in the soil solutions. This may be accomplished by acid treatment or by thermal treatment. The treatment of the phosphate rock with acids such as sulfuric or phosphoric acid is commonly used for the production of normal or triple superphosphates [1]. The phosphate rock composition changes from one deposit to another. Therefore, phosphate rock from different sources is expected to behave differently in acidulation processes, which are the basis of the phosphate fertilizer industry. The phosphatic formations in Mazıdağ 1 were investigated. It was found that these phosphorite deposits occurred as a result of the following steps: erosion of calcitized deposits, redeposition in a phosphatizing environment, phosphatization of this detritus material and weathering causing an enrichment of the ores [2]. The Mazıdağı phosphate rock mainly consists of calcite, fluorapatite and carbonate-fluorapatite [3].

Acidic dissolution of natural phosphate rocks is a basic process in the production of inorganic phosphorus fertilizers, such as superphosphate (mono and double), wet-process phosphoric acid (the main semiproduct for subsequent fabrication of various kinds of inorganic fertilizers, e.g., ammonium phosphate or amorphous), nitroamorphous, and other complex and mixed fertilizers $[4,5]$. The phosphate ores used in wet

\footnotetext{
* To whom all correspondence should be sent:

E-mail: melikesinirkaya@gmail.com
}

processes today are mainly sedimentary phosphates or francolites. They are mainly produced by digestion of phosphate ore (fluorapatite) with sulfuric acid. This yields phosphoric acid as a product and hydrated calcium sulfate as a byproduct. The ore also contains traces of heavy metal ions, the most worrisome of which are cadmium and radium. They can cause serious environmental problems. When a mixture of phosphoric acid and sulfuric acid is used for the digestion of the ore, two processes occur more or less simultaneously: digestion of the ore and precipitation of calcium sulfate hemihydrate. At high sulfate concentrations, the calcium sulfate tends to precipitate not only upon the hemihydrate crystals but also upon the ore particles. Such a coating is often called blinding [6].

Ultrasound has been successfully used to enhance the rates of mass transfer and reaction in several homogeneous $[7,8]$ and heterogeneous $[9,10]$ media. The true chemical effects of ultrasound are attributed to the implosive collapse of microbubbles which are formed during the rarefaction, or negative pressure period, of sound waves. The implosive collapse of microbubbles, typically referred to as cavitation, results in a variety of mechanical effects as well. When solid particles are in the vicinity of the cavitation bubble, the implosion may occur symmetrically, depending on the proximity of the solids. Symmetric cavitations create shock waves which propagate to the surrounding solid causing microscopic turbulence and thinning of the solid-liquid film. This phenomenon is called microstreaming and is believed to be responsible for increasing the mass transfer rate of the reactants and products through the film [11]. 
M. Sinırkaya: Effect of ultrasound on the dissolution of Mardin-Mazıdağ (Turkey) phosphate ore in dilute $\mathrm{H}_{3} \mathrm{PO}_{4} \ldots$

Table 1. Analysis of the phosphate rock samples (wt\%)

\begin{tabular}{|ccccc|}
\hline Components & $710-1000 \mu \mathrm{m}$ & $500-710 \mu \mathrm{m}$ & $355-500 \mu \mathrm{m}$ & $250-355 \mu \mathrm{m}$ \\
\hline $\mathrm{CaO}$ & 50.50 & 50.27 & 50.18 & 50.36 \\
\hline $\mathrm{P}_{2} \mathrm{O}_{5}$ & 22.34 & 23.27 & 24.53 & 25.09 \\
\hline $\mathrm{MgO}$ & 1.59 & 1.6 & 1.59 & 1.62 \\
\hline $\mathrm{Fe}_{2} \mathrm{O}_{3}$ & 0.01 & 0.03 & 0.04 & 0.06 \\
\hline $\mathrm{Al}_{2} \mathrm{O}_{3}$ & 1.14 & 1.13 & 1.13 & 1.14 \\
\hline $\mathrm{SiO}_{2}$ & 4.26 & 4.05 & 3.34 & 2.86 \\
\hline $\mathrm{F} 2$ & 3.28 & 3.6 & 3.77 & 3.85 \\
\hline Loss on & 15.41 & 14.85 & 13.86 & 13.09 \\
ignition $\left(\mathrm{CO}_{2}\right)$ & $(12.06)$ & $(11.23)$ & $(8.57)$ & $(6.98)$ \\
\hline
\end{tabular}

Zhu et al. discovered that ultrasound could improve gold extraction from refractory gold ores by reducing the diffusion resistance and enhance the gold leaching rate [12]. Zhang et al. observed that the leaching rates of $\mathrm{Sb}$ and $\mathrm{Pb}$ in lead-rich and antimony-rich oxidizing slag were significantly improved by using ultrasound radiation and the reaction time was sharply shortened [13]. Avvaru et al. [14] found that the ultrasound clearly improved the uranium leaching rate in nitric acid and sulfuric acid solutions. Shen et al. [15] employed hightemperature ultrasonic acid leaching to improve the extraction of indium from blast furnace sludge compared with that without ultrasonic assistance. They found that ultrasound had a positive effect on the rate of leaching. The purpose of this work was to investigate the effect of ultrasonic energy on the dissolution of phosphate ore in dilute $\mathrm{H}_{3} \mathrm{PO}_{4}$ solutions.

$$
\begin{aligned}
& \mathrm{C}_{\mathrm{a} 10}\left(\mathrm{PO}_{4}\right)_{6} \mathrm{~F}_{2}+4 \mathrm{H}_{3} \mathrm{PO}_{4} \rightarrow 10 \mathrm{CaHPO}_{4}+2 \mathrm{HF} \\
& \mathrm{Ca}_{10}\left(\mathrm{PO}_{4}\right)_{6} \mathrm{~F}_{2}+14 \mathrm{H}_{3} \mathrm{PO}_{4} \rightarrow 10 \mathrm{Ca}\left(\mathrm{H}_{2} \mathrm{PO}_{4}\right)_{2}+2 \mathrm{HF}
\end{aligned}
$$

\section{EXPERIMENTAL PROCEDURE}

Phosphate rock samples were obtained from Mardin-Mazıdag1 phosphate rock concentration plant, Turkey. The sample was crushed, ground, and then sieved to give 250-355, 355-500, 500710 and $710-1000 \mu \mathrm{m}$ size fractions using ASTM standard sieves. The chemical analysis of the phosphate rock was carried out by standard gravimetric, volumetric, and spectrometric methods, and the results of chemical analysis are given in Table 1.

The dissolution experiments were carried out with phosphate samples having particle sizes of 500-710 $\mu \mathrm{m}$. The set-up for dissolution experiments is shown in Fig. 1. It consists of an ultrasonic generator (Cole Parmer, Ultrasonic homogenizer, $400 \mathrm{~W}, 20 \mathrm{kHz}$ ), a probe with tip radius of $1 \mathrm{~cm}$ and a thermocouple. The probe was covered with a Teflon band for hindering the probable corrosion of the probe in the $\mathrm{H}_{3} \mathrm{PO}_{4}$. Five hundred $\mathrm{ml}$ of phosphoric acid solution prepared from different concentrations of chemically pure phosphoric acid (85 mass $\% \mathrm{H}_{3} \mathrm{PO}_{4}$ ) and distilled water were placed in a batch reactor and heated to the desired temperature. For each run, $5 \mathrm{~g}$ of phosphate sample was quickly transferred into the reactor. A 3-ml sample was withdrawn from the reactor at predetermined time intervals and was filtered immediately. The $\mathrm{CaO}$ content of the filtrate was analyzed by volumetric titration [16].

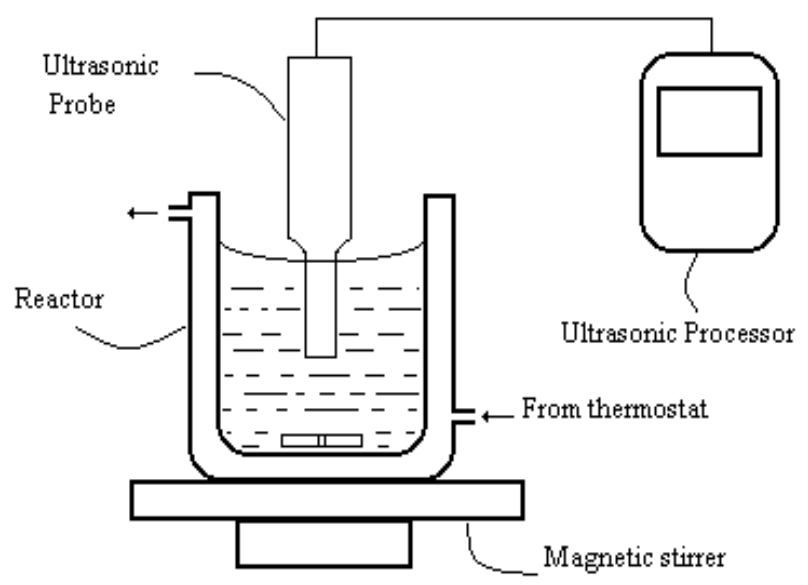

Figure 1. Scheme of the experimental set-up for dissolution.

\section{RESULTS AND DISCUSSION}

In this study, the effects of the temperature and phosphoric acid concentration on the dissolution rate were investigated in both the presence and absence of ultrasound. Experiments were performed for the concentrations of $0.1 \mathrm{M}, 0.25 \mathrm{M}$ and $0.5 \mathrm{M}$ at reaction temperatures of 30,50 and $75^{\circ} \mathrm{C}$, a stirring speed of $800 \mathrm{rpm}$ and a particle size of $500-710 \mu \mathrm{m}$. The results of the experiments in the absence of ultrasound are shown in Figs. 2, 4 and 6.

It is seen that the dissolved fraction decreased with increasing temperature, as shown in Figs. 2 and 4. The phosphoric acid used for the digestion is a recycled product acid and $\mathrm{CaHPO}_{4}$ forms at a low acid concentration. This layer is stronger at high temperatures $[17,18]$. That is why the lowest $\mathrm{CaO}$ conversion in absence of ultrasound was obtained at 
M. Sinırkaya: Effect of ultrasound on the dissolution of Mardin-Mazıdağ (Turkey) phosphate ore in dilute $\mathrm{H}_{3} \mathrm{PO}_{4} \ldots$

$75^{\circ} \mathrm{C}$ and this ratio was found to be $40.75 \%$ in 0.1 $\mathrm{M}$ phosphoric acid solution for 30 min reaction time, as shown in Fig 2.

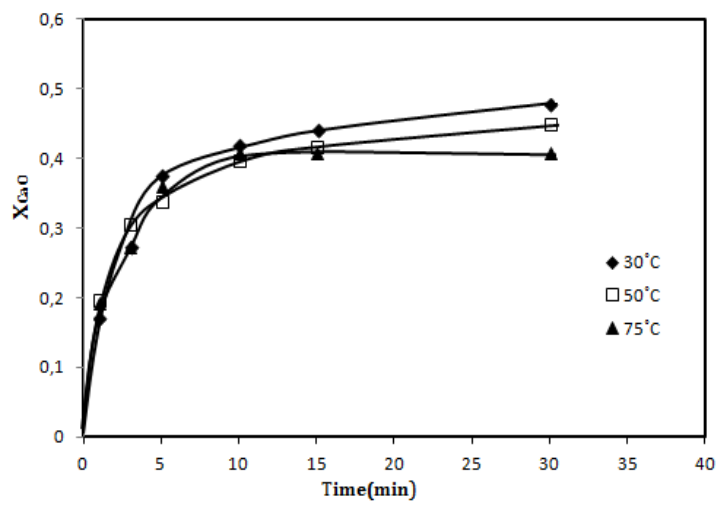

Figure 2. Effect of reaction temperature on the dissolution of $\mathrm{CaO}$ in $0.1 \mathrm{M}\left(0.71\right.$ wt $\left.\% \mathrm{P}_{2} \mathrm{O}_{5}\right)$ phosphoric acid in the absence of ultrasound.

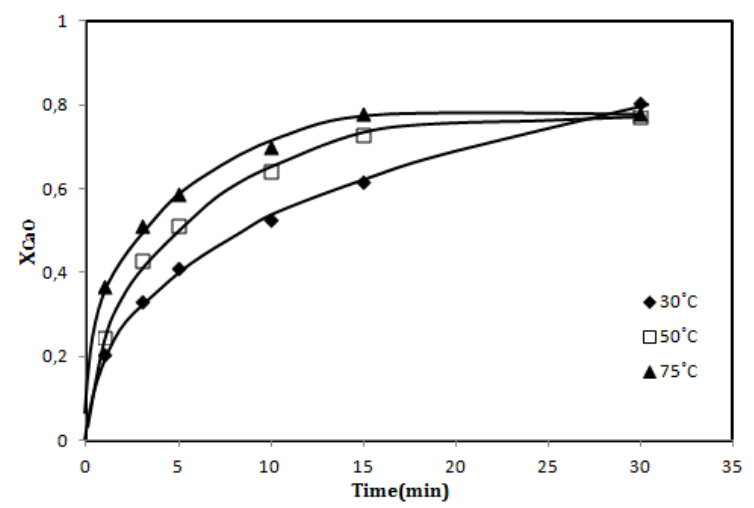

Figure 4. Effect of reaction temperature on the dissolution of $\mathrm{CaO}$ in $0.25 \mathrm{M}\left(1.76\right.$ wt $\left.\% \mathrm{P}_{2} \mathrm{O}_{5}\right)$ phosphoric acid in the absence of ultrasound.

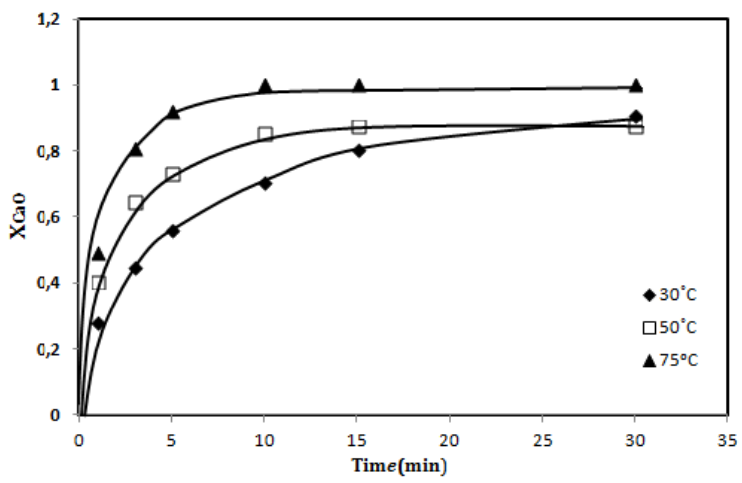

Figure 6. Effect of reaction temperature on the dissolution of $\mathrm{CaO}$ in $0.5 \mathrm{M}\left(3.97\right.$ wt $\left.\% \mathrm{P}_{2} \mathrm{O}_{5}\right)$ phosphoric acid in the absence of ultrasound.

The solubility increased with increasing acid concentration. As the concentration of $\mathrm{H}_{3} \mathrm{PO}_{4}$ increased, the dissolved fraction of $\mathrm{CaO}$ increased in both the absence and presence of ultrasound. As the concentration of $\mathrm{H}_{3} \mathrm{PO}_{4}$ increased, the migration of $\mathrm{Ca}^{+2}$ ions from the ash film $\left(\mathrm{CaHPO}_{4}\right)$ to the liquid medium increased.
The experimental results with ultrasound are shown in Figs. 3, 5 and 7. In these experiments the solubility increased with increasing reaction temperature.

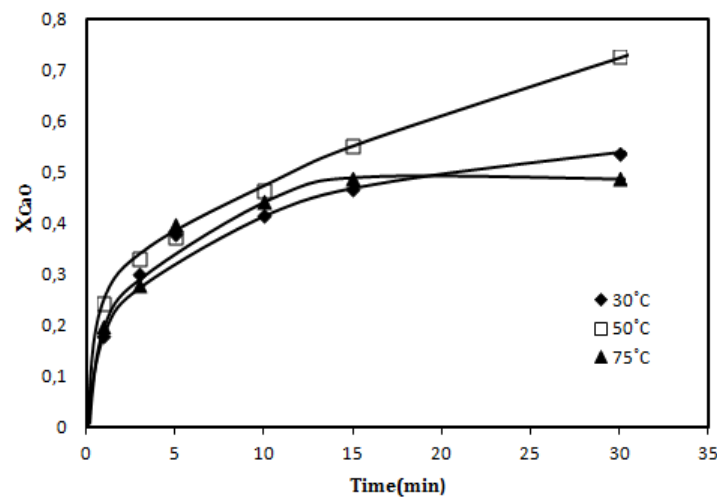

Figure 3. Effect of reaction temperature on the dissolution of $\mathrm{CaO}$ in $0.1 \mathrm{M}\left(0.71 \mathrm{wt} \% \mathrm{P}_{2} \mathrm{O}_{5}\right)$ phosphoric acid in the presence of ultrasound.

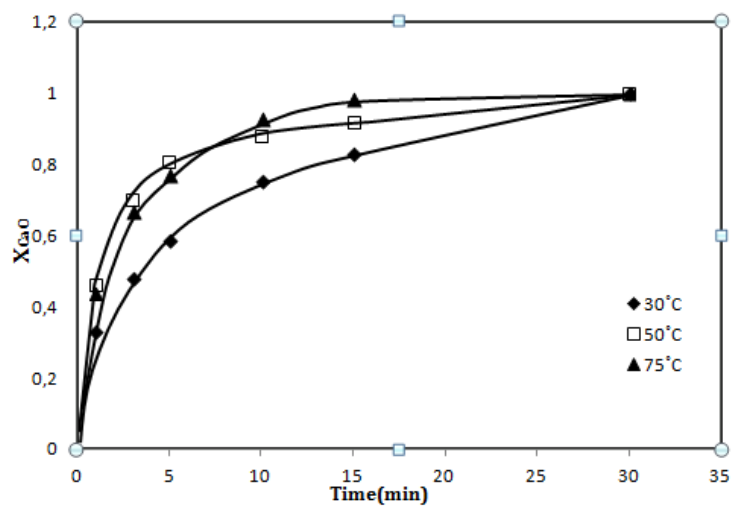

Figure 5. Effect of reaction temperature on the dissolution of $\mathrm{CaO}$ in $0.25 \mathrm{M}\left(1.76\right.$ wt $\left.\% \mathrm{P}_{2} \mathrm{O}_{5}\right)$ phosphoric acid in the presence of ultrasound.

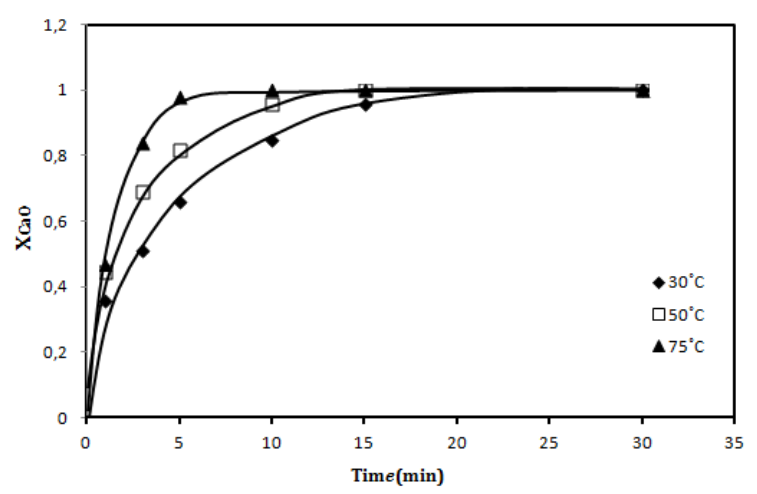

Figure 7. Effect of reaction temperature on the dissolution of $\mathrm{CaO}$ in $0.5 \mathrm{M}\left(3.97\right.$ wt $\left.\% \mathrm{P}_{2} \mathrm{O}_{5}\right)$ phosphoric acid in the presence of ultrasound.

The dissolved fraction of $\mathrm{CaO}$ in presence of ultrasound increased because the ash layer thickness $\left(\mathrm{CaHPO}_{4}\right)$, which reduces the dissolution rate, decreased with increasing time. Hagenson and Doraiswamy observed that ultrasound could enhance the rates of mass transfer and reaction in heterogeneous systems, since ultrasound caused a 
M. Sinırkaya: Effect of ultrasound on the dissolution of Mardin-Mazıdağ (Turkey) phosphate ore in dilute $\mathrm{H}_{3} \mathrm{PO}_{4} \ldots$ decrease in the diffusion layer thickness on the solid-liquid interfaces in the suspension [11]. As shown in Figure 2, the dissolution rate of $\mathrm{CaO}$ was $45.04 \mathrm{wt} \%$ for $30 \mathrm{~min}$ at $50^{\circ} \mathrm{C}$ in the absence of ultrasound, whereas, this rate was $72.54 \mathrm{wt} \%$ at the same conditions in the presence of ultrasound (Fig. $3)$.

\section{CONCLUSIONS}

In this study, the dissolution kinetics of phosphate ore from Mardin-Mazıdaği, Turkey, in dilute phosphoric acid solutions was investigated in both the presence and absence of ultrasound. The precipitation of $\mathrm{CaHPO}_{4}$ on the mineral surface created difficulty for $\mathrm{H}_{3} \mathrm{O}^{+}$to diffuse to the unreacted mineral surface in absence of ultrasound. According to the results of the study, the use of ultrasound increased the conversion fraction.

This result can be attributed to the reduction of the liquid film thickness around the solid particle, due to the increase in the velocity of the solid relative to the liquid velocity. The application of ultrasound has affected the controlling step of the dissolution. Our opinion is that ultrasound has enhanced the attack of $\mathrm{H}_{3} \mathrm{O}^{+}$on cationic sites on the rock which facilitates the migration of $\mathrm{Ca}^{+2}$ ions to the liquid medium.

Phosphoric acid is used for fertilizer applications. To minimize environmental pollution by heavy metals, a new process was being designed. One of the important steps in this process is the complete digestion of phosphate ore in phosphoric acid.

Phosphoric acid is a major component of many fertilizers. Since the digestion process is dominated by the diffusion of calcium ions from the surface of the ore into the bulk of the solution, no influence of impurities present in the process acid is expected. This was confirmed by using real product acid where the impurities did not hamper the digestion. Only if the impurities present in the acid (like excess sulfate ions) give rise to blinding of the ore particles can a reduction of the digestion process be expected [19]. In this study, a fast complete digestion of phosphate ore was achieved with dilute phosphoric acid solutions in presence of ultrasound at lower concentrations.

As a result, in the light of these observations, ultrasound would be a helpful tool for phosphoric acid production because the dissolution experiments performed in the presence of ultrasound needs much less phosphoric acid. Thus, the utization of ultrasound in phosphoric acid production as a product and calcium hydrogen phosphate as a byproduct not only decreases the acid consumption but also gives a useful product, which helps to eliminate environmental damages.

\section{REFERENCES}

1. G. L. Bridger, D.R. Boylan, Industrial and Engineering Chemistry, 45, 646 (1953).

2. J. Naktiyok, A. K. Özer, M.Çelik, M. Ş. Gülaboğlu, J. Chem. Soc. Pak., 35, 1138 (2013).

3. A. K. Özer, M. Ş. Gülaboğlu, S. Bayrakçeken, W. Weisweiler, Advanced Powder Technology, 17, 481 (2006).

4. W. R. Hamilton, A. R. Wooley, A. C. Bishop, Minerals, Rocks and Fossils, 4th ed., Country Life Books, Twickenham, Middlesex, England, 1987.

5. M. M. Seckler, O.S.L. Bruinsma, G.M. van Rosmalen, Water Res., 30, 1585 (1996).

6. P. Becker, Phosphate and Phosphoric Acid, Fertiliser Science and Technology Series, Marcel Dekker, New York, 1983.

7. J. Berlan, F. Trabelsi, H. Delmas, Ultrasonics Sonochem., 1, 97 (1994).

8. G. Cum, R. Gallo, A. Spadaro, J. Chem. Soc. Perkin Trans., 11, 375 (1998).

9. M.S.F. Lie Ken Jie, C.K. Lam, Ultrasonics Sonochem., 2, 11 (1972).

10. J.L. Lunche, Ultrasonics Sonochem., 1, 111 (1994).

11. L.C. Hagenson, L.K. Doraiswamy, Chemical Engineering Science, 53, 131 (1998).

12. P. Zhu, X.J. Zhang, K.F. Li, G.R. Qian, Int. J. Miner. Metall. Mater., 19, 473 (2012).

13. R. Zhang, X. Zhang, S. Tang, A. Huang, Ultrason. Sonochem., 27, 187 (2015).

14. B. Avvaru, S.B. Roy, Y. Ladola, S. Chowdhury, K.N. Hareendran, A.B. Pandit, Chem. Eng. Process., 47, 2107 (2008).

15. X.M. Shen, L.S. Li, Z.J. Wu, H.H. Lu, J. Lu, Metall. Mater. Trans. B, 44, 1324 (2013).

16. H. Gulensoy, Complexometric Titrations, Istanbul Univ. Pub., İstanbul, 1977.

17. K. L. Elmore, T.D. Farr, Ind. Eng. Chem., 32, 580 (1940).

18. M. Sinırkaya, A.K. Özer, M.S. Gulaboglu, Minerals Metallurgical processing, 27,110 (2010).

19. S. van der Sluis, Y. Meszaros, W.G.J. Marchee, H. A. Wesselingh, G. M. van Rosmalen, Ind. Eng. Chem. Res., 26, 2501 (1987). 


\title{
Thermo-diffusion effects on the magnetohydrodynamic natural convection flow of a chemically reactive Brinkman type nanofluid in a porous medium
}

\author{
G. S. Seth ${ }^{1 *}$ R. Kumar' ${ }^{1}$ R. Tripathi ${ }^{2}$ \\ ${ }^{1}$ Department of Applied Mathematics, Indian Institute of Technology (Indian School of Mines), Dhanbad-826004, India \\ ${ }^{2}$ Department of Mathematics, National Institute of Technology Jamshedpur, Jamshedpur-831014, India
}

Received May 21, 2017; Revised December 30, 2018

An investigation on the unsteady MHD natural convection heat and mass transfer flow of an electrically conducting, viscous, incompressible, chemically reactive and heat-absorbing nanofluid of Brinkman type past an exponentially accelerated moving vertical plate with ramped wall temperature and ramped surface concentration is carried out. Governing equations are non-dimensionalized and Laplace Transform Technique is used to find the exact solutions for fluid velocity, fluid temperature and species concentration. The quantities of physical interest, i.e. skin friction, rates of heat and mass transfers at the plate are also calculated. Numerical results for the velocity, temperature and species concentration of the fluid are demonstrated with the help of graphs whereas those of skin friction, rate of heat and mass transfers at the plate are displayed in tables for various flow parameters.

Keywords: Brinkman type nanofluid, Natural convection, Heat absorption, Ramped temperature, Ramped surface concentration

\section{INTRODUCTION}

The study on the boundary layer flow of a nanofluid finds numerous applications in various engineering, as well as industrial problems, viz. industrial cooling application, smart fluids, nuclear reactors, nanofluid coolant, cooling of microchips, etc. Nanofluids have higher thermal conductivity as compared with some other fluids such as water, mineral oils, and ethylene glycol. Eastman et al. [1] observed in an experiment that when $\mathrm{CuO}$ nanoparticles are added to the base fluid having volume fraction of $5 \%$, the thermal conductivity of the base fluid (water) increased up to $60 \%$. He indicated that this enhancement is because of increasing surface area of the base fluid due to suspension of nanoparticles. Choi et al. [2] noticed that when carbon nanotubes are added to ethylene glycol or oil, there is $150 \%$ increment in the thermal conductivity. Makinde and Aziz [3] have made an investigation of the convective boundary layer flow and heat transfer of nanofluids past a linearly stretching sheet considering effects of thermophoresis and Brownian motion. A numerical investigation for natural convection flow and heat transfer of nanofluids in a vertical rectangular duct was demonstrated by Umavathi et al. [4] considering the Darcy-Forchheimer Brinkman model. Hayat et al. [5] described the effect of Marangoni convection in the flow of a carbonwater nanofluid taking thermal radiation into account.

The study of magnetohydrodynamic (MHD)

\footnotetext{
* To whom all correspondence should be sent:

E-mail: gsseth.ism@gmail.com

flow has essential applications in physics, chemistry and engineering. Industrial equipment, such as magnetohydrodynamic (MHD) generators, pumps and bearings are affected by the interaction between the electrically conducting fluid and a magnetic field. Many researchers have studied the behavior of incompressible, viscous and electrically conducting nanofluids, such as water mixed with a little acid and other ingredients in the presence of a magnetic field, past a moving surface or a stretching sheet in a quiescent fluid. Hamad and Pop [6] have presented the unsteady MHD free convection flow of a nanofluid past a vertical permeable flat plate in a rotating frame of reference with constant heat source. Effect of magnetic field on free convection flow of a nanofluid past a vertical semi-infinite flat plate was studied by Hamad et al. [7]. Sheikholeslami et al. [8] analysed a simulation of MHD $\mathrm{CuO}$-water nanofluid flow taking into account the Lorentz force. Sheikholeslami et al. [9] have described the MHD free convection heat transfer nanofluid flow using Lattice Boltzmann method. Mehrez et al. [10] studied hydromagnetic effects on heat transfer for a nanofluid flow within an open cavity. Recently, Sheikholeslami [11] revealed the effect of Lorentz force on nanofluid flow in a porous cylinder taking Darcy model into the account.

A chemical reaction is the process by means of which a set of chemical substances are transformed into another. Moreover, the process of chemical reaction takes place between the fluid and a foreign mass. Study of mass transfer flow taking chemical reaction into account has found numerous 
G. S. Seth et al.: Thermo-diffusion effects on the magnetohydrodynamic natural convection flow ...

important applications in several chemical and hydrometallurgical industries such as catalytic chemical reactors, production of glassware and ceramics, food processing, undergoing endothermic or exothermic chemical reaction, etc. Chamkha [12] presented an analysis of MHD flow for uniformly stretched vertical permeable surface in presence of chemical reaction and heat generation/absorption. Afify [13] discussed the impact of heat radiation on the natural convection flow of a chemically reactive fluid past an isothermal vertical cone surface. Interesting results regarding chemical reaction are described in the research works of Muthucumaraswamy et al. [14], Ibrahim et al. [15], Rashad et al. [16] and Bhattacharya and Layek [17].

When heat and mass transfer take place simultaneously in a moving fluid, the relations between the fluxes and driving potentials become more intricate in nature. Mass fluxes can also be created by temperature gradients and this results in a thermal - diffusion (Soret) effect. In most of the studies related to heat and mass transfer processes, Soret effect is neglected on the ground that it is of smaller order of magnitude than the effect described by Ficks law. But this effect is considered as a second-order phenomenon and may become significant in areas such as hydrology, petrology, geosciences, etc. Mabood et al. [18] studied the Soret effect on MHD non-Darcian convective flow past a stretching sheet in a micropolar fluid with radiation. Effects of Hall current and rotation along with Soret effect on hydromagnetic free convection heat and mass transfer flow in a porous medium past an accelerated vertical plate were described by Sarma and Pandit [19]. Recently, Zueco et al. [20] analysed a two-dimensional free convective Newtonian Hartmann flow with thermal diffusion and Soret effects. Relevant study regarding Soret effect is published by Seth et al. [21].

Natural convection flows are generally modelled by the researchers under the assumptions of uniform surface temperature or constant surface heat flux. But in many physical situations, the temperature of the bounding surface may require non-uniform or arbitrary wall conditions. Moreover, there may occur step discontinuities in the surface temperature. In recent years, several researchers investigated unsteady hydromagnetic free convection flow past a vertical plate with ramped temperature considering different aspects of the problem. Some of the relevant research studies are performed by Rajesh and Chamkha [22], Kundu et al. [23], Seth and Sarkar [24], Seth et al. [25] and Hussain et al. [26].
Purpose of the present investigation is the study of an unsteady hydromagnetic natural convection heat and mass transfer flow of an electrically conducting, viscous, incompressible, chemically reactive and heat absorbing nanofluid past an exponentially accelerated moving vertical plate with ramped wall temperature and ramped surface concentration.

\section{MATHEMATICAL FORMULATION}

Let us consider unsteady MHD free convection flow of an electrically conducting, incompressible, viscous, heat generating/absorbing, chemically reactive nanofluid of Brinkman type, past a vertical plate embedded in a fluid-saturated porous medium. Cartesian co-ordinate system is chosen in such a fashion that the length of the plate is taken along the $x$ axis which is perpendicular to the $y$ axis. The $z$ axis is taken in a direction normal to the $x-y$ plane. The flow region is exposed to a uniform magnetic field of intensity $B_{0}$ applied in a direction parallel to the $y$ axis. Initially, i.e. at $t^{\prime} \leq 0$, the plate is at rest, the temperature and concentration of species are maintained at constant values $T_{\infty}$ and $C_{\infty}^{\prime}$, throughout the flow region. At time $t^{\prime}>0$, the plate is accelerated exponentially with the velocity $U_{0} e^{a^{\prime} t^{\prime}}$ in the $x$-direction. The flow is solely induced due to the movement of the plate in $x$-direction. The temperature and concentration of the species are raised or lowered to $T_{\infty}+\left(T_{w}-T_{\infty}\right) t^{\prime} / t_{0}$ and $C_{\infty}^{\prime}+\left(C_{w}^{\prime}-C_{\infty}^{\prime}\right) t^{\prime} / t_{0}$, respectively, for $0<t^{\prime} \leq t_{0}$. Thereafter, i.e. for $t^{\prime}>t_{0}$, the plate is maintained at uniform temperature $T_{w}$ and the level of concentration at the surface of the plate is maintained at uniform concentration $C_{w}^{\prime}$. It is assumed that a homogenous chemical reaction of first order exists among the species. Fluid is considered to be a metallic liquid or partially ionized gas having a very small magnetic Reynolds number. Hence, the magnetic field which is induced due to fluid motion is negligible in comparison to the applied one. The existence of a flux in species concentration due to a gradient in the fluid temperature is also considered. Usually this effect is important where more than one chemical species are present under a very large temperature gradient. The schematic diagram of the physical model of the problem is shown in Figure 1.

In view of the above assumptions, the governing equations for unsteady hydromagnetic natural convection flow of a heat generating/absorbing and chemically reactive nanofluid of Brinkman type 
G. S. Seth et al.: Thermo-diffusion effects on the magnetohydrodynamic natural convection flow ...

with the consideration of Soret effect, in a fluidsaturated porous medium are presented as:

$$
\begin{aligned}
& \rho_{n f}\left(\frac{\partial u_{1}}{\partial t^{\prime}}+\beta u_{1}\right)=\mu_{n f} \frac{\partial^{2} u_{1}}{\partial y^{2}}-\left(\sigma_{n f} B_{0}^{2}+\frac{\mu_{n f} \psi}{k}\right) u_{1} \\
& +g\left(\rho \beta_{T}\right)_{n f}\left(T-T_{\infty}\right)+g\left(\rho \beta_{C}\right)_{n f}\left(C^{\prime}-C_{\infty}^{\prime}\right), \\
& \left(\rho c_{p}\right)_{n f} \frac{\partial T}{\partial t^{\prime}}=k_{n f} \frac{\partial^{2} T}{\partial y^{2}}-Q\left(T-T_{\infty}\right), \\
& \frac{\partial C^{\prime}}{\partial t^{\prime}}=D_{n f} \frac{\partial^{2} C^{\prime}}{\partial y^{2}}-k_{2}\left(C^{\prime}-C_{\infty}^{\prime}\right)+\frac{D_{m} k_{t}}{T_{m}} \frac{\partial^{2} T}{\partial y^{2}},
\end{aligned}
$$

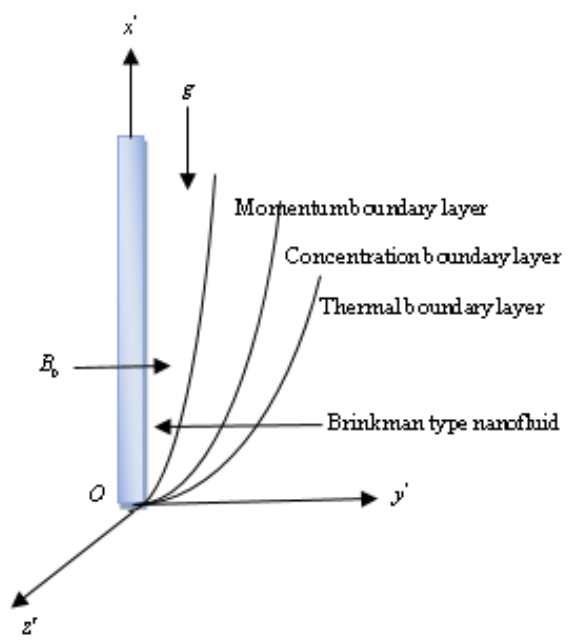

Fig. 1. Physical model of the problem

The corresponding initial and boundary conditions are presented as:

$$
\begin{aligned}
& u_{1}=0, T=T_{\infty}, C^{\prime}=C_{\infty}^{\prime} \text { for } y>0 \text { and } t^{\prime} \leq 0, \\
& u_{1}=U_{0} e^{a^{\prime} t^{\prime}} \text { at } y=0 \text { for } t^{\prime}>0, \\
& T= \begin{cases}T_{\infty}+\left(T_{w}-T_{\infty}\right) t^{\prime} / t_{0} \text { at } y=0 \text { for } 0<t^{\prime} \leq t_{0}, \\
T_{w} & \text { at } y=0 \text { for } t^{\prime}>t_{0},\end{cases} \\
& C^{\prime}= \begin{cases}C_{\infty}^{\prime}+\left(C_{w}^{\prime}-C_{\infty}^{\prime}\right) t^{\prime} / t_{0} & \text { at } y=0 \text { for } 0<t^{\prime} \leq t_{0}, \\
C_{w}^{\prime} & \text { at } y=0 \text { for } t^{\prime}>t_{0},\end{cases} \\
& u_{1} \rightarrow 0, T \rightarrow T_{\infty}, C^{\prime} \rightarrow C_{\infty}^{\prime} \text { as } y \rightarrow \infty \text { for } t^{\prime}>0
\end{aligned}
$$

where $\quad u_{1}, T, C^{\prime}, \rho_{n f}, \mu_{n f}, \sigma_{n f},\left(\beta_{T}\right)_{n f}$, $\left(\beta_{C}\right)_{n f}, g, \psi, \beta,\left(\rho c_{p}\right)_{n f}, k_{n f}, D_{n f}$ and $k_{2} \quad$ are, respectively, fluid velocity along $x$ - direction, nanofluid temperature, nanofluid concentration, density of the nanofluids, dynamic viscosity of nanofluids, electrical conductivity of nanofluids, thermal expansion coefficient, coefficient of volumetric expansion, acceleration due to gravity, porosity of porous medium, material parameter of Brinkman type fluid, specific heat capacity of the nanofluids, thermal conductivity of nanofluids, mass diffusivity and chemical reaction coefficient.

For nanofluids, the expressions of $\mu_{n f},\left(\rho c_{p}\right)_{n f}$, $\rho_{n f},\left(\rho \beta_{T}\right)_{n f},\left(\rho \beta_{C}\right)_{n f}, \sigma_{n f} / \sigma_{f}$ are defined as: $\mu_{n f}=\frac{\mu_{f}}{(1-\varphi)^{2.5}},\left(\rho c_{p}\right)_{n f}=(1-\varphi)\left(\rho c_{p}\right)_{f}+\varphi\left(\rho c_{p}\right)_{s}$,

$\rho_{n f}=(1-\varphi) \rho_{f}+\varphi \rho_{s}$,

$\left(\rho \beta_{T}\right)_{n f}=(1-\varphi)\left(\rho \beta_{T}\right)_{f}+\varphi\left(\rho \beta_{T}\right)_{s}$,

$\frac{\sigma_{n f}}{\sigma_{f}}=1+\frac{3(\sigma-1) \varphi}{(\sigma+2)-(\sigma-1) \varphi}$,

where $\varphi$ is the solid volume fraction of the nanoparticles, $\rho_{f}$ is density of the base fluid, $\rho_{s}$ is density of the nanoparticles, $\sigma_{f}$ is electrical conductivity of the base fluid, $\sigma_{s}$ is electrical conductivity of the nanoparticles, $\mu_{f}$ is viscosity of the base fluid, $\left(\rho c_{p}\right)_{f}$ is heat capacitance of the base fluid and $\left(\rho c_{p}\right)_{s}$ is heat capacitance of the nanoparticles. The effective thermal conductivity (Oztop and Abu-Nada [27]) is given as:

$$
\frac{k_{n f}}{k_{f}}=\frac{k_{s}+2 k_{f}-2 \varphi\left(k_{f}-k_{s}\right)}{k_{s}+2 k_{f}+\varphi\left(k_{f}-k_{s}\right)},
$$

where $k_{f}$ is thermal conductivity of the base fluid and $k_{s}$ is thermal conductivity of the nanoparticles.

The governing equations (1) to (3) along with initial and boundary conditions (4a) to (4e) are presented in dimensional form. In order to nondimensionalize these equations and conditions, following non-dimensional variables and parameters are introduced:

$$
\begin{aligned}
& u=\frac{u_{1}}{U_{0}}, \eta=\frac{U_{0} y}{v_{f}}, t=\frac{U_{0}^{2} t^{\prime}}{v_{f}}, a=\frac{a^{\prime} v_{f}}{U_{0}^{2}}, \\
& \theta=\frac{T-T_{\infty}}{T_{w}-T_{\infty}}, C=\frac{C^{\prime}-C_{\infty}^{\prime}}{C_{w}^{\prime}-C_{\infty}^{\prime}}, \\
& M^{2}=\frac{y_{5} v_{f} \sigma_{f} B_{0}^{2}}{\rho_{n f} U_{0}^{2}}, \beta_{1}=\frac{\beta v_{f}}{U_{0}^{2}}, K=\frac{k U_{0}^{2}}{v_{n f} \psi v_{f}}, \\
& \operatorname{Pr}=\frac{\left(\mu c_{p}\right)_{f}}{k_{f}}, \mathrm{Gr}=\frac{v_{f} g \beta_{T f}}{U_{0}^{3}}\left(T_{w}-T_{\infty}\right) \text {, } \\
& \mathrm{Gm}=\frac{v_{f} g \beta_{C f}}{U_{0}^{3}}\left(C_{w}^{\prime}-C_{\infty}^{\prime}\right), \mathrm{S}_{c}=\frac{v_{f}}{D_{n f}}, \\
& \gamma=\frac{k_{2} v_{f}}{U_{0}^{2}}, S_{r}=\frac{D_{m} k_{t}}{T_{m} v_{f}} \frac{\left(T_{w}-T_{\infty}\right)}{\left(C_{w}^{\prime}-C_{\infty}^{\prime}\right)} .
\end{aligned}
$$


G. S. Seth et al.: Thermo-diffusion effects on the magnetohydrodynamic natural convection flow ...

Using equation (6), equations (1) to (3) are converted into non-dimensional forms which are given as:

$\frac{\partial u}{\partial t}=\frac{1}{\operatorname{Re}} \frac{\partial^{2} u}{\partial \eta^{2}}-K u+\mathrm{Gr}_{0} \theta+\mathrm{Gm}_{0} C$,

$\frac{\partial \theta}{\partial t}=\frac{1}{b_{0}} \frac{\partial^{2} \theta}{\partial \eta^{2}}-Q_{1} \theta$

$\frac{\partial C}{\partial t}=\frac{1}{\mathrm{~S}_{c}} \frac{\partial^{2} C}{\partial \eta^{2}}-\gamma C+\mathrm{S}_{r} \frac{\partial^{2} \theta}{\partial \eta^{2}}$,

where $M^{2}, \beta_{1}, K, \mathrm{Pr}, \mathrm{Gr}, \mathrm{Gm}, \mathrm{S}_{c}, \gamma$ and $\mathrm{S}_{r}$ are, respectively, magnetic field parameter, dimensionless Brinkman parameter, permeability of porous medium, Prandtl number, thermal Grashof number, solutal Grashof number, Schmidt number, chemical reaction parameter and Soret number. The other parameters and variables appearing in equations (7) to (9) are given by: $\operatorname{Re}=(1-\varphi)^{2.5} y_{1}, \lambda_{n f}=\frac{k_{n f}}{k_{f}}, \mathrm{Gr}_{0}=y_{3} \mathrm{Gr}, \mathrm{Gm}_{0}=y_{4} \mathrm{Gm}$, $b_{0}=\frac{\operatorname{Pr} y_{6}}{\lambda_{n f}}, Q_{1}=\frac{Q}{\left(\rho c_{p}\right)_{n f}} \frac{v_{f}}{U_{0}^{2}}, y_{2}=M^{2}+\frac{1}{K}+\beta_{1}$,

$y_{1}=\left[(1-\varphi)+\varphi\left(\frac{\rho_{s}}{\rho_{f}}\right)\right], y_{3}=\frac{(1-\varphi) \rho_{f}-\varphi \rho_{s}\left(\frac{\beta_{T s}}{\beta_{T f}}\right)}{\rho_{n f}}$,

$y_{4}=\frac{(1-\varphi) \rho_{f}-\varphi \rho_{s}\left(\frac{\beta_{C s}}{\beta_{C f}}\right)}{\rho_{n f}}$,

$y_{5}=1+\frac{3(\sigma-1) \varphi}{(\sigma+2)-(\sigma-1) \varphi}, y_{6}=(1-\varphi)+\varphi \frac{\left(\rho c_{p}\right)_{s}}{\left(\rho c_{p}\right)_{f}}$.

The corresponding initial and boundary conditions in non-dimensional form are given as:

$u=0, \theta=0, C=0$ for $\eta \geq 0$ and $\mathrm{t} \leq 0$,

$u=e^{a t} \quad$ at $\eta=0$ for $\mathrm{t}>0$,

$\theta= \begin{cases}t & \text { at } \eta=0 \text { for } 0<t \leq 1, \\ 1 & \text { at } \eta=0 \text { for } t>1,\end{cases}$

$C= \begin{cases}t & \text { at } \eta=0 \text { for } 0<t \leq 1, \\ 1 & \text { at } \eta=0 \text { for } t>1,\end{cases}$

$u \rightarrow 0, \theta \rightarrow 0, C \rightarrow 0$ as $\eta \rightarrow \infty$ for $\mathrm{t}>0$. (10e)

According to above non-dimensionalization process, the characteristic time $t_{0}$ may be defined as:

$t_{0}=\frac{v_{f}}{U_{0}^{2}}$
Table 1. Thermophysical properties of nanofluids

\begin{tabular}{|c|c|c|c|c|}
\hline & $\rho\left(\mathrm{kgm}^{-3}\right)$ & $c_{p}\left(\mathrm{~kg}^{-1} \mathrm{~K}^{-1}\right)$ & $k\left(\mathrm{Wm}^{-1} \mathrm{~K}^{-1}\right)$ & $\beta \times 10^{-5}\left(\mathrm{~K}^{-1}\right)$ \\
\hline $\mathrm{Al}_{2} \mathrm{O}_{3}$ & 3970 & 765 & 40 & 0.85 \\
\hline $\mathrm{Cu}$ & 8933 & 385 & 401 & 1.67 \\
\hline $\mathrm{TiO}$ & 4250 & 686.2 & 8.9528 & 0.9 \\
\hline $\mathrm{Ag}$ & 10.500 & 235 & 429 & 1.89 \\
\hline
\end{tabular}

Equations (7) to (9) along with the initial and boundary conditions represent a system of initial and boundary value problems which can be solved by Laplace transform technique. The solutions for fluid temperature $\theta$ species concentration $C$ and fluid velocity $u$ are presented as:

$$
\begin{aligned}
\theta(\eta, t)= & P(\eta, t)-H(t-1) P(\eta, t-1), \\
C(\eta, t)= & G(\eta, t)-H(t-1) G(\eta, t-1), \\
u(\eta, t)= & e^{a t} f_{1}\left(\eta, t, \operatorname{Re}, y_{2}, a\right)+R(\eta, t) \\
& -H(t-1) R(\eta, t-1),
\end{aligned}
$$

where

$$
\begin{aligned}
& P(\eta, t)=f_{2}\left(\eta, t, b_{0}, Q_{1}\right), \\
& \begin{aligned}
& G(\eta, t)=\alpha\left(1-\frac{Q_{1}}{b_{1}}\right)\left[f_{1}\left(\eta, t, \mathrm{~S}_{c}, \gamma, 0\right)-\right. \\
& f_{1}\left(\eta, t, b_{0}, Q_{1}, 0\right)-e^{b_{1} t}\left\{f_{1}\left(\eta, t, \mathrm{~S}_{c}, \gamma,-b_{1}\right)\right. \\
&\left.\left.f_{1}\left(\eta, t, b_{0}, Q_{1},-b_{1}\right)\right\}\right]+\left(1+\alpha Q_{1}\right) f_{2}\left(\eta, t, \mathrm{~S}_{\mathrm{c}}, \gamma\right) \\
& \quad-\alpha Q_{1} f_{2}\left(\eta, t, b_{0}, Q_{1}\right), \\
& R(\eta, t)=g_{1}(\eta, t)+g_{2}(\eta, t)+g_{3}(\eta, t)-g_{4}(\eta, t) \\
&-g_{5}(\eta, t)-g_{6}(\eta, t)-g_{7}(\eta, t)+g_{8}(\eta, t),
\end{aligned}
\end{aligned}
$$

and

$$
\begin{aligned}
& \alpha=\frac{\mathrm{S}_{c} \mathrm{~S}_{r}}{\left(b_{0}-\mathrm{S}_{c}\right)} \frac{b_{0}}{b_{1}}, a_{0}=\frac{\operatorname{Re} y_{2}-b_{0} Q_{1}}{b_{0}-\operatorname{Re}}, \\
& b_{1}=\frac{b_{0} Q_{1}-\mathrm{S}_{c} \gamma}{b_{0}-\mathrm{S}_{c}}, b_{2}=\frac{\mathrm{S}_{c} \gamma-\operatorname{Re} y_{2}}{\mathrm{~S}_{c}-\operatorname{Re}}, \\
& G r_{1}=\frac{\operatorname{Re} G r_{0}}{b_{0}-\operatorname{Re}}, G m_{1}=\frac{\operatorname{Re} G m_{0}}{\mathrm{~S}_{c}-\operatorname{Re}} \\
& \text { and } G m_{2}=\frac{\operatorname{Re} G m_{0}}{b_{0}-\operatorname{Re}} .
\end{aligned}
$$

$H(t-1)$ represents Heaviside unit step function.

The expressions for $f_{1}, f_{2}, g_{1}, g_{2}, g_{3}, g_{4}$, $g_{4}, g_{5}, g_{6}, g_{7}$ and $g_{8}$ are provided in the Appendix. 
G. S. Seth et al.: Thermo-diffusion effects on the magnetohydrodynamic natural convection flow ...

Solution when fluid is in contact to isothermal plate with uniform surface concentration

Equations (12) to (14) represent the analytical solutions for fluid temperature, species concentration and fluid velocity, respectively, for an unsteady hydromagnetic free convection heat and mass transfer flow of a viscous, incompressible, electrically conducting, chemically reactive and heat generating/absorbing nanofluid of Brinkman type, past an exponentially accelerated moving vertical plate with ramped temperature and ramped surface concentration, through a porous medium. To emphasize the effect of the rampedness in temperature as well as on species concentration of the fluid, it is worthwhile to compare such flows with the one near an exponentially accelerated moving vertical plate with uniform temperature and uniform surface concentration. Accordingly, the fluid temperature, species concentration and fluid velocity take the following forms:

$$
\begin{aligned}
& \theta(\eta, t)=f_{1}\left(\eta, t, b_{0} \mathrm{Q}_{1}, 0\right), \\
& C(\eta, t)=f_{1}\left(\eta, t, \mathrm{~S}_{c}, \gamma, 0\right)+\alpha\left[\left(b_{1}-Q_{1}\right) e^{\left(-b_{1} t\right)}\right. \\
& \left.\quad f_{1}\left(\eta, t, \mathrm{~S}_{c}, \gamma,-b_{1}\right)+Q_{1} f_{1}\left(\eta, t, \mathrm{~S}_{c}, \gamma, 0\right)\right] \\
& -\alpha\left[\left(b_{1}-Q_{1}\right) e^{\left(-b_{1} t\right)} f_{1}\left(\eta, t, b_{0}, \mathrm{Q}_{1},-b_{1}\right)+\right. \\
& \left.\quad Q_{1} f_{1}\left(\eta, t, b_{0}, \mathrm{Q}_{1}, 0\right)\right] \\
& u(\eta, t)=h_{1}(\eta, t)+h_{2}(\eta, t)+h_{3}(\eta, t) \\
& \quad+h_{4}(\eta, t)-h_{5}(\eta, t)-h_{6}(\eta, t)-h_{7}(\eta, t) \\
& \quad-h_{8}(\eta, t)+h_{9}(\eta, t),
\end{aligned}
$$

The expressions for $f_{1}, f_{2}, h_{1}, h_{2}, h_{3}, h_{4}$, $h_{5}, h_{6}, h_{7}, h_{8}$ and $h_{9}$ are provided in the Appendix.

\section{Skin friction}

The expression for skin friction $\tau$ is obtained and given in the following simplified form:

For ramped temperature plate with ramped surface concentration:

$$
\begin{aligned}
& \begin{aligned}
\tau=\left.\frac{\partial u}{\partial \eta}\right|_{\eta=0}= & e^{a t} f_{3}\left(t, \operatorname{Re}, y_{2}, a\right)+R_{1}(0, t) \\
- & H(t-1) R(0, t-1),
\end{aligned} \\
& \qquad \begin{aligned}
& R_{1}(0, t)=g_{1}^{\prime}(0, t)+g_{2}^{\prime}(0, t)+g_{3}^{\prime}(0, t) \\
&-g_{4}^{\prime}(0, t)-g_{5}^{\prime}(0, t)-g_{6}^{\prime}(0, t) \\
& \text { where } \quad-g_{7}^{\prime}(0, t)+g_{8}^{\prime}(0, t),
\end{aligned}
\end{aligned}
$$

The expressions for $g_{1}^{\prime}, g_{2}^{\prime}, g_{3}^{\prime}, g_{4}^{\prime}, g_{5}^{\prime}, g_{6}^{\prime}, g_{7}^{\prime}$ and $g_{8}^{\prime}$ are provided in the Appendix.

For isothermal plate with uniform surface concentration:

$$
\begin{aligned}
\tau=\left.\frac{\partial u}{\partial \eta}\right|_{\eta=0} & =h_{1}^{\prime}(0, t)+h_{2}^{\prime}(0, t)+h_{3}^{\prime}(0, t) \\
& +h_{4}^{\prime}(0, t)-h_{5}^{\prime}(0, t)-h_{6}^{\prime}(0, t)-h_{7}^{\prime}(0, t) \\
& h_{8}^{\prime}(0, t)+h_{9}^{\prime}(0, t),
\end{aligned}
$$

where the expressions for $f_{3}, f_{4}, h_{1}^{\prime}, h_{2}^{\prime}, h_{3}^{\prime}$, $h_{4}^{\prime}, h_{5}^{\prime}, h_{6}^{\prime}, h_{7}^{\prime}, h_{8}^{\prime}$ and $h_{9}^{\prime}$ are provided in the Appendix.

\section{Rate of heat transfer at the plate}

Expression for rate of heat transfer at the plate which is denoted as $N_{u}$ (Nusselt number), is presented as:

For ramped temperature plate:

$N_{u}=\left.\frac{\partial \theta}{\partial \eta}\right|_{\eta=0}=P_{1}(0, t)-H(t-1) P_{1}(0, t-1)$,

where

$P_{1}(0, t)=f_{4}\left(t, b_{0}, Q_{1}\right)$,

For isothermal plate:

$$
N_{u}=\left.\frac{\partial \theta}{\partial \eta}\right|_{\eta=0}=f_{3}\left(t, b_{0} \mathrm{Q}_{1}, 0\right) .
$$

\section{Rate of mass transfer at the plate}

Expression for rate of mass transfer at the plate which is denoted as $S_{h}$ (Sherwood number), is presented as:

For ramped surface concentration:

$S_{h}=\left.\frac{\partial C}{\partial \eta}\right|_{\eta=0}=G_{1}(0, t)-H(t-1) G_{1}(0, t-1)$,

where

$$
\begin{aligned}
& G_{1}(0, t)=\alpha\left(1-\frac{Q_{1}}{b_{1}}\right)\left[\left\{f_{3}\left(t, \mathrm{~S}_{c}, \gamma, 0\right)\right.\right. \\
& \left.-f_{3}\left(t, b_{0}, Q_{1}, 0\right)\right\}-e^{b_{1} \tau}\left\{f_{3}\left(t, \mathrm{~S}_{c}, \gamma,-b_{1}\right)\right. \\
& \left.\left.-f_{3}\left(t, b_{0}, Q_{1},-b_{1}\right)\right\}\right]+\left(1+\alpha Q_{1}\right) f_{4}\left(t, \mathrm{~S}_{\mathrm{c}}, \gamma\right) \\
& -\alpha Q_{1} f_{4}\left(t, b_{0}, Q_{1}\right),
\end{aligned}
$$

For uniform surface concentration:

$$
\begin{array}{r}
S_{h}=\left.\frac{\partial C}{\partial \eta}\right|_{\eta=0}=f_{3}\left(t, S_{c}, \gamma, 0\right)+\alpha\left(b_{1}-Q_{1}\right) \\
e^{\left(-b_{1} t\right)} f_{3}\left(t, S_{c}, \gamma,-b_{1}\right)+\alpha Q_{1} f_{3}\left(t, S_{c}, \gamma, 0\right)
\end{array}
$$


G. S. Seth et al.: Thermo-diffusion effects on the magnetohydrodynamic natural convection flow ...

$-\alpha\left(b_{1}-Q_{1}\right) e^{\left(-b_{1} t\right)} f_{3}\left(t, b_{0}, \mathrm{Q}_{1},-b_{1}\right)$

$-\alpha Q_{1} f_{3}\left(t, b_{0}, \mathrm{Q}_{1}, 0\right)$,

\section{RESULTS AND DISCUSSION}

To understand the physics of the flow regime, the influence of various physical parameters involved in the flow-field are analysed. The numerical computations for fluid velocity, fluid temperature and species concentration together with skin friction coefficient, heat and mass transfer rates at the plate were carried out and are presented in graphical and tabular forms in Figures 2 to 12 and Tables 2 to 4 . The default values of all the governing flow parameters are selected as $M^{2}=3, \mathrm{Gr}=10, \mathrm{Gm}=4, \gamma=4, \mathrm{t}=0.5, \mathrm{~S}_{c}=0.6, \mathrm{~S}_{r}=0.3$, $Q_{1}=3$ and $\beta_{1}=0.5$.

To know the effects of Brinkman parameter $\beta_{1}$, magnetic parameter $M$, thermal Grashof number $G r$ and solutal Grashof number $G m$ on the flow-field, the numerical values of the fluid velocity $u$, computed from the solutions (14) and (17), are displayed graphically in Figures 2 to 5 for both ramped temperature plate with ramped surface concentration and isothermal plate with uniform surface concentration. It is interesting to observe that the impact of pertinent flow parameters on the fluid velocity is invariant under the thermal and solutal conditions at the plate, i.e. the characteristics of fluid velocity remain unchanged for both ramped temperature plate with ramped surface concentration and isothermal plate with uniform surface concentration. Figure 2 depicts the influence of Brinkman parameter $\beta_{1}$ on the fluid velocity $u$. It is evident from Figure 2 that $u$ is getting decreased as we increase $\beta_{1}$ throughout the boundary layer region. This is justified with the fact that $\beta_{1}$ is the ratio of drag force and density. So, an increment in the value of $\beta_{1}$ means an enhancement in drag force, which consequently, retards the fluid velocity. Figure 3 displays the behaviour of fluid velocity $u$ with respect to magnetic field parameter $M$. It is evident from Figure 3 that $u$ is getting reduced with the increment in $M$. This is due to the fact that an application of magnetic field to an electrically conducting fluid gives rise to a mechanical force, called Lorentz force, which has a tendency to resist fluid motion in the flow-field. Figures 4 and 5 portray the influence of thermal and solutal buoyancy forces on the fluid velocity. It is noted from these two figures that there is an enhancement in $u$ as we increase the values of $\mathrm{Gr}$ and $G m$ since $G r$ measures the relative strength of thermal buoyancy force to viscous force and $\mathrm{Gm}$ measures the relative strength of solutal buoyancy force to viscous force. Thus, an increase in $G r$ and $G m$ leads to an increase in thermal and solutal buoyancy forces, respectively. Therefore, thermal and solutal buoyancy forces tend to accelerate the fluid flow.

In order to analyze the influences of heat absorption parameter $Q_{1}$ and time $t$ on the temperature field $\theta$, the numerical values of fluid temperature $\theta$, computed from the solutions (12) and (15) are shown graphically versus boundary layer coordinate $\eta$ in Figures 6 and 7. It is inferred from Figure 6 that the value of $\theta$ decreases on increasing heat absorption parameter $Q_{l}$ for both ramped temperature and isothermal plates. This suggests that an increment in heat absorption results in a significant fall in fluid temperature. Again from Figure 7 one can see that for both ramped temperature and isothermal plates, fluid temperature is getting enhanced as we increase the time $t$ throughout the boundary layer region.

To study the effects of Soret number $S_{r}$, chemical reaction parameter $\gamma$, Schmidt number $S_{c}$ and time $t$ on the concentration field $C$ for both ramped and uniform surface concentrations, the numerical values of species concentration $C$, computed from the analytical solutions (13) and (16) are depicted graphically versus boundary layer coordinate $\eta$ in Figures 8 to 11 . Figure 8 reveals that with the increase of chemical reaction parameter $\gamma$, species concentration is getting reduced for both ramped and uniform surface concentrations. From Figure 9 it is observed that for both ramped and uniform surface concentrations, Schmidt number $S_{c}$ has a tendency to reduce the species concentration throughout the boundary layer region. It is revealed from Figure 10 that $C$ is getting increased with the increment in Soret number $S_{r}$ for both ramped and uniform surface concentrations. Figure 11 displays the effect of time on species concentration. It is perceived from Figure 11 that there is an increment in $C$ with the progress of time throughout the boundary layer region for both ramped and uniform surface concentrations. Comparison of velocity profile for different nanofluids is shown in Figure 12. This figure shows that $\mathrm{Al}_{2} \mathrm{O}_{3}$ water-based nanofluid has the highest velocity followed by $\mathrm{TiO}_{2}, \mathrm{Cu}$ and $\mathrm{Ag}$.

The nature of skin friction coefficient $\tau$, under the actions of Brinkman parameter $\beta_{1}$ magnetic parameter $M$ thermal Grashof number $G r$ and solutal Grashof number $\mathrm{Gm}$ is presented in Table 2. It is seen from Table 2 that $\tau$ is getting reduced on increasing $\beta_{1}$ and magnetic field parameter $M$ 
G. S. Seth et al.: Thermo-diffusion effects on the magnetohydrodynamic natural convection flow ...

whereas it is getting enhanced on increasing $G r$ and $G m$ for both ramped temperature plate with ramped surface concentration and isothermal plate with uniform surface concentration.

It is noticed from Table 3 that for ramped temperature plate, the rate of heat transfer $N_{u}$ increases on increasing the values of both $Q_{1}$ and $t$. On the other hand, in case of isothermal plate $N_{u}$ gets increased as we increase the value of $Q_{l}$ but an adverse effect is observed with the progress of time.

Numerical values of rate of mass transfer at the plate $S_{h}$ are presented in Table 4 for various values of $S_{c}, S_{r}, \gamma$ and $t$. It is observed from Table 4 that the value of $S_{h}$ increases on increasing either $S_{c}$ or $\gamma$ in both the cases, i.e. when the concentration of species at the surface of the plate has a ramped profile and when it has a uniform concentration at the surface of the plate. On the other hand, $S_{h}$ is getting decreased on increasing the value of $S_{r}$. On increasing time $t$, the value of $S_{h}$ increases when the concentration of species at the surface of the plate has a ramped profile and it decreases when species concentration at the surface of the plate is uniform.

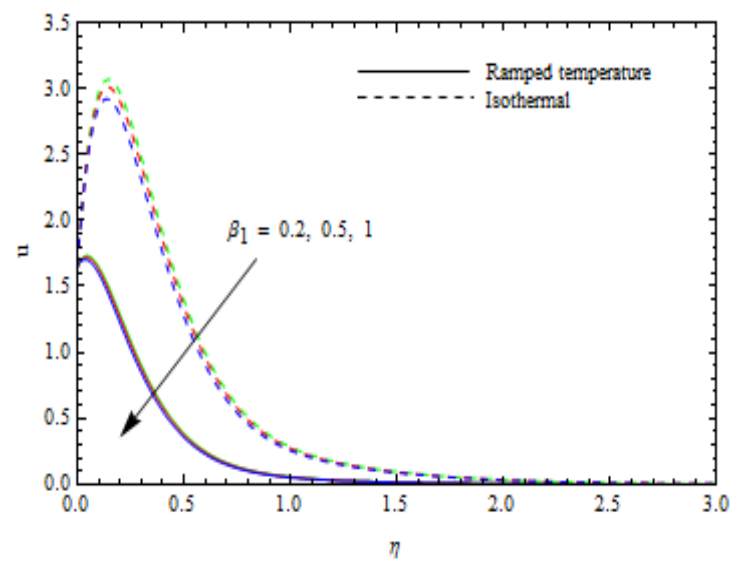

Fig. 2. Velocity profiles for $\beta_{1}$.

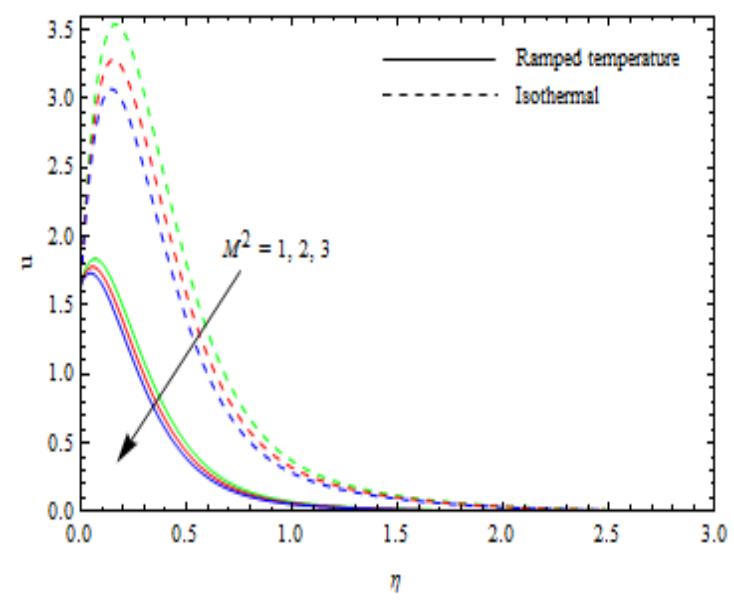

Fig. 3. Velocity profiles for $M^{2}$.

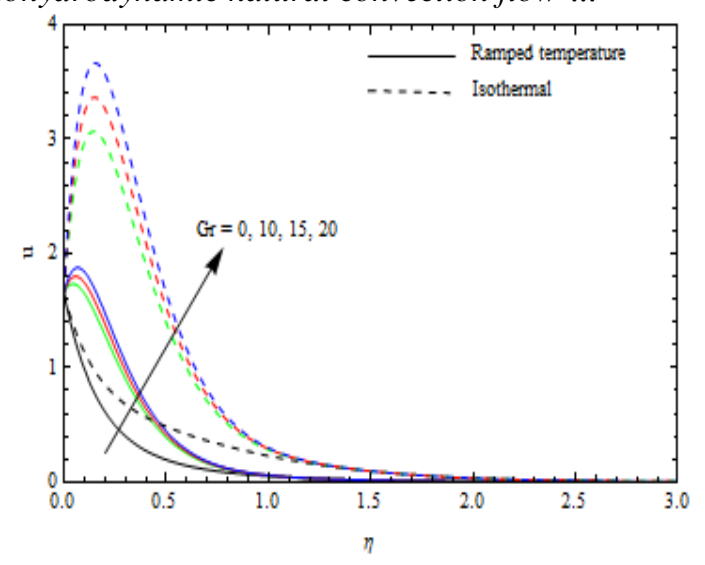

Fig. 4. Velocity profiles for Gr.

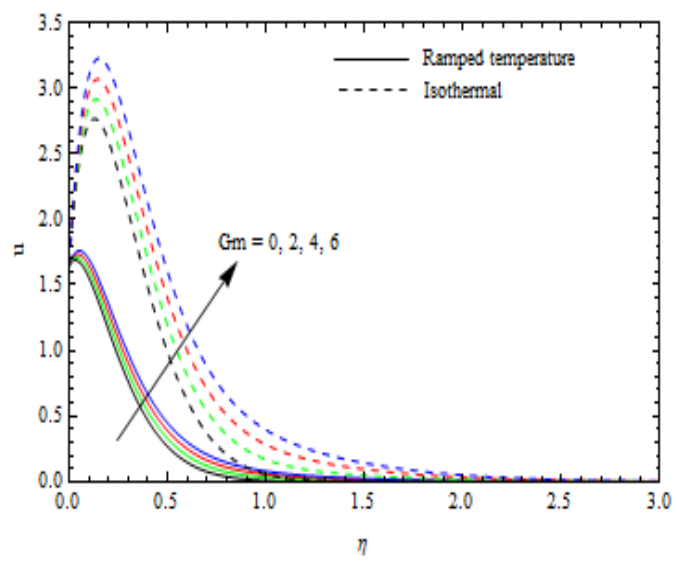

Fig. 5. Velocity profiles for Gm.

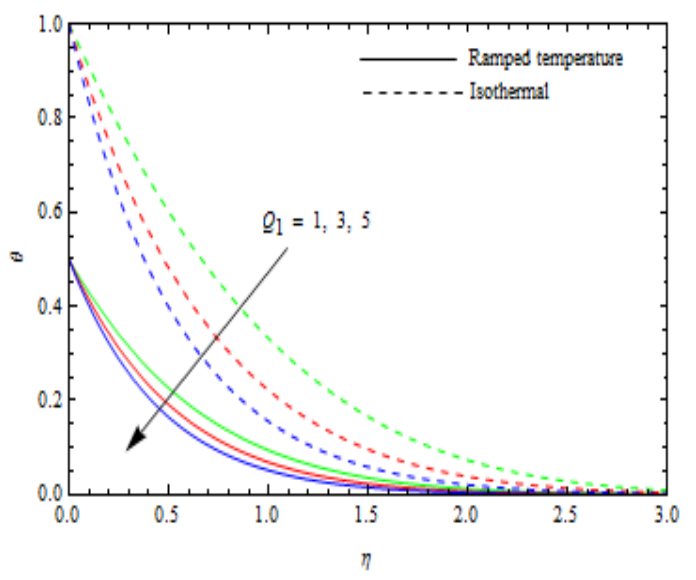

Fig. 6. Temperature profiles for $Q_{1}$.

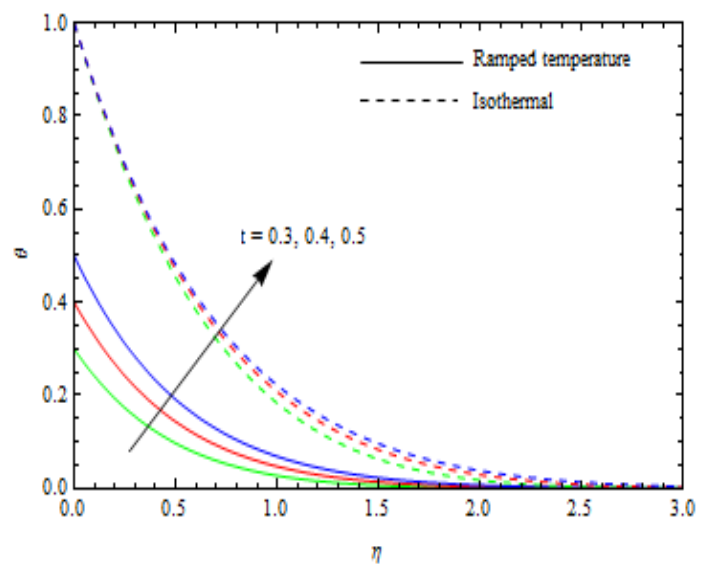

Fig. 7. Temperature profiles for $\boldsymbol{t}$. 
G. S. Seth et al.: Thermo-diffusion effects on the magnetohydrodynamic natural convection flow ...

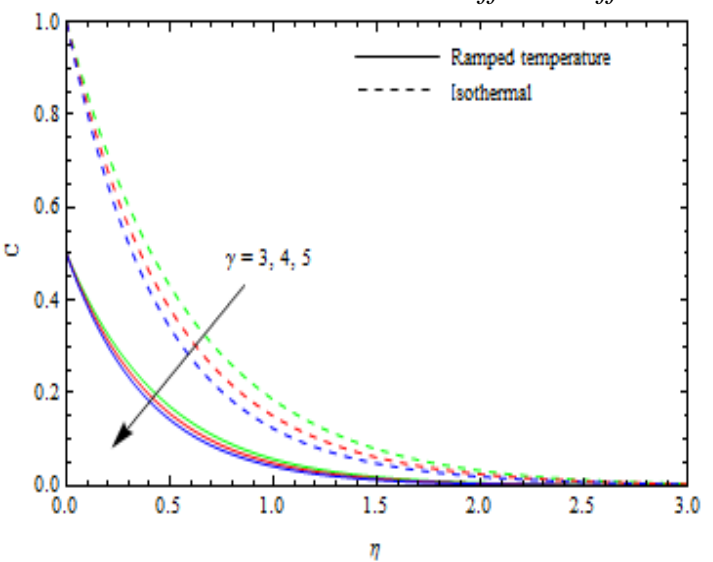

Fig. 8. Concentration profiles for $\gamma$.

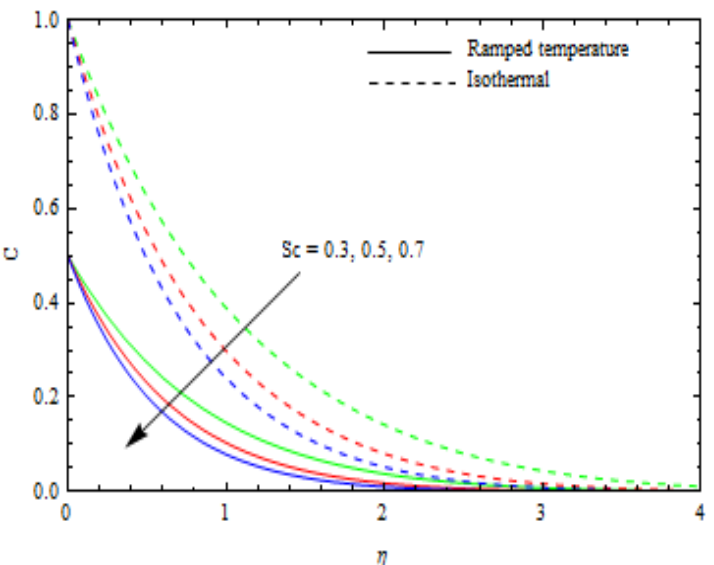

Fig. 9. Concentration profiles for $\mathrm{S}_{c}$.

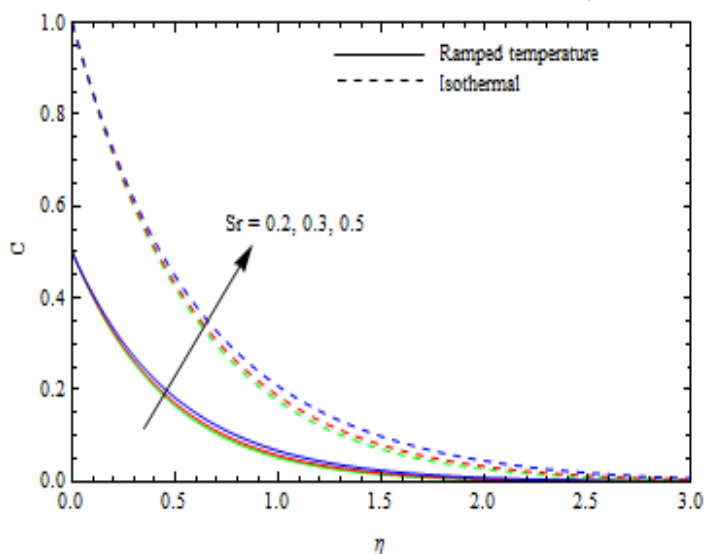

Fig. 10. Concentration profiles for $\mathrm{S}_{r}$.

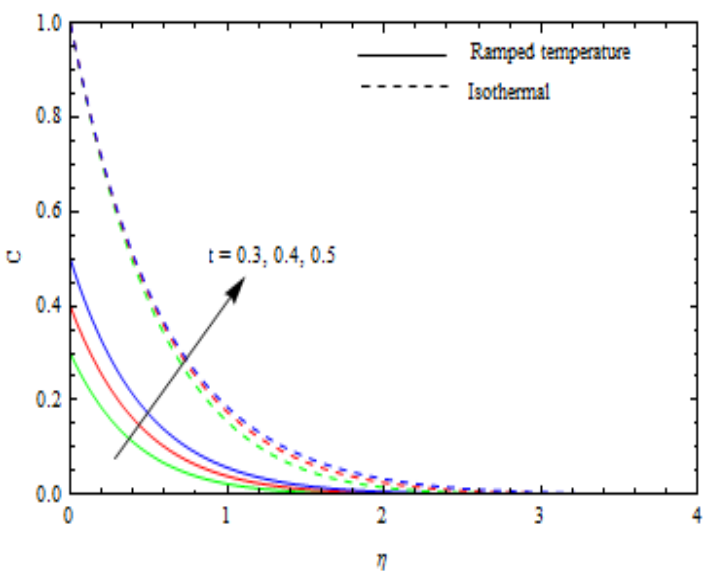

Fig. 11. Concentration profiles for $t$

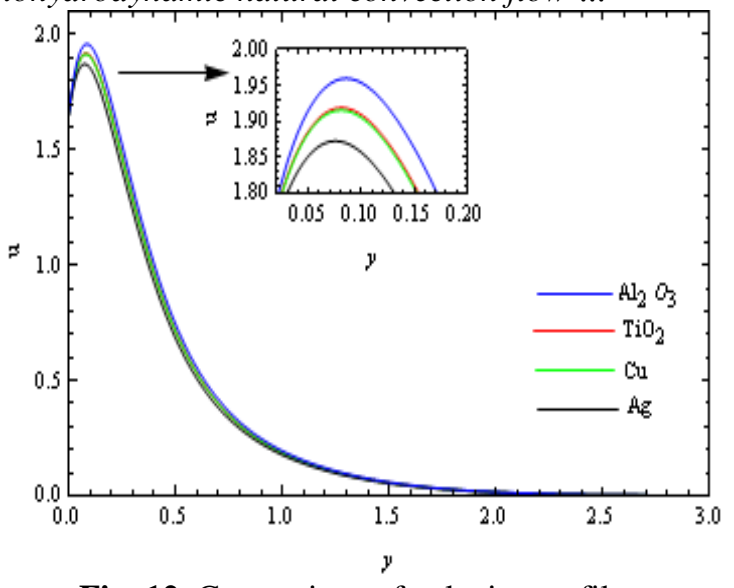

Fig. 12. Comparison of velocity profiles for different nanofluids

Table 2. Skin friction coefficient $\tau$ for $\beta_{1}, M^{2}$, Gr and $\mathrm{Gm}$

\begin{tabular}{|c|c|c|c|c|c|}
\hline$\beta_{1}$ & $M^{2}$ & $\mathrm{Gr}$ & $\mathrm{Gm}$ & $\begin{array}{c}\tau \text { for } \\
\text { ramped } \\
\text { temperature } \\
\text { plate }\end{array}$ & $\begin{array}{c}\tau \text { for } \\
\text { isothermal } \\
\text { plate }\end{array}$ \\
\hline $\mathbf{0 . 2}$ & & \multirow{2}{*}{$\mathbf{0 . 5}$} & \multirow{2}{*}{4} & $\begin{array}{c}2.33402 \\
2.02391\end{array}$ & 1.85488 \\
$\mathbf{1 . 0}$ & & & & 1.52471 & 0.95141 \\
\hline & $\mathbf{1}$ & & & 4.85153 & 4.44652 \\
0.2 & $\mathbf{2}$ & 15 & 4 & 3.44202 & 3.08423 \\
& $\mathbf{3}$ & & & 2.33402 & 1.85488 \\
\hline & & $\mathbf{1 0}$ & & 1.41593 & 1.85488 \\
0.2 & 3 & $\mathbf{1 5}$ & 4 & 2.33402 & 4.10068 \\
& & $\mathbf{2 0}$ & & 3.25211 & 6.34648 \\
\hline & & & $\mathbf{2}$ & 1.61280 & 0.35629 \\
0.2 & 3 & 15 & $\mathbf{4}$ & 2.33402 & 1.85488 \\
& & & $\mathbf{6}$ & 3.05524 & 3.35347 \\
\hline
\end{tabular}

Table 3. Rate of heat transfer $N_{u}$ at the plate.

\begin{tabular}{|c|c|c|c|}
\hline$Q_{1}$ & $t$ & $\begin{array}{c}\text { Ramped } \\
\text { temperature plate } \\
-N_{u}\end{array}$ & $\begin{array}{c}\text { Isothermal plate } \\
-N_{u}\end{array}$ \\
\hline $\mathbf{1}$ & & 0.75526 & 0.95290 \\
$\mathbf{3}$ & 0.5 & 0.93734 & 1.44236 \\
$\mathbf{5}$ & & 1.09482 & 1.83362 \\
\hline & $\mathbf{0 . 3}$ & 0.64418 & 1.50256 \\
3 & $\mathbf{0 . 4}$ & 0.79220 & 1.46254 \\
& $\mathbf{0 . 5}$ & 0.93734 & 1.44236 \\
\hline
\end{tabular}


G. S. Seth et al.: Thermo-diffusion effects on the magnetohydrodynamic natural convection flow ...

Table 4. Rate of mass transfer $S_{h}$ at the plate.

\begin{tabular}{|c|c|c|c|c|c|}
\hline$S_{c}$ & $S_{r}$ & $\gamma$ & $t$ & $\begin{array}{c}-S_{h} \text { for } \\
\text { ramped } \\
\text { temperature } \\
\text { plate }\end{array}$ & $\begin{array}{c}-S_{h} \text { for } \\
\text { iso- } \\
\text { thermal } \\
\text { plate }\end{array}$ \\
\hline $\mathbf{0 . 3 0}$ & & & & 0.57805 & 0.88950 \\
$\mathbf{0 . 6 0}$ & 3 & 3 & 4 & 0.80231 & 1.23458 \\
$\mathbf{0 . 7 0}$ & & & & 0.86289 & 1.32780 \\
\hline & $\mathbf{0 . 1}$ & & & 0.86004 & 1.32341 \\
0.2 & $\mathbf{0 . 3}$ & 3 & 4 & 0.80231 & 1.23458 \\
& $\mathbf{0 . 5}$ & & & 0.74458 & 1.14574 \\
\hline & & $\mathbf{3}$ & & 0.80231 & 1.23458 \\
0.2 & 3 & $\mathbf{4}$ & 4 & 0.88295 & 1.43785 \\
& & $\mathbf{5}$ & & 0.95829 & 1.62155 \\
\hline & & & $\mathbf{0 . 3}$ & 0.55139 & 1.28611 \\
0.2 & 3 & 3 & $\mathbf{0 . 4}$ & 0.67808 & 1.25185 \\
& & & $\mathbf{0 . 5}$ & 0.80231 & 1.23458 \\
\hline
\end{tabular}

\section{CONCLUSIONS}

An investigation of the unsteady magnetohydrodynamic free convection flow of an incompressible, viscous, heat absorbing/generating, electrically conducting and chemically reactive nanofluid of Brinkman type, taking Soret effect into account, past a vertical plate embedded in a fluid saturated porous medium was carried out. The significant outcomes of the present study are as follows:

- For both ramped temperature plate with ramped surface concentration and isothermal plate with uniform surface concentration:

○ Brinkman parameter and magnetic parameter have the tendency to decelerate the fluid velocity whereas a reverse trend is observed for thermal and solutal buoyancy forces.

- Fluid temperature gets reduced with an increment in the heat absorption parameter whereas an adverse effect is noted with the progress of time.

- Species concentration of the fluid is enhanced with increasing the values of Soret number, as well as with the progress of time, but completely opposite patterns are followed for chemical reaction parameter and Schmidt number.

- Shear stresses at the plate decrease as we increase the values of Brinkman parameter and magnetic parameter. On the other hand, thermal and solutal buoyancy forces tend to enhance the shear stress components at the plate.

- Rate of heat transfer at the plate gets improved with an increment in the heat absorption parameter. For ramped temperature plate heat transfer rate at the plate gets enhanced with the progress of time whereas in case of isothermal plate an adverse effect is noticed.

- Chemical reaction parameter and Schmidt number improve the rate of mass transfer at the plate whereas Soret number reduces the mass transfer rate at the plate. For ramped temperature plate rate of mass transfer at the plate gets enhanced with the progress of time whereas a reverse effect is noted in case of isothermal plate.

\section{REFERENCES}

1. J. A. Eastman, S. U. S. Choi, S. Li, L. J. Thompson, S. Lee, Nanophase and Nanocomposite Materials II, MRS Pittsburgh, PA, 1997, p. 3.

2. S. U. S. Choi, Z. G. Zhang, W. Yu, F. E. Lockwood, E. A. Grulke, Appl. Phys. Lett., 79, 2252 (2001).

3. O. D. Makinde, A. Aziz, Int. J. Therm. Sci., 50, 1326 (2011).

4. J. C. Umavathi, O. Ojjela, K. Vajravelu, Int. J. Therm. Sci. 111, 511 (2017).

5. T. Hayat, M. I. Khan, M. Farooq, A. Alsaedi, T. Yasmeen, Int. J. Heat and Mass Transf., 106, 810 (2017).

6. M. A. A. Hamad, I. Pop, Heat Mass Transf., 47, 1517 (2011).

7. M. A. A. Hamad, I, Pop, A. I. Md Ismail, Nonlinear Anal: Real World Appl. 12, 1338 (2011).

8. M. Sheikholeslami, M. Gorji-Bandpy, R. Ellahi, A. Zeeshan, J. Magn. Magn. Mater., 369, 69 (2014).

9. M. Sheikholeslami, M. Gorji- Bandpy, D. D. Ganji, Powder Tech., 254, 82 (2014).

10. Z. Mehrez, A. El Cafsi, A. Belghith, P. L. Quere, J. Magn. Magn. Mater., 374, 214 (2015).

11. M. Sheikholeslami, J. Molecular Liquids, 225, 903 (2017).

12. A. J. Chamkha, Int. Comm. in Heat and Mass Transf., 30, 413 (2003).

13. A. A. Afify, Canad. J. Phys., 82, 447 (2004).

14. R. Muthucumaraswamy, P. Chandrakala, S. A. Raj, Int. J. Appl. Mech. Eng. 11, 639 (2006).

15. F. S. Ibrahim, A. M. Elaiw, A. Bakr, Comm. Nonlin. Sci. Numer. Simul., 13, 1056 (2008).

16. A. M. Rashad, A. J. Chamkha, S. M. M. El-Kabeir, Int. J. Numer. Methods Heat Fluid Flow, 21, 418 (2011).

17. K. Bhattacharyya, G. C. Layek, Meccanica, 47, 1043 (2012).

18. F. Mabood, S. M. Ibrahim, M. M. Rashidi, M. S. Shadloo, G. Lorenzini, Int. J. Heat Mass Transf., 93, 674 (2016).

19. D. Sarma, K. K. Pandit, Ain Shams Eng. J., (2016)

20. J. Zueco, S. Ahmed, L. M. Lopez-Gonzalez, Int. J. Heat Mass Transf., 110, 467 (2017).

21. G. S. Seth, B. Kumbhakar, S. Sarkar, Int. J. Eng. Sci. and Tech., 7, 767 (2015).

22. V. Rajesh, A. J. Chamkha, Comm. Num. Anal., Article ID cna-00218 (2014). 
G. S. Seth et al.: Thermo-diffusion effects on the magnetohydrodynamic natural convection flow ...

23. P. K. Kundu, K. Das, N. Acharya, J. Mech., 30, 277 (2014).

24. G. S. Seth, S. Sarkar, Bulg. Chem. Comm., 47, 66 (2015).

25. G. S. Seth, R. Tripathi, R. Sharma, Bulg. Chem. Comm., 48, 770 (2016).

26. S. M. Hussain, J. Jain, G. S. Seth, Bulg. Chem. Comm., 48, 659 (2016).

27. H. Oztop, E. Abu-Nada, Int. J. Heat Fluid Flow, 29, 1326 (2008).

\section{APPENDIX}

$$
\begin{aligned}
& f_{1}\left(d_{1}, d_{2}, d_{3}, d_{4}, d_{5}\right)=\frac{1}{2}\left[e^{d_{1} \sqrt{d_{3}\left(d_{4}+d_{5}\right)}}\right. \\
& \times \operatorname{erfc}\left(\frac{d_{1}}{2} \sqrt{\frac{d_{3}}{d_{2}}}+\sqrt{d_{2}\left(d_{4}+d_{5}\right)}\right)+ \\
& \left.\times e^{-d_{1} \sqrt{d_{3}\left(d_{4}+d_{5}\right)}} \operatorname{erfc}\left(\frac{d_{1}}{2} \sqrt{\frac{d_{3}}{d_{2}}}-\sqrt{d_{2}\left(d_{4}+d_{5}\right)}\right)\right] \\
& f_{2}\left(d_{1}, d_{2}, d_{3}, d_{4}\right)=\frac{1}{2} \operatorname{erfc}\left(\frac{d_{1}}{2} \sqrt{\frac{d_{3}}{d_{2}}}+\sqrt{d_{2} d_{4}}\right) \\
& \times e^{d_{1} \sqrt{d_{3} d_{4}}}\left(d_{2}+\frac{d_{1}}{2} \sqrt{\frac{d_{3}}{d_{4}}}\right)+\frac{1}{2} e^{-d_{1} \sqrt{d_{3} d_{4}}} \\
& \times\left(d_{2}-\frac{d_{1}}{2} \sqrt{\frac{d_{3}}{d_{4}}}\right) \operatorname{erfc}\left(\frac{d_{1}}{2} \sqrt{\frac{d_{3}}{d_{2}}}-\sqrt{d_{2} d_{4}}\right) \\
& f_{3}\left(d_{1}, d_{2}, d_{3}, d_{4}\right)=\left\{\operatorname{erfc}\left(\sqrt{d_{1}\left(d_{3}+d_{4}\right)}\right)-1\right\} \\
& \times \sqrt{d_{2}\left(d_{3}+d_{4}\right)}-e^{-d_{1}\left(d_{3}+d_{4}\right)} \sqrt{\frac{d_{2}}{\pi d_{1}}} \\
& f_{4}\left(d_{1}, d_{2}, d_{3}\right)=\frac{1}{2}\left\{\operatorname{erfc}\left(\sqrt{d_{1} d_{3}}\right)-1\right\} \\
& \times\left(\sqrt{\frac{d_{2}}{d_{3}}}+2 d_{1} \sqrt{d_{2} d_{3}}\right)-e^{-d_{1} d_{3}} \sqrt{\frac{d_{1} d_{2}}{\pi}} \\
& g_{1}(\eta, t)=\frac{G r_{1}}{a_{0}^{2}}\left[e^{a_{0} t} f_{1}\left(\eta, t, \operatorname{Re}, y_{2}, a_{0}\right)\right. \\
& \left.-f_{1}\left(\eta, t, \operatorname{Re}, y_{2}, 0\right)-a_{0} f_{2}\left(\eta, t, \operatorname{Re}, y_{2}\right)\right] \text {, } \\
& g_{2}(\eta, t)=\frac{G m_{1}}{b_{2}^{2}}\left[e^{-b_{2} t} f_{1}\left(\eta, t, \operatorname{Re}, y_{2},-b_{2}\right)\right. \\
& \left.-f_{1}\left(\eta, t, \operatorname{Re}, y_{2}, 0\right)+b_{2} f_{2}\left(\eta, t, \operatorname{Re}, y_{2}\right)\right] \text {, } \\
& g_{3}(\eta, t)=\frac{\alpha G m_{1}}{b_{2}}\left[f_{1}\left(\eta, t, \operatorname{Re}, y_{2}, 0\right)\left\{1-\frac{Q_{1}}{b_{2}}\right\}\right. \\
& -e^{-b_{2} t} f_{1}\left(\eta, t, \operatorname{Re}, y_{2},-b_{2}\right)\left\{1-\frac{Q_{1}}{b_{2}}\right\}+Q_{1}
\end{aligned}
$$

$$
\left.\times f_{2}\left(\eta, t, \operatorname{Re}, y_{2}\right)\right]-\frac{\alpha G m_{1}}{\left(b_{2}-b_{1}\right)}\left[f_{1}\left(\eta, t, \operatorname{Re}, y_{2},-b_{1}\right)\right.
$$$$
\times e^{-b_{1} t}\left\{1-\frac{Q_{1}}{b_{1}}\right\}-\left\{1-\frac{Q_{1}}{b_{2}}\right\} f_{2}\left(\eta, t, \operatorname{Re}, y_{2},-b_{2}\right)
$$$$
\left.\times e^{-b_{2} t}+Q_{1} \frac{\left(b_{2}-b_{1}\right)}{b_{1} b_{2}} f_{1}\left(\eta, t, \operatorname{Re}, y_{2}, 0\right)\right],
$$$$
g_{4}(\eta, t)=\frac{\alpha G m_{2}}{a_{0}}\left[e^{a_{0} t} f_{1}\left(\eta, t, \operatorname{Re}, y_{2}, a_{0}\right)\right.
$$$$
\times\left\{1+\frac{Q_{1}}{a_{0}}\right\}-f_{1}\left(\eta, t, \operatorname{Re}, y_{2}, 0\right)\left\{1+\frac{Q_{1}}{a_{0}}\right\}
$$$$
\left.-Q_{1} f_{2}\left(\eta, t, \operatorname{Re}, y_{2}\right)\right]-\frac{\alpha G m_{2}}{\left(a_{0}+b_{1}\right)}\left[e^{a_{0} \tau}\left\{1+\frac{Q_{1}}{a_{0}}\right\}\right.
$$$$
\times f_{1}\left(\eta, t, \operatorname{Re}, y_{2}, a_{0}\right)-f_{1}\left(\eta, t, \operatorname{Re}, y_{2},-b_{1}\right) e^{-b_{1} t}
$$$$
\left.\left.\times\left\{1-\frac{Q_{1}}{b_{1}}\right\}-Q_{1} \frac{\left(a_{0}+b_{1}\right)}{a_{0} b_{1}} f_{1}\left(\eta, t, \operatorname{Re}, y_{2}, 0\right)\right\}\right] \text {, }
$$$$
g_{5}(\eta, t)=\frac{G r_{1}}{a_{0}^{2}}\left[e^{a_{0} t} f_{1}\left(\eta, t, b_{0}, \mathrm{Q}_{1}, a_{0}\right)\right.
$$$$
\left.-f_{1}\left(\eta, t, b_{0}, \mathrm{Q}_{1}, 0\right)-a_{0} f_{2}\left(\eta, t, b_{0}, \mathrm{Q}_{1}\right)\right],
$$$$
g_{6}(\eta, t)=\frac{G m_{1}}{b_{2}^{2}}\left[b_{2} f_{2}\left(\eta, t, \mathrm{~S}_{c}, \gamma\right)\right.
$$$$
\left.-f_{1}\left(\eta, t, \mathrm{~S}_{c}, \gamma, 0\right)+e^{\left(-b_{2} t\right)} f_{1}\left(\eta, t, \mathrm{~S}_{c}, \gamma,-b_{2}\right)\right],
$$$$
g_{7}(\eta, t)=\frac{\alpha G m_{1}}{b_{2}}\left[f_{1}\left(\eta, t, \mathrm{~S}_{c}, \gamma, 0\right)\left\{1-\frac{Q_{1}}{b_{2}}\right\}\right.
$$$$
-\left\{1-\frac{Q_{1}}{b_{2}}\right\} e^{-b_{2} t} f_{1}\left(\eta, t, \mathrm{~S}_{c}, \gamma,-b_{2}\right)+Q_{1}
$$$$
\left.\times f_{2}\left(\eta, t, \mathrm{~S}_{c}, \gamma\right)\right]-\frac{\alpha G m_{1}}{\left(b_{2}-b_{1}\right)}\left[e^{-b_{1} t}\left\{1-\frac{Q_{1}}{b_{1}}\right\}\right.
$$$$
\times f_{1}\left(\eta, t, \mathrm{~S}_{c}, \gamma,-b_{1}\right)-e^{-b_{2} t} f_{1}\left(\eta, t, \mathrm{~S}_{c}, \gamma,-b_{2}\right)
$$$$
\left.\times\left\{1-\frac{Q_{1}}{b_{2}}\right\}+Q_{1} \frac{\left(b_{2}-b_{1}\right)}{b_{1} b_{2}} f_{1}\left(\eta, t, \mathrm{~S}_{c}, \gamma, 0\right)\right],
$$$$
g_{8}(\eta, t)=\frac{\alpha G m_{2}}{a_{0}}\left[e^{a_{0} t} f_{1}\left(\eta, t, b_{0}, Q_{1}, a_{0}\right)\right.
$$$$
\times\left\{1+\frac{Q_{1}}{a_{0}}\right\}-f_{1}\left(\eta, t, b_{0}, Q_{1}, 0\right)\left\{1+\frac{Q_{1}}{a_{0}}\right\}-Q_{1}
$$

$\left.\times f_{2}\left(\eta, t, b_{0}, Q_{1}\right)\right]-\frac{\alpha G m_{2}}{\left(a_{0}+b_{1}\right)}\left[f_{1}\left(\eta, t, b_{0}, Q_{1}, a_{0}\right)\right.$ 
G. S. Seth et al.: Thermo-diffusion effects on the magnetohydrodynamic natural convection flow ...

$$
\begin{aligned}
& \times e^{a_{0} t}\left\{1+\frac{Q_{1}}{a_{0}}\right\}-e^{-b_{1} t} f_{1}\left(\eta, t, b_{0}, Q_{1},-b_{1}\right) \\
& \times \frac{G m_{2} Q_{1}}{a_{0}}\left[f_{1}\left(\eta, t, b_{0}, Q_{1}, 0\right)-f_{1}\left(\eta, t, b_{0}, Q_{1}, a_{0}\right)\right. \\
& \left.\times\left\{1-\frac{Q_{1}}{b_{1}}\right\}-Q_{1} \frac{\left(a_{0}+b_{1}\right)}{a_{0} b_{1}} f_{1}\left(\eta, t, b_{0}, Q_{1}, 0\right)\right], \\
& h_{1}(\eta, t)=e^{a t} f_{1}\left(\eta, t, \operatorname{Re}, y_{2}, a\right) \text {, } \\
& h_{2}(\eta, t)=\frac{G r_{1}}{a_{0}}\left[e^{a_{0} t} f_{1}\left(\eta, t, \operatorname{Re}, y_{2}, a_{0}\right)\right. \\
& \left.-f_{1}\left(\eta, t, \operatorname{Re}, y_{2}, 0\right)\right] \text {, } \\
& h_{3}(\eta, t)=\frac{G m_{1}}{b_{2}}\left[f_{1}\left(\eta, t, \operatorname{Re}, y_{2}, 0\right)-e^{-b_{2} t}\right. \\
& \left.\times f_{1}\left(\eta, t, \operatorname{Re}, y_{2},-b_{2}\right)\right], \\
& h_{4}(\eta, t)=\alpha G m_{1}\left\{f_{1}\left(\eta, t, \operatorname{Re}, y_{2},-b_{2}\right)\right. \\
& \times e^{\left(-b_{2} t\right)}\left(1-\frac{b_{2}}{b_{2}-b_{1}}\right)+\frac{b_{1}}{b_{2}-b_{1}} e^{\left(-b_{1} t\right)} \\
& \left.\times f_{1}\left(\eta, t, \operatorname{Re}, y_{2},-b_{1}\right)\right\}, \\
& h_{5}(\eta, t)=\alpha G m_{2}\left\{f_{1}\left(\eta, t, \operatorname{Re}, y_{2}, a_{0}\right)\right. \\
& \times e^{\left(a_{0} t\right)}\left(1-\frac{a_{0}}{a_{0}+b_{1}}\right)-f_{1}\left(\eta, t, \operatorname{Re}, y_{2},-b_{1}\right) \\
& \left.\times e^{\left(-b_{1} t\right)} \frac{b_{1}}{a_{0}+b_{1}}\right\}-\alpha \frac{G m_{2} Q_{1}}{a_{0}} \\
& {\left[f_{1}\left(\eta, t, \operatorname{Re}, y_{2}, 0\right)-f_{1}\left(\eta, t, \operatorname{Re}, y_{2}, a_{0}\right) \times e^{\left(a_{0} t\right)}\right.} \\
& \left.\left\{1-\frac{1}{a_{0}+b_{1}}\right\}-\frac{e^{\left(-b_{1} t\right)}}{a_{0}+b_{1}} f_{1}\left(\eta, t, \operatorname{Re}, y_{2},-b_{1}\right)\right], \\
& h_{6}(\eta, t)=\frac{G r_{1}}{a_{0}}\left[e^{a_{0} t} f_{1}\left(\eta, t, b_{0}, Q_{1}, a_{0}\right)\right. \\
& \left.-f_{1}\left(\eta, t, b_{0}, Q_{1}, 0\right)\right], \\
& h_{7}(\eta, t)=\frac{G m_{1}}{b_{2}}\left[f_{1}\left(\eta, t, \mathrm{~S}_{c}, \gamma, 0\right)\right. \\
& \left.-e^{\left(-b_{2} t\right)} f_{1}\left(\eta, t, \mathrm{~S}_{c}, \gamma,-b_{2}\right)\right], \\
& h_{8}(\eta, t)=\alpha G m_{1}\left\{e^{\left(-b_{2} t\right)} f_{1}\left(\eta, t, \mathrm{~S}_{c}, \gamma,-b_{2}\right)\right. \\
& \left.\times\left(1-\frac{b_{2}}{b_{2}-b_{1}}\right)+\frac{b_{1}}{b_{2}-b_{1}} e^{\left(-b_{1} t\right)} f_{1}\left(\eta, t, \mathrm{~S}_{c}, \gamma,-b_{1}\right)\right\}, \\
& +\alpha \frac{G m_{1} Q_{1}}{b_{2}}\left[f_{1}\left(\eta, t, \mathrm{~S}_{c}, \gamma, 0\right)-f_{1}\left(\eta, t, \mathrm{~S}_{c}, \gamma,-b_{1}\right)\right. \\
& \left.\times \frac{e^{\left(-b_{1} t\right)}}{b_{2}-b_{1}}-e^{\left(-b_{2} t\right)} f_{1}\left(\eta, t, \mathrm{~S}_{c}, \gamma,-b_{2}\right)\left\{1-\frac{e^{\left(-b_{2} t\right)}}{b_{2}-b_{1}}\right\}\right], \\
& h_{9}(\eta, t)=\alpha G m_{2}\left\{\left(1-\frac{a_{0}}{a_{0}+b_{1}}\right) f_{1}\left(\eta, t, b_{0}, Q_{1}, a_{0}\right)\right. \\
& \left.\times e^{\left(a_{0} t\right)}-\frac{b_{1}}{a_{0}+b_{1}} e^{\left(-b_{1} t\right)} f_{1}\left(\eta, t, b_{0}, Q_{1},-b_{1}\right)\right\}-\alpha \\
& \left.\times e^{\left(a_{0} t\right)}\left\{1-\frac{1}{\left(a_{0}+b_{1}\right)}\right\}-\frac{e^{\left(-b_{1} t\right)}}{\left(a_{0}+b_{1}\right)} f_{1}\left(\eta, t, b_{0}, Q_{1},-b_{1}\right)\right] \text {. } \\
& g_{1}^{\prime}(0, t)=\frac{G r_{1}}{a_{0}^{2}}\left[e^{a_{0} t} f_{3}\left(t, \operatorname{Re}, y_{2}, a_{0}\right)\right. \\
& \left.-f_{3}\left(t, \operatorname{Re}, y_{2}, 0\right)-a_{0} f_{4}\left(t, \operatorname{Re}, y_{2}\right)\right], \\
& g_{2}^{\prime}(0, t)=\frac{G m_{1}}{b_{2}^{2}}\left[e^{-b_{2} t} f_{3}\left(t, \operatorname{Re}, y_{2},-b_{2}\right)\right. \\
& \left.-f_{3}\left(t, \operatorname{Re}, y_{2}, 0\right)+b_{2} f_{4}\left(t, \operatorname{Re}, y_{2}\right)\right] \text {, } \\
& g_{3}^{\prime}(0, t)=\frac{\alpha G m_{1}}{b_{2}}\left[f_{3}\left(t, \operatorname{Re}, y_{2}, 0\right)\left\{1-\frac{Q_{1}}{b_{2}}\right\}\right. \\
& -\left\{1-\frac{Q_{1}}{b_{2}}\right\} e^{-b_{2} t} f_{3}\left(t, \operatorname{Re}, y_{2},-b_{2}\right) \\
& \left.+Q_{1} f_{4}\left(t, \operatorname{Re}, y_{2}\right)\right]-\frac{\alpha G m_{1}}{\left(b_{2}-b_{1}\right)}\left[e^{-b_{1} t}\left\{1-\frac{Q_{1}}{b_{1}}\right\}\right. \\
& \times f_{3}\left(t, \operatorname{Re}, y_{2},-b_{1}\right)-\left\{1-\frac{Q_{1}}{b_{2}}\right\} f_{3}\left(t, \operatorname{Re}, y_{2},-b_{2}\right) \\
& \left.\times e^{-b_{2} t}+Q_{1} \frac{\left(b_{2}-b_{1}\right)}{b_{1} b_{2}} f_{3}\left(t, \operatorname{Re}, y_{2}, 0\right)\right], \\
& g_{4}^{\prime}(0, t)=\frac{\alpha G m_{2}}{a_{0}}\left[f_{3}\left(t, \operatorname{Re}, y_{2}, a_{0}\right)\left\{1+\frac{Q_{1}}{a_{0}}\right\}\right. \\
& e^{a_{0} t}-f_{3}\left(t, \operatorname{Re}, y_{2}, 0\right)\left\{1+\frac{Q_{1}}{a_{0}}\right\}-f_{4}\left(\tau, \operatorname{Re}, y_{2}\right) \\
& \left.\times Q_{1}\right]-\frac{\alpha G m_{2}}{\left(a_{0}+b_{1}\right)}\left[\left\{f_{3}\left(t, \operatorname{Re}, y_{2}, a_{0}\right)\left\{1+\frac{Q_{1}}{a_{0}}\right\}\right.\right. \\
& \times e^{a_{0} t}-\left\{1-\frac{Q_{1}}{b_{1}}\right\} e^{-b_{1} t} f_{3}\left(t, \operatorname{Re}, y_{2},-b_{1}\right) \\
& \left.-Q_{1} \frac{\left(a_{0}+b_{1}\right)}{a_{0} b_{1}} f_{3}\left(t, \operatorname{Re}, y_{2}, 0\right)\right], \\
& g_{5}^{\prime}(0, t)=\frac{G r_{1}}{a_{0}^{2}}\left[e^{a_{0} t} f_{3}\left(t, b_{0}, \mathrm{Q}_{1}, a_{0}\right)\right. \\
& \left.-f_{3}\left(t, b_{0}, \mathrm{Q}_{1}, 0\right)-a_{0} f_{4}\left(t, b_{0}, \mathrm{Q}_{1}\right)\right] \text {, } \\
& g_{6}^{\prime}(0, t)=\frac{G m_{1}}{b_{2}^{2}}\left[b_{2} f_{4}\left(t, \mathrm{~S}_{c}, \gamma\right)\right. \\
& \left.-f_{3}\left(t, \mathrm{~S}_{c}, \gamma, 0\right)+e^{\left(-b_{2} t\right)} f_{3}\left(t, \mathrm{~S}_{c}, \gamma,-b_{2}\right)\right] \text {, }
\end{aligned}
$$


G. S. Seth et al.: Thermo-diffusion effects on the magnetohydrodynamic natural convection flow ...

$$
\begin{aligned}
& g_{7}^{\prime}(0, t)=\frac{\alpha G m_{1}}{b_{2}}\left[\left\{f_{3}\left(t, \mathrm{~S}_{c}, \gamma, 0\right)\left\{1-\frac{Q_{1}}{b_{2}}\right\}\right.\right. \\
& h_{5}^{\prime}(0, t)=\alpha G m_{2}\left\{\left(1-\frac{a_{0}}{a_{0}+b_{1}}\right) f_{3}\left(t, \operatorname{Re}, y_{2}, a_{0}\right)\right. \\
& -\left\{1-\frac{Q_{1}}{b_{2}}\right\} e^{-b_{2} t} f_{3}\left(t, \mathrm{~S}_{c}, \gamma,-b_{2}\right)+f_{4}\left(t, \mathrm{~S}_{c}, \gamma\right) \\
& \left.\times e^{\left(a_{0} t\right)}-\frac{b_{1}}{a_{0}+b_{1}} e^{\left(-b_{1} t\right)} f_{3}\left(t, \operatorname{Re}, y_{2},-b_{1}\right)\right\} \\
& \left.\times Q_{1}\right]-\frac{\alpha G m_{1}}{\left(b_{2}-b_{1}\right)}\left[e^{-b_{1} t} f_{3}\left(t, \mathrm{~S}_{c}, \gamma,-b_{1}\right)\right. \\
& \times\left\{1-\frac{Q_{1}}{b_{1}}\right\}-\left\{1-\frac{Q_{1}}{b_{2}}\right\} e^{-b_{2} t} f_{3}\left(t, \mathrm{~S}_{c}, \gamma,-b_{2}\right) \\
& \left.+Q_{1} \frac{\left(b_{2}-b_{1}\right)}{b_{1} b_{2}} f_{3}\left(t, \mathrm{~S}_{c}, \gamma, 0\right)\right] \text {, } \\
& g_{8}^{\prime}(0, t)=\frac{\alpha G m_{2}}{a_{0}}\left[f_{3}\left(t, b_{0}, Q_{1}, a_{0}\right) e^{a_{0} t}\right. \\
& \times\left\{1+\frac{Q_{1}}{a_{0}}\right\}-f_{3}\left(t, b_{0}, Q_{1}, 0\right)\left\{1+\frac{Q_{1}}{a_{0}}\right\}-Q_{1} \\
& \left.\times f_{4}\left(t, b_{0}, Q_{1}\right)\right]-\frac{\alpha G m_{2}}{\left(a_{0}+b_{1}\right)}\left[e^{a_{0} t}\left\{1+\frac{Q_{1}}{a_{0}}\right\}\right. \\
& \times f_{3}\left(t, b_{0}, Q_{1}, a_{0}\right)-\left\{1-\frac{Q_{1}}{a_{1}}\right\} f_{3}\left(t, b_{0}, Q_{1},-b_{1}\right) \\
& \left.\times e^{-b_{1} t}-Q_{1} \frac{\left(a_{0}+b_{1}\right)}{a_{0} b_{1}} f_{3}\left(t, b_{0}, Q_{1}, 0\right)\right] . \\
& h_{1}^{\prime}(0, t)=e^{a t} f_{3}\left(t, \operatorname{Re}, y_{2}, a\right) \text {, } \\
& h_{2}^{\prime}(0, t)=\frac{G r_{1}}{a_{0}}\left[e^{a_{0} t} f_{3}\left(t, \operatorname{Re}, y_{2}, a_{0}\right)\right. \\
& \left.-f_{3}\left(t, \operatorname{Re}, y_{2}, 0\right)\right], \\
& h_{3}^{\prime}(0, t)=\frac{G m_{1}}{b_{2}}\left[f_{3}\left(t, \operatorname{Re}, y_{2}, 0\right)\right. \\
& \left.-e^{-b_{2} t} f_{3}\left(t, \operatorname{Re}, y_{2},-b_{2}\right)\right] \text {, } \\
& h_{4}^{\prime}(0, t)=\alpha G m_{1}\left\{e^{\left(-b_{2} t\right)} f_{3}\left(t, \operatorname{Re}, y_{2},-b_{2}\right)\right. \\
& \times\left(1-\frac{b_{2}}{b_{2}-b_{1}}\right)+\frac{b_{1}}{b_{2}-b_{1}} f_{3}\left(t, \operatorname{Re}, y_{2},-b_{1}\right) \\
& \left.\times e^{\left(-b_{1} t\right)}\right\}+\alpha \frac{G m_{1} Q_{1}}{b_{2}}\left[f_{3}\left(t, \operatorname{Re}, y_{2}, 0\right)-\frac{e^{\left(-b_{1} t\right)}}{\left(b_{2}-b_{1}\right)}\right. \\
& \times f_{3}\left(t, \operatorname{Re}, y_{2},-b_{1}\right)-e^{\left(-b_{2} t\right)} \\
& \left.\times f_{3}\left(t, \operatorname{Re}, y_{2},-b_{2}\right)\left\{1-\frac{1}{\left(b_{2}-b_{1}\right)}\right\}\right] \text {, } \\
& -\alpha \frac{G m_{2} Q_{1}}{a_{0}}\left[f_{3}\left(t, \operatorname{Re}, y_{2}, 0\right)-\left\{1-\frac{1}{\left(a_{0}+b_{1}\right)}\right\} e^{\left(a_{0} t\right)}\right. \\
& \left.\times f_{3}\left(t, \operatorname{Re}, y_{2}, a_{0}\right)-\frac{e^{\left(-b_{1} t\right)}}{\left(a_{0}+b_{1}\right)} f_{3}\left(t, \operatorname{Re}, y_{2},-b_{1}\right)\right] \text {, } \\
& h_{6}^{\prime}(0, t)=\frac{G r_{1}}{a_{0}}\left[e^{\left(a_{0} t\right)} f_{3}\left(t, b_{0}, Q_{1}, a_{0}\right)\right. \\
& \left.-f_{3}\left(t, b_{0}, Q_{1}, 0\right)\right] \text {, } \\
& h_{7}^{\prime}(0, t)=\frac{G m_{1}}{b_{2}}\left[f_{3}\left(t, \mathrm{~S}_{c}, \gamma, 0\right)\right. \\
& \left.-e^{\left(-b_{2} t\right)} f_{3}\left(t, \mathrm{~S}_{c}, \gamma,-b_{2}\right)\right], \\
& h_{8}^{\prime}(0, t)=\alpha G m_{1}\left\{\left(1-\frac{b_{2}}{b_{2}-b_{1}}\right) e^{\left(-b_{2} t\right)}\right. \\
& \times f_{3}\left(t, \mathrm{~S}_{c}, \gamma,-b_{2}\right)+f_{3}\left(t, \mathrm{~S}_{c}, \gamma,-b_{1}\right) \\
& \left.\times \frac{b_{1}}{b_{2}-b_{1}} e^{\left(-b_{1} t\right)}\right\}+\alpha \frac{G m_{1} Q_{1}}{b_{2}}\left[f_{3}\left(t, \mathrm{~S}_{c}, \gamma, 0\right)\right. \\
& -\left\{1-\frac{1}{\left(b_{2}-b_{1}\right)}\right\} e^{\left(-b_{2} t\right)} f_{3}\left(t, \mathrm{~S}_{c}, \gamma,-b_{2}\right) \\
& \left.-\frac{e^{\left(-b_{1} t\right)}}{\left(b_{2}-b_{1}\right)} f_{3}\left(t, \mathrm{~S}_{c}, \gamma,-b_{1}\right)\right], \\
& h_{9}^{\prime}(0, t)=\alpha G m_{2}\left\{e^{\left(a_{0} t\right)} f_{3}\left(t, b_{0}, Q_{1}, a_{0}\right)\right. \\
& \times\left(1-\frac{a_{0}}{a_{0}+b_{1}}\right)-\frac{b_{1}}{a_{0}+b_{1}} f_{3}\left(t, b_{0}, Q_{1},-b_{1}\right) \\
& \left.\times e^{\left(-b_{1} t\right)}\right\}-\alpha \frac{G m_{2} Q_{1}}{a_{0}}\left[f_{3}\left(t, b_{0}, Q_{1}, 0\right)-e^{\left(a_{0} t\right)}\right. \\
& \times f_{3}\left(t, b_{0}, Q_{1}, a_{0}\right)\left\{1-\frac{1}{\left(a_{0}+b_{1}\right)}\right\} \\
& \left.-\frac{e^{\left(-b_{1} t\right)}}{\left(a_{0}+b_{1}\right)} f_{3}\left(t, b_{0}, Q_{1},-b_{1}\right)\right] \text {. }
\end{aligned}
$$

where $\operatorname{erfc}(x)$ denotes complementary error function. 


\title{
Thermal properties and time-dependent flow behavior of a viscous fluid
}

\author{
M. Imran Khan ${ }^{1, *}$, T. Hayat ${ }^{2,3}$, M. Ijaz Khan ${ }^{2}$, T. Yasmeen ${ }^{4}$ \\ ${ }^{1}$ Heriot Watt University, Edinburgh EH14 4AS, United Kingdom \\ ${ }^{2}$ Department of Mathematics, Quaid-I-Azam University 45320 Islamabad 44000, Pakistan \\ ${ }^{3}$ Nonlinear Analysis and Applied Mathematics (NAAM) Research Group, Department of Mathematics, Faculty of \\ Science, King Abdulaziz University, P. O. Box 80257, Jeddah 21589, Saudi Arabia \\ ${ }^{4}$ University of Engineering \& Technology, Peshawar, Pakistan
}

Received June 17, 2017; Accepted February 7, 2018

\begin{abstract}
Our goal in this attempt is to model a nonlinear stretchable flow of a radiative viscous liquid with magnetohydrodynamics. Flow caused is due to a unsteady stretching surface with variable thickness. Consideration of thermal radiation effect characterizes the heat transfer process. Induced electric and magnetic fields are not accounted for. Appropriate transformations gave nonlinear systems. Modern methodology, i.e., HAM, is implemented for the computational process. Velocity and temperature are plotted for influential variables which are important in this problem. Moreover, surface drag force and heat transfer rate are computed and discussed. Velocity field is noted to decay the function of the larger Hartman number whereas opposite situation for temperature is examined via larger radiation parameter.
\end{abstract}

Keywords: Thermal radiation; time-dependent flow; viscous liquid; variable sheet thickness

\section{INTRODUCTION}

The consideration of heat transport in stretchable flows is influential due to its broad utilizations in chemical engineering. Numerous processes in chemical engineering comprising polymer extrusion and metallurgical processes involved paper production, glass-fiber and chilling of molten liquid being extended into a chilling system [1]. The rates of cooling/stretching in such processes affect the excellence of the ultimate product. Various investigations in this direction have been reported under distinct physical aspects. For instance a magnetohydrodynamic (MHD) stretchable flow of viscous material considering velocity slip characteristics is reported by Mukhopadhyay [2]. Hayat et al. [3] modeled convected Jeffrey liquid with Joule heating and dissipation effects. Analysis of nonlinear micropolar and generalized Burgers liquids towards stretched surface are developed by Waqas et al. [4, 5]. Very recently Hayat et al. [6] scrutinized a stretchable flow of magneto-third-grade liquid with chemical reaction.

Several researchers nowadays are involved in exploring heat transfer characteristics via consideration of thermal radiation. This aspect regularly arises in several environmental and scientific processes. Few applications of thermal radiation comprise material dyeing processes, hot wiring, space/solar technology, glass fiber production, transpiration process, heat exchangers, prevention of energy and high temperature plasmas. Olajuwon et al. [7] explored Hall current and thermal radiation aspects in MHD micropolar liquid flow. Numerical solutions for thermally radiating threedimensional (3D) stretchable flow of magneto viscous nanoliquid with convective heating condition are

* To whom all correspondence should be sent:

E-mail: mk42@hw.ac.uk constructed by Mahanthesh et al. [8]. Hayat et al. [9, 10] reported a radiation impact in stagnation point flow of thixotropic and viscous materials towards stretched surface utilizing homotopic and numerical approach.

A magnetohydrodynamic (MHD) phenomenon has distinct industrial implication in mathematics, physics, engineering and chemistry. It has also applications in biological transportation, mixing of samples, cooling of strips, drug delivery, etc. This mechanism is fruitful to control both flow and heat transport. Various investigations have been reported in this context. For instance Sandeep and Sulochana [11] developed dual solutions for unsteady micropolar material with mixed convection and magnetohydrodynamics. Analysis of a three-dimensional dissipative stretchable flow of magneto Eyring-Powell nanoliquid with radiation and convective heating effects is reported by Mahanthesh et al. [12]. Khan and Khan [13] and Hayat et al. [14, 15] examined MHD impact in flow of power-law and Carreau materials through different geometries. Hayat et al. [16] explored thermal radiation and heat generation/absorption characteristics in convected Eyring-Powell nanoliquid towards stretched cylinder.

Aforestated literature survey assures that a stretchable flow regarding Newtonian/non-Newtonian materials is reported mostly considering negligible thickness. To our knowledge stretchable flows with variable thickness are not addressed extensively. Only few analyses in this direction are reported (for details see [17-19]). Thus our goal here is to venture further towards this aspect by considering unsteady stretched surface with variable thickness. Here we adopted the Newtonian fluid model with magnetohydrodynamics (MHD) and thermal radiation. The nonlinear developed systems are computed analytically via homotopy theory [20-25]. Moreover, the results are scrutinized graphically for distinct sundry variables. 


\section{Imran Khan et al.: Thermal properties and time-dependent flow behavior of a viscous fluid}

\section{FORMULATION}

Here the magnetohydrodynamic (MHD) flow of a viscous fluid towards unsteady stretching sheet with variable thickness is accounted for. The flow is generated due to a nonlinear stretching phenomenon with velocity $u_{w}(x)\left(=\frac{u_{0}(x+b)^{n}}{1-\gamma t}\right)$. Liquid is supposed in compression. Viscous liquid is conducted electrically subject to magnetic field of the form $B(x)\left(=B_{0}(x+b)^{\frac{n-1}{2}}\right)$ imposed in $y$ - direction. The effect of thermal radiation is retained. Besides this, viscous dissipation impact in heat process is neglected. The resulting boundary layer expressions of viscous fluid for present flow situation are:

$\frac{\partial u}{\partial x}+\frac{\partial v}{\partial y}=0$

$u \frac{\partial u}{\partial x}+v \frac{\partial u}{\partial y}=v \frac{\partial^{2} u}{\partial y^{2}}-\frac{\sigma B^{2}(x)}{\rho} u$,

$u \frac{\partial T}{\partial x}+v \frac{\partial T}{\partial y}=\frac{k}{\rho c_{p}} \frac{\partial^{2} T}{\partial y^{2}}$

$+\frac{16 \sigma^{*}}{3 k^{*} \rho c_{p}} \frac{\partial}{\partial y}\left(T^{3} \frac{\partial T}{\partial y}\right)$

with the subjected conditions:

$$
\left.\begin{array}{c}
u=u_{w}(x)=\frac{u_{0}(x+b)^{n}}{1-\gamma t}, v=0, \\
T=T_{w} \text { at } y=\delta(x+b)^{\frac{1-n}{2}}, \\
u \rightarrow 0, T \rightarrow T_{\infty} \text { as } y \rightarrow \infty .
\end{array}\right\}
$$

Here $(u, v)$ are the velocity components in the $(x, y)$ directions, respectively, $v$ the kinematic viscosity, $\sigma$ the electrical conductivity, $k$ the thermal conductivity, $\rho$ the fluid density, $\sigma^{*}$ the Stefan-Boltzmann constant, $k^{*}$ the mean absorption coefficient, $c_{p}$ the specific heat, $n$ the velocity power index, $B(x)=B_{0}(x+b)^{\frac{n-1}{2}}$ the non-uniform magnetic field, $\left(T, T_{w}, T_{\infty}\right)$ the (fluid, surface, ambient fluid) temperatures, $\delta$ the small variable w.r.t surface adequately thin, $u_{0}$ the reference velocity and $(b, \gamma)$ the positive constants.

Utilizing the following transformations:

$$
\begin{aligned}
u= & \frac{u_{0}(x+b)^{n}}{1-\gamma t} F^{\prime}(\xi), \\
v & =-\sqrt{\frac{n+1}{2} \frac{v u_{0}(x+b)^{n-1}}{1-\gamma t}}\left(F(\xi)+\xi \frac{n-1}{n+1} F^{\prime}(\xi)\right), \\
\xi= & y \sqrt{\frac{n+1}{2} \frac{u_{0}}{v(1-\gamma t)}(x+b)^{n-1}}, \\
& \Theta(\xi)=\frac{T-T_{\infty}}{T_{w}-T_{\infty}},
\end{aligned}
$$

incompressibility condition is satisfied trivially and Eqs. (2)-(5) become:

$$
\begin{aligned}
& F^{\prime \prime \prime}+F F^{\prime \prime}-\frac{2}{n+1} S\left(F^{\prime}-\frac{\eta}{2} F^{\prime \prime}\right)-\frac{2}{n+1} H a^{2} F^{\prime}=0, \\
& \left(1+\frac{4}{3} R\right) \Theta^{\prime \prime}+\operatorname{Pr} F \Theta^{\prime}-\frac{2}{n+1} S\left(2 \Theta+\frac{\eta}{2} \Theta^{\prime}\right)=0, \\
& F^{\prime}(\alpha)=1, F(\alpha)=\alpha \frac{1-n}{1+n}, \Theta(\alpha)=1, \\
& F^{\prime}(\infty)=0, \Theta(\infty)=0 \text {. }
\end{aligned}
$$

Now we define $F(\xi)=f(\xi-\alpha)=f(\eta)$ and $\Theta(\xi)=\theta(\xi-\alpha)=\theta(\eta)$ then Eqs. (7-9) become:

$$
\begin{aligned}
& \begin{array}{l}
f^{\prime \prime \prime}+f f^{\prime \prime}-\frac{2 n}{n+1} f^{\prime 2} \\
-\frac{2}{n+1} S\left(f^{\prime}-(\xi+\alpha) f^{\prime \prime}\right)-\frac{2}{n+1} H a^{2} f^{\prime}=0,
\end{array} \\
& \begin{array}{l}
\left(1+\frac{4}{3} R\right) \theta^{\prime \prime}+\operatorname{Pr} f \theta^{\prime} \\
-\frac{2}{n+1} S\left(2 \theta+\frac{\xi+\alpha}{2} \theta^{\prime}\right)=0,
\end{array} \\
& \left.\begin{array}{l}
f^{\prime}(0)=1, f(0)=\alpha \frac{1-n}{1+n}=0, \\
f^{\prime}(\infty)=0, \theta(0)=1, \theta(\infty)=0,
\end{array}\right\}
\end{aligned}
$$

where $S\left(=\frac{\gamma}{u_{0}(x+b)^{m-1}}\right)$ represents the unsteadiness parameter, $H a\left(=\frac{\sigma B_{0}^{2}}{\rho u_{0}}\right)$ the Hartman number, $R\left(=\frac{4 \sigma^{*} T_{\infty}^{3}}{k k^{*}}\right)$ the thermal radiation parameter, $\operatorname{Pr}\left(=\frac{\mu c_{p}}{k}\right)$ the Prandtl number and $\alpha\left(\delta \sqrt{\frac{n+1}{2} \frac{u_{0}}{v}}\right)$ the wall thickness parameter.

The skin friction coefficient and local Nusselt number are:

$$
C_{f}=\frac{\tau_{w}}{\rho u_{w}^{2}}, N u_{x}=\frac{(x+b) q_{w}}{k\left(T_{w}-T_{\infty}\right)},
$$

in which surface shear stress $\left(\tau_{w}\right)$ and surface heat flux $\left(q_{w}\right)$ are:

$$
\begin{aligned}
& \tau_{w}=\left.\mu \frac{\partial u}{\partial y}\right|_{y=\delta(x+b)^{\frac{1-n}{2}}}, \\
& q_{w}=\left.\left(-k\left(\frac{\partial T}{\partial y}\right)-\frac{16 \sigma^{*}}{3 k^{*} \rho c_{p}} \frac{\partial T}{\partial y}\right)\right|_{y=\delta(x+b)^{\frac{1\llcorner n}{2}}} .
\end{aligned}
$$

Utilizing Eq. (13) in Eq. (12) the skin friction coefficient and local Nusselt number in dimensionless forms are:

$$
\left.\begin{array}{l}
\sqrt{\operatorname{Re}_{x}} C_{f}=\sqrt{\frac{n+1}{2}} f^{\prime \prime}(0), \\
N u_{x} \operatorname{Re}_{x}^{-1 / 2}=-\left(1+\frac{4}{3} R\right) \sqrt{\frac{n+1}{2}} \theta^{\prime}(0),
\end{array}\right\}
$$

with $\operatorname{Re}_{x}=\frac{u_{w}(x)}{v}(x+b)$ as the local Reynolds number. 


\section{Imran Khan et al.: Thermal properties and time-dependent flow behavior of a viscous fluid SOLUTION METHODOLOGY}

Here we employed the HAM technique in order to acquire the convergent solutions of expressions (9) and (10) subject to the conditions given in Eq. (11). The relevant initial guesses and operators have the following forms:

$$
\begin{aligned}
& \left.\begin{array}{l}
f_{0}(\eta)=\alpha \frac{1-n}{1+n}+\left(1-e^{-\eta}\right), \\
\theta_{0}(\eta)=e^{-\eta},
\end{array}\right\} \\
& \left.\begin{array}{l}
£_{f}=f^{\prime \prime \prime}-f^{\prime}, £_{\theta}=\theta^{\prime \prime}-\theta, \\
\text { with } \\
£_{f}\left[C_{1}+C_{2} e^{\eta}+C_{3} e^{-\eta}\right]=0, \\
£_{\theta}\left[C_{4} e^{\eta}+C_{5} e^{-\eta}\right]=0,
\end{array}\right\}
\end{aligned}
$$

in which $C_{i}(i=1-5)$ elucidate the arbitrary constants. These constants are given as:

$$
\left.\begin{array}{l}
C_{2}=C_{4}=0, C_{3}=\left.\frac{\partial f_{m}^{*}(\eta)}{\partial \xi}\right|_{\eta=0}, \\
C_{1}=-C_{3}-f_{m}^{*}(0), C_{5}=-\theta_{m}^{*}(0) .
\end{array}\right\}
$$

\section{CONVERGENCE ANALYSIS}

The homotopic technique offers extraordinary choice to choose the auxiliary variables $\mathrm{h}_{f}$ and $\mathrm{h}_{\theta}$ concerning control and adjustment about the convergence. Now to determine the suitable values of $\mathrm{h}_{f}$ and $\mathrm{h}_{\theta}$ the $\hbar$-curves have been portrayed in Fig. 1. It is observed that allowable values are $-1.85 \leq \mathrm{h}_{f} \leq-0.15$ and $-1.6 \leq \mathrm{h}_{\theta} \leq$ 0.4 . Moreover, convergence of derived solutions is also ensured numerically through Table 1 . Clearly, $15^{\text {th }}$ order approximations are sufficient for the convergence of momentum and energy expressions.

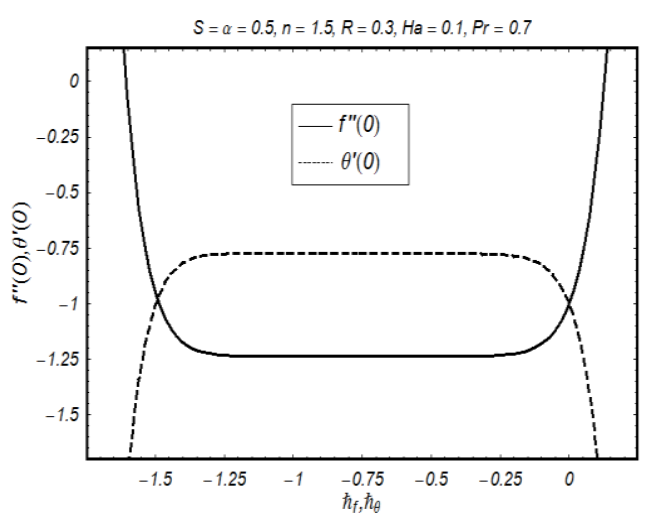

Fig. 1. h-curves for $\boldsymbol{f}^{\prime \prime}(\mathbf{0})$ and $\boldsymbol{\theta}^{\prime}(\mathbf{0})$.

Table 1. Homotopy solutions convergence when $\mathrm{S}=$ $\alpha=0.5, n=1.5, H a=0.1, R=0.3$ and $\operatorname{Pr}=0.7$.

\begin{tabular}{ccc}
\hline $\begin{array}{c}\text { Order of } \\
\text { approximations }\end{array}$ & $-f^{\prime \prime}(0)$ & $-\theta^{\prime}(0)$ \\
\hline 1 & 1.1895 & 0.8122 \\
\hline 5 & 1.2358 & 0.7745 \\
\hline 10 & 1.2359 & 0.7745 \\
\hline 15 & 1.2359 & 0.7745 \\
\hline 20 & 1.2359 & 0.7745 \\
\hline 25 & 1.2359 & 0.7745 \\
\hline
\end{tabular}

This section interprets the effects of influential variables like unsteadiness parameter $(S)$ shape parameter $(\alpha)$ velocity power index $(n)$ Hartman number (Ha) Prandtl number (Pr) and thermal radiation parameter $(R)$ on velocity $\left(f^{\prime}\right)$ temperature $(\theta)$ skin friction $\left(C_{f} \operatorname{Re}_{x}^{1 / 2}\right)$ and local Nusselt number $\left(N u \operatorname{Re}_{x}^{-1 / 2}\right)$. Figs. 2-9 and Tables 2 and 3 are presented for this purpose.

Impact of $S$ on $f^{\prime}$ is indicated in Fig. 2. Here larger $S$ shows smaller rate of stretching in the $x$-direction which ultimately reduce the velocity and thickness of boundary layer. Fig. 3 reveals the characteristics of $\alpha$ on $f$ ' Clearly $f$ ' and related thickness layer are reduced via larger $\alpha$. Physically when $\alpha$ is enhanced for $n<1$, stretching velocity decays which corresponds to a reduction of $f$ ' and related layer thickness. The role of $n$ on $f^{\prime}$ is reported in Fig. 4. Clearly, $f$ ' illustrates growing behavior via larger $n$. Higher $n$ corresponds to an enhancement in stretching rate which yields more deformation in the liquid. Thus velocity distribution boosts. The impact of $\mathrm{Ha}$ on $f^{\prime}$ is disclosed through Fig. 5. Clearly increase in $H a$ decays the velocity ( $\left.f^{\prime}\right)$ Since Lorentz force behaves as retarding force which increments the frictional resistance differing the liquid motion in the momentum layer thickness. Fig. 6 illustrates the effect of $\operatorname{Pr}$ on temperature $(\theta)$ Larger $\operatorname{Pr}$ considerably reduces temperature $(\theta)$ and the associated thermal boundary layer. As thermal diffusivity decays through larger Pr thus heat diffuses away slowly from the heated surface. Characteristics of radiation parameter on temperature $(\theta)$ are interpreted in Fig. 7. An increment in $R$ augments the heat flux from the sheet which corresponds to higher fluid temperature. Hence, the temperature $(\theta)$ and associated thermal layer boost with an increase in $R$. Fig. 8 describes the behavior of $S$ on temperature $(\theta)$ Clearly, temperature $(\theta)$ and associated layer thickness are decaying functions of $S$. Characteristics of $n$ on temperature $(\theta)$ are depicted in Fig. 9. As expected, both temperature and related thickness layer are enhanced via larger $n$.

The role of $S, \alpha$ and $H a$ on surface drag force is computed in Table 2. Here surface drag force enhances via larger $S$ and $H a$ while it decays when $\alpha$ is increased. Table 3 exhibits the impacts of $S, \operatorname{Pr}, R$ and $\alpha$ on Nusselt number. Clearly, Nusselt number augments via larger $S$; for $\operatorname{Pr}$ and $R$, however, opposite situation is noted when $\alpha$ is enhanced. 


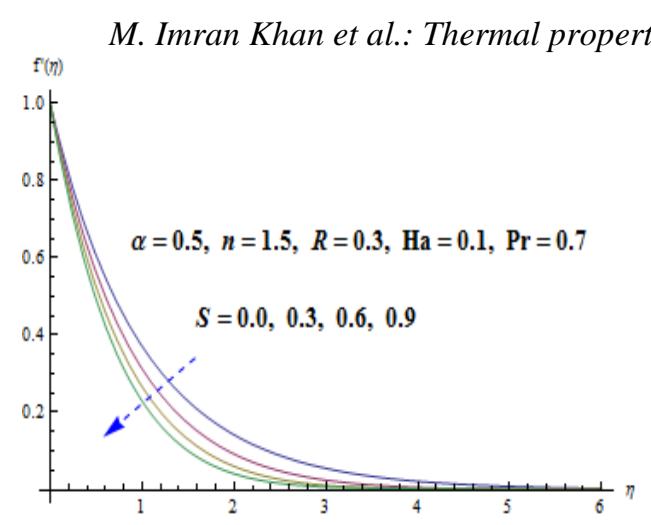

Fig. 2. $\boldsymbol{f}^{\prime}$ against $\mathrm{S}$.

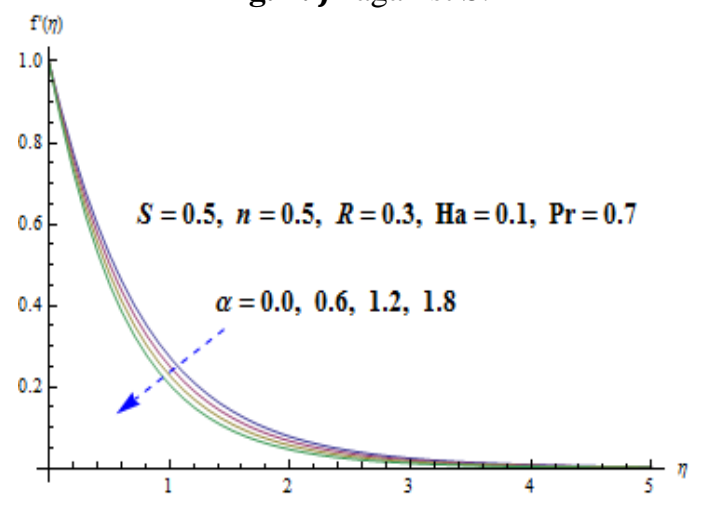

Fig. 3. $\boldsymbol{f}^{\prime}$ against $\alpha$.

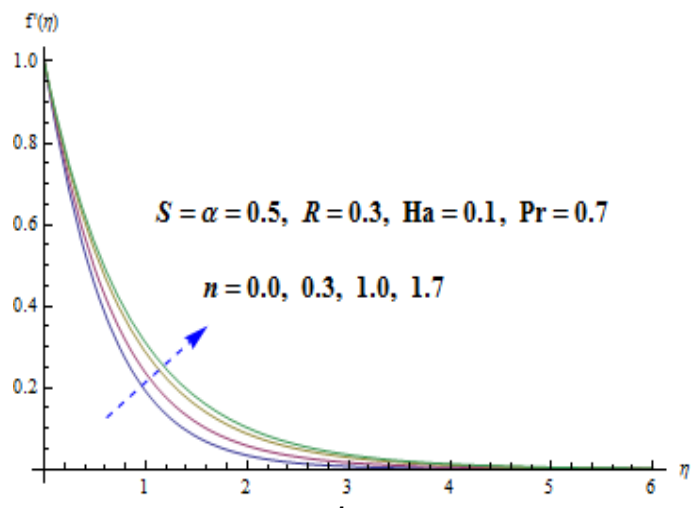

Fig. 4. $\boldsymbol{f}^{\prime}$ against $\mathrm{n}$.

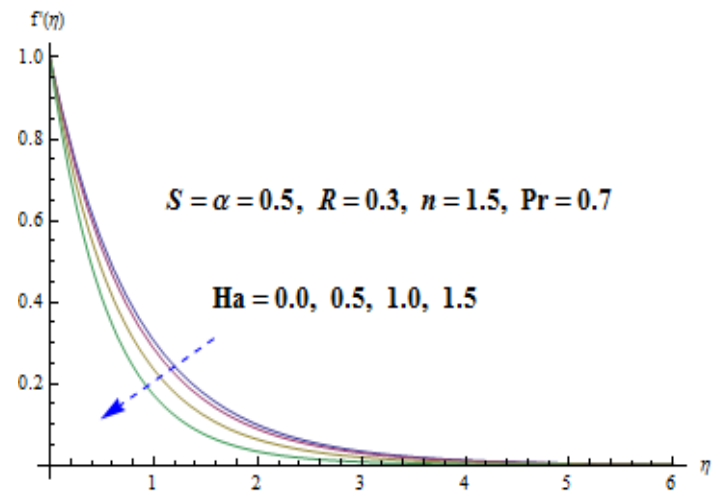

Fig. 5. $\boldsymbol{f}^{\prime}$ against Ha.

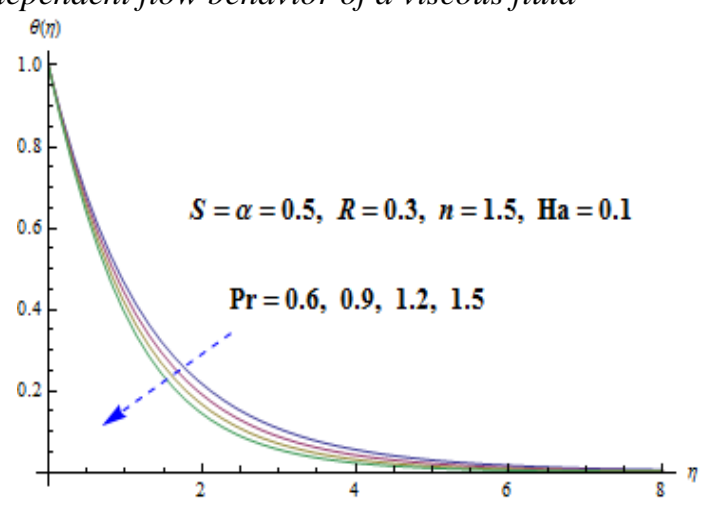

Fig. 6. $\boldsymbol{\theta}$ against $\operatorname{Pr}$.

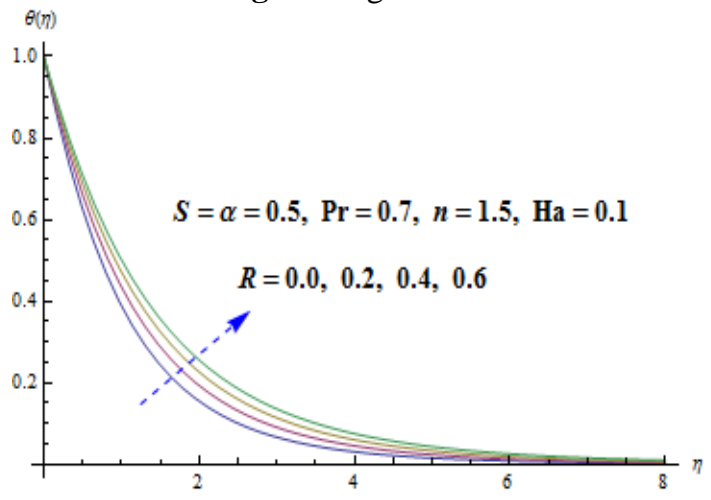

Fig. 7. $\boldsymbol{\theta}$ against R.

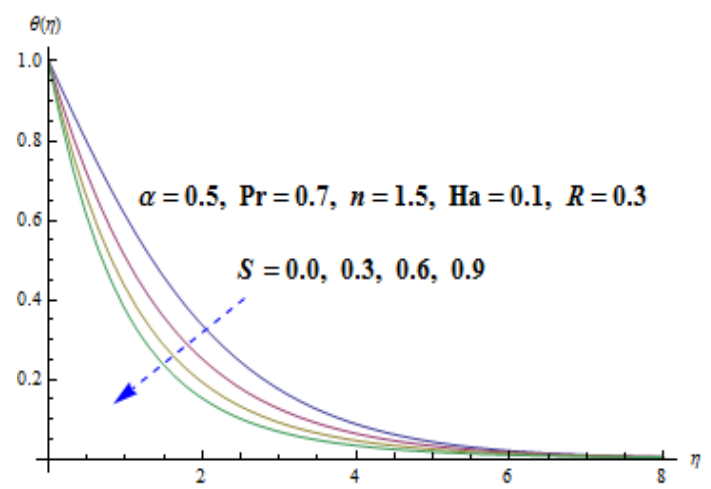

Fig. 8. $\boldsymbol{\theta}$ against $S$.

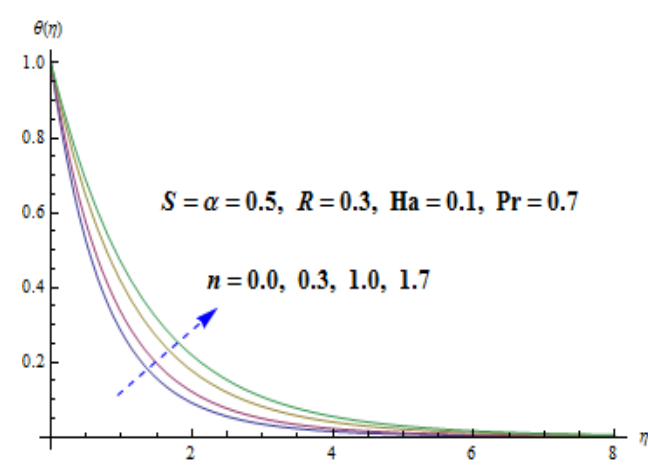

Fig. 9. $\boldsymbol{\theta}$ against $n$. 
M. Imran Khan et al.: Thermal properties and time-dependent flow behavior of a viscous fluid

Table 2. Effects of $S, \alpha$ and $H a$ on skin friction $\left(C_{\mathrm{j}} R e_{\mathrm{x}}{ }^{-1 / 2}\right)$ when $n=1.5, R=0.3$ and $\operatorname{Pr}=0.7$.

\begin{tabular}{cccc}
\hline$S$ & $\alpha$ & $\mathrm{Ha}$ & $-\theta^{\prime}(0)$ \\
\hline 0.0 & & & 1.1377 \\
\hline 0.3 & & & 1.2899 \\
\hline 0.6 & & & 1.4255 \\
\hline 0.5 & 0.0 & & 1.4377 \\
\hline & 0.6 & & 1.3709 \\
\hline & 1.2 & & 1.3073 \\
\hline & 0.5 & 0.0 & 1.3784 \\
\hline & & 0.4 & 1.4327 \\
\hline & & 0.8 & 1.5852 \\
\hline
\end{tabular}

Table 3. Effects of $S, \operatorname{Pr}, R$ and $\alpha$ on Nusselt number $\left(N u_{\mathrm{x}} R e_{\mathrm{x}}^{-1 / 2}\right)$ when $\mathrm{Ha}=0.1$ and $n=1.5$.

\begin{tabular}{|c|c|c|c|c|}
\hline$S$ & $P r$ & $R$ & $\alpha$ & $N u_{x} R e_{x}^{-0.5}$ \\
\hline 0.0 & & & & 1.3081 \\
\hline 0.3 & & & & 1.3981 \\
\hline 0.6 & & & & 1.4825 \\
\hline \multirow[t]{9}{*}{0.5} & 0.8 & & & 1.2265 \\
\hline & 0.9 & & & 1.2416 \\
\hline & 1.0 & & & 1.2569 \\
\hline & 0.7 & 0.0 & & 1.0271 \\
\hline & & 0.2 & & 1.1536 \\
\hline & & 0.4 & & 1.2666 \\
\hline & & 0.3 & 0.0 & 1.2496 \\
\hline & & & 0.6 & 1.2041 \\
\hline & & & 1.2 & 1.1602 \\
\hline
\end{tabular}

\section{CONCLUSIONS}

This research aims to explore the impact of unsteady variably thick surface in a magnetohydrodynamic (MHD) radiative flow of a viscous liquid. The presented research has the following key points:

- Unsteadiness parameter (S) has similar impact on velocity and temperature.

- Larger wall thickness parameter $(\alpha)$ and Hartman number $(\mathrm{Ha})$ reduce both the velocity and the related thickness of boundary layer.

- Temperature of the liquid improves via larger radiation factor $(R)$.

- Impacts of unsteadiness parameter $(S)$ and Prandtl number (Pr) on temperature distribution are similar in a qualitative manner.

- Surface drag force is noted to have a decaying function on larger wall thickness parameter $(\alpha)$, however, it boosts when unsteadiness parameter $(S)$ and Hartman number $(H \alpha)$ are enhanced.

- $\quad$ Impact of $S, \operatorname{Pr}$ and $R$ on Nusselt number is opposite to $\alpha$.

- The case for steady variably thick surface can be recovered by putting $\gamma=0$.

\section{REFREENCES}

1. H. Ajam, S. S. Jafari, N. Freidoonimehr, Ain Shams Eng. J., (2016), DOI: 10.1016/j.asej.2016.03.006
2. S. Mukhopadhyay, Ain Shams Eng. J., 4, 317 (2013).

3. T. Hayat, M. Waqas, S. A. Shehzad, A. Alsaedi, J. Hydrol. Hydromech., 63, 311 (2015).

4. M. Waqas, M. Farooq, M. I. Khan, A. Alsaedi, T. Hayat, T. Yasmeen, Int. J. Heat Mass Transf., 102, 766 (2016).

5. M. Waqas, T. Hayat, M. Farooq, S. A. Shehzad, A. Alsaedi, Cattaneo-Christov, J. Mol. Liq., 220, 642 (2016).

6. T. Hayat, M. I. Khan, M. Waqas, A. Alsaedi, T. Yasmeen, Chinese J. Chem. Eng., 25, (2017) 10.1016/j.cjche.2016.06.008.

7. B. I. Olajuwon, J. I. Oahimire, M. Ferdow, Eng. Sci. Tech. an Int. J., 17, 185 (2014).

8. B. Mahanthesh, B. J. Gireesha, R. S. R. Gorla, J. Nigerian Math. Soc., 35, 178 (2016).

9. T. Hayat, M. Waqas, S. A. Shehzad, A. Alsaedi, $J$. Mol. Liq., 215, 704 (2016).

10. T. Hayat, M. I. Khan, M. Waqas, A. Alsaedi, M. Farooq, Computer Methods Appl. Mech. Eng., 315, 1011 (2017).

11. N. Sandeep, C. Sulochana, Eng. Sci. Tech. an Int. J., 18, 738 (2015)

12. B. Mahanthesh, B. J. Gireesha, R. S. R. Gorla, J. Assoc. Arab Univ. Basic Appl. Sci., 23, (2017) DOI: 10.1016/j.jau as.2016.05.004.

13. M. Khan, W. A. Khan, AIP Adv., 6, 025211 (2016).

14. T. Hayat, S. Farooq, A. Alsaedi, B. Ahmad, Results Phys., 7, (2017). DOI: 10.1016/j.rinp.2016.12.048.

15. T. Hayat, M. I. Khan, M. Waqas, A. Alsaedi, Colloid Surf. A-Physicochem. Eng. Asp., 518, 263 (2017).

16. T. Hayat, M. I. Khan, M. Waqas, A. Alsaedi, J. Mol. Liq., 231, 126 (2017).

17. T. Fang, J. Zhang, Y. Zhong, Appl. Math. Comput., 218, 7214 (2012)

18. M. M. Khader, A. M. Megahed, European Physical Journal Plus, 100, 128 (2013).

19. M. I. Khan, T. Hayat, M. Waqas, A. Alsaedi, J. Mol. Liq., 230, 143 (2017).

20. S. J. Liao, Homotopic analysis method in nonlinear differential equations, Springer, Heidelberg, Germany, 2012.

21. T. Hayat, M. I. Khan, M. Farooq, T. Yasmeen, A. Alsaedi, J. Mol. Liq., 220, 1121 (2016).

22. T. Hayat, M. Waqas, M. I. Khan, A. Alsaedi, Int. J. Heat Mass Transf., 102, 1123 (2016).

23. M. Turkyilmazoglu, Filomat, 30, 1633 (2016).

24. T. Hayat, M. Waqas, S. A. Shehzad, A. Alsaedi, J. Mol. Liq., 215, 704 (2016).

25. T. Hayat, M. I. Khan, M. Farooq, A. Alsaedi, M. Waqas, T. Yasmeen, Impact of Cattaneo-Christov heat flux model in flow of variable thermal conductivity fluid over a variably thick surface, Int. J. Heat Mass Transfer, 99, 702 (2016).

26. T. Hayat, M. I. Khan, M. Waqas, A. Alsaedi, Results Phys., 7, 256 (2017). 


\title{
The eliminatory effects of cold argon plasma jet on aflatoxin B1 produced by different isolates of Aspergillus section nigri
}

\author{
S. Hassanpour ${ }^{1}$, M. Bayat ${ }^{1 *}$, A. Chaichi Nosrati ${ }^{2}$, M. Ghorannevis ${ }^{3}$, S. Hashemi ${ }^{4}$ \\ ${ }^{I}$ Department of Microbiology, Science and Research Branch, Islamic Azad University, Tehran, Iran \\ ${ }^{2}$ Department of Molecular \& Cell Biology, Lahijan Branch, Islamic Azad University, Lahijan, Iran \\ ${ }^{3}$ Plasma Physics Research Center, Science and Research Branch, Islamic Azad University, Tehran, Iran \\ ${ }^{4}$ Department of Medical Parasitology and Mycology, School of Public Health, Tehran University of Medical Science, \\ Tehran, Iran
}

Received December 16, 2017; Revised August 21, 2018

\begin{abstract}
Aflatoxin and ochratoxin are among the most resistant and severe toxins produced by Aspergillus spp. The aim of this study was an evaluation of eliminatory effects of cold argon plasma jet on aflatoxin B1. Sampling of wheat, corn, oatmeal, flour and rice products from northern cities of Iran was carried out. The fungal species were cultured on CHAPK medium and next to sabouraud dextrose broth + malt extract (SB + ME) and also SB + yeast extract (SB + YE) media to obtain aflatoxin B1. The ELISA test was conducted to measure the aflatoxin level. The mean initial concentration of the aflatoxin from charts analysis in the SB +ME medium was $16.106 \mu \mathrm{g} / \mathrm{kg}(10.4,8.32$ and $7.55 \mu \mathrm{g} /$ $\mathrm{kg}$ at 30, 60 and $360 \mathrm{sec}$, respectively), and in the $\mathrm{SB}+\mathrm{YE}$ medium from $23.699 \mu \mathrm{g} / \mathrm{kg}(12.82,9.93$ and $9.54 \mu \mathrm{g} / \mathrm{kg}$, respectively), at the same time. Furthermore, the mean concentrations of aflatoxin B1 by tables analysis in the SB+ ME medium changed from $20.02 \mu \mathrm{g} / \mathrm{kg}$ to $6.87,10.46$ and $7.55 \mu \mathrm{g} / \mathrm{kg}$, at 30,60 and $360 \mathrm{sec}$, respectively, and in the medium SB +YE from $28.85 \mu \mathrm{g} / \mathrm{kg}$ reached $6.56 .6,8.43$ and $7.02 \mu \mathrm{g} / \mathrm{kg}$, respectively, at the same time. In this study, the change or decrease in the concentration of aflatoxin B1, from Lin/Log and Log/Lin analyses of charts was significant $(\mathrm{p}<0.05)$ at $60 \mathrm{~s}$ and $360 \mathrm{~s}$ of application of the cold argon plasma jet in both media. The cold plasma as a new technology in various fields of food and agriculture can provide effective and suitable solutions in order to promote the goals of food industry, especially in the areas of mycotoxin elimination.
\end{abstract}

Keywords: Aspergillus nigri, Aflatoxins, Cold plasma jet, ELISA

\section{INTRODUCTION}

Aspergillus species are classified among the most important group of pathogenic and destructive fungi with over 900 species present, and 50 of which produce toxic metabolites. The economic importance of this genus is also because of the production of acids or enzymes by a number of species [1]. Aspergillosis, a disease caused by these species, is a major cause of degradation of agricultural products before and after cultivation via the production of various mycotoxins, and this is the most crucial harmful effect. The species produce highly potent toxins with long-lasting and severe effects $[2,3]$. The most important toxins of this species which function in human and animal food industries include aflatoxin, ochratoxin A, citrinin, cyclopiazonic acid, patulin, sterigmatocystin and some other thermogenic toxins $[4,5]$.

Mycotoxins, along with other fungal metabolites, such as antibiotics and alkaloids, are compounds produced by the fungal cells in the late stages of growth of the fungus. These secondary metabolites apparently act as a defense mechanism and are produced in human and animal food when suitable conditions exist. Obtaining of polyacetyl-derived mycotoxins is the most important pathway for mycotoxin biosynthesis due to the involvement of acetyl coenzyme A, which produces mycotoxins such as aflatoxin, ochratoxin, patulin, citrinin, and pentanedioic acid $[6,7]$.

The most important mycotoxins produced by Aspergillus species include aflatoxin and ochratoxin. Aflatoxins are a large group of mycotoxins produced by certain species of Aspergillus. This group of fungal toxins is considered the head of all mycotoxins [8]. These toxins are common pollutants of cereals and oilseeds, starch, dried fruit, and coffee. The metabolites produced by aflatoxins include aflatoxin M1 and M2. Aflatoxin M1 and M2 are mono-hydroxyl derivatives of aflatoxins $\mathrm{B} 1$ and B2, respectively, stored in human milk and lactating animals and formulated and secreted through diets contaminated of aflatoxins B1 and B2 [9].

The aflatoxin $\mathrm{B} 1$ has a molecular weight of 312 Dalton and its structural formula is $\mathrm{C}_{17} \mathrm{H}_{12} \mathrm{O}_{6}$. This compound is destroyed at $268-269{ }^{\circ} \mathrm{C}$ without melting. Its maximum optical absorption is at 265 , 262, and $223 \mathrm{~nm}$. Aflatoxins, in spite of the high resistance to human and animal nutrition, are inactive at $\mathrm{pH}$ values above 10 and below 3 . Likewise, oxidizing compounds and ultraviolet

* To whom all correspondence should be sent:

E-mail: Dr_mansour_bayat@yahoo.com

(C) 2019 Bulgarian Academy of Sciences, Union of Chemists in Bulgaria 
S. Hassanpour et al.: The eliminatory effects of cold argon plasma jet on aflatoxin $B 1$ produced by different isolates ...

radiation in the presence of oxygen cause the inactivation of some types of aflatoxins. According to the food and drug organization (FAO) annual report, $20 \%$ of the world's food products are contaminated by fungal toxins, mostly including aflatoxins $[10,11]$.

The plasma is, in fact, the fourth state of matter and is a set of particles produced by an energy source such as an electrical power supply in the environment. The cold or non-thermal plasma is a kind of plasma that can be produced simply and quickly using gas evacuation. The use of plasma systems is a new method of sterilization. The ability of sterilization at relatively low temperatures and in short periods leads to non-toxicity of the plasma. In this way, all microorganisms are killed without causing pathogenesis. In plasma sterilization, in addition to killing bacteria, dead bacteria are removed from the surface of the material due to a process similar to that of plasma engraving [12-14].

Until now, efforts have been performed to use different types of plasma to eliminate existing microorganisms in the food. However, no research has been conducted in Iran on the use of atmospheric plasma jet to mycotoxins elimination. The aim of this study was to investigate the effects of cold argon plasma jet on aflatoxin B1 produced by different isolates of Aspergillus nigri.

\section{METHODOLOGY}

\section{Sampling}

Sampling of wheat, corn, oatmeal, flour and rice products from the northern cities of Iran was carried out. Samples were transferred to the laboratory immediately after collection in order to determine the amount of aflatoxin B1 and to monitor the effect of plasma jet on mycotoxin at $4^{\circ} \mathrm{C}$.

\section{Culture conditions}

In this study, a photochemical culture medium was used for enrichment, isolation, and production of mycotoxin of Aspergillus from Sabouraud dextrose agar broth environments, malt extract and yeast extract (Merck, Germany).

Culturing the specimens in a CHAPK agar medium. For isolation of aflatoxin $\mathrm{B} 1$ bearing fungi, the samples were cultured in a CHAPK agar medium and incubated for 2 weeks at $25{ }^{\circ} \mathrm{C}$. In order to identify fungi, their morphological characteristics were determined, such as colony color, landscape view of the colony for the presence of folding and breaker, radial lines or concentric circles, smooth or folded colony levels, and also the colony level with respect to the identification keys of Barnett and Hunter, Click and Dugan.
Cultivation in a liquid medium. In order to increase mycotoxin production, the sabouraud dextrose broth + malt extract $(\mathrm{SB}+\mathrm{ME})$ and also sabouraud dextrose broth + yeast extract $(\mathrm{SB}+\mathrm{YE})$ media were applied. Isolated species were transferred to the liquid medium and incubated at $25^{\circ} \mathrm{C}$ for 2 weeks in a shaker incubator. To prevent drying of liquid media, phosphate salt buffer was added to the culture media.

Preparation of slide culture from selected fungal species. The slide culture was prepared for each species previously grown in CHAPK media. Using a sterile scalp, a portion of the medium was cut into square centimeters and placed on a slide. Then, it was put on a U-shaped tube inside a glass plate; this U-shaped tube was used to prevent the contact of the plate with the slide on it. Then the isolation of species was inoculated into 4 dots of the medium and the sterilized small slide on the tray. The agar was incubated for 14 days at $25{ }^{\circ} \mathrm{C}$. After growth, the fungal species were investigated using lactophenol cotton blue on the slide under the optical microscope.

\section{Extraction of mycotoxins from culture media}

After incubation for two weeks, extraction and purification of mycotoxins were performed from pure strains in a non-cellular medium. For this purpose, the tubes containing the liquid medium were subjected to vortex with an angle of 35 degrees for at least $15 \mathrm{~min}$ and then kept at $-70^{\circ} \mathrm{C}$. After defrosting at the laboratory temperature, 2.5 $\mathrm{ml}$ of extraction solvent was added and the sample was vortexed twice for $15 \mathrm{~min}$. At the final stage, the mycotoxins were separated from the supernatant using a funnel and filter paper, transferred into a sterile micro-tube and stored at $4^{\circ} \mathrm{C}$ for further study.

Extraction of mycotoxins from the CHAPK medium. The CHAPK agar was cut by a sterile scalpel to $3 \times 1 \mathrm{~cm}$ sized portions, inoculated into a sterile falcon tube $(10 \mathrm{cc})$ containing $5 \mathrm{cc}$ of phosphate salt solution and placed in a freezer at 70 ${ }^{\circ} \mathrm{C}$ after being vortexed for a minimum of $15 \mathrm{~min}$. The next step, the same as the extraction of mycotoxin from the broth medium, was performed using an extraction solvent and Whatman filter paper.

\section{Analysis of aflatoxin B1 by ELISA test}

The ELISA test was implemented with the Biotech-ELX 800 device. An enzyme kit and aflatoxin B1 standards were provided from the RBiopharm Corporation, Germany. Aflatoxin B1 kits (R1211) contain competitive immunoassay enzyme to determine the amounts of these toxins in foods. 


\section{S. Hassanpour et al.: The eliminatory effects of cold argon plasma jet on aflatoxin B1 produced by different isolates ...}

The extraction protocol was used according to the instruction.

Accordingly, $50 \mu \mathrm{l}$ of the standard aflatoxin solutions $(0,1,5,10,20$ and $50 \mathrm{ppb})$ and $50 \mu \mathrm{l}$ of the specimen were added separately to microtiter wells in duplicate. Then $50 \mu \mathrm{l}$ of the conjugated enzyme was added to each well and incubated for $10 \mathrm{~min}$ at $20-25^{\circ} \mathrm{C}$. In the next step, $50 \mu \mathrm{L}$ of antiaflatoxin antibody solution was added to each well and incubated at 25 to $20{ }^{\circ} \mathrm{C}$. Wells were washed three times with deionized water. In the next step, $100 \mu \mathrm{l}$ of the substrate / chromogenic solution was added to each well and incubated in dark space at 20-25 ${ }^{\circ} \mathrm{C}$. In the final step, $100 \mu \mathrm{l}$ of the stopping solution was added and after $10 \mathrm{~min}$, absorption was measured with ELISA reader at OD $=450 \mathrm{~nm}$. The standard calibration curve was drawn based on the percentage of absorption of standard samples and the amount of aflatoxin in the samples. Subsequently, aflatoxin content of each sample was obtained on the basis of the absorbance of each sample and its correlation with the calibration curve in a $\mu \mathrm{g} / \mathrm{kg}$ scale.

\section{Elimination of mycotoxins with the cold atmospheric plasma jet}

Argon gas was used to create the plasma and to eliminate mycotoxins. In this device, the chamber in which the plasma is formed is helix-shaped to ensure better contact of the plasma jet with the level of mycotoxin. The length of the helix pipe is $12 \mathrm{~cm}$ and its internal and external diameter is 2 and 3 $\mathrm{mm}$, respectively. Its core diameter is $3 \mathrm{~cm}$ and its distance is $1.5 \mathrm{~cm}$. The two ends of the helix are divided into three ways to allow the simultaneous entry of gas and mycotoxin and can be separated from each other at the exit site.

The output of the power supply was adjusted to $50 \mathrm{kV}, 100$ Watt and the electron frequency to 30 $\mathrm{kHz}$, and its values were measured with high voltage probes and oscilloscope. Then, at a gas flow rate of 6 liters per min and a high potential difference between the two electrodes, the effect of the cold atmospheric argon gas plasma jet were investigated at 30,60 and $360 \mathrm{sec}$.

\section{Statistical analysis}

The obtained data were analyzed by SPSS 18 software and one-way ANOVA for comparison of aflatoxin B1 levels in wheat, corn, barley, flour and rice samples. P-value $<0.05$ determined a significant result.

\section{RESULTS}

\section{Adoption of mycotoxin producing Aspergillus spp.}

In this study, five different food sources (wheat, corn, oats, flour, and rice) were sampled and 12 different Aspergillus species were identified among them. To compare the results and control the production of mycotoxins among isolates, the $A$. nigri standard strain was used.

ELISA assay. The ELISA assay was implemented before and after the application of the cold atmospheric plasma jet pressure on aflatoxin B1 mycotoxin. The concentration of aflatoxin B1 was higher than the limit determined in the national standard of Iran among more than $90 \%$ of wheat, corn, oatmeal, flour and rice samples. The permitted concentration of aflatoxin B1 according to the national standard of Iran, entitled "Human Feed - Livestock - Maximum Tolerability of Mycotoxins", with the number: 5925 is equal to 5 ppb.

\section{Aflatoxin B1 concentration measurement by the analysis of charts by Lin/Log and Log/Lin analysis}

The aflatoxin B1 concentration measurement was obtained by the analysis of Lin / Log charts in sabouraud dextrose broth + malt extract $(\mathrm{SB}+\mathrm{ME})$ and yeast extract $(\mathrm{SB}+\mathrm{YE})$.

The mean initial concentration of aflatoxin from charts analysis in the SB +ME medium was 16.106 $\mu \mathrm{g} / \mathrm{kg}$ and it was $10.4,8.32$ and $7.55 \mu \mathrm{g} / \mathrm{kg}$ at 30 , 60 and $360 \mathrm{sec}$, respectively. In the SB +YE medium, from $23.699 \mu \mathrm{g} / \mathrm{kg}$ it reached 12.82, 9.93 and $9.54 \mu \mathrm{g} / \mathrm{kg}$, respectively, at the same time.

Furthermore, the mean concentration of aflatoxin B1 by tables analysis of $\log /$ Lin charts in SB+ ME medium altered from $20.02 \mu \mathrm{g} / \mathrm{kg}$ to 6.87 , 10.46 and $7.55 \mu \mathrm{g} / \mathrm{kg}$, at 30,60 and $360 \mathrm{sec}$, respectively, and in the SB +YE medium from $28.85 \mu \mathrm{g} / \mathrm{kg}$ it reached 6.56.6, 8.43 and $7.02 \mu \mathrm{g} /$ $\mathrm{kg}$, respectively, at the same time.

As noted above, the change or decrease in the concentration of aflatoxin B1, from $\mathrm{Lin} / \mathrm{Log}$ and $\mathrm{Log} / \mathrm{Lin}$ analyses of charts was significant $(\mathrm{p}<0.05)$ at $60 \mathrm{~s}$ and $360 \mathrm{~s}$ of the application of the cold argon plasma jet in both media and likewise, from the analysis of tables results, it was significantly decreased at all the times at which the cold argon gas plasma jet was applied. The Log/Lin, Lin/Log results and their comparison are shown in diagrams 1,2 and 3. The concentrations of aflatoxin in dextrose broth + malt extract and dextrose broth + yeast extract are shown in Table 1.

According to the obtained data from the analysis of $\mathrm{Log} / \mathrm{Lin}$ and $\mathrm{Lin} / \mathrm{Log}$ aflatoxin B1, at 30 and 360 $\mathrm{s}$, the $\mathrm{Log} / \mathrm{Lin}$ analysis exhibits a stronger decrease compared to the Lin/Log analysis. However, at the $60 \mathrm{~s}$ treatment, the Lin/Log analysis demonstrated a stronger decrease than $\mathrm{Log} / \mathrm{Lin}$ analysis. According to the results analysis, the $\operatorname{Lin} / \log$ showed that 
S. Hassanpour et al.: The eliminatory effects of cold argon plasma jet on aflatoxin B1 produced by different isolates ... aflatoxin after $60 \mathrm{sec}$ in the ME+SB decreased by $56 / 65 \%$ and in YE+SB decreased by $41.85 \%$. Likewise, after $360 \mathrm{~s}$ in the ME+SB $46.87 \%$ and from $60 \mathrm{~s}-360 \mathrm{~s}$ it decreased by $9.25 \%$. Moreover, the reduction of toxin in the YE+SB after $360 \mathrm{~s}$ was $40.26 \%$ and after $60 \mathrm{~s}-360 \mathrm{~s}$ the reduction was $3.83 \%$

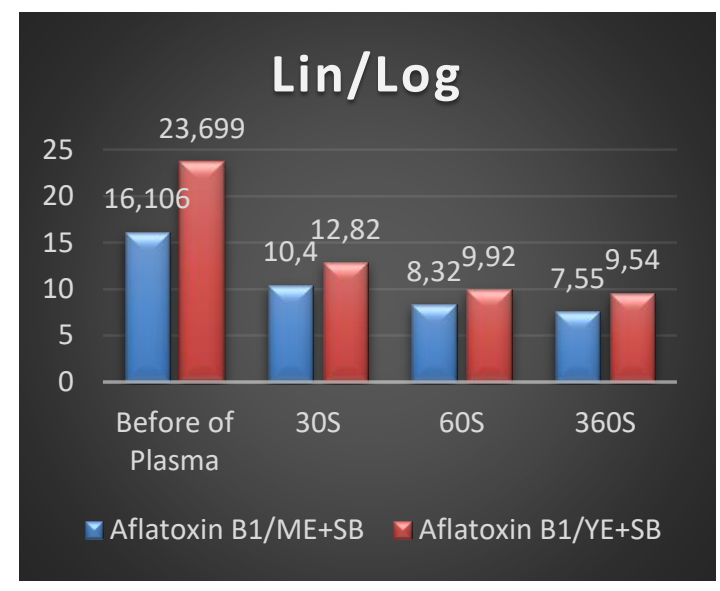

Diagram 1. Average concentration of aflatoxin B1 before and after exposure to plasma (Lin/Log results)

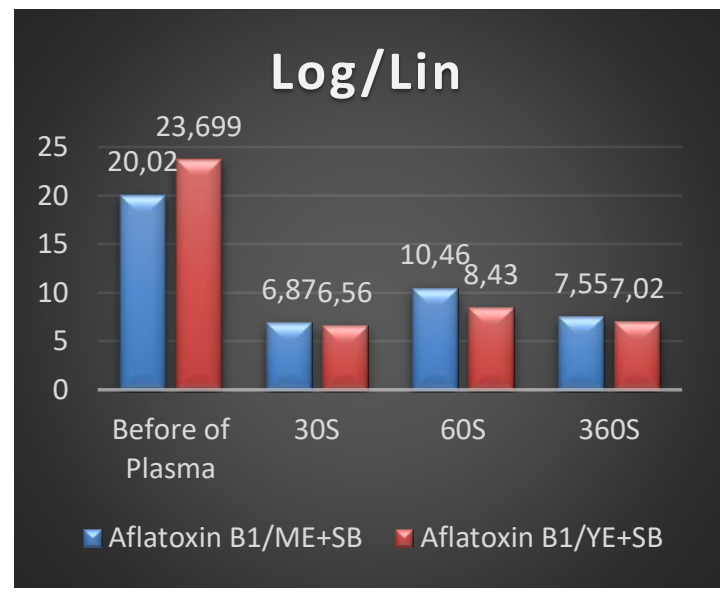

Diagram 2. Average concentration of aflatoxin B1 before and after exposure to plasma ( $\mathrm{Log} / \mathrm{Lin}$ results)

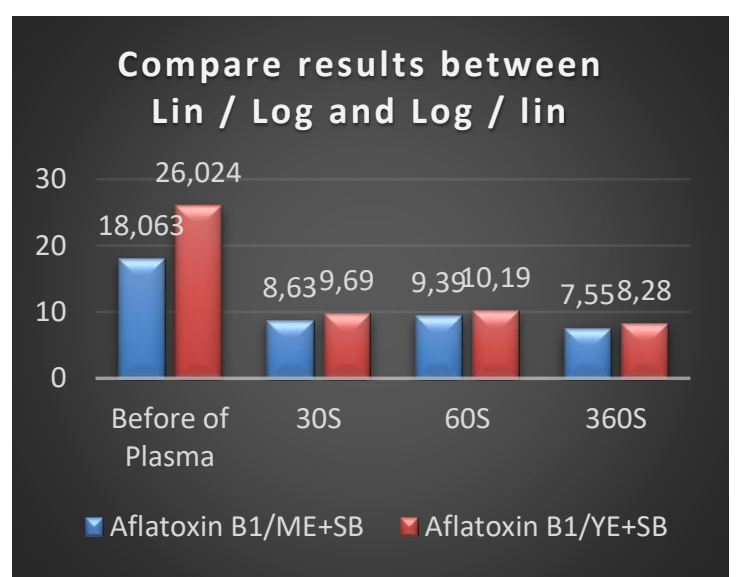

Diagram 3. Average concentration of aflatoxin B1 before and after exposure to plasma (Comparison of results between $\operatorname{Lin} / \log$ and $\log / \operatorname{Lin}$ )
Table 1. Concentrations of aflatoxin in dextrose broth + malt extract and dextrose broth + yeast extract

\begin{tabular}{|c|c|c|c|c|}
\hline \multirow{3}{*}{ SB+ME } & \multicolumn{4}{|c|}{ AFB1 } \\
\cline { 2 - 5 } & & $\mathrm{c} / \mathrm{m} 30$ & $\mathrm{c} / \mathrm{m} 60$ & $\mathrm{c} / \mathrm{m} \mathrm{360}$ \\
\cline { 2 - 5 } & Lin/Log & 7.87 & 8.78 & 8.78 \\
\cline { 2 - 5 } & $\mathrm{Log} / \mathrm{Lin}$ & 6.87 & 10.46 & 7.55 \\
\hline \multirow{3}{*}{$\mathrm{SB}+\mathrm{YE}$} & & $\mathrm{c} / \mathrm{y} 30$ & $\mathrm{c} / \mathrm{y} 60$ & $\mathrm{c} / \mathrm{y} 360$ \\
\cline { 2 - 5 } & $\mathrm{Lin} / \mathrm{Log}$ & 7.73 & 7.29 & 7.29 \\
\cline { 2 - 5 } & $\mathrm{Log} / \mathrm{Lin}$ & 6.56 & 8.43 & 7.02 \\
\hline
\end{tabular}

\section{DISCUSSION}

The inhibitory effect of the cold plasma was firstly demonstrated by Kung et al. (2009) against bacteria [15]. The cold atmospheric plasmas have the ability to inactivate microorganisms under controlled laboratory conditions. In 2001, Moissan et al. performed sterilization using jet plasma. They used a plasma jet machine at a frequency of $13 / 56$ $\mathrm{MHz}$ against a highly resistant spore-forming bacillus species [16].

In 2005, Larusi examined the sterilization using argon microwave plasma at atmospheric pressure at a frequency of $2.45 \mathrm{GHz}$ on Escherichia coli and Staphylococcus aureus bacterial species. The results of this study confirmed that the effects of this plasma at atmospheric pressure needed less sterilization time due to free radicals and ultraviolet radiation produced during plasma motility, for the high microwave plasma density, high free radicals and high ultraviolet light. The results showed that both bacteria were completely sterilized in less than one second, regardless of bacterial strains [17].

Bassaran observed that cold low-pressure plasma was developed using sulfur hexafluoride gases (SF6) and they tested it for its antifungal effect on Aspergillus parasiticus in nuts and treated them with both gas plasma and SF6 plasma for $20 \mathrm{~min}$. When the effect of plasma treatment on aflatoxins was tested, the gas plasma exhibited 50\% reduction in the total aflatoxins (AFB1, AFB2, AFG1, AFG2), but only $20 \%$ reduction was measured in the total aflatoxin by SF6 plasma treatment after 20 min. In these experiments, they concluded that the use of plasma is a more efficient approach to eliminate aflatoxin produced by fungi [18].

In our study, toxin decontamination (aflatoxin B1) by the cold plasma jet was investigated at atmospheric pressure of argon gas. In summary, it can be stated that many disinfection methods, including chemical, physical, dry or wet heat, are used to disinfect solid surfaces or liquids and biological surfaces such as skin with functional limitations. The use of non-aggressive plasma technology in cleansing microbes and toxin removal has advantages over existing methods, including high decontamination properties during 
S. Hassanpour et al.: The eliminatory effects of cold argon plasma jet on aflatoxin $B 1$ produced by different isolates ...

short flow, lack of remaining toxicity, and the costeffectiveness of argon gas. In the Pearson correlation ( Lin / Log) analysis of the aflatoxin B1 concentrations in $\mathrm{ME}+\mathrm{SB}$ and $\mathrm{YE}+\mathrm{SB}$ media after treatment with the plasma jet the aflatoxin $\mathrm{B} 1$ reduction in $\mathrm{ME}+\mathrm{SB}$ medium was stronger than that in the $\mathrm{YE}+\mathrm{SB}$ medium and there was a significant statistical correlation.

Furthermore, a significant correlation was observed between the levels of aflatoxin B1 toxicity and the increase in the plasma treatment time in both ME + SB and YE + SB media. The analysis of the results showed that aflatoxin $\mathrm{B} 1$ concentrations in $\mathrm{ME}+\mathrm{SB}$ and $\mathrm{YE}+\mathrm{SB}$ media decreased with increasing the cold plasma jet treatment time. As it was stated, the comparison of the reduction ratio and correlation of changes in the amount of aflatoxin $\mathrm{B} 1$ toxin in the two $\mathrm{ME}+\mathrm{SB}$ and $\mathrm{YE}+$ SB media after $60 \mathrm{sec}$, the plasma effect was statistically confirmed to be more efficient in the $\mathrm{ME}+\mathrm{SB}$ than in the YE + SB medium.

In similar studies in 2017, Devi et al. used the effect of cold plasma jet at atmospheric pressure with argon gas on the elimination of mycotoxins produced by Aspergillus species. In their study, they were able to remove more than $97 \%$ of aflatoxin B1 produced by Aspergillus parasiticus and more than 95 aflatoxins produced by Aspergillus flavus by plasma emission at 15 and 12 min, respectively [19].

Ouf et al. [20] employed the low-pressure cold plasma for toxin elimination in 2015 and demonstrated removal of both aflatoxin and ochratoxin produced by Aspergillus nigri at a rate of 3.5 and 7.5 liter per min, respectively [20].

\section{CONCLUSION}

According to the results obtained from this study, the non-thermal (cold) atmospheric plasma jet can exert fungicidal and mycotoxin eliminatory effects. Due to the presence of charged particles of electrons and ions, ultraviolet radiation, free radicals and reactive chemical species, the plasma can cause changes in the cell wall, morphology, or genetic characteristics of microorganisms and destroy them. As a result, the cold plasma as a new technology in various fields of food and agriculture can provide effective and suitable solutions in order to promote the goals of the food industry, especially in the areas of mycotoxin elimination.

It can be deduced from Lin/Log charts and tables that aflatoxin B1 decontamination was performed with the help of compression of argon gas over time and the mean initial concentration of samples was significantly comparable with the mean concentration after each plasma radiation. It was concluded that the aflatoxin B1 detoxification was successful in all samples, indicating the high efficiency of the cold atmospheric plasma jet, especially in the food industry.

\section{REFERENCES}

1. C. Paulussen, J. E. Hallsworth, S. Álvarez-Pérez, W. C. Nierman, P. G. Hamill, D. Blain, H. Rediers, B. Lievens, Microbial Biotechnology, 10, 296 (2017).

2. D. W. Denning, Clin. Infect. Dis., 26, 781 (1998).

3. B. H. Segal, N. Engl. J. Med., 360, 1870 (2009).

4. R. M. Duran, J.W. Cary, A. M. Calvo, Appl. Microbiol. Biotechnol., 73, 1158 (2007).

5. A. M. Abdelhamid, Arch. Tierernahr., 40, 647 (1990).

6. J. W. Bennett, M. Klich, Clin. Microbiol. Rev., 16, 497 (2003).

7. N. Chamkouri, S., Khodadoust, F. Ghalavandi, Journal of Chromatographic Science, 53, 10 (2015).

8. C. P. Wild, Y. Y. Gong, Carcinogenesis, 31, 71, (2010).

9. M. F. Dutton, K. Ehrlich, J. W. Bennett, Appl. Environ. Microbiol., 49, 1392 (1985).

10. L. A. Loeb, C. C. Harris, Cancer Research, 68, 6863 (2008).

11. M. C. Smith, S. Madec, E. Coton, N. Hymery, Toxins, 8, 94 (2016).

12. K. Matra, S. Wongkuan, Procedia Computer Science, 86, 313 (2016).

13. A. Akbari, N. Chamkouri, A. Zadabdollah, Oriental Journal of Chemistry, 32, 6 (2016).

14. J. Napp, G. Daeschlein, M. Napp, S. von Podewils, D. Gümbel, R. Spitzmueller, P. Fornaciari, P. Hinz, M. Jünger, GMS Hygiene and Infection Control, 10, Doc08 (2015).

15. M. G. Kong, G. Kroesen, G. Morfill, T. Nosenko, T. Shimizu, J. van Dijk, J. L. Zimmermann, New Journal of Physics, 11, 115012 (2009).

16. M. Moisan, J. Barbeau, S. Moreau, J. Pelletier, M. Tabrizian, L. H. Yahia, Int. J. Pharm., 226, 1 (2001).

17. M. Laroussi, Plasma Processes and Polymers, 2, 391 (2005).

18. P. Basaran, N. Basaran-Akgul, L. Oksuz, Food Microbiol., 25, 626 (2008).

19. Y. Devi, Food Control, 77, 187 (2017).

20. S. A. Ouf, A. H. Basher, A. A. H. Mohamed, J. Sci. Food Agric., 95, 3204 (2015). 


\title{
Comparison of ANFIS and ANN modeling for predicting the behavior of a catalytic methane reformer
}

\author{
B. Parvizi ${ }^{1 *}$, A. Khanlarkhani ${ }^{1}$, Y. Palizdar ${ }^{1}$, A.Farshad ${ }^{2}$ \\ ${ }^{I}$ Institute of Material and Energy (MERC), Karaj, Iran \\ ${ }^{2}$ Iranian Ghadir Iron \& Steel Co., Iran
}

Received January 9, 2018, Revised December 4, 2018

\begin{abstract}
Adaptive neuro-fuzzy inference system (ANFIS) and artificial neural network (ANN) were applied in modeling of methane mixed reforming in a packed bed catalytic reactor. These methods were developed by use of data collected from a methane reforming pilot plant using $\mathrm{CO}_{2}$ and steam and in process conditions near to MIDREX reforming plant in sponge Iron production. Different reaction temperatures from 700 to $1100 \mathrm{C}$ with different values of carbon dioxide, steam, hydrogen, methane and carbon monoxide, were randomly selected and used to generate around 5000 data set of input- output data. Both networks achieve quite satisfying scientific results with acceptable deviations. However, it is hard to say which one is better as they have close output values but ANN marginally outperformed ANFIS in predicting the reaction outputs by varying the inputs. The prediction performances of these models are compared. The accuracies of the two models were evaluated in terms of square correlation coefficient $\left(\mathrm{R}^{2}\right)$ and mean square error (MSE).
\end{abstract}

Keywords: Neural network, Neuro-Fuzzy, Catalytic reforming, Modeling

\section{INTRODUCTION}

Natural gas reforming reaction is one of the most important processes in oil, gas, petrochemical and steel production plants. The main process in each application is to convert hydrocarbons, mainly methane, to syngas with different $\mathrm{H}_{2}$-to- $\mathrm{CO}$ ratios. For example the ratio in an ammonia plant should be higher than 4 and in a MIDREX plant it should be 1.5-2 [1-3]. Among the variety of methods for syngas production, reforming with steam and carbon dioxide is commonly used due to its higher efficiency and cost effectiveness compared with other competing processes. This reaction takes place in a tubular reactor filled of mostly nickel alloy catalyst at around $900-1100{ }^{\circ} \mathrm{C}$ via an extremely endothermic reaction. Besides these reactions, the probability for occurrence of some undesirable reactions like production of coke will be high if the process parameters are not fixed at the optimized ones and lead to formation of hot spot and rupture of reforming tubes [1].

Main parameters for optimization of the process are: the composition of reactants which are methane, carbon dioxide, carbon monoxide, hydrogen, water and the temperature of the reaction zone. For finding the optimized region and controlling each parameter, developing a process model that describes the dynamic relations of inlet and outlet conditions is a must. Then this model can be used in a digital control system to predict the online behavior, return and keep the total system in

\footnotetext{
* To whom all correspondence should be sent: E-mail: behzadparvizi@gmail.com
}

optimized conditions [4-6].

Generally modeling methods are divided in two main groups: fundamental and empirical. The first group includes theoretical and mathematical relations and focuses on mass, energy and momentum balance and kinetics of reactions. Such methods are very useful in case of availability of mechanistic information. The second one includes data-driven models which can relate the inputoutput data in a dynamic mode via black box estimators. New digital control systems are mainly using an artificial intelligence based model which allows simulation and online prediction of system's behavior by processing online or offline inputoutput databases. Among the empirical AI methods artificial neural network (ANN) and adaptive neuro-fuzzy inference systems are the most promising ones and are applied to many real world systems [4]. These two methods have been applied in many researches and were compared. In some cases ANN was more accurate than fuzzy inference systems and in some cases vice versa [7-19].

C. Ozel used these methods for estimation of the thermal conductivity coefficient of some materials and revealed that ANFIS has less RMSE than ANN and is more accurate [7]. Areerachakul has used these two methods for estimation of biochemical oxygen demand parameter in surface waters, and as a result ANN was more accurate than the ANFIS model [8]. In another study conducted by Chauhan, these models were used in a dynamic control model for basic oxygen furnace steel making process with a higher yield for the neuro-fuzzy model [11].

The motivation behind this paper was to develop 
B. Parvizi et al.: Comparison of ANFIS and ANN modeling for predicting the behavior of a catalytic methane reformer

a method for modeling empirical relations of input (reactant composition and temperature) and output data (outlet compositions) from a pilot unit of a mixed reformer to use in online implemented control. ANN was used with BP algorithm and ANFIS by dividing data space into rule patches and the versatility, robustness and predictive accuracy of each method was compared.

\section{MODEL DEVELOPMENT Material and methods}

A pilot plant was built specifically to evaluate experimental data gathering of methane reforming with steam and carbon dioxide. The basic design of this pilot was scaled down from an industrial unit of DRI production using MIDREX technology. It essentially consists of a tubular reactor with 2" diameter and $2 \mathrm{~m}$ height, filled of three levels of catalysts, inert, semi-active and active with different percentage of nickel oxide. The reactor is fixed in a cubic electrical heater designed to reach $1400{ }^{\circ} \mathrm{C}$ with three heating zones. Five temperature sensors (TT) were implemented on inlet, outlet and three sections of the heater for accurate control of the reaction temperature. Also five mass flow controllers (model: Alicat- MCR) for gas cylinder lines and one vortex flow meter (model: YokogawaDY015) on the steam line were used for accurate control of the reactant flow rates. This plant is schematically shown in Figure 1.

Methane, steam, carbon dioxide, carbon monoxide, hydrogen and nitrogen were mixed and preheated up to around $500{ }^{\circ} \mathrm{C}$ through electrical heaters, and then the mixture flows to the reactor on three levels of catalysts. The reaction occurs at
900-1100 ${ }^{\circ} \mathrm{C}$ and produced syngas is then cooled via a water condenser and then goes online through a gas chromatograph for composition analysis. The typical operational reaction conditions and catalyst properties in the MIDREX plant used in sizing of the equipment are reported in table 1.

To know the whole process well and find out the contribution of each process parameter on outlet condition, a sufficient and most informative data set should be generated to learn the ANN and ANFIS structures, which could cover the whole data space in experimental conditions. Therefore, around 5000 sets of randomly selected input data, comprising gas compositions from 0 to $3 \mathrm{Nm}^{3} / \mathrm{h}$ and reaction temperature from 700 to $1100{ }^{\circ} \mathrm{C}$, were used to generate the input-output dataset.

\section{Neural Network Modeling}

An artificial neural network (ANN) is composed of simple calculation nodes or neurons which are connected to each other and operate in a parallel manner just like the network of neurons in the human brain. Each neuron represents an activation function with its specific output. Figure 2 shows the basic structure of the ANN [20]. In brief the method will be applied in the project in five steps:

(1) choosing proper input/output parameters;

(2) splitting the data bank into training and testing sets;

(3) creating a proper structure of neural network;

(4) training and optimizing the network architecture and

(5) performance analysis after being trained.

A schematic view of the basic structure of ANN is depicted in Fig. 2 [21].

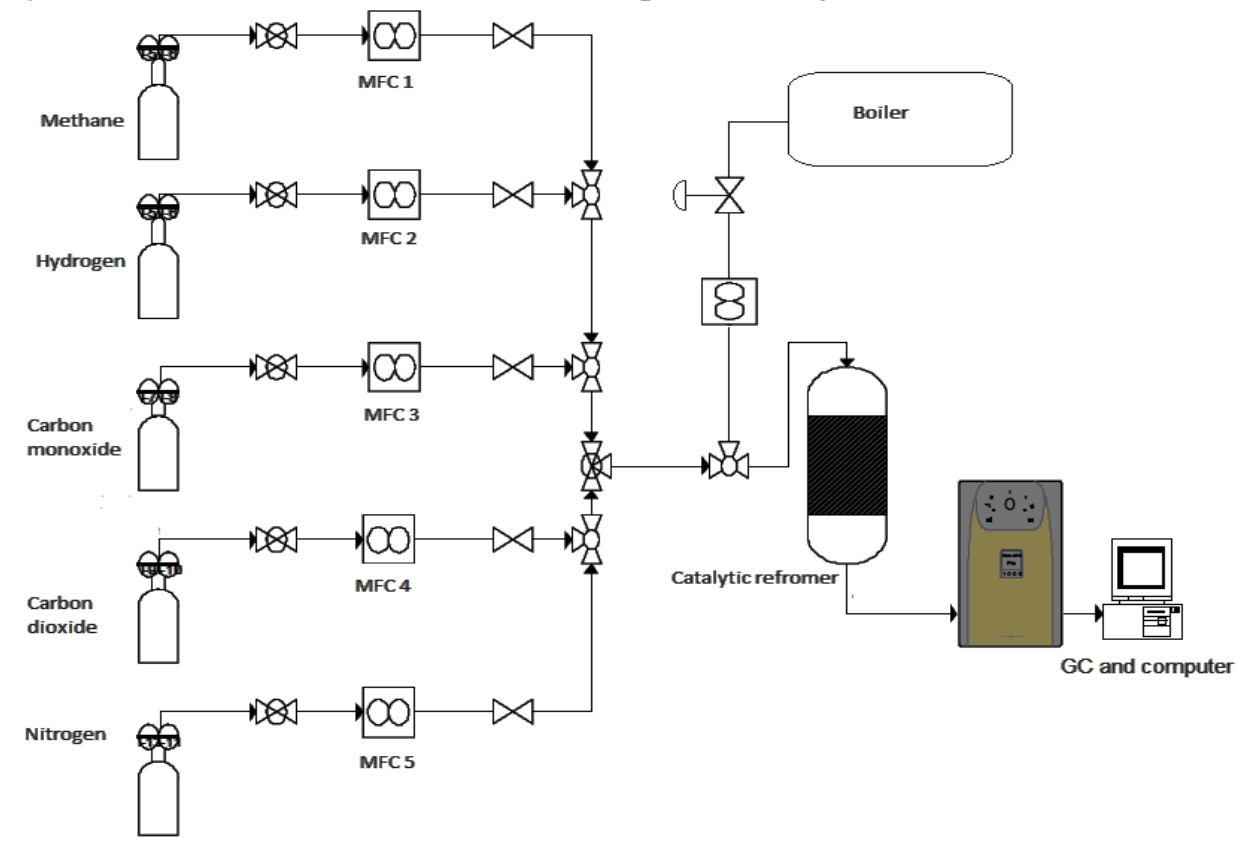

Fig. 1. Schematic diagram of the experimental system. 


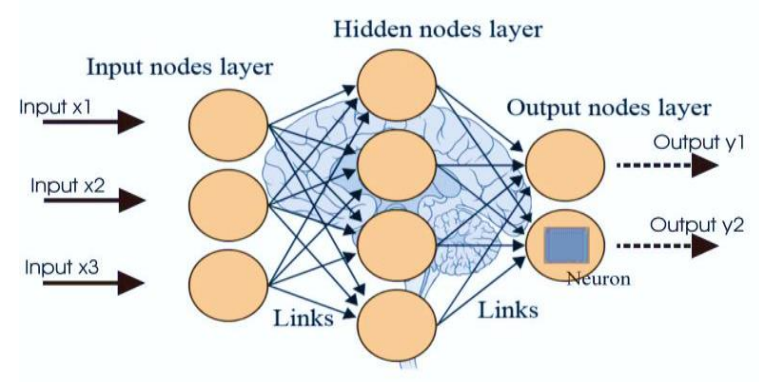

Figure 2. Basic structure of ANN

Adaptive Neuro-Fuzzy Inference System (ANFIS)

Another identification and modeling methoc is the adaptive neuro-fuzzy inference systen (ANFIS) which is a neuro-fuzzy modelin technique that applies a hybrid learning algorithm combining back propagation (BP) and leas square estimate (LSE) during the modelins process. Data-driven procedures for the construction of ANFIS networks are typically based on clustering a training set of numerica samples of the unknown function to bs approximated [22].

Table 1. Reaction conditions in the MIDREX reformer.

\begin{tabular}{|c|c|c|c|}
\hline \multicolumn{4}{|c|}{ Catalyst parameters } \\
\hline Type & $\begin{array}{l}\text { Particle size } \\
(\mathrm{mm})\end{array}$ & Porosity & Sphericity \\
\hline $\begin{array}{c}\text { Raschig } \\
\text { ring }\end{array}$ & $16 \times 6 \times 16$ & 0.52 & 0.656 \\
\hline Tortuosity & $\begin{array}{l}\text { Loose } \\
\text { density } \\
\left(\mathrm{kg} / \mathrm{m}^{3}\right)\end{array}$ & $\begin{array}{c}\text { Bed } \\
\text { density } \\
\left(\mathrm{kg} / \mathrm{m}^{3}\right)\end{array}$ & \\
\hline 2.74 & 2390 & 1362 & \\
\hline \multicolumn{4}{|c|}{ Reaction conditions } \\
\hline $\begin{array}{c}\text { Pressure } \\
\text { (bar g) }\end{array}$ & $\begin{array}{c}\text { Temperature } \\
\left({ }^{\circ} \mathrm{C}\right)\end{array}$ & $\mathrm{CO}_{2} \%$ & $\mathrm{CO} \%$ \\
\hline 2 & $900-1100$ & 15.21 & 17.4 \\
\hline $\mathrm{H}_{2} \%$ & $\mathrm{H}_{2} \mathrm{O} \%$ & $\mathrm{~N}_{2} \%$ & $\mathrm{CH}_{4} \%$ \\
\hline 31.09 & 13.94 & $1.5-2$ & 19.73 \\
\hline
\end{tabular}

Each layer in the network corresponds to a part of the fuzzy inference system (FIS) called: input fuzzification, rule inference and fire strength computation, and output defuzzification. The main advantage of this kind of representation is that the
FIS parameters are encoded as weights in the neural network and, thus, can be optimized via powerful well-known neural network learning methods.

This model is mostly suited to the modeling of nonlinear systems. Figure 3 shows a typical example of ANFIS architecture [22]. Each node in layer $\mathbf{1}$ is an adaptive node, with a node function that may be a Gaussian membership function or any other membership function. Each node in layer 2 is a fixed node labeled $\pi$, representing the firing strength of each rule. Each node in layer 3 is a fixed node labeled $\mathrm{N}$, representing the normalized firing strength of each rule. Each node in layer $\mathbf{4}$ is an adaptive node with a node function. The single node in layer 5 is a fixed node labeled $\Sigma$, indicating the overall output $(Z)$ as the summation of all incoming signals [23].

ANFIS systems are often applied in conjunction with the Takagi-Sugeno (TSK) fuzzy system, its main purpose being to utilize fuzzy modeling using measured data. In the fuzzy TSK method, for a system bearing two input parameters $X$ and $Y$ each describing a membership function, are depicted in premise and consequent parts of Fig. 3(a). Where $x$ and $\mathrm{y}$ are the input to node, $i, p, q$, and $k$ are consequence parameters resulting from the training; and $A$ and $B$ are labels of the fuzzy linguistic set [24].

\section{Modeling scenario}

Most of the time, data gathering techniques are well controlled, which results in outliers, missing values and different types of errors. Analyzing data that have not been carefully separated for these problems can produce confusing results. In order to avoid such problems, min-max normalization method was implemented to the gathered data before mathematical modeling.

A multilayer perceptron feed-forward neural network with back-propagation algorithms was used to predict the reformer behavior and syngas produced. The considered learning rule and training function are Levenberg Marquardt and trainBR, respectively. A number of around 3461 data were utilized for training session. By using the trained structure and remaining experimental data, the model was tested and the difference between the real and model data was calculated. Among structures and configurations tested, 4 hidden layers predicted the best and nearest result to the actual conditions. The log sigmoid function was employed as an activation function and 872 numbers of epochs were considered to overcome the over- and underfitting of data 


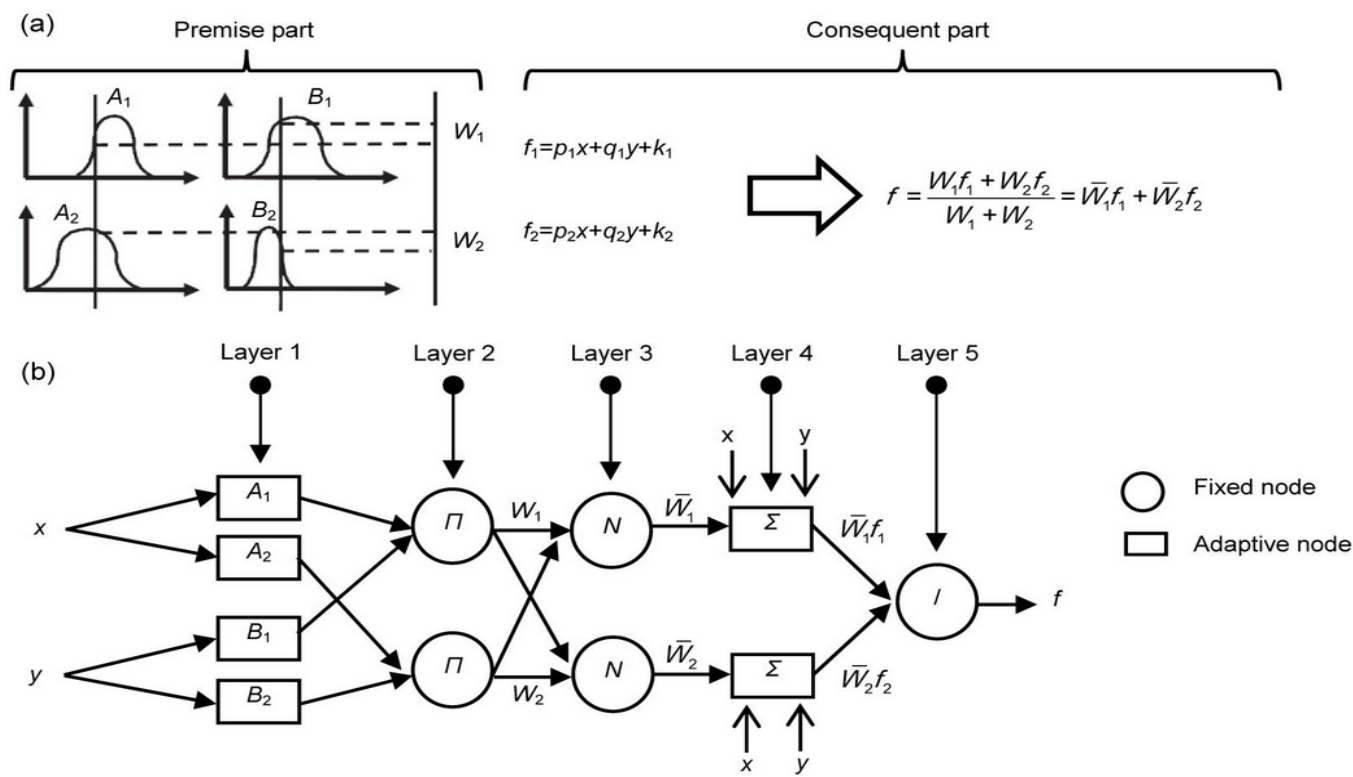

Figure 3. Basic structure of ANFIS [24].

Table 2. ANN - ANFIS model properties

\begin{tabular}{|c|c|c|c|}
\hline \multicolumn{2}{|c|}{ ANN model structure } & \multicolumn{2}{c|}{ ANFIS model structure } \\
\hline Parameter & Description & Parameter & Description \\
\hline Total layer & 6 & Total layer & 6 \\
\hline Hidden layer & 4 & Hidden layer & 4 \\
\hline $\begin{array}{c}\text { Input layer } \\
\text { neuron }\end{array}$ & 6 & Type of MF & Gaussian \\
\hline Output layer & 5 & Number of MF & 6 \\
\hline Learning rate & 0.001 & Type of output parameter & Linear \\
\hline Epoch number & 872 & Epoch numbers & $27-62$ \\
\hline Error goal & $1 \mathrm{e}^{-5}$ & Error goal & $1 \mathrm{e}^{-5}$ \\
\hline
\end{tabular}

Similar numbers of training and test data were used for the ANFIS model and tuning of the membership function of each input was performed by the hybrid method of back propagation for input membership function and the least square estimation for the parameters output membership functions. The computations of the membership function parameters are facilitated by a gradient vector which provides a measure of how well the FIS system is modeling the input/output data. The comparative properties of each model are shown in table 2.

As the most suitable method for training a neural network, the MATLAB-Simulink neural network function fitting was employed for the implementation of the ANN model. In contrast, the MATLAB command was also used to apply a fuzzy system into the forecasting model. Also the ANFIS Editor GUI applications enable the parameters of the membership functions to be automatically used. To analyse and evaluate the performance and proficiency of developed ANN and ANFIS methods in predicting the catalytic reformer two of most common error factors, $\mathrm{R}^{2}$, coefficient of determination, and MSE, mean square error, were selected and used in this work. Definition and formula of each method are denoted with the equations below:

$$
\begin{aligned}
& R^{2}=1-\frac{\sum_{i=1}^{N}\left(Y_{i}^{\text {real }}-Y_{i}^{\text {model }}\right)^{2}}{\sum_{i=1}^{N}\left(Y_{i}^{\text {real }}-Y_{a v}^{\text {real }}\right)^{2}} \\
& M S E=\frac{1}{N} \sum_{i=1}^{N}\left(Y_{i}^{\text {real }}-Y_{i}^{\text {model }}\right)^{2}
\end{aligned}
$$

where $\mathrm{N}, \mathrm{Y}(\mathrm{i})^{\text {real }}, \mathrm{Y}(\mathrm{i})^{\text {model }}$ and $\mathrm{Y}(\mathrm{av} .)^{\text {model }}$ are number of data, real data, predicted value and average of real data, respectively. It should be noted that unlike $\mathrm{R}^{2}$ which being to 1 is desirable, MSE being close to zero is desirable and shows better performance of the developed model.

\section{SIMULATION RESULTS}

In the current study, two models of ANN and ANFIS were developed by estimation of the output parameters of the catalytic reformer, which are $\mathrm{CH}_{4}$, $\mathrm{CO}_{2}, \mathrm{CO}, \mathrm{H}_{2}, \mathrm{H}_{2} \mathrm{O}$ composition, from input 
B. Parvizi et al.: Comparison of ANFIS and ANN modeling for predicting the behavior of a catalytic methane reformer parameters, which are normal flow rate of each of the above gases added by reaction temperatures. The same training and testing data sets were used to train and test both models to extract better conclusions from the comparison results. About 70 percent of the datasets were used for training of the neural network. The error histogram plot for these data is shown in figure 4 . This analysis can be an effective means for investigation and resolving the inconsistent networks performance. In this graph, blue, green and red bars represent the training, validation and testing data, respectively. As it is depicted, most of the data fall on the zero error line and there is a large central peak which indicates very small errors or outputs which are close to the target values. The performance indicators related to the training, test and validation of the ANN model including the $\mathrm{R}^{2}$ and MSE are reported in Table 3 . A comparison between the real experimental and predicted data separately is presented and discussed in the following. In parallel the ANFIS model was also implemented for input/output datasets. 6 numbers of FIS were generated. Genfis1 was used as the initial training of fuzzy sets. Training and testing process using ANFIS model were also done.

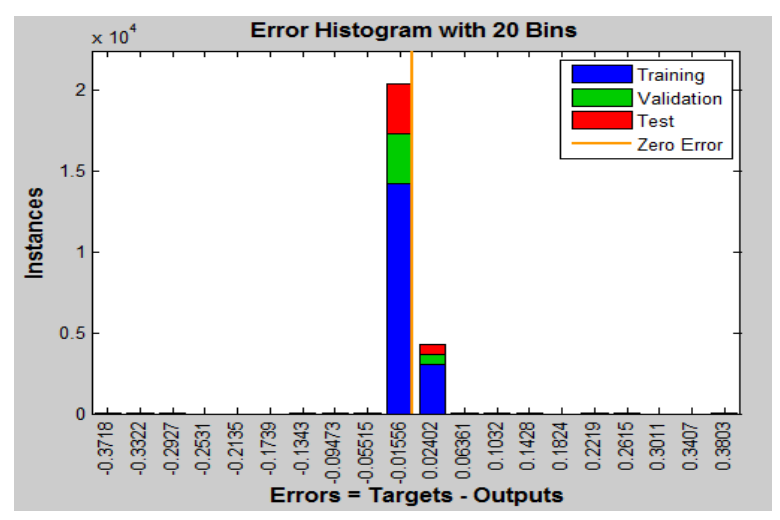

Table 3. Performance of developed ANN network

\begin{tabular}{|c|c|c|c|}
\cline { 2 - 4 } \multicolumn{1}{c|}{} & Number of data & MSE & $\mathrm{R}^{2}$ \\
\hline Training & 3461 & $7.19 \mathrm{e}^{-5}$ & $9.99 \mathrm{e}^{-1}$ \\
\hline Validation & 742 & $1.83 \mathrm{e}^{-4}$ & $9.97 \mathrm{e}^{-1}$ \\
\hline Test & 742 & $5.43 \mathrm{e}^{-5}$ & $9.99 \mathrm{e}^{-1}$ \\
\hline
\end{tabular}

Analysis of training - test in different epoch numbers was also done for each 5 different outputs in ANFIS models to find the best numbers of the epochs in each case.

Figure 5 (a-e) depicts the RMSE-Epoch numbers for all outlets. In this graph the blue line refers to training sets and green line to testing sets. In Table 4 the performance of the developed ANFIS model including MSE and $\mathrm{R}^{2}$ is reported, which can be compared with Table 3 .

Table 4. Performance of developed ANFIS model

\begin{tabular}{|c|c|c|c|}
\cline { 2 - 4 } \multicolumn{1}{c|}{} & Number of data & MSE & $\mathrm{R}^{2}$ \\
\hline Training & 3461 & $5.54 \mathrm{e}^{-5}$ & $9.99 \mathrm{e}^{-1}$ \\
\hline Validation & 742 & $5.28 \mathrm{e}^{-4}$ & $9.67 \mathrm{e}^{-1}$ \\
\hline Test & 742 & $6.68 \mathrm{e}^{-4}$ & $9.54 \mathrm{e}^{-1}$ \\
\hline
\end{tabular}

Figures 6-10 show the comparison graphs between predictions from the two networks, ANN and ANFIS against actual values obtained from the experiments. These sets of graphs are related to each outlet gas composition, respectively, methane, carbon monoxide, carbon dioxide, hydrogen and water and also each set comprised 4 sub-plots which show the comparative result of ANN with real data (a), related calculated error (b), comparative result of ANFIS with real data (c) and related calculated error (d). With regard to the huge number of input-output data, to have clearer plots, the first 200 data were considered and compared to each other.

Figure 4. Error histogram for the neural network
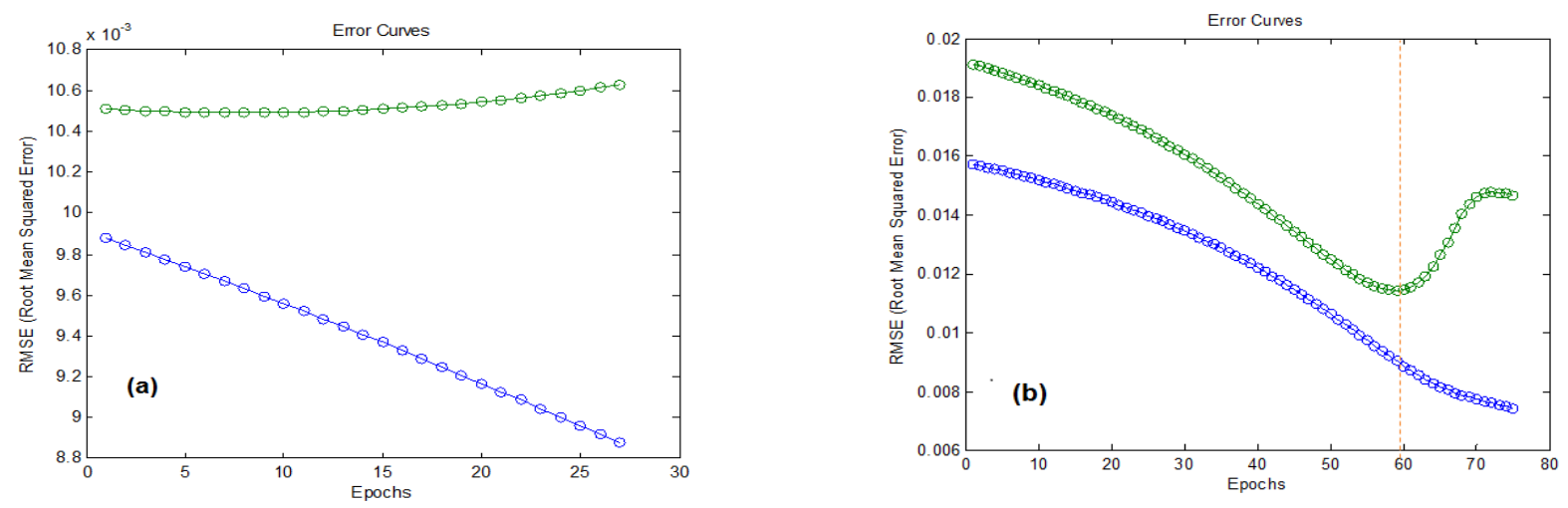
B. Parvizi et al.: Comparison of ANFIS and ANN modeling for predicting the behavior of a catalytic methane reformer
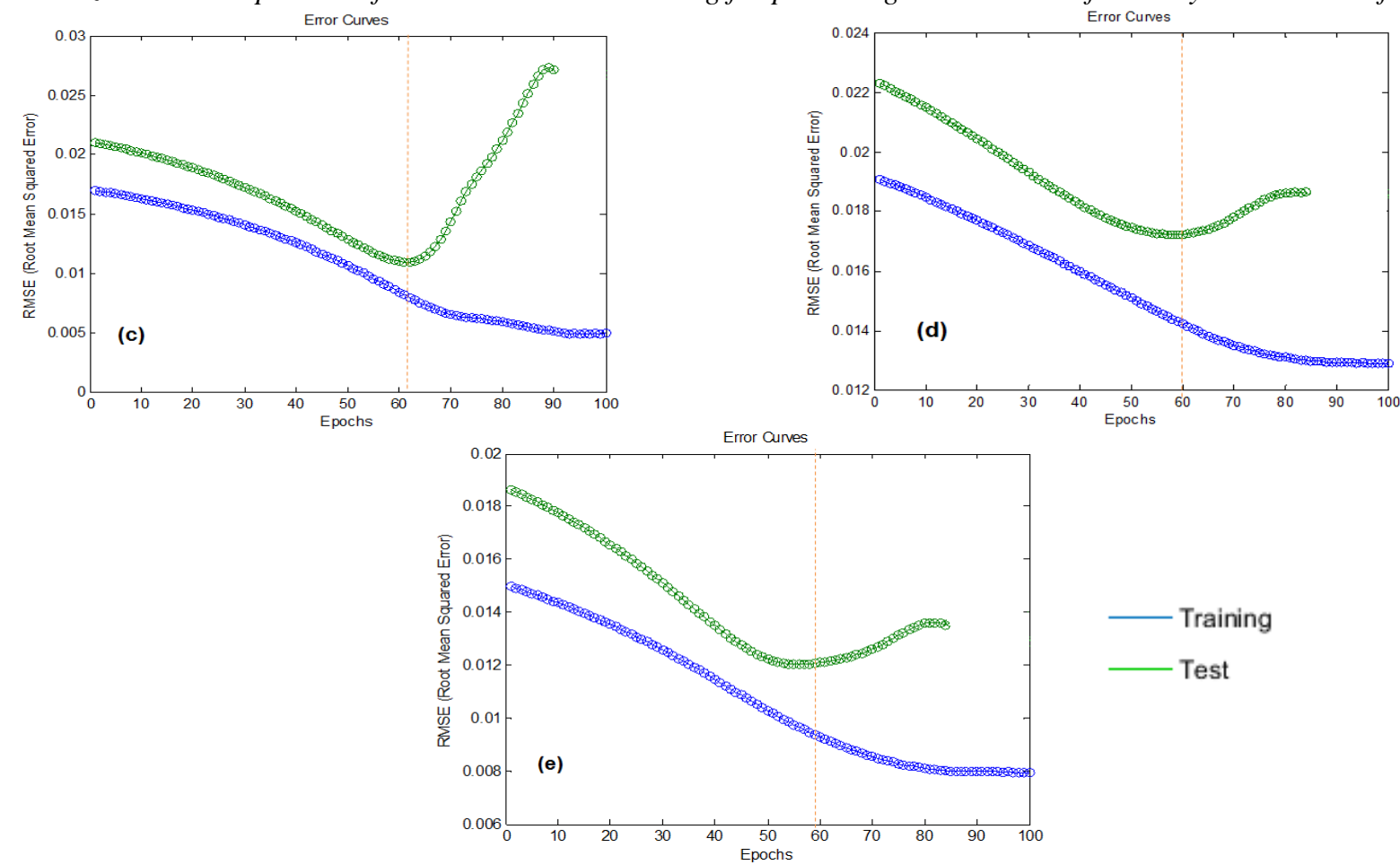

Figure 5. Test/ Train performance for ANFIS model in predicting each output and finding number of epochs. (a) $\mathrm{CH}_{4}$,

(b) $\mathrm{CO}$, (c) $\mathrm{CO}_{2}$, (d) $\mathrm{H}_{2}$, (e) $\mathrm{H}_{2} \mathrm{O}$
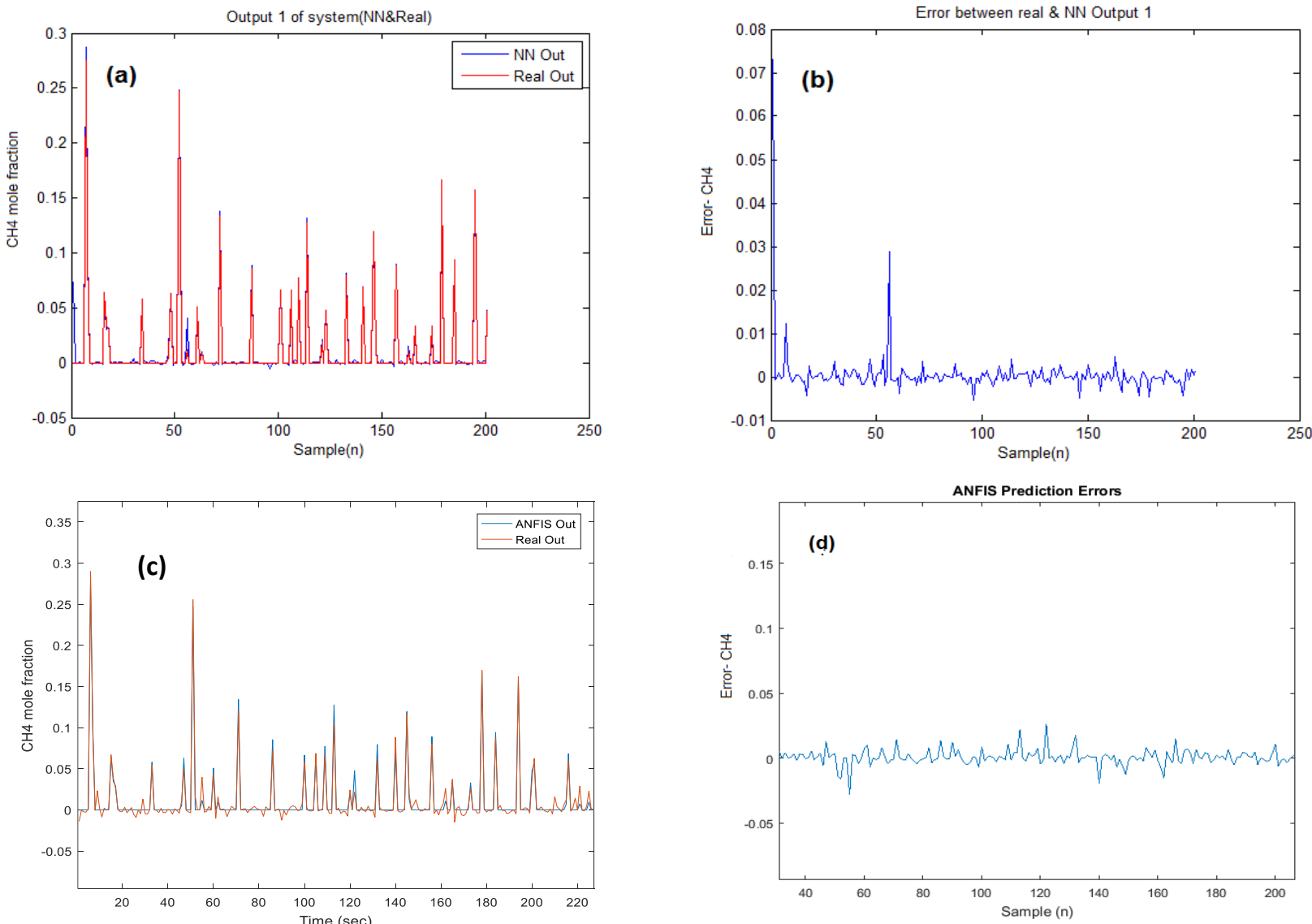

Figure 6. Comparative results for prediction performance of outlet methane composition using ANN (a,b), ANFIS $(\mathrm{c}, \mathrm{d})$ and real data

As can be seen in these graphs, there is excellent agreement between the predicted results by both ANN and ANFIS with real datasets from experimental setup. It is obvious that the ANN and ANFIS both fit and follow the real data diagram in a consistent way. Also the error plot shows the 
B. Parvizi et al.: Comparison of ANFIS and ANN modeling for predicting the behavior of a catalytic methane reformer deviation more clearly which means that all of them are in an acceptable range for both approaches.

Based on the two criteria mentioned above, $\mathrm{R}^{2}$ and MSE for the developed model, reported in tables 3 and 4 , it is obvious that, although $\mathrm{R}^{2}$ values for both models are sufficiently near to 1 , for ANN the situation is better, moreover this observation can be proven by checking the MSE. Although the

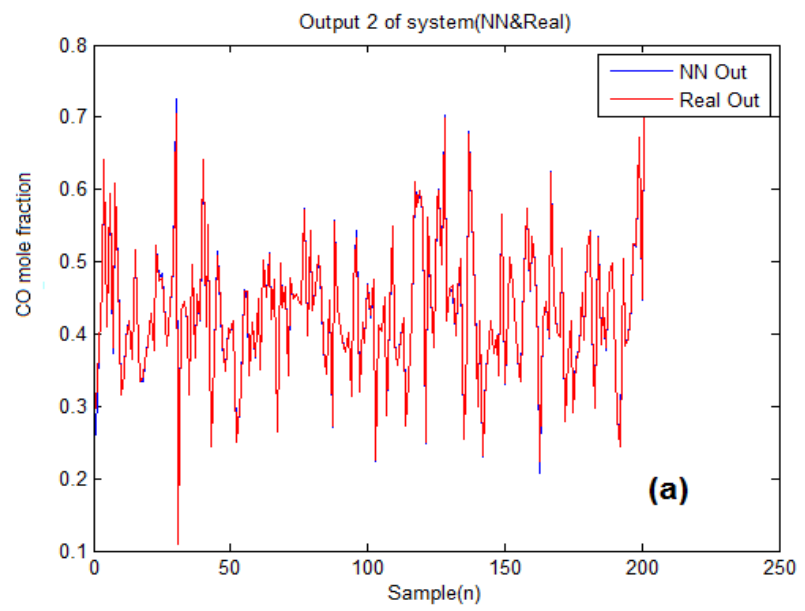
values of both are near to zero and both model outputs follow the trend of the actual ones, for ANN the values are more close to zero and so, ANN is more favorable. Despite some of its general demerits, like overfitting problems, high processing time, etc., in this special case utilizing the fuzzy logic beside the neural network did not make any added value and forward movement.
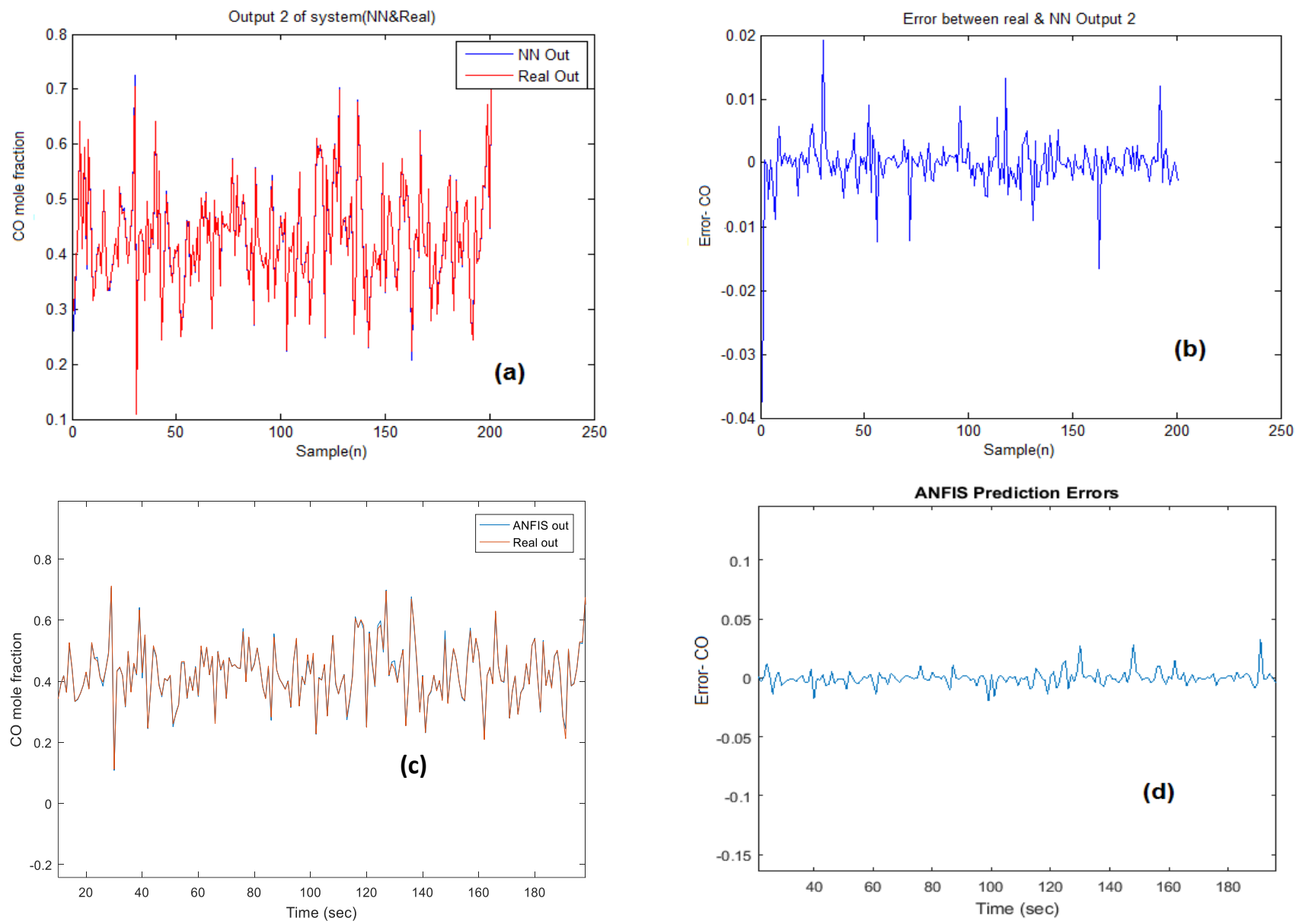

Figure 7. Comparative results for prediction performance of outlet CO composition using ANN (a,b), ANFIS $(\mathrm{c}, \mathrm{d})$ and real data. 
B. Parvizi et al.: Comparison of ANFIS and ANN modeling for predicting the behavior of a catalytic methane reformer
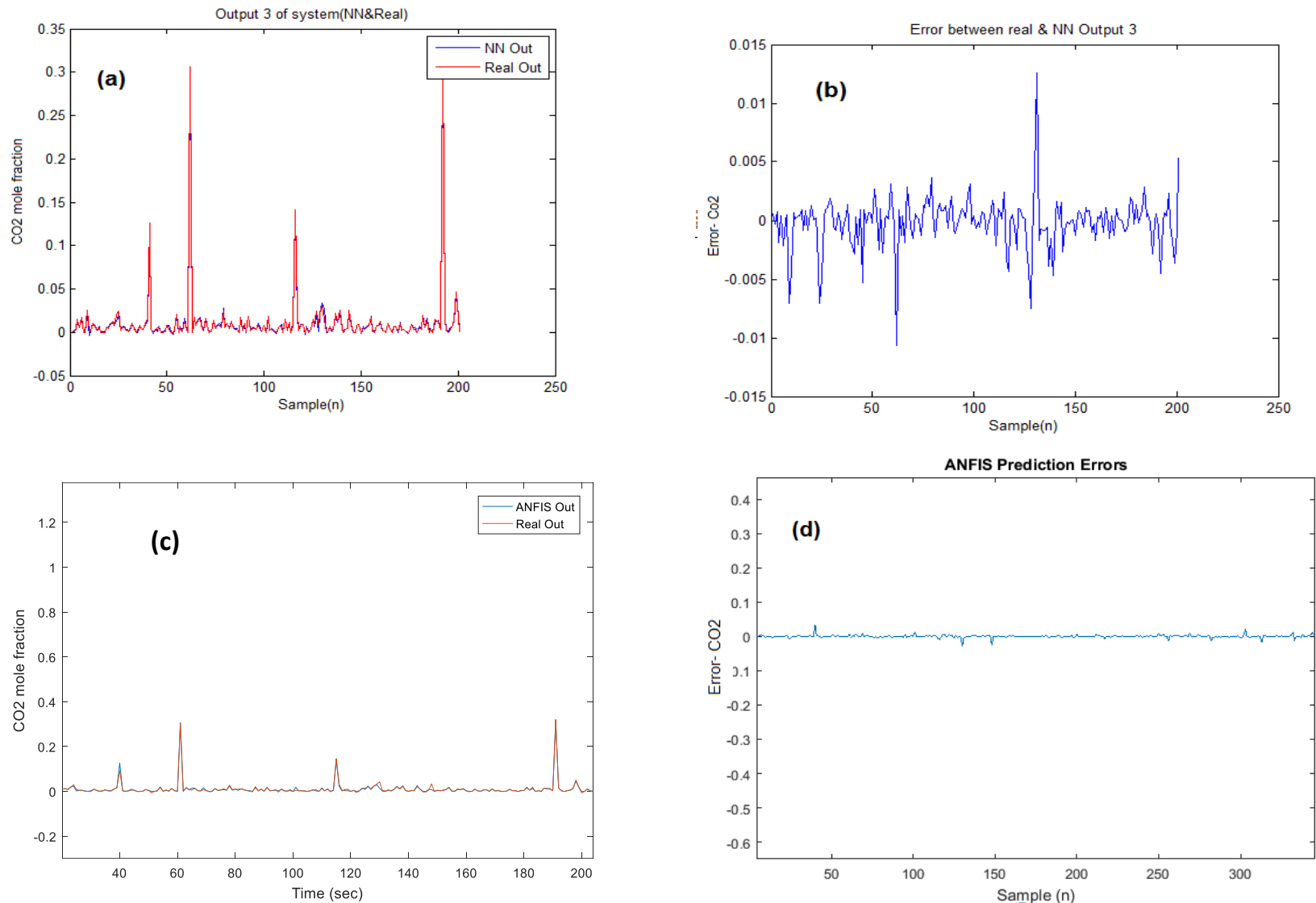

Figure 8. Comparative results for prediction performance of outlet $\mathrm{CO}_{2}$ composition using ANN (a,b), ANFIS (c,d) and real data
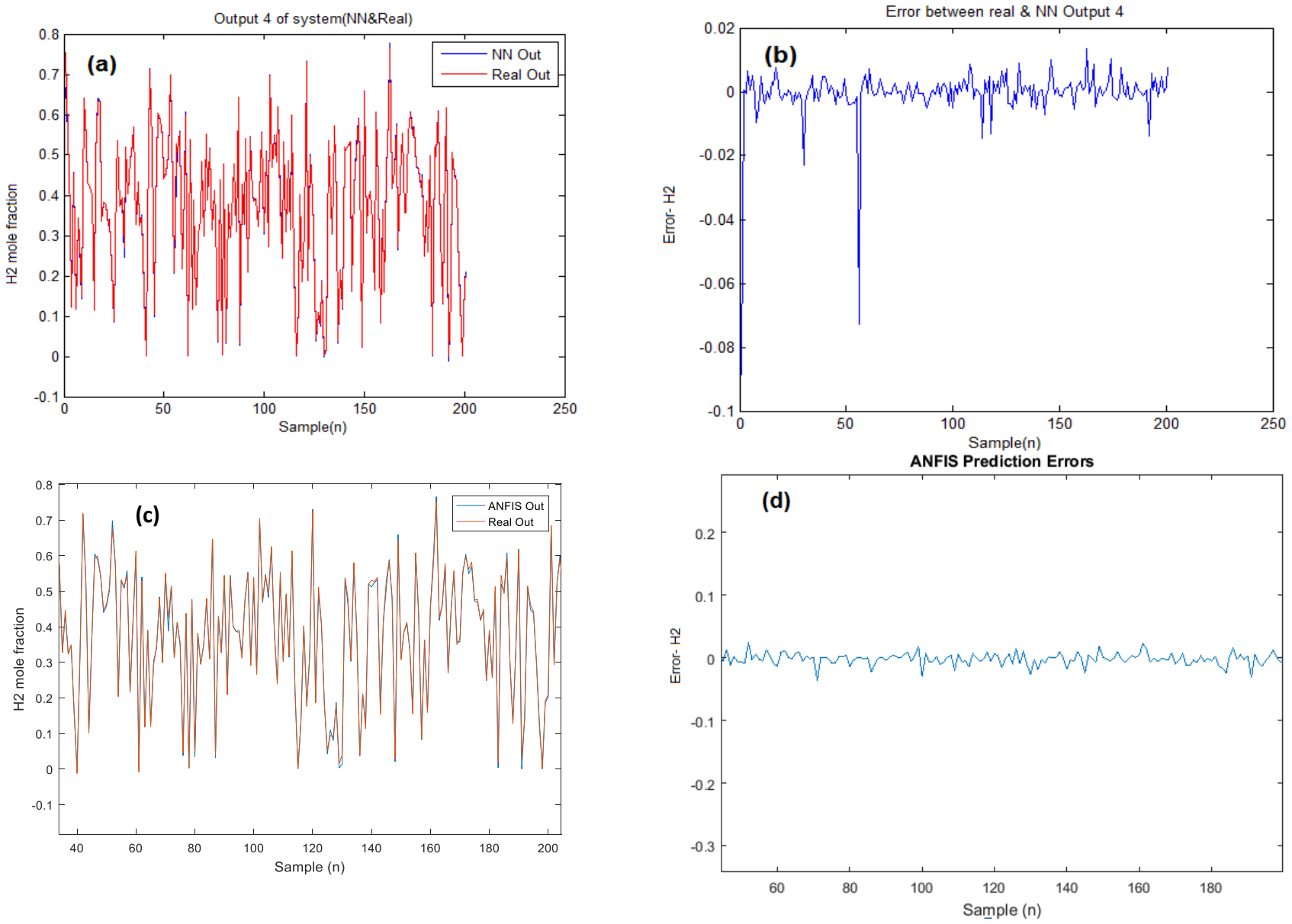

Figure 9. Comparative results for prediction performance of outlet hydrogen composition using ANN (a,b), ANFIS 

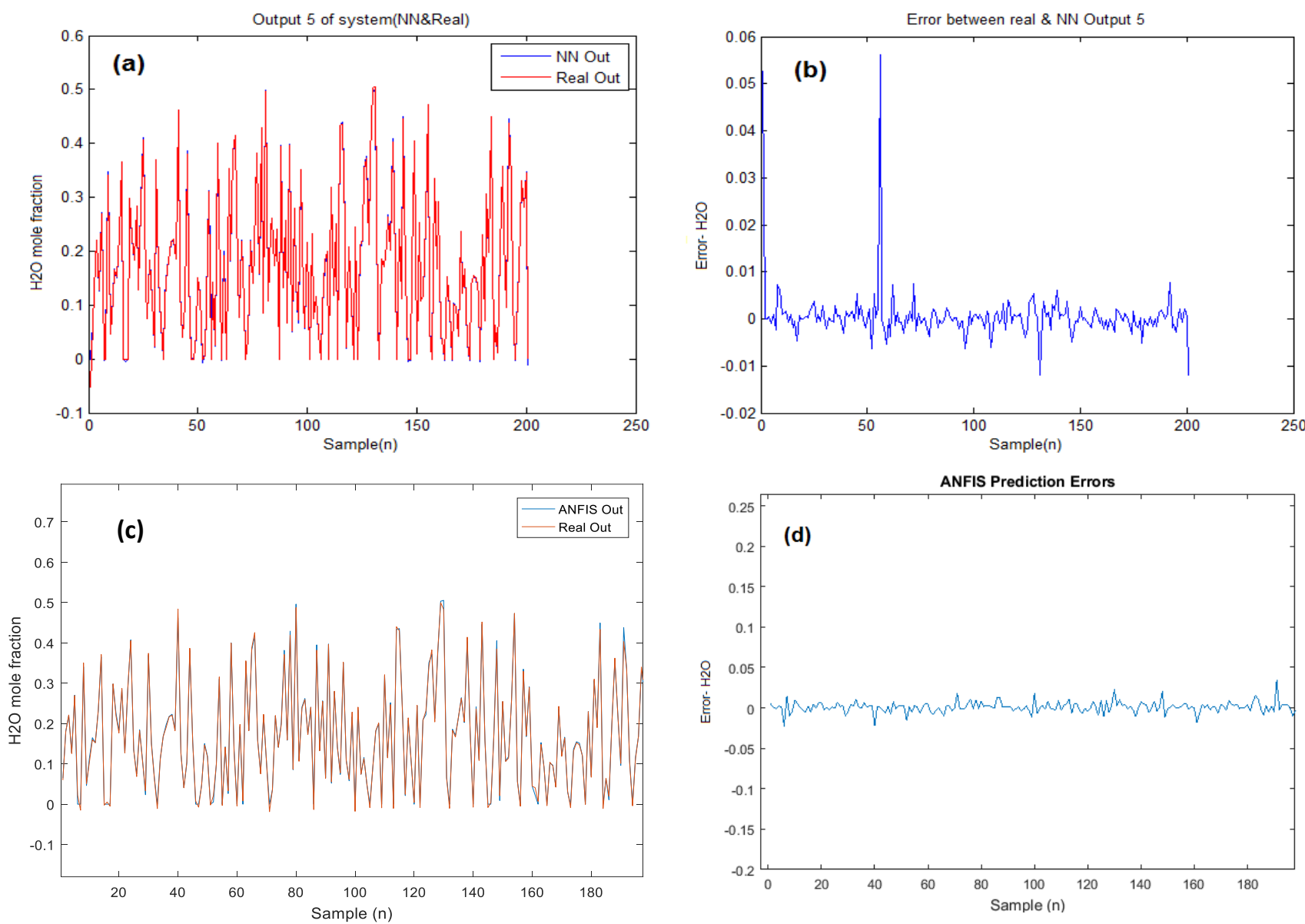

Figure 10. Comparative results for prediction performance of outlet $\mathrm{H}_{2} \mathrm{O}$ composition using ANN (a,b), ANFIS (c,d) and real data

\section{CONCLUSIONS}

Due to the importance of methane catalytic reforming for producing syngas in different industries, especially in DRI plants, which this study is focused on, for producing sponge iron, a pilot plant with the design of a MIDREX reformer was constructed. At different inlet gas flow rates and temperatures, around 5000 data were tested and their outlet data including gas compositions were acquired in an input- output databank. Two smart approaches, ANN and ANFIS were utilized for prediction of the outlet parameters from each inlet data. The predicted data were then compared with real data from the experimental ones. As to the result, both models followed the trend of real data with an acceptable accuracy and their $\mathrm{R}^{2}$ and MSE values were sufficiently near to 1 and zero, respectively. But as to these values calculated for each case, the maximum error of the ANN model was around 0.3 and this value for the ANFIS model was around 0.5, so the ANN model was more favorable and had more accurate data in comparison to addition of fuzzy system to it.

\section{REFERENCES}

1. Direct Reduction Plant, MIDREX Company Operations Manual, vol. 1, 1981, p. 8.

2. B. Cañete, C. E. Gigola, N. B. Brignole, Synthesis Gas Processes for Methanol Production via $\mathrm{CH}_{4}$ Reforming with $\mathrm{CO}_{2}, \mathrm{H}_{2} \mathrm{O}$, and $\mathrm{O}_{2}$, Ind. Eng. Chem. Res., 53, 7103 (2014).

3. A. Masaaki, MIDREX Processes, Kobelco Technology Review No. 29, 2010.

4. D. Sliškovi'c, R. Grbić, Z. Hocenski, Automatika, 52, 306 (2011).

5. M. Jajarmizadeh, S. Harun, M. Salarpour, J. Environ. Sci. Technol., 5, 249 (2012).

6. S. F. M. Jalli, S. Al-Asheh, H. E. Alfadala, Use of artificial neural network black-box modeling for the prediction of wastewater treatment plants performance, J. Environ. Manag., 83, 329 (2007).

7. C. Ozel, Scientia Iranica Transactions A: Civil Engineering, 22, 2001 (2015).

8. S. Areerachakul, Int. J. Chem. Biol. Eng., 6, 286 (2012).

9. E. K. Lafdani, A. R. Moghaddam Nia,, Int. J. Eng. Techno. Sci., 1, 32 (2013). 
B. Parvizi et al.: Comparison of ANFIS and ANN modeling for predicting the behavior of a catalytic methane reformer

10. M. K. Tiwari, S. Bajpai, Int. J. Eng. Innovative Technol. 6, 192 (2012).

11. S. Chauhan, N. K. Singh, Int. J. Eng. Innovative Technol., 2, 198 ( 2013).

12. O. Kisi, A. Murat, Comparison of ANN and ANFIS techniques in modeling dissolved oxygen", Sixteenth International Water Technology Conference, IWTC-16, Istanbul, Turkey, 2012.

13. M. T. Dastorani, A. R. Moghadamnia, J. Piri, Environ. Monitoring Assess, 166, 421 (2010).

14. P. Lotfi Anari, H. Sharifi, Res. J. Environ. Sci., 5, 236 (2011).

15. M. Aqil, I. Kita, A. Yano, J. Hydrol., 337, 22 (2007).

16. J. Kim, N. Kasabov, Neural Networks, 12, 1301 (1999).

17. T. Hussain, Z. Ali Malik, Z. Arshad, A. Nazir, J. Textile Institute, 106, 934 (2015).
18. S. Uzuner, D. Cekmecelioglu, J. BioResources, 11 8676 (2016).

19. M.T. Yilmaz, J. Texture Stud., 43, 384 (2012)

20. R. Rojas, Neural Networks, Springer-Verlag, Berlin, 1996.

21. A. Zendehboudi, X. Li, Int. J. Refrigeration, 74, 475 (2017).

22. J.-S. R. Jang, Ch.-Ts. Sun, Neuro-fuzzy modeling and control, Proceedings of IEEE, 83(3), 378 (1995).

23. A. Vasičkaninová, B. Monica, Neural Network Predictive Control and Neuro-Fuzzy Control of Heat Exchanger, Slovak University of Technology Press, 7, 2011, p. 28.

24. D. Behnia, K. Ahangari, A. Noorzad, J. Zhejiang Univ.-Sci. A, (Appl. Phys. \& Eng.), 1, 589 (2013). 


\title{
Biotransformation of oleic acid and antimicrobial and anticancer activities of its biotransformation extracts
}

\author{
Ö. Özşen Batur*, Ö. Atlı, İ. Kıran \\ Department of Chemistry, Faculty of Arts and Sciences, Eskişehir Osmangazi University, 26480, Eskişehir, Turkey
}

Submitted March 6, 2018; Revised March 17, 2018

Oleic acid is an unsaturated fatty acid found in significant quantities in various edible oils. Scientific studies have shown that oleic acid and its derivatives exhibit a variety of biological activities including antimicrobial and anticancer activities. In the present work, biotransformation of oleic acid was carried out initially using 27 different microbial strains. Extracts obtained from biotransformation with Alternaria alternata (clinical isolate) and Aspergillus terreus var. africanus (clinical isolate) were used in antimicrobial and anticancer activity studies. The in vitro antimicrobial activities of the extracts were evaluated against 9 different pathogenic microorganisms. The results indicated that the microbial extracts were more active than oleic acid itself and showed good inhibitory activity against all tested microorganisms. In in vitro anticancer activity studies, extract 2 obtained from biotransformation with Alternaria alternata exhibited notable anticancer activity against A549 cell line with an $\mathrm{IC}_{50}$ value of $62.5 \mu \mathrm{g} / \mathrm{ml}$ whereas positive control cisplatin showed an $\mathrm{IC}_{50}$ value of $43.5 \mu \mathrm{g} / \mathrm{ml}$. The anticancer activity of extract 2 was also found to be selective according to its higher $\mathrm{IC}_{50}$ value $(122.7 \mu \mathrm{g} / \mathrm{ml})$ obtained against the healthy cell line, mouse embryonic fibroblasts, NIH3T3. Due to its anticancer effect, extract 2 is considered to participate in further research.

Keywords: Antimicrobial activities, Biotransformation, Cytotoxic activities, Minimum inhibitory concentration, Oleic acid

\section{INTRODUCTION}

Oleic acid, also known as 9-octadecenoic acid, is an unsaturated fatty acid present in various vegetable oils such as hazelnut and olive oil as glyceryl esters. It is used commercially as a pharmaceutical solvent and in the preparation of oleates and lotions [1, 2]. Scientific studies have shown that oleic acid and oleic acid-containing extracts exhibit a variety of biological activities including antibacterial activity [3-5], antifungal activity [6, 7], antioxidant activity [8-10], antiinsecticidal activity [11], antiviral activity [12], hemolytic activity against human erythrocyte [13] and anticancer activity against MCF-7 and HT-29 cancer cells [14]. Therefore, molecular modifications of oleic acid have been carried out by biotransformation reactions in recent years in the hope that the addition of functional groups may enhance its activity.

The literature reveals several microbial transformations of oleic acid by using fungi, bacteria and recombinant microorganisms. These include conversion to ricinoleic acid by a soil bacterium [15] and to 10-hydroxyoctadecanoic and 10-oxo-octadecanoic acids by Rhodococcus rhodochrous (Nocardia aurantia) [16], Nocardia cholesterolicum [16, 17] and Pseudomonas sp. [16, 18-21]; hydroxylation to 10-hydroxystearic acid by

\footnotetext{
* To whom all correspondence should be sent. E-mail: oozsen@ogu.edu.tr
}

Pseudomonas sp. [17, 22, 23], by recombinant Escherichia coli [24] and several enterobacteria [25]; hydroxylation to 10-hydroxyoctadecenoic acid by Pseudomonas aeruginosa [2, 26-28] and dihydroxylation to 7,10-dihydroxy-8-(E)octadecenoic acid [29, 30].

The study was aimed to obtain oleic acid extracts through microbial biotransformation using fungi and evaluate their antimicrobial activity and in vitro anticancer activity on human lung carcinoma cell line (A549). The cytotoxicity of oleic acid extracts on normal mouse embryonic fibroblast cell line (NIH3T3) was also investigated to determine the selectivity of the extracts.

\section{MATERIALS AND METHODS Chemicals}

All chemicals and solvents were used in high purity, obtained from commercial sources. The solvents used for purification were distilled prior to use. Silica gel type 60 (Merck, 230-400 mesh) was used for column chromatography. Thin layer chromatography (TLC) was carried out on a 0.25 $\mathrm{mm}$ thick silica gel plate (Merck, $60 \mathrm{G} \mathrm{F254)}$ in $n$ hexane (Merck)/ethyl acetate (Merck) $(1: 1, \mathrm{v} / \mathrm{v})$. Compounds were detected either under UV light (at $254 \mathrm{~nm})$ or by spraying with sulfuric acid (1:1) solution followed by heating on a hot plate.

\section{Microorganisms and media}

Fungal cultures used in the present study were obtained from the USA Agriculture Research (C) 2019 Bulgarian Academy of Sciences, Union of Chemists in Bulgaria 


\section{Ö. Özşen et al.: Biotransformation of oleic acid and antimicrobial and anticancer actıvittes ...}

Service Culture Collection (NRRL), American Type Culture Collection (ATCC) and the culture collections at both Faculties of Pharmacy and Science at Anadolu University and the Faculty of Science of Eskisehir Osmangazi University in Turkey.

Fungi used in biotransformation studies were as follows: Aspergillus parasiticus NRRL 2999, A. niger NRRL 326, A. niger ATCC 10549, A. alliaceus NRRL 317, A. terreus var. africanus (isolate), A. nidulans (isolate), Penicillium claviforme MR 376, P. adametzii NRRL 737, P. chrysogenum NRRL 792, $P$. primulinum (isolate), $P$. valentinum (isolate), Fusarium solani ATCC 1284, F. moniliforme NRRL 2374, F. culmorum (isolate), $F$. heterosporium DSM 62719, Saccharomyces cerevisiae ATCC 9763, Hansenula anomala ATCC 20170, Sporobolomyces pararoseus ATCC 11385, Mucor ramannianus ATCC 1839, Neurospora crassa (isolate), Corynespora cassiicola DSM 62475, Alternaria alternata (isolate), Trametes versicolor ATCC 200801, Phanerachaete chrysosporium ME 446, Pycnoporus cinnabarinus (isolate), Trichotesium roseum (isolate) and Botrytis cinerea AHU 9424.

Fungal and bacterial strains used in antimicrobial studies were as follows: Aspergilllus niger ATCC 10549, Fusarium heterosporium DSM 62719, Penicillium valentinum (isolate), Saccharomyces cerevisiae ATCC 9763, Hansenula anomala ATCC20170, Sporobolomyces pararoseus ATCC 11385, Bacillus subtilis NRRL B-4378, Staphylococcus aureus ATCC 6538, Escherichia coli ATCC 8739.

All microorganisms were stored at $-85^{\circ} \mathrm{C}$ (Ultrafreezer, New Brunswick) in 15\% glycerol and maintained on nutrient agar (Merck) and malt extract agar (Merck) slants at $4^{\circ} \mathrm{C}$, respectively. Prior to testing, the purity of microorganisms was checked by subculturing twice on nutrient agar and malt extract agar.

\section{Biotransformation}

The cultures were precultured on potato dextrose agar (PDA) (Merck) slants at $25^{\circ} \mathrm{C}$ for 7 days prior to biotransformation experiments. A medium for growing fungi used in biotransformation experiments was prepared by mixing glucose $(20 \mathrm{~g})$ (Merck), yeast extract $(5 \mathrm{~g})$ (Merck), polypeptone (5 g) (Sigma), $\mathrm{NaCl}$ (5 g) (Merck) and $\mathrm{Na}_{2} \mathrm{HPO}_{4}(5 \mathrm{~g})$ (Merck) in distilled water (1 1). Spores were aseptically transferred into 20 Erlenmeyer flasks $(250 \mathrm{ml})$ containing $100 \mathrm{ml}$ of freshly prepared and autoclaved medium described above and left on an orbital shaker at $25^{\circ} \mathrm{C}$ for 2 days for the full growth. A solution of oleic acid
(500 mg) (Roth) in ethanol (20 ml) was then evenly distributed between these Erlenmeyer flasks containing the fungi. The fermentations were continued for a further 7 days.

\section{Extraction and purification}

After 7 days, the mycelium was filtered off and the broth was extracted with ethyl acetate. The extract was dried over $\mathrm{Na}_{2} \mathrm{SO}_{4}$ (Merck) and the solvent was evaporated to give an oily residue which was dissolved in acetone. Silica powder was then added to this solution and excess acetone was removed on an evaporator. The resulting powder containing biotransformation mixture was loaded into the glass column $(3 \mathrm{~cm}$ wide and $60 \mathrm{~cm}$ long) followed by purification via column chromatography. Approximately $120 \mathrm{~g}$ of powdery silica gel (Merck) was used as stationary phase and glass tubes with a capacity of $10 \mathrm{ml}$ of eluent solution $(1.5 \mathrm{~cm}$ wide and $12 \mathrm{~cm}$ long) were used to collect the fraction. Increasing concentrations of ethyl acetate (Merck) in light petroleum (Merck) were used as an eluent system.

\section{In vitro antimicrobial activity}

The broth microdilution method recommended by the Clinical Laboratory Standards Institute (CLSI) was used for assessing in vitro antibacterial and antifungal activities of extracts [31]. Ampicillin and amphotericin $\mathrm{B}$ were used as standard antibacterial and antifungal agents, respectively. They were purchased from Sigma. All tests were assayed in duplicate in two independent experiments.

\section{Broth microdilution test for bacteria}

Broth microdilution testing was performed in accordance with the guidelines of CLSI M100-S16 [32]. The minimum inhibitory concentrations (MIC) of oleic acid and its metabolites were studied by broth micro dilution method using 96-well microtiter plates (Sigma). Overnight grown microbial suspensions in double strength MuellerHinton broth (MHB) (Merck) were standardized turbidometrically to approximately $10^{8} \mathrm{CFU} 1 / \mathrm{ml}$ (using Mac Farland No: 0.5). Test extracts were dissolved in DMSO (50\%) and diluted in MHB to get a concentration range of $0.98-2000 \mu \mathrm{g} / \mathrm{ml}$. The solution was then two-fold diluted in MHB (100 $\mu 1)$, inoculated with bacterial strains and then incubated at $37^{\circ} \mathrm{C}$ for $24 \mathrm{~h}$. Resazurin solution (Sigma) was added to confirm the MICs. The MIC endpoint was defined as the lowest concentration with complete $(100 \%)$ growth inhibition. The results of antimicrobial testing were compared with those of standard ampicillin (Sigma) as 


\section{Ö. Özşen et al.: Biotransformation of oleic acid and antimicrobial and anticancer actıvittes ...}

antibacterial agent. The final concentrations were between 0.04 and $40 \mu \mathrm{g} / \mathrm{ml}$ and DMSO was assayed as the negative control.

\section{Broth microdilution test for yeasts}

CLSI broth microdilution testing was also performed exactly as outlined in document M27-A2 by using 96-well microtiter plates in RPMI-1640 medium (Sigma) and inocula of $0.5-2.5 \times 10^{3}$ cells $/ \mathrm{ml}$ (Mac Farland 0.5). The final concentrations of oleic acid and its extracts were between $0.98-2000$ $\mu \mathrm{g} / \mathrm{ml}$. MIC values were determined for $24 \mathrm{~h}$ at $37^{\circ} \mathrm{C}$ incubation. Resazurin solution was added to confirm the MICs. The MIC endpoint was defined as the lowest concentration with complete $(100 \%)$ growth inhibition [33].

\section{Broth microdilution test for fungi}

Similarly, in vitro susceptibility testing of fungal strains was also performed with broth microdilution methods as described in CLSI document M38-A2 [34]. Spore counts were made in a Thoma counting chamber (Hawskley) and spore solutions were prepared $\left(10^{8}\right.$ spore $\left.1 / \mathrm{ml}\right)$ by using sterile $0.02 \%$ Tween-80 (Merck) applied for collecting spores from plate.

\section{Cytotoxicity test}

NIH3T3 and A549 cells line were used for cytotoxicity tests. NIH3T3 cells were incubated in Dulbecco's modified Eagle's medium (DMEM; Hyclone, Thermo Scientific, USA) supplemented with fetal calf serum (Hyclone, Thermo Scientific, USA), $100 \mathrm{IU} / \mathrm{ml}$ penicillin and $100 \mathrm{mg} / \mathrm{ml}$ streptomycin (Hyclone, Thermo Scientific, USA) and $7.5 \% \quad \mathrm{NaHCO}_{3}$ at $37^{\circ} \mathrm{C}$ in a humidified atmosphere of $95 \%$ air and $5 \% \mathrm{CO}_{2}$. A549 cells were incubated in RPMI medium (Hyclone, Thermo Scientific, USA) supplemented with fetal calf serum, $100 \mathrm{IU} / \mathrm{ml}$ penicillin and $100 \mathrm{mg} / \mathrm{ml}$ streptomycin and $7.5 \% \quad \mathrm{NaHCO}_{3}$ at $37^{\circ} \mathrm{C}$ in a humidified atmosphere of $95 \%$ air and $5 \% \mathrm{CO}_{2}$. NIH3T3 and A549 cells were seeded at 10000 cells into each well of 96-well plates. After $24 \mathrm{~h}$ of incubating period, the culture media were removed and extracts were added to culture media at 8 concentrations $(500 ; 250 ; 125 ; 62.5 ; 31.2 ; 15.6 ; 7.8$; $3.9 \mu \mathrm{g} / \mathrm{ml}$ ). After $24 \mathrm{~h}$ of incubation, cytotoxicity test was performed using the In Cytotox-XTT 1 Parameter Cytotoxicity Kit (Xenometrix AG, Switzerland), which measures mitochondrial activity (tetrazolium hydroxide (XTT)) in NIH3T3 and A549 cells. The cells were washed with phosphate buffer saline (PBS) and then added to $200 \mu \mathrm{l} /$ well of fresh culture medium. XTTI and XTTII solution were mixed at 1:100 ratio. Then, 50 $\mu 1$ of this mixture was added to all wells. The plate was incubated for $3 \mathrm{~h}$ at $37^{\circ} \mathrm{C}, 5 \% \mathrm{CO}_{2}$. Then, the content of the well was mixed by pipetting up and down. OD of the plate was read at $480 \mathrm{~nm}$ with a reference wavelength at $680 \mathrm{~nm}$. Inhibition \% was calculated for each concentration of the biotransformation extracts and $\mathrm{IC}_{50}$ values were estimated by plotting a dose response curve of the inhibition \% equation (1) versus test compound concentrations.

Inhibition $\%=100-($ corrected mean OD sample $\times$ $100 /$ corrected mean OD solvent controls)

Cisplatin was used as positive control. Stock solutions of extracts were prepared in dimethyl sulfoxide (DMSO) and further dilutions were made with fresh culture medium. The final DMSO concentration was under $0.1 \%$. All experiments were performed in triplicate [35].

\section{Biotransformation reactions}

Pre-biotransformation screening experiments with oleic acid were carried out with 27 different microorganisms. Among the evaluated, the fungi Alternaria alternata (isolate) and Aspergillus terreus var. africanus (isolate) showed the presence of polar compounds when compared with oleic acid used as a reference compound according to chromatographic analyses which urged further preparative scale biotransformation studies for 7 days at $25^{\circ} \mathrm{C}$. From these biotransformation reactions, a pure single metabolite of oleic acid could not be obtained and metabolites were collected from the column as a mixture of at least two or more by eluting the column with increasing concentrations of ethyl acetate in petroleum ether. These fractions were named as 1, 2, 3, 4, 5 and 6 and used for antimicrobial and cytotoxic activity studies (Table 1).

\section{Antimicrobial activities}

The MIC values of oleic acid biotransformation extracts (1-6) are presented in Table 2. According to the MICs results, the most effective antifungal activity was shown by extract 5 at $62.5-125 \mu \mathrm{g} / \mathrm{ml}$ concentration for both mold and yeasts. Extracts 1, 2 and 4 gave better results than oleic acid itself against Hansenula anomala ATCC 20170 and Sporobolomyces pararoseus ATCC 11385. Extracts 1,2 and 4 at a concentration of $250 \mu \mathrm{g} / \mathrm{ml}$ were more effective than oleic acid $(500 \mu \mathrm{g} / \mathrm{ml}$ concentration) against all fungi. Among all extracts, the most effective antibacterial activity was shown by extract 5 at $125 \mu \mathrm{g} / \mathrm{ml}$ concentration. 
M. Manoochehri et al .: Cadmium (II) organomettalic complex with 4-choloro-N ((pyridine - 2 yl) methylene)...

Table 1. The biotransformation extracts

\begin{tabular}{cccc}
\hline $\begin{array}{c}\text { Extract } \\
\text { No }\end{array}$ & Fungus & Eluent (\%) & Eluent volume (ml) \\
\hline 1 & A. alternata & Ethyl acetate (25): Petroleum ether (75) & 200 \\
2 & A. alternata & Ethyl acetate (45): Petroleum ether (55) & 100 \\
3 & A. alternata & Ethyl acetate (55): Petroleum ether (45) & 200 \\
4 & A. terreus var. africanus & Ethyl acetate (50): Petroleum ether (50) & 200 \\
5 & A. terreus var. africanus & Ethyl acetate (55): Petroleum ether (45) & 100 \\
6 & A. terreus var. africanus & Ethyl acetate (60): Petroleum ether (40) & 100 \\
\hline
\end{tabular}

Table 2. Antimicrobial activity of the biotransformation extracts $(1-6)$ as mic $(\mu \mathrm{g} / \mathrm{ml})$

\begin{tabular}{c|cccccccc}
\hline Fungal Strains & 1 & 2 & 3 & 4 & 5 & 6 & $\begin{array}{c}\text { Oleic } \\
\text { Acid }\end{array}$ & Amf. B. \\
& & & & & & & & \\
\hline A. niger ATCC & 250 & 250 & 500 & 250 & 125 & 500 & 500 & 128 \\
F. & 250 & 250 & 500 & 250 & 125 & 500 & 500 & 64 \\
heterosporium & & & & & & & & \\
P. valentinum & 250 & 250 & 500 & 250 & 125 & 500 & 500 & 128 \\
S. cerevisiae & 125 & 125 & 250 & 125 & 62.5 & 250 & 125 & 128 \\
H. anomala & 62.5 & 62.5 & 500 & 62.5 & 62.5 & 125 & 125 & 64 \\
S. pararoseus & 250 & 250 & 500 & 250 & 125 & 500 & 500 & 64 \\
Bacterial Strains & & & & & & & 500 & Ampicillin \\
B. subtilis & 250 & 250 & 500 & 250 & 125 & 250 & 500 & 1 \\
S. aureus & 250 & 250 & 500 & 250 & 125 & 500 & 500 & 4 \\
$\quad$ E. coli & 250 & 250 & 500 & 250 & 125 & 500 & 500 & 2 \\
\hline
\end{tabular}

Table 3. Cytotoxic activity of the biotransformation extracts against cell lines

\begin{tabular}{c|c|c}
\hline Extract No & \multicolumn{2}{|c}{ IC 50 values for cell lines $(\mu \mathrm{g} / \mathrm{ml})$} \\
& NIH3T3 & A549 \\
\hline 1 & $>500$ & 463.2 \\
2 & 122.7 & 62.5 \\
3 & $>500$ & 500 \\
4 & 90.5 & $>500$ \\
5 & 46.3 & 89.6 \\
6 & 280.6 & 117 \\
Cisplatin & ND & 43.5 \\
\hline
\end{tabular}

Extracts 1 and 2 were more effective than oleic acid at $250 \mu \mathrm{g} / \mathrm{ml}$ concentration against all bacteria. On the other hand, extract 6 was effective against B. subtilis only. As a result, the microbial extracts of oleic acid were more active than oleic acid itself and showed good inhibitory activity against all tested microorganisms.

\section{Cytotoxic activities}

It is reported in the last world cancer report that lung cancer is the first cause of newly estimated cancer-related deaths. The most commonly diagnosed cancer types are prostate, lung and colorectal in males; breast, colorectal and lung in females in developed countries whereas they are lung, liver and stomach among males and breast, cervix uteri and lung among females in developing countries [36]. Therefore the anticancer activities of the biotransformation extracts were determined against human lung adenocarcinoma cell line, A549, by XTT assay, which were not studied before. Extracts 1, 2, 3, 4, 5 and 6 showed anticancer activity against $\mathrm{A} 549$ with $\mathrm{IC}_{50}$ values of $463.2,62.5,500,>500,89.6$ and $117 \mu \mathrm{g} / \mathrm{ml}$, respectively. According to these results, extract 2 exhibited the highest cytotoxic activity and its $\mathrm{IC}_{50}$ value is comparable with that of the positive control, cisplatin $\left(\mathrm{IC}_{50}=43.5 \mu \mathrm{g} / \mathrm{ml}\right)$.

Selectivity is the major problem of chemotherapy limiting drug discovery process. Primarily, an effective anticancer agent should selectively kill cancer cells without causing unacceptable toxicity on healthy cells [37]. Therefore, the cytotoxicity of these extracts was evaluated in a normal mouse embryonic fibroblast cell line, NIH3T3, by performing XTT test for determining selectivity. Based on the results of the XTT test against NIH3T3 cell line, extracts 2 and 6 were found to be selectively cytotoxic against A549 cell line. Extract 2 showed higher $\mathrm{IC}_{50}$ value against NIH3T3 cell line than A549 cell line which were 
Ö. Özşen et al.: Biotransformation of oleic acid and antimicrobial and anticancer activtties ...

122.7 and $62.5 \mu \mathrm{g} / \mathrm{ml}$, respectively whereas extract 6 showed higher $\mathrm{IC}_{50}$ value of $280.6 \mu \mathrm{g} / \mathrm{ml}$ against NIH3T3 cell line than its $\mathrm{IC}_{50}$ value of $117 \mu \mathrm{g} / \mathrm{ml}$ against A549 cell line. The $\mathrm{IC}_{50}$ values of the extracts against cell lines are presented in Table 3.

\section{CONCLUSIONS}

Microbial biotransformation of oleic acid with Alternaria alternate (clinical isolate) and Aspergillus terreus var. africanus (clinical isolate) produced extracts which exhibited anticancer and antimicrobial activities. The in vitro antimicrobial activities of the extracts were evaluated against 9 different pathogenic microorganisms. The microbial extracts of oleic acid were more active than oleic acid itself and showed good inhibitory activity against all tested microorganisms. In vitro anticancer evaluation studies revealed that extract 2 showed notable anticancer activity against A549 cells with an $\mathrm{IC}_{50}$ value of $62.5 \mu \mathrm{g} / \mathrm{ml}$, similar to our positive control, cisplatin, and further studies are required to evaluate the anticancer activities of extract 2.

Acknowledgements: This work is part of the PhD thesis of Özge ÖZŞEN and was supported by Eskişehir Osmangazi University Research Fund (Grant number: 2014-654). The authors would like to thank Prof. Fatih Demirci from Anadolu University for providing the standard microorganisms. Also special thanks to the Faculty of Science both in Anadolu and Eskisehir Osmangazi Universities in Turkey for providing their microbial isolates.

Conflict of interest: The authors declare no conflict of interest.

\section{REFERENCES}

1. T.L. Stedman, Stedman's Medical Dictionary, 7th edn., Vol. 26, Lipincott Williams \& Wilkins, USA, 1995, p. 2439.

2. A. Bódalo, J. Bastida, M.F. Maximo, A.M. Hidalgo, M.D. Murcia, Am. J. Biochem. Biotechnol., 1, 1, (2005).

3. A. Taqi, K.A. Askar, L. Mutihac, I. Stamatin, Food Agric. Immunol., 24, 2 (2013).

4. C.-M. Huang, C.-H. Chen, D. Pornpattananangkul, L. Zhang, M. Chan, M.-F. Hsieh, L. Zhang, Biomaterials, 32, 1 (2010).

5. G. Bergsson, Ó. Steingrímsson, H. Thormar, Int. J. Antimicrob. Agents, 20, 4 (2002).

6. V. Kesari, A. Das, L. Rangan, Biomass Bioenerg., 34, 1 (2010).

7. K. Thillairajasekar, V. Duraipandiyan, P. Perumal, S. Ignacimuthu, Int. J. Integr. Biol., 5, 3 (2009).
8. A.B. Hsouna, A.S. Alayed, Afr. J. Biotechnol., 11, 47 (2012).

9. J.d.D. Tamokou, M.D.J. Simo, L.P. Keilah, M. Tene, P. Tane, J.R. Kuiate, BMC complement. Altern. Med., 12, 99 (2012).

10. S. Martín-Peláez, M.I. Covas, M. Fitó, A. Kušar, I. Pravst, Mol. Nutr. Food Res., 57, 5 (2013).

11. N. Senthilkumar, S. Murugesan, K.B. Vijayalakshmi, Asian J. Plant Sci. Res., 2, 2 (2012).

12. I. Orhan, B. Özçelik, B. Şener, Turk J. Biol., 35, 2, (2011).

13. M. Sellami, A. Chaari, I. Aissa, M. Bouaziz, Y. Gargouri, N. Miled, Process Biochem., 48, 10, (2013).

14. O.D.J. Dailey, X. Wang, F. Chen, G. Huang, Anticancer Res., 31, 10 (2011).

15. K. Soda, From Proc.- World Conf. Biotechnol. Fats Oils Ind., 178, 9 (1988).

16. S.H. El-Sharkawy, W. Yang, L. Dostal, J.P.N. Rosazza, J. Pharm. Sci., 6, 2 (1992).

17. S. Koritala, L. Hosie, C.T. Hou, C.W. Hesseltine, M.O. Bagby, Appl. Microbiol. Biotechnol., 32, 3 (1989).

18. E.N. Davis, L.L. Wallen, J.C. Goodwin, W.K. Rohwedder, R.A. Rhodes, Lipids, 4, 5 (1969).

19. G.J.J. Schroepfer, J. Am. Chem. Soc., 87, 6, (1965).

20. G.J.J. Schroepfer, K. Bloch, J. Biol. Chem., 240, 1 (1964).

21. L.L. Wallen, US Patent, 3115442 (1963).

22. W.G.J. Niehaus, A. Kisic, A. Torkelson, D.J. Bednarczyk, G.J.J. Schroepfer, J. Biol. Chem., 245, 15 (1970).

23. S. Gocho, N. Tabogami, M. Inagaki, C. Kawabata, T. Komai, Biosci. Biotechnol. Biochem., 59, 8 (1995).

24. E.-Y. Jeon, J.-H. Lee, K.-M. Yang, Y.-C. Joo, D.-K. Oh, J.-B. Park, Proc. Biochem., 47, 6, (2012).

25. N. Esaki, S. Ito, W. Blank, K. Soda, Biosci. Biotechnol. Biochem., 58, 2 (1994).

26. J. Bastida, C.D. Andres, J. Cullere, M. Busquets, A. Manresa, Biotechnol. Lett., 21, 12 (1999).

27. T.M. Kuo, J.-K. Huang, D. Labeda, L. Wen, G. Knothe, Curr. Microbiol., 57, 5 (2008).

28. I. Martin-Arjol, M. Busquets, T.A. Isbell, A. Manresa, Appl. Microbiol. Biotechnol., 97, 18 (2013).

29. C.T. Hou, M.O. Bagby, J. Ind. Microbiol., 7, 2 (1991).

30. C.T. Hou, M.O. Bagby, R.D. Plattner, S. Koritala, J. Am. Oil Chem. Soc., 68, 2 (1991).

31.D. Amsterdam, Susceptibility testing of antimicrobials in liquid media, in: Antibiotics in Laboratory Medicine, 4th edn., Williams \&Wilkins, Baltimore, 1996.

32. Performance standards for antimicrobial susceptibility testing, Sixteenth informational supplement, CLLS document M100-S16, Clinical and Laboratory Standards Institute (CLSI), Wayne, PA, USA. 2006.

33. Reference method for broth dilution antifungal susceptibility testing of yeast approved standard, 2nd edn., CLLS document M27-A2, Clinical and 
Ö. Özşen et al.: Biotransformation of oleic acid and antimicrobial and anticancer actıvities ...

Laboratory Standards Institute (CLSI), Wayne, PA, USA, 2002.

34. Reference method for broth dilution antifungal susceptibility testing of filamentous fungi, approved standard, 2nd edn., CLLS document M38-A2, Clinical and Laboratory Standards Institute (CLSI), Wayne, PA, USA. 2008.
35. Ö. Özşen, İ. Kıran, İ. Dağ, Ö. Atlı, G.A. Çiftçi, F. Demirci, Process Biochem., 52, 130 (2017).

36. Global Cancer Facts and Figures, 3rd edn., American Cancer Society, Atlanta, 2015.

37. F.A. Khorshid, S.S. Mushref, N.T. Heffny, JKAU: Med. Sci., 12, (2005). 


\title{
Spectrophotometric determination of significant minerals in milk samples found in Lahore (Pakistan) for ensuring food safety
}

\author{
R. Rehman*, T. Tanveer \\ Institute of Chemistry, University of the Punjab, Lahore-54590, Pakistan
}

Received March 28, 2018; Revised February 27, 2019

\begin{abstract}
This work was carried out for determination of essential and toxic metals ions in different milk powder samples commonly found in Lahore (Pakistan). Samples were collected from local markets and after dry ashing, they were dissolved in $2 \mathrm{M}$ nitric acid for complete mineralization. Then the concentration of metal ions was determined by flame photometry and atomic absorption spectrometry. The concentration levels of cadmium, lead and nickel were found to be below detection limits in all samples, whereas the maximum values of sodium, potassium, calcium, magnesium, iron, and zinc were 48.55, 0.96, $4.94 \mathrm{mg} / \mathrm{g}$, and 768, 4.12 and $3.02 \mu \mathrm{g} / \mathrm{g}$ respectively. Minimum amounts of these metal ions were: $3.69,0.07,0.85 \mathrm{mg} / \mathrm{g}$ and $214,0.21,1.02 \mu \mathrm{g} / \mathrm{g}$, respectively. The amounts of all metal ions were found to be within safe limits as recommended by WHO.
\end{abstract}

Key words: Milk samples, powder milk, toxic metals, essential metals, flame emission spectroscopy, atomic absorption spectrometry

\section{INTRODUCTION}

Milk is a white fluid created by the mammary organs of vertebrates. It is an essential nourishment for infants of mammals before they can process different sorts of nutrition. Early-lactation drain contains colostrum, which conveys the mother's antibodies to its young and can decrease the danger of numerous ailments. It contains numerous other nutrients [1].

Milk is a perplexing material comprising different components which have a noteworthy role despite the fact that they are present in low amounts. Expanded consciousness of the impact of eating routine on human fitness has encouraged making food items of higher quality which must be rich in supplements and vitamins. Great quality estimations are fundamental to control, keep up items and process quality, in assembling, exchange and research [2]. For healthy nourishment and proper growth of the human body, milk and dairy items have been perceived worldwide as a good source [3]. Enlarged environmental contamination has enhanced the effects of milk uncleanness and doubts about the quality of milk. Because of xenobiotic compounds and environmental pollution milk contamination is registered worldwide which exerts bad influence on public health. Metals of high density and toxicity at low quantity are referred to as 'heavy metals'. Their harmful effects on humans are constantly growing in the previous few decades [4]. Their importance for human beings cannot be denied, but excessive amounts are detrimental. For example: high amount of iron (II) leads to Alzheimer's or Parkinson's diseases [6]. $\mathrm{Cu}(\mathrm{II})$ is essential for respiration and proper growth of body, but its excess leads to muscles injury, lungs irritation, liver cancer ending up to Wilson disease [7]. $\mathrm{Zn}$ (II) is a central atom of carbonic anhydrase that controls $\mathrm{CO}_{2}$ concentration in the body, but its excess leads to rectum cancer [8]. $\mathrm{Cr}(\mathrm{VI})$ is enlisted in the top sixteen carcinogenic elements and its excess results in dizziness, abdominal pain, liver CNS and heart tissues toxicity [9]. $\mathrm{Ni}$ (II) is a central atom of urease enzyme which plays an important role during amino acid digestion. But its excess leads to renal failure and lungs diseases [10]. Excess of $\mathrm{Co}(\mathrm{II})$ causes DNA fragmentation [11]. $\mathrm{Cd}(\mathrm{II})$ and $\mathrm{Pb}$ (II) are non-essential elements in biological systems [12]. Cd(II) excess leads to gastrointestinal tract irritation, joint pain and Itai Itai disease. $\mathrm{Pb}(\mathrm{II})$ excess leads to Alzheimer's, kidney failure, CNS and reproductive systems infections [14]. So, the contents of these metals should be regularly analyzed for continuous monitoring of milk and dairy products for their safe limits [15]. The goal of the current study is to estimate the amount of essential and heavy metals in milk powder and infant milk formulas purchased from different areas of Lahore (Pakistan). The amount measured was compared with the Recommended Dietary Allowance (RDA) given by different international organizations.

* To whom all correspondence should be sent:

E-mail: grinorganic@yahoo.com, 


\section{EXPERIMENTAL}

\section{Materials and instrumentation}

Analytical grade chemicals (Merck, Germany) were used for analysis. For standard solution preparation certified standard solutions (Fluka) were employed. Flame photometer (Corning 410), atomic absorption spectrometer (AAnalyst100),
Furnace (Nabertherm, Germany) were used. Five milk powder samples from different localities were collected from various areas of Lahore (Pakistan) and categorized as shown in table 1. Then, after estimating their metal ions concentration, data were analyzed statistically for validation of the results by Microsoft Excel (2010). Average values were reported in the results.

Table 1. Samples of milk powder.

\begin{tabular}{|c|l|c|l|}
\hline Sample ID & Sampling area of Lahore & Sample ID & Sampling area of Lahore \\
\hline 1. & Sadar Cantt & 2. & Pakistan Mint Stop \\
\hline 3. & Johar Town & 4. & Nishat Colony \\
\hline 5. & Wapda Town & 6. & DHA phase 5 \\
\hline 7. & NFC Society & 8. & Taj Bagh \\
\hline 9. & Darogawala & 10. & Sui gas society \\
\hline 11. & Bahria Town & 12. & Shalimar Link Road \\
\hline 13. & Al-Raheem Garden & 14. & PIA housing society \\
\hline 15. & EME Society & 16. & Awan Town \\
\hline 17. & Harbanspura & 18. & Near General Hospital \\
\hline 19. & Canal Bank Housing Scheme & 20. & Mazung Chungi \\
\hline
\end{tabular}

\section{Procedure and measurement}

Chemical treatment of the milk samples was carried out as followed: $1 \mathrm{~g}$ of milk powder was taken in a china dish. It was heated until it was converted into black ash. After cooling, 3-5 mL of concentrated nitric acid was added and heated again till all nitric acid was evaporated. Same procedure was repeated till the emitted brown fumes turned to white fumes. Then, the resulting black ash was kept in a furnace (Nabertherm) at $550{ }^{\circ} \mathrm{C}$, for $2 \mathrm{~h}$ for decomposition of organic components of the sample and complete mineralization [15]. The resulting white ash was dissolved in $3-5 \mathrm{~mL}$ of $2 \mathrm{M}$ $\mathrm{HNO}_{3}$. After filtration, the filtrate was diluted with distilled water in a $100-\mathrm{mL}$ flask. The instrument was calibrated using standard solutions of metal ions and all samples were analyzed in triplicate. Average values were used for graphical representation of metal ions in various samples.

\section{RESULTS AND DISCUSSION}

Milk is the most important diet of human nutrition especially for infants, because it contains a lot of minerals which are required for their proper growth.

In this work the concentration of metal ions $\mathrm{Na}(\mathrm{I}), \mathrm{K}(\mathrm{I}), \mathrm{Ca}(\mathrm{II}), \mathrm{Mg}(\mathrm{II}), \mathrm{Fe}(\mathrm{II}), \mathrm{Zn}(\mathrm{II}), \mathrm{Pb}(\mathrm{II})$, $\mathrm{Cd}(\mathrm{II})$ and $\mathrm{Ni}$ (II) in different brands of milk formula and commercially available milk powder samples obtained from different areas of Lahore (Pakistan) was determined. Some preliminary physico-chemical analyses were done for comparing percentage volatile organic contents and inorganic ash contents of these samples before metal ion determination and results are reported in Table 2

It indicates that lower moisture contents are found in those samples which are properly packed and sealed in metallic containers, which leads to their long shelf life, whereas open samples and those which are sold as in shopping bags contain higher moisture leading to deterioration by bacterial and fungal growth. Similar trend is observed for ash contents or inorganic contents. Milk samples packed in plastic or cloth bag samples have higher inorganic contents and less volatile organic contents.

After ashing of the milk powder samples, their metal contents were estimated and reported in Table 3. Concentration of essential elements was found to be higher as compared to toxic elements. In most of the samples, no toxic elements were found and the samples are safe for human use. Concentrations of essential metals, like: sodium and calcium, were higher in all samples as compared to other metal ions. In some milk powder samples, lead, cadmium and nickel were detected in minute amounts. Sodium was found in samples in the 4.25$48.55 \mathrm{mg} / \mathrm{g}$ range, as is obvious from Fig. 1. Average amount was $11.94 \mathrm{mg} / \mathrm{g}$. Sodium is an important component of extracellular fluids in the organism. Osmotic pressure of body is also controlled by sodium [8-10]. In Addison's diseases, diarrhea, intestinal obstruction and vomiting, 
R. Rehman, T. Tanveer: Spectrophotometric determination of significant minerals in milk samples found in Lahore... hyponatremia occurs (deficiency of sodium in blood serum) while in Cushion's disease hypernatremia occurs [8]. Its deficiency may cause growth retardation [11]. $1500 \mathrm{mg}$ of sodium is required per day.

Potassium was found in all samples in the 0.6$0.96 \mathrm{mg} / \mathrm{g}$ range, as shown in Fig. 2. Average value was $0.528 \mathrm{mg} / \mathrm{g}$. It plays a role in acid-base balance, osmotic pressure regulation, nerve impulse conduction, contraction of muscles [12]. Potassium acts as a cofactor in protein synthesis.

Calcium was found in the range of about $0.852-$ $4.942 \mathrm{mg} / \mathrm{g}$ and the average value in all samples was $2.43 \mathrm{mg} / \mathrm{g}$, as shown in Fig. 3. Large number of enzymes like adenosine triphosphates (ATPase), succinic dehydrogenase and lipase are activated by calcium. As a constituent of bones and teeth calcium plays an important role. Excess calcium is excreted because extra calcium leads to respiratory and cardiac failure [12].

Magnesium amount was in the range of 214-768 samples was $598.7 \mu \mathrm{g} / \mathrm{g}$. Magnesium is the major component of bones and teeth [9]. Magnesium depletion also occurs due to chronic or excessive diarrhea and vomiting [12]. The best sources include leafy green vegetables.

Iron was in the range of $0.21-3.02 \mu \mathrm{g} / \mathrm{g}$ and the average value in all samples was $1.771 \mu \mathrm{g} / \mathrm{g}$, as shown in Fig. 5. The amount of iron found in a previously studied milk sample was $3.2-12.91 \mu \mathrm{g} / \mathrm{g}$ [13]. In animals and humans, iron is an essential component. Muscle, hemoglobin and myoglobin have iron as a major component. Our food contains considerable amounts of iron which satisfy our needs. Anemia may result due to iron deficiency, which can treated by artificial medication [14]. The largest quantity of iron is present in the liver and spleen. Constipation and vomiting occur by taking orally iron salts [16]. It was observed that iron was in higher amount in infant milk formula than in commercially available milk powders. $\mu \mathrm{g} / \mathrm{g}$, as shown in Fig. 4. Its average value in all

Table 2. Physicochemical analysis of milk powder samples.

\begin{tabular}{|c|c|c|c|c|}
\hline $\begin{array}{l}\text { Sample } \\
\text { ID }\end{array}$ & Powder packaging material & $\begin{array}{l}\text { Moisture } \\
(\%)\end{array}$ & $\begin{array}{l}\text { Volatile organic contents } \\
\qquad(\%)\end{array}$ & $\begin{array}{c}\text { Inorganic ash contents } \\
(\%)\end{array}$ \\
\hline 1. & Plastic bag & 4.5 & 82.9 & 6.4 \\
\hline 2. & Metallic container & 2.1 & 86.5 & 5.2 \\
\hline 3. & Cloth bag & 6.3 & 79.9 & 7.6 \\
\hline 4. & Plastic bag & 4.1 & 82.9 & 6.8 \\
\hline 5. & Cloth bag & 5.5 & 81.1 & 7.2 \\
\hline 6. & Metallic container & 2.3 & 86.9 & 4.6 \\
\hline 7. & Metallic container & 1.7 & 87.3 & 4.8 \\
\hline 8. & Metallic container & 1.8 & 86.9 & 5.1 \\
\hline 9. & Metallic container & 1.6 & 88 & 4.2 \\
\hline 10. & Cloth bag & 5.4 & 80.1 & 8.3 \\
\hline 11. & Plastic bag & 4.2 & 82.1 & 7.5 \\
\hline 12. & Cloth bag & 5.3 & 84.3 & 4.2 \\
\hline 13. & Plastic bag & 4.3 & 82.3 & 7.2 \\
\hline 14. & Plastic bag & 3.9 & 82.3 & 7.6 \\
\hline 15. & Plastic bag & 3.6 & 82.6 & 7.6 \\
\hline 16. & Metallic container & 1.8 & 87.2 & 4.8 \\
\hline 17. & Plastic bag & 4.1 & 82.5 & 7.2 \\
\hline 18. & Plastic bag & 3.7 & 82.8 & 7.3 \\
\hline 19. & Plastic bag & 3.6 & 83.9 & 6.3 \\
\hline 20. & Cloth bag & 4.6 & 83.1 & 6.1 \\
\hline \multicolumn{5}{|c|}{ Descriptive Statistical Analysis of Data } \\
\hline & Average & 3.72 & 96.18 & 6.3 \\
\hline & Maximum & 1.6 & 92.3 & 4.2 \\
\hline & Minimum & 6.3 & 88 & 8.3 \\
\hline & Standard deviation $( \pm)$ & 1.42 & 2.49 & 1.31 \\
\hline
\end{tabular}


Table 3. Concentration of metal ions in milk powder samples found in Lahore (Pakistan).

\begin{tabular}{|c|c|c|c|c|c|c|c|c|c|}
\hline \multirow[b]{3}{*}{ Sample ID } & \multicolumn{9}{|c|}{ Concentration } \\
\hline & \multicolumn{2}{|c|}{ Alkali metals } & \multicolumn{2}{|c|}{ Alkaline earth metals } & \multicolumn{2}{|c|}{ Transition metals } & \multicolumn{3}{|c|}{ Toxic metals } \\
\hline & $\begin{array}{c}\mathrm{Na}(\mathrm{I}) \\
(\mathrm{mg} / \mathrm{g})\end{array}$ & $\begin{array}{c}\mathrm{K}(\mathrm{I}) \\
(\mathrm{mg} / \mathrm{g})\end{array}$ & $\begin{array}{l}\mathrm{Ca}(\mathrm{II}) \\
(\mathrm{mg} / \mathrm{g})\end{array}$ & $\begin{array}{c}\mathrm{Mg}(\mathrm{II}) \\
(\mu \mathrm{g} / \mathrm{g})\end{array}$ & $\begin{array}{l}\mathrm{Fe}(\mathrm{II}) \\
(\mu \mathrm{g} / \mathrm{g})\end{array}$ & $\begin{array}{l}\mathrm{Zn}(\mathrm{II}) \\
(\mu \mathrm{g} / \mathrm{g})\end{array}$ & $\begin{array}{l}\mathrm{Pb}(\mathrm{II}) \\
(\mu \mathrm{g} / \mathrm{g})\end{array}$ & $\begin{array}{l}\mathrm{Cd}(\mathrm{II}) \\
(\mu \mathrm{g} / \mathrm{g})\end{array}$ & $\begin{array}{l}\mathrm{Ni}(\mathrm{II}) \\
(\mu \mathrm{g} / \mathrm{g})\end{array}$ \\
\hline 1. & 16.65 & 0.07 & 2.052 & 214 & 0.21 & 1.22 & BDL & BDL & BDL \\
\hline 2. & 12.96 & 0.15 & 2.73 & 422 & 3.2 & 1.11 & BDL & 0.01 & BDL \\
\hline 3. & 4.92 & 0.29 & 1.06 & 414 & 1.1 & 1.17 & BDL & BDL & 0.01 \\
\hline 4. & 3.82 & 0.47 & 1.47 & 324 & 4.1 & 2.03 & BDL & BDL & BDL \\
\hline 5. & 3.69 & 0.56 & 1.79 & 428 & 3.1 & 1.71 & BDL & 0.01 & BDL \\
\hline 6. & 9.91 & 0.59 & 1.76 & 523 & 0.41 & 1.22 & BDL & BDL & BDL \\
\hline 7. & 14.30 & 0.65 & 1.06 & 514 & 0.56 & 1.17 & BDL & BDL & BDL \\
\hline 8. & 48.55 & 0.81 & 0.88 & 614 & 0.64 & 1.71 & 0.01 & BDL & BDL \\
\hline 9. & 12.35 & 0.89 & 0.85 & 267 & 4.12 & 1.02 & BDL & BDL & BDL \\
\hline 10. & 16.03 & 0.96 & 1.63 & 718 & 2.11 & 1.11 & BDL & BDL & BDL \\
\hline 11. & (8.97 & 0.68 & 3.53 & 567 & 1.11 & 3.01 & BDL & BDL & 0.01 \\
\hline 12. & 4.67 & 0.32 & 2.99 & 522 & 2.13 & 2.13 & 0.01 & BDL & BDL \\
\hline 13. & 14.80 & 0.6 & 4.18 & 655 & 2.11 & 2.11 & BDL & BDL & BDL \\
\hline 14. & 17.26 & 0.7 & 2.58 & 455 & 3.02 & 3.02 & BDL & BDL & BDL \\
\hline 15. & 5.17 & 0.19 & 4.94 & 567 & 1.11 & 1.11 & BDL & BDL & BDL \\
\hline 16. & 9.01 & 0.46 & 3.34 & 436 & 1.01 & 1.75 & 0.01 & BDL & BDL \\
\hline 17. & 8.52 & 0.48 & 3.02 & 567 & 1.11 & 1.11 & BDL & BDL & BDL \\
\hline 18. & 4.25 & 0.36 & 4.46 & 345 & 1.21 & 1.21 & BDL & BDL & BDL \\
\hline 19. & 11.61 & 0.37 & 3.65 & 455 & 1.32 & 1.32 & BDL & BDL & BDL \\
\hline 20. & 11.51 & 0.96 & 2.47 & 768 & 1.74 & 1.74 & BDL & BDL & BDL \\
\hline \multicolumn{10}{|c|}{ Descriptive Statistical Analysis of Data } \\
\hline Mean & 11.94 & 0.53 & 2.52 & 488.75 & 1.771 & 1.599 & BDL & BDL & BDL \\
\hline Minimum & 3.69 & 0.07 & 0.85 & 214 & 0.21 & 1.02 & BDL & BDL & $\mathrm{BDL}$ \\
\hline Maximum & 48.55 & 0.96 & 4.94 & 768 & 4.12 & 3.02 & 0.01 & 0.01 & 0.01 \\
\hline $\begin{array}{l}\text { Standard } \\
\text { deviation }\end{array}$ & 9.72 & 0.068 & 1.52 & 203.37 & 1.398 & 0.37 & BDL & BDL & BDL \\
\hline
\end{tabular}

*BDL= Below detection limit

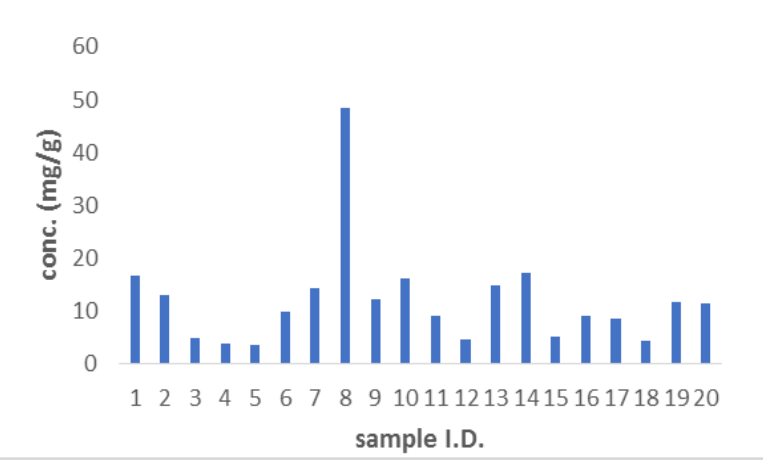

Fig. 1. Concentration of sodium in samples. 


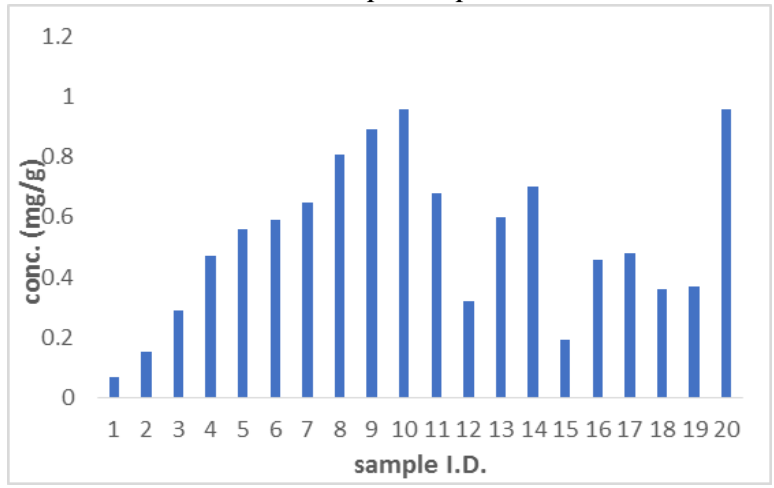

Fig. 2. Concentration of potassium in samples.

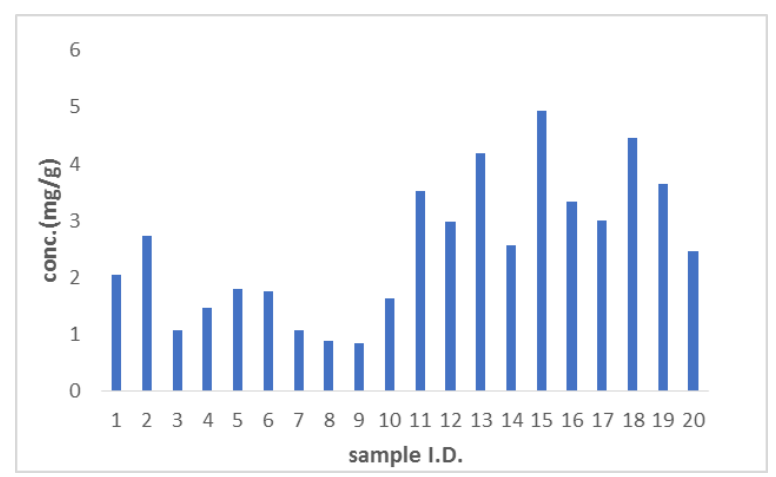

Fig. 3. Concentration of calcium in samples

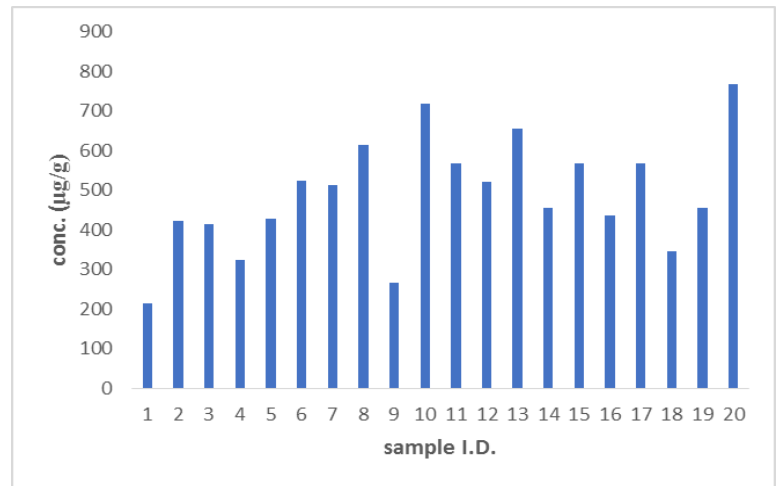

Fig. 4. Concentration of magnesium in samples.

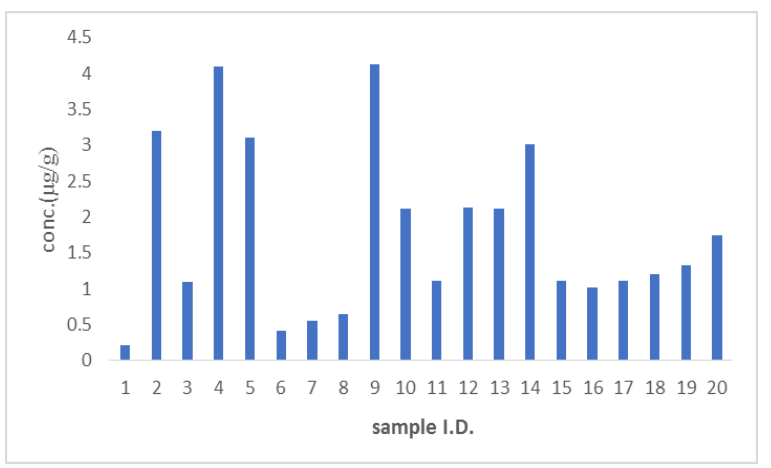

Fig. 5. Concentration of iron in samples.

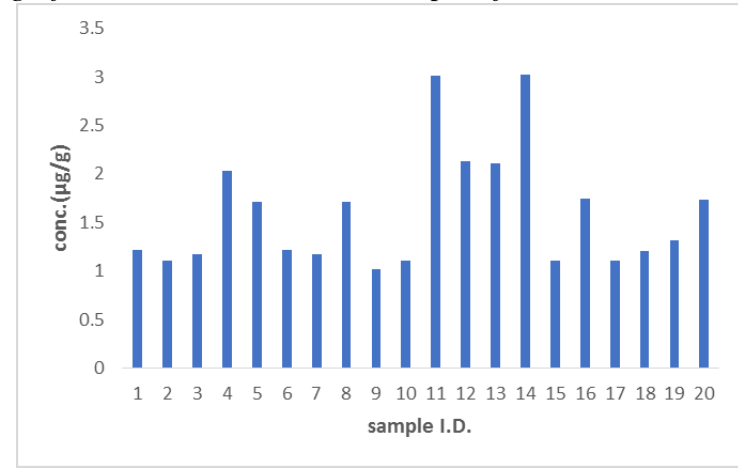

Fig. 6. Concentration of zinc in samples.

Zinc was present in the range of 1.02-3.01 $\mu \mathrm{g} / \mathrm{g}$ and the average value of zinc was $1.59 \mu \mathrm{g} / \mathrm{g}$, as shown in Fig. 6. It is important for proper working of carbonic anhydrase enzyme which regulates carbon dioxide concentration in blood. Its excess, however, leads to "fever foundry". The concentration of zinc was lower than that found in a previous study which was $2.016 \mu \mathrm{g} / \mathrm{g}$ [17].

Lead is the most dangerous metal for all living organisms, mostly for plants, animals and specifically microorganisms. Main source of lead are the lead mines, fuel combustion sewage sludge applications and farm yard manure. Continuous contact with lead can result in anemia, pale skin, paralysis, nausea, and vomiting. The nervous system may also be affected by prolonged exposure, resulting in severe headache, coma and death. Fertility lost and birth defects may also occur due to prolonged exposure to lead [18]. In all milk powder samples, $\mathrm{Pb}$ (II) was not found except for samples collected from Taj Bagh and Shalimar Link Road area. So, all samples of milk were safe to use. Lungs and kidney may be damaged due to prolonged inhalation [19]. Due to nickel exposure, itching of the fingers, hands, and forearms may occur. Respiratory effects, lung and nasal cancer had been linked to nickel inhalation [20]. Nickel was almost absent in all milk samples except for samples collected from Johar Town and Bahria Town. Serious health problems may cause cadmium [21], like: kidney and heart failure [22]. Chronic poisoning leads to muscle, lungs and skeleton damage disorders in humans [23]. Fortunately, cadmium was also absent in most of the milk samples found in Lahore, except for those samples collected from Pakistan Mint stop and Wapda Town.

\section{CONCLUSION}

It was found in the present study that packaging of milk powder samples in plastic, cloth or metallic containers had great effect on their composition. Samples packed in metallic containers have less 
moisture and more nutritional elements as compared to those packed in plastic or cloth. The concentration levels of cadmium, lead and nickel were below detection limits in all samples, whereas the maximum values of sodium, potassium, calcium, magnesium, iron, zinc were 48.55, 0.96, $4.94 \mathrm{mg} / \mathrm{g}$, and $768,4.12$ and $3.02 \mu \mathrm{g} / \mathrm{g}$, respectively. Minimum amounts of these metal ions were: $3.69,0.07,0.85 \mathrm{mg} / \mathrm{g}$ and $214,0.21,1.02$ $\mu \mathrm{g} / \mathrm{g}$, respectively. All samples contained essential metals in considerable amounts, especially the most important nutrients while toxic metals, like $\mathrm{Pb}$ (II), $\mathrm{Cd}(\mathrm{II})$ and $\mathrm{Ni}$ (II) were absent in most of the samples. Hence, all these milk samples were safe for drinking and usage in dairy food products.

\section{REFERENCES}

1. P. Pehrsson, D. Haytowitz, J. Holden, C. Perry, D. Beckler, J. Food Compos. Anal., 13, 379 (2000).

2. S. Birghila, S. Dobrinas, G. Stanciu, A. Soceanu, Env. Eng. Manage. J., 7 (2008).

3. Z. Hussain, A. Nazir, U. Shafique M. Salman, J. Sci. Res., 40, 1 (2010).

4. M. I. Muhib, M. A. Z. Chowdhury, N. J. Easha, M. M. Rahman, M. Shammi, Z. Fardous, M. L. Bari, M. K. Uddin, M. Kurasaki, M. K. Alam, Int. J. Food Contamin., 3, 16 (2016).

5. J. Alan, Phytoremed. Contamin. Soil Water, 85, (2000).

6. V. Lacronique, A. Boureux, R. Monni, S. Dumon, M. Mauchauffé, P. Mayeux, F. Gouilleux, R. Berger, S. Gisselbrecht, J. Ghysdael, Blood, 95, 2076 (2000).

7. M. J. Blaylock, J. W. Huang, Phytoextraction of metals, in: Phytoremediation of toxic metals using plants to clean up the environment, I. Raskin, B.D. Ensley (eds.). Wiley, New York. 2000, p. 53.
8. V. Hays, M. Swenson, Dukes' physiology of domestic animals, Cornell University Press, London, UK, 1985, p. 449.

9. R. T. Greenlee, T. Murray, S. Bolden, P. A. Wingo, CA: a Cancer Journal for Clinicians, 50, 7 (2000).

10. A. Leri, P. P. Claudio, Q. Li, X. Wang, K. Reiss, S. Wang, A. Malhotra, J. Kajstura, P. Anversa, J. Clinical Invest., 101, 1326 (1998).

11. J. Soulie, M.-F. Rousseau-Merck, H. Mouly, C. Nezelof, Virchows Archiv B Cell Pathology ZellPathologie, 50, 339 (1986).

12. K. Soetan, C. Olaiya, O. Oyewole, Afric. J. Food Sci., 4, 200 (2010).

13. A. W. Ernst, Appl. Geochem, 11, 163 (1996).

14. A.Kabata-Pendias, H. Pedias, Biogeochemistry of trace elements, 2nd edn. Wyd. Nauk PWN, Warszawa (in Polish), 1999.

15. S. Moncayo, S. Manzoor, J.D. Rosales, J. Anzano, J.O. Caceres, Food Chem., 232, 322 (2017).

16. P. Kijewski, Rocz. Glebozn, 29, 98 (1994).

17. P. Licata, D. Trombetta, M. Cristani, F. Giofre, D. Martino, M. Calo, F. Naccari, Env. Int., 30, 1 (2004).

18. Z. Dobrzanski, R. Kolacz, H. Górecka, K. Chojnacka, A. Bartkowiak, Polish J. Env. Stud., 14, 685 (2005).

19. J. T. Zelikoff, L. C. Chen, M. D. Cohen, R. B. Schlesinger, J. Toxic Env. Health B: Crit. Rev., 5, 269 (2002).

20. Bletsas, A. Khisti, D. P. Reed, A. Lippman, IEEE J. on Select Area Commun, 24, 659 (2006).

21. D. M. Stetson, A. Mazur, Sage Publications, 154 (1995).

22. G. Brümmer, J. Gerth, U. Herms, J. Plant Nut. Soil Sc., 149, 382 (1986).

23. E. Gorlach, F. Gambuś, Rocz. Glebozn., 42, 207 (1991). 


\title{
The influence of pigment proportions and calendering of coated paperboards on dot
}

\author{
gain \\ S. Sönmez ${ }^{1 *}$ Ö. Özden ${ }^{2}$ \\ ${ }^{1}$ Marmara University, School of Applied Sciences, Department of Printing Technologies, Goztepe-34722, Istanbul, \\ Turkey \\ ${ }^{2}$ Istanbul University-Cerrahpaşa, Faculty of Forest, Department of Forest Product Chemistry and Technology, \\ Bahcekoy-34473, Istanbul, Turkey
}

Received, January 2, 2018; Accepted, December 5, 2018

\begin{abstract}
Dot gain is called Tone Value Increase (TVI). Low dot gain and rounder dot shape are important properties to obtain a good print. Dot gain is a measure of how much extra weight a given percent dot or tone has gained on the final printed substrate by comparison to the actual dot area on a press plate. Dot gain value depends on many factors. The interactions of paper, ink and press conditions are important determinates for a good print. In addition, the pigment coating process and calendering conditions have a significant effect on the printability of paperboards. Pigment coating formulations including mineral pigments, binders and additives improve the gloss, brightness, opacity and smoothness of the paperboards. As a result, print quality of paperboards increase. A uniform paperboard surface is needed to obtain a high quality surface smoothness after the coating process. High surface smoothness improves the uniformity of the dot shape and size. Pigment coating formulation parameters; pigment selection, binder selection and binder level influence print quality. The aims of this study - pigment selection and ratio - are to determine the effect on dot gain in lithography printing. To this aim, base paperboards were coated using five pigment coating formulations which included different combinations of kaolin, calcium carbonate and titanium dioxide pigment with a styreneln-butyl acrylate copolymer binder using a bar application. After coating, the samples were air-dried overnight under TAPPI conditions. Then, half of the coated-paperboards were calendered. Tone scales from 1 to $100 \%$ were offset printed using black ink on the uncalendered and calenderedcoated paperboards. Then, from the printed tone scale, the tone area values were measured with the Gretagmacbeth Spectrolino spectrophotometer. These values showed that pigment coating improved the surface optical and physical properties of paperboards. After calendering, the roughness values of coated paperboards decreased. In addition, the obtained dot and line sharpness on calendered-coated paperboards were better than on uncalenderedcoated paperboards. However, the dot gain values of calendered-coated paperboards had fewer dot gains than uncalendered-coated paperboards. It was established that the variation of pigment proportions in the coating formulations had no significant effect on dot gain.
\end{abstract}

Keywords: Pigment, Coating, Calendering, Offset, TVI, Dot gain

\section{INTRODUCTION}

Considerable differences in tone value can occur during both platemaking and printing and they have a detrimental effect on print quality [1]. However, constant halftone value transfer can be achieved in platemaking and in printing by standardization of the offset process [2-3]. Deformations of the halftone dots in the halftone image can very easily lead to dot gain and color shifting. Slurring and doubling are the two most important influential factors when printing [4].

Dot gain/tone value increase $(Z)[5,6]$ is calculated from the area coverage of the film (FF) as a master for platemaking and the tone value (FD) is transferred to/printed on the substrate via the printing form, printing process, and printing units as follows (Eq. 1):

\footnotetext{
* To whom all correspondence should be sent:

E-mail: ssonmez@marmara.edu.tr
}

$$
\mathrm{Z}[\%]=\mathrm{FD}[\%]-\mathrm{FF}[\%]
$$

Using the color control bar black can be determined by measuring the solid and halftone patches (e. g., $40 \%$ or $80 \%$ ) [7].

The halftone areas should have clean, well defined individual dots in prints. A good halftone print is said to have high printing snap [8]. Dot gain refers to the undesirable condition when the printed halftone dots are larger than the size of the dots on the image carrier. Dot gain depends to a large extent on the characteristics of the ink and the pressure in the printing nip [9]. On the part of the paper, the dot gain is greater on uncoated papers than on hard coated papers because of the absorbent nature of the former grades [10]. Similarly, the printing snap is better on coated paper than on uncoated paper [11]. Dot gain essentially depends on the paper's surface and its absorption/ink setting behavior, the ink rheology, 
the blanket and printing pressure [12].

Surface coating improves smoothness, ink receptivity and surface structure [13]. Therefore, the ingredients in the coating formulations have an important part in obtaining good print quality with a high printing brightness, a wide-tonal range, a uniformity of ink transfer, a high optical density of ink and good dot shapes [14].

The quality of the print depends greatly on the preparatory work done in prepress [15], the printing process, the machine engineering employed, and the materials used for creating the printed product such as paper and ink.

\section{EXPERIMENTAL}

\section{Methodology}

This study is divided into three phases: (1) to develop coating, (2) to apply onto base paperboard using \#4 bar, and (3) to characterize the base paperboard coated sample's dot gain properties.

\section{Materials}

A commercial base paperboard was used as the base substrate for coating. The pigment characteristics are given in Table 1. The binders' properties according to commercial firms are given in Table 2.

\section{Coating Formulations and Application Methods}

For this study, five different coatings were prepared with pigments of different ratios and latex. The coating formulation is given in Table 3 . Prepared formulations were coated on base paperboard by a K-Control Coater laboratory coater using \#4 bar. Coating colors were prepared using a dispersion, their dry solid content was $60 \%$ and $\mathrm{pH}$ was 8-9 (ISO 6588:1981). Viscosities of coating colors were measured by a Brookfield viscometer (TAPPI T666 om-91). After mixing for $30 \mathrm{~min}$, $\mathrm{pH}$, percentage of coating solids and viscosity were measured. After the coating process, the coated base paperboards (TAPPI T402) were calendered at calendering pressures of $300 \mathrm{PLI}, 2$-nip smooth side.

Table 1. Mineral pigment properties

\begin{tabular}{|l|c|c|c|c|}
\hline Pigments and binders & Solid $(\%)$ & Particle size $(\mu \mathrm{m})$ & Brightness $(\%)$ & $\mathrm{pH}$ \\
\hline $\begin{array}{l}\text { Kaolin } \\
(\mathrm{BASF}, \text { Nuclay) }\end{array}$ & 68 & $\begin{array}{c}78-82 \% \\
\text { (below } 2 \mu \mathrm{m})\end{array}$ & 88 & 7.5 \\
\hline $\begin{array}{l}\mathrm{CaCO} \\
(\text { Omya, Hydrocarb 90) }\end{array}$ & 76 & $\begin{array}{c}90 \% \\
\text { (below } 2 \mu \mathrm{m})\end{array}$ & 93 & 9.5 \\
\hline $\begin{array}{l}\mathrm{TiO}_{2} \\
(\text { Tronox, R-KB-2) }\end{array}$ & 94 & $0.3 \mu \mathrm{m}$ & 95 & 7.5 \\
\hline
\end{tabular}

Table 2. Binder properties

\begin{tabular}{|l|c|c|c|c|}
\hline Binders & Dry matter $(\%)$ & Viscosity (mPa.s) & Density $\left(\mathrm{g} / \mathrm{cm}^{3}\right)$ & $\mathrm{pH}$ \\
\hline $\begin{array}{l}\text { Latex } \\
\text { (BASF, }\end{array}$ & $50 \pm 1$ & 370 & 1.02 & $8 \pm 0.50$ \\
Acronal S 360 D) & & & & \\
\hline
\end{tabular}

Table 3. Used pigments and binder parts in the formulations

\begin{tabular}{|c|c|c|c|c|c|}
\hline Coating Formulations & F1 & F2 & F3 & F4 & F5 \\
\hline Ingredients & \multicolumn{5}{|c|}{ Dry Parts Added } \\
\hline Kaolin & 50 & 25 & 75 & 30 & 30 \\
\hline $\mathrm{CaCO}_{3}$ & 50 & 75 & 25 & 65 & 60 \\
\hline Titanium dioxide & & & & 5 & 10 \\
\hline Latex & 8 & 8 & 8 & 8 & 8 \\
\hline
\end{tabular}

\section{Paper Testing}

All the coated base paperboards were conditioned for $24 \mathrm{~h}$ at $50 \% \mathrm{RH}$ and $23{ }^{\circ} \mathrm{C}(73.4$ $\left.{ }^{\circ} \mathrm{F}\right)$ before any measurements were made. Paper roughness was measured by PPS ME-90 (1000 Pa, soft backing) based on TAPPI T555-OM-99. 


\section{S. Sönmez, Ö. Özden: The influence of pigment proportions and calendering of coated paperboards on dot gain}

\section{Printing Testing}

The tone scale from 1 to $100 \%$ was printed on the calendered and uncalendered-coated base paperboard's surface with offset printing, using black ink and a screen frequency of $60 \mathrm{lpc}$. The following printing properties were analyzed after the press trial. Dot gains of printed-uncalendered or calendered-coated paperboards were measured using a calibrated Gretagmacbeth Spectrolino spectrophotometer $\left(\mathrm{D}_{50}, 2\right.$-degree observer, UV included, with white backup) according to ISO $12647-2$ just after the printing, $6 \mathrm{~h}, 12 \mathrm{~h}, 18 \mathrm{~h}$ and $24 \mathrm{~h}$ after the printing. For image analysis of screened dots, the line and surface were zoomed 190 times with an optical microscope (Olympus SZ Pt) and then were transferred into a digital platform and analyzed.

\section{RESULTS AND DISCUSSION}

The surface roughness values of paperboard are a specific property of prime importance for the determination of the printability. The surface roughness affects the printing resolution, i.e. the capability to transfer the thinnest printed lines, dots and their combinations without breaks and distortions. Eventually it defines the quality of a final printed product. Lower roughness value proved a good print surface. Increasing the kaolin ratio in the formulations decreased roughness values. After calendering, the roughness values decreased (Figure 1).

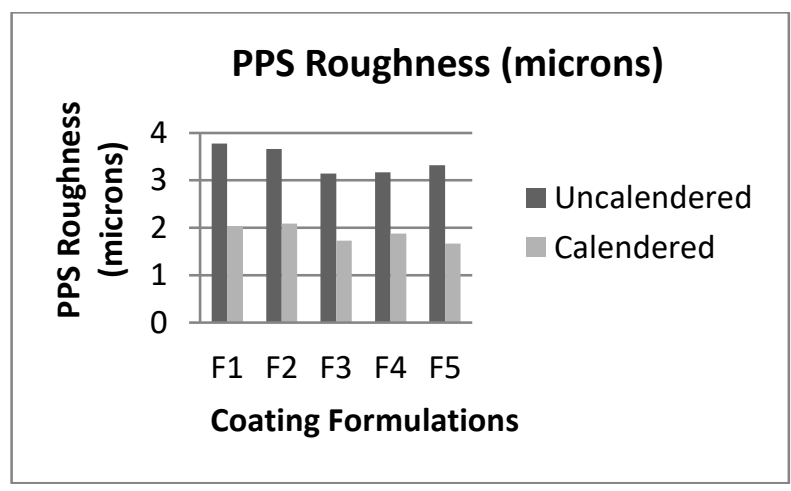

Figure 1. The surface roughness.

In Figures 2-6, for the paperboards overcoated with different formulations that were offset printed, dot areas were determined with a spectrophotometer before and after calendering in the time interval. Line sharpness and surface smoothness views are given at $40 \%$ and $80 \%$ screen tints that were displayed with the optical microscope.

According to ISO standards, the dot gain results of all measurements on printed tone scales' spaces of $40 \%$ and $80 \%$ screen tints are seen to be acceptable; however, uncalendered-coated paperboards have fewer dot gains than calendered-coated paperboards. After the calendering process, the surface roughness values of coated paperboards are decreased, by contrast, dot gains are increased.

The highest dot gain value on $40 \%$ screen tint is obtained with F3 using 75 parts of kaolin and 25 parts of calcium carbonate, which results in an $18 \%$ dot gain. By contrast, when using dot gain values of the $80 \%$ screen tint on all formulations, a dot gain of $12 \%$ was observed.

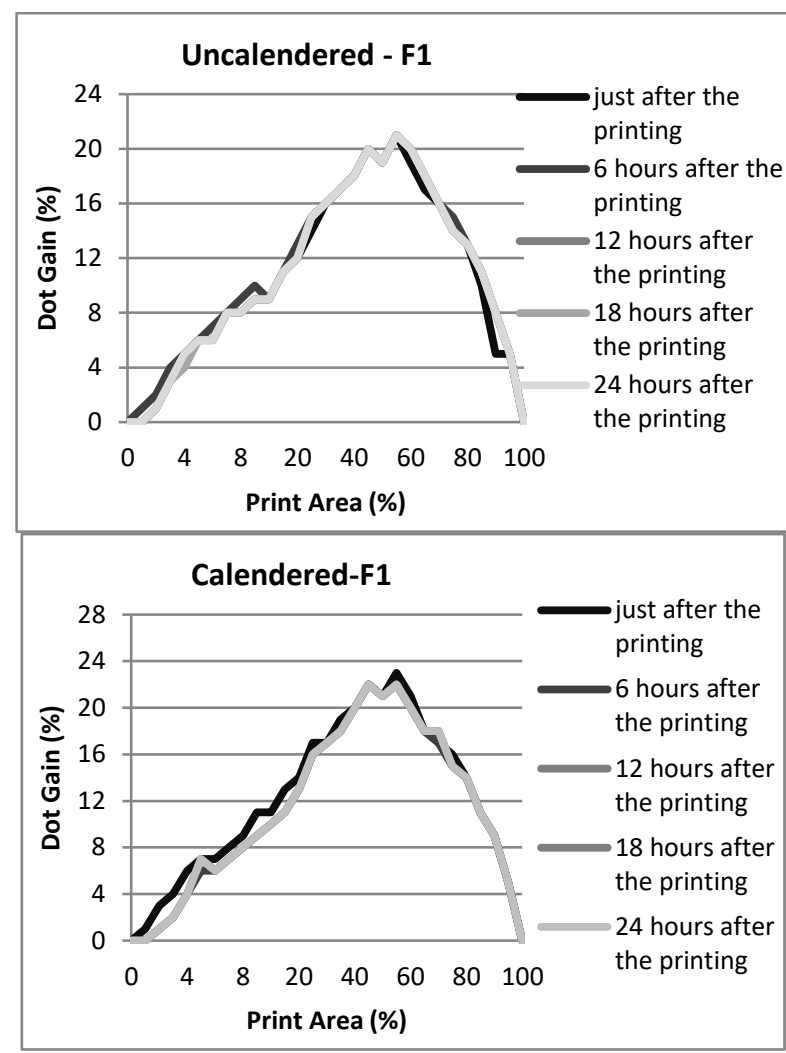

Figure 2. The dot gain values of black printing uncalendered lcalendered-coated paperboards using F1.

Dot gain increased by linking the surface smoothness that affected pigments parameters, calendering conditions and base paperboard properties. Pigments have an important surface characteristic. Especially, their particle shape changed smoothness, ink absorption, brightness, etc. So, the pigment ratio in the coating formulation should be adjusted according to the properties expected from the surface. Calcium carbonate is a coating pigment ensuring good ink absorption, opacity, brightness, smoothness and improved printability [16]. Kaolin pigments in the formulation increased the surface smoothness values of paperboard due to the plate-shaped particles [17]. 

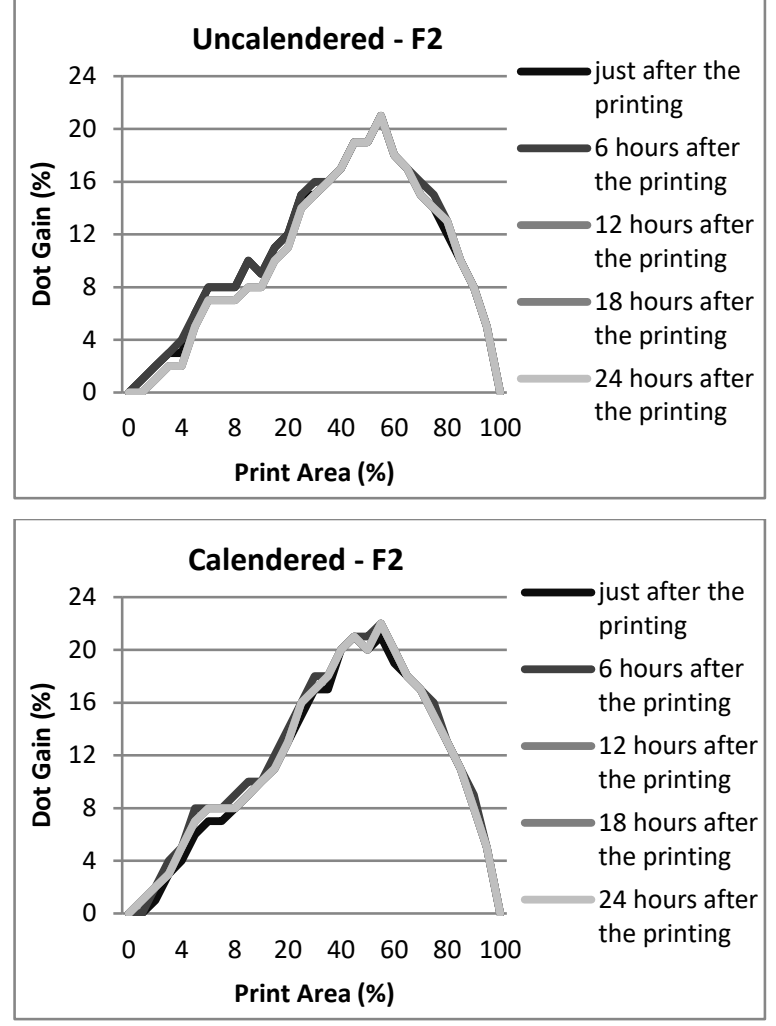

Figure 3. The dot gain values of black printing uncalenderedlcalendered-coated paperboards using F2.
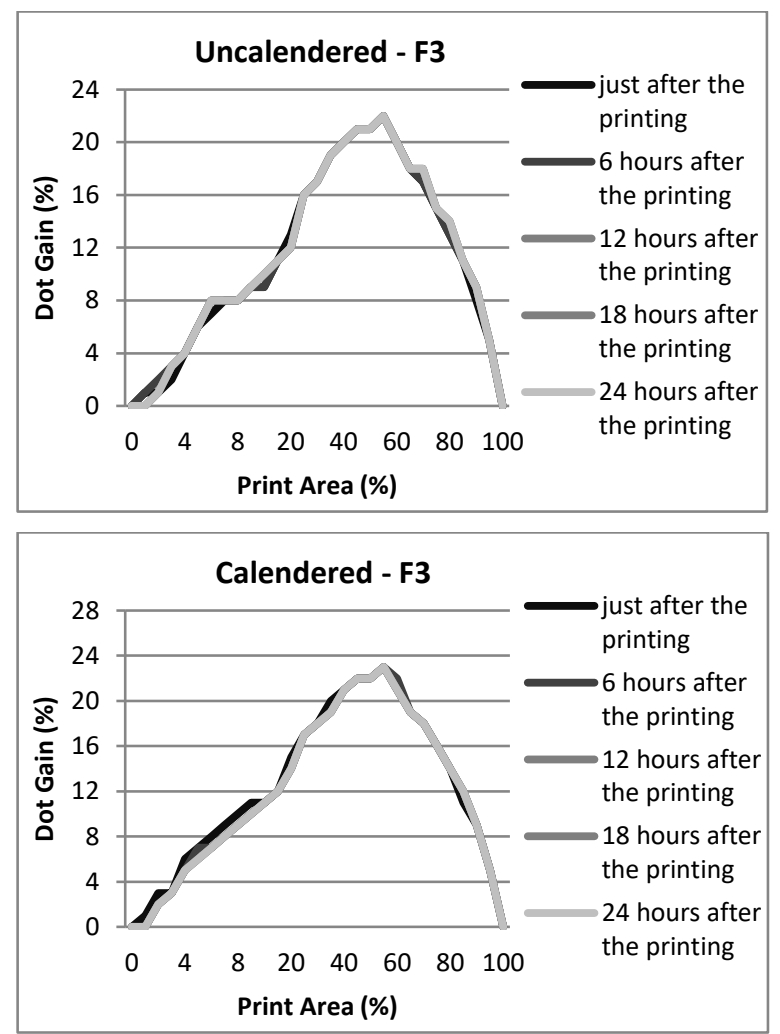

Figure 4. The dot gain values of black printing uncalenderedlcalendered-coated paperboards using F3.
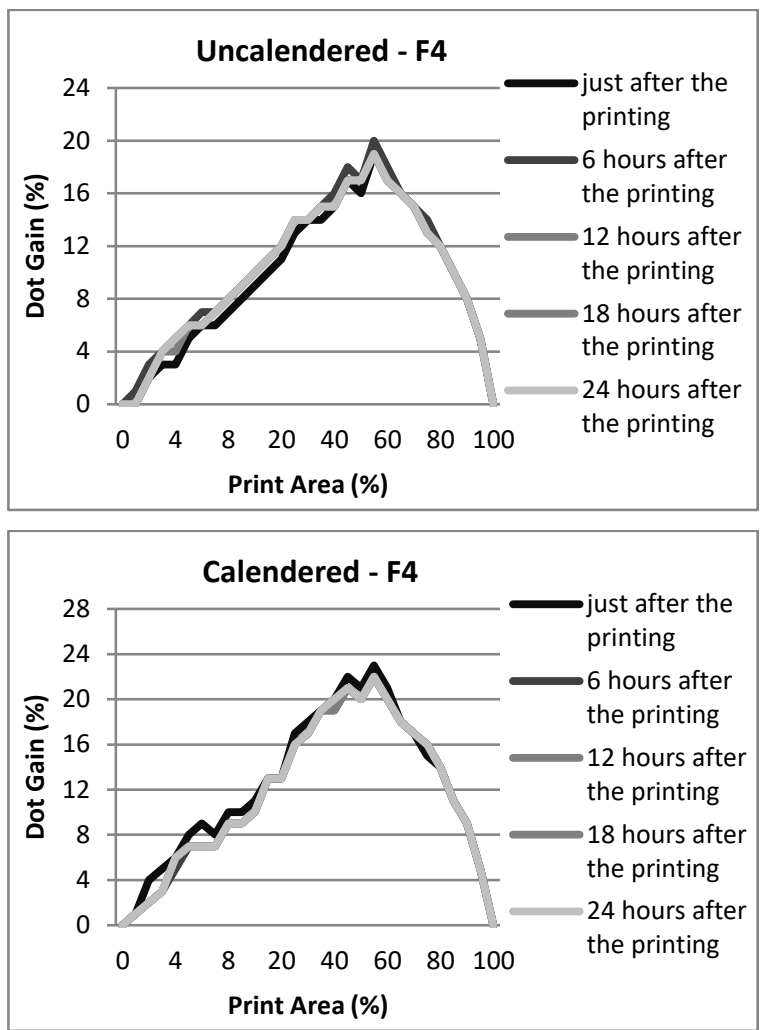

Figure 5. The dot gain values of black printing uncalenderedlcalendered-coated paperboards using F4.
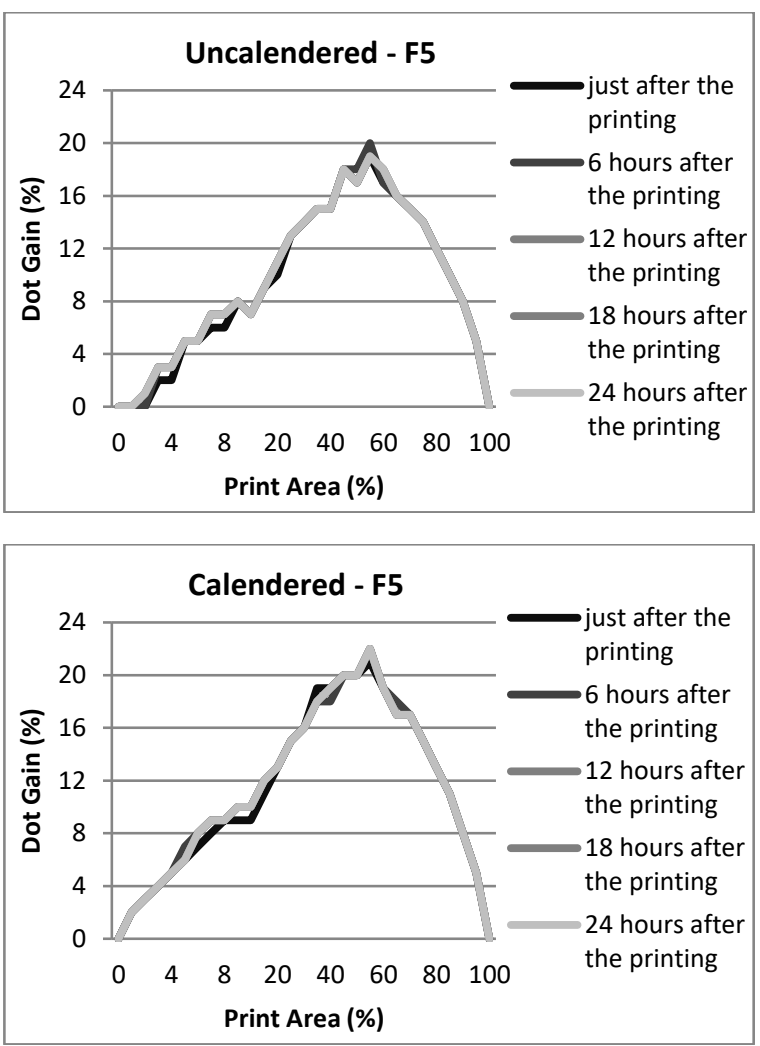

Figure 6. The dot gain values of black printing uncalenderedlcalendered-coated paperboards using F5. 


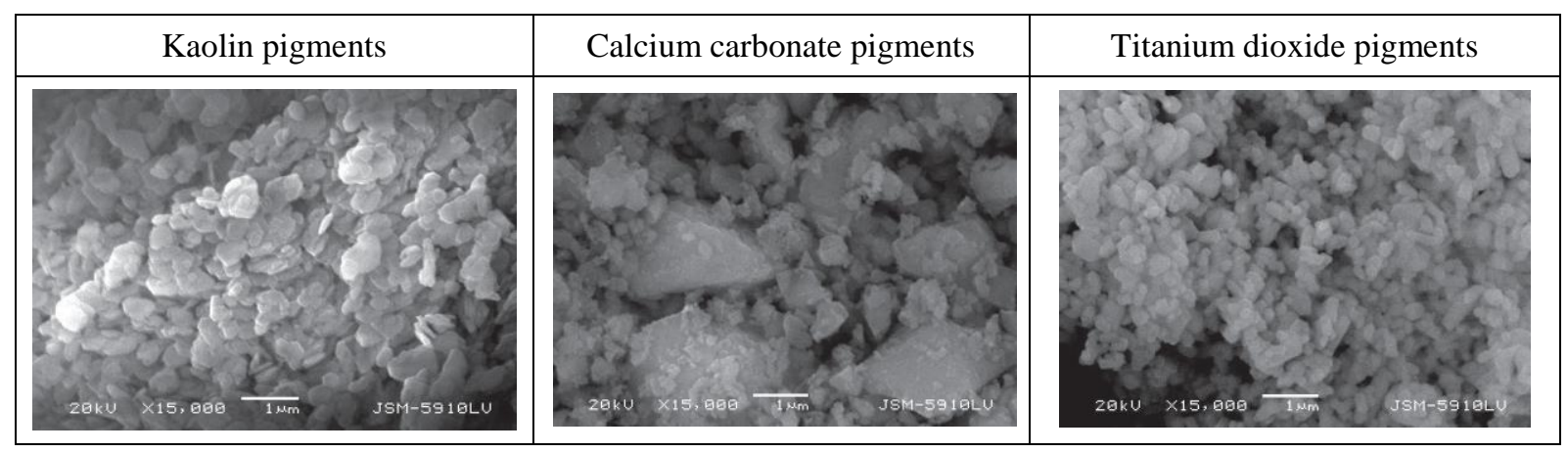

Image 1. SEM images of pigments in the formulations at $\times 15000$ magnification [15]

Image 1 demonstrates the SEM images of pigments in the coating formulations at $15000 \times$ magnification Images 2-6 were observed for the uncalendered and calendered printed $40 \%$ ink tones, $80 \%$ ink tones and paperboard-ink bound with unprinted uncalendered - calendered paperboard surface, zoomed $190 \times$ with microscope. After the calendering process, sharpness is seen in all formulations. These views showed that pigments type and ratios do not affect dot shape and line sharpness.

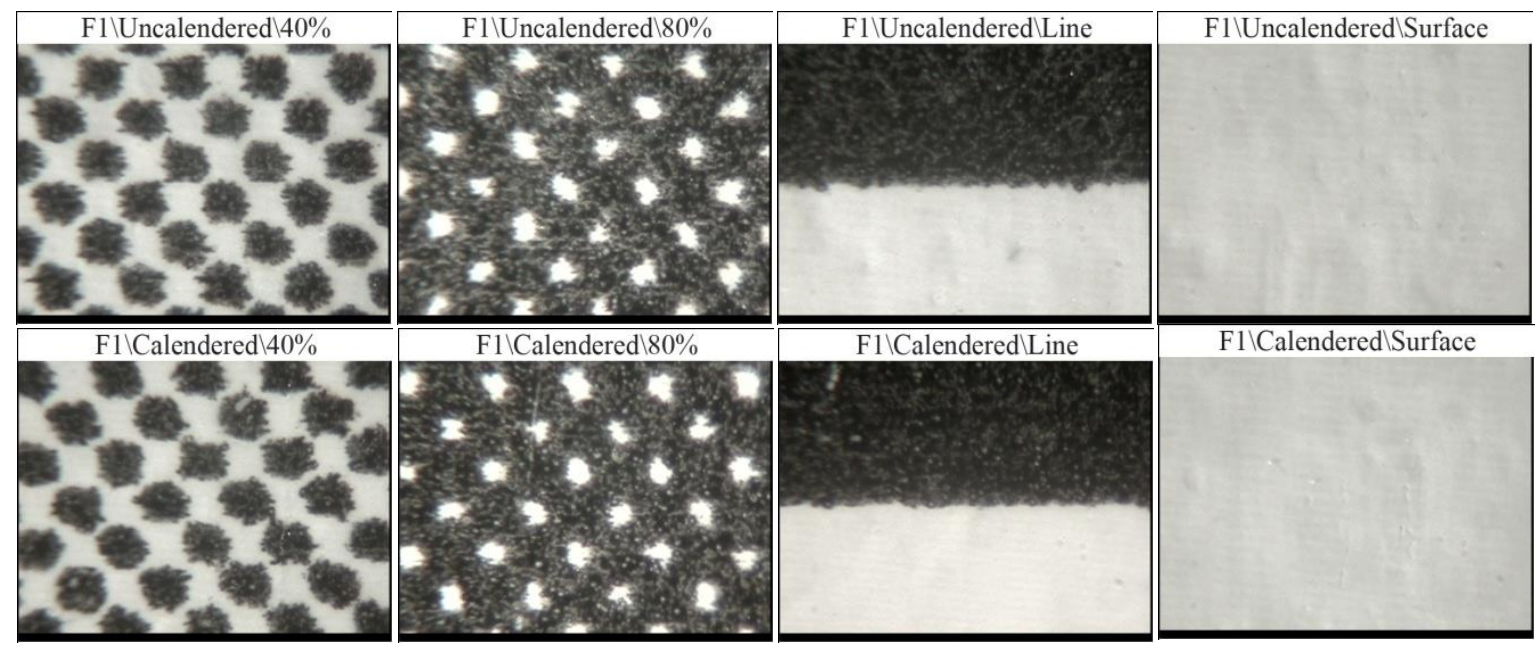

Image 2. The optical microscope views of $40 \%$ dots, $80 \%$ dots, ink-line sharpness and surfaces of uncalenderedlcalendered-coated paperboards using F1

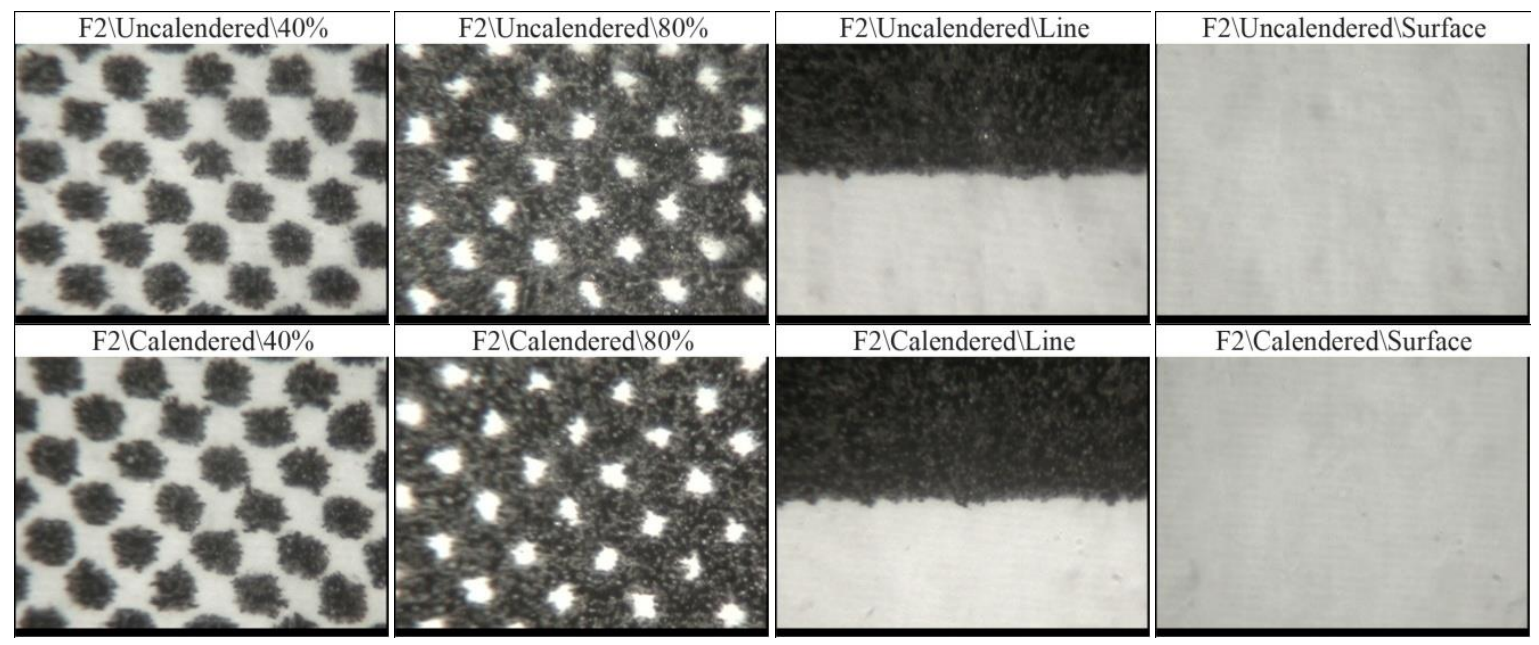

Image 3. The optical microscope views of $40 \%$ dots, $80 \%$ dots, ink-line sharpness and surfaces of uncalenderedlcalendered-coated paperboards using F2 


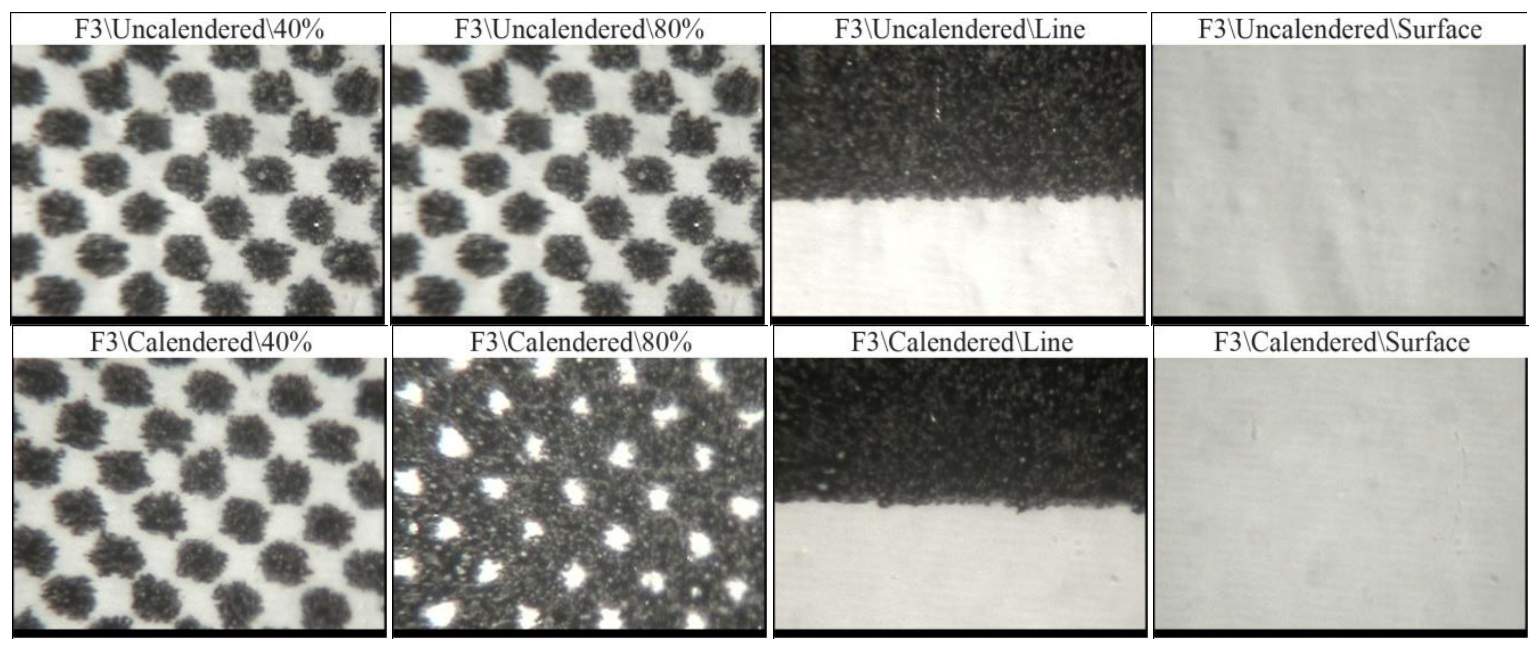

Image 4. The optical microscope views of $40 \%$ dots, $80 \%$ dots, ink-line sharpness and surfaces of uncalenderedlcalendered -coated paperboards using F3.

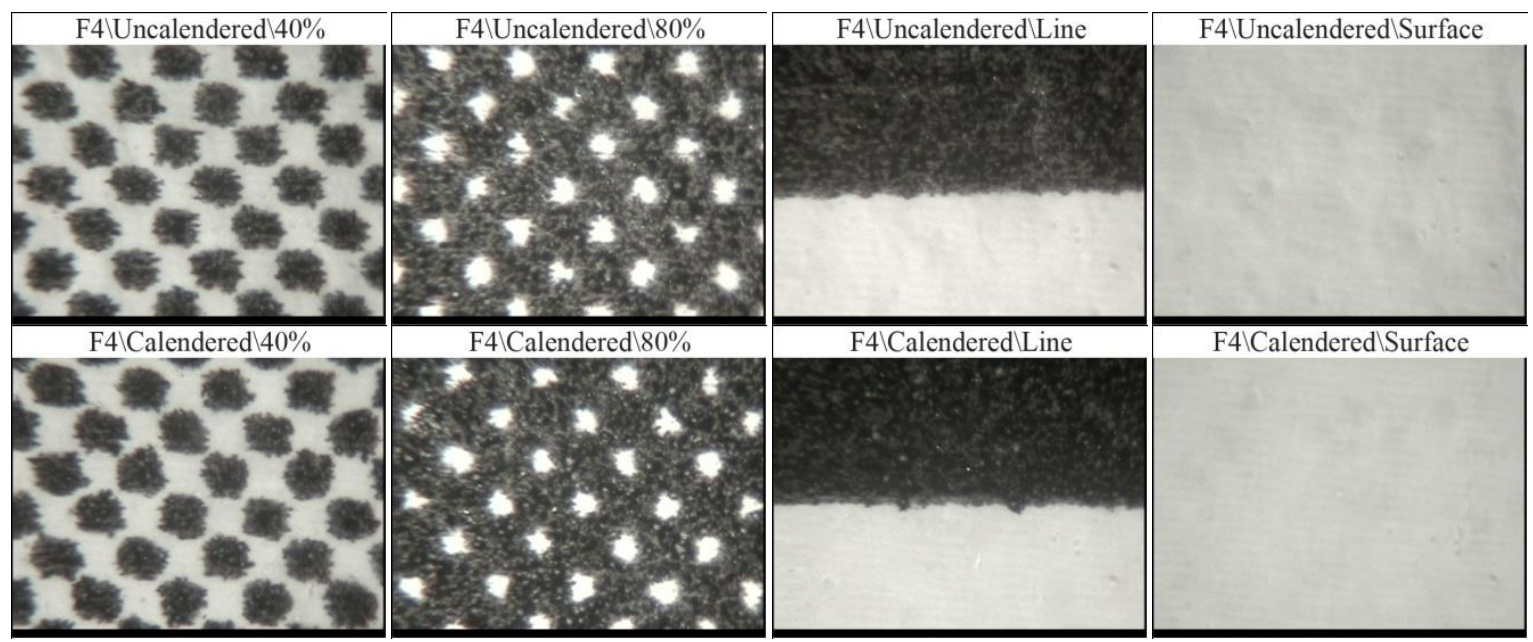

Image 5. The optical microscope views of 40\% dots, $80 \%$ dots, ink-line sharpness and surfaces of uncalenderedlcalendered-coated paperboards using F4.

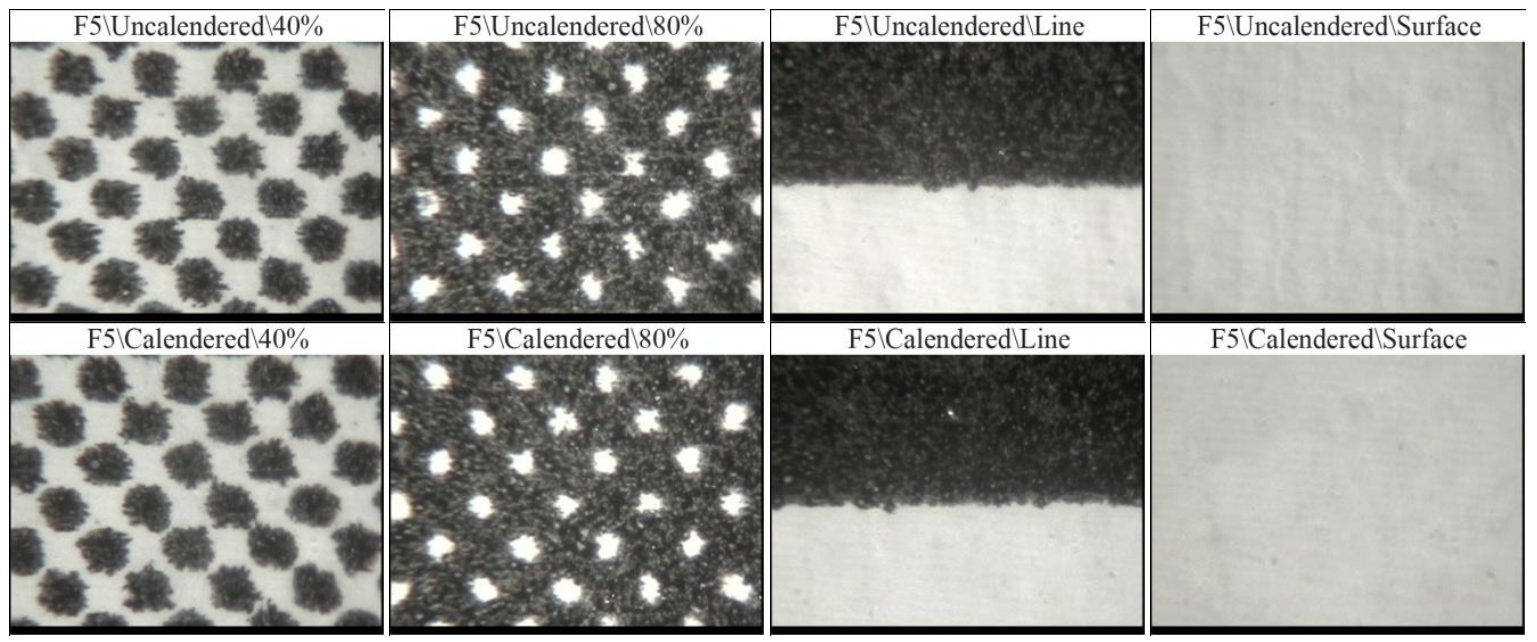

Image 6. The optical microscope views of $40 \%$ dots, $80 \%$ dots, ink-line sharpness and surfaces of uncalenderedlcalendered -coated paperboards using F5.

\section{CONCLUSIONS}

The conclusions of this study are based upon the analysis of data and findings. The specific effect of dot gain of different coating pigments and calendering process is studied. Dot gain is increased linking the surface smoothness that is affected by 
S. Sönmez, Ö. Özden: The influence of pigment proportions and calendering of coated paperboards on dot gain pigments parameters, calendering conditions and base paperboard properties. With kaolin pigments the highest surface smoothness is obtained due to kaolin particular shape. So the image sharpness and definition is increased. Therefore, the contrast is sharper and the color gamut is broader. Dot gain is increasing in the paperboards coated with formulations containing high amounts of kaolin; this increase is acceptable according to ISO standards.

\section{REFERENCES}

1. Y. C. Hsieh, Factors Affecting Dot Gain On Sheet-Fed Offset Presses, Journal of Visual Communications, University of Houston, Houston, TX, 39 (1997).

2. H. N. Dharavath, T. M. Bensen, B. Gaddam, Analysis of Print Attributes of Amplitude Modulated (AM) vs. Frequency Modulated (FM) Screening of Multicolor Offset Printing, Journal of Industrial Technology, 21(3), 2 (2005).

3. A. Verikas, J. Lundström, M. Bacauskiene, A. Gelzinis, Advances in Computational Intelligencebased Print Quality Assessment and Control in Offset Colour Printing, Expert Systems with Applications, 38, 13441 (2011).

4. D. Kumpar, I. Zjakic, I. Bates, Deviation of Deformation of the Screen Elements Through Circulation in the Newspaper Printing, Chapter 34, in: DAAAM International Scientific Book 2010, B. Katalinic (ed.), DAAAM International, Vienna, 2010, p. 357.

5. R. S. M. Abouzeid, A Toner technology \& Image Quality in Electrophotography Printing, The International Design Journal, 3 (2), 1 (2013).

6. F. Miroslav, Separation Model of Colour Regions in A Halftone Print, Journal of Computers \& Graphics, 27 (3), 801 (2003).

7. H. Kipphan, Handbook of Print Media, Springer, Heidelberg, 2001.

8. Y. V. Kuznetsov, Halftoning Myths and Reality.

What is Adaptive to What in Screening?, International Scientific-Practical Conference, Ukraine, 2017, p. 61.

9. R. H. Leach, The Printing Ink Manual, Springer, England, 1988.

10. A. Jurkiewicz, Y. Pyryev, J. Kowalczyk, Printouts' Quality Depending on Too Small Pressure of a Blanket Cylinder Against an Impression Cylinder and a Plate Cylinder in Offset Machine, Challenges of Modern Technology, 4(4), 12 (2013).

11. S. Sonmez, Interactive Effects of Copolymers and Nano-Sized Pigments on Coated Recycled Paperboards in Flexographic Print Applications. Asian Journal of Chemistry, 23(6), 2609 (2011).

12. A. A. Elwan, Influence of Ink Viscosity Level on tone Value Increase in Sheet-fed Offset Printing, International Design Journal, 7(2), 307 (2017).

13. J. S. Preston, A. G. Hiorns, D. J. Parsons, P. J. Heard, Design of Coating Structure for Flexographic Printing, Coating and Graphic Arts Conference, Miami, Florida, USA, 2007, p. 85.

14. H. K. Lee, H.J. Youn, K.H. Lee, C.H. Kim, J.D. Kim, C.Y. Chen, Development of New Coated Linerboard by Combining Condebelt Drying and Curtain Coating Technologies, 63rd Appita Annual Conference and Exhibition, Melbourne, Australia, 2009, p. 203.

15. S. Sonmez, Effects of Calendering on Print Densities of Coated Paperboards, Marmara Journal of Pure and Applied Sciences, 28(4), 164 (2016).

16. J. K. D. Kralj, L. Breèeviæ, G. Falini, Influence of Some Polysaccharides on The Production of Calcium Carbonate Filler Particles, Journal of Crystal Growth, 310(21), 4554 (2008).

17. S. Sonmez, Interactive Effects of Copolymers and Nano-sized Pigments on Coated Recycled Paperboards in Flexographic Print Applications, Asian Journal of Chemistry, 23(6), 2609 (2011). 


\title{
Optical spectroscopic study of $\mathrm{Ru}$ and $\mathrm{Rh}$ doped $\mathrm{Bi}_{12} \mathrm{TiO}_{20}$ crystals
}

\author{
P. M. Rafailov ${ }^{1 *}$, R. Todorov ${ }^{2}$, V. Marinova 2 , D. Z. Dimitrov ${ }^{1,2}$, M. M. Gospodinov ${ }^{1}$ \\ ${ }^{1}$ Institute of Solid State Physics, Bulgarian Academy of Sciences, 72 Tzarigradsko Chaussee Blvd., 1784 Sofia, Bulgaria \\ ${ }^{2}$ Institute of Optical Materials and Technologies, Bulgarian Academy of Sciences, Sofia 1113, Bulgaria
}

Received, April 18, 2918; Accepted, December 19, 2018

$\mathrm{Bi}_{12} \mathrm{TiO}_{20}$ (BTO) single crystals in pristine state and doped with ruthenium and rhodium are grown by the top-seeded solution growth method and characterized by optical and Raman spectroscopy. The effect of doping on the vibrational and optical properties is studied. The doped crystals show higher absorption in the visible spectral range and higher transmission in the near infrared region as compared to pristine BTO. The performed spatially resolved polarized Raman measurements reveal no significant doping-induced shift of vibrational modes while differences in the LO/TO intensity ratio of the tetrahedral asymmetric stretching vibration are encountered. The observations are discussed in terms of lattice ordering and dopant oxidation states.

Keywords: Crystal growth, $\mathrm{Bi}_{12} \mathrm{TiO}_{20}$, Doping, Optical spectroscopy, Raman analysis

\section{INTRODUCTION}

Sillenite type crystals $\mathrm{Bi}_{12} \mathrm{MO}_{20}(\mathrm{M}=\mathrm{Si}$, Ge and Ti) attract special interest due to their excellent photosensitivity (remarkable high photoconductivity) and high charge carrier mobility, which permit achievement of very fast response time. Based on their outstanding properties, they find applications in real-time holography, optical information processing, light amplification, optical phase conjugation, optical interconnection and communications, optical metrology, etc. [1-4]. Moreover, sillenites are appropriate media for optical correlation of spatially-frequency-shifted images and correlation filtering in acoustic-optical imaging for optical tomography $[5,6]$.

Amongst sillenites, bismuth titanate $\mathrm{Bi}_{12} \mathrm{TiO}_{20}$ (BTO) is more attractive due to the higher photoconductivity, larger electro-optical coefficient, lower optical activity and shifted holographic sensitivity to the red and near infrared range in comparison with BSO and BGO. For example, the low optical activity makes BTO an appropriate medium for optical spatial soliton propagation, wave-guides fabrications and correlations [7]. The provoked interest to BTO relays on the advanced photoconductivity (one order of magnitude higher than that of BSO and BGO), which is due to the increased presence of lattice defects in the so called "tetrahedral" positions in the structure [8-11]. Namely, the tetrahedral positions are the lattice sites which the doping elements preferentially occupy. The effect of doping BTO with transition metals has been intensively studied and improvements of the holographic performance have

* To whom all correspondence should be sent:

E-mail: <rafailov@issp.bas.bg> been reported [12-19]. Therefore, the influence of doping on the crystal's structure and properties of BTO crystals becomes a topic of high interest, especially when doping elements are $4 \mathrm{~d}$ metals like $\mathrm{Ru}$ and $\mathrm{Rh}$.

In this communication $\mathrm{n}$ we study the effect of $\mathrm{Ru}$ - and Rh-doping in BTO single crystals using optical and Raman spectroscopy and compare the results with non-doped BTO. The polarized Raman measurements indicate differences in the LO/TO intensity ratio of the asymmetric stretching vibration: $\mathrm{Rh}$ seems to enhance and $\mathrm{Ru}$ to diminish the relative intensity of the LO component, which demonstrates the strength of long-range polarization fields. The results are attributed to the different oxidation states of the dopants.

\section{EXPERIMENTAL}

Pure and doped BTO crystals were grown along the [001] crystallographic axis by the top-seeded solution growth method (TSSG). More details of the crystal growth and doping processes are reported in Ref. [11]. Crystal plates of about $1 \mathrm{~mm}$ thickness were cut nearly perpendicularly to the growth axis, precisely lapped and optically polished. The concentration of the doping elements was estimated at about $5.2 \times 10^{-18} \mathrm{~cm}^{-3}$ by electrothermal and flame atomic absorption spectrometry with ZEEMAN 3030 and VARIAN 240 instruments, respectively.

The Raman spectra were measured in backscattering geometry using HORIBA Jobin Yvon Labram HR visible spectrometer equipped with a Peltier-cooled charge-coupled device (CCD) detector. The 633-nm line of a He-Ne-laser was used for excitation. The laser beam was focused on spots of different size using microscope optics. 
Spectroscopic ellipsometry was applied for the determination of the complex refractive index of the samples in the spectral range of 190-2000 $\mathrm{nm}$. The measurements were conducted by UV-Visible phase modulated spectroscopic ellipsometric platform UVISEL2 (HORIBA Jobin Yvon) at $70^{\circ}$ incident angle. The instrument operates in a rotating compensator configuration, with a white light source and a CCD detector providing fast data acquisition capabilities.

Optical transmission $(T)$ spectra were measured at room temperature in the range of 300-2000 nm on double-side polished parallel plates using a Varian UV-VIS spectrophotometer Carry 5E within an accuracy of $\pm 0.5 \mathrm{~nm}$.

\section{RESULTS AND DISCUSSION}

It is well known that BTO crystallizes in sillenite type cubic structure (space group I23) $[1,8]$. The framework of the structure is based on two structural elements: Bi-O5 polyhedra and [TiO4] tetrahedra. The Bi-polyhedra are represented usually as $\left[\mathrm{BiO}_{5}\right]$ and form dimers by means of a common edge. The $\left[\mathrm{TiO}_{4}\right]$ tetrahedra are situated in the corners and in the centre of the unit cell, surrounded by four equidistant oxygen atoms.

According to neutron diffraction data [9], the main difference between BSO and BTO is the fact that in the case of $\mathrm{Bi}_{12} \mathrm{TiO}_{20}$, the occupation factor of the tetrahedral positions is approximately 0.9 and that of $\mathrm{O}_{3}$-positions approximately 0.97 , while in the case of $\mathrm{Bi}_{12} \mathrm{GeO}_{20}$ and $\mathrm{Bi}_{12} \mathrm{SiO}_{20}$ the occupancy of both tetrahedral and oxygen positions are equal to unity. The vacancies in tetrahedral positions are related to the substantially larger ionic radius of $\mathrm{Ti}^{4+}(0.68 \AA)$ in comparison to that of $\mathrm{Ge}^{4+}$ and $\mathrm{Si}^{4+}$. The presence of such vacancies contributes to the change from an "ideal" bismuth octahedron $\mathrm{BiO}_{7}$ (typical for BSO and BGO) to the "defective" $\mathrm{BiO}_{5}$ polyhedron in case of BTO. To preserve the electro-neutrality, the $\mathrm{Ti}^{4+}$ vacancies in $\mathrm{TiO}_{4}$ are occupied by $\mathrm{Bi}^{3+}$-atoms, which gives rise to two additional oxygen $\mathrm{O}(3)$ vacancies in the tetrahedron, which are simultaneously in polyhedral positions [8]. Therefore, doping with $\mathrm{Ru}$ and $\mathrm{Rh}$ elements is expected to create an even more complex interplay with the vacancies due to the variety of possible oxidation states of $\mathrm{Ru}$ and $\mathrm{Rh}$.

The results of the optical measurements are presented as absorption coefficients $\alpha$ calculated using Beer-Lambert's formula,

$\alpha=d^{-1} \ln (1 / T)$,

where $d$ stands for the sample thickness.

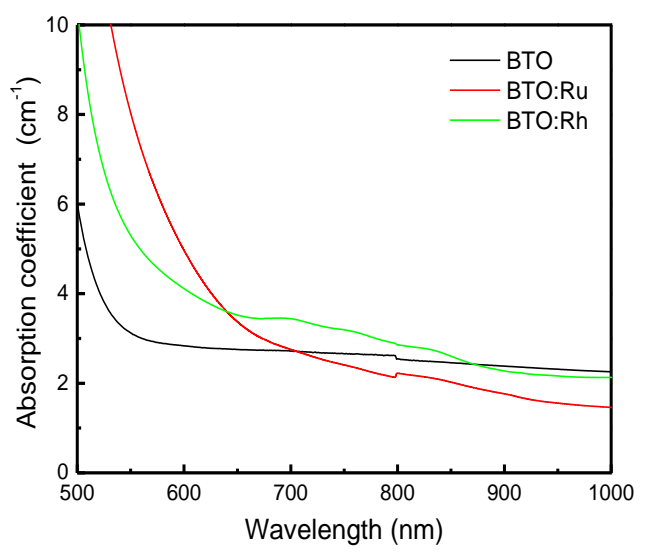

Figure 1. Optical absorption coefficients of BTO, BTO:Ru and BTO:Rh crystals.

Fig. 1 presents the wavelength dependence of the optical absorption coefficients of BTO, BTO:Ru and BTO:Rh. A typical shoulder at about $550 \mathrm{~nm}$ is observed for undoped BTO, supposed to be due to the contribution of an intrinsic antisite defects $\left(\mathrm{Bi}^{3+}\right.$ $+h^{+}$) formed by occupation of a tetrahedrally coordinated $\mathrm{Ti}^{4+}$ site by a $\mathrm{Bi}^{3+}$, coupled with a hole, mainly localized at the oxygen neighbors $\left(h^{+}\right.$ denoting a positive hole on a neighbor oxygen) [8]. Therefore, the absorption edge of non-doped BTO is attributed to the so called "anti-site" Bi-defect, supposed also as a reason of the yellow color, typical for non-doped sillenite crystals [9].

The addition of $\mathrm{Ru}$ and $\mathrm{Rh}$ elements in a sillenite crystal structure caused a shift of absorption edge from blue-green to the red and near infrared spectral range (Fig. 1). Moreover, the BTO:Rh sample has an additional absorption band between 650 and $900 \mathrm{~nm}$. The additionally introduced absorption that increases close to the fundamental absorption edge of sillenite crystals is probably due to photochromism, which is increased in the doped crystals $[2,8]$.

Ramaz et al. [16] demonstrated by magnetic circular dichroism (MCD) that Ru substitutes Bisites in pseudo-octahedral position (formed by $\mathrm{Bi}$ and oxygen atoms) under three valence states $\mathrm{Ru}^{3+} /$ $\mathrm{Ru}^{4+} / \mathrm{Ru}^{5+}$. Usually, $\mathrm{Ru}^{4+}$ possesses an amphoteric behavior; therefore it can accept holes or electrons to produce $\mathrm{Ru}^{5+}$ or $\mathrm{Ru}^{3+}$, respectively. An evidence of doping-related changes in the concentration of trap levels (positioned shallower than the deep levels typical for sillenites), acting as acceptor centers for photo-excited electrons is discussed later by Raman analysis.

Figure 2 (a) and (b) shows real and imaginary parts of the complex refractive index of pure and doped BTO crystals calculated by ellipsometric 


\section{P. M. Rafailov et al.: Optical spectroscopic study of Ru and Rh doped Bi $i_{2}$ Tio ${ }_{20}$ crystals}

measurements. In our calculations the sample was modeled as an isotropic slab with rough surfaces. To obtain the best fit of the experimental data for the ellipsometric angles $\Psi$ and $\Delta$, the dispersion of the optical constants (refractive index and extinction coefficient) of the samples was presented as a sum of three Lorentz oscillators.

The result showed that in case of Rh doping of BTO crystal both the refractive index and the extinction coefficient are slightly decreased in the UV region while increasing of the refractive index by 0.03 in comparison with pure BTO crystal was observed in the visible and near-infrared spectral ranges. $\mathrm{Ru}$ doping causes only insignificant deviations from the pertinent values for the pristine non-doped BTO sample.

The Raman spectra from all regions in the examined crystals turned out to be practically identical to those of undoped BTO, except for the high-energy stretching $\mathrm{F}$ mode of the Ti-centered tetrahedra. This is the only $\mathrm{F}$ mode in BTO exhibiting TO-LO splitting (TO at $662 \mathrm{~cm}^{-1}$ and LO at $\left.685 \mathrm{~cm}^{-1}\right)$, and the intensity ratio $\rho=\mathrm{I}(\mathrm{TO}) / \mathrm{I}(\mathrm{LO})$ may serve as a measure of the influence of the dopant. It is known [20] that doping of sillenites with metal ions takes place predominantly in tetrahedral positions. Via the dopant ion charge and ionic radius this should govern the balance between the short-range and long-range interactions in the crystal and hence the electron-phonon coupling which determines the Raman efficiency of the vibrational modes [20,21]. Each of the Figs. 3a (pure BTO), 3b (BTO:Ru) and 3c (BTO:Rh) contains two Raman spectra of the high-energy stretching $\mathrm{F}$ mode in polarization geometries chosen to allow only the TO and only the LO component, respectively. However, in almost every spectrum, the respective forbidden component emerges with small intensity depending on its Raman efficiency and the lattice disorder. Thus the Raman selection rules are strictly complied with in the BTO:Ru crystal and slightly relaxed in the BTO:Ru crystal due to different degree of lattice disorder in those crystals. Nevertheless, a comparison of Figs. 3 (a), (b) and (c) can be made by juxtaposition of the intensities of the allowed LO/TO components in each of the three cases. The results reveal that $\mathrm{Rh}$ enhances the long-range polarization fields governing the LO intensity while $\mathrm{Ru}$ weakens them leading to relative enhancement of the TO intensity as compared to non-doped BTO. It is obvious that these changes are due to the influence of the central tetrahedrally coordinated ion because the nearby lying forbidden modes $\mathrm{E}$ (620) and A (718), both comprising vibrations of the $\mathrm{O} 3$ atoms at the tetrahedral corners without involving the central ion, are not enhanced by the doping. The better depreciation of the E-intensity in the BTO:Ru spectra may even imply a general improvement of the crystal order and possibly diminished number of vacancies in tetrahedral positions upon $\mathrm{Ru}$ doping. This finding is particularly intriguing because the opposite behavior was reported for Ru doping of BSO [22], i.e. an $\mathrm{LO}$ enhancement was found for BSO:Ru and, furthermore, doped BTO crystals are considered more defective than the pure ones [13]. The observed deviations may point to different distributions of $\mathrm{Ru}$ and $\mathrm{Rh}$ dopants over their possible oxidations states $\mathrm{Ru}^{+3 /+4 /+5}$ or $\mathrm{Rh}^{+3 /+4 /+5}$.
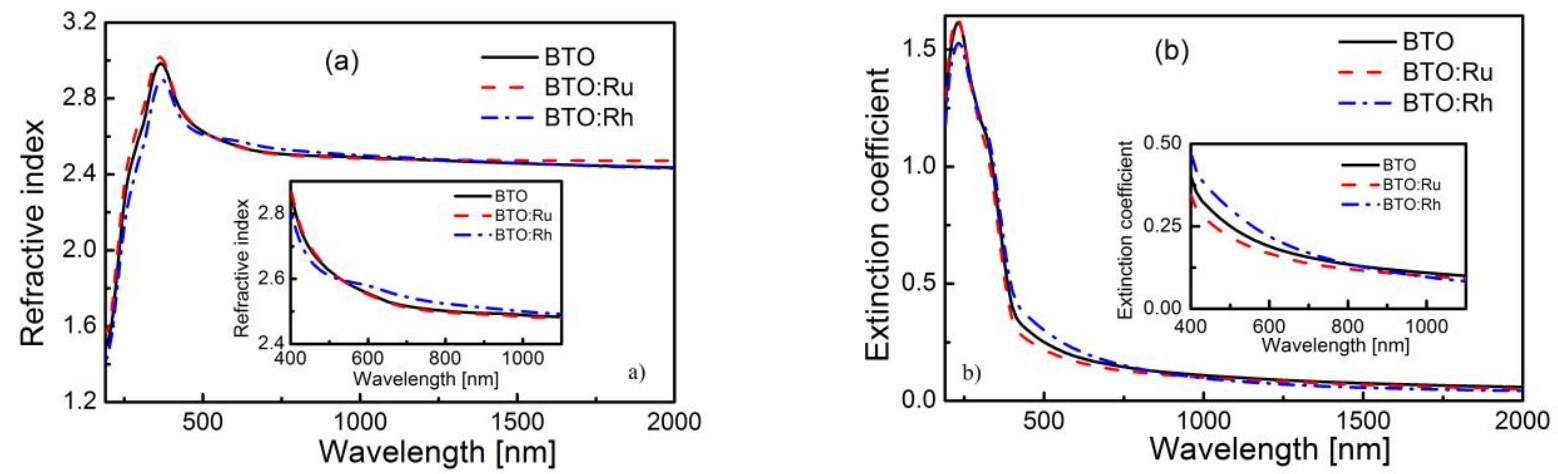

Figure 2. Spectra of the refractive index (a) and the extinction coefficient (b) of BTO, BTO:Ru and BTO:Rh in the 190-2000 nm spectral range. 


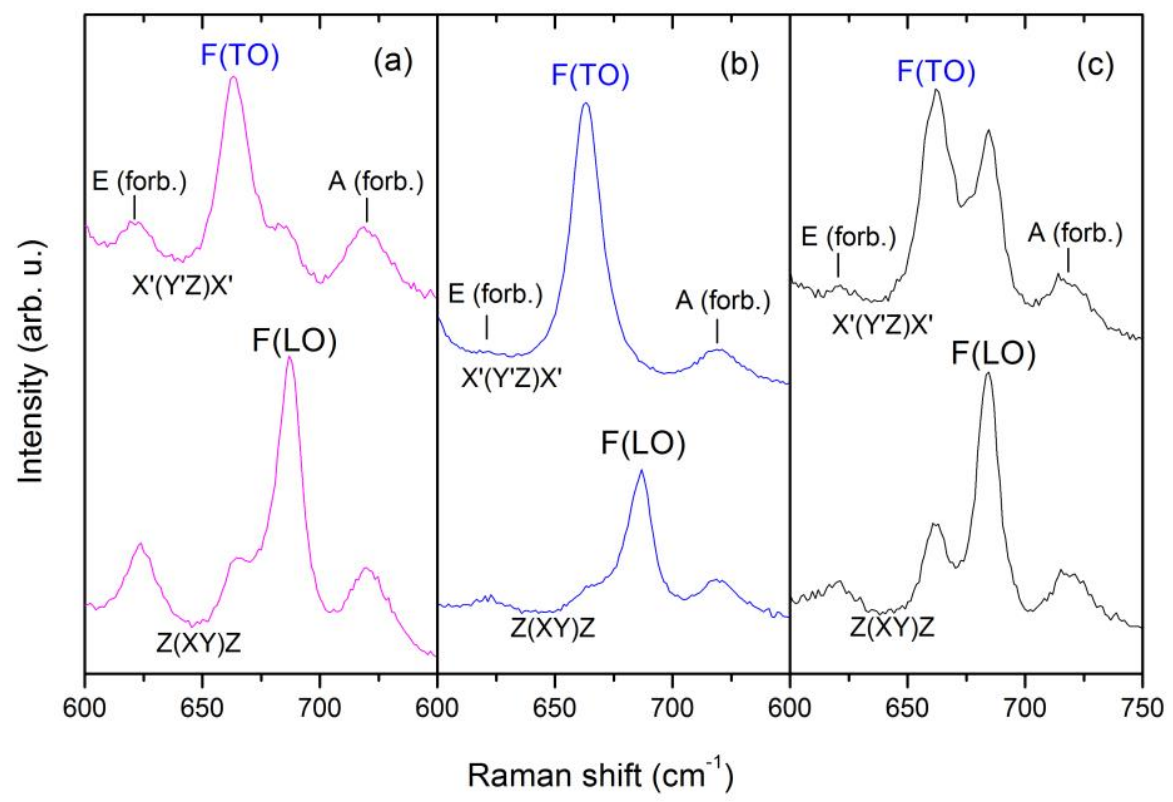

Figure 3. Raman spectra of pristine BTO (a), BTO:Ru (b) and BTO:Rh (c). The scattering configurations are given in Porto notations below each spectrum.

\section{CONCLUSIONS}

Single crystals of $\mathrm{Bi}_{12} \mathrm{TiO}_{20}$ (BTO) doped with ruthenium and rhodium are successfully grown and characterized by optical and Raman spectroscopy. The effect of the doping on the vibrational and optical properties is studied. The doped crystals exhibit higher absorption in the visible region with respect to pristine BTO due to activation of intrinsic defect levels. The polarized Raman measurements reveal differences in the LO/TO intensity ratios of the tetrahedral asymmetric stretching vibration with $\mathrm{Rh}$ enhancing and $\mathrm{Ru}$ diminishing the LO component reflecting the strength of long-range polarization fields. We attribute these observations to different distributions of the dopants over their possible oxidation states.

Acknowledgment: Authors gratefully acknowledge the financial support of this work by the Bulgarian national scientific research fund by contract DFNIT02/26.

\section{REFERENCES}

1. L. Arizmendi, J. Cabrera, F. Agullo-Lopez, Int. J. Optoelectonics, 7, 149 (1992).

2. B. Briat, V. G. Grachev, G. I. Malovichko, O. F. Schirmer, M. Wöhlecke, in: Photorefractive Materials and Their Applications, P. Günter, J. P. Huignard (eds.), Springer-Verlag, Berlin, vol. 2, 2007, 9.

3. M. R. R. Gesualdi, M. Mori, M. Muramatsu, E. A. Liberti, E. Munin, Appl. Optics, 46, 5419 (2007).
4. M. R. R. Gesualdi, D. Soga, M. Muramatsu, Opt. \& Laser Tech., 39, 98 ( 2007).

5. G. Caroena, M. Mori, M. R. R. Gesualdi, E. A. Liberti, E. Ferrara, M. Muramatsu, J. Biomechanics, 43, 680 (2010).

6. E. B. a la Guillaume, U. Bortolozzo, J. P. Huignard, S. Residori, F. Ramaz, Opt. Lett., 38, 287 (2013).

7. A. Tavassoli, M. F. Becker, Appl. Optics, 43, 1695 (2004).

8. R. Oberschmid, Absorption Centers of $\mathrm{Bi}_{12} \mathrm{GeO}_{20}$ and $\mathrm{Bi}_{12} \mathrm{SiO}_{20}$ crystals, Phys. Stat. Solidi A, 89, 263 (1985).

9. Y. F. Kargin, V. I. Burkov, A. A. Maryn, A. V. Egorysheva, Crystals $\mathrm{Bi}_{12} \mathrm{M}_{\mathrm{x}} \mathrm{O}_{20 \pm \delta}$ with sillenite structure. Synthesis, composition, properties, (in Russian), Russian Academy of Sciences, Moscow, 2004.

10. M. Gospodinov, S. Haussuhl, P. Sveshtarov, V. Tassev, N. Petkov, Bulg. J. Phys., 15, 140 (1988).

11. D. Petrova, M. Gospodinov, P. Sveshtarov, Cryst. Res. Technol., 31, 577 (1996).

12. T.S. Yeh, W. J. Lin, I. N. Lin, L. J. Hu, S. P. Lin, S. L. Tu, C. H. Lin, S. E. Hsu, Appl. Phys. Lett., 65 (10), 1213 (1994).

13. V. Marinova, M.-L. Hsieh, Sh. H. Lin, K. Y. Hsu, Opt. Comm., 203 (3-6), 377 (2002).

14. V. Marinova, Optical properties of $\mathrm{Bi}_{12} \mathrm{TiO}_{20}$ doped with $\mathrm{Al}, \mathrm{P}, \mathrm{Ag}, \mathrm{Cu}, \mathrm{Co}$ and co-doped with $\mathrm{Al}+\mathrm{P}$ single crystals, Optical Materials, 15(2), 149 (2000).

15. V. Marinova, M. Veleva, D. Petrova, I. Kourmoulis, D. Papazoglou, A. Apostolidis, E. Vanidhis, N. Deliolanis, J. Appl. Phys., 89, 2686 (2001).

16. F. Ramaz, L. Rakitina, M. Gospodinov, B. Briat, Optical Materials, 27, 1547-1554 (2005).

17. V. Marinova, R. Ch. Liu, Sh. H. Lin, K. Y. Hsu, Optics Letters, 36, 1981 (2011). 
P. M. Rafailov et al.: Optical spectroscopic study of Ru and Rh doped Bi ${ }_{12}$ Tio ${ }_{20}$ crystals

18. V. Marinova, R. Ch. Liu, Sh. H. Lin, M.-S. Chen, Y. H. Lin, K. Y. Hsu, Optics Letters, 38, 495 (2013).

19. V. Marinova, K. Y. Hsu, Sh. H. Lin, R. Ch. Liu, Y. H. Lin, Ruthenium and rhodium doped sillenite crystals: holographic properties and applications at near-infrared spectral range, Proc. SPIE, 8776, 17th ISQE, 877605-1-6 (2013).
20. B. Mihailova, G. Bogachev, V. Marinova, L. Konstantinov, J. Phys. \& Chem. Solids, 60, 1829 (1999).

21. K. Stoychev, L. Konstantinov, R. Titorenkova, Enhanced Raman Scattering from LO Phonons in Doped Semiconductors, AIP Conference Proceedings 1203, 277 (2010).

22. B. Mihailova, L. Konstantinov, D. Petrova, M. Gospodinov, Solid State Commun., 102, 441 (1997). 


\title{
Theoretical insight to intermolecular hydrogen bond interactions between methyl N-(2-pyridyl) carbamate and acetic acid: substituent effects, cooperativity and energy decomposition analysis
}

\author{
S. M. Chalanchi, A. Ebrahimi*, A. Nowroozi \\ Department of Chemistry, Computational Quantum Chemistry Laboratory, University of Sistan and Baluchestan, \\ P.O. Box 98135-674, Zahedan, Iran
}

Received June 24, 2018; Revised July 12, 2018

\begin{abstract}
In the present work, the hydrogen bond (HB) interactions between substituted syn and anti rotamers of methyl N-(2pyridyl) carbamate and acetic acid were investigated using quantum mechanical (QM) calculations. The rotamers have two typical active sites to form hydrogen bonds with acetic acid, such that four stable complexes are found on the potential energy surface. The complexes in which the oxygen atom of carbamate acts as proton acceptor are stabilized by EWSs and are destabilized by EDSs. The trend in the effects of substituents is reversed in the other two complexes, in which the nitrogen atom of ring is involved in the interaction. According to energy data, the substituent effects on the interaction energy can be expressed by Hammett constants. The natural resonance theory (NRT) model was used to investigate the charge distribution on the carbamate group and to discuss the interaction energies. The individual HB energies were estimated to evaluate their cooperative contributions on the interaction energies of the complexes. In addition, the localized molecular orbital energy decomposition analyses (LMO-EDA) demonstrate that the electrostatic interactions are the most important stabilizing components of interactions.
\end{abstract}

Keywords: Carbamate; Hydrogen bond; Substituent effects; Localized molecular orbital energy decomposition analysis (LMO-EDA).

\section{INTRODUCTION}

The carbamate functionality is a common motif in biologically active natural products, functional materials and pharmaceuticals [1-7]. Structurally, the carbamate functionality is related to amide-ester hybrid properties, which generally represent excellent chemical and proteolytic stabilities. Carbamates are extensively used as a peptide bond successor in medicinal chemistry. This is mainly due to their chemical stability and capability to penetrate cell membranes. Another unique property of carbamates is their ability to modulate inter- and intramolecular interactions with the target enzymes or receptors. The carbamate functionality imposes a degree of conformational restriction owing to the delocalization of non-bonded electrons on nitrogen into the carboxyl group. Also, the carbamate functionality takes part in hydrogen bonding via the carboxyl group and the backbone NH. Hence, substitution on the $\mathrm{O}$ - and $\mathrm{N}$-termini of a carbamate offers opportunities for modulation of biological features and improvement in stability and pharmacokinetic features [8].

The carbamate moiety plays a significant role in medicinal chemistry, not only because it is found in drugs but also for its attendance to many prodrugs [9]. In recent years, carbamate derivatives have motivated a large number of theoretical and

* To whom all correspondence should be sent: E-mail: ebrahimi@chem.usb.ac.ir experimental studies due to their application in drug design and discovery [10-24].

In the present work, the HB interactions between the syn and anti rotamers of methyl $\mathrm{N}$-(2pyridyl) carbamate (CA) and acetic acid (AA), as shown in Scheme 1, were evaluated by quantum chemical calculation. The selected carbamate can act as an important starting point for drug discovery, particularly in the design of enzyme inhibitors $[8,13]$. In addition, acetic acid has been chosen as a model of carboxyalkyl side chains of proteins [25-27]. For example, it can be considered the simplest molecular model of aspartic and glutamic acid side chains involved in protein-DNA pairs [28]. These amino acids are known to play key roles in maintaining the spatial structure of various proteins [29]. The structural features of complexes and the effects of substituents on the characteristics of $\mathrm{H}$-bond interactions were also especially considered.<smiles>[R]NC(=O)O[R]</smiles>

anti

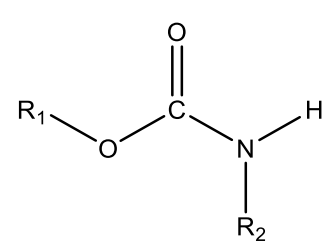

syn
Scheme 1. The syn and anti rotamers of secondary carbamates obtained by rotation about $\mathrm{C}$ (carbonyl)-N single bond. 
S. M. Chalanchi et al.: Theoretical insight to intermolecular hydrogen bond interactions between ...

The individual $\mathrm{O}-\mathrm{H} \cdots \mathrm{O}, \mathrm{O}-\mathrm{H} \cdots \mathrm{N}$ and $\mathrm{N}-\mathrm{H} \cdots \mathrm{O}$ $\mathrm{HB}$ energies were estimated to evaluate the cooperative contributions to the overall stabilization of the complexes. It is worthwhile noting that these kinds of hydrogen bonds play an important role in energetically stabilizing a ligand at the interface of an enzyme structure [30,31]. In addition, localized molecular orbital energy decomposition analyses (LMO-EDA) [32] were carried out to elucidate the strengths and properties of interactions in these hydrogen bonded systems. This information may be beneficial in further design of carbamate-based molecules as drugs or prodrugs.

\section{Computational details and methodology}

The geometry of the simulated complexes was optimized at the B3LYP/6-311++G(d,p) computational level using Gaussian 09 suite of programs [33]. The B3LYP/6-31++G(d,p) methodology was used to locate the stationary points along the potential energy surface because it is a cost-effective method and has widely been applied to H-bonded complexes as model systems [34-39]. The basis set superposition error (BSSE) was calculated by the counterpoise method of Boys and Bernardi [40]. Frequency calculations at the same level were performed to confirm that all structures are local minima on the potential energy surfaces, and to calculate the Gibbs free energies. In order to estimate the dependence of energy changes to method and basis set, the HB interaction energies were also calculated by single-point calculations using MP2 [41], M06-2X [42] and B3LYP-D3 [43] methods in conjunction with the 6$311++\mathrm{G}(\mathrm{d}, \mathrm{p})$ and aug-cc-pVTZ [44] basis sets. The solvent effects were also considered in the selfconsistent reaction-field (SCRF) calculations using the polarizable continuum model (PCM) [45]. The atoms in molecules (AIM) [46] calculations were performed by the AIM2000 program [47]. The natural resonance theory (NRT) models $[48,49]$ were used to analyze the molecular electron density in terms of resonance structures.

Two methods were considered to estimate the individual $\mathrm{HB}$ energies $\left(\Delta \mathrm{E}_{\mathrm{HB}}\right)$ in the complexes. In the first method, the individual $\mathrm{HB}$ energies $\left(\Delta \mathrm{E}_{\mathrm{HB}}^{\mathrm{a}}\right)$ were estimated using models structures that keep one $\mathrm{HB}$ at a time by rotating the acetic acid around the axis of that HB by approximately 90 degrees. The geometries of twisted structures (single $\mathrm{H}$ bonded, SHB) were optimized using two constraints indicated in Scheme $2, \phi \approx 90^{\circ}$ and $\theta$ is equal to the angle value obtained upon full optimization of the double H-bonded (DHB) complexes. The cooperative part of H-bond interactions $\left(\Delta \mathrm{E}_{\text {coop, }}\right.$ tot $)$ was obtained as the difference between the total binding energies of DHB complexes and the sum of the individual $\mathrm{H}$ bond energies of SHB structures (eq. 1) [3]:

$$
\Delta \mathrm{E}_{\text {coop, tot }}=\Delta \mathrm{E}-\left(\Delta \mathrm{E}_{\mathrm{HB} 1}^{\mathrm{a}}+\Delta \mathrm{E}_{\mathrm{HB} 2}^{\mathrm{a}}\right)
$$

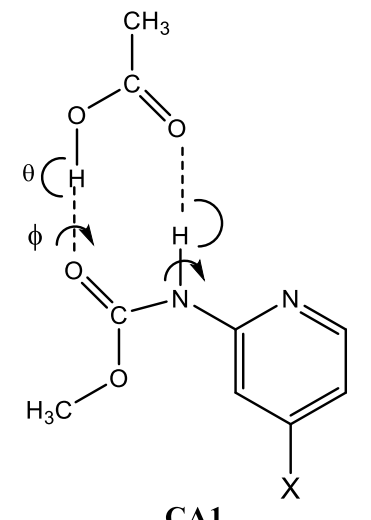<smiles></smiles>

CA2

$\mathrm{S} 1$

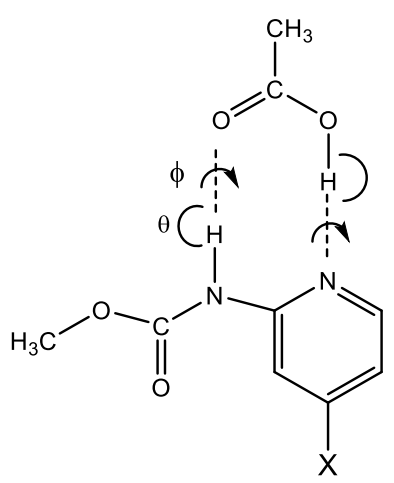

CA3

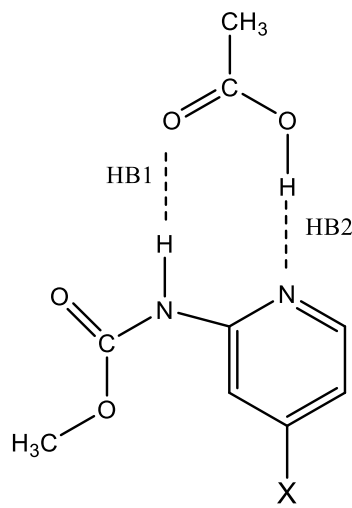

CA4
S2

$\mathrm{X}=\mathrm{F}, \mathrm{Cl}, \mathrm{COH}, \mathrm{CN}, \mathrm{NO}_{2}, \mathrm{H}, \mathrm{OH}, \mathrm{OCH}_{3}, \mathrm{NH}_{2}$, $\mathrm{NHCH}_{3}$

Scheme 2. HB interactions between the syn and anti rotamers of methyl N-(2-pyridyl) carbamate (CA) and acetic acid (AA), In the S1 series of complexes (CA1 and CA2), the oxygen atom of carbamate acts as proton acceptor in the interaction between $\mathrm{CA}$ and $\mathrm{AA}$, and in the $\mathrm{S} 2$ series of complexes (CA3 and CA4), the nitrogen atom of the ring is involved in the interaction.

In the second one, the individual $\mathrm{HB}$ energies $\left(\Delta \mathrm{E}_{\mathrm{HB}}^{b}\right)$ were estimated using the electron densities $(\rho)$ calculated using the AIM method at the H-bond critical points (HBCPs) on the basis of the following equation [51-53].

$$
\Delta \mathrm{E}_{\mathrm{HB}, \mathrm{i}}^{b}=100 \mathrm{a}_{\mathrm{i}}\left(1-\mathrm{e}^{\rho_{i}}\right)
$$

where $\rho_{i}$ is the electronic charge density calculated at the related BCP and $a_{i}$ is a fitting parameter obtained by fitting the $\Delta\left(=\Delta \mathrm{E}-\Sigma \Delta \mathrm{E}_{\mathrm{HB}, \mathrm{i}}^{b}\right)$ value to zero by the least-square method, where $\Delta \mathrm{E}$ is the total binding energy corrected with the BSSE. The cooperative part of the total HB 
S. M. Chalanchi et al.: Theoretical insight to intermolecular hydrogen bond interactions between ...

interaction $\left(\Delta \mathrm{E}_{\text {coop,tot }}\right)$ and the individual $\mathrm{HB}$ interactions $\left(\Delta \mathrm{E}_{\text {coop, }} \mathrm{HBi}\right)$ can be estimated through the following eqs.:

$$
\begin{gathered}
\Delta \mathrm{E}_{\text {coop, tot }}=\left(\Delta \mathrm{E}_{\mathrm{HB} 1}^{b}+\Delta \mathrm{E}_{\mathrm{HB} 2}^{b}\right)-\left(\Delta \mathrm{E}_{\mathrm{HB} 1}^{\mathrm{a}}+\Delta \mathrm{E}_{\mathrm{HB} 2}^{\mathrm{a}}\right) \\
=\Delta \mathrm{E}_{\text {coop, } \mathrm{HB} 1}+\Delta \mathrm{E}_{\text {coop, } \mathrm{HB} 2} \\
\Delta \mathrm{E}_{\text {coop, } \mathrm{HBi}}=\Delta \mathrm{E}_{\mathrm{HBi}}^{b}-\Delta \mathrm{E}_{\mathrm{HBi}}^{a} \mathrm{i}=1,2
\end{gathered}
$$

The localized molecular orbital energy decomposition analyses (LMO-EDA) scheme [30], as implemented in the GAMESS software [54], was applied to examine the factors contributing to binding energies. LMO-EDA scheme offers to fragment the total binding energy $(\Delta \mathrm{E})$ into electrostatic $\left(\Delta \mathrm{E}_{\text {ele }}\right)$, exchange $\left(\Delta \mathrm{E}_{\text {ex }}\right)$, repulsion $\left(\Delta \mathrm{E}_{\text {rep }}\right)$, polarization $\left(\Delta \mathrm{E}_{\mathrm{pol}}\right)$ and dispersion $\left(\Delta \mathrm{E}_{\text {disp }}\right)$ components.

$$
\Delta \mathrm{E}=\Delta \mathrm{E}^{\text {ele }}+\Delta \mathrm{E}^{\text {ex }}+\Delta \mathrm{E}^{\text {rep }}+\Delta \mathrm{E}^{\mathrm{pol}}+\Delta \mathrm{E}^{\text {disp }}
$$

Herein, the LMO-EDA scheme was also utilized for energy decomposition analysis in the SHB structures to obtain insight into the origin of the cooperative contributions. According to eqs. (1) and (5), $\Delta \mathrm{E}_{\text {coop, tot }}$ can be decomposed into the following terms:

$$
\Delta \mathrm{E}_{\text {coop, tot }}=\Delta \Delta \mathrm{E}^{\text {ele }}+\Delta \Delta \mathrm{E}^{\text {exrep }}+\Delta \Delta \mathrm{E}^{\mathrm{pol}}+\Delta \Delta \mathrm{E}^{\text {disp }}
$$

The terms on the right side of eq. (6) are the electrostatic, exchange-repulsion, polarization, and dispersion contribution, respectively, which are defined by eqs. (7) $-(10)$ :

$$
\begin{aligned}
& \Delta \Delta \mathrm{E}^{\text {ele }}=\Delta \mathrm{E}^{\text {ele }}-\left(\Delta \mathrm{E}_{\mathrm{HB} 1}^{\text {ele }}+\Delta \mathrm{E}_{\mathrm{HB} 2}^{\text {ele }}\right) \\
& \Delta \Delta \mathrm{E}^{\mathrm{pol}}=\Delta \mathrm{E}^{\mathrm{pol}}-\left(\Delta \mathrm{E}_{\mathrm{HB} 1}^{\mathrm{pol}}+\Delta \mathrm{E}_{\mathrm{HB} 2}^{\mathrm{pol}}\right) \\
& \Delta \Delta \mathrm{E}^{\text {exrep }}=\Delta \mathrm{E}^{\text {exrep }}-\left(\Delta \mathrm{E}_{\mathrm{HB} 1}^{\text {exrep }}+\Delta \mathrm{E}_{\mathrm{HB} 2}^{\text {exrep }}\right) \\
& \Delta \Delta \mathrm{E}^{\text {dis }}=\Delta \mathrm{E}^{\text {disp }}-\left(\Delta \mathrm{E}_{\mathrm{HB} 1}^{\text {disp }}+\Delta \mathrm{E}_{\mathrm{HB} 2}^{\text {disp }}\right)
\end{aligned}
$$

In eq. (9), the exchange and repulsion interactions are grouped as one exchange-repulsion term to describe the Pauli repulsion [55].

\section{RESULTS AND DISCUSSION}

\section{Geometrical parameters and interaction energies}

Quantum mechanical calculations were used to investigate the interaction between $\mathrm{CA}$ and $\mathrm{AA}$. Considering the fact that CA can simultaneously act as both proton acceptor and proton donor, four cyclic double H-bonded complexes could be obtained on the potential energy surface which were named as CA1-CA4. Scheme 2 depicts the molecular structures of the complexes CA1-CA4. In the S1 series of complexes (CA1 and CA2) the oxygen atom of carbamate acts as proton acceptor in the interaction between $\mathrm{CA}$ and $\mathrm{AA}$, whereas in the S2 series of complexes (CA3 and CA4), the nitrogen atom of the ring is involved in the interaction. Because the AIM, NRT, and LMOEDA results on both rotamers in each series are similar, the data of the complexes CA1 and CA3 were employed to show the salient features of the interactions. The results obtained for the complexes CA2 and CA4 are summarized in the Supplementary Materials. Of

The total binding energies $(\triangle \mathrm{E})$ of the complexes calculated using B3LYP, B3LYP-D3, M06-2X and MP2 methods in conjunction with the 6-311++G (d,p) and aug-cc-pVTZ basis set on the structures optimized at B3LYP/6-311++G(d,p) level and corrected for BSSE are reported in Tables 1 and S1. The BSSE correction decreases the binding energies by approximately $2 \mathrm{kcal} \mathrm{mol}^{-1}$. According to Tables 1 and $\mathrm{S} 1$, the trend in the $\Delta \mathrm{E}$ values obtained at various levels is B3LYP-D3 > MP2 > M06-2X > B3LYP. As seen, the $|\Delta E|$ values calculated using the M06-2X, MP2 and B3LYP-D3 methods with the $6-311++\mathrm{G}(\mathrm{d}, \mathrm{p})$ basis set are by 1.93-2.85, 2.11-2.98 and 3.15-3.95 larger than those calculated at the B3LYP/6-311++G(d,p) level, respectively. So the binding energies significantly depend on the method. On the other hand, changing the basis set from $6-311++\mathrm{G}(\mathrm{d}, \mathrm{p})$ to aug-cc-pVTZ increases the $|\Delta \mathrm{E}|$ values using all mentioned methods. The effect of method on the $\Delta \mathrm{E}$ values is higher than that of the basis set. Although the $\Delta \mathrm{E}$ values of complexes calculated with the B3LYPD3, MP2 and M06-2X methods are more negative than those calculated by the B3LYP method, similar trends are also observed at those levels of theory. Therefore, the results obtained at the B3LYP/6-311++G(d,p) level are used in the subsequent discussions.

As can be seen in Tables 1 and S1, interaction between CAs and AA in the S2 series is stronger than that in the S1 series, where the trend in the relative stability is CA3 (12.21) > CA4 (10.96) > CA1 $(10.61)>$ CA2 $\left(6.03 \mathrm{kcal} \mathrm{mol}^{-1}\right)$ and $\mathrm{X}=\mathrm{H} . \Delta \mathrm{E}$ of the $\mathrm{S} 1$ series of complexes becomes smaller with the electron-withdrawing substituents (EWSs) and becomes larger in the presence of the electrondonating substituents (EDSs). A reverse trend is observed for the S2 series of complexes. The highest and lowest values of $\Delta \mathrm{E}$ calculated at the B3LYP/6- 311++G (d,p) computational level in the $\mathrm{S} 1$ series of complexes correspond to the $\mathrm{NO}_{2}$ and $\mathrm{NHCH}_{3}$ substituents, respectively, and in the $\mathrm{S} 2$ series of complexes to the $\mathrm{NHCH}_{3}$ and $\mathrm{NO}_{2}$ substituents, respectively. The stabilization free energies $(\Delta \Delta G)$ of complexes calculated at the B3LYP/6-311++G(d,p) computational level are also collected in Table 1. A look at the obtained free energy values indicates that the S2 series of 
S. M. Chalanchi et al.: Theoretical insight to intermolecular hydrogen bond interactions between ...

complexes are formed more easily as compared to the $\mathrm{S} 1$ series of complexes. The large binding energies and negative Gibbs free energy changes imply that the S2 series of complexes are very stable and thermodynamically favourable (spontaneous process) to form intermolecular hydrogen bonding. Considering the $\Delta \mathrm{E}$ and $\Delta \Delta \mathrm{G}$ values, the most and the least stable complexes, i.e. CA3 and $\mathbf{C A 2}$ in the presence of $\mathrm{NHCH}_{3}$ substituent, respectively, have the most negative and the most positive value of $\Delta \Delta \mathrm{G}$ (see Table 1).

The results obtained using PCM method in the water solvent $(\varepsilon=78.39)$ at the B3LYP/6$311++\mathrm{G}(\mathrm{d}, \mathrm{p})$ computational level are gathered in Table S2. As can be seen, the binding energies of the complex CA2 suffer only a small reduction, from 0.5 to $1.5 \mathrm{kcal} / \mathrm{mol}$. It decreases by $30-35 \%$ in the complexes CA1, CA3 and CA4, which is larger than that of complex CA2. The trend in the relative stability is CA3 (10.10) > CA4 (9.86) > CA1 (8.38) $>$ CA2 $\left(5.14 \mathrm{kcal} \mathrm{mol}^{-1}\right)$ where $\mathrm{X}=\mathrm{H}$. This trend is quite similar to that predicted at the same level in the gas phase.

The substituent effects can be discussed using the resonance structures presented in Scheme 3.

The NRT model was applied within the NBO methodology [56], where four resonance structures A, B, C and D, shown in Scheme 3, were explicitly taken into account. The NRT results and the most important bond lengths of CA are gathered in Table S3. The NRT results are also graphically illustrated in Fig. 1. As can be seen, the neutral resonance weighting of CA (A) is remarkably reduced in the presence of both EWSs and EDSs. The EWSs located at the ring decrease the populations of resonance structures $\mathrm{B}$ and increase the populations of resonance structures $\mathrm{C}$ and especially $\mathrm{D}$ with higher negative charges near to the substituents. A reverse behaviour is observed in the presence of EDSs.

As shown in Fig. 1, the changes in the resonance weight of structure $\mathrm{D}$, which is in a reliable correlation with the Hammett constants $(\sigma)$, are almost twice larger than the changes in the two other ionic structures. Moreover, the changes in the resonance weight of structure D are in good correlation with the changes in the geometrical parameters of CA (see Fig. S1). Therefore, it is expected that the effects of substituents on the total interaction energy of complexes and the estimated individual $\mathrm{HB}$ energies should be in correlation with the changes in the resonance weight of structure D. In general, an increase in the resonance weight of structure D by EWSs is accompanied with an increase in the positive charge on the N3 atom (see Scheme 3) and an increase in the tendency of the $\mathrm{N}-\mathrm{H}$ group of $\mathrm{CA}$ for H-bonding with the $\mathrm{O}$ atom of $\mathrm{AA}(\mathrm{NH} . . \mathrm{O}$ bond).

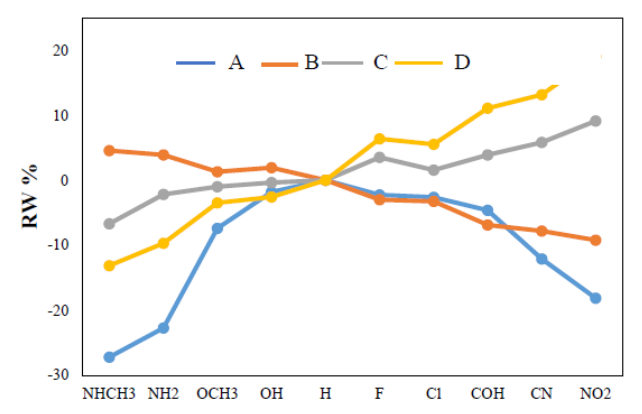

Fig. 1. Changes in the resonance weights (RW \%) calculated from natural resonance theory (NTR) for resonance structures shown in Scheme 3 in the presence of both EWSs and EDSs. [RW\% $\left.=\left(\frac{\mathrm{RW}_{\mathrm{X}}-\mathrm{RW}_{\mathrm{H}}}{\mathrm{RW}_{\mathrm{H}}}\right) \times 100\right]$

The tendency of $\mathrm{C}=\mathrm{O}$ and $-\mathrm{C}-\mathrm{O}-\mathrm{R}$ groups of $\mathrm{CA}$ for $\mathrm{H}$-bonding with the $\mathrm{O}-\mathrm{H}$ group of $\mathrm{AA}$ is reduced with increasing the resonance weight of structure $\mathrm{D}$ (O...HO bond). Also, the EWSs in CA pull the lone pair of the nitrogen atom of pyridyl inside the ring and decrease its interaction with the $\mathrm{H}$ atom of AA (N...HO bond). According to this evidence, in the $\mathrm{S} 1$ series of complexes, the EWSs have a much greater effect on the $\mathrm{NH}$... O hydrogen bond than the $\mathrm{O} \ldots \mathrm{HO}$ one (closer to $\mathrm{NH}$... O bond); thereby increasing the stability of these complexes, while in the $\mathrm{S} 2$ series the N...HO bond is more affected than the $\mathrm{NH}$... O bond and hence decreases its stability. The results are reversed for EDSs.

In addition, the relationship between the $\Delta \mathrm{E}$ values and Hammett constants $(\sigma)$ were considered to explore the generality of substitution effects on the interactions [57]. The substituent effects can be attributed to the inductive and resonance effects that exclusively correlate to the values of $\sigma_{\mathrm{m}}$ and $\sigma_{\mathrm{p}}$ constants, respectively [58]. The linear correlation coefficients ( $\mathrm{R}$ ) between the $\Delta \mathrm{E}$ values of the complexes and the $\sigma_{\mathrm{m}}$ and $\sigma_{\mathrm{p}}$ constants are equal to 0.90 and 0.98 , respectively. Herein, it would be more realistic to use $\sigma_{\mathrm{t}}\left(\sigma_{\mathrm{t}}=\left(\sigma_{\mathrm{p}}+\sigma_{\mathrm{m}}\right)\right)$ [59] as a parameter to describe the interactions. There are good linear correlations between the $\Delta \mathrm{E}$ values of complexes and $\sigma_{\mathrm{t}}$ as shown in Figs. 2 and $\mathrm{S} 2$. The higher correlation coefficients for the $\Delta \mathrm{E}-\sigma_{\mathrm{t}}$ pair $(\mathrm{R}=0.95)$ demonstrate that both inductive and resonance effects of substituents play vital roles in these intermolecular interactions. 
S. M. Chalanchi et al.: Theoretical insight to intermolecular hydrogen bond interactions between ...

Table 1. BSSE corrected binding energies $(-\Delta \mathrm{E})$ and free energy differences $(\Delta \Delta \mathrm{G})$ of complexes calculated at the B3LYP/6-311++G (d,p) ccomputational level. All energy data are given in $\mathrm{kcal} \mathrm{mol}^{-1}$.

\begin{tabular}{lllll}
\hline & CA1 & CA2 & CA3 & CA4 \\
\hline $\mathrm{NHCH}_{3}$ & $10.03(0.57)$ & $5.74(6.43)$ & $13.30(-2.84)$ & $11.92(-1.04)$ \\
$\mathrm{NH}_{2}$ & $10.16(0.50)$ & $5.79(4.77)$ & $13.13(-1.62)$ & $11.82(-0.86)$ \\
$\mathrm{OCH}_{3}$ & $10.31(0.44)$ & $5.93(4.21)$ & $12.94(-1.36)$ & $11.28(-0.54)$ \\
$\mathrm{OH}$ & $10.45(0.39)$ & $5.98(3.98)$ & $12.47(-1.23)$ & $11.19(-0.16)$ \\
$\mathrm{H}$ & $10.61(0.39)$ & $6.03(3.82)$ & $12.21(-1.21)$ & $10.96(-0.07)$ \\
$\mathrm{F}$ & $10.75(0.32)$ & $6.28(3.77)$ & $12.15(-1.13)$ & $10.90(0.08)$ \\
$\mathrm{Cl}$ & $10.86(0.25)$ & $6.30(3.79)$ & $12.03(-0.86)$ & $10.78(0.21)$ \\
$\mathrm{COH}$ & $11.03(0.13)$ & $6.34(3.70)$ & $11.57(-0.31)$ & $10.62(0.34)$ \\
$\mathrm{CN}$ & $11.13(0.04)$ & $6.40(3.69)$ & $11.48(0.08)$ & $10.43(0.42)$ \\
$\mathrm{NO}_{2}$ & $11.21(0.00)$ & $6.42(3.60)$ & $11.37(0.19)$ & $10.22(0.45)$ \\
\hline
\end{tabular}

The data in parentheses correspond to the free energy differences $(\Delta \mathrm{G})$ of the complexes.<smiles>[X]c1ccnc(N(C)C(=O)OC)c1</smiles>

A<smiles>[X]c1ccnc(N=[N+]([O-])OC)c1</smiles>

B<smiles>[X]c1ccnc(NC(=O)[O-])c1</smiles>

$\mathrm{C}$<smiles></smiles>

$\mathrm{D}$

Scheme 3. Some resonance structures of the syn rotamer of methyl N-(2-pyridyl) carbamate.

\section{AIM analysis}

The AIM analysis was carried out at the B3LYP/6-311++G (d,p) computational level to characterize the interactions.

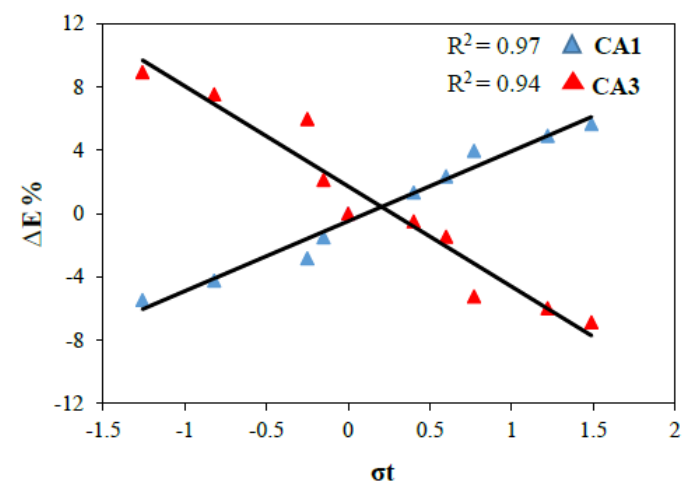

Fig. 2. Correlation between the change in the binding energies $(\Delta \mathrm{E} \%)$ and the Hammett constants $\sigma_{\text {tot }}$ of substituents for the complexes CA1 and CA3.

$$
\left[\Delta \mathrm{E} \%=\left(\frac{\Delta \mathrm{E}_{\mathrm{X}}-\Delta \mathrm{E}_{\mathrm{H}}}{\Delta \mathrm{E}_{\mathrm{H}}}\right) \times 100\right]
$$

The values of electronic charge density $(\rho)$ calculated at the bond critical points (BCPs) are listed in Table S4.

The topological properties of $\rho$ calculated at the intermolecular BCPs may be treated as the measures of $\mathrm{HB}$ strengths [60]. The $\rho$ values at the $\mathrm{NH} \cdots \mathrm{O}$ and $\mathrm{O}(\mathrm{N}) \cdots \mathrm{HO}$ hydrogen BCPs (HBCPs) 228 vary from 0.0231 to 0.0291 au and 0.0314 to $0.0560 \mathrm{au}$, respectively, which are within the ranges of hydrogen-bonded complexes values. It is well proven that the higher $\rho$ values imply the existence of stronger interactions. Hence, as presented in Table S3, the greatest $\rho_{\mathrm{BCP}}$ value of complex CA3 is observed in the presence of $\mathrm{NHCH}_{3}$ substituent, which is in agreement with its highest interaction energy. Linear correlations are observed between $\Delta \mathrm{E}$ values and the sum of $\rho$ values calculated at HBCPs $\left(\sum \rho\right)$, as is illustrated in Figs. 3 and S3.

\section{Individual hydrogen bond energies and cooperativity}

A proper evaluation of individual $\mathrm{HB}$ energies $\left(\Delta \mathrm{E}_{\mathrm{HB}}\right)$ and the cooperative contributions $\left(\Delta \mathrm{E}_{\text {coop }}\right)$ can be carried out by the characterization of mutual interplay of HBs in the CA $\cdots$ AA complexes [61]. As mentioned above, two methods were considered to estimate the $\Delta \mathrm{E}_{\mathrm{HB}}$ of complexes. The values estimated using both methods are gathered in Tables 2 and S5, and are also graphically illustrated in Figs. 4 and S4.

As can be seen in Figs. 4a and S4 (a-c), a very good correlation is observed between values estimated using the two methods. The $\Delta \mathrm{E}_{\mathrm{HB}}$ values obtained from both methods are completely in agreement with the nature of substituents and the role of atoms involved in the HBs. 
S. M. Chalanchi et al.: Theoretical insight to intermolecular hydrogen bond interactions between ...
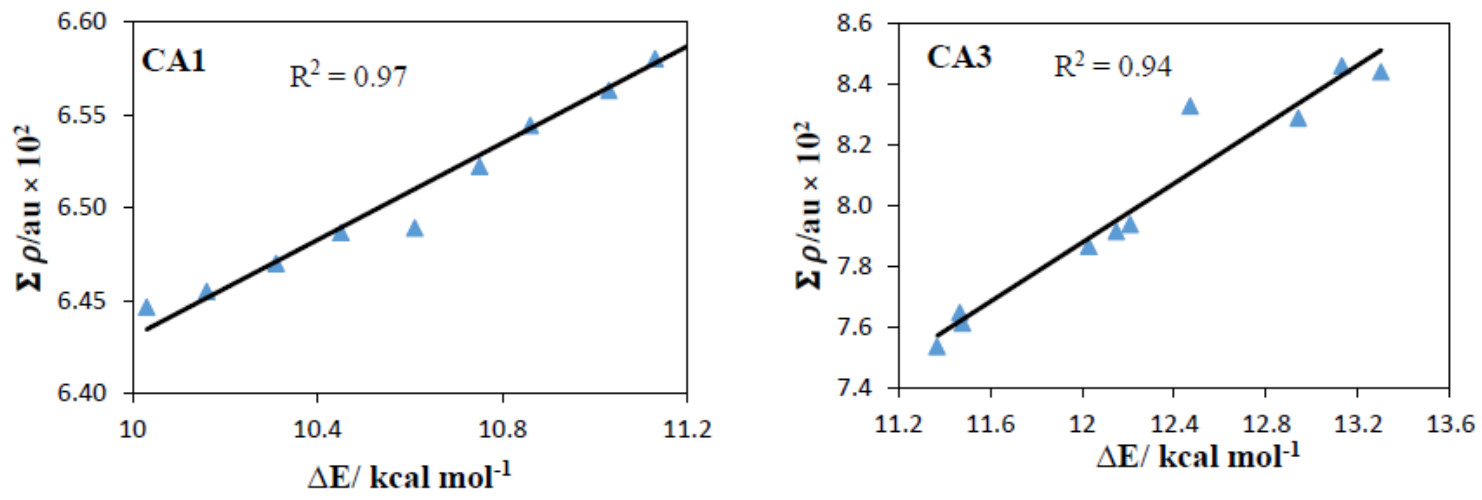

Fig. 3. Linear relationship between the sum of electron densities $\sum \rho$ calculated at HBCPs and the binding energies $\left(-\Delta \mathrm{E}\right.$ in $\left.\mathrm{kcal} \mathrm{mol}^{-1}\right)$ in the complexes CA1 and CA3.
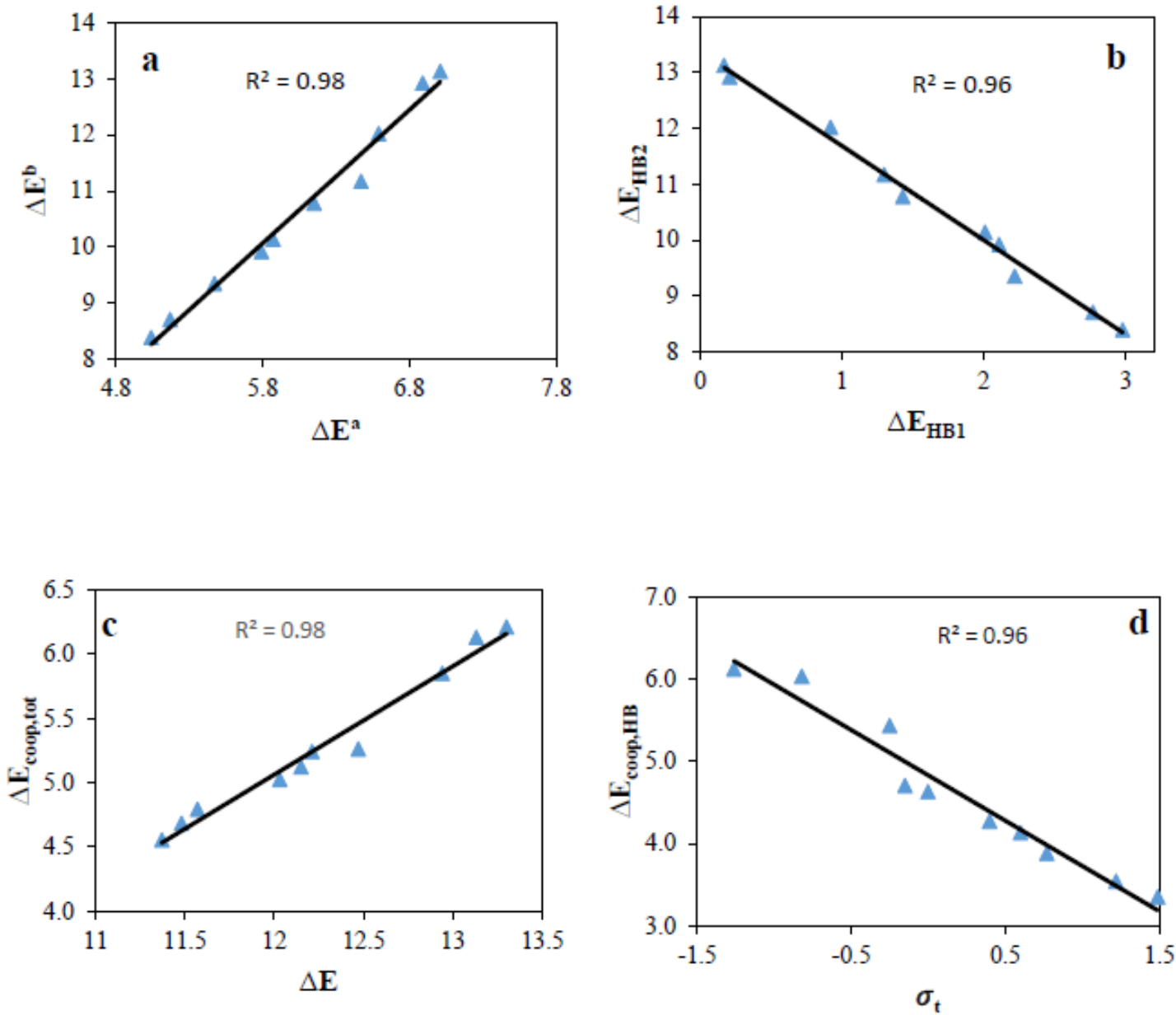

Fig. 4. (a) Correlation between the individual $\mathrm{HB}$ energies estimated using the $\mathrm{SHB}$ structures, $\Delta \mathrm{E}_{\mathrm{HB}}^{\mathrm{a}}$ and the $\rho$ values of the DHB complexes, $\Delta \mathrm{E}_{\mathrm{HB}}^{\mathrm{b}}$ for $\mathrm{HB} 2$ interaction; (b) Correlation between the $\Delta \mathrm{E}_{\mathrm{HB} 1}^{\mathrm{b}}$ and $\Delta \mathrm{E}_{\mathrm{HB} 2}^{\mathrm{b}}$; (c) Correlation between the cooperativity of total HB interaction $\left(-\Delta \mathrm{E}_{\mathrm{coop}}\right.$, tot $)$ and the binding energy $(-\Delta \mathrm{E})$; (d) Correlation between $\Delta \mathrm{E}_{\text {coop }}$ for HB2 interaction $\left(-\Delta \mathrm{E}_{\text {coop, } \mathrm{HB} 1)}\right)$ and the Hammett constants $\left(\sigma_{\text {tot }}\right)$ in the complex CA3. The HB1 and $\mathrm{HB} 2$ correspond to the $\mathrm{NH} \cdots \mathrm{O}$ and $\mathrm{N} . . . \mathrm{OH}$ interactions, respectively. All energy data are given in $\mathrm{kcal}^{\mathrm{mol}}{ }^{-1}$.

The decrease in the electron donation (or increase in the electron acceptor) power of the substituent makes $\mathrm{CA}$ a stronger $\mathrm{HB}$ donor in $\mathrm{NH} \cdots \mathrm{O}$ and rises the $\Delta \mathrm{E}_{\mathrm{HB} 1}$ value, while making $\mathrm{CA}$ a weaker $\mathrm{HB}$ acceptor in $\mathrm{O} \cdots \mathrm{HO}$ and $\mathrm{N} \cdots \mathrm{HO}$ and reducing the $\Delta \mathrm{E}_{\mathrm{HB} 2}$ value as shown in Figs. $4 \mathrm{~b}$ and S4 (d-f). In all categories, the highest and lowest values of $\Delta \mathrm{E}_{\mathrm{HB} 1}$ correspond to the strongest EWS
$\left(\mathrm{NO}_{2}\right)$ and EDS $\left(\mathrm{NHCH}_{3}\right)$, respectively; a reverse trend is observed for the $\Delta \mathrm{E}_{\mathrm{HB} 2}$ values.

The sums of the magnitudes of interactions for the individual HBs are 3.84, 1.92, 5.24 and 4.42, $\mathrm{kcal} \mathrm{mol}^{-1}$, less than the total interaction energy of the complexes CA1-CA4, respectively, where $\mathrm{X}=\mathrm{H}$ (see Tables 1 and 2). Thus, according to eq. (1), the $\Delta \mathrm{E}_{\text {coop, tot }}$ value is $36,31,42$ and $40 \%$ of the total 
S. M. Chalanchi et al.: Theoretical insight to intermolecular hydrogen bond interactions between ...

interaction energy of the complexes CA1-CA4, respectively. The $\Delta \mathrm{E}_{\text {coop, tot }}$ tends to have more negative values as the electron-withdrawing power of the substituents increases in the S1 series of the complexes. An opposite trend is observed in the S2 series of the complexes. A reliable relationship is observed between the total interaction energy of the complexes and the calculated cooperative energies as shown in Figs. 4c and S4 (g-i). As can be seen, the cooperative energies rise by the increase in the stability of complexes.

In all cases, the individual $\mathrm{HB}$ energies estimated using the $\rho$ values of the DHB complexes, $\Delta \mathrm{E}_{\mathrm{HB}}^{\mathrm{b}}$, are higher than those estimated using the SHB structures, $\Delta \mathrm{E}_{\mathrm{HB}}^{\mathrm{a}}$, in accordance with the positive cooperativity for both HBs. The trend in $\Delta \mathrm{E}_{\text {coop }}$ values estimated using eq. (4) is CA2 $(0.84)>$ CA1 $(0.74)>$ CA3 $(0.63)>$ CA4 $(0.53)$ for $\Delta \mathrm{E}_{\mathrm{HB} 1}$, where the data in parentheses are the related values in $\mathrm{kcal} \mathrm{mol}^{-1}$, which include 43, 19, 12 and $11 \%$ of the $\Delta \mathrm{E}_{\text {coop, tot }}$, respectively, where $\mathrm{X}=\mathrm{H}$ (see Table 2). The order can be discussed by comparing $\Delta \mathrm{E}_{\mathrm{HB} 1}$ and $\Delta \mathrm{E}_{\mathrm{HB} 2}$, and the related $\mathrm{HB}$ angles in each case. With respect to the weak HB2 interaction, HB1 has a better orientation and higher binding energy $\left(\Delta \mathrm{E}_{\mathrm{HB}}^{\mathrm{b}}\right)$ in the complex $\mathbf{C A 2}$ as compared with that in other complexes. Therefore, according to eq. (4), this higher binding energy makes the cooperativity more effective for HB1 in the complex CA2. In all cases, the $\Delta \mathrm{E}_{\text {coop, }} \mathrm{HB1}$ values increase as the group changes from the strongest EDS to the strongest EWS. A reverse trend is observed for the $\Delta \mathrm{E}_{\text {coop-HB2 }}$ values. These correlations are shown using $\sigma$ values in Figs. $4 d$ and S4 (j-1).

\section{Energy decomposition analysis}

To understand the nature of interactions in terms of meaningful physical components, binding energies were decomposed using the localized molecular orbital energy decomposition analyses (LMO-EDA) scheme [62]. The LMO-EDA results are summarized in Tables 3 and S6-S9, and are also graphically illustrated in Figs. 5 and S5. As can be seen, the most important stabilizing component and driving force of the interactions between CAs and $\mathrm{AA}$ is $\Delta \mathrm{E}^{\text {ele }}$, which approximately includes $41-44 \%$ of the total attraction terms; the trend in the magnitude of the terms is $\Delta \mathrm{E}^{\text {ele }}>\Delta \mathrm{E}^{\mathrm{pol}}>\Delta \mathrm{E}^{\mathrm{ex}}>$ $\Delta \mathrm{E}^{\text {disp }}$. The $\Delta \mathrm{E}^{\mathrm{pol}}, \Delta \mathrm{E}^{\mathrm{ex}}$ and $\Delta \mathrm{E}^{\text {disp }}$ values are 24,23 , and $9 \%$ of the total interaction energy in the complexes CA1, CA3 and CA4; although the $\Delta \mathrm{E}^{\mathrm{ex}}$ contribution for CA2 is not different from the rest, those of $\Delta \mathrm{E}^{\mathrm{pol}}$ and $\Delta \mathrm{E}^{\text {disp }}$ change to 19 and $14 \%$, respectively.
As can be seen in Figs. 5 and S5, the electronic effect of substituents on the $\Delta \mathrm{E}^{\mathrm{disp}}$ value is lower than other terms. Closer examination of terms reveals that the change in $\Delta \mathrm{E}^{\text {ele }}$ is larger than the values of $\Delta \mathrm{E}^{\mathrm{pol}}$ and $\Delta \mathrm{E}^{\mathrm{ex}}$. The $\Delta \mathrm{E}^{\text {ele }}$ value is mainly dependent on the charges of atoms that compose the interaction, which are directly affected by the electron donating or withdrawing nature of the substituents, whereas the covalent components $\left(\Delta \mathrm{E}^{\mathrm{pol}}\right.$ and $\Delta \mathrm{E}^{\mathrm{ex}}$ ) depend on the overlapping of orbitals that are hardly affected by the nature of the substituents. The $\Delta \mathrm{E}^{\mathrm{disp}}$ value is slightly affected by the nature of the substituent; herein, the maximum change is $0.7 \mathrm{kcal} \mathrm{mol}^{-1}$ in the complex CA3. Finally, the effect of substituent on the repulsion contribution is between those of exchange and polarization. The LMO-EDA scheme can also be used to determine the contributions of terms in the cooperativity of DHB complexes. The contributions of terms in the cooperativity estimated by eqs. (7) (10) as the difference between the LMO-EDA terms in the DHB complexes and two SHB structures, are listed in Tables 3 and S6-S9. As can be seen, the electrostatic $\left(\Delta \Delta \mathrm{E}^{\mathrm{ele}}\right)$, polarization $\left(\Delta \Delta \mathrm{E}^{\mathrm{pol}}\right)$ and repulsion interactions $\left(\Delta \Delta \mathrm{E}^{\mathrm{rep}}\right)$ make a positive contribution to the total cooperativity of the complexes, while the exchange $\left(\Delta \Delta \mathrm{E}^{\mathrm{ex}}\right)$ and dispersion $\left(\Delta \Delta \mathrm{E}^{\text {disp }}\right)$ interactions make a negative contribution to it. As mentioned above, the $\Delta \Delta \mathrm{E}^{\mathrm{ex}}$ and $\Delta \Delta \mathrm{E}^{\text {rep }}$ interactions are grouped as one exchange-repulsion $\left(\Delta \Delta \mathrm{E}^{\mathrm{exrep}}\right)$ term to describe the Pauli repulsion [Error! Bookmark not defined.]. In all cases, the $\Delta \Delta \mathrm{E}^{\text {exrep }}$ term is the largest one among all LMO-EDA terms while the $\Delta \Delta \mathrm{E}^{\text {ele }}$ term is the second-large contribution. The large negative value of $\Delta \Delta \mathrm{E}^{\text {exrep }}$ shows that the Pauli repulsion referring to the repulsive interactions between fragments is remarkably reduced when two hydrogen bond interactions operate simultaneously in DHB complexes. The negative value of the $\Delta \Delta \mathrm{E}^{\text {ele }}$ term shows that the electrostatic interaction energies between fragments are enhanced when two hydrogen bond interactions work in concert with each other in DHB complexes.

The negative value of the $\Delta \Delta \mathrm{E}^{\mathrm{pol}}$ term indicates that the orbitals in DHB complexes undergo a stronger change in their shapes in order to maximize the strength of hydrogen bonds. Finally, the positive value of the $\Delta \Delta \mathrm{E}^{\text {disp }}$ term shows that the dispersion energies are decreased in the DHB complexes. In complex CA2, the contribution of $\Delta \Delta \mathrm{E}^{\text {exrep }}$ is larger than that of $\Delta \mathrm{E}_{\text {coop, }}$ tot (see Table $3)$. It is due to the larger dispersion contribution in the formation of complex CA2. 
S. M. Chalanchi et al.: Theoretical insight to intermolecular hydrogen bond interactions between ...

Table 2. The estimated values of individual hydrogen bond energies $\left(-\Delta \mathrm{E}_{\mathrm{HB}}\right.$ in $\left.\mathrm{kcal} \mathrm{mol}^{-1}\right)$ and the cooperativity energy $\left(-\Delta \mathrm{E}_{\mathrm{COOP}}\right.$ in $\left.\mathrm{kcal} \mathrm{mol}^{-1}\right)$ in the unsubstituted complexes $(\mathrm{X}=\mathrm{H})$.

\begin{tabular}{cccccccc}
\hline & $\Delta \mathrm{E}_{\mathrm{HB} 1}^{\mathrm{a}}$ & $\Delta \mathrm{E}_{\mathrm{HB} 2}^{\mathrm{a}}$ & $\Delta \mathrm{E}_{\mathrm{HB} 1}^{\mathrm{b}}$ & $\Delta \mathrm{E}_{\mathrm{HB} 2}^{\mathrm{b}}$ & $\Delta \mathrm{E}_{\mathrm{COOP}(\mathrm{H}}$ & $\Delta \mathrm{E}_{\mathrm{COOP}(\mathrm{H}}$ & $\Delta \mathrm{E}_{\mathrm{COOP}(\mathrm{T}}$ \\
\hline $\mathrm{CA} 1$ & 1.43 & 5.39 & 2.22 & 8.39 & 0.79 & 3.00 & 3.79 \\
$\mathrm{CA} 2$ & 1.79 & 2.32 & 2.63 & 3.4 & 0.84 & 1.08 & 1.92 \\
$\mathrm{CA} 3$ & 0.82 & 6.15 & 1.43 & 10.78 & 0.62 & 4.63 & 5.24 \\
$\mathrm{CA} 4$ & 0.77 & 5.77 & 1.30 & 9.66 & 0.53 & 3.89 & 4.42 \\
\hline
\end{tabular}

$\mathrm{HB} 1$ and $\mathrm{HB} 2$ correspond to the $\mathrm{NH} \cdots \mathrm{O}$ and $\mathrm{O}(\mathrm{N}) \cdots \mathrm{H}$ hydrogen bonds, respectively; $\mathrm{a}$ and $\mathrm{b}$ correspond to the individual HB energies estimated using the SHB structures and estimated from the $\rho$ values calculated at the HBCPs of DHB complexes, respectively.

Table 3. The LMO-EDA results of unsubstituted complexes $(\mathrm{X}=\mathrm{H})$ calculated at the B3LYP/6-311++G(d,p) computational level $\left(\mathrm{kcal} \mathrm{mol}^{-1}\right)$.

\begin{tabular}{cccccccc}
\hline \multirow{2}{*}{ CA1 } & $\Delta \mathrm{E}^{\text {ele }}$ & $\Delta \mathrm{E}^{\text {ex }}$ & $\Delta \mathrm{E}^{\text {pol }}$ & $\Delta \mathrm{E}^{\text {disp }}$ & $\Delta \mathrm{E}^{\text {rep }}$ & $\Delta \mathrm{E}$ \\
& $\mathrm{DHB}$ & -24.34 & -12.66 & -12.73 & -5.78 & 42.99 & -12.52 \\
& $\mathrm{SHB} 2$ & -14.88 & -8.17 & -7.92 & -3.62 & 27.85 & -6.75 \\
& $\mathrm{SHB} 1$ & -8.01 & -4.56 & -3.77 & -2.85 & 16.55 & -2.64 \\
& $\Delta \Delta \mathrm{E}^{\mathrm{X}}$ & -1.45 & 0.07 & -1.04 & 0.69 & -1.41 & -3.13 \\
& $\mathrm{DH} 2$ & -16.6 & -8.93 & -8.23 & -5.17 & 31.56 & -7.36 \\
& $\mathrm{SHB} 2$ & -9.15 & -5.46 & -4.74 & -3.32 & 19.78 & -2.89 \\
& $\mathrm{SHB} 1$ & -7.28 & -3.79 & -3.23 & -2.61 & 14.06 & -2.86 \\
& $\Delta \Delta \mathrm{E}^{\mathrm{X}}$ & -0.17 & 0.32 & -0.26 & 0.76 & -2.28 & -1.61 \\
& $\mathrm{DHB}$ & -31.30 & -17.99 & -18.34 & -6.76 & 59.40 & -14.99 \\
& $\mathrm{SHB} 2$ & -20.47 & -12.99 & -12.63 & -4.63 & 42.76 & -7.96 \\
& $\mathrm{SHB} 1$ & -9.64 & -5.78 & -4.70 & -3.24 & 20.52 & -2.84 \\
& $\Delta \Delta \mathrm{E}^{\mathrm{X}}$ & -1.19 & 0.79 & -1.01 & 1.12 & -3.88 & -4.17 \\
& $\mathrm{DHB}$ & -29.43 & -17.27 & -17.32 & -6.63 & 57.12 & -13.52 \\
& $\mathrm{SHB} 2$ & -19.63 & -12.54 & -12.01 & -4.59 & 41.4 & -7.37 \\
& $\mathrm{SHB} 1$ & -9.09 & -5.53 & -4.50 & -3.17 & 19.67 & -2.62 \\
& $\Delta \Delta \mathrm{E}^{\mathrm{X}}$ & -0.71 & 0.80 & -0.81 & 1.13 & -3.95 & -3.53 \\
\hline
\end{tabular}

The $\Delta \Delta \mathrm{E}^{\mathrm{X}}$ values were estimated using Eqs. (7) - (10). DHB corresponds to the double H-bonded complexes and SHB1 and SHB2 correspond to the $\mathrm{NH} \cdots \mathrm{O}$ and $\mathrm{O}(\mathrm{N}){ }^{\cdots} \mathrm{H}$ hydrogen bonds, respectively.
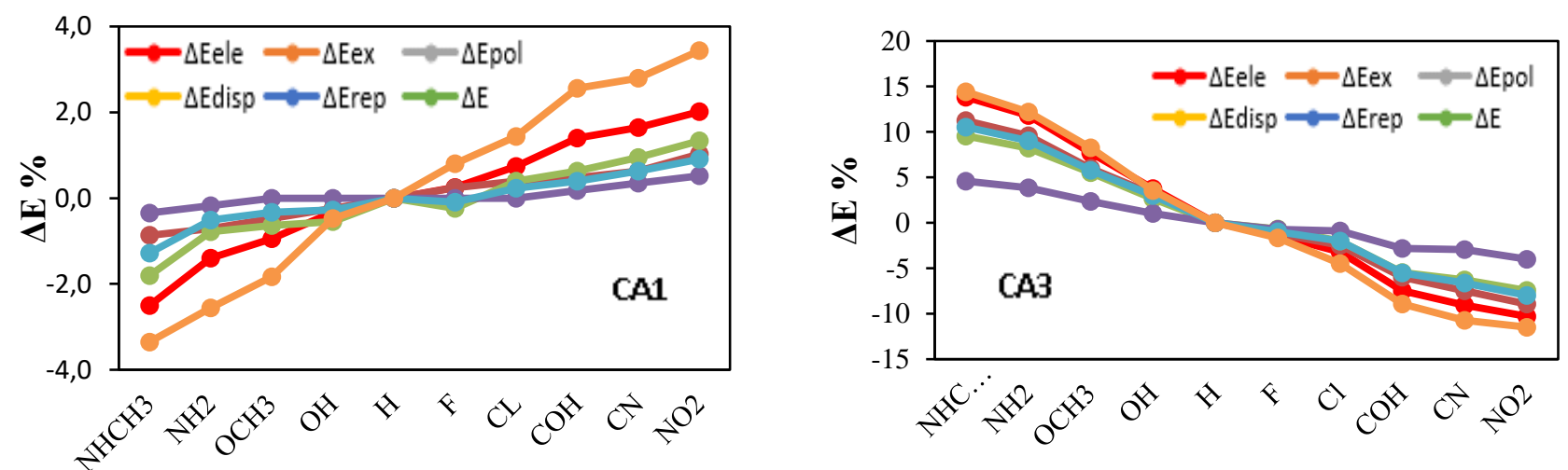

Fig. 5. The changes in energy terms $(\Delta \mathrm{E} \%)$ obtained using the LMO-EDA scheme for the complexes CA1 and CA3. $\left[\Delta \mathrm{E} \%=\left(\frac{\Delta \mathrm{E}_{\mathrm{X}}-\Delta \mathrm{E}_{\mathrm{H}}}{\Delta \mathrm{E}_{\mathrm{H}}}\right) \times 100\right]$

The negative value of $\Delta \Delta \mathrm{E}^{\text {exrep }}$ is almost cancelled out by the large positive value of the $\Delta \Delta \mathrm{E}^{\text {disp }}$, resulting in the very small $\Delta \mathrm{E}_{\text {coop, tot }}$ value for complex CA2.

\section{CONCLUSIONS}

Four cyclic double H-bonded complexes between the syn and anti rotamers of CA and AA, 
S. M. Chalanchi et al.: Theoretical insight to intermolecular hydrogen bond interactions between ...

named as CA1-CA4, were investigated on the potential energy surface. The complexes CA1 and CA2, in which the oxygen atom of CA acts as a proton acceptor, are stabilized by EWSs and are destabilized by EDSs. The behaviors of substituents are reversed in the complexes CA3 and CA4, in which the nitrogen atom of the ring is involved in the interaction. The energy data and geometrical parameters are in excellent correlations with the $\sigma$ coefficients of substituents. Very good linear correlation is observed between the individual $\mathrm{HB}$ energies estimated using (a) the SHB structures and (b) the $\rho$ values of the $\mathrm{DHB}$ complexes. The $\Delta \mathrm{E}_{\mathrm{HB}}$ values obtained from both methods are completely in agreement with the nature of substituents and the role of atoms involved in the HBs. A positive cooperativity is observed between $\mathrm{HB} 1$ and $\mathrm{HB} 2$ interactions in the DHB complexes. In addition, a linear relationship is found between the total cooperative energies and the total interaction energy of complexes. The most stable complexes are the ones showing the larger absolute values for cooperativity. The LMO-EDA scheme shows that the order of energy contribution in the stabilization of complexes is $\Delta \mathrm{E}^{\text {ele }}>\Delta \mathrm{E}^{\mathrm{pol}}>\Delta \mathrm{E}^{\mathrm{ex}}>\Delta \mathrm{E}^{\text {disp }}$, which clearly shows that the interactions are all electrostatic dominant. The LMO-EDA scheme also shows that cooperativity is mainly caused by $\Delta \Delta \mathrm{E}^{\text {exrep}}$, which indicates that the Pauli repulsion is remarkably reduced when two hydrogen bond interactions operate simultaneously.

Acknowledgement: We are grateful to the University of Sistan and Baluchestan for financial support and the Computational Quantum Chemistry Laboratory for computational facilities.

\section{REFERENCES}

1. V. J. Stella, Prodrugs: Challenges and Rewards, Springer: New York, 2007.

2. J. E. Hennessy, M. J. Latter, S. Fazelinejad, A. Philbrook, D. M. Bartkus, H. K Kim, C. J. Easton, Appl Environ Microbiol., 84, e02250-17 (2018).

3. A .K. Ghosh, B. D. Chapsal, I. T. Weber, H. Mitsuya, Acc. Chem. Res., 41, 78 (2008).

4. P. E. Chen, N.C. Anderson, Z.M. Norman, J. S. Owen, J. Am. Chem. Soc., 139, 3227 (2017).

5. I. Dierynck, H. Van Marck, M.V. Ginderen, T. H. Jonckers, M. N. Nalam, C. A. Schiffer, A. Raoof, G. Kraus, G. Picchio, Antimicrob. Agents Chemother., 55, 5723 (2011).

6. A. K. Ghosh, B. D. Chapsal, A. Baldridge, M. P. Steffey, D. E. Walters, Y. Koh, M. Amano, H. Mitsuya, J. Med. Chem., 54, 622 (2011).

7. A. K. Ghosh, C. X. Xu, K. V. Rao, A. Baldridge, J. Agniswamy, Y. F. Wang, I. T. Weber, M. Aoki, S. G. Miguel, M. Amano, H. Mitsuya, Chem. Med. Chem., 5, 1850 (2010).
8. A. K. Ghosh, M. Brindisi, J. Med. Chem., 58, 2895, (2015).

9. B. Testa, J. M. Mayer, Hydrolysis in Drug and Prodrug Metabolism-Chemistry, Biochemistry, and Enzymology; Wiley-VCH: Weinheim, Germany, 2003.

10. D. Aydin, M. Arslan, A. Sanyal, R. Sanyal, Bioconjugate Chem., 28, 1443 (2017).

11. T. K. Das, S. Mondal, R. G. Gonnade, A. T. Biju, Org. Lett., 19, 5597 (2017).

12. Y. G. Li, L. Li, M. Y. Yang, G. He, E. A. B. Kantchev, J. Org. Chem., 82, 4907 (2017).

13. H. Du, Z. Song, Y. Xu, J. Agric. Food Chem., 66, 387 (2018).

14. J. Tang, L. Hong, A. K. Ghosh, Wiley-VCH: Weinheim, Germany, 45, 413 (2010).

15. J. M. Ahn, J. C. Peters, G. C. Fu, J. Am. Chem. Soc., 139, 18101 (2017).

16. B. Z. Kurt, I. Gazioglu, A. Dag, R. E. Salmas, G. Kayı, S. Durdagi, F. Sonmez, Bioorganic Med. Chem., 25, 1352 (2017).

17. L. B. Dong, J. D. Rudolf, L. Lin, C. Ruiz, M. D. Cameron, B. Shen, Bioorganic Med. Chem., 25, 1990 (2017).

18. A. K. Ghosh, D. W. Shin, G. Koelsch, X. Lin, J. Ermolieff, J. Tang, J. Am. Chem. Soc., 122, 3522 (2000).

19. V. Gowd, H. Su, P. Karlovsky, W. Chen, Food Chem., 248, 312 (2018).

20. W. Zhou, R. Fang, Q. Chen, Food Chem., 233, 174 (2017).

21. H. Kurouchi, A. Sumita, Y. Otani, T. Ohwada, Chem. Eur. J., 20, 8682 (2014).

22. U. Das, B. Chattopadhyay, D. K. Hazra, V. V. Sureshbabu, A. K Mukherjee, J. Mol. Struct., 1122, 290 (2016).

23. C. Dugave, L. Demange, Chem. Rev., 103, 2475 (2003).

24. M. J. Deetz, M. Jonas, J. P. Malerich, B. D. Smith, Supramol. Chem., 14, 487 (2002).

25. A. C. Cheng, W. W. Chen, C. N. Fuhrmann, A. D. Frankel, J. Mol. Biol., 327, 781 (2003).

26. E. M. Basílio Janke, H. H. Limbach, K. Weisz, J. Am. Chem. Soc., 126, 2135 (2004).

27. S. Schlund, M. Mladenovic, E. M. Basílio Janke, B. Engels, K. Weisz, J. Am. Chem. Soc., 127, 16151 (2005).

28. J. Allers, Y. Shamoo, J. Mol. Biol., 311, 75 (2001).

29. O. H. O. Brovarets, Y. P. Yurenko, I. Y. Dubey, D. M. Hovorun, J Biomol Struct Dyn., 29, 1101 (2012).

30. S. Sarkhel, G. R. Desiraju, Proteins: Structure, Function, and Bioinformatics, 54, 247 (2004).

31. D. Chen, N. Oezguen, P. Urvil, C. Ferguson, S. M. Dann, T. C. Savidge, Science Advances, 2(3), e1501240 (2016).

32. P. F. Su, H. Li, J. Chem. Phys., 131, 014102 (2009).

33. M.J. Frisch, G.W. Trucks, H.B. Schlegel, G.E. Scuseria, M.A. Robb, J.R. Cheeseman, G. Scalmani, V. Barone, B. Mennucci, G.A. Petersson, H. Nakatsuji, M. Caricato, X. Li, H.P. Hratchian, A.F. Izmaylov, J. Bloino, G. Zheng, J.L. Sonnenberg, M. 
S. M. Chalanchi et al.: Theoretical insight to intermolecular hydrogen bond interactions between ...

Hada, M. Ehara, K. Toyota, R. Fukuda, J. Hasegawa, M. Ishida, T. Nakajima, Y. Honda, O. Kitao, H. Nakai, T. Vreven, J.A. Montgomery, J.E. Peralta, F. Ogliaro, M. Bearpark, J.J. Heyd, E. Brothers, K.N. Kudin, V.N. Staroverov, R. Kobayashi, J. Normand, K. Raghavachari, A. Rendell, J.C. Burant, S.S. Iyengar, J. Tomasi, M. Cossi, N. Rega, J.M. Millam, M. Klene, J.E. Knox, J.B. Cross, V. Bakken, C. Adamo, J. Jaramillo, R. Gomperts, R.E. Stratmann, O. Yazyev, A.J. Austin, R. Cammi, C. Pomelli, J.W. Ochterski, R.L. Martin, K. Morokuma, V.G. Zakrzewski, G.A. Voth, P. Salvador, J.J. Dannenberg, S. Dapprich, A.D. Daniels, O. Farkas, J.B. Foresman, J.V. Ortiz, J. Cioslowski, D.J. Fox. Gaussian09, revision A.02. Gaussian Inc., Wallingford, CT, 2009.

34. G. Subhapriya, S. Kalyanaraman, N. Surumbarkuzhali, S. Vijayalakshmi, V. Krishnakumar, S. Gandhimathi, J. Mol. Struct., 1128, 534 (2017).

35. Z. Moosavi-Tekyeh, F. Taherian, S. F. Tayyari, J. Mol. Struct., 1111, 185 (2016).

36. A. E. Shchavlev, A. N. Pankratov, A. V. Shalabay, J. Phys. Chem. A, 109, 4137 (2005).

37. I. Saada, J. K. Pearson, Comput. Theor. Chem., 969, 76 (2011).

38. R. Improta, V. Barone, J. Comput. Chem., 25, 1333 (2004).

39. A. E. Shields, T. van Mourik, J. Phys. Chem. A, 111, 13272 (2007).

40. S. F. Boys, F. Bernardi, Mol. Phys., 19, 553 (1970).

41. C. Møller, M. S. Plesset, Phys. Rev., 46, 618 (1934).

42. E. G. Hohenstein, S. T. Chill, C. D. Sherrill, J. Chem. Theory Comput., 4, 1996 (2008).

43. S. Grimme, J. Antony, S. Ehrlich, H. Krieg, J. Chem. Phys., 132, 154104 (2010).

44. J. Dunning, H. Thom, J. Chem. Phys., 901007 (1989).

45. J. Tomasi, B. Mennucci, R. Cammi, Quantum mechanical continuum solvation models, Chem. Rev., 105, 2999 (2005).
46. R. F. W. Bader, Atoms in Molecules: A Quantum Theory, Oxford University Press, Oxford, 1990.

47. K. F. Biegler, J. Schonbohm, D. Bayles, J. Comput. Chem., 22, 545 (2001).

48. E. D. Glendening, F. Weinhold, J. Comput. Chem., 19, 593 (1998).

49. E. D. Glendening, F. Weinhold, J. Comput. Chem., 19, 610 (1998)

50. A. Asensio, N. Kobko, J. J. Dannenberg, J. Phys. Chem. A, 107, 6441 (2003).

51. A. Ebrahimi, S. H. Khorassani, H. Delarami, Chemical Physics, 365, 18 (2009).

52. A. Ebrahimi, S. H. Khorassani, H. Delarami, H. Esmaeeli, J. Comput. Aided Mol., 24,409 (2010).

53. N. Hesabi, A. Ebrahimi, A. Nowroozi, J. Mol. Graph. Model., 77, 86 (2017).

54. M. W. Schmidt, K. K. Baldridge, J. A. Boatz, S. T. Elbert, M. S. Gordon, J. H. Jensen, S. Koseki, N. Matsunaga, K. A. Nguyen, S. Su, T. L. Windus, M. Dupuis, J. A. Montgomery, J. Comput. Chem., 14, 1347 (1993).

55. X. Chang, P. Su, W. Wu, Chem. Phys. Lett., 610, 246 (2014).

56. E. D. Glendening, J. K. Badenhoop, A. E. Reed, J. E. Carpenter, J. A. Bohmann, C. M. Morales, F. Weinhold, NBO 5.G, Theoretical Chemistry Institute, University of Wisconsin, Madison, WI, 2001.

57. C. Hansch, A. Leo, R.W. Taft, Chem. Rev., 97, 165 (1991).

58. F. Cozzi, F. Ponzini, R. Annunziata, M. Cinquini, J. S. Siegel, Angewandte Chemie, 34, 1019 (1995).

59. A. Ebrahimi, M. Habibi, R. S. Neyband, A. R. Gholipour, Phys. Chem. Chem. Phys., 11, 11424 (2009).

60. S. J. Grabowski, M. Małecka, J. Phys. Chem. A, 110, 11847 (2006).

61. W. Xu, X. C. Li, H. Tan, G. J. Chen, Phys. Chem. Chem. Phys., 8, 4427 (2006).

62. A. Bharti, T. Banerjee, Comput. Theor. Chem., 1067, 48 (2015). 


\title{
Synthesis, antimicrobial and antimalarial activityof1, 4-benzothiazepine and pyrazolinederivatives incorporating carbazolemoiety
}

\author{
V. A. Kadnor ${ }^{1}$, S. N. Shelke ${ }^{2 *}$ \\ ${ }^{1}$ Department of Chemistry, A. C. S. College Satral, Rahuri, Ahmednagar, MS 413711, India \\ ${ }^{2}$ Department of Chemistry, S. S. G. M. College, Kopargaon, Ahmednagar, MS 423601, India
}

Received July 18, 2018; Revised August 30, 2018

\begin{abstract}
A series ofcarbazole-based 1,4-benzothiazepine and pyrazoline derivatives weresynthesized and the structures of the newly synthesized compoundswere confirmed by FT-IR, ${ }^{1} \mathrm{H}$ NMR, ${ }^{13} \mathrm{C}$ NMR and mass spectral studies. All new derivatives $\mathbf{4 ( a - f ) a n d ~} \mathbf{5 ( a - e ) w e r e ~ s c r e e n e d ~ f o r ~ t h e i r i n ~ v i t r o ~ a n t i m i c r o b i a l ~ a c t i v i t y , ~ a n d ~ a l s o ~ f o r ~ t h e i r ~ a n t i m a l a r i a l ~}$ activity.Compounds $\mathbf{4 a}, \mathbf{4 b}, \mathbf{4 d}, \mathbf{5 a}, \mathbf{5 b}$ and $\mathbf{5 c e x h i b i t e d p r o m i s i n g a n t i m i c r o b i a l ~ a n d ~ a n t i m a l a r i a l ~ a c t i v i t i e s ~ a s ~ c o m p a r e d ~}$ to positive control. Notably, compounds $\mathbf{4 a}, \mathbf{4 b a n d} \mathbf{4 d}$ showedexcellent antifungal activity against Penicillium $s p$.comparable to that of a standard drug.
\end{abstract}

Key words:Carbazole, 1,4-Benzothiazepine,Pyrazoline,Antimicrobial andantimalarial activities

\section{INTRODUCTION}

A large number of natural and synthetic carbazole derivatives have been reported to exhibit diverse biological activities such as antimicrobial $[1,2]$, antiviral [3], antimalarial [4] and potential application as pharmacological agents $[5,6]$. Recentlycarbazole-substitutedchalcone and its urea derivatives have been reported to exhibit antimicrobial, radical scavenger, cancer chemopreventive and polyphenol oxidase enzyme activities [7, 8]. Chalcones are also key precursors in the synthesis of many biologically important heterocyclic compounds such as benzothiazepines and pyrazolines.

Thiazepinesbelong to the important class of heterocyclic compoundsfor the synthesis of pharmaceutical agents, as well as biologically active compounds [9]. Benzothiazepinesplay an important role in drug discovery, as they show bioactivities such as anticonvulsant [10], endogenous natriuretic factors [11], potential central nervous system agents [12], antibiotics [13], antimicrobials [14], antihypertensive [15], antidiabetic [16] and cytotoxic agents [17].Novel carbazole assembled 1,4-thiazepine derivatives have been reported, which not only have significant antioxidant activities, but also exhibit remarkably selective cytotoxicity to carcinoma cell line HCT 116 [18]. Pyrazolines and their derivatives have been found to possess a wide spectrum of biological activities such as antimicrobial [19-22], antimalarial [23, 24], anti-inflammatory [25] and antioxidant [26].3-(substituted)-aryl-5-(9-methyl-3carbazole)-1 $H$-2-pyrazolines are reported as a novel class of anti-inflammatory and antioxidant agents

* To whom all correspondence should be sent:

E-mail: snshelke@yahoo.co.in

234
[27], thus literature survey reveals that carbazole is a useful starting material for pharmacologically important products.

Therefore, in continuation of our efforts to synthesize biologically active heterocyclic compounds $[28,29]$, herein we report the synthesis of carbazole-containing 1,4-benzothiazepineand pyrazolinederivatives with their antimicrobial and antimalarial activities.

\section{RESULTS AND DISCUSSION}

\section{Chemistry}

In view of the emerging biological importance of carbazole, we synthesized a series of carbazolechalcones and its corresponding 1,4benzothiazepine and pyrazolinederivatives from 3formyl-9-ethyl carbazole2 as shown in scheme1 on the hope of obtaining more antimicrobial and antimalarial agents. Thus, the starting compound 3formyl-9-ethylcarbazole $\mathbf{2}$ was prepared byVilsmeier-Haack formylation of carbazole1. 3formyl-9-ethylcarbazole 2 was obtainedbyClaisenSchmidt condensation with various substituted 2hydroxyacetophenones in ethanolic potassium hydroxide afforded carbazolechalcones 3 . The 1,4benzothiazepine4(a-f)derivatives were synthesized by Michael addition of 2-aminothiophenol to carbazolechalcones3in acetic acid and ethanol. Carbazolepyrazolines5(a-e)were prepared from the compounds 3 on treatment with hydrazine hydrate in ethanol and acetic acid, the reaction most likely takes place through the intervention of an appropriate $\alpha, \beta$-unsaturated hydrazone, which instantly cyclizes to give a pyrazoline ring, at reflux temperature cyclizing agent is acetic acid. 
V. A. Kadnor, S. N. Shelke: Synthesis, antimicrobial and antimalarial activity of 1, 4-benzothiazepine and ...

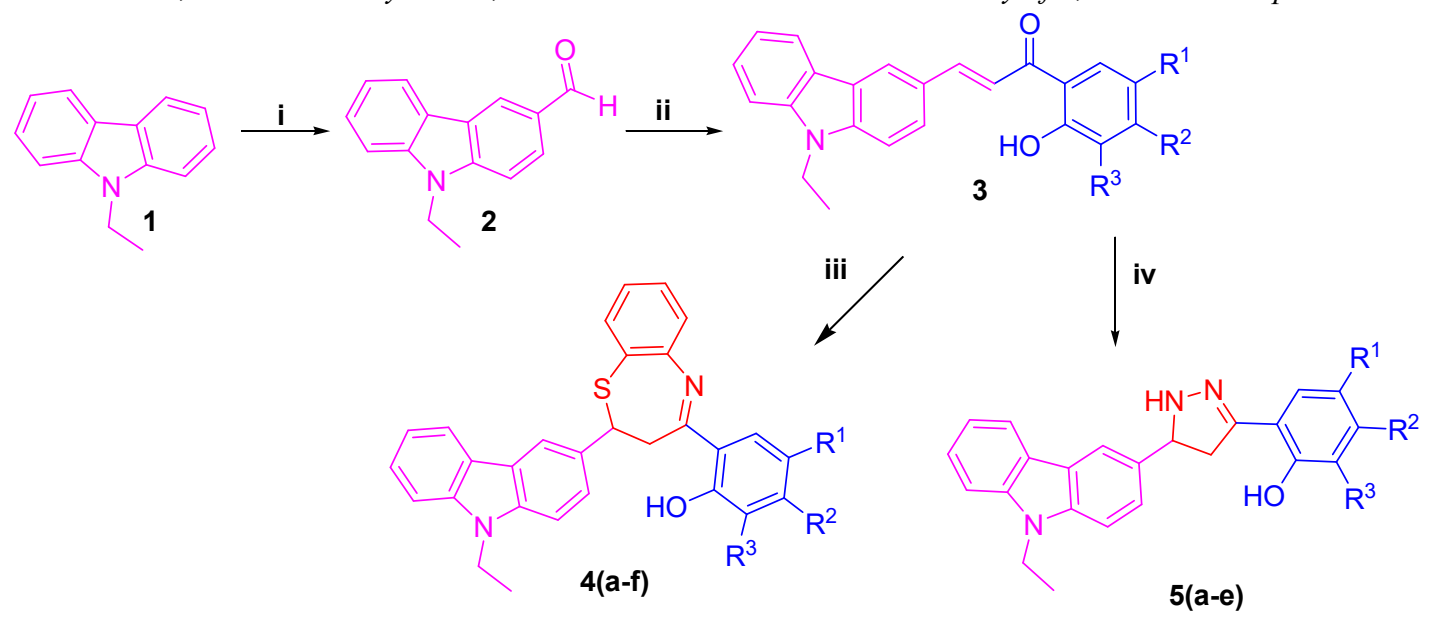

Scheme1. Reagents and conditions: (i) DMF, $\mathrm{POCl}_{3}, 80^{\circ} \mathrm{C}$, $4 \mathrm{~h}$ (ii) Substituted2-hydroxyacetophenones, $\mathrm{KOH}, \mathrm{EtOH}$, rt., 24-36 h. (iii) 2-Aminothiophenol, $\mathrm{AcOH}$, EtOH, Reflux, 8h. (iv) $\mathrm{NH}_{2} \mathrm{NH}_{2} \cdot \mathrm{H}_{2} \mathrm{O}$, EtOH, AcOH, reflux 6 h.

The structures of $\mathbf{4 ( a - f )}$ and $\mathbf{5 ( a - e ) ~ w e r e ~}$ confirmed by FT-IR, ${ }^{1} \mathrm{H}$ and ${ }^{13} \mathrm{C}$ NMR, and mass spectroscopic technique.For example, the infrared spectra of compounds 4(a-f) showed characteristic signals at 1688 and $3350 \mathrm{~cm}^{-1}$ for $\mathrm{C}=\mathrm{N}$ and hydroxyl group absorption, respectively.In general, three thiazepine protons of carbazole-based benzothiazepines showed similar patterns of signals in the ${ }^{1} \mathrm{H}$ NMR spectra. They displayed a doublet of a doublet at $\mathrm{C}_{17}$ for two protons and a triplet at $\mathrm{C}_{16}$ for one proton. The methine proton at $\mathrm{C}_{16}$ of the thiazepine nucleus resonates at around $\delta 3.25 \mathrm{ppm}$ as a triplet with coupling constant $(J)$ of nearly 12.6 $\mathrm{Hz}$. This signal is observed as a triplet instead of a doublet of a doublet because two $J$-values accidentally are the same and two inner lines of the quartet occur at the same point, appearing as a single line of double intensity [30]. The two methylene protons at $\mathrm{C}_{17}$ displayed two signals as a doublet of doublet at around $\delta 3.45 \mathrm{ppm}$ with coupling constantsof nearly $9.5 \mathrm{~Hz}$ and $3.8 \mathrm{~Hz}$ and a doublet of doublet at around $\delta 5.16 \mathrm{ppm}$ with coupling constants of nearly $9.4 \mathrm{~Hz}$ and $3.9 \mathrm{~Hz}$. The ${ }^{13} \mathrm{C}$ NMR spectrum of compounds $4(\mathbf{a}-\mathbf{f})$ showedaromatic carbon signals in the region of $108.68-157.89 \mathrm{ppm}$. In the mass spectrum in all cases, peaks corresponding to molecular ions were observed which confirmed their molecular weights.

IR spectra of the compounds 5(a-e) revealed a characteristic strong intensity band due to $-\mathrm{OH}$ and $-\mathrm{NH}$ stretching at 3668 and $3205 \mathrm{~cm}^{-}$ ${ }^{1}$ respectively, whilea pyrazoline $-\mathrm{C}=\mathrm{N}$ band was observed around $1614 \mathrm{~cm}^{-1}$. The ${ }^{1} \mathrm{H}$ NMR spectrum of these compounds exhibited an $\mathrm{ABX}$ pattern for the presence of two diastereotopic protons at $\mathrm{C}_{17}$ and one single proton at the $\mathrm{C}_{16}$ position. Asymmetric - $\mathrm{CH}$ proton displayed a triplet at $\delta$ $5.12 \mathrm{ppm}$ with $J=10.8 \mathrm{~Hz}$, whereas the pro-chiral methylene $\left(\mathrm{CH}_{2}\right)$ protons appeared as two characteristic doublets of a doublet at $\delta 3.16$ and $3.66 \mathrm{ppm}$ with $J=10.8$ and 5.7 Hzwhich indicates the magnetic non-equivalence of the two protons. According to the high resolution mass spectrum (HRMS) of the representative compound 5acalculated for $\mathrm{C}_{23} \mathrm{H}_{20} \mathrm{ON}_{3} \mathrm{Cl}_{2} \quad(\mathrm{M}+\mathrm{H})^{+}$ws 424.0981, found 424.0978.

\section{Antibacterial and antifungal evaluation}

The synthesized carbazole-assembled 1,4benzothiazepine4(a-f)and pyrazoline 5(ae)derivativeswere tested for their in vitro antimicrobial activity against two gram negative (Escherichia coli, Pseudomonas putida), two gram positive (Bacillus subtilis, Streptococcus lactis) bacterialstrains and three (Aspergillus niger, Penicillium sp., Candida albicans) fungal strains usingampicillin and greseofulvin as standard drugs, respectively.The inhibition zone diameters were measured in millimeters ( $\mathrm{mm}$ )andminimal inhibitory concentration (MIC) was expressed as $\mu \mathrm{g} / \mathrm{mL}$ of all synthesized compounds, the results obtained are enclosed in Table 1. Among the synthesized compounds, 4a, 4b, 4d,5a, 5b and 5ccould effectively inhibit the growth of most tested bacterial and fungal strains with considerable MIC $(\mu \mathrm{g} / \mathrm{mL})$ values. Carbazole-tethered 1,4benzothiazepines $\mathbf{4}(\mathbf{a}-\mathbf{f})$, three derivatives $4 a, 4 b$ and 4d exhibited a significant activity against P.putida with MIC valuesof 50,40 and $45 \mu \mathrm{g} / \mathrm{mL}$, respectively as compared with positive control. Three compounds $\mathbf{4 c}, \mathbf{4 e}$ and $\mathbf{4 f}$ also displayed moderate antibacterial activities $(65-100 \mu \mathrm{g} / \mathrm{mL})$ against all evaluated bacterial strains. Notably, compounds $\mathbf{4 a}, \mathbf{4 b}$ and $\mathbf{4 d}$ gave remarkable broader antifungal bioactive spectrum with MIC values in the range of $40-45 \mu \mathrm{g} / \mathrm{mL}$ against Penicillium $s p$. while two compounds $\mathbf{4 c}$ and4ehad satisfying activities against all screened fungal strains with 
V. A. Kadnor, S. N. Shelke: Synthesis, antimicrobial and antimalarial activity of 1, 4-benzothiazepine and ... considerable MIC values. It was found that carbazole pyrazolines5(a-e), compounds $\mathbf{5 a}, \mathbf{5 b}$ and 5cshowed strong activities $(45-65 \mu \mathrm{g} / \mathrm{mL})$ against gram positive $B$. subtilis and gram negative $P$. putida bacteria, while compounds $\mathbf{5 d}$ and $\mathbf{5 e}$ showedgood activities $(70-110 \mu \mathrm{g} / \mathrm{mL})$ against allfour bacterial strains as compared with standard drug ampicillin. As for antifungal activities, compound5a exhibited significant activity against Penicillium sp. and C. albicanswith MIC valuesof 55 and $60 \mu \mathrm{g} / \mathrm{mL}$, respectively, while $\mathbf{5 b}, \mathbf{5 c}, \mathbf{5 d}$ and 5e showed moderate activities (70-100 $\mu \mathrm{g} / \mathrm{mL}$ )against all testedfungal strainscompared to that of standard drug greseofulvin.

\section{Antimalarial activity}

The synthesized compounds $\mathbf{4}$ and $\mathbf{5}$ were also screened for theirin vitro antimalarial activity against Plasmodium falciparumstrain using chloroquine and quinine as reference drugs. The mean $\mathrm{IC}_{50}(\mu \mathrm{g} / \mathrm{mL})$ values of the test compounds against the test microbe are presented in Table 2.The results revealed that the majority of the synthesized compounds showed significant degrees of inhibition against $P$. falciparum as compared with positive control quinine than that of chloroquine. Carbazole benzothiazepine derivatives 4(a-f), 4a and 4b showed moderate growth inhibition activities with $\mathrm{IC}_{50}$ values of 0.75 and $0.80 \mu \mathrm{g} / \mathrm{mL}$ as compared with standard drug quinine, while compounds $\mathbf{4 c}, \mathbf{4 d}, \mathbf{4 e}$ and $\mathbf{4 f}$ showed the lowest inhibition activities against $P$. falciparum comparable to that of reference compounds. The carbazole-pyrazoline derivatives 5(a-e), compound 5a exhibiteda good antimalarial spectrum with $\mathrm{IC}_{50}$ value of $0.56 \mu \mathrm{g} / \mathrm{mL}$ as compared with standard drug quinine, the remaining four compounds $\mathbf{5 b}, \mathbf{5 c}, \mathbf{5 d}$ and 5eshowed considerable inhibition activities withIC $_{50}$ values in the range of $0.76-1.25 \mu \mathrm{g} / \mathrm{mL}$.

\section{CONCLUSION}

As structure-activity relationships (SAR) of all compounds were taken into account, it was observed that compounds $4 \mathbf{a}, \mathbf{4 b}, \mathbf{4 d}, \mathbf{5 a}, \mathbf{5 b}$ and 5chaving electron withdrawing groups like chloro and bromo substituents on the phenyl ring showed excellent potential of antibacterial and antifungal activities. The antimalarial evaluation of $\mathbf{4 ( a - f )}$ and 5(a-e)revealed that, as the electronegativity nature of the substituents attached to an aromatic ring decreased, activity also decreased. Two derivatives4cand $\mathbf{5 d}$ containing electron releasing methyl and electron withdrawing chlorine groupattached to phenyl ring were able to display moderate growth inhibitory activity against all tested microorganisms. In addition, carbazole derivatives4eand 5econtaining methyl and methoxy group on the phenyl ring also inhibited thegrowth of the tested bacterial and fungal strains. Furthermore, compound4f without substituent in the phenyl ring showed the lowest activities against all tested bacterial, fungal and antimalarial strains. In general, all synthesized compounds $\mathbf{4}$ and $\mathbf{5}$ exhibited only moderate antimalarial activity $\mathrm{IC}_{50}$ values ranging $0.56-1.25 \mu \mathrm{g} / \mathrm{mL}$.High potency and promising antimicrobial and antimalarial activity of the newly synthesized compounds $\mathbf{4}(\mathbf{a}-\mathbf{f})$ and $\mathbf{5 ( a -}$ e)suggest that these compounds could serve as good leads for further optimization and development.

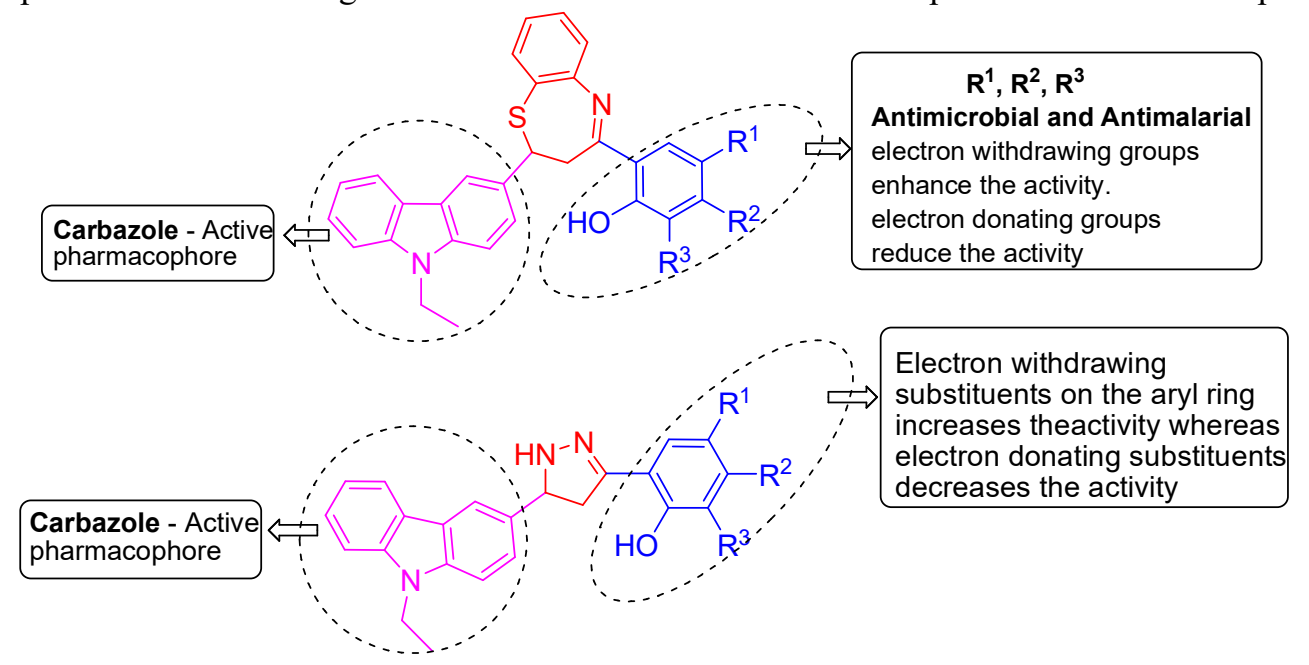

Figure 1. The structure- activity relationship in the target compounds 
V. A. Kadnor, S. N. Shelke: Synthesis, antimicrobial and antimalarial activity of 1, 4-benzothiazepine and ... Table 1.Antimicrobial activities of the synthesized compounds $\mathbf{4}$ and $\mathbf{5}$ against pathological organisms expressed

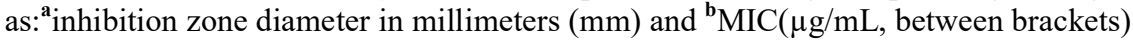

\begin{tabular}{|c|c|c|c|c|c|c|c|}
\hline \multirow{3}{*}{ Compounds } & \multicolumn{7}{|c|}{ Microorganisms } \\
\hline & \multicolumn{2}{|c|}{ Gram negative bacteria } & \multicolumn{2}{|c|}{ Gram positive bacteria } & \multicolumn{3}{|c|}{ Fungi } \\
\hline & E. coli & P. putida & B. subtilis & S. lactis & A. niger & $\begin{array}{l}\text { Penicillium } \\
\text { sp. }\end{array}$ & C. albicans \\
\hline $4 \mathrm{a}$ & $16(50)$ & $18(50)$ & $15(100)$ & $20(110)$ & $17(80)$ & $14(45)$ & $12(100)$ \\
\hline $4 b$ & $16(65)$ & $17(40)$ & $16(80)$ & $16(80)$ & $18(100)$ & $14(40)$ & $12(130)$ \\
\hline $4 c$ & $17(80)$ & $16(65)$ & $15(80)$ & 19(100) & $17(65)$ & $13(100)$ & $11(100)$ \\
\hline $4 d$ & $17(50)$ & $18(45)$ & $16(100)$ & $15(110)$ & $17(100)$ & $14(40)$ & $12(80)$ \\
\hline $4 \mathrm{e}$ & $15(80)$ & $14(80)$ & $12(65)$ & $17(80)$ & $14(80)$ & $11(80)$ & $11(100)$ \\
\hline $4 \mathrm{f}$ & $14(100)$ & $13(80)$ & $13(100)$ & $13(100)$ & $12(65)$ & $12(80)$ & $10(80)$ \\
\hline $5 a$ & $15(80)$ & $16(45)$ & $17(50)$ & $18(45)$ & $17(80)$ & $12(55)$ & $12(60)$ \\
\hline $5 b$ & $14(80)$ & $16(50)$ & $17(50)$ & $16(50)$ & $16(100)$ & $12(70)$ & $12(80)$ \\
\hline $5 c$ & 14(100) & $15(65)$ & $16(45)$ & $14(65)$ & $16(100)$ & 11(90) & 11(90) \\
\hline $5 d$ & $15(90)$ & $14(80)$ & $16(70)$ & $18(80)$ & 13(90) & $11(100)$ & $11(80)$ \\
\hline $5 \mathrm{e}$ & 11(110) & $12(100)$ & 11(100) & $15(80)$ & $12(100)$ & $09(100)$ & $11(80)$ \\
\hline Ampicillin & $24(25)$ & $20(25)$ & $19(25)$ & $22(25)$ & .. & ... & $\ldots$ \\
\hline Greseofulvin & $\ldots$ & ... & $\ldots$ & ... & $24(25)$ & $14(25)$ & $14(25)$ \\
\hline $\begin{array}{c}\text { Control } \\
(1 \% \text { DMSO })\end{array}$ & NA & NA & NA & NA & NA & NA & NA \\
\hline
\end{tabular}

Table 2.Substitution pattern andin vitro antimalarial activity of the target compounds $\mathbf{4}$ and $\mathbf{5}$

\begin{tabular}{ccccc}
\hline & & & $\mathrm{R}^{3}$ & P. falciparum \\
\cline { 5 - 5 } Compounds & $\mathrm{R}^{1}$ & $\mathrm{R}^{2}$ & $\mathrm{Cl}$ & 0.75 \\
$4 \mathrm{a}$ & $\mathrm{Cl}$ & $\mathrm{H}$ & $\mathrm{H}$ & 0.80 \\
$4 \mathrm{~b}$ & $\mathrm{Cl}$ & $\mathrm{H}$ & $\mathrm{H}$ & 0.85 \\
$4 \mathrm{c}$ & $\mathrm{Cl}$ & $\mathrm{CH}_{3}$ & $\mathrm{H}$ & 0.90 \\
$4 \mathrm{e}$ & $\mathrm{Br}$ & $\mathrm{H}$ & $\mathrm{H}$ & 1.10 \\
$4 \mathrm{f}$ & $\mathrm{CH}_{3}$ & $\mathrm{H}$ & $\mathrm{H}$ & 1.30 \\
$5 \mathrm{a}$ & $\mathrm{H}$ & $\mathrm{H}$ & $\mathrm{H}$ & 0.56 \\
$5 \mathrm{~b}$ & $\mathrm{Cl}$ & $\mathrm{Cl}$ & $\mathrm{H}$ & 0.76 \\
$5 \mathrm{c}$ & $\mathrm{Cl}$ & $\mathrm{H}$ & $\mathrm{H}$ & 0.88 \\
$5 \mathrm{~d}$ & $\mathrm{Br}$ & $\mathrm{H}$ & $\mathrm{H}$ & 1.20 \\
$5 \mathrm{e}$ & $\mathrm{Cl}$ & $\mathrm{CH}$ & $\mathrm{H}$ & 1.25 \\
Quinine & $\mathrm{H}$ & $\mathrm{OCH}_{3}$ & --- & 0.268 \\
Chloroquine & --- & --- & -- & 0.020 \\
\hline
\end{tabular}




\section{A. Kadnor, S. N. Shelke: Synthesis, antimicrobial EXPERIMENTAL}

The recorded melting points were determined in an open capillary and are uncorrected. IR spectra were recorded on a PerkinElmer Fourier-transform infrared (FTIR) spectrophotometer with ATR. The ${ }^{1} \mathrm{H}$ NMRand ${ }^{13} \mathrm{C}$ NMR spectrawere recorded on a Bruker Avance II (400 MHz) and Bruker (125 $\mathrm{MHz}$ ) spectrometer respectively, using TMS as internal standard.Mass spectra were recorded on a Waters, Q-Tof micromass spectrometer andhighresolution mass spectra (ESI) were recorded on a Thermo scientific Q-Exactive, Accela 1250 pump. The thin layer chromatography (TLC) was carried out on precoated silica gel aluminum plates to check compounds purity. Starting compound 9ethyl-9H-carbazole is of Sigma Aldrich make.

In vitro antimicrobial assay

The antimicrobial activity was evaluated by the agar well diffusion method [31]. The activity was determined by measuring the diameter of inhibition zone (in $\mathrm{mm}$ ). The samples of the tested compound concentrations ( $50 \mu \mathrm{L}, 1 \mathrm{mg} / \mathrm{mL})$ were loaded into wells on the plates. All solutions were prepared in DMSO, and pure DMSO was loaded as a control. The plates were incubated at $37^{\circ} \mathrm{C}$ for $1-5$ days and then were examined for the formation of inhibition zone. Each inhibition zone was measured three times to get an average value. The test was performed three times for each bacterium culture [32].

\section{Minimal inhibitory concentration (MIC) measurement}

The potato dextrose broths and microorganism susceptibility tests in nutrient media were used for the determination of MIC. Tested compounds stock $1000 \mu \mathrm{g} / \mathrm{mL}$ solutions, ampicillin and greseofulvin were prepared in DMSO followed by dilutions to 250-25 $\mu \mathrm{g} / \mathrm{mL}$ concentrations. Inoculated microorganism suspensions were incubated at $37^{\circ} \mathrm{C}$ for 1-5 days for MIC determination.

\section{Antimalarial activity}

A stock solution of $5 \mathrm{mg} / \mathrm{mL}$ of each of the test samples, as well as standards was prepared in DMSO and subsequent dilutions were prepared with the culture medium. The diluted samples in 20 $\mu \mathrm{L}$ volumes were added to the test wells so as to obtain final concentrations (at five-fold dilutions) ranging between 0.4 and $100 \mu \mathrm{g} / \mathrm{mL}$ in duplicate well containing parasitized cell preparation. The in vitro antimalarial assay was carried out in 96 well plates according to the micro assay protocol with minor modifications [33]. The cultures of $P$. falciparumstrain were maintained in a medium of
RPMI 1640 supplemented with 25 mM HEPES, $1 \%$ D-glucose, $0.23 \%$ sodium bicarbonate and $10 \%$ heat-inactivated human serum. The asynchronous parasites of $P$. falciparum were synchronized after $5 \%$ D-sorbitol treatment to obtain only the ring stage parasitized cells. For carrying out the assay, an initial ring stage parasitaemia of $0.8-1.5 \%$ at $3 \%$ haematocrit in a total volume of $200 \mu \mathrm{L}$ of medium RPMI-1640 was determined by Jaswant Singh Bhattacharya (JSB) staining[34]to assess the percent parasitaemia (rings) and uniformlymaintained with $50 \%$ RBCs $\left(\mathrm{O}^{+v e}\right)$. The culture plates were incubated at $37^{\circ} \mathrm{C}$ in a candle jar. After 36-40 h of incubation, thin blood smears from each well were prepared stained with JSB stain. The slides were microscopically observed to record maturation of the ring stage parasites into trophozoites and schizonts in the presence of different concentrations of the test agents. The test concentrations which inhibited the complete maturation in to schizonts were recorded as the minimum inhibitory concentrations (MIC). Chloroquine and quinine were used as the reference drugs.

\section{General procedure for the synthesis of 3-formyl-9- ethylcarbazole(2)}

9-ethyl carbazole 1 (1.95 g, $10 \mathrm{mmol})$ was dissolved in dry DMF $(20 \mathrm{~mL})$ under anhydrous conditions. It was cooled to $0^{\circ} \mathrm{C}$, and $\mathrm{POCl}_{3}(1.89$ $\mathrm{mL}$ ) was added dropwise and stirring continued for $4 \mathrm{~h}$ at $80^{\circ} \mathrm{C}$. Completion of reaction was monitored by TLC.The reaction mass was poured over crushed ice, neutralized with $\mathrm{NaHCO}_{3}$, the white colored precipitate was filtered off and purified through recrystallization using ethyl alcohol to afford compound 2 .

\section{General procedure for the synthesis of benzothiazepine derivatives 4(a-f)}

Chalcone 3 (2 mmol) was dissolved in a minimum quantity of ethanol. To this, 2aminothiophenol $(2 \mathrm{mmol})$ was added and the resulting reaction mixture was refluxed at $60-70{ }^{\circ} \mathrm{C}$ for $3 \mathrm{~h}$. Then, the mixture was acidified with 5-6 drops of glacial acetic acid and heating was continued for further 4-5 h. After completion of the reaction (monitored by TLC), the reaction mixture was cooled and poured over crushed ice. The obtained solid was filtered and purified by recrystallization from methanol to afford compounds4(a-f).

2,4-Dichloro-6-((E)-2-(9-ethyl-9H-carbazol-3yl)-2,3-dihydrobenzo[b] [1,4] thiazepin-4yl)phenol

(4a): Light yellow colored solid; Yield $(69 \%) ; \mathrm{R}_{\mathrm{f}}=$ 0.54 (6\% ethylacetate in $n$-hexane);m. p. 222- 
V. A. Kadnor, S. N. Shelke: Synthesis, antimicrobial and antimalarial activity of 1, 4-benzothiazepine and ... $223^{\circ} \mathrm{C}$; IR $\tilde{v}_{\max } / \mathrm{cm}^{-1}: 3559(\mathrm{OH}), 2976(\mathrm{CH}), 1593$ $(\mathrm{C}=\mathrm{N}) ;{ }^{1} \mathrm{H}$ NMR $\left(\mathrm{CDCl}_{3}\right) \delta / \mathrm{ppm}: 1.42(\mathrm{t}, 3 \mathrm{H}$, $\left.J=6.4 \mathrm{~Hz}, \mathrm{CH}_{3}\right), 3.25$ (t, $1 \mathrm{H}, J=12.5 \mathrm{~Hz}$, thiazepine ring), $3.41(\mathrm{dd}, 1 \mathrm{H}, J=9.5 \& 3.8 \mathrm{~Hz}$, thiazepine ring), $4.40\left(\mathrm{q}, 2 \mathrm{H}, J=6.7 \mathrm{~Hz}, \mathrm{~N}-\mathrm{CH}_{2}\right), 5.36(\mathrm{dd}, 1 \mathrm{H}$, $J=9.2 \& 3.8 \mathrm{~Hz}$, thiazepine ring), 7.22-7.30 (m, $2 \mathrm{H}$, Ar-H), 7.35-7.42 (m, 3H, Ar-H), 7.48-7.55 (m, 4H, Ar-H), 7.70- 8.05 (m, 4H, Ar-H), 15.92 (s, 1H, Ar$\mathrm{OH}),{ }^{13} \mathrm{C}$ NMR $\left(\mathrm{CDCl}_{3}\right) \delta / \mathrm{ppm}: 13.84,37.68$, $60.99,108.68,108.86,117.85,119.08,119.54$, $120.49,122.40,122.63,122.97,123.84,124.00$, $125.34,125.74,126.08,126.62,127.22,130.09$, $133.12,133.56,135.44,139.71,140.42,147.48$, 157.89, 172.25; MS $(m / z): 517(\mathrm{M}+\mathrm{H})^{+}$.

4-Chloro-2-((E)-2-(9-ethyl-9H-carbazol-3-yl)2,3-dihydrobenzo[b] [1,4]thiazepin-4-yl)phenol

(4b): Light yellow colored solid; Yield (70\%); $\mathrm{R}_{\mathrm{f}}=$ 0.52 (6\% ethylacetate in n-hexane);m. p. 226$228^{\circ} \mathrm{C}$; IR $\tilde{v}_{\max } / \mathrm{cm}^{-1}$ : $3376(\mathrm{OH}), 3055(\mathrm{CH})$, 1611 $(\mathrm{C}=\mathrm{N}) ;{ }^{1} \mathrm{H}$ NMR $\left(\mathrm{CDCl}_{3}\right) \delta / \mathrm{ppm}: 1.43(\mathrm{t}, 3 \mathrm{H}$, $\left.J=7.0 \mathrm{~Hz}, \mathrm{CH}_{3}\right), 3.24(\mathrm{t}, 1 \mathrm{H}, J=12.6 \mathrm{~Hz}$, thiazepine ring), $3.42(\mathrm{dd}, 1 \mathrm{H}, J=8.8 \& 4.5 \mathrm{~Hz}$, thiazepine ring), 4.37 (q, $\left.2 \mathrm{H}, J=7.0 \mathrm{~Hz}, \mathrm{~N}-\mathrm{CH}_{2}\right), 5.32(\mathrm{dd}, 1 \mathrm{H}$, $J=8.2 \& 4.4 \mathrm{~Hz}$, thiazepine ring), 7.03 (m, 1H, ArH), 7.22-7.28 (m, 3H, Ar-H), 7.32-7.37 (m, 3H, ArH), 7.40-7.46 (m, 2H, Ar-H), 7.51-7.53 (m, 2H, Ar$\mathrm{H})$, 7.69-8.06 (m, 3H, Ar-H), 14.63 (s, 1H, Ar$\mathrm{OH}) ;{ }^{13} \mathrm{C} \mathrm{NMR}\left(\mathrm{CDCl}_{3}\right) \delta / \mathrm{ppm}: 14.19,37.75$, $60.80,108.16,108.60,108.83,109.25,110.47$, $119.80,120.02,120.76,121.23,122.10,123.08$, $123.31,124.94,126.04,126.82,127.48,133.30$, $137.03,138.44,140.98,141.49,142.39,144.25$, $145.45, \quad 168.95, \quad 169.25, \quad 175.02 ; \mathrm{MS}$ $(\mathrm{m} / \mathrm{z}): 483(\mathrm{M}+\mathrm{H})^{+}$.

4-Chloro-2-((E)-2-(9-ethyl-9H-carbazol-3-yl)2,3-dihydrobenzo[b] [1,4] thiazepin-4-yl)-5-

methylphenol (4c): Light yellow colored solid; Yield $(68 \%) ; \mathrm{R}_{\mathrm{f}}=0.58(6 \%$ ethylacetate in $\mathrm{n}$ hexane);m. p. $196-197^{\circ} \mathrm{C}$; IR $\tilde{v}_{\max } / \mathrm{cm}^{-1}: 3550$ (OH), $2935(\mathrm{CH}), 1688(\mathrm{C}=\mathrm{N}){ }^{1} \mathrm{H}$ NMR $\left(\mathrm{CDCl}_{3}\right) \delta$ / ppm: $1.48\left(\mathrm{t}, 3 \mathrm{H}, J=7.0 \mathrm{~Hz}, \mathrm{CH}_{3}\right), 2.35$ (s, 3H, Ar$\left.\mathrm{CH}_{3}\right), 3.20$ (t, $1 \mathrm{H}, J=12.6 \mathrm{~Hz}$, thiazepine ring), 3.44 (dd, $1 \mathrm{H}, J=8.8 \& 4.5 \mathrm{~Hz}$, thiazepine ring), 4.39 (q, $\left.2 \mathrm{H}, J=7.0 \mathrm{~Hz}, \mathrm{~N}-\mathrm{CH}_{2}\right), 5.30(\mathrm{dd}, 1 \mathrm{H}, J=7.8 \& 4.4$ $\mathrm{Hz}$, thiazepine ring), 7.05 (m, 1H, Ar-H), 7.20-7.29 (m, 2H, Ar-H), 7.33-7.42 (m, 3H, Ar-H), 7.40-7.48 (m, 2H, Ar-H), 7.55-7.65 (m, 2H, Ar-H), 7.73-7.85 (m, 1H, Ar-H), 8.05-8,10 (m, 2H, Ar-H), 14.60 (s, $1 \mathrm{H}, \mathrm{Ar}-\mathrm{OH}) ;{ }^{13} \mathrm{C} \mathrm{NMR}\left(\mathrm{CDCl}_{3}\right) \delta / \mathrm{ppm}: 13.74$, $37.50,60.85,108.66,108.83,117.77,119.25$, $119.58,120.39,122.55,122.65,122.90,123.80$, $124.10,125.25,125.72,126.23,126.68,127.49$, $130.08,133.17,133.47,135.49,139.75,140.48$, 147.40, 157.85, 170.85; MS $(\mathrm{m} / \mathrm{z}): 497(\mathrm{M}+\mathrm{H})^{+}$.

4-Bromo-2-((E)-2-(9-ethyl-9H-carbazol-3-yl)2,3-dihydrobenzo[b] [1,4] thiazepin-4-yl)phenol

(4d): Light yellow colored solid; Yield $(71 \%) ; \mathrm{R}_{\mathrm{f}}=$ $0.50(6 \%$ ethylacetate in n-hexane);m. p. 188$189^{\circ} \mathrm{C}$; IR $\tilde{v}_{\max } / \mathrm{cm}^{-1}: 3545(\mathrm{OH}), 2935(\mathrm{CH}), 1688$ $(\mathrm{C}=\mathrm{N}) ;{ }^{1} \mathrm{H}$ NMR $\left(\mathrm{CDCl}_{3}\right) \delta / \mathrm{ppm}: 1.45(\mathrm{t}, 3 \mathrm{H}$, $\left.J=7.3 \mathrm{~Hz}, \mathrm{CH}_{3}\right), 3.28(\mathrm{t}, 1 \mathrm{H}, J=12.3 \mathrm{~Hz}$, thiazepine ring), $3.45(\mathrm{dd}, 1 \mathrm{H}, J=8.9 \& 4.2 \mathrm{~Hz}$, thiazepine ring), 4.32 (q, 2H, J=7.1 Hz, N-CH $), 5.30(\mathrm{dd}, 1 \mathrm{H}$, $J=8.7 \& 4.3 \mathrm{~Hz}$, thiazepine ring), 7.05 (m, 1H, ArH), 7.20-7.29 (m, 3H, Ar-H), 7.34-7.38 (m, 3H, Ar$\mathrm{H})$, 7.43-7.49 (m, 2H, Ar-H), 7.55-7.63 (m, 2H, Ar$\mathrm{H})$, 7.70-8.09 (m, 3H, Ar-H), 14.33 (s, 1H, Ar$\mathrm{OH}) ;{ }^{13} \mathrm{C}$ NMR $\left(\mathrm{CDCl}_{3}\right) \delta / \mathrm{ppm}: 13.79,37.59$, 60.89 , 108.62, 108.81, 117.88, 119.07, 119.52, $120.44,122.47,122.68,122.92,123.89,124.09$, $125.37,125.72,126.06,126.63,127.21,130.08$, $133.28,133.59,135.49,139.72,140.48,147.41$, $157.75,172.85 ; \mathrm{MS}(\mathrm{m} / z): 527(\mathrm{M}+\mathrm{H})^{+}$.

2-((E)-2-(9-ethyl-9H-carbazol-3-yl)-2,3-

dihydrobenzo[b][1,4]thiazepin-4-yl)-4-

methylphenol (4e):Light yellow colored solid; Yield $(69 \%) ; \mathrm{R}_{\mathrm{f}}=0.56(6 \%$ ethylacetate in $\mathrm{n}$-hexane);m. p. $215-216^{\circ} \mathrm{C}$; IR $\tilde{v}_{\max } / \mathrm{cm}^{-1}: 3363(\mathrm{OH}), 2973$ (CH), $1594(\mathrm{C}=\mathrm{N}) ;{ }^{1} \mathrm{H}$ NMR $\left(\mathrm{CDCl}_{3}\right) \delta / \mathrm{ppm}: 1.44$ $\left(\mathrm{t}, 3 \mathrm{H}, J=7.0 \mathrm{~Hz}, \mathrm{CH}_{3}\right), 1.55\left(\mathrm{~s}, 3 \mathrm{H}, \mathrm{Ar}-\mathrm{CH}_{3}\right), 3.23$ (t, $1 \mathrm{H}, J=12.6 \mathrm{~Hz}$, thiazepine ring), $3.42(\mathrm{dd}, 1 \mathrm{H}$, $J=8.8 \& 4.5 \mathrm{~Hz}$, thiazepine ring), 4.37 (q, $2 \mathrm{H}, J=7.0$ $\left.\mathrm{Hz}, \mathrm{N}-\mathrm{CH}_{2}\right), 5.33$ (dd, $1 \mathrm{H}, J=7.8$ \& $4.4 \mathrm{~Hz}$, thiazepine ring), $7.01(\mathrm{~m}, 1 \mathrm{H}, \mathrm{Ar}-\mathrm{H}), 7.25-7.27(\mathrm{~m}$, 3H, Ar-H), 7.32-7.40 (m, 3H, Ar-H), 7.42-7.44 (m, 2H, Ar-H), 7.45-7.51 (m, 2H, Ar-H), 7.53-7.70 (m, 1H, Ar-H), 8.02-8.06 (m, 2H, Ar-H), 14.59 (s, 1H, Ar-OH $) ;{ }^{13} \mathrm{C}$ NMR $\left(\mathrm{CDCl}_{3}\right) \delta / \mathrm{ppm:} \mathrm{13.78,} \mathrm{37.58,}$ $60.87,108.65,108.80,117.87,119.04,119.58$, $120.49,122.44,122.67,122.96,123.88,124.06$, $125.39,125.70,126.03,126.69,127.29,130.09$, $133.27,133.57,135.48,139.70,140.44,147.43$, 157.72, 172.80; MS $(\mathrm{m} / \mathrm{z}): 463(\mathrm{M}+\mathrm{H})^{+}$.

2-((E)-2-(9-ethyl-9H-carbazol-3-yl)-2,3dihydrobenzo $[b][1,4]$ thiazepin-4-yl)phenol $\quad(4 f)$ : Light yellow colored solid; Yield $(68 \%) ; \mathrm{R}_{\mathrm{f}}=0.55$ ( $6 \%$ ethylacetate in n-hexane);m. p. $226-227^{\circ} \mathrm{C}$; IR $\tilde{v}_{\max } / \mathrm{cm}^{-1}$ : $3555(\mathrm{OH}), 2935(\mathrm{CH}), 1688(\mathrm{C}=\mathrm{N}){ }^{1}{ }^{1} \mathrm{H}$ NMR $\left(\mathrm{CDCl}_{3}\right) \delta / \mathrm{ppm}: 1.28(\mathrm{t}, 3 \mathrm{H}, J=7.2 \mathrm{~Hz}$, $\left.\mathrm{CH}_{3}\right), 3.12(\mathrm{t}, 1 \mathrm{H}, \mathrm{J}=12.8 \mathrm{~Hz}$, thiazepine ring), 3.65 (dd, $1 \mathrm{H}, J=9.4 \& 3.7 \mathrm{~Hz}$, thiazepine ring), 4.41 (q, $\left.2 \mathrm{H}, J=6.8 \mathrm{~Hz}, \mathrm{~N}-\mathrm{CH}_{2}\right), 5.45(\mathrm{dd}, 1 \mathrm{H}, J=9.4 \& 3.9$ $\mathrm{Hz}$, thiazepine ring), 6.95-6.99 (m, 2H, Ar-H), 7.18 (m, 1H, Ar-H), 7.29 (m, 1H, Ar-H), 7.38-7.45 (m, 4H, Ar-H), 7.53-7.64 (m, 4H, Ar-H), 7.91 (m, 3H, Ar-H), 14.32 (s, 1H, Ar-OH); ${ }^{13} \mathrm{C} \mathrm{NMR}\left(\mathrm{CDCl}_{3}\right) \delta /$ ppm: 13.74, 37.48, 60.92, 108.58, 108.83, 117.81, $119.06,119.53,120.45,122.42,122.67,122.90$, $123.80,124.08,125.35,125.74,126.07,126.65$, $127.20,130.07,133.10,133.57,135.46,139.73$, $140.47, \quad 147.42, \quad 157.80, \quad 172.23 ; \mathrm{MS}$ $(\mathrm{m} / z): 449(\mathrm{M}+\mathrm{H})^{+}$. 
V. A. Kadnor, S. N. Shelke: Synthesis, antimicrobial and antimalarial activity of 1, 4-benzothiazepine and ...

\section{General procedure for the synthesis of pyrazoline derivatives $5(a-e)$}

Chalcone3 (2 mmol)was dissolved in ethanol $(15 \mathrm{~mL})$ under stirring. To this reaction mixture, 0.5 $\mathrm{mL}$ of hydrazine hydrate and $0.2 \mathrm{~mL}$ of acetic acid was added. The reaction mixture was heated at reflux temperature for $6 \mathrm{~h}$. Completion of reaction was monitored by TLC. Thereaction mixture was cooled to room temperature. Then slowly $15 \mathrm{~mL}$ of cold water were added to the flask, the white solid obtained was washed with cold water several times.The crude compounds were recrystallizedfromethanol to afford the target compounds 5(a-e).

4,5-Dichloro-2-(5-(9-ethyl-9H-carbazol- 239 3-yl)-4,5-dihydro-1H-pyrazol-3-yl)phenol

$(5 a)$ : White colored solid; Yield $(65 \%) ; \mathrm{R}_{\mathrm{f}}=0.48$ ( $6 \%$ ethylacetate in n-hexane);m. p. $155-156^{\circ} \mathrm{C}$; IR $\tilde{\mathrm{v}}_{\max } / \mathrm{cm}^{-1}: 3668(\mathrm{OH}), 3205(\mathrm{NH}), 3051(\mathrm{CH})$, $1614(\mathrm{C}=\mathrm{N}) ;{ }^{1} \mathrm{H} \mathrm{NMR}\left(\mathrm{CDCl}_{3}\right) \delta / \mathrm{ppm}: 1.36(\mathrm{t}$, $\left.3 \mathrm{H}, J=7.5 \mathrm{~Hz}, \mathrm{CH}_{3}\right), 3.16(\mathrm{dd}, 1 \mathrm{H}, J=10.7 \mathrm{~Hz} \& 5.9$ $\mathrm{Hz}$, pyrazoline ring), $3.65(\mathrm{dd}, 1 \mathrm{H}, J=10.7 \& 5.9 \mathrm{~Hz}$, pyrazoline ring), 4.43 (q, 2H, $J=7.5 \mathrm{~Hz}, \mathrm{~N}-\mathrm{CH}_{2}$ ), $5.12(\mathrm{t}, 1 \mathrm{H}, J=10.7 \mathrm{~Hz}$, pyrazoline ring), 7.15-7.35 (m, 3H, Ar-H), 7.40-7.55 (m, 4H, Ar-H), 8.01 (m, 2H, Ar-H), 8.13 (m, 1H, NH), 12.02 (s, 1H, Ar$\mathrm{OH}) ;{ }^{13} \mathrm{C}$ NMR $\left(\mathrm{CDCl}_{3}\right) \delta / \mathrm{ppm}: 13.82,37.67$, $41.70,63.62,108.67,109.03,118.25,118.65$, $119.10,120.53,122.05,122.54,123.22,123.58$, $123.77,125.48, \quad 126.08, \quad 129.85,131.72, \quad 139.76$, 140.76, 152.26; HRMS (ESI): calculated for $\mathrm{C}_{23} \mathrm{H}_{20} \mathrm{ON}_{3} \mathrm{Cl}_{2}(\mathrm{M}+\mathrm{H})^{+} 424.0981$, found 424.0978 .

4-Chloro-2-(5-(9-ethyl-9H-carbazol-3-yl)-4,5dihydro-1H-pyrazol-3-yl)phenol (5b):White colored solid; Yield $(69 \%) ; \mathrm{R}_{\mathrm{f}}=0.52 \quad(6 \%$ ethylacetate in n-hexane);m. p. $141-142^{\circ} \mathrm{C}$; IR $\tilde{v}_{\max } /$ $\mathrm{cm}^{-1}$ : $3363(\mathrm{OH}), 3055(\mathrm{NH}), 2950(\mathrm{CH}), 1593$ $(\mathrm{C}=\mathrm{N}) ;{ }^{1} \mathrm{H} \mathrm{NMR}\left(\mathrm{CDCl}_{3}\right) \delta / \mathrm{ppm}: 1.35(\mathrm{t}, 3 \mathrm{H}$, $\left.J=8.5 \mathrm{~Hz}, \mathrm{CH}_{3}\right), 3.18$ (dd, $1 \mathrm{H}, J=12.5 \mathrm{~Hz} \& 6.5 \mathrm{~Hz}$, pyrazoline ring), $3.69(\mathrm{dd}, 1 \mathrm{H}, J=12.5 \& 6.5 \mathrm{~Hz}$, pyrazoline ring), 4.45 (q, $2 \mathrm{H}, J=8.5 \mathrm{~Hz}, \mathrm{~N}-\mathrm{CH}_{2}$ ), $5.12(\mathrm{t}, 1 \mathrm{H}, J=12.5 \mathrm{~Hz}$, pyrazoline ring), 7.16-7.29 (m, 2H, Ar-H), 7.41-7.48 (m, 3H, Ar-H), 7.50-7.55 (m, 2H, Ar-H), 8.10-8.23 (m, 4H, Ar-H, NH), 9.70 (s, $1 \mathrm{H}, \mathrm{Ar}-\mathrm{OH}) ;{ }^{13} \mathrm{C} \mathrm{NMR}\left(\mathrm{CDCl}_{3}\right) \delta / \mathrm{ppm}: 13.73$, 37.98, 41.93, 62.96, 99.07, 108.61, 109.06, 111.14, $117.71,118.38,119.54,120.78,122.63,123.74$, $126.06,126.57,127.26,128.89,131.43,148.96$, 154.79, 155.45, HRMS (ESI): calculated for $\mathrm{C}_{23} \mathrm{H}_{21} \mathrm{ON}_{3} \mathrm{Cl}(\mathrm{M}+\mathrm{H})^{+} 390.11856$, found 390.11876 .

4-Bromo-2-(5-(9-ethyl-9H-carbazol-3-yl)-4,5-

dihydro-1H-pyrazol-3-yl)phenol (5c): White colored solid; Yield (71\%); $\mathrm{R}_{\mathrm{f}}=0.60(6 \%$ ethylacetate in $\mathrm{n}$ hexane);m. p. $183-184^{\circ} \mathrm{C}$; IR $\tilde{v}_{\max } / \mathrm{cm}^{-1}: 3655$ $(\mathrm{OH}), 3225(\mathrm{NH}), 3065(\mathrm{CH}), 1635(\mathrm{C}=\mathrm{N}) ;{ }^{1} \mathrm{H}$ 240 NMR $\left(\mathrm{CDCl}_{3}\right) \delta / \mathrm{ppm}: 1.40(\mathrm{t}, 3 \mathrm{H}, J=7.5 \mathrm{~Hz}$, $\left.\mathrm{CH}_{3}\right), 3.12$ (dd, 1H, J=10.7 Hz \& $5.9 \mathrm{~Hz}$, pyrazoline ring), 3.61 (dd, $1 \mathrm{H}, J=10.7 \& 5.9 \mathrm{~Hz}$, pyrazoline ring), 4.44 (q, $2 \mathrm{H}, J=7.5 \mathrm{~Hz}, \mathrm{~N}-\mathrm{CH}_{2}$ ), $5.14(\mathrm{t}, 1 \mathrm{H}, J=10.7 \mathrm{~Hz}$, pyrazoline ring), 7.11-7.35 (m, 4H, Ar-H), 7.48-7.75 (m, 4H, Ar-H), 8.10(m, 2H, Ar-H), 8.18 (m, 1H, NH), 12.10 (s, 1H, Ar$\mathrm{OH}) ;{ }^{13} \mathrm{C}$ NMR $\left(\mathrm{CDCl}_{3}\right) \delta / \mathrm{ppm}: 14.82,37.55$, $41.68,63.65,108.77,109.23,118.25,118.89$, $119.63,120.68,122.45,122.83,123.26,123.78$, $123.89,125.69, \quad 126.28,129.79,131.80,139.76$, 140.68, 154.10; HRMS (ESI): calculated for $\mathrm{C}_{23} \mathrm{H}_{21} \mathrm{ON}_{3} \mathrm{Br}(\mathrm{M}+\mathrm{H})^{+} 434.0478$, found 434.0485 .

4-Chloro-2-(5-(9-ethyl-9H-carbazol-3-yl)-4,5dihydro-1H-pyrazol-3-yl)-5-methylphenol (5d): White colored solid; Yield (67\%); $\mathrm{R}_{\mathrm{f}}=0.46(6 \%$ ethylacetate in n-hexane);m. p. $138-139^{\circ} \mathrm{C}$; IR $\tilde{v}_{\max } /$ $\mathrm{cm}^{-1}$ : $3650(\mathrm{OH}), 3238(\mathrm{NH}), 3029(\mathrm{CH}), 1650$ $(\mathrm{C}=\mathrm{N}) ;{ }^{1} \mathrm{H} \mathrm{NMR}\left(\mathrm{CDCl}_{3}\right) \delta / \mathrm{ppm}: 1.39(\mathrm{t}, 3 \mathrm{H}$, $\left.J=7.5 \mathrm{~Hz}, \mathrm{CH}_{3}\right), 2.30$ (s, 3H, Ar- $\left.\mathrm{CH}_{3}\right), 3.16$ (dd, $1 \mathrm{H}, J=10.8 \mathrm{~Hz} \& 5.9 \mathrm{~Hz}$, pyrazoline ring), 3.67 (dd, $1 \mathrm{H}, J=10.8 \& 5.9 \mathrm{~Hz}$, pyrazoline ring), 4.45 (q, $2 \mathrm{H}$, $\left.J=7.5 \mathrm{~Hz}, \mathrm{~N}-\mathrm{CH}_{2}\right), 5.12(\mathrm{t}, 1 \mathrm{H}, J=10.8 \mathrm{~Hz}$, pyrazoline ring), 7.24-7.40 (m, 3H, Ar-H), 7.517.75 (m, 4H, Ar-H), 8.10 (m, 2H, Ar-H), 8.25 (m, $1 \mathrm{H}, \mathrm{NH}), 12.15$ (s, 1H, Ar-OH); ${ }^{13} \mathrm{C} \mathrm{NMR}\left(\mathrm{CDCl}_{3}\right)$ $\delta$ / ppm: 13.95, 37.55, 41.68, 63.69, 108.80, 109.23, $117.29,118.78,119.12,120.68,122.65,122.83$, $123.90,124.80,124.95,125.69,126.78,128.79$, 131.80, 139.72, 140.68, 155.25; HRMS (ESI): calculated for $\mathrm{C}_{24} \mathrm{H}_{23} \mathrm{ON}_{3} \mathrm{Cl} \quad(\mathrm{M}+\mathrm{H})^{+} 403.12514$, found 403.12516 .

2-(5-(9-Ethyl-9H-carbazol-3-yl)-4,5-dihydro1H-pyrazol-3-yl)-5-methoxyphenol (5e): White colored solid; Yield $(68 \%) ; \quad \mathrm{R}_{\mathrm{f}}=0.55(6 \%$ ethylacetate in n-hexane);m. p. $121-122^{\circ} \mathrm{C}$; IR $\tilde{\mathrm{v}}_{\max } /$ $\mathrm{cm}^{-1}$ : $3325(\mathrm{OH}), 3056(\mathrm{NH}), 2973(\mathrm{CH}), 1678$ $(\mathrm{C}=\mathrm{N}) ;{ }^{1} \mathrm{H}$ NMR $\left(\mathrm{CDCl}_{3}\right) \delta / \mathrm{ppm}: 1.36(\mathrm{t}, 3 \mathrm{H}$, $\left.J=8.8 \mathrm{~Hz}, \mathrm{CH}_{3}\right), 3.18(\mathrm{dd}, 1 \mathrm{H}, J=13.3 \mathrm{~Hz} \& 7.4 \mathrm{~Hz}$, pyrazoline ring), $3.33\left(\mathrm{~s}, 3 \mathrm{H}, \mathrm{Ar}-\mathrm{OCH}_{3}\right), 3.65$ (dd, $1 \mathrm{H}, J=13.3 \& 7.4 \mathrm{~Hz}$, pyrazoline ring), 4.43 (q, $2 \mathrm{H}$, $\left.J=8.8 \mathrm{~Hz}, \mathrm{~N}-\mathrm{CH}_{2}\right), 5.14 \quad(\mathrm{t}, 1 \mathrm{H}, J=13.3 \mathrm{~Hz}$, pyrazoline ring), 7.16-7.21 (m, 2H, Ar-H), 7.337.45 (m, 3H, Ar-H), 7.47-7.55 (m, 3H, Ar-H), 8.04$8.10(\mathrm{~m}, 2 \mathrm{H}, \mathrm{ArH}), 8.13$ (s, 1H, NH), 12.00 (s, 1H, Ar-OH $) ;{ }^{13} \mathrm{C}$ NMR $\left(\mathrm{CDCl}_{3}\right) \delta / \mathrm{ppm}: 13.96,37.98$, $44.77,63.41,68.08, \quad 107.94,110.03,117.48$, $120.24,121.22,122.10,123.30,123.76,125.39$, $125.61,126.57,127.00,128.47,128.90,129.35$, 140.53, 141.95, 149.41, 157.31; HRMS (ESI): calculated for $\mathrm{C}_{24} \mathrm{H}_{24} \mathrm{O}_{2} \mathrm{~N}_{3}(\mathrm{M}+\mathrm{H})^{+} 386$. 1904, found 386.1908 .

Acknowledgement: $V . A$. K. is grateful to UGC, WRO, Pune for providing a teacher fellowship under the Faculty development programme of the 
V. A. Kadnor, S. N. Shelke: Synthesis, antimicrobial and antimalarial activity of 1, 4-benzothiazepine and ...

$U G C$ 's $12^{\text {th }}$ plan. The authors are also grateful to the Principal of ACS College, Satral and SSGM College, Kopargaon for providing the necessary facilities and SAIF, University of Punjab, Chandigarh for providing the characterization.

View supporting data here

\section{REFERENCES}

1. K. Thevissen, A. Marchand, P. Chaltin, E. M. K. Meert, B. P. A. Cammue, Curr. Med. Chem., 16, 2205 (2009).

2. M. M. Rahman, A. I. Gray, Phytochemistry, 66, 1601 (2005).

3. I. J. Kang, L. W. Wang, S. J. Hsu, C. C. Lee, Y. C. Lee, Y. S. Wu, A. Yueh, J. C. Wang, T. A. Hsu, Y. S. Chao, J. H. Chern, Bioorg. Med. Chem. Lett., 19, 6063 (2009).

4. C. Yenjai, S. Sripontan, P. Sriprajun, P. Kittakoop, A. Jintasirikul, M. Tanticharoen, Y. Thebtaranonth, Planta Med., 66, 277 (2000).

5. H. Knolker, K. Reddy, Chem. Rev., 102, 4303 (2002).

6. A. Gluszynska, Eur. J. Med. Chem., 94, 405 (2015).

7. B. P. Bandgar, L. K. Adsul, S. V. Lonikar, H. V. Chavan, S. N. Shringare, S. A. Patil, S. S. Jalde, B. A. Koti, N. A. Dhole, R. N. Gacche, A. Shirfule, J. Enzyme Inhib. Med.Chem.,28(3), 593(2013).

8. A. R. Nixha, M. Arslan, Y. Atalay, N. Gencer, A. Ergün, O. Arslan, J. Enzyme Inhib. Med. Chem., 28(4), 808 (2013).

9. H. J. Bo Hrisc, H. Faltz, M. Patzel, J. Liebsc Her, Tetrahedron, 50, 1070 (1994).

10. G. De Sarro, A. Chimirri, A. De Sarro, R. Gitto, S. Grasso, M. Zappala, Eur. J. Med. Chem.,30, 925 (1995).

11. D. Kantoci, E. D. Murray, D. D. Quiggle, W. J. Wechter, J. Med. Chem., 39, 1196 (1996).

12. J. F. F. Liegeois, F. A. Rogister, J. Bruhwyler, J. Damas, T. P. Nguyen, M. O. Inarejos, E. M. G. Chleide, M. G. A. Mercier, J. E. Delarge, J. Med. Chem., 37, 519 (1994).

13. S. V. Karthikeyan, S. Perumal, Tetrahedron Lett., 1, 2261 (2007)

14. U. C. Pant, A. Dandia, H. Chandra, S. Goyal, S. Pant, Phosphorus, Sulfur, Silicon, Relat. Elem., 180, 559 (2005).

15. I. V. Patricio, M. Raquel, M. D. Ivorra, M. P. D’Ocon, B. K. Assels, J. Nat. Prod., 66, 954 (2003).
16. J. B. Bariwal, K. D. Upadhyay, A. T. Manvar, J. C. Trivedi,J. S. Singh, K. S. Jain, A. K. Shah, Eur. J. Med. Chem., 43, 2279 (2008).

17. K. Arya, A. Dandia, Med. Chem., 18, 114 (2008).

18. F. Shi, Z. Xiao-Ning, C. Xu-Dong, Z. Shu, J. Bo, Z. Wei-Fa, T. Shu-Jiang, Bioorg. Med. Chem. Lett., 22, 743 (2012).

19. A. Rahman, A. A. Siddiqui, Int. J. Pharm. Sci. Drug Res., 2, 165 (2010).

20. P. M. Sivakumar, S. Ganesan, P. Veluchamy, M. Doble., Chem. Biol. Drug Des., 76, 407 (2010).

21. P. M. Sivakumar, S. Prabhu Seenivasan, V. Kumar, M. Doble, Bioorg. Med. Chem. Lett., 20, 3169 (2010).

22. P. K. Sharma, S. Kumar, P. Kumar, Eur. J. Med. Chem., 45, 2650 (2010).

23. B. N. Acharya, D. Saraswat, A. K. Shrivastava, R. Ghorpade, S. Bapna, M. P. Kaushik, Eur. J. Med. Chem., 45,430 (2009).

24. A. K Pandey, S. Sharma, M. Pandey, M. M. Alam, M. Shaquiquzzaman, M. Akhter, Eur. J. Med.Chem.,123, 476 (2016).

25. E. Bansal, V. K. Srivastava, A. Kumar, Eur. J. Med. Chem.,36, 81 (2001).

26. T. S. Jeong, K. S. Kim, J. R. Kim, K. H. Cho, S. Lee, W. Lee, Bioorg. Med. Chem. Lett., 14, 2719 (2004).

27. B. P. Bandgar, L. K. Adsul, H. V. Chavan, S. S. Jalde, S. N. Shringare, R. Shaikh, R. J. Meshram, R. N. Gacche, V. Masand, Bioorg. Med. Chem. Lett., 22, 5839 (2012).

28. S. N. Shelke, G. R. Mhaske, S. Gadakh, C. Gill, Bioorg. Med. Chem. Lett., 24(20), 7200 (2010).

29. S. N. Shelke, G. R. Mhaske, D. B. Bonifacio Vasco, M. Gawande, Bioorg. Med. Chem. Lett., 17(22), 5727 (2012).

30. P. S. Kalsi, Spectroscopy of Organic Compounds, $6^{\text {th }}$ edn., New Age International Publishers, New Delhi, 2010, p. 282.

31. A. P. Keche, G. D. Hatnapure, R.T. Tale, A. H. Rodge, S. S. Birajdar, V. M. Kamble,Med. Chem. Res., 22, 14(2013).

32. M. A. Patel, V. G. Bhila, N. H. Patel, A. K. Patel, D. I. Brahmbhatt, Med. Chem. Res., 21, 4381 (2012).

33. K. H. Reickmann, G. H. Campbell, L. J. Sax, J. E. Mrema, Lancet, 1, 221(1978).

34. J. J. S. B. Singh, Indian J.Malariology, 10, 117(1956). 


\section{The effects of temperature and ultrasound on the migration of di-(2-ethylhexyl) phthalate from plastic packaging into dairy products

\author{
${ }^{1}$ University of Niš, Faculty of Sciences and Mathematics, Višegradska 33, 18000 Niš, Serbia \\ ${ }^{2}$ University of Niš, Faculty of Medicine, Bulevar dr Zorana Đinđića 81, 18000 Niš, Serbia
} \\ D. S. Bogdanović ${ }^{*}$, D. H. Anđelković ${ }^{1}$, I. S. Kostić ${ }^{1}$, G. M. Kocić ${ }^{2}$, T. D. Anđelković ${ }^{1}$}

Received, December 13, 2018; Revised, January 22, 2019

The aim of this paper is to define migration of phthalates from plastic packaging into dairy products of different fat content caused by increasing temperature and application of ultrasound on dairy product. The most common used phthalate, di-(2-ethylhexyl) phthalate (DEHP) was investigated for migration into following food-recipients with different fat content: infant formula, milk powder, whey powder and water. The results obtained by liquid-liquid extraction followed by gas chromatography-mass spectrometry (GC-MS) were compared. The base peak ion of DEHP $(\mathrm{m} / \mathrm{z}, 149)$ was selected for further research and recording was performed in the selected ion monitoring mode (SIM). For the considered range of phthalate concentrations, $0.25-2.50 \mu \mathrm{g} \mathrm{mL} \mathrm{m}^{-1}$, the response of the mass-selective detector (MSD) was linear. Results showed that the migration of DEHP from packaging material is higher in dairy products with higher percentage of fat. The mass of migrated DEHP was the highest in the sample of infant formula, followed by powdered milk, whey powder and water. Phthalates being just incorporated into plastics, and not chemically bound to plastics, show increased migration when ultrasound is applied in addition to increased temperature.

Keywords: phthalate; milk samples; leachability; gas chromatography-mass spectrometry.

\section{INTRODUCTION}

Phthalates are organic chemical compounds that make up the basic component of all plastic packaging to make it more flexible, more elastic and softer to use and for that reason they are also called plasticizers. The proportion of phthalate in a plastic product can be up to $45 \%$ of its total weight, depending on the type and purpose of the product itself [1-3]. The most commonly used phthalates are dimethyl phthalate (DMP), dibutyl phthalate (DBP), butyl benzyl phthalate (BBP), di(2-ethylhexyl) phthalate (DEHP), di- $n$-octyl phthalate (DOP), diisononyl phthalate (DINP), diisodecyl phthalate (DIDP). Although there is a large number of phthalates, only some of them are produced massively, at industrial level, such as the production of DEHP (50\% of the total annual production of phthalates belongs to the production of DEHP). The World Health Organization (WHO) estimated that the annual industrial production of phthalate approached the amount of 8 million tons [4]. Long-chain phthalates or high molecular weight ones, such as DEHP, are mainly found in flexible polyvinyl chloride materials (PVC) used in consumer products such as food packaging and medical equipment (peritoneal dialysis bags and tubing, intravenous bags, infusion tubing, blood bags, catheters, oxygen masks, etc.) [5-6].

Because of their physical and chemical properties, phthalates are very mobile and easily

\footnotetext{
* To whom all correspondence should be sent:

E-mail: dannica.milojkovic@gmail.com
}

migrate from plastic products to the surrounding environment. Phthalates are just incorporated into plastics; they are not chemically bound to the plastic material, which causes their ability to subsequently leach out [7]. Small amounts of fatty foods, oils or dairy products are enough for almost complete extraction of these lipophilic softeners and migration into food. Due to the large use of these plasticizers, exposure to humans is inevitable. People can be exposed to phthalates in several ways: dermal, inhalation, oral, intravenous, while the highest exposure takes place through ingestion of contaminated food. A study in Canada estimates that a man (20-70 years) of an average body weight of $70 \mathrm{~kg}$ receives most phthalates via food (4900 ng of DEHP kg-1 body weight / day) [8]. The estimated total daily exposure of humans to DEHP is $5800 \mathrm{ng}$ $\mathrm{kg}^{-1}$ body weight / day.

Considering that phthalates affect human health [9-12] and that phthalates are found in more than 95 $\%$ of human urine samples worldwide by biomonitoring studies, the European Food Safety Authority (EFSA) lists the tolerable daily intake values (TDI) for phthalates. The TDI values for DBP, DEHP and BBP are 10, 50 and $500 \mu \mathrm{g} \mathrm{kg}^{-1}$ body weight / day, respectively [13]. For DINP and DIDP, TDI amount is $150 \mu \mathrm{g} \mathrm{kg}^{-1}$ body weight / day [14].

In this study, whey powder, powdered milk and infant formula were used, as a food that is very important in the nutrition of infants, children, athletes and sick people. Milk powder that contains 
$2-3 \%$ of water is a very long-lasting milk product that has great application in the diet of people, especially children. Whey powder is a product of cheese production that represents a source of important minerals and proteins. Infant formula is a milk product suitable for feeding babies since their birth and for this reason it is crucial that it does not comprise contaminants (e.g. phthalates). The migration of DEHP into water was also examined in order to compare DEHP leachability into a polar matrix without fat with its leachability into dairy products of different fat content.

Previous experiments showed that phthalates migration from packaging materials is influenced by storage time, storage temperature, exposure to heat / light, $\mathrm{pH}$ of the sample, PET bottle volume [15-17]. However, according to the detailed literature search, this is the first systematic report that investigates the influence of temperature and ultrasound on the migration of DEHP from plastic material into dairy products. In this paper, the ultrasound-assisted migration with influence of temperature was tested. It was also examined how the composition of dairy products (fat content) influenced the degree of the migration of DEHP. As high-level phthalate source for this investigation a dialysis bag was used with predefined content of DEHP [18].

\section{EXPERIMENTAL}

\section{Reagents and materials}

HPLC grade $n$-hexane was purchased from Sigma-Aldrich (St. Louis, MO, USA). Di(2ethylhexyl) phthalate (DEHP) was purchased, in the highest available purity, from Sigma-Aldrich (St. Louis, MO, USA). Dibutyl adipate (DBA) was purchased from Fluka (Buchs, Switzerland) and used as an internal standard. Water from a Milli-Q system (Millipore, Bedford, MA, USA) was used. Dialysis bag (Baxter, USA) was used. Milk powder was purchased from Gold pack (Serbia), whey powder was from Madico (Poland) and infant formula was purchased from Humana (Germany).

In order to minimize the contamination by phthalates from the laboratory, only glassware equipment was used. All glassware was washed with soap, tap water and ultrapure water, then washed with acetone and $n$-hexane and dried at 200 ${ }^{\circ} \mathrm{C}$ in the oven for 4 hours [19].

All stock, intermediate and working solutions were prepared in $n$-hexane. The stock solutions of DEHP and DBA were prepared at a concentration of $1.0 \mathrm{mg} \mathrm{mL}$. The stock standard was diluted stepwise with $n$-hexane to prepare working solutions of DEHP. The calibration standard series was obtained with DEHP in the concentration range of $0.25,0.50,1.00,1.50$ and $2.50 \mu \mathrm{g} \mathrm{mL}^{-1}$ with DBA as the internal standard, at a concentration of $1 \mu \mathrm{g} \mathrm{mL}^{-1}$. The calibration curve was linear in the range from 0.25 to $2.5 \mu \mathrm{g} \mathrm{mL}^{-1}$ with a correlation coefficient higher than 0.99 . The linear dynamic range was broader and covered the range from 2.50 to $50 \mu \mathrm{g} \mathrm{mL}^{-1}$.

\section{GC-MS analysis}

Determination of DEHP was performed on a gas chromatograph 6890 (Hewlett-Packard) equipped with a mass selective detector (MSD) 5973 (Agilent, USA) and a DB-5 MS capillary column $(30 \mathrm{~m} \times 250 \mathrm{~mm} \times 0.25 \mathrm{~mm})$ (Agilent, USA). The gas chromatograph was operated in the splitless injection mode. The oven temperature was programmed from $60{ }^{\circ} \mathrm{C}(1 \mathrm{~min})$ to $220^{\circ} \mathrm{C}(1 \mathrm{~min})$ at a rate of $20^{\circ} \mathrm{C} \mathrm{min}-1$ and then to $280{ }^{\circ} \mathrm{C}(4 \mathrm{~min})$ at a rate of $5{ }^{\circ} \mathrm{C} \mathrm{min}{ }^{-1}$. Helium was the carrier gas $\left(1.0 \mathrm{~mL} \mathrm{~min}^{-1}\right)$ and the inlet temperature was 250 ${ }^{\circ} \mathrm{C}$. The operating temperature of the MSD was 280 ${ }^{\circ} \mathrm{C}$ with the electronic impact of $70 \mathrm{eV}$. The dwell time was $100 \mathrm{~ms}$. The MSD was used in the single ion-monitoring mode (SIM) at $m / z$ 149. The identification and quantification of the target compound was based on the relative retention time, the presence of target ions and its relative abundance. The quantification ion is $\mathrm{m} / \mathrm{z} 149$ for DEHP and ion $m / z \quad 185$ was chosen as representative ion of DBA internal standard.

\section{Samples preparation}

In order to test the degree of migration of DEHP from the plastic material under the influence of temperature and ultrasound, the following dairy products were used: milk powder, whey powder, infant formula. Water was taken for comparison as a phthalate recipient of high polarity and no fat content. Nutritional values of milk powder, whey powder and infant formula are given in Table 1.

Preparation of milk samples was done according to the producer's instruction. Water from a Milli-Q system was used to prepare the solutions of dairy samples. Milk powder (45 g), powdered whey (65 g) and infant formula (45 g) were dissolved in $1 \mathrm{~L}$ of water, respectively. Fresh samples of dairy products were prepared immediately before analysis. 
D. S. Bogdanović et al.: The effects of temperature and ultrasound on the migration of di-(2-ethylhexyl) phthalate ...

Table 1. Nutritional values of infant formula, milk powder and whey powder

\begin{tabular}{cccc}
\hline & Fats $(\%)$ & Lactose $(\%)$ & Milk proteins $(\%)$ \\
\hline Infant formula & 22.8 & 39.0 & 10.1 \\
Milk powder & 20.0 & 55.0 & 9.0 \\
Whey powder & 2.0 & 61.0 & 11.0 \\
\hline
\end{tabular}

A dialysis bag with a known content of DEHP was used to examine phthalate migration, as a reliable source of phthalate. The used dialysis bag contains about $35.8 \%$ of DEHP compared to the total weight [18]. Sample of the dialysis bag was measured to provide the same weight-to-volume ratio of dialysis bag and the sample of diary product, as if the dairy product was kept in the dialysis bag.

The exactly weighed dialysis bag was cut into pieces of $1 \mathrm{~cm}^{2}$ and added into $35 \mathrm{~mL}$ of dairy samples. The conditions for migration tests were set in two ways: (1) for determination of effects of exposure time and temperature heating up to $60{ }^{\circ} \mathrm{C}$ for up to $60 \mathrm{~min}$ with total heating time up to 60 hours was applied; (2) determination of effects of ultrasound-assisted migration was applied whenever heating was applied.

The influence of temperature was investigated as follows: samples were vigorously stirred and heated to a temperature up to $60{ }^{\circ} \mathrm{C}$ for $60 \mathrm{~min}$. After heating, the samples were left to cool at room temperature and filtered into vessels of $50 \mathrm{~mL} . n$ Hexane $(20 \mathrm{~mL})$ was added to the samples. The method of extraction of DEHP from dairy samples by $n$-hexane was shown to be a simple and effective procedure [20]. Manual shaking for $15 \mathrm{~min}$ and extraction agitation on the ultrasonic bath for 30 min were done. Blurred extracts were left for clarification. After separation of layers, the hexane layer was concentrated to a volume of $1 \mathrm{~mL}$. DBA was added to the evaporated hexane extract so that the concentration of DBA was $1 \mu \mathrm{g} \mathrm{mL}^{-1}$ and immediately analyzed by GC-MS. Pieces of dialysis bag were subjected to repeated migration tests into dairy products, providing a total heating time of $60 \mathrm{~h}$. The test was performed until a plateau was reached on the curve $m=f(t)$, where $m$ is mass of the migrated DEHP and $t$ is time.

The effect of ultrasound-assisted migration was studied in the same way as for temperature investigation with additional agitation that was performed on an ultrasonic bath.

All analyses were performed in triplicate and GC-MS quantification was repeated twice. The laboratory contamination was monitored with blank samples obtained from dairy samples without plastic pieces that were treated in the same way as the dairy samples with plastic pieces from the dialysis bag.

\section{RESULTS AND DISCUSSION}

\section{GC-MS acquisition}

The chromatogram of a standard solution of DEHP and DBA at a concentration of $2.5 \mu \mathrm{g} \mathrm{mL} L^{-1}$ and $1.00 \mu \mathrm{g} \mathrm{mL}^{-1}$, respectively, is given in Fig. 1. The chromatogram shows that the separation of DEHP and DBA as internal standard, occurred within a running time of $20 \mathrm{~min}$ and retention times for DBA and DEHP were 9.990 and 18.426 min, respectively.

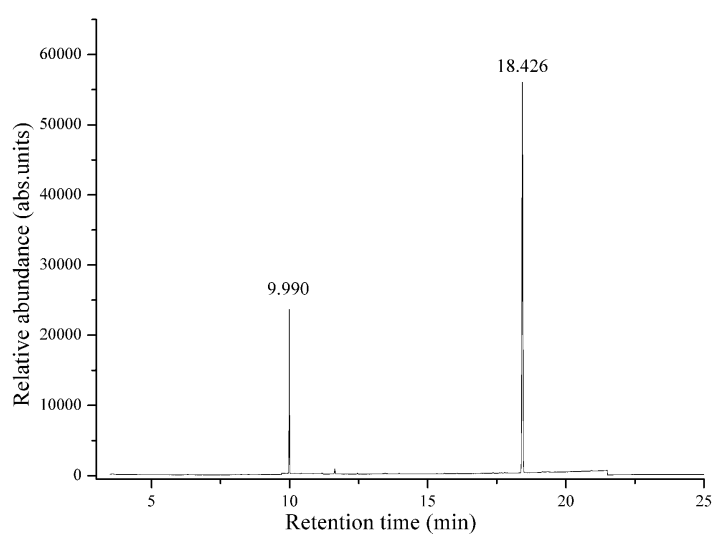

Fig. 1. GC-MS chromatogram of a standard solution of DEHP and DBA at concentrations of $2.5 \mu \mathrm{g} \mathrm{mL} \mathrm{m}^{-1}$ and $1.00 \mu \mathrm{g} \mathrm{mL} \mathrm{mL}^{-1}$, respectively.

The analytical curve obtained for DEHP within the concentration range $0.25-2.5 \mu \mathrm{g} \mathrm{mL}^{-1}$ is linear with a correlation coefficient $\mathrm{R}^{2}=0.99$ and linear equation $\mathrm{y}=(505228 \pm 9612) \mathrm{x}-(8473 \pm 13465)$, as shown on Fig. 2. The limit of determination (LOD) and limit of quantification (LOQ) were calculated from the signal/noise ratios which were multiplied with the factor 3 and 10 , respectively [21]. The LOD and LOQ values for DEHP are 0.04 $\mu \mathrm{g} \mathrm{mL} \mathrm{m}^{-1}$ and $0.12 \mu \mathrm{g} \mathrm{mL} \mathrm{mL}^{-1}$, respectively, with relative standard deviation (RSD) value of $3.6 \%$ (n $=3$ ).

Comparison of dairy products as recipients of DEHP and evaluation of influence of temperature and ultrasound on the migration of DEHP

The aim of this work is to determine the total mass of the migrated DEHP from plastic dialysis bag into recipient after repeated migration, related to temperature and ultrasound. 


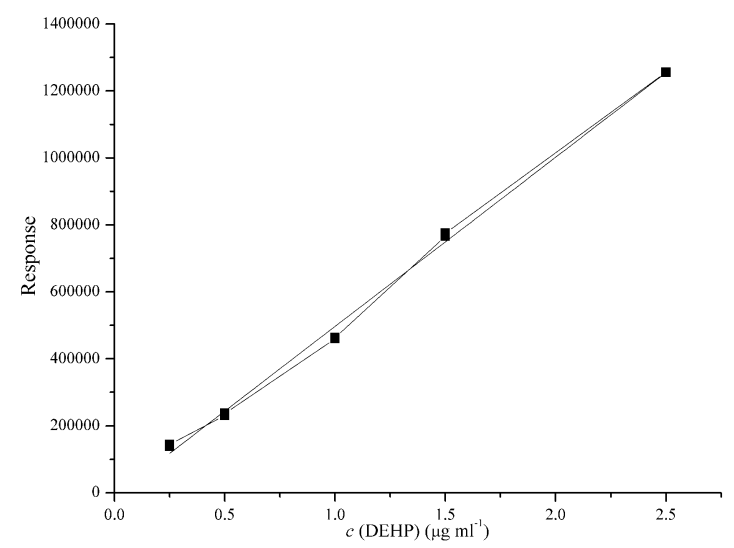

Fig. 2. The analytical curve for DEHP within the concentration range $0.25-2.5 \mu \mathrm{g} \mathrm{mL}^{-1}$

The initial phthalate concentrations were below detection limits in the samples of infant formula, milk powder, whey powder and water. The effects of exposure time, temperature, ultrasound and fat content of dairy products on the release of DEHP are summarized in Table 2.

The highest percentage of migrated DEHP was found in infant formula, which is associated with the fact that infant formula contains the highest percentage of fat $(22.8 \%)$, followed by milk powder (with a percentage of fat of $20 \%$ ), whey powder (with a fat content of $2 \%$ ) and water (without fat). The same trend is observed in both condition sets, for elevated temperature and for ultrasound-assisted migration, as shown in Fig. 3, with indication that ultrasound-assisted migration had a greater effect on the migration of DEHP.
Figures 4 and 5 show the graphs of the mass of the migrated DEHP as a function of exposure time.

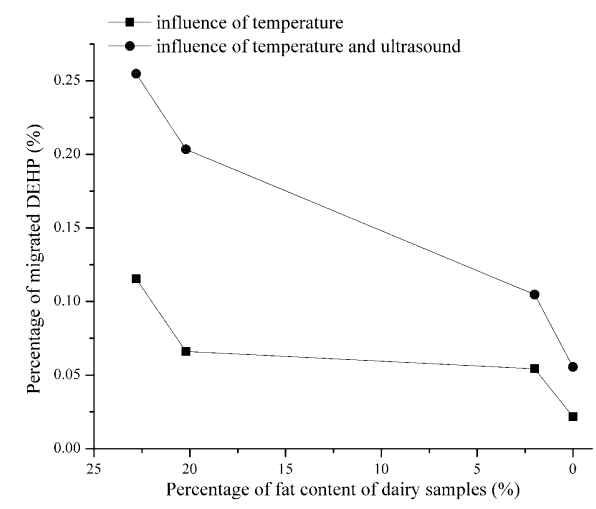

Fig. 3. The effects of temperature, ultrasoundassisted migration and fat content of dairy products on DEHP migration.

The graphs show a linear increase of the mass of migrated DEHP over time under the influence of temperature and ultrasound, until the plateau is reached. Plateau indicates that the maximum migration of DEHP has been achieved and DEHP no longer migrates into the samples under the given influence.

Maximal amount of migrated DEHP to water as recipient was reached after $1450 \mathrm{~min}$ due to the combined effect of elevated temperature and ultrasound and $2500 \mathrm{~min}$ by the effect of elevated temperature alone. Plateau was reached almost 1000 min faster in the case when ultrasound was applied beside temperature.

Table 2. Migration of DEHP influenced by (1) temperature and (2) ultrasound into recipients with different fat content

\begin{tabular}{lccccc}
\hline $\begin{array}{c}\text { Food } \\
\text { recipient } \\
\text { type }\end{array}$ & $\begin{array}{c}\text { Mass of part } \\
\text { of dialysis } \\
\text { bag }(\mathrm{g})\end{array}$ & $\begin{array}{c}\text { Mass of DEHP in } \\
\text { part of dialysis } \\
\text { bag }(\mathrm{g})^{19}\end{array}$ & $\begin{array}{c}\text { Total migration } \\
\text { time of DEHP } \\
(\mathrm{min})\end{array}$ & $\begin{array}{c}\text { Mass of } \\
\text { migrated } \\
\text { DEHP }(\mu \mathrm{g})\end{array}$ & $\begin{array}{c}\text { Percentage } \\
\text { of migrated } \\
\text { DEHP }(\%)\end{array}$ \\
\hline $\begin{array}{l}\text { Water } \\
\text { Whey }\end{array}$ & 1.7410 & 0.6233 & 2500 & $135.67 \pm 1.23$ & 0.02 \\
powder & 1.6113 & 0.5768 & 2950 & $311.83 \pm 1.40$ & 0.05 \\
Milk & 1.6200 & 0.5800 & 2900 & $384.66 \pm 3.61$ & 0.07 \\
$\begin{array}{l}\text { powder } \\
\text { Infant } \\
\text { formula }\end{array}$ & 1.4560 & 0.5212 & 3000 & $601.29 \pm 2.73$ & 0.12 \\
\hline $\begin{array}{l}\text { Water } \\
\text { Whey } \\
\text { powder }\end{array}$ & 1.4709 & 0.5266 & 1450 & $292.16 \pm 1.37$ & 0.06 \\
Milk & 1.4924 & 0.5343 & 2700 & $559.45 \pm 0.78$ & 0.10 \\
powder & 1.6049 & 0.5745 & 2800 & $1170.33 \pm 1.96$ & 0.20 \\
$\begin{array}{l}\text { Infant } \\
\text { formula }\end{array}$ & 1.4502 & 0.5192 & 2700 & $1323.36 \pm 1.87$ & 0.25 \\
\hline
\end{tabular}


D. S. Bogdanović et al.: The effects of temperature and ultrasound on the migration of di-(2-ethylhexyl) phthalate ...
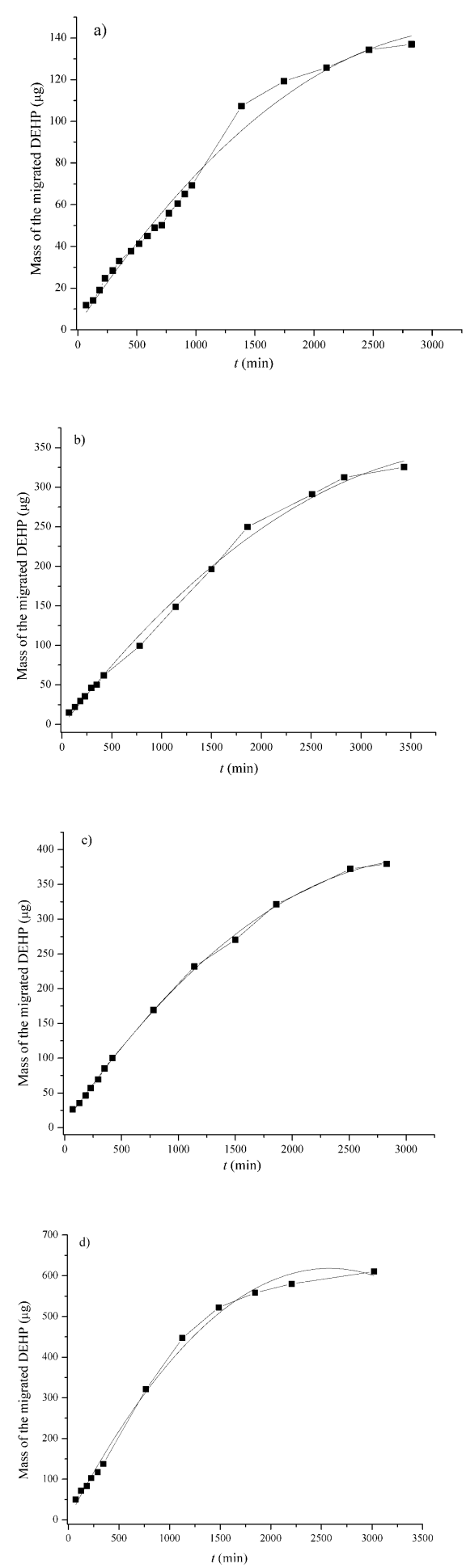

Fig. 4. The influence of temperature on the migration of DEHP in a) water, b) whey powder, c) milk powder and d) infant formula.

The time to achieve saturation, when ultrasound was applied, was shorter than when only temperature was applied for about $200 \mathrm{~min}$ for whey powder, milk powder and infant formula as recipients.
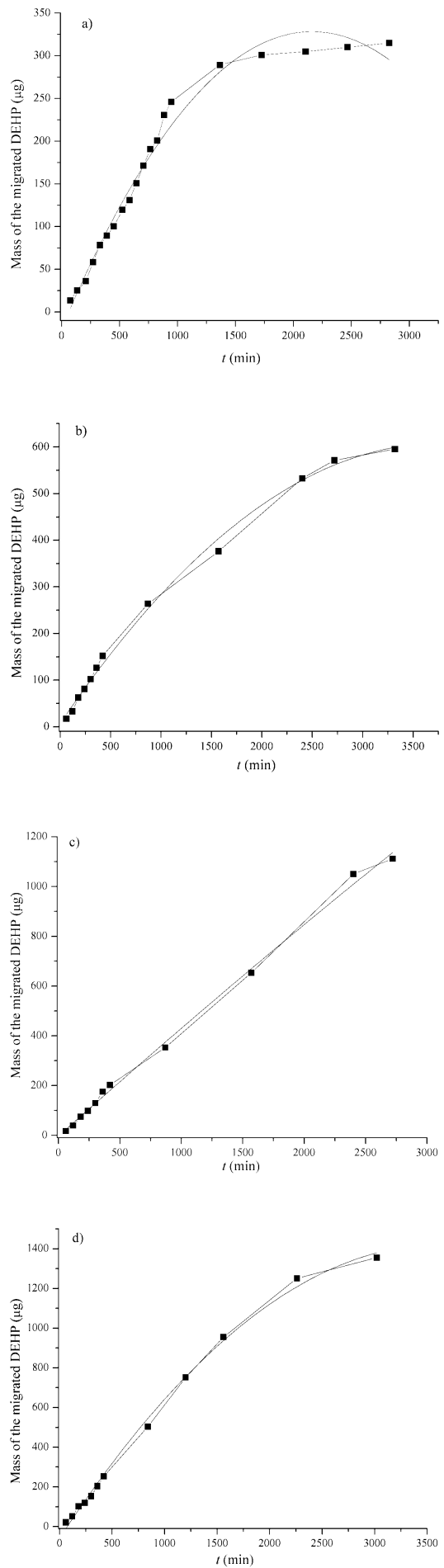

Fig. 5. The influence of ultrasound and temperature on the migration of DEHP in a) water, b) whey powder, c) milk powder and d) infant formula.

Expected reason is that water, as polar molecule, only "washed" molecules of DEHP from the plastic surface. On the other hand, non-polar milk samples can dissolve DEHP from the whole plastic material and for this a longer period is necessary.

Figures 6 and 7 show two time regions, one up to $450 \mathrm{~min}$ with a higher slope of the curve of 
migrated DEHP over time and the other from 450 up to 3500 min with a smaller slope. The graphs in (a) show the dependence of the mass of the migrated DEHP as a function of the total time of exposure of the samples to the effect of temperature and ultrasound and the graphs in (b) show the dependence of the mass of migrated DEHP in the first $450 \mathrm{~min}$. It is interesting to note that in the first $450 \mathrm{~min}$ the correlation between mass of migrated DEHP and time of extraction is almost linear, and the result is similar under both influences. This period is one sixth part of the total exposure time, and during this period about $30 \%$ of the total amount of migrated DEHP migrates into the recipients (Table 3). This migration was expressed, unlike the rest of the migration that takes place slower, until saturation is achieved.

It is interesting to note that in the first $450 \mathrm{~min}$ the correlation between mass of migrated DEHP and time of extraction is almost linear and the result is similar under both influences (Figs. 6 and 7). In this time period about $30 \%$ of the total migrated amount of DEHP migrates into the recipients (Table 3).

Obtained results showed that ultrasound has a greater effect on DEHP migration from the dialysis bag than temperature. Taking any time period during which the migration of DEHP from the dialysis bag was performed under both influences, a higher mass of DEHP was always obtained, under the influence of ultrasound, as can be seen in Table 3 , as in the graphs (Figs. 6 and 7). The degree of migration of DEHP under the influence of ultrasound increased by $223.34 \%$ for milk powder, by $128.79 \%$ for infant formula, by $92.93 \%$ for water and by $84.65 \%$ for whey powder. Increased efficiency of migration of DEHP due to the effect of ultrasound is a consequence of the power and energy provided by oscillations produced by ultrasound. In this way, the plastic pieces of the dialysis bag come in better contact with the sample; the sample under the influence of the energy of the ultrasound infiltrates deeper into the plastic, and soils DEHP in a larger quantity.
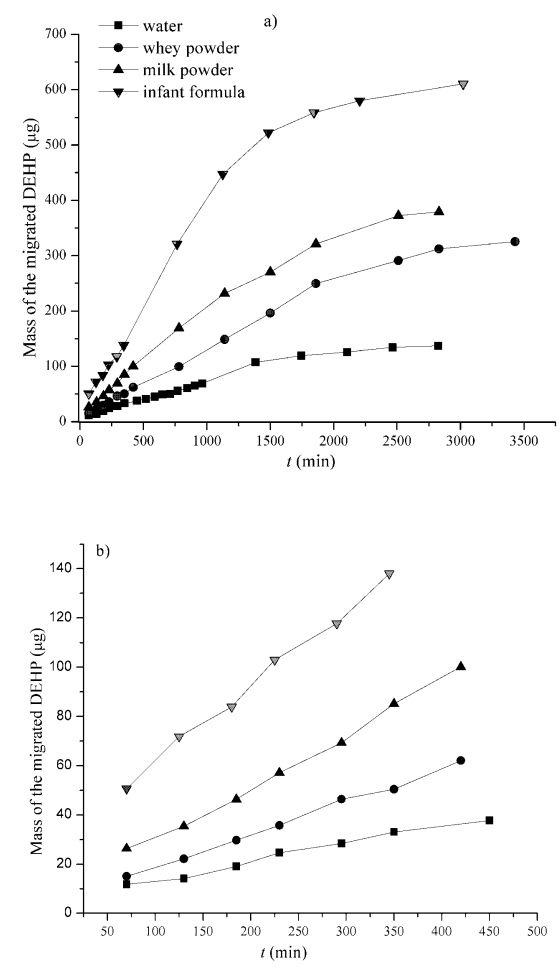

Fig. 6. Mass of the migrated DEHP under the influence of temperature a) in a time period of $3500 \mathrm{~min}$ and $b$ ) in a time period of $450 \mathrm{~min}$.
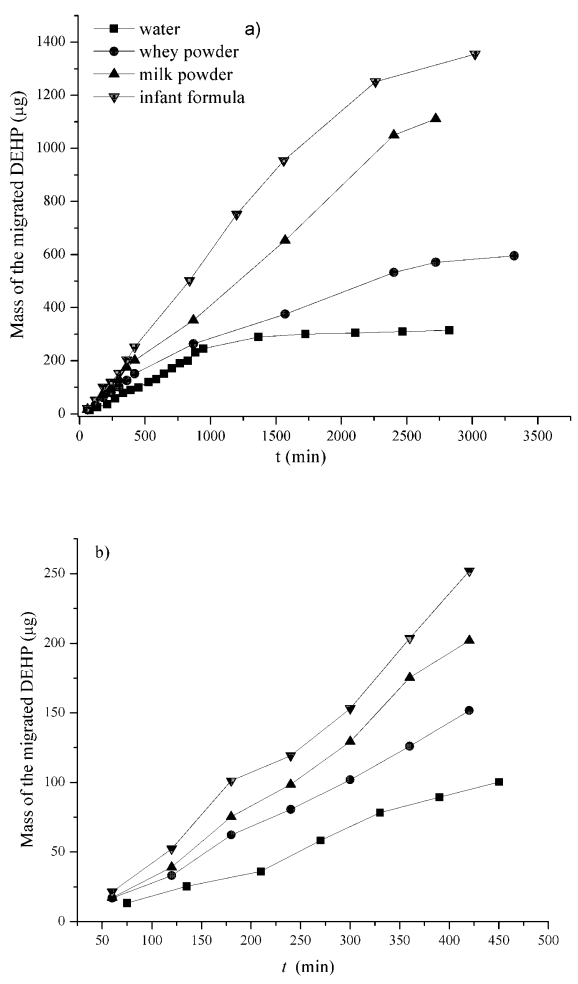

Fig. 7. Mass of the migrated DEHP under the influence of ultrasound a) in a time period of $3500 \mathrm{~min}$ and $b$ ) in a time period of $450 \mathrm{~min}$. 
D. S. Bogdanović et al.: The effects of temperature and ultrasound on the migration of di-(2-ethylhexyl) phthalate ... Table 3. Mass of migrated DEHP under the influence of temperature and ultrasound for different time periods

\begin{tabular}{|c|c|c|c|c|}
\hline & \multicolumn{4}{|c|}{ Mass of the migrated DEHP $(\mu \mathrm{g})$} \\
\hline & \multicolumn{2}{|c|}{$\begin{array}{l}\text { Under the influence of temperature } \\
\text { after a period of }\end{array}$} & \multicolumn{2}{|c|}{$\begin{array}{l}\text { Under the influence of temperature and } \\
\text { ultrasound after a period of }\end{array}$} \\
\hline & $450 \mathrm{~min}$ & $3000 \mathrm{~min}$ & $450 \mathrm{~min}$ & $3000 \mathrm{~min}$ \\
\hline Water & $38.55 \pm 0.79$ & $144.19 \pm 1.85$ & $110.34 \pm 0.99$ & $275.25 \pm 1.09$ \\
\hline \multicolumn{5}{|l|}{ Whey powder } \\
\hline & $67.67 \pm 1.56$ & $315.52 \pm 0.83$ & $142.29 \pm 1.06$ & $582.58 \pm 0.52$ \\
\hline Milk powder & $104.63 \pm 0.86$ & $385.43 \pm 0.96$ & $193.48 \pm 1.03$ & $1247.06 \pm 1.01$ \\
\hline Infant formula & $199.12 \pm 0.61$ & $601.41 \pm 1.08$ & $283.57 \pm 0.51$ & $1376.56 \pm 0.51$ \\
\hline
\end{tabular}

\section{CONCLUSIONS}

Obtained data for the migration of DEHP from packaging material to dairy products due to the effects of temperature, ultrasound and fat content of the dairy product show that migration depends on all three factors. The analysis has shown that in both cases when temperature and ultrasound were applied, the amount of migrated DEHP decreased in the following order: infant formula $>$ powdered milk > whey powder $>$ water, indicating that the fat content affects migration, the highest migration being observed for the dairy product with the highest fat content. By comparing the effects of temperature and ultrasound, the conclusion is that the influence of ultrasound on the migration of DEHP from plastic packaging into dairy products is higher than the effect of temperature, for all dairy products, due to the fact that ultrasound disrupts the structure of plastic material and accelerates diffusion and release of phthalate.

Acknowledgement: This study was supported by the Ministry of Education, Science and Technological Development of the Republic of Serbia and was performed as a part of Project TR 31060 .

\section{REFERENCES}

1. D. N. Brookle, S. Dobson, P.D. Howe, J.R. Nielsen, Environmental hazard assessment: di-(2-ethylhexyl) phthalate, United Kingdom Department of the Environment, Toxic Substances Division, Report TSD/2, London, 1991.

2. World Health Organization: Guidelines for Drinking-water Quality, 2008.

3. K. Bouma, D.J. Schakel, Food Addit. Contam. 19, 602 (2002).

4. T. Wenzl, Methods for the determination of phthalates in food, European Commission Joint
Research Centre, Institute for Reference Materials and Measurements, 2009.

5. X-L. Cao, Compr. Rev. Food. Sci. F. 9, 21 (2010).

6. M. Wormuth, M. Scheringer, M. Vollenweider, K. Hungerbuhler, Risk Anal., 26, 803 (2006).

7. HAH - Hrvatska agencija za hranu: Znanstveno mišljenje o prisutnosti ftalata u hrani, Osijek, 2014.

8. M.E. Meek, P.K.L. Chan, Environ. Carcinog. Ecotoxicol., 12, 179 (1994).

9. H.M. Koch, A.M. Calafat, Philos. Trans. R. Soc. Lond. Ser. B. Biol. Sci., 364, 2063 (2009).

10. I.K. Mahood, H.M. Scott, R. Brown, N. Hallmark, M. Walker, R.M. Sharpe, Environ. Health Perspect., 115, 55 (2007).

11. S.M. Duty, M.J. Silva, D.B. Barr, J.W. Brock, L. Ryan, Z. Chen, Epidemiology, 14, 269 (2003).

12. B.A. Johnsson, J. Richthoff, L. Rylander, A. Giwercman, L. Hagmar, Epidemiology, 16, 487 (2005).

13. European Food Safety Authority, EFSA J. 241, 1 (2005).

14. European Food Safety Authority, EFSA J. 243, 1 (2005)

15. V.G. Mihucz, G. Záray, Appl. Spectrosc. Rev., 51, 183 (2016).

16. M. Farhoodi, Z. Emam-Djomeh, M. Reza Ehsani, A. Oromiehie, Arab. J. Sci. Eng., 33, 279 (2008).

17. J. Bošnir, D. Puntarić, A. Galić, I. Škes, T. Dijanić, M. Klarić, et al. Food. Technol. Biotechnol., 45, 91 (2007).

18. I. Kostić, T. Anđelković, D. Anđelković, A. Bojić, T. Cvetković, D. Pavlović, Bulg. Chem. Commun. 49, 360 (2017).

19. A. Fankhauser-Noti, K. Grob, Anal. Chim. Acta, 582, 353 (2007).

20. D. Milojković, D. Anđelković, G. Kocić, T. Andjelković, J. Serb. Chem. Soc., 80, 983 (2015).

21. Wisconsin Department of Natural Resources Laboratory Certification Program: Analytical Detection Limit Guidance \& Laboratory Guide for Determining Method Detection Limits, 1996. (http://dnr.wi.gov/regulations/labcert/documents/gui dance/-lodguide.pdf). 


\title{
In vitro study on the antitumor activity of Tanacetum vulgare L. extracts
}

\author{
A.M. Vasileva ${ }^{1 *}$, I. A. Iliev², V. S. Lozanov ${ }^{1}$, M. B. Dimitrova ${ }^{2}$, V. I. Mitev¹, I. P. Ivanov ${ }^{1}$ \\ ${ }^{1}$ Department of Medical Chemistry and Biochemistry, Medical University of Sofia, 2, Zdrave Str., Sofia 1431, Bulgaria \\ ${ }^{2}$ Institute of Experimental Morphology, Pathology and Anthropology with Museum, Bulgarian Academy of Sciences, \\ Acad. G. Bonchev Str., Bl. 25, 1113 Sofia
}

Received December 20, 2018; Revised January 24, 2019

\begin{abstract}
The major nonvolatile compounds derived from extracts and fractions from Tanacetum vulgare L. flowers were determined by LC-HRMS. Major compounds in the crude extract were determined to be: six hydroxycinnamoyl quinic acids with 4,5-dicaffeoylquinic acid and twelve flavonoids and their derivatives, six of which were in the form of flavonoid-O-glucuronides. Generally, the major flavonoid aglycone in tansy was luteolin. Extracts and fractions were tested under in vitro conditions in nine cell lines - one control non-tumorogenic and eight tumor lines, whereby antitumor activity was observed after 72 hours of incubation with the aforementioned substances as determined by an MTT assay. The obtained results show the highest selectivity index for the ethyl acetate extract from Flores Tanaceti (EAFT) and for the ethyl acetate fraction of the crude extract (EACE). EAFT extract was found to exert the highest antitumor effect, followed by EACE. From the above results it becomes evident that ethyl acetate extracts of $T$. vulgare contain substances with high selective activity against tumor cells.
\end{abstract}

Key words: Tanacetum vulgare L.; phenolic compounds; cancer cell line; cell viability; antitumor activity

\section{INTRODUCTION}

Globally, the incidence of oncological diseases increases compared to other life-threatening pathologies. Therefore, there is a high demand for high-efficiency low-toxicity therapeutic agents with anti-tumor activity. Natural products are a good alternative to commonly used cytotoxic agents because of their good biological tolerance and high metabolic breakdown. Medicinal plants are capable of synthesizing thousands of diverse bioactive constituents. These compounds may elicit a diverse range of different effects on humans and animals. Chemical compounds of herbal origin and those of the conventional drugs accomplish their effects on the human body through similar processes and mechanisms of action. Medicinal plants are a promising source for the development of novel therapeutics for various diseases [1], including cancer [2, 3]. In recent years, there has been a growing interest in natural phenolic compounds and their presumed roles in the prevention and treatment of various degenerative diseases, such as cancer and cardiovascular disease $[4,5]$.

Tanacetum vulgare $\mathrm{L}$. (common tansy) is a perennial, herbaceous plant species (Asteraceae family) native to temperate Europe and Asia but invasive to other parts of the world. Tansy is widely used in traditional medicine in different parts of Eastern Europe. Extracts of the herb show antioxidant, anti-inflammatory, antiulcerogenic, antiviral, antimicrobial and anticancer activities [6]. Overdosing with teas containing tansy is considered

\footnotetext{
* To whom all correspondence should be sent
}

E-mail: vasileva_anelia@abv.bg to be dangerous due to the presence of the toxic monoterpene, $\alpha$-thujone in the plant. Tansy is also an abortifacient herb. Nevertheless, there appears to be a wide margin of safety for the therapeutic use of the aqueous extract of herb leaves. Previous studies on the crude extract from $T$. vulgare have shown antitumor activity against MCF-7 breast cancer cell line [7]. The chloroform fraction of the crude extract of the herb possesses a significantly stronger inhibitory effect on the proliferation of tumor cell lines HeLa, MCF-7 and A2780 compared to the total extract [8]. The main nonvolatile components in $T$. vulgare flower extract are caffeoylquinic and dicaffeoylquinic acids [9, 10], flavonoids and their O-glycosylated (Oglucosides and O-glucuronides) derivatives [9 11]. The antitumor potential demonstrated by the chloroform fraction of T. vulgare [8] suggests that future in-depth studies are required to gain better understanding of the antitumor properties exhibited by various extracts and fractions of the herb, as well as for the identification of the active ingredients contained within them. The aim of the present work was to determine the main components in different extracts and fractions from the flowers of $T$. vulgare and to evaluate their effects on the viability of various tumor cell lines as compared to a control non-tumorogenic cell line.

\section{EXPERIMENTAL}

\section{Chemicals and reagents}

Acetonitrile of mass spectrometry grade was purchased from Merck (Germany). Ethyl acetate, 
diethyl ether, diisopropyl ether and hexane were from Fisher Chemical (UK); formic acid, 98\%, was from Fluka (Germany). High-purity water was obtained using a Purelab UHQ II system from ELGA (Netherland). All reagents were of the highest purity available. The flowers of $T$. vulgare and the dried powdered crude extract of the herb were kindly provided by Vemo 99 Ltd (Sofia, Bulgaria).

\section{Preparation of the ethyl acetate fraction from the crude extract (EACE)}

Twenty $\mathrm{mL}$ of water were added to $5 \mathrm{~g}$ of the powdered crude tansy extract while stirring. $6 \mathrm{~N}$ $\mathrm{HCl}$ was then added in a dropwise manner until a $\mathrm{pH} 3.0$ was achieved. Ethyl acetate $(15 \mathrm{~mL})$ was applied to the aqueous phase while stirring. The organic phase was separated and the aqueous phase was extracted with $10 \mathrm{~mL}$ of ethyl acetate. The combined organic phases were filtered, washed with brine and dried using $\mathrm{Na}_{2} \mathrm{SO}_{4}$. Ethyl acetate was removed under vacuum and a small amount of diisopropyl ether was added. The formed dark yellow solid residue was filtered off and dried.

\section{Preparation of dicyclohexylammonium salts fraction (DCHAS)}

Solid dicyclohexylammonium salts were obtained from the ethyl acetate extract, as follows: The volume of the ethyl acetate extract was reduced to $1 / 4$ and dicyclohexylamine was added dropwise. The obtained precipitate was filtered, washed with diisopropyl ether and dried.

\section{Isolation of solid substance from diethyl ether/hexane (DEHS)}

The filtrate obtained after removal of the dicyclohexylammonium salts was concentrated in vacuo giving a thick oily residue. Diethyl ether was added to the residue, followed by hexane, resulting in a dark yellow precipitate.

\section{Preparation of ethyl acetate extract from Flores Tanaceti (EAFT)}

Sixteen $\mathrm{mL}$ of water and $48 \mathrm{~mL}$ of ethyl acetate were added to $4 \mathrm{~g}$ of Flores Tanaceti while stirring. Then, $6 \mathrm{~N} \mathrm{HCl}$ was added dropwise until the aqueous phase reached a $\mathrm{pH} 3.0$ and the mixture was stirred for an additional hour. Following filtration, the organic phase was separated and processed as above. Finally, diisopropyl ether was added and the obtained precipitate was filtered and dried.

\section{LC-HRMS analysis}

Analysis was carried out using Q Exactive hybrid quadrupole-Orbitrap mass spectrometer
(Thermo Scientific Co, USA) equipped with TurboFlow ${ }^{\circledR}$ LC system, heated electrospray model HESI II on IonMax ${ }^{\circledR}$ (Thermo Scientific Co, USA).

The chromatographic separation of analytes was carried out by Hypersil Gold column $(100 \mathrm{~mm} \times$ $2.1 \mathrm{~mm}$ i.d., $1.9 \mu \mathrm{m}$ ) using the following mobile phases: A: $0.1 \%$ formic acid in water and B: $0.1 \%$ formic acid in acetonitrile at a flow rate of 300 $\mu \mathrm{L} /$ min and gradient: $0 \% \mathrm{~B}$ for $1 \mathrm{~min}, 30-90 \% \mathrm{~B}$ for $30 \mathrm{~min}, 90 \% \mathrm{~B}$ for $5 \mathrm{~min}, 90-0 \% \mathrm{~B}$ for $2 \mathrm{~min}$ and $0 \% \mathrm{~B}$ for $2 \mathrm{~min}$. Injection volume was $10.0 \mu \mathrm{L}$.

Full-scan spectra over the $\mathrm{m} / \mathrm{z}$ range $80-1200$ were acquired in negative ion mode at resolution settings of 70 000. All MS parameters were optimized for sensitivity to the target analytes using the instrument control software program. Q Exactive parameters were: spray voltage $4.0 \mathrm{kV}$, Sheath gas flow rate 32, Auxiliary gas flow rate 10, Spare gas flow rate 3, Capillary temperature 320 ${ }^{\circ} \mathrm{C}$, Probe heater temperature $300^{\circ} \mathrm{C}$ and S-lens RF level 50. All Ion Fragmentation (AIF) mode of operation of mass analyzer was used for extracts compound identification. Optimized values of the collision energy were HCD $25 \%$. Data acquisition and processing were carried out with Xcalibur 2.4® software package (Thermo Scientific Co, USA). Calculations for theoretical $\mathrm{m} / \mathrm{z}$ values were made by Mass Frontier 5.1 Software program (Thermo Scientific Co, USA). Extracts of tansy ( $3 \mathrm{mg}$ ) were dissolved in $1 \mathrm{~mL}$ of $0.1 \%$ formic acid buffer by ultrasound-assisted extraction for $15 \mathrm{~min}$ and $10 \mu \mathrm{L}$ were injected for LC-HRMS analyses.

\section{Cell lines}

In our in vitro experiments, we used several human cancer cell lines as models of common oncological diseases: invasive ductal adenocarcinoma of the breast (MCF-7), triple negative breast adenocarcinoma (MDA-MB-231), non-small cell lung carcinoma (H1299), alveolar non-small cell adenocarcinoma (A549), cervical cancer cell line (HeLa), hepatocellular carcinoma (HepG2), colon carcinoma (HT-29) and prostate carcinoma cell line (PC3). As a model of normal tissue we used the non-tumorigenic epithelial breast cell line (MCF-10A).

\section{In vitro antitumor activity}

The antitumor activity testing was performed on cell cultures from several human cancer cell lines using the standard MTT-dye reduction assay, described by Mosmann [12]. The cell cultures were routinely grown as monolayers in $75 \mathrm{~cm}^{2}$ tissue culture flasks (Orange Scientific), in Dulbecco's Modified Eagle's Medium - high glucose 
A. M. Vasileva et al.: In vitro study on the antitumor activity of Tanacetum vulgare L. extracts

(DMEM), supplemented with $10 \%$ fetal calf serum and antibiotics. Cultures were maintained at 37.5 ${ }^{\circ} \mathrm{C}, 5 \% \mathrm{CO}_{2}$, in a humidified atmosphere. Cells were plated at a density of $1 \times 10^{3}$ cells per well in 96-well flat-bottomed microplates and allowed to adhere for $24 \mathrm{~h}$ before treatment with the test compounds, dissolved in ethanol, and further diluted in culture medium to the final concentrations. A concentration range from 10 to $500 \mu \mathrm{g} / \mathrm{mL}$ was tested over an incubation period of $72 \mathrm{~h}$. All experiments were performed in triplicate. The MTT-formazan absorption was measured using a microplate reader (TECAN, Sunrise TM, Groedig/Salzburg, Austria) at $580 \mathrm{~nm}$. Antiproliferative activities were expressed as $\mathrm{IC}_{50}$ values (concentrations required for $50 \%$ inhibition of cell growth), calculated using non-linear regression analysis (GraphPad Prizm4 Software).
There was a good reproducibility between replicates with standard deviation below $\pm 10 \%$. The statistical analysis involved One-way ANOVA followed by Bonferroni's post hoc test. $\mathrm{p}<0.05$ was accepted as the lowest level of statistical significance.

\section{RESULTS AND DISCUSSION}

\section{Characterization of compounds in the crude extract}

We determined the major nonvolatile compounds in the extract of $T$. vulgare, provided by Vemo 99 Ltd., by LC-HRMS method in negative ionization mode. Compounds were identified via $\mathrm{MS}^{2}$ analyses and compared with literature data as is discussed below and summarized in Table 1. The total ion chromatogram (TIC) of the crude extract is presented in Fig. 1.

Table 1. Identification of phytochemical compounds in $T$. vulgare crude extract by LC-HRMS in negative mode

\begin{tabular}{|c|c|c|c|c|}
\hline $\begin{array}{l}\text { Peak } \\
\text { № }\end{array}$ & $\begin{array}{l}{[\mathrm{M}-\mathrm{H}]^{-} \mathrm{m} / \mathrm{z}} \\
\text { Delta }(\mathrm{ppm})\end{array}$ & $\begin{array}{l}\text { Molecular } \\
\text { formula }\end{array}$ & $\mathrm{MS}^{2}$ data $\mathrm{m} / \mathrm{z},($ R.I., $\%)$ & $\begin{array}{l}\text { Proposed } \\
\text { compound }^{\mathrm{a}}\end{array}$ \\
\hline & & & Hydroxycinnamoylquinic acids & \\
\hline 1 & $\begin{array}{l}353.0873 \\
(0.79)\end{array}$ & $\mathrm{C}_{16} \mathrm{H}_{18} \mathrm{O}_{9}$ & $\begin{array}{c}191.0548(73), 179.0336(18), \\
135.0435(100), 85.0277(12)\end{array}$ & $\begin{array}{l}\text { 3-caffeoylquinic acid } \\
\qquad[9,10]\end{array}$ \\
\hline 2 & $\begin{array}{l}353.0871 \\
(1.01)\end{array}$ & $\mathrm{C}_{16} \mathrm{H}_{18} \mathrm{O}_{9}$ & $191.0548(100), 173.0441(8)$ & $\begin{array}{c}\text { 5-caffeoylquinic acid } \\
\qquad[9,10]\end{array}$ \\
\hline 3 & $\begin{array}{l}515.1185 \\
\quad(0.25)\end{array}$ & $\mathrm{C}_{25} \mathrm{H}_{24} \mathrm{O}_{12}$ & $\begin{array}{c}353.0873(9), 335.0770(7), 191.0548 \\
(50), 179.0336(91), 173.0442(100), \\
161.0229(27), 155.0334(11) \\
135.0435(34)\end{array}$ & $\begin{array}{c}\text { 3,4-dicaffeoylquinic acid } \\
{[9,10]}\end{array}$ \\
\hline 4 & $\begin{array}{l}515.1186 \\
\quad(0.36)\end{array}$ & $\mathrm{C}_{25} \mathrm{H}_{24} \mathrm{O}_{12}$ & $\begin{array}{c}353.0872(33), 191.0548(100), \\
179.0336(66), 173.0442(4), \\
161.0229(5), 135.0435(23)\end{array}$ & $\begin{array}{c}\text { 3,5-dicaffeoylquinic acid } \\
{[9,10]}\end{array}$ \\
\hline 5 & $\begin{array}{c}515.1186 \\
(0.48)\end{array}$ & $\mathrm{C}_{25} \mathrm{H}_{24} \mathrm{O}_{12}$ & $\begin{array}{c}191.0548(100), 179.0336(12) \\
161.0229(8), 135.0435(6)\end{array}$ & $\begin{array}{c}\text { 1,5-dicaffeoylquinic acid } \\
{[13,14]}\end{array}$ \\
\hline 6 & $\begin{array}{l}515.1186 \\
(0.36)\end{array}$ & $\mathrm{C}_{25} \mathrm{H}_{24} \mathrm{O}_{12}$ & $\begin{array}{c}353.0874(46), 191.0549(34), \\
179.0337(73), 173.0442(100), \\
161.0230(5), 135.0436(22)\end{array}$ & $\begin{array}{c}\text { 4,5-dicaffeoylquinic acid } \\
{[9,10]}\end{array}$ \\
\hline 7 & $\begin{array}{l}285.0402 \\
(0.89)\end{array}$ & $\mathrm{C}_{15} \mathrm{H}_{10} \mathrm{O}_{6}$ & $\begin{array}{c}\text { Flavonoids } \\
151.0021(10), 133.0279(100), \\
107.0122(11)\end{array}$ & Luteolin [9 - 11] \\
\hline 8 & $\begin{array}{l}345.0613 \\
(2.27)\end{array}$ & $\mathrm{C}_{17} \mathrm{H}_{14} \mathrm{O}_{8}$ & $\begin{array}{l}330.0379(22), 315.0144(72), \\
287.0194(100), 149.0229(50)\end{array}$ & $\begin{array}{l}\text { Quercetagetin dimethyl } \\
\text { ether }[9,10]\end{array}$ \\
\hline 9 & $\begin{array}{l}329.0663 \\
(0.76)\end{array}$ & $\mathrm{C}_{17} \mathrm{H}_{14} \mathrm{O}_{7}$ & $\begin{array}{c}299.0189(22), 271.0244(100), \\
243.0289(7), 227.0340(6), 199.0389 \\
(10)\end{array}$ & Eupalitin $[10,11]$ \\
\hline 10 & $\begin{array}{l}463.0877 \\
(0.60)\end{array}$ & $\mathrm{C}_{21} \mathrm{H}_{20} \mathrm{O}_{12}$ & $\begin{array}{l}\text { Flavonoid-O-glucuronides } \\
287.0556(13), 151.0021(100), \\
135.0435(40), 113.0228(9)\end{array}$ & $\begin{array}{l}\text { Eriodictyol-O- } \\
\text { glucoronide [11] }\end{array}$ \\
\hline 11 & $\begin{array}{l}477.0662 \\
\quad(0.25)\end{array}$ & $\mathrm{C}_{21} \mathrm{H}_{18} \mathrm{O}_{13}$ & $\begin{array}{c}301.0347(100), 178.9973(5), \\
151.0021(15)\end{array}$ & $\begin{array}{l}\text { Quercetin-3-O- } \\
\text { glucuronide [15] }\end{array}$ \\
\hline 12 & $\begin{array}{l}461.0720 \\
\quad(0.21)\end{array}$ & $\mathrm{C}_{21} \mathrm{H}_{18} \mathrm{O}_{12}$ & $285.0399(100)$ & $\begin{array}{c}\text { Luteolin-7-O-glucuronide } \\
{[9-11]}\end{array}$ \\
\hline
\end{tabular}




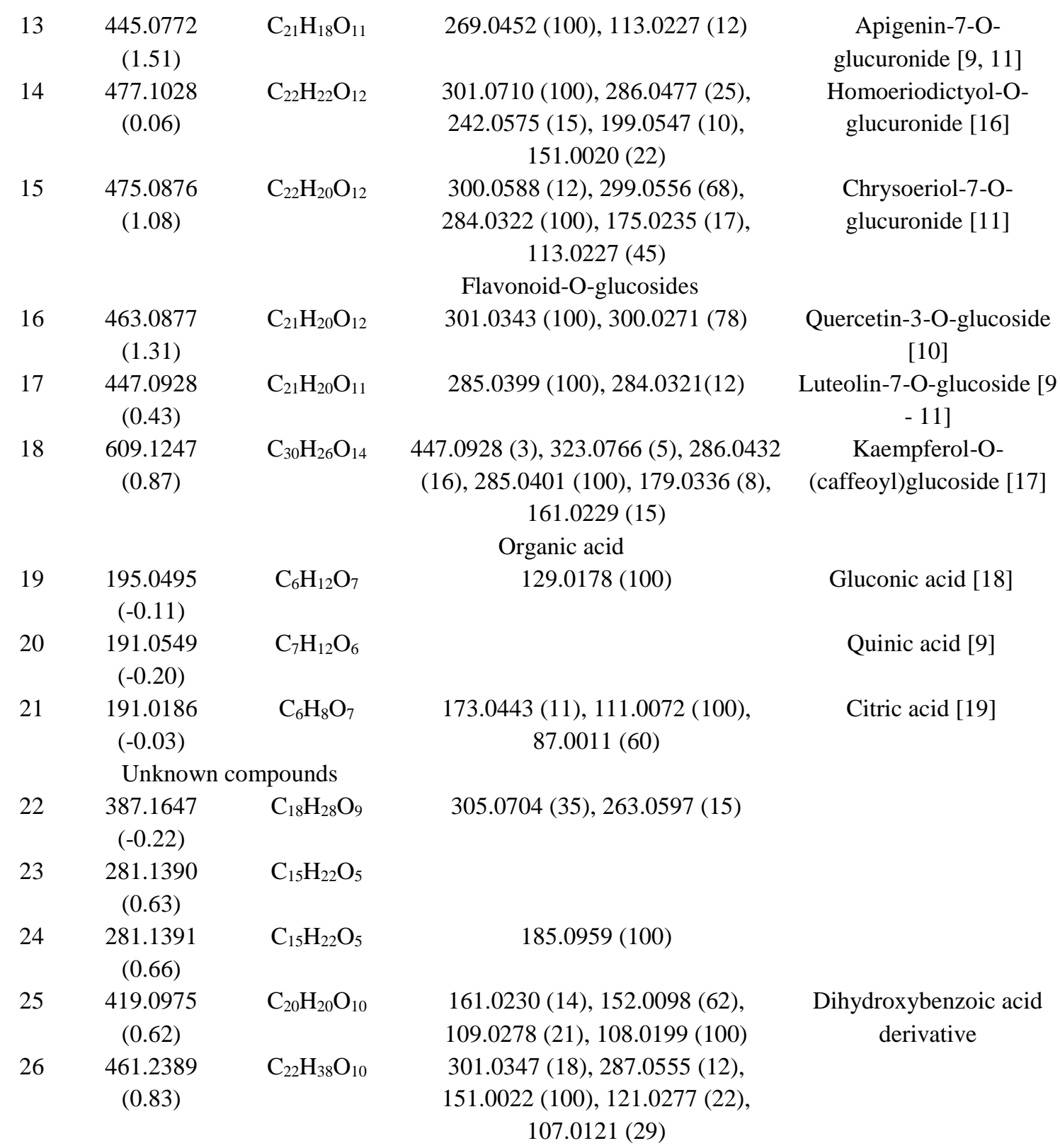

${ }^{a}$ Consistent with the literature on the presence of these compounds in the tansy or based on the comparison of $\mathrm{m} / \mathrm{z}$ values from the $\mathrm{MS}^{2}$ spectra with the literature data.

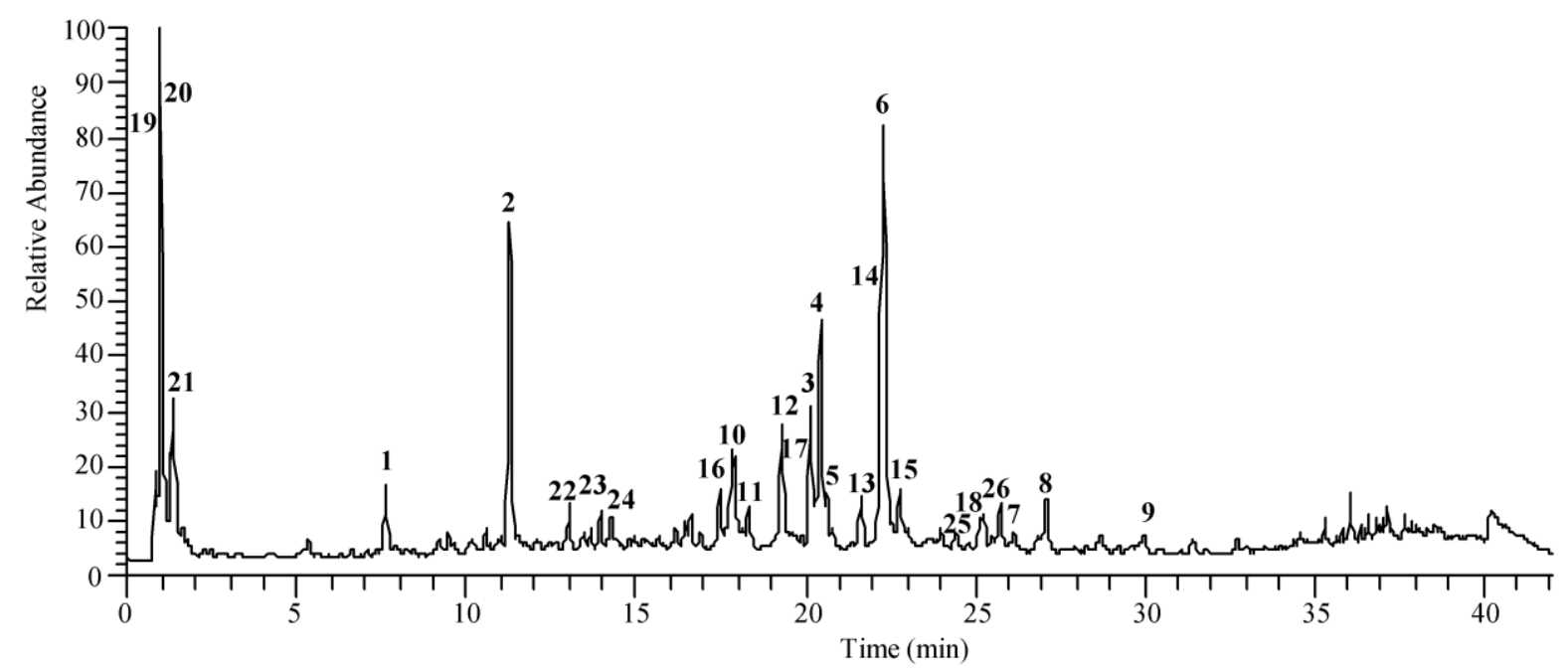

Fig. 1. Total ion chromatogram (TIC) of T. vulgare crude extract in negative ion mode. The numbering of the compounds corresponds to Table 1. 
A. M. Vasileva et al.: In vitro study on the antitumor activity of Tanacetum vulgare L. extracts

Compounds 1 and 2 showed a $[\mathrm{M}-\mathrm{H}]^{-}$ion at $\mathrm{m} / \mathrm{z}$ 353.08 in negative-ion mode, enabling the identification of the two isomers of caffeoylquinic acid (CQA). The primary degradation pathways of isomeric CQAs generate fragment ions with $\mathrm{m} / \mathrm{z}$ 191 corresponding to [quinic acid-H]; $\mathrm{m} / \mathrm{z} 179$ corresponding to [caffeic acid-H],$\quad \mathrm{m} / \mathrm{z} 173$ corresponding to [quinic acid- $\left.\mathrm{H}-\mathrm{H}_{2} \mathrm{O}\right]^{-}$and $\mathrm{m} / \mathrm{z} 135$ corresponding to [caffeic acid- $\left.\mathrm{CO}_{2}\right]^{-}$, which are characteristic and the differences in their intensity could be used to correctly identify the respective CQAs [13, 14]. Thus, compounds 1 and $\mathbf{2}$ were identified as 3-CQA and 5-CQA, respectively. Compounds 3 - 6 gave the $[\mathrm{M}-\mathrm{H}]^{-}$ions at $\mathrm{m} / \mathrm{z}$ 515.12, indicating that they were dicaffeoylquinic acid (diCQA) isomers. The specific product ions mentioned above were observed in the $\mathrm{MS}^{2}$ spectrum, as well as an ion with $\mathrm{m} / \mathrm{z} 353.08$ corresponding to [CQA-H] $]^{-}$A specific ion with $\mathrm{m} / \mathrm{z} 335.08$ was observed in the spectrum of 3,4diCQA (compound 3). The isomers of diCQAs could be distinguished by the intensity of product ions $[13,14]$. A total of twelve flavonoids and their derivatives were characterized in $T$. vulgare, including two aglycone flavonoids (compounds $\mathbf{7}$, 9), one flavonoid dimethyl ether (compound 6), six flavonoid-O-glucuronides (compounds 10 -15) and three flavonoid-O-glucosides (compounds 16 -18). Generally, the flavonoid aglycones in tansy were luteolin, quercetin and apigenin. The sugar type can be easily deduced from the difference between the mass of parent ions and the mass of aglycone fragments. The loss of 162 and $176 \mathrm{Da}$ corresponded to hexoside and glycuronide moieties, respectively. Identification of the aglycone part of flavonoid-O-glycosides and the structures of flavonoid-O-glycosides could be determined by performing analyses of the $\mathrm{MS}^{2}$ spectrum and comparison with the corresponding spectra in a related reference (see Table 1). The presence of three organic acids (gluconic, quinic and citric acid) and five unknown compounds is detected in the crude extract of $T$. vulgare.

\section{Characterization of compounds in the ethyl acetate fraction}

The extractability of the phenolic compounds is known to be influenced by the polarity of the solvent used. Ethyl acetate was proposed by Ignat et al. [20] as the organic solvent for extraction of polyphenols. Powder of the crude tansy extract was treated with a two-phase solvent system consisting of ethyl acetate and water. Aqueous phase was acidified to $\mathrm{pH} 3$, resulting in precipitation of acidic substances such as isomeric caffeoylquinic, dicaffeoylquinic acids and flavonoid-O- glucuronides, which led to an increase of the partition coefficients of these compounds. The LCHRMS analysis of the ethyl acetate fraction showed that relatively more hydrophobic components were extracted in the organic phase. Hydrophilic components such as gluconic, quinic and citric acid were not detected in this fraction.

\section{Characterization of dicyclohexylammonium salts}

After reduction of the ethyl acetate fraction volume, a dicyclohexylamine was added. A yellow precipitate was formed as a result of the reaction. The analysis of the dried solid substance by LCHRMS indicated the presence of 3- and 5-CQA, 3,4-, 3,5-, 4,5- and 1,5-diCQA, flavonoid-Oglucuronides and luteolin. No flavonoid-Oglucosides were detected. This is a simple method for separation of acidic from other substances, in particular flavonoid-O-glucuronides from other flavonoid-O-hexosides.

\section{Characterization of the solid from diethyl ether/hexane}

Analysis of the obtained dark yellow solid substance after precipitation with diethyl ether/hexane revealed the presence of quercetagetin dimethyl ether, eupalitin, an unknown compound with $\mathrm{m} / \mathrm{z}$ 457.21, residual quantity of 5-CQA, luteolin, homoeriodictyol-O-glucuronide and other unidentified compounds.

\section{Characterization of compounds in the ethyl acetate extract}

Flores Tanaceti were treated with a two-phase solvent system consisting of ethyl acetate and water ( $\mathrm{pH}$ of aqueous phase was 3). Water induced swelling of the plant particles and increased the porosity of the cell wall and thus facilitated the diffusion of extracted substances into the organic solvent. The use of ethyl acetate resulted in a significant improvement of the extraction of compounds and resulted in considerable reduction of the amount of used solvent. Through LC-HRMS we found that hydrophobic components were extracted more efficiently with ethyl acetate than by the traditionally used approach with alcohol-water mixtures. Hydrophilic components such as gluconic, quinic and citric acid were not detected in this extract. The above extraction method has many advantages over the traditionally used approach using alcohol-water mixtures. Separation of hydrophilic and relatively hydrophobic components is accomplished in a single step and the organic phase was practically free of tannins. 


\section{Antitumor activity}

Tansy extracts and fractions were tested in vitro on a panel of 9 cell lines - 1 control and 8 tumor lines. Antitumor activity was determined after $72 \mathrm{~h}$ of incubation with the respective substances at concentrations from 10 to $5000 \mu \mathrm{g} / \mathrm{mL}$. The observed antiproliferative effect showed a doseresponse sigmoidal curve (data not shown). Using these curves we calculated the $\mathrm{IC}_{50} \pm \mathrm{SD}$ values (Table 2). For the control MCF-10A cell line, the lowest toxicities were observed with CETV, EACE and EAFT $\left(\mathrm{IC}_{50}=875 \pm 21.9 \mu \mathrm{g} / \mathrm{mL}, 438 \pm 16.8\right.$ $\mu \mathrm{g} / \mathrm{mL}$ and $174.1 \pm 8.4 \mu \mathrm{g} / \mathrm{mL}$, respectively). In tumor cells, the lowest $\mathrm{IC}_{50}$ was observed with the DCHAS, DEHS, EAFT $\left(\mathrm{IC}_{50}=18 \pm 3.4 \mu \mathrm{g} / \mathrm{mL}\right.$ for $\mathrm{H} 1299, \mathrm{IC}_{50}=29 \pm 2.1 \mu \mathrm{g} / \mathrm{mL}$ for MDA-MB-231 $\mu \mathrm{g} / \mathrm{mL}$ and $\mathrm{IC}_{50}=22.8 \pm 1 \mu \mathrm{g} / \mathrm{mL}$ for MCF-7 respectively). The highest selectivity index $\left(\mathrm{IC}_{50}\right.$ of MCF-10A $/ \mathrm{IC}_{50}$ of the tumor cell line) was observed with EACE and EAFT (Fig. 2).

The highest selectivity index was found for MCF-7 with EAFT (7.6) and for the same cell line with EACE (4.71). Analysis of the obtained results revealed that the EAFT from $T$. vulgare exhibited the highest potential as an antitumor agent, followed by the EACE. From the above results becomes evident that ethyl acetate extracts contain substances bearing the highest selective activity towards tumor cells. On the other hand, those substances are obviously concentrated in tansy flowers.

Table 2. $\mathrm{IC}_{50}$ values obtained by MTT assay for extracts and fractions of $T$. vulgare after $72 \mathrm{~h}$ of treatment. Data are expressed as mean \pm SD values of three independent experiments, each made in triplicate.

\begin{tabular}{cccccc}
\hline \multirow{2}{*}{ Cell lines } & \multicolumn{5}{c}{${\text { Mean } \mathrm{IC}_{50} \pm \mathrm{SD}(\mu \mathrm{g} / \mathrm{mL})}$} \\
\cline { 2 - 6 } & CETV $^{\mathrm{a}}$ & EACE $^{\mathrm{b}}$ & DCHAS $^{\mathrm{c}}$ & DEHS $^{\mathrm{d}}$ & EAFT $^{\mathrm{e}}$ \\
\hline MCF-10 & $875 \pm 21.9$ & $438 \pm 16.8$ & $53 \pm 6.4$ & $36 \pm 1.9$ & $174.1 \pm 8.4$ \\
MCF-7 & $380 \pm 13.6$ & $93 \pm 5.7$ & $294 \pm 19.3$ & $91 \pm 7$ & $22.84 \pm 1.0$ \\
MDA-MB-231 & $943 \pm 12.8$ & $443 \pm 21.9$ & $47 \pm 11$ & $29 \pm 2.1$ & $60.6 \pm 0.7$ \\
H1299 & $410 \pm 1.6$ & $121 \pm 1.7$ & $18 \pm 3.4$ & $40 \pm 5.2$ & $60.5 \pm 0.6$ \\
A549 & $391 \pm 5.2$ & $117 \pm 5.7$ & $199 \pm 15.9$ & $91 \pm 8.1$ & $35.6 \pm 1.8$ \\
HeLa & $483.9 \pm 26.4$ & $227.1 \pm 2.2$ & $473.6 \pm 5.1$ & $203.7 \pm 10.4$ & $57.46 \pm 2.0$ \\
HepG2 & $453.7 \pm 7.4$ & $177.9 \pm 18.9$ & $297.7 \pm 12.4$ & $160.3 \pm 6.6$ & $73.5 \pm 2.4$ \\
HT-29 & $329.7 \pm 16.2$ & $128.4 \pm 6.5$ & $153 \pm 9.8$ & $193.3 \pm 15.1$ & $42.5 \pm 3.1$ \\
PC3 & $231.9 \pm 9.6$ & $101.2 \pm 8.0$ & $195 \pm 5.0$ & $124.6 \pm 7.8$ & $37.4 \pm 1.2$ \\
\hline
\end{tabular}

${ }^{\mathrm{a}} \mathrm{CETV}$ - crude extract of T. vulgare; ${ }^{\mathrm{b}} \mathrm{EACE}$ - ethyl acetate fraction from the crude extract of T. vulgare; ${ }^{\mathrm{c} D C H A S}-$ dicyclohexylammonium salts fraction; ${ }^{\mathrm{d} D E H S}$ - solid substance from diethyl ether/hexane separation; ${ }^{\mathrm{e}} \mathrm{EAFT}-\mathrm{ethyl}$ acetate extract from Flores Tanaceti.

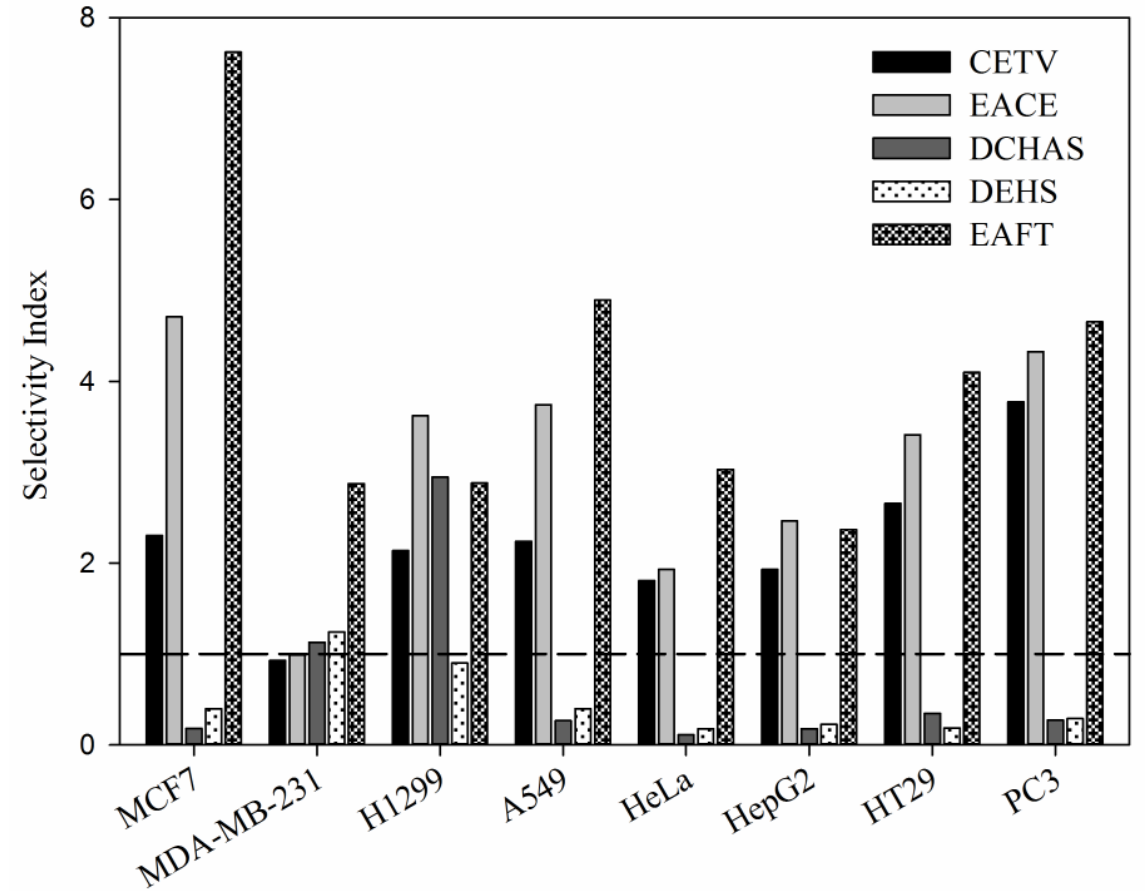

Fig. 2. The selectivity index (SI) of tumor cell lines obtained using $\mathrm{IC}_{50}$ values. SI represents $\mathrm{IC}_{50}$ for $\mathrm{MCF}-10 \mathrm{cell}$ line $/ \mathrm{IC}_{50}$ for cancerous cell lines, after $72 \mathrm{~h}$ of treatment. 
A. M. Vasileva et al.: In vitro study on the antitumor activity of Tanacetum vulgare L. extracts

According to a previous study [8] the main antitumor activity of $T$. vulgare flowers in MCF-7 cell line was demonstrated by the chloroform fraction. In our study we used an ethyl acetate extract from tansy flowers which contained a different set of compounds. Our results showed that EAFT had the highest antitumor activity and selectivity towards MCF-7 and other tumor cell lines in comparison to the control MCF-10A cell line (Table 2 and Fig. 2). Further study of individual compounds in this ethyl acetate extract should be conducted to identify the main active substance(s) exhibiting antitumor properties.

Acknowledgement: This work is financially supported by the Medical University-Sofia, Scientific Fund, Grant № D-129/2018 and the National Science Fund of the Bulgarian Ministry of Education and Science, Grant № T02/25 2014.

\section{REFERENCES}

1. M. R Goyal, A. O Ayeleso (eds.), Bioactive Compounds of Medicinal Plants: Properties and Potential for Human Health, CRC Press, 2018.

2. G. M. Cragg, J. M Pezzuto, Med Princ Pract., 25 (Suppl. 2), 41 (2016).

3. R. Arpita, A. Shruti, B. Navneeta, J. Plant Sci. Agric. Res., 2(1), 008 (2017).

4. K. Pandey, S. Rizvi, Oxid. Med. Cell Longev. NovDec, 2 (5), 270 (2009).

5. C. G. Fraga (ed.), Plant phenolics and human health: biochemistry, nutrition and pharmacology, vol. 1, John Wiley \& Sons, 2009.
6. T. K. Lim (ed.), Edible medicinal and non-medicinal plants. Vol. 7, Flowers, Springer, 2014, p. 501.

7. Z. Gospodinova, G. Antov, S. Angelova, M. Krasteva, Int. J. Pharma. Sci., 4 (2), 468 (2014).

8. Z. Gospodinova, N. Bózsity, I. Ocsovszki, O. Orbán-Gyapai, M. Krasteva, I. Zupkó, Int. J. Pharma. Sci., 5 (2), 986 (2015).

9. R. Baranauskienè, R. Kazernavičiūtè, M. Pukalskienè, R. Maždžierienė, P. R. Venskutonis, Ind. Crops Prod., 60, 113 (2014).

10. N. Devrnja, B. Anđelković, S. Aranđelović, S. Radulović, M. Soković, D. Krstić-Milošević, M. Ristić, D. Ćalić, S. Afr. J. Bo., 111, 212 (2017).

11. A. Uehara, S. Akiyama, T. Iwashina, Nat. Prod. Commun., 10 (3), 403 (2015).

12. T. Mosmann, J. Immunol. Methods, 65 (1-2), 55 (1983).

13. M. N. Clifford, S. Knight, N. Kuhnert, J. Agric. Food Chem., 53 (10), 3821 (2005).

14. J. Y. Zhang, Q. Zhang, N. Li, Z.J. Wang, J.Q. Lu, Y.J. Qiao, Talanta, 104, 1 (2013).

15. M. Kajdžanoska, V. Gjamovski, S. Stefova, Maced. J. Chem. Chem. Eng., 29, 181 (2010).

16. J. Kang, W.E. Price, J. Ashton, L.C. Tapsell, S. Johnson, Food Chem., 211, 215 (2016).

17. I. Parejo, O. Jáuregui, F. Viladomat, J. Bastida, C. Codina, Rapid Commun. Mass Spectrom., 18 (23), 2801 (2004).

18. S. Li, Z. Lin, H. Jiang, L. Tong, H. Wang, S. Chen, J. Chromatogr. Sci., 54 (7), 1225 (2016).

19. E. J. Llorent-Martínez, G. Zengin, ML. Fernándezde Córdova, O. Bender, A. Atalay, R. Ceylan, A. Mollica, A. Mocan, S. Uysal, G.O. Guler, A. Aktumsek, Front Pharmacol., 8, 83 (2017).

20. I. Ignat, I. Volf, V.I. Popa, Food Chem., 126 (4), 1821 (2011). 


\title{
Air pollution monitoring with mosses in Western Rhodopes, Bulgaria
}

\author{
N.P. Gribacheva ${ }^{1 *}$, G.M. Gecheva ${ }^{2}$, V.M. Stefanova ${ }^{3}$ \\ ${ }^{1}$ Forest Research Institute, Bulgarian Academy of Sciences, Kliment Ohridski Blvd. 132, 1756 Sofia, Bulgaria, \\ ${ }^{2}$ University of Plovdiv, Faculty of Biology, Tsar Asen Str. 24, 4000 Plovdiv, Bulgaria \\ ${ }^{3}$ University of Plovdiv, Faculty of Chemistry, Tsar Asen Str. 24, 4000 Plovdiv, Bulgaria
}

Received January 17, 2018; Revised January 30, 2019

For the first time a complex study of the bioaccumulation of 22 macro- and microelements, as well as of 18 rare earth and radioactive elements in mosses was applied in the territory of Western Rhodopes. The research is a part of the international program (ICP Vegetation, UNECE) that has been carried out in most of the European countries since 1987. Certain local emitters like old and open mines, serpentines, as well as cross-border transmission were identified. In addition, highest concentrations of radioactive elements were registered in the area of an old uranium mine and most elevated sites, probably due to increased wet deposition. Both heavy metals and toxic elements, as well as radioactive elements maxima were lower than measured highest levels for Bulgaria and Europe. In such studies, inductively coupled plasma spectrometry (ICP-OES, ICP-MS) could be recommended.

Keywords: atmospheric deposition; trace elements; ICP-OES; ICP-MS; NAA

\section{INTRODUCTION}

In recent decades mosses have been successfully used as biomonitors of atmospheric deposition of heavy metals in Europe [1-3].

Western Rhodopes were selected as a research area because they cover a territory of low population density: $28.5 \mathrm{~km}^{2}$ [4], a poorly developed industry and protected areas (about $11 \%)$. At the same time, there is a significant number of old and open mines (Table 1).

Table 1. Geographical and economical characteristics of the sampling sites.

\begin{tabular}{|c|c|c|c|c|c|c|}
\hline \multirow[b]{2}{*}{ No } & \multirow[b]{2}{*}{ Sites } & \multicolumn{2}{|c|}{ Coordinates } & \multirow{2}{*}{$\begin{array}{c}\text { Altitude, } \\
\text { m a.s.l. }\end{array}$} & \multirow[b]{2}{*}{ Industry } & \multirow{2}{*}{$\begin{array}{l}\text { Environmental } \\
\text { protection of } \\
\text { the region }\end{array}$} \\
\hline & & $\mathbf{N}$ & $\mathbf{E}$ & & & \\
\hline 1 & Kiselchovo village & 41.53461 & 24.576694 & 1066 & $\begin{array}{l}\text { Old uranium } \\
\text { mine }\end{array}$ & Natura 2000 \\
\hline 2 & Luki town & 41.76622 & 24.809278 & 1000 & Mine works & Natura 2000 \\
\hline 3 & Rudozem town & 41.50292 & 24.855611 & 930 & Mine works & Natura 2000 \\
\hline 4 & Madan town & 41.46781 & 24.959278 & 788 & Mine works & Natura 2000 \\
\hline 5 & Erma reka village & 41.42085 & 25.036028 & 580 & Tailing pond & Natura 2000 \\
\hline 6 & Svetulka village & 41.56639 & 25.101583 & 705 & Background & \\
\hline 7 & Belite brezi & 41.57831 & 25.161667 & 930 & Background & \\
\hline 8 & $\begin{array}{c}\text { Tsankov kamak } \\
\text { Dam }\end{array}$ & 41.73703 & 24.419722 & 677 & Background & Natura 2000 \\
\hline 9 & Barutin village & 41.59006 & 24.148611 & 1040 & $\begin{array}{l}\text { Old uranium } \\
\text { mine }\end{array}$ & Natura 2000 \\
\hline 10 & $\begin{array}{c}\text { Goliam Beglik } \\
\text { Dam }\end{array}$ & 41.81478 & 24.130528 & 1560 & Background & Natura 2000 \\
\hline 11 & Batak Dam & 41.95022 & 24.147694 & 1143 & Background & Natura 2000 \\
\hline 12 & Yundola & 42.03417 & 23.907444 & 1070 & Background & Natura 2000 \\
\hline 13 & $\begin{array}{l}\text { Momchilovtsi } \\
\text { village }\end{array}$ & 41.65906 & 24.774694 & 1215 & Background & Natura 2000 \\
\hline 14 & Rozhen & 41.67001 & 24.735722 & 1430 & Background & \\
\hline 15 & Bachkovo village & 41.95001 & 24.868944 & 425 & Background & Natura 2000 \\
\hline
\end{tabular}

* To whom all correspondence should be sent: 
The combination of background and impacted sites, as well as the lack of data on pollutant deposition, determined the interest in a comprehensive study and the selection of the Western Rhodopes as a region for implementing the biomonitoring approach with mosses.

\section{MATERIALS AND METHODS}

The sampling net includes fifteen sites in the region of Western Rhodopes $\left(8732 \mathrm{~km}^{2}\right)$ : two sites near to old uranium mines (No 1, No 9, Table 1), three in the range of lead-zinc mines (No 2, No 3, No 4), one to a tailing pond (No 5) and nine were accepted as background sites in the initial analysis of the potential sources of impact (No 6, No7, No 8, No 10 , No 11, No 12, No 13, No 14, No 15).

Sampling was carried out during the dry season of summer 2015. Hypnum cupressiforme Hedw. was collected at 14 sites. At one site (Barutin village, No 9), a representative sample could only be formed by Homalothecium lutescens (Hedw.) H.Rob.

The moss sampling followed the requirements of the ICP-V manual [5]: each sample consisted of up to 5 sub-samples after the standardized European methodology. Sampling, transport, storage and pre-treatment followed the methodological recommendations of the European Program [6].

The treatment of the moss samples included the following procedures. The samples were carefully cleaned from mechanical particles and other organic material, dried at $40^{\circ} \mathrm{C}$ and wet-ashed. About $1 \mathrm{~g}$ of moss material was treated with nitric acid (65\%) overnight, followed by addition of $2 \mathrm{ml}$ portions of hydrogen peroxide. Samples were sealed and irradiated in Milestone Ethos One microwave digestion system. A portion of moss reference material (M2 or M3) was digested together with every sample series and corresponding blank samples were prepared as well.

The elements $\mathrm{P}, \mathrm{K}, \mathrm{Ca}, \mathrm{S}, \mathrm{Na}, \mathrm{Mg}, \mathrm{Mn}, \mathrm{Fe}, \mathrm{Al}$, $\mathrm{Zn}, \mathrm{Cu}, \mathrm{Pb}$ and $\mathrm{Sr}$ were determined by inductively coupled plasma atomic emission spectrometry (ICP-OES) using iCAP 6300 Duo, Thermo Scientific, carried out in the Department of Analytical and Computer Chemistry, Faculty of Chemistry, University of Plovdiv. The elements Cr, $\mathrm{Co}, \mathrm{Cd}, \mathrm{V}, \mathrm{Ni}, \mathrm{As}, \mathrm{Hg}, \mathrm{Se}$ and $\mathrm{Sb}$ were determined by ICP-MS (Agilent 7700).

The calibration solutions for both methods of analysis (ICP-OES and ICP-MS), were prepared by appropriate dilution of Merck stock solutions: ICP multi-element standard solution IV (23 elements in dilute nitric acid, $1000 \mathrm{mg} \mathrm{L}^{-1}$ Certipur () and respectively for ICP-MS multi-element standard solution VI (30 elements in dilute nitric acid Certipur $($ ). For the ICP-OES analysis spectral-free analytical lines of the following elements were selected: $\mathrm{Na}(589.592 \mathrm{~nm}) ; \mathrm{K}(766.490 \mathrm{~nm}) ; \mathrm{Mg}$ $(285.213 \mathrm{~nm}) ; \mathrm{Ca}(422,673 \mathrm{~nm}) ; \mathrm{Zn}(202.548 \mathrm{~nm})$; Mn (257,610 nm); Fe (238,204 nm); Al (167.079 $\mathrm{nm}) ; \mathrm{Cu}(324.754 \mathrm{~nm}) ; \mathrm{Sr}(421.552 \mathrm{~nm}) ; \mathrm{Pb}$ $(220.353 \mathrm{~nm})$; P $(177.495 \mathrm{~nm})$ and $\mathrm{S}(182.034 \mathrm{~nm})$. Measurements were performed by axial plasma monitoring.

Two measurement modes were used to determine trace elements by ICP-MS. In the standard mode (without collision gas), the signals of ${ }^{51} \mathrm{~V},{ }^{111} \mathrm{Cd}$ and ${ }^{202} \mathrm{Hg}$ were measured. A collision cell (operating with 4,8 $\mathrm{ml} . \mathrm{min}^{-1} \mathrm{He}$ ) was applied to eliminate spectral matrix-induced polyatomic interferences for determination of ${ }^{52} \mathrm{Cr},{ }^{59} \mathrm{Co},{ }^{62} \mathrm{Ni}$, ${ }^{75} \mathrm{As},{ }^{78} \mathrm{Se}$ and ${ }^{121} \mathrm{Sb}$. Rh was added to all samples and calibrants as an internal standard for dynamic correction of the non-spectral matrix effect during ICP-MS analysis.

For quality assurance purposes moss reference materials $\mathrm{M} 2$ and $\mathrm{M} 3$ were applied [7, 8]. All concentrations were presented as $\mathrm{mg} \mathrm{kg}^{-1}$ dry weight.

The elements $\mathrm{Na}, \mathrm{Mg}, \mathrm{Al}, \mathrm{Cl}, \mathrm{K}, \mathrm{Ca}, \mathrm{Sc}, \mathrm{Ti}, \mathrm{V}$, $\mathrm{Cr}, \mathrm{Mn}, \mathrm{Fe}, \mathrm{Ni}, \mathrm{Co}, \mathrm{Zn}, \mathrm{I}, \mathrm{Ba}, \mathrm{Cs}, \mathrm{La}, \mathrm{Ce}, \mathrm{Sm}, \mathrm{Tb}$, $\mathrm{Tm}, \mathrm{Hf}, \mathrm{Ta}, \mathrm{W}, \mathrm{Th}, \mathrm{U}$ were determined by neutron activation analysis (NAA). The analysis was performed at the pulsed fast reactor IBR-2 at the Frank Laboratory of Neutron Physics, Joint Institute for Nuclear Research, Dubna, Moscow Region, Russia. At the laboratory the samples were cleaned from extraneous plant material and airdried to constant weight at $30 \div 40^{\circ} \mathrm{C}$ for $48 \mathrm{~h}$. The samples were not washed and not homogenised. The concentrations are presented as $\mathrm{mg} \mathrm{kg}^{-1}$ dry weight.

Principal component analysis (PCA) in programme CANOCO was used to study relationships between the different elements in each site [9]. The element concentrations were divided by their standard deviation and PCA was speciescentered. The spatial trends were assessed. The maps were produced using ArcMAP, part of ArcGIS, an integrated geographical information system (GIS) and display the mean element concentration per $50 \times 50 \mathrm{~km}^{2}$ EMEP grid cell $[8$, $10]$.

\section{RESULTS AND DISCUSSION}

From the group of macroelements in moss tissues $\mathrm{Ca}, \mathrm{K}, \mathrm{Al}$ and $\mathrm{P}$ dominated, while $\mathrm{Pb}$ was in the highest amount as microelement. The difference 
N.P. Gribacheva et al.: Air pollution monitoring with mosses in Western Rhodopes, Bulgaria

between $\mathrm{Pb}$ levels in the regions of open and old mines (No 1-5; No 9) in comparison with more pristine sites was more than 60 times, while for $\mathrm{Cd}$ it was 5 times (Fig. 1).

PCA showed that the first axis (eigenvalue 0.420) correlated with 12 macro- and microelements, while the second axis was positively correlated $(0.200)$ with macroelements $\mathrm{K}$, $\mathrm{Mg}, \mathrm{Mn}, \mathrm{P}$ and $\mathrm{S}$. In the lower left part of the ordination plot were located sites with significant anthropogenic impact (No 2-5; No 15) and increased atmospheric deposition, in particular of strontium, lead, cadmium and zinc (Fig. 2) Additionally a serpentine small spot - site № 6, in the region of Svetulka village, joined this group of hotspots. Two potential background sites (No 10, 14) with highest altitude, located in a similar longitude line, were affected by transboundary pollution, also known as the Southeastern European gradient (at the upper left part of the ordination). With lowest pollutant deposition were two sampling sites in the western part of the sampling net, near Barutin and Batak villages (upper right of Fig. 2). Comparison with data for the same elements and sites from the previous year [4] showed that in 2015 higher levels were found for 4 elements: $\mathrm{Co}, \mathrm{Fe}, \mathrm{Hg}$ and $\mathrm{Ni}$. a)

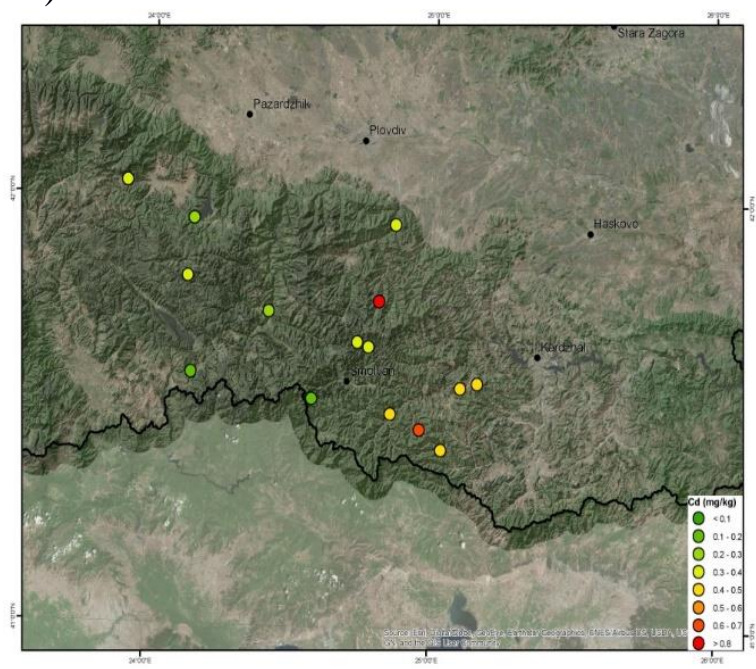

b)

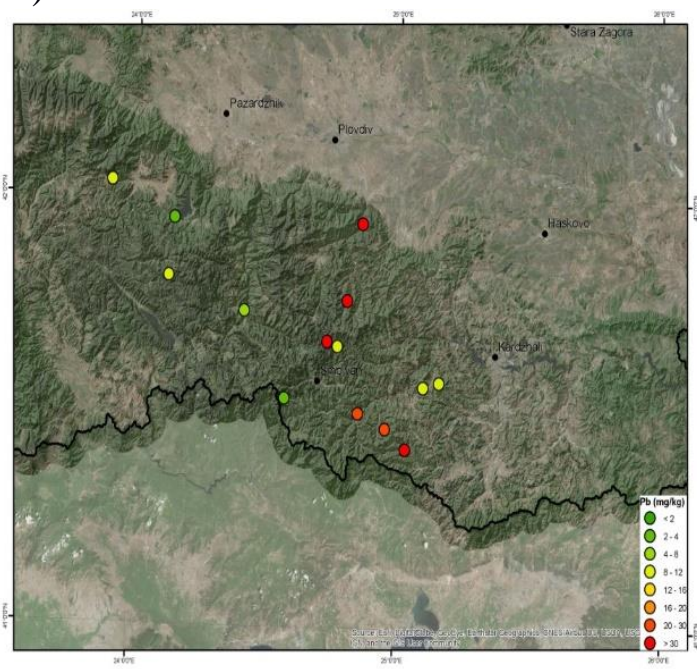

Fig. 1. Element concentration in mosses in Western Rhodopes, Bulgaria for a) cadmium, b) lead.

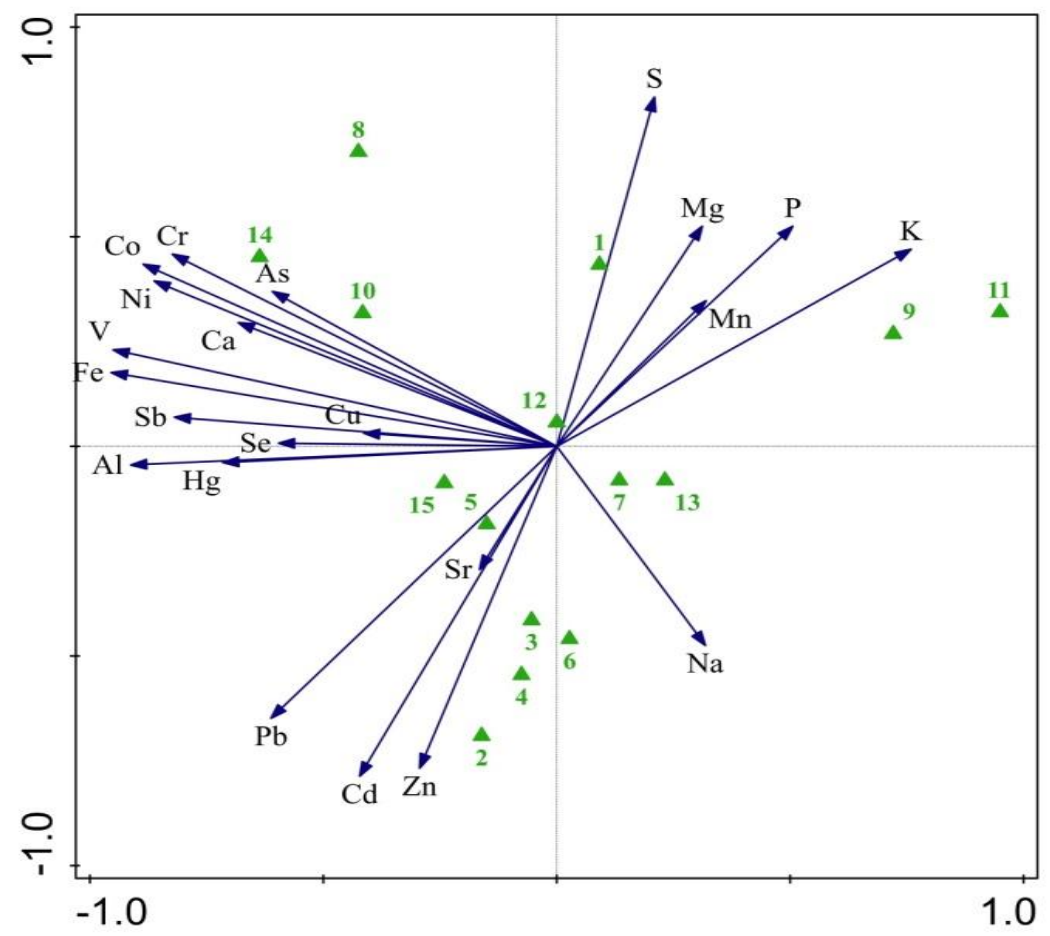

Fig. 2. PCA-ordination diagram of studied sites and analysed elements. 
N.P. Gribacheva et al.: Air pollution monitoring with mosses in Western Rhodopes, Bulgaria

Comparison with heavy metals and toxic elements ( $\mathrm{Al}, \mathrm{As}, \mathrm{Cd}, \mathrm{Cr}, \mathrm{Cu}, \mathrm{Fe}, \mathrm{Ni}, \mathrm{Pb}, \mathrm{V}, \mathrm{Zn}$ ) deposition in Europe [3] illustrated lower element accumulation in mosses in Western Rhodopes. Nevertheless, elements' medians from the studied region were higher than medians for both Bulgaria and Europe, except for copper.

Among the rest of the analyzed elements, more pronounced was the variation of U (32 times), followed by Hf (23 times) and Sc (19 times), Cs and $\mathrm{W}$ (16 times), Th (14 times), Sm and Tb (13 times), Ta (12 times), La (10 times), No Tm (8 times), $\mathrm{Ti}, \mathrm{Ba}$, и $\mathrm{Ce}$ (7 times), $\mathrm{Cl}$ (4 times), $\mathrm{Br}$ and $\mathrm{Rb}$ (5 times) and I (3 times) (Table 2). A positive correlation was assessed between bioaccumulation of Th and $U\left(R^{2}=0.957\right)$.

Table 2. Minimum, maximum concentrations and median of radioactive and rare earth elements.

\begin{tabular}{|c|c|c|c|}
\hline $\begin{array}{l}\text { Element, } \\
\mathrm{mg} \mathrm{kg}^{-1}\end{array}$ & Minimum & Maximum & Median \\
\hline $\mathrm{Cl}$ & 50.2 & 211 & 100 \\
\hline $\mathrm{Sc}$ & 0.042 & 0.83 & 0.29 \\
\hline $\mathrm{Ti}$ & 25 & 303 & 106 \\
\hline $\mathrm{Br}$ & 0.90 & 4.4 & 2.5 \\
\hline $\mathrm{Rb}$ & 5.2 & 26.5 & 9.6 \\
\hline $\mathrm{I}$ & 0.63 & 1.6 & 1.2 \\
\hline $\mathrm{Ba}$ & 15.5 & 103 & 44.8 \\
\hline Cs & 0.05 & 0.81 & 0.36 \\
\hline $\mathrm{La}$ & 0.23 & 2.2 & 0.83 \\
\hline $\mathrm{Ce}$ & 0.6 & 4.2 & 1.77 \\
\hline $\mathrm{Sm}$ & 0.03 & 0.38 & 0.134 \\
\hline $\mathrm{Tb}$ & 0.004 & 0.05 & 0.018 \\
\hline $\mathrm{Tm}$ & 0.004 & 0.03 & 0.014 \\
\hline Hf & 0.013 & 0.3 & 0.106 \\
\hline $\mathrm{Ta}$ & 0.006 & 0.07 & 0.024 \\
\hline $\mathrm{W}$ & 0.02 & 0.32 & 0.093 \\
\hline Th & 0.05 & 0.68 & 0.21 \\
\hline $\mathrm{U}$ & 0.012 & 0.38 & 0.073 \\
\hline
\end{tabular}

Maxima of 7 of the above elements ( $\mathrm{Sc}, \mathrm{Ti}, \mathrm{Cs}$, $\mathrm{Ce}, \mathrm{Sm}, \mathrm{Tb}$ and $\mathrm{W}$ ) were found in mosses from Golyam Beglik Dam, 6 in the area of an old uranium mine (U, Th, Ba, I, Rb, Br) and 3 (Tm, Hf and $\mathrm{Ta}$ ) in the potential background Rozhen region. High values in mosses in both potential background sites (Golyam Beglik and Rozhen) could be linked to increased radioactive deposition with elevation due to higher wet deposition.

Maximum measured $U$ concentration was 17 times lower than $U$ values for Bulgaria, detected in $2005\left(6.23 \mathrm{mg} \mathrm{kg}^{-1)}[11]\right.$ and about 4 times lower than maximum for neighboring FYRM. High $U$ values were registered also in samples from Rozhen $\left(0.30 \mathrm{mg} \mathrm{kg}^{-1}\right)$ and Tsankov kamak Dam $(0.25 \mathrm{mg}$ $\left.\mathrm{kg}^{-1}\right)$.

Thorium maximum level was 34 times lower than the maximum value for the country of $23 \mathrm{mg}$ $\mathrm{kg}^{-1}$ and 11 times lower than in FYRM $\left(7.6 \mathrm{mg} \mathrm{kg}^{-}\right.$ $\left.{ }^{1}\right)$.

Caesium maximum in the studied region was 7 times lower in comparison with the maximum for Bulgaria (5.71 mg kg-1) and 22 times lower than the maximum in Serbia (18.2 $\left.\mathrm{mg} \mathrm{kg}^{-1}\right)$ [10].

Highest concentrations of $\mathrm{U}$ and $\mathrm{Th}$ were found in moss samples from an old uranium mine near Kiselchovo village; maximum of Cs was analyzed determined in moss tissues at the region of Golyam Beglik Dam. Minimum levels for cesium were found in the Barutin village; for thorium and uranium in the samples from Batak Dam.

\section{CONCLUSIONS}

Presence of natural geochemical anomalies for Western Rhodopes, mainly associated with accumulation of minerals, was illustrated by hotspots near open way of mining and old mines. Despite lower maximum concentration in the studied region compared to those for Bulgaria and Europe in 2010, medians of all 10 heavy metals and toxic elements except $\mathrm{Cu}$ were higher.

Measured maximum values of $\mathrm{U}$, Th and Cs were lower than maximum levels for Bulgaria and neighboring countries.

Based on the results, inductively coupled plasma techniques (ICP-OES, ICP-MS) could be recommended because they permit to analyze macroelements with an important physiological role such as $\mathrm{Mg}, \mathrm{Na}$ and micro elements as $\mathrm{Sb}$ and $\mathrm{Sr}$ with sufficient accuracy.

Annual sampling at each sampling point for three consecutive years could be recommended in regions with lack of previous data, significant elevation changes and with local emission sources. This will ensure analysis of tendencies and will reveal average pollutant levels.

Acknowledgment: We would like to thank Prof. Marina Frontasyeva, United Institute for Nuclear Research (JINR), Dubna, Russian Federation, for the conducted NAA of moss samples.

\section{REFERENCES}

1. H.G. Zechmeister, K. Grodzinkska, G. SzarekLukaszewska. Principles, concepts and applications. Elsevier, Amsterdam, The Netherlands, ISBN 0-08044177-7, 2003, p. 329.

2. H. Harmens, D.A. Norris, G.R. Koerber, A. Buse, E. 
N.P. Gribacheva et al.: Air pollution monitoring with mosses in Western Rhodopes, Bulgaria

Steinnes, Å. Rühling. Atmos. Environ., 41, 6673 (2007).

3. H. Harmens, D.A. Norris, D. Cooper, J. Hall and the participants of the moss survey. Programme Coordination Centre for the ICP Vegetation, Centre for Ecology and Hydrology, Bangor, UK. http://icpvegetation.ceh.ac.uk, 2008.

4. G. Gecheva, N. Gribacheva, L. Yurukova†, V. Stefanova, V. Kmetov, M. Frontasyeva, G. Popgeorgiev. Atmospheric pollution assessment with mosses in Western Rhodopes, Bulgaria, $J$. BioSci. Biotechnol., 5(2), 125 (2016).

5. M. Frontasyeva, H. Harmens, the participants of the ICP Vegetation, 2014, Heavy metals, nitrogen and POPs in European mosses: 2015 survey, Monitoring Manual.

6. ICP Vegetation (2010), Heavy metals in European mosses: 2010 survey. Monitoring manual, ICP Vegetation Programme Coordination Centre, CEH Bangor, UK. http://icpvegetation.ceh.ac.uk.

7. E. Steinnes, A. Rühling, H. Lippo, A. Mäkinen, Accred. Qual.Assur., 2, 243 (1997).

8. H. Harmens, D.A. Norris, E. Steinnes, E. Kubin, J. Piispanen, R. Alber, Y. Aleksiayenak, O. Blum, M. Coşkun, M. Dam, L. De Temmerman, J.A.
Fernández, M. Frolova, M. Frontasyeva, L. González-Miqueo, K. Grodzinska, Jeran, S. Korzekwa, M. Krmar, K. Kvietkus, S. Leblond, S. Liiv, B. Mankovská, R. Pesch, Å. Rühling, J.M. Santamaria, W. Schröder, Z. Spiric, I. Suchara, L Thöni, V. Urumov, L. Yurukova, H.G. Zechmeister, Environ. Pollut., 158, 3144 (2010).

9. C.J.F. Ter Braak, P. Smilauer. CANOCO reference manual and CanoDraw for Windows user's guide: software for canonical community ordination (version 5). Microcomputer Power, Ithaca, New York, 2002.

10. H. Harmens, D.A. Norris, D. Cooper, G. Mills, E. Steinnes, E. Kubin, L. Thöni, J.R. Aboal, R. Alber, A. Carballeira, M. Coșkun, L. De Temmerman, M. Frolova, L. Gonzáles-Miqueo, Z. Jeran, S. Leblond, S. Liiv, B. Mankovská, R. Pesch, J. Poikolainen, Å. Rühling, J. M. Santamaria, P. Simonèiè, W. Schröder, I. Suchara, L. Yurukova, H. G. Zechmeister, Environ. Pollut., 159, 2852 (2011).

11. S. Marinova, L. Yurukova, M. Frontasyeva, E. Steinnes, L. Strelkova, A. Marinov, A. Karadzinova, Ecol. Chem. Eng., 17, 37 (2010).

12. B. Smodiš, A. Bleise, J. Radioanal. Nucl. Chem., 271, 269 (2007). 


\title{
Optimization of high ultrasound-assisted extraction (INEFU) of active components from natural materials by response HPLC-PDA analysis
}

\author{
S.B. Seo*, Y.M. Kim \\ SKEDERM Cosmetic R\&D Center, 240 Teheran-ro, Gangnam-gu, Seoul, South Korea 06221
}

Received January 16, 2019; Revised, January 31, 2019

\begin{abstract}
High ultrasonic-assisted extraction technology (INEFU) was employed to extract the active components from natural materials (green coffee beans, Citrus madurensis, Centella asiatica, Laminaria Japonica). The extraction conditions were optimized by a response surface method and Box-Behnken design. The active component yields were obtained under the optimum parameters: ultrasound power (1800 watts), ultrasonication time, and particle size. After INEFU of natural materials (green coffee beans, Citrus madurensis, Centella asiatica and Laminaria Japonica) the products were analyzed with high performance liquid chromatography (HPLC). HPLC analysis showed that the four natural materials were composed of different combinations of vitamin $\mathrm{C}$, polyphenols, chlorogenic acid, caffein, caffeic acid, asiaticoside and alginic acid. In addition, the INEFU results showed that natural materials can yield more active components during a simulated extraction process.
\end{abstract}

Keywords: INEFU, Natural materials, Green coffee beans, Citrus madurensis, Centella asiatica, Laminaria Japonica, HPL

\section{INTRODUCTION}

Currently, ultrasound-assisted extraction has been applied to effectively extract natural components from different kinds of materials $[1,2]$. Compared with the conventional hot water extraction method, ultrasound-assisted extraction can enhance the extraction yield, save operation time and streamline the operation process [3]. Response surface method is a practical statistical method used for optimizing processing parameters [4]. The major advantage of Response surface method is that it can reduce the number of experimental groups and investigate the relation between the response and variables [5]. Therefore, Response surface method has been widely applied to accelerate and optimize the operation process for saving time, energy, and raw materials [6]. Green coffee beans have been commonly used in functional foods or medicines, which was ascribed to the valuable functional components [7]. Green coffee beans are rich in caffeins, polyphenols, and trace elements [8]. Phenols are a class of plant compounds with the potential to eliminate free radicals because of their stable structure after freeradical capture as hydroxycinnamic acid, with chlorogenic acid and caffeic acid being the most abundant in coffee $[9,10]$.

Moreover, these compounds, found mainly in green coffee, are important and biologically active dietary polyphenols. [11]. Calamondin orange (Citrus madurensis, Citrofortunella microcarpa), which belongs to Citrus Metacitrus
Pseudofortunella, ranges from tropical to subtropical areas, including China, Philippines, Central America, Japan, and Hawaii [12]. Calamondin orange contains vitamin $\mathrm{C}$, including tangeretin, and sinensetin, in large quantities in the peel part of this citrus orange [13]. Pure juice extracted from calamondin orange resembles the juice from shiikuwasha in color and flavor $[14,15]$. Moreover, the adulteration of fruit juices has been a serious economic problem. This problem has been detrimental to consumers and the food industry for many years [16]. Centella asiatica has active compounds such as pentacyclic triterpenes and has been used in treating small wounds, burns, psoriasis, and scleroderma [17]. Centella asiatica is also called guta kola. The mechanism of guta kola action involves increasing fibroblast production, synthesis of collagen and fibronectin content [18]. Brinkhaus et al. reported that application of Centella asiatica showed a beneficial effect on reducing the progression of cellulite, with significant improvement in $85 \%$ of the participants with no adverse reactions [19]. Moreover, researchers reported that it can improve the tensile strength of newly formed skin and reduces the inflammatory phase of hypertrophic scars and keloids. Laminaria Japonica, also called kelp in Chinese medicines, is a kind of large marine plant [20]. L. japonica, a brown alga, is very popular in East Asia due to its unique flavor, taste and biological activities, in which polysaccharides are the major active components, e.g. alginates,

* To whom all correspondence should be sent:

(C) 2019 Bulgarian Academy of Sciences, Union of Chemists in Bulgaria

E-mail: chrismoon@ classys.com 
S.B. Seo, Y.M. Kim: Optimization of high ultrasound-assisted extraction (INEFU) of active components ...

fucoidan, and laminarian [21]. By comparing 4 types of edible seaweeds, it is found that $L$. japonica has potential anti-diabetic and antiinflammatory effects in diet-induced obesity [22,23]. Some recent studies showed that $L$. japonica has many beneficial effects to the human health, such as antiviral, anticoagulant, anticancer, hypolipidemic, anti-inflammatory and immunomodulatory activities [24].

In this paper, high ultrasonic-assisted extraction technology (INEFU) of the natural active components of four natural materials was investigated. The effects of four variables (extraction time, extraction temperature, ultrasonic power, and liquid-to-solid ratio) on the yields of four natural materials were optimized by Response surface method. After HPLC-PDA analysis of green coffee beans, Citrus madurensis, Centella asiatica, and Laminaria Japonica, INEFU was applied to accelerate and optimize the operation process for saving time, energy, and raw materials

\section{MATERIALS AND METHODS}

\section{Materials}

Green coffee beans, Citrus madurensis, Centella asiatica and Laminaria Japonica were purchased from G-market (Gmarket, South Korea). Before extraction, the green coffee beans, Citrus madurensis, Centella asiatica and Laminaria Japonica were dried in a forced convection oven dry box (Jeio Co., Ltd., South Korea) at $60{ }^{\circ} \mathrm{C}$, ground into powder in a universal high-speed smashing machine (Jeio Ltd., South Korea), and screened through a 80 mesh sieve. Dry powder of Green coffee bean, Citrus madurensis, Centella asiatica and Laminaria Japonica was stored for further natural active components extraction. Analytical standards of different compounds (vitamin C, polyphenol mixer, chlorogenic acid, caffein, caffeic acid, asiaticoside, and alginic acid) were purchased from Sigma Co. (USA). HPLC solvents and chemicals were purchased from Daejungchem Ltd. (Shiheung, South Korea). All other reagents were of analytical grade.

\section{High ultrasonic and heat-assisted extraction of the active components}

Active component extraction with high ultrasonic assisted extraction was performed in a high ultrasonic cartriage (HUC, Classys Co., Ltd., Seoul, South Korea). The dandelion powder (5.0 g) was placed into a beaker, soaked with deionized water at a ratio of 1:20. The $\mathrm{pH}$ value was adjusted to $5( \pm 0.05)$. Then the mixture was placed in a high ultrasonic cartridge (INEFU: high ultrasonic cartridge power ranging from 1800 to 2000 watts) for a certain time (extraction time $42 \mathrm{~min}$ ) at $35^{\circ} \mathrm{C}$, and heat-assisted extraction (HAE) for a certain time (extraction time $42 \mathrm{~min}$ ) at $60{ }^{\circ} \mathrm{C}$. The extract was concentrated and then precipitated with $80 \%$ $(v / v)$ ethanol. The precipitate was collected by centrifugation (3000 rpm for $5 \mathrm{~min}$ ) and washed by anhydrous ethanol and acetone. Then the crude active components were prepared after freeze drying process. The extraction yield of crude active components $Y(\%)$ was calculated according to the following equation:

$$
\mathrm{Y}(\%)=((\mathrm{cXV}) / \mathrm{m}) \times 100
$$

where $c(\mathrm{mg} / \mathrm{mL})$ represents the concentration of the active components solution, $V(\mathrm{~mL})$ is the volume of solution, and $m(\mathrm{mg})$ is the mass of powder.

\section{HPLC}

HPLC instrument such as ultraviolet-PDA detector used a Shimadzu LC-6AD pump with DGU-20A5 degasser, communication module-20A, and PDA detector SPD-M20A with FRC-10A fraction collector (Kyoto, Japan).

Vitamin C: The HPLC was set at isocratic method at $254 \mathrm{~nm}$ with a reverse-phase ODS C18 column (LiChrospher ${ }^{\circledR} 100 \mathrm{RP}-18$; diameter, $5 \mu \mathrm{m}$ ) under a $20^{\circ} \mathrm{C}$-controlled column chamber. Rheodyne $^{\circledR}$ sample loops of $50 \mu \mathrm{L}$ capacity were used. The mobile phase was $0.05 \mathrm{M} \mathrm{KH}_{2} \mathrm{PO}_{4} / \mathrm{ACN}$ $(60: 40)$ at $\mathrm{pH} 6.8$. The flow rate of injection into the system of HPLC was $1 \mathrm{~mL} / \mathrm{min}$.

Polyphenol: The HPLC was set at $280 \mathrm{~nm}$ with a reverse-phase ODS C18 column (LiChrospher ${ }^{\circledR}$ $100 \mathrm{RP}-18$; diameter, $5 \mu \mathrm{m}$ ) under a $30^{\circ} \mathrm{C}$ controlled column chamber. Rheodyne ${ }^{\circledR}$ sample loops of $20 \mu \mathrm{L}$ capacity were used. The mobile phase consisted of $0.1 \%$ acetic acid (A) and $0.1 \%$ acetic acid in acetonitrile (B) filtered through a membrane filter $(0.2 \mu \mathrm{m})$ prior to use. A gradient program starting at $\mathrm{A}: \mathrm{B}(89: 5, \mathrm{v} / \mathrm{v})$ and ending at $\mathrm{A}: \mathrm{B}(65: 35, \mathrm{v} / \mathrm{v})$ was applied over $60 \mathrm{~min}$ at a flow rate of injection into the system of HPLC of 1 $\mathrm{mL} / \mathrm{min}$.

Chlorogenic acid, caffein, caffeic acid: The HPLC was set at isocratic method at $280 \mathrm{~nm}$ with a reverse-phase ODS C18 column (LiChrospher ${ }^{\circledR}$ $100 \mathrm{RP}-18$; diameter, $5 \mu \mathrm{m}$ ) under a $20^{\circ} \mathrm{C}$ controlled column chamber. Rheodyne ${ }^{\circledR}$ sample loops of $20 \mu \mathrm{L}$ capacity were used. The mobile phase was $\mathrm{MeOH} / 5 \mathrm{mM} \mathrm{KH} 2 \mathrm{PO}_{4}\left(\mathrm{pH} 2.5, \mathrm{H}_{3} \mathrm{PO}_{4}\right)$ (30:70). The flow rate of injection into the system of HPLC was $0.4 \mathrm{~mL} / \mathrm{min}$. 
S.B. Seo, Y.M. Kim: Optimization of high ultrasound-assisted extraction (INEFU) of active components ...

Asiaticoside: The HPLC was set at Photo diode array detector/UV detector with a reverse-phase ODS C18 column (LiChrospher ${ }^{\circledR} 100$ RP-18; diameter, $5 \mu \mathrm{m})$ under a $30^{\circ} \mathrm{C}$-controlled column chamber. Rheodyne ${ }^{\circledR}$ sample loops of $20 \mu \mathrm{L}$ capacity were used. The mobile phase consisted of $0.3 \%$ orthophosphoric acid (A)/ acetonitrile (B) filtered through a membrane filter $(0.2 \mu \mathrm{m})$ prior to use. A gradient program starting at A:B $(95: 5, \mathrm{v} / \mathrm{v})$ and ending at $\mathrm{A}: \mathrm{B}(5: 5, \mathrm{v} / \mathrm{v})$ was applied over 40 min at a flow rate of injection of $1.8 \mathrm{~mL} / \mathrm{min}$ into the system of HPLC.

Alginic acid: The HPLC was set at Photo diode array detector/UV detector with a reverse-phase ODS C18 column (LiChrospher ${ }^{\circledR} 100$ RP-18; diameter, $5 \mu \mathrm{m}$ ) under a $30^{\circ} \mathrm{C}$-controlled column chamber. Rheodyne ${ }^{\circledR}$ sample loops of $20 \mu \mathrm{L}$ capacity were used. The mobile phase, consisting of $0.5 \mathrm{~mL}$ phosphoric acid in 1-L distilled water adjusted to $\mathrm{pH} 7.00$ with sodium hydroxide, was applied over $30 \mathrm{~min}$ at a flow rate of $0.7 \mathrm{~mL} / \mathrm{min}$ into the system of HPLC.

\section{Data analysis}

The ratio of the peak area of the standard (vitamin C, polyphenol mixer, chlorogenic acid, caffein, caffeic acid, asiaticoside, and alginic acid) was used as the assay parameter. Peak area ratios were plotted against analyte concentrations, and standard calibration curves were obtained from least-squares linear regression analysis of the data. The linearity of the method was confirmed via evaluation of the calibration y-intercept and correlation coefficients.

\section{Statistical analysis}

All data were expressed as mean \pm standard deviation (SD) of three separate experiments. Design-Expert software (8.6 Statease Inc., Minneapolis, USA) was used for the experimental design and statistical analysis. Statistical significance was set at $\mathrm{p}<0.05$.

\section{RESULTS AND DISCUSSION}

\section{Verification of the INEFU and HAE models}

The suitability of the model equation was examined under the modified conditions: high ultrasound power(INEFU) of $1800 \mathrm{~W}$, extraction time of $42 \mathrm{~min}$, extraction temperature of $35^{\circ} \mathrm{C}$, and particle size of 80 mesh, and the final mean values obtained from the experiments were $18.11 \pm 0.42 \%$, $19.51 \pm 0.31 \%, 20.19 \pm 0.12 \%, 31.23 \pm 1.83 \%$, ( for green coffee beans, Citrus madurensis, Centella asiatica and Laminaria Japonica, respectively), which demonstrated that the model was suitable and precise for the actual extraction process. As shown in Table 1, different extraction methods including heat-assisted extraction (HAE) were also evaluated for comparison. Under the same extraction time, the active components of INEFU and HAE were $18.11 \pm 0.42 \%, 6.34 \pm 0.12 \%(\mathrm{n}=3$, green coffee beans), $19.51 \pm 0.31 \%, 8.34 \pm 0.14 \%$, $(\mathrm{n}=3$, Citrus madurensis), $20.19 \pm 0.12 \%, 9.21 \pm$ $0.91 \%(\mathrm{n}=3$, Centella asiatica $), 31.23 \pm 1.83 \%$, $19.24 \pm 1.09 \% \quad(\mathrm{n}=3$, Laminaria Japonica $)$, respectively. Apparently, INEFU was more efficient in comparison with HAE.

\section{Identification and quantification of vitamin $C$ in Citrus madurensis (Citrofortunella microcarpa)}

Analytes were tentatively identified in Citrus madurensis samples by our combining the information obtained with PDA detectors and by comparison with literature data. When possible, the identification of compounds was confirmed by comparison with standards commercially available. The UV-vis chromatograms of the INEFU and HAE extracts at a wavelength of $254 \mathrm{~nm}$ are shown in Fig. 1.

Table 1. Comparison of the active component yields by different extraction methods.

\begin{tabular}{ccccccc}
\hline \multirow{2}{*}{ Natural materials } & \multirow{2}{*}{$\begin{array}{c}\text { Extraction } \\
\text { methods }\end{array}$} & \multicolumn{3}{c}{$\begin{array}{c}\text { Extraction } \\
\text { conditions }\end{array}$} & \multirow{2}{*}{ The yield of components (\%) } \\
\cline { 3 - 5 } & & $\mathrm{A}$ & $\mathrm{B}$ & $\mathrm{C}$ & \\
\hline \multirow{2}{*}{ Green coffee beans } & INEFU & 1800 & 42 & 35 & $18.11 \pm 0.42 \%$ \\
& $\mathrm{HAE}$ & - & 42 & 60 & $6.34 \pm 0.12 \%$ \\
\hline \multirow{2}{*}{ Citrus madurensis } & INEFU & 1800 & 42 & 35 & $19.51 \pm 0.31 \%$ \\
& $\mathrm{HAE}$ & - & 42 & 60 & $8.34 \pm 0.14 \%$ \\
\multirow{2}{*}{ Centella asiatica } & INEFU & 1800 & 42 & 35 & $20.19 \pm 0.12 \%$ \\
& $\mathrm{HAE}$ & - & 42 & 60 & $9.21 \pm 0.91 \%$ \\
\hline \multirow{2}{*}{ Laminaria Japonica } & INEFU & 1800 & 42 & 35 & $31.23 \pm 1.83 \%$ \\
& HAE & - & 42 & 60 & $19.24 \pm 1.09 \%$ \\
\hline
\end{tabular}

A: Ultrasound power (watt); B: Extraction time (min); C: Extraction temperature $\left({ }^{\circ} \mathrm{C}\right)$. 
S.B. Seo, Y.M. Kim: Optimization of high ultrasound-assisted extraction (INEFU) of active components ...

The INEFU and HAE extracts of Citrus madurensis yielded similar qualitative HPLC profiles for each kind of sample. Vitamin C concentration is expressed as number of times for INEFU and HAE extract, and INEFU extracted 27 times more vitamin $\mathrm{C}$ than HAE for Citrus adurensis samples. Citrus madurensis juice usually contains vitamin $\mathrm{C}$, between 3\% (for INEFU extracts in Citrus madurensis) and $0.2 \%$ (for HAE extracts in Citrus madurensis) of the total concentration of detected analyte [25]. In addition, $\mathrm{HAE}$ extracts contained vitamin $\mathrm{C}$ at a very lower percentage than in INEFU extracts (range $0.12 \sim 0.23 \%$ ).

\section{Identification and quantification of polyphenols in green coffee beans}

Analytes were tentatively identified in green coffee beans samples by our combining the information obtained with PDA detectors and by comparison with literature data. When possible, the identification of compounds was confirmed by comparison with standards commercially available. The UV-vis chromatograms of the INEFU and HAE extract at a wavelength of $280 \mathrm{~nm}$ are shown in Fig. 2. Six phenolic compounds belonging to different classes were found, and six of them were tentatively identified. Among them, the following compounds were identified: sinapic aldehyde, Pcoumaric acids, rutin, feruric acid, epicatechin gallate and naringin. The INEFU and HAE extracts of the green coffee beans yielded similar qualitative HPLC profiles for each kind of sample. Polyphenols concentration is expressed as number of times for INEFU and HAE extract, and INEFU extracted 10.7 times (based on the feruric acid) more than HAE for green coffee beans samples. INEFU samples were quantitatively the richest ones in the phenolic compounds studied (medium total concentration of $121.1 \mathrm{mg} / \mathrm{g}$ ), whereas HAE samples were the poorest ones in the molecules of interest (medium total concentration of $0.5 \mathrm{mg} / \mathrm{mL}$ ) even though they had a large number of detected compounds.

Identification and quantification of chlorogenic acid, caffein and caffeic acid in green coffee beans

Analytes were tentatively identified in green coffee beans samples by our combining the information obtained with PDA detectors and by comparison with literature data. When possible, the identification of compounds was confirmed by comparison with standards commercially available. The UV-vis chromatograms of the INEFU and HAE extracts at a wavelength of $280 \mathrm{~nm}$ are shown in Fig. 3. The INEFU and HAE extracts of the 264 green coffee beans yielded similar qualitative HPLC profiles for each kind of sample. Green coffee beans concentration is expressed as number of times for INEFU and HAE extract, and INEFU extracted 2.2 times (based on the caffein), 2.1 times (based on the chlorogenic acid) more than HAE for green coffee beans. Also, caffeic acid was only detected for INEFU. Green coffee beans usually contain caffeine between 0.7 and $1 \%$, So, $80 \%$ of caffeine amounts from the green coffee beans can be extracted with INEFU methods. In addition, HAE extract contained caffein and chlorogenic acid at a very lower percentage than the INEFU extract.

\section{Identification and quantification of asiaticoside and madecassoside in Centella asiatica}

Analytes were tentatively identified in Centella asiatica samples by our combining the information obtained with PDA detectors and by comparison with literature data. When possible, the identification of compounds was confirmed by comparison with standards commercially available. The UV-vis chromatograms of the INEFU and HAE extracts at wavelengths of 254, $280 \mathrm{~nm}$ are shown in Fig. 4. The INEFU and HAE extracts of Centella asiatica yielded similar qualitative HPLC profiles for each kind of sample. Asiaticoside and madecassoside concentrations are expressed as $\mu \mathrm{g} / \mathrm{ml}$ for INEFU and HAE extract, and INEFU extracted $30 \mu \mathrm{g} / \mathrm{ml}$ (madecassoside) $43 \mu \mathrm{g} / \mathrm{ml}$ (asiaticoside) for Centella asiatica samples. Asiaticoside and madecassoside can be extracted at $38 \sim 50 \mu \mathrm{g} / \mathrm{ml}$ and $28 \sim 39 \mu \mathrm{g} / \mathrm{ml}$ when methanol is used as a solvent, when water solvent is hardly extracting. In addition, HAE extract contained asiaticoside and madecassoside at a very lower percentage than INEFU extract.

\section{Identification and quantification of alginic acid in Laminaria Japonica}

Analytes were tentatively identified in Laminaria Japonica samples by our combining the information obtained with PDA detectors and by comparison with literature data. When possible, the identification of compounds was confirmed by comparison with standards commercially available. The UV-vis chromatograms of the INEFU and HAE extracts at a wavelength of $200 \mathrm{~nm}$ are shown in Fig. 5. The INEFU and HAE extracts of Laminaria Japonica yielded similar qualitative HPLC profiles for each kind of sample. Alginic acid concentration is expressed as number of times for INEFU and HAE extract, and INEFU extracted 1.72 times more than HAE for Laminaria Japonica samples. Laminaria Japonica juice usually mainly contains alginic acid. 
S.B. Seo, Y.M. Kim: Optimization of high ultrasound-assisted extraction (INEFU) of active components ...
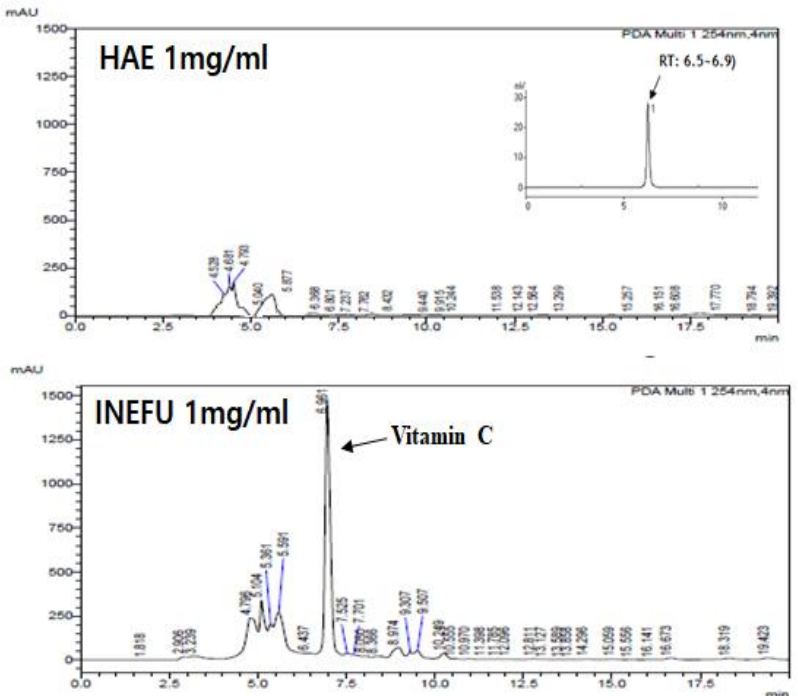

Fig. 1. High-performance liquid chromatographyphotodiode array (HPLC-PDA) chromatogram of vitamin $\mathrm{C}$ in Citrus madurensis (Citrofortunella microcarpa).
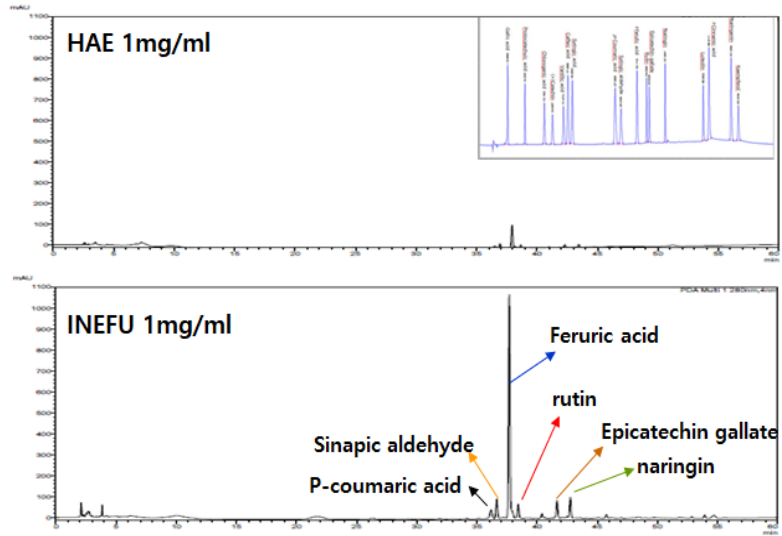

Fig. 2. High-performance liquid chromatographyphotodiode array (HPLC-PDA) chromatogram of polyphenols in Citrus madurensis (Citrofortunella microcarpa).
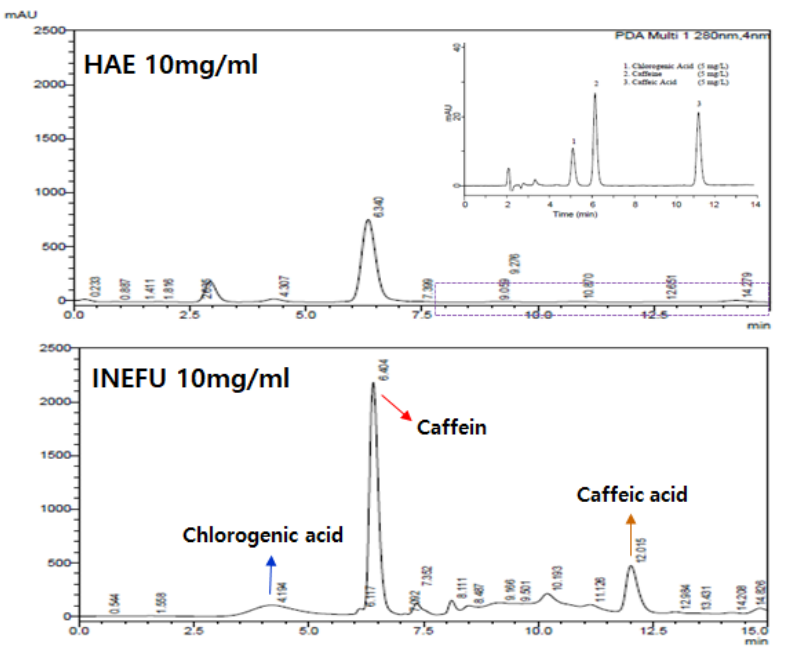

Fig. 3. High-performance liquid chromatographyphotodiode array (HPLC-PDA) chromatogram of chlorogenic acid, caffein, caffeic acid in green coffee beans.
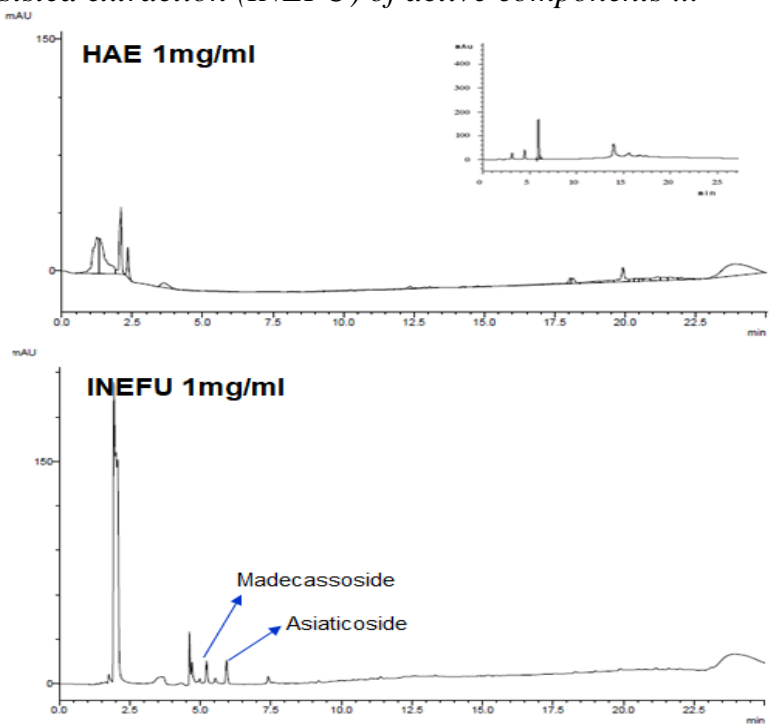

Fig. 4. High-performance liquid chromatographyphotodiode array (HPLC-PDA) chromatogram of asiaticoside and madecassoside in Centella asiatica.
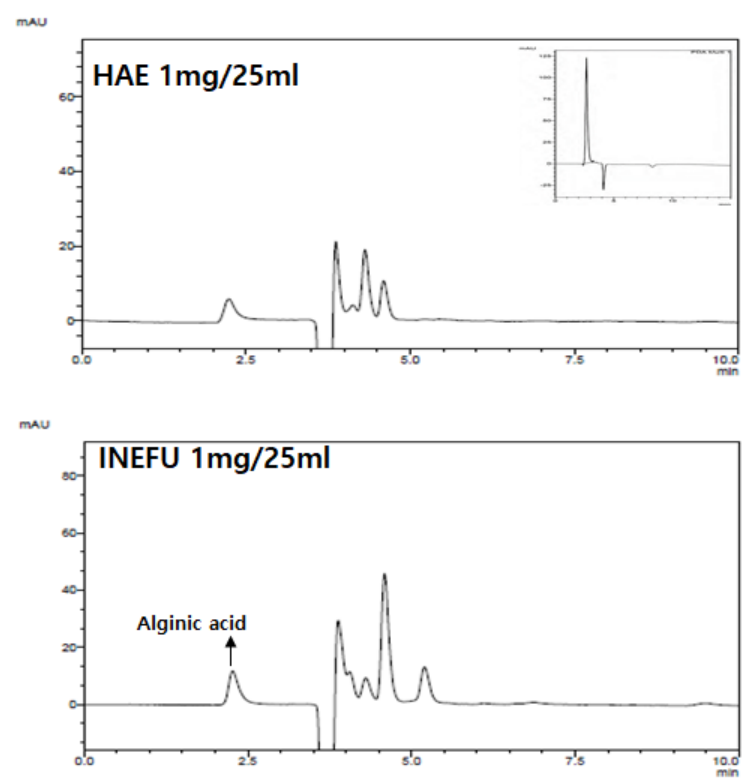

Fig. 5. High-performance liquid chromatographyphotodiode array (HPLC-PDA) chromatogram of alginic acid in Laminaria Japonica.

\section{CONCLUSION}

A method for the analysis of natural materials (green coffee beans, Citrus madurensis, Centella asiatica and Laminaria Japonica) belonging to different classes was developed and validated. The analyses were performed by HPLC-PDA directly after INEFU and HAE extractions of the natural materials and after freeze drying for five important active index components. The method was fully validated and applied to the analysis of the active index components in samples from natural materials varieties and one well-known international cultivar, Wonderful. The method allowed qualitative and quantitative analysis of the principal active index component in the different 
S.B. Seo, Y.M. Kim: Optimization of high ultrasound-assisted extraction (INEFU) of active components ...

extraction methods (INEFU and HAE) of green coffee bean, Citrus madurensis, Centella asiatica and Laminaria Japonica. Differences in active index component profile and concentration can be evidenced, allowing green coffee beans, Citrus madurensis, Centella asiatica and Laminaria Japonica to be distinguished on the basis of the concentration of compounds from INEFU extraction methods.

Acknowledgment: This work was carried out with the support of Classys INC. (Project title: Construction of high purity, concentration extracts techniques from natural materials).

\section{REFERENCES}

1. J.P. Maran, B. Priya, Int. J. Biol. Macromol., 70, 530 (2014).

2. K. Ponmurugan, N.A. Al-Dhabi, J.P. Maran, K. Karthikeyan, I.G. Moothy, N. Sivarajasekar, J.J.B. Manoj, Carbohydr. Polym., 173, 707 (2017).

3. J.H. Xie, M.Y. Shen, M.Y. Xie, S.P. Nie, Y. Chen, C. Li, D.F. Huang, Y.X. Wang, Carbohydr. Polym., 89, 177 (2012).

4. M. Yolmeh, M.B.H. Najafi, R. Farhoosh, Food Chem., 155, 319 (2014).

5. S.P. Wang, X.F. Dong, J.M. Tong, Int. J. Biol. Macromol., 62, 387 (2013).

6. Y. Zhu, Q. Li, G.H. Mao, Y. Zou, W.W. Feng, D.H. Zheng, W. Wang, L.L. Zhou, T.X. J. Zhang, et al. Carbohydr. Polym., 101, 606 (2014).

7. P. Esquivel, V.M. Jiménez, Food Res. Int., 46, 488 (2012).

8. C. Xie, L. Cui, J. Zhu, K. Wang, N. Sun, C. Sun, J. Hum. Hypertens., 32, 83 (2018).

9. M.L.G. Bizzo, A. Farah, J.A. Kemp, L.B. Scancetti, Elsevier, Amsterdam, The Netherlands, 2015, p. 11.

10. A. Riedel, C.M. Hochkogler, R. Lang, G. Bytof, I. Lantz, T. Hofmann, V. Somoza, Food Funct., 5, 454 (2014).
11. F.A. Khan, A. Maalik, G. Murtaza, J. Food Drug Anal., 24, 695 (2016).

12. K. Yamamoto, A. Yahada, K. Sasaki, K. Ogawa, N. Koga, H. Ohta, Journal of Agricultural and Food Chemistry, 60(44), 11182 (2012).

13. Y. Nogata, K. Sakamoto, H. Shiratsuchi, T. Ishii, M. Yano, H. Ohta, Biosci., Biotechnol., Biochem., 70, 178 (2006).

14. A. Murakami, Y. Nakamura, K. Torikai, T. Tanaka, T. Koshiba, K. Koshimizu, S. Kuwahara, Y. Takahashi, K. M. Ogawa, H. Yano, H. Tokuda, H. Nishino, Y. Mimaki, Y. Sashida, S. Kitanaka, Cancer Res., 60, 5059 (2000)

15. A. Minagawa, Y. Otani, T. Kubota, N. Wada, T. Furukawa, K. Kumai, K. Kameyama, Y. Okada, M. Fujii, M. Yano, T. Sato, M. Kitajima, Jpn. J. Cancer Res., 92, 1322 (2001).

16. T. Akachi, Y. Shiina, Y. Ohishi, T. Kawaguchi, H. Kawagishi, T. Morita, M. Mori, K. Sugiyama, J. Nutr. Vitaminol., 56, 60 (2010).

17. F.L. Greenway, G.A. Bray, D. Heber, Obes Res., 3(4), 561 (1995).

18. M.K. Caruso, S. Pekarovic, W.J. Raum, F. Greenway, Diabetes Obes. Metab., 9, 300 (2007).

19. B. Brinkhaus, M. Lindner, D Schuppan, E.G. Hahn, Phytomedicine, 75, 427 (2000).

20. L. Huang, K. Wen, X. Gao, Y. Liu, Pharm. Biol., 48, 422 (2010).

21. H.T.V. Lin, W.J. Lu, G.J. Tsai, C.T. Chou, H.I. Hsiao, P.A. Hwang, Process. Biochem., 51, 1945 (2016).

22. L. Qiong, L. Jun, Y. Jun, Z. Yinzhu, C. Xiaoyan, Y. Mingliang, Environ. Toxicol. Phar., 31, 286 (2011).

23. X.Q. Zha, C.Q. Lu, S.H. Cui, L.H. Pan, H.L. Zhang, J.H. Wang, J.P. Luo, Int. J. Biol. Macromol., 78, 429 (2015).

24. Q. Zhu, J. Chen, Q. Li, T. Wang, H. Li, Int. J. Biol. Macromol., 92, 156 (2016).

25. K. Yamamoto, A. Yahada, K. Sasaki, K. Ogawa, N. Koga, H. Ohta, J. Agric. Food Chem., 60, 1182 (2012). 


\title{
Design, synthesis, structural characterization and cell cytotoxicity of a new derivative poly(maleic anhydride-co-vinyl acetate)/miltefosine polymer/drug conjugate
}

\author{
G. Karakus ${ }^{1 *}$, Z. Akin Polat ${ }^{2}$, M. Karahan ${ }^{3}$ \\ ${ }^{1}$ Division of Pharmaceutical Chemistry, Faculty of Pharmacy, Cumhuriyet University, Sivas, Turkey \\ ${ }^{2}$ Division of Medical Parasitology, School of Medicine, Cumhuriyet University, Sivas, Turkey \\ ${ }^{3}$ Division of Bioengineering, Faculty of Engineering and Natural Sciences, Üsküdar University, Uskudar-Istanbul, \\ Turkey
}

Received, May, 31, 2018; Accepted, January 18, 2019

\begin{abstract}
In recent years, polymeric systems are selected as biomaterials because of their desired biocompatible properties and easy design/preparation of a number of different structures with lower toxicity and good solubility. Nontoxic polymeric drug carrier, maleic anhydride-co-vinyl acetate copolymer (MAVA), was prepared via free-radical chain polymerization at $80 \pm 0.1{ }^{\circ} \mathrm{C}$. MEK (methyl ethyl ketone) and BPO (benzoyl peroxide) were used as the organic medium and radical initiator, respectively. Copolymer was conjugated with a broad-spectrum antimicrobial agent, miltefosine (MF, an oral drug in the treatment of leishmaniosis), Impavido ${ }^{\circledR}$ and Miltex ${ }^{\circledR}, 1: 1$ molar ratio of copolymer:drug for $48 \mathrm{~h}$ at $60{ }^{\circ} \mathrm{C}$ in aqueous medium in presence of $\mathrm{N}$-(3-dimethylaminopropyl)-N'-ethylcarbodiimide hydrochloride (EDAC). Fourier transform infrared (FTIR) and nuclear magnetic resonance $\left({ }^{1} \mathrm{H}\right.$ - and $\left.{ }^{31} \mathrm{P}-\mathrm{NMR}\right)$ were used to characterize the structure of the copolymer and MAVA/MF conjugate. Molecular weights were measured via size-exclusion chromatography (SEC). Results, obtained from the spectroscopic and SEC analysis, verified that conjugation was successfully carried out with good water-solubility. WST-1 cytotoxicity tests, $24 \mathrm{~h}$ by quantitative analysis, were carried out for copolymer, miltefosine, and MAVA/MF. The cytotoxicity values, by comparing with control group, were found statistically significantly different $(\mathrm{P}<0.05)$. MAVA/MF copolymer/drug couple was successfully designed with lower cytotoxicity than the free drug (MF).
\end{abstract}

Keywords: maleic anhydride-co-vinyl acetate copolymer conjugation, miltefosine, cytotoxic activity, FTIR, ${ }^{1} \mathrm{H}-\mathrm{NMR}$, ${ }^{31} \mathrm{P}-\mathrm{NMR}, \mathrm{SEC}$

\section{INTRODUCTION}

Anhydride containing polyanhydride copolymers have been commonly applied, specifically as solubilizing agents, nanoparticulate forms, surface modifiers, drug carriers, and also for other purposes such as diagnostic imaging agents and implants [1]. Generally, water-soluble conjugates could be well and easy designed as drug carriers for drug delivery. N-(2-hydroxypropyl)methacrylamide (HPMA) copolymer-based drug delivery systems (DDSs) are recently used for clinical applications as anticancer agents. Biologically inert poly(ethylene glycol) (PEG), approved by Food and Drug Administration (FDA) for pharmaceutical trials that is preferred in many pharmaceutical, cosmetic, and industrial applications because of its water solubility, biocompatibility, nontoxicity, and nonimmunogenicity characteristics [2]. Maleic anhydride (MA) copolymers mainly consist of MA and vinyl-based monomers. Modification/ conjugation of the anhydride copolymers is accompanied by a chemical process in which the active anhydride unit can be opened after nucleophilic attack by the ring opening reaction with amino $\left(\mathrm{R}-\mathrm{NH}_{2}\right)$ or hydroxyl functional groups (R-OH) of nucleophilic reagents [3,4-6] to obtain amide/ester and carboxylic acid structures. We often encounter the amide/carboxyl structure because of its more favorable properties in our studies. Another main goal for this conjugation reaction was getting a copolymer/drug couple with better water solubility and lower cytotoxicity properties than the free drug for improvement of its main biological activity.

For more than 50 years, several techniques have been applied in pharmaceutical industry to combine biologically active agents with polymeric materials including PEG, poly( $N$-vinyl pyrrolidone), and cellulose derivatives [7]. Ringsdorf first proposed a model (schematized in Figure 1) known by its name in 1975 for design of polymer base prodrugs [8-10]. Commercially available vinyl base poly(styrene-comaleic acid/anhydride) (SMA) is a well-known copolymer $[3,11]$. SMANCS conjugate consist of a SMA copolymer and the antitumor protein NCS (neocarcinostatin) [10,12-16].

The conjugate has also been applied in Japan for hepatocellular carcinoma treatment with significant therapeutic results and quite few side effects [17].

\footnotetext{
* To whom all correspondence should be sent: 
G. Karakus et al.: Design, synthesis, structural characterization and cell cytotoxicity of a new derivative ...

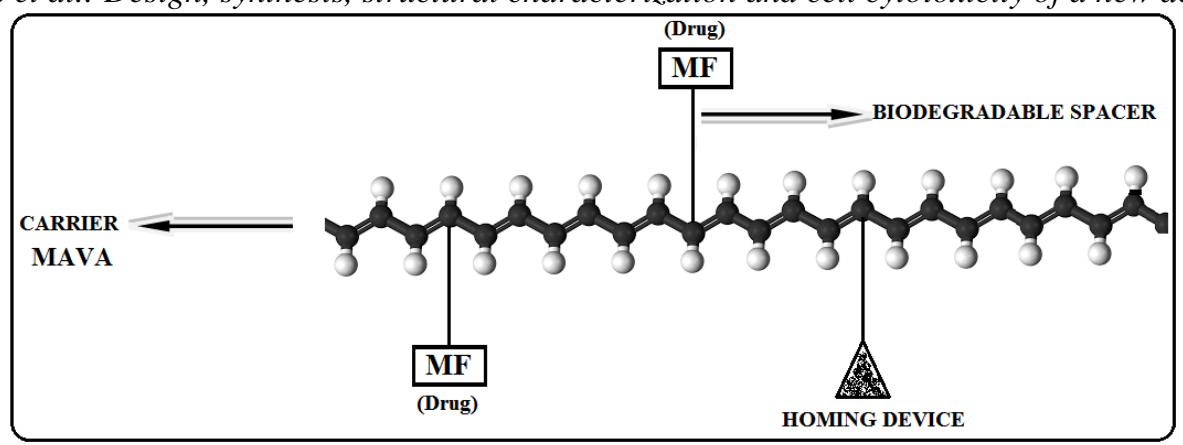

Figure 1. The Ringsdorf model.

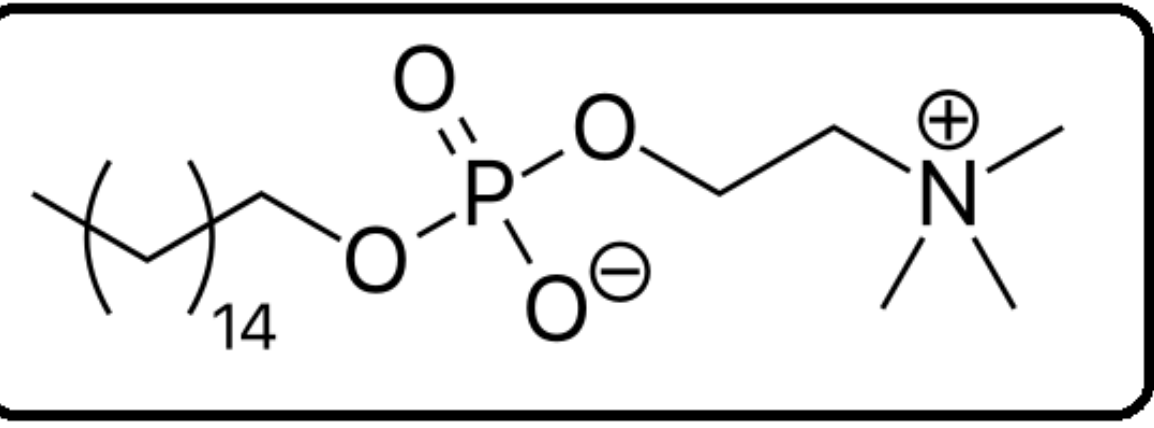

Figure 2. Chemical structure of miltefosine, MF, [2-(hexadecoxy-oxido-phosphoryl)oxyethyl-trimethyl-azanium].

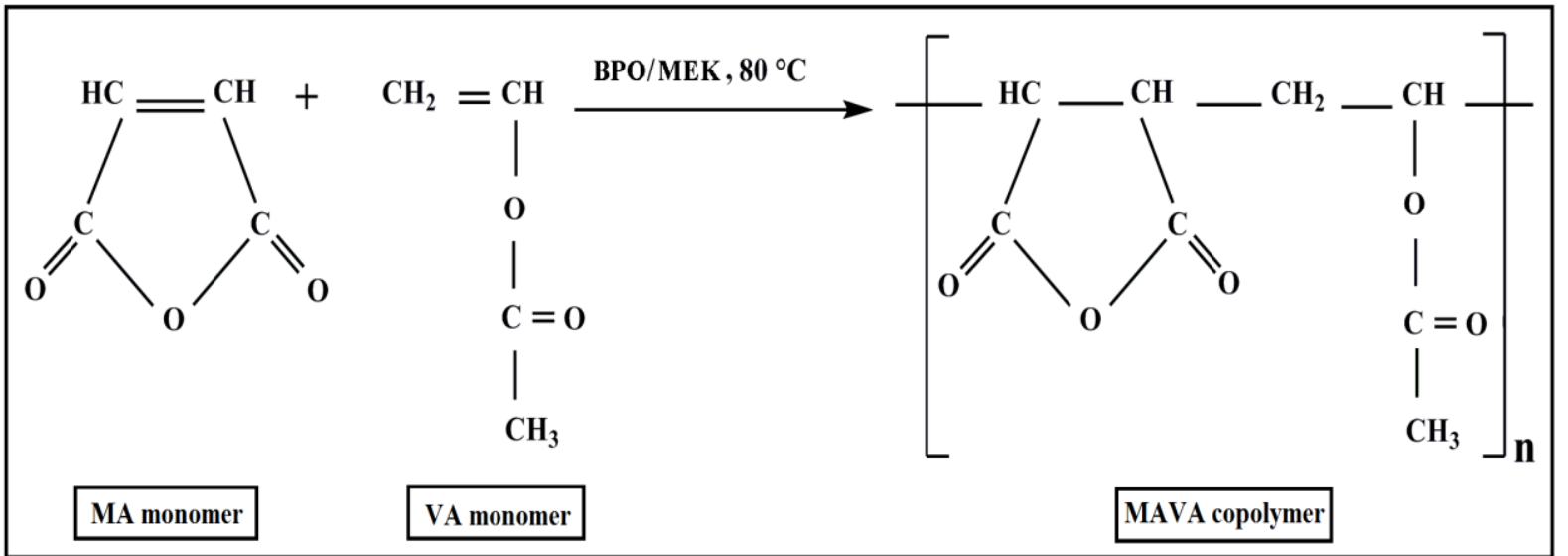

Scheme 1. Polymerization reaction mechanism of the copolymer.

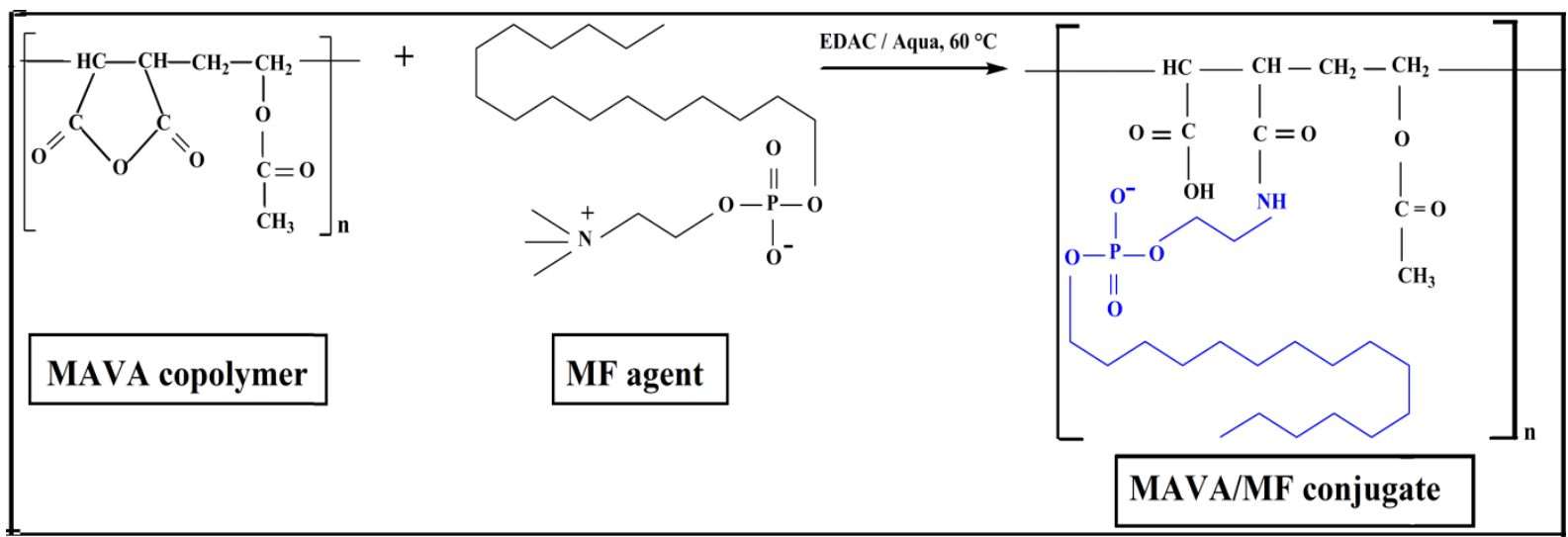

Scheme 2 Conjugation reaction of the copolymer with MF agent.

An antileishmanial drug, miltefosine [1- inhibitor of CTP: phosphocholine cytidylyl hexadecylphosphorylcholine $(\mathrm{HePC})]$ is an transferase and has antimetastatic properties. It is 
G. Karakus et al.: Design, synthesis, structural characterization and cell cytotoxicity of a new derivative... also more than $90 \%$ effective in eradicating visceral infections of Leishmania species. World Health Organization (WHO) reported that Leishmaniases, tropical diseases caused by the protozoan parasite Leishmania, are one of the six major diseases in developing countries [18]. Its systematic IUPAC name, chemical formula and molecular mass as follows: 2-(hexadecoxy-oxide-phosphoryl) oxyethyl-tri-methyl-azanium, $\mathrm{C}_{21} \mathrm{H}_{46} \mathrm{NO}_{4} \mathrm{P}, 407.568$ $\mathrm{g} / \mathrm{mol}$. Miltefosine (MF) lateral chain consists of a phosphate $\left(\mathrm{PO}_{4}{ }^{3-}\right)$ group, an intermediate ethyl chain $\left(-\mathrm{CH}_{2}-\mathrm{CH}_{2}-\right)$, known with trade names Impavido ${ }^{\circledR}$, Miltex ${ }^{\circledR}$, a trimethylamine $\mathrm{N}^{+}\left(\mathrm{CH}_{3}\right)_{3}$ group and a terminated carbon side chain (Figure 2).

In this study, as a drug carrier maleic anhydridevinyl acetate copolymer (MAVA) (Scheme 1) was chemically modified, through its reactive anhydride ring [19], by conjugation of the pharmaceutically active agent miltefosine, MF (Scheme 2). Conjugated product was named MAVA/MF. Cell cytotoxic effect of MAVA/MF was also studied with MAVA and MF cytotoxicity by using a healthy L929 mouse fibroblast cell line. Cytotoxicity experiments showed that MAVA/MF conjugate had no cytotoxicity when cell viability was taken into consideration even at $250 \mu \mathrm{g} / \mathrm{mL}$ concentration.

\section{EXPERIMENTAL}

\section{Materials}

Synthesis, Conjugation and Purification: Chemicals were obtained from Merck (Schuchardt,

Table 1 Synthetic reaction conditions of copolymer and conjugated product.

\begin{tabular}{|c|c|c|c|c|c|}
\hline 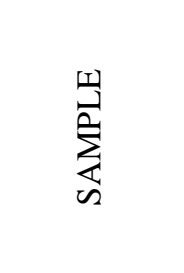 & 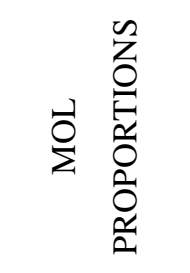 & 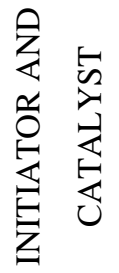 & 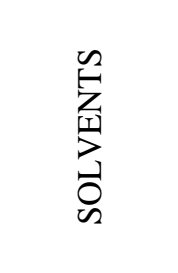 & $\sum_{ٍ}^{\Perp}$ & 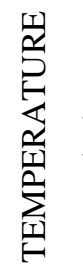 \\
\hline \multirow{2}{*}{ MAVA } & MA:VA & \multirow{2}{*}{$\mathrm{BPO}$} & \multirow{2}{*}{ MEK } & \multirow{2}{*}{24} & \multirow{2}{*}{80} \\
\hline & $\sim(1: 1)$ & & & & \\
\hline \multirow{2}{*}{ MAVA/MF } & MAVA:MF & \multirow{2}{*}{ EDAC } & \multirow{2}{*}{$\begin{array}{c}\text { Water } \\
\text { (ultra pure) }\end{array}$} & \multirow{2}{*}{48} & \multirow{2}{*}{60} \\
\hline & $\sim(1: 1)$ & & & & \\
\hline
\end{tabular}

\section{Synthesis and Purification of MAVA/MF Conjugate}

MAVA/MF conjugate was designed by the modification of MAVA copolymer with miltefosine (MF, mol. mass: $407.568 \mathrm{~g} / \mathrm{mol}$ ), at a 1:1 [6,22] molar ratio for $48 \mathrm{~h}$ at $60{ }^{\circ} \mathrm{C}$ in ultra-pure water, using EDAC [N-(3-dimethyl-aminopropyl)- $N$ '-
Germany) are as follows: MA (maleic anhydride), MEK (methyl ethyl ketone), EDAC [N-(3dimethyl-aminopropyl)-N'-ethylcarbodiimide hydrochloride] and BPO (benzoyl peroxide). Ethyl alcohol (95\%) was purchased from Carlo-Erba (Rodano, Italy). $\mathrm{NaOH}$ (sodium hydroxide), $\mathrm{HCl}$ (hydrochloric acid), VA (vinyl acetate), ethyl acetate and petroleum ether were ordered from Sigma-Aldrich (St. Louis, USA). Miltefosine was supplied by Orphanidis Pharma Research $\mathrm{GmbH}$ (Vienna, Austria).

Cultivation of L929 Mouse Fibroblast Cells and Cell Proliferation Assay: DMEM (Dulbecco's minimum Eagle medium) and fetal calf serum (10\%) were obtained from Sigma (St. Louis, MO, USA), and WST-1 labeling reagent was purchased from Roche (Mannheim, Germany).

\section{Synthesis and Purification of MAVA Copolymer}

As described in our previous study [20], MAVA was synthesized traditionally by free radical chain polymerization reaction in the presence of MA and VA monomers. Table 1 summarizes the reaction conditions (briefly: 1:1 monomer molar ratio, MEK organic solvent, BPO free radical initiator, $24 \mathrm{~h}$, and $80^{\circ} \mathrm{C}$ ). Unreacted or homopolymer products were gently removed by incubating the white precipitated conjugate in ethyl acetate $(24 \mathrm{~h})$. Petroleum ether precipitation, under vacuum filtration, and vacuum incubator drying at $55{ }^{\circ} \mathrm{C}, 24 \mathrm{~h}$ were carried out for purification [21]. 
G. Karakus et al.: Design, synthesis, structural characterization and cell cytotoxicity of a new derivative ...

room for interacting all of the anhydride repeated units with concentrated $\mathrm{NaOH}$ [11]. Copolymer was expected to dissolve/hydrolyze in alkaline water medium and turn to a homogeneous transparent mixture which was immediately cooled again to room temperature, then the $\mathrm{pH}$ was reduced to 5 by drop-wise adding of $1 \mathrm{M} \mathrm{HCl}$. Modified sample was prepared using the mixed solvent (5\% ethyl alcohol/injectable water, stored at $-20{ }^{\circ} \mathrm{C}$ until the adding step) [18] by drop-wise addition of $\sim 10 \mathrm{mg} / \mathrm{mL}$ solution of MF. Initiation reaction was started by adding EDAC $(20 \mathrm{mg} / \mathrm{mL}$ solution) over $15 \mathrm{~min}, \mathrm{pH}$ was kept at 5 by adding $0.01 \mathrm{M} \mathrm{HCl}$ [11]. The reaction medium was then allowed to proceed for $24 \mathrm{~h}$ at $50{ }^{\circ} \mathrm{C}$ in a continuously shaking condition to get a viscous blurry white solution in a dark room, and allowed further for $48 \mathrm{~h}$ at $60^{\circ} \mathrm{C}$. The products were washed several times with excess of cold ethanol and incubated/cooled for $1 \mathrm{~h}$ at $-20^{\circ} \mathrm{C}$. White precipitate was then collected (centrifugation, $10 \mathrm{~min}, 3,000$ $\mathrm{rpm}$ ) in powder form after the liquid phase was removed and purified in a vacuum incubator $(24 \mathrm{~h}$, $\left.55^{\circ} \mathrm{C}\right)[23]$.

\section{Structural Characterization}

The infrared spectrum of the samples (copolymer and MAVA/MF conjugate) were recorded as $\mathrm{KBr}$ pellets on a FTIR spectrophotometer (Unicam MATTSON 1000, USA) at $400-4000 \mathrm{~cm}^{-1}$ with increments adjusted to $4 \mathrm{~cm}^{-1}$. The nuclear magnetic resonance analyses, ( ${ }^{1} \mathrm{H}-\mathrm{NMR}$ and $\left.{ }^{31} \mathrm{P}-\mathrm{NMR}\right)$ were performed on a Bruker Avance III instrument (Karlsruhe, Germany) at $400 \mathrm{MHz}$ using $6 \mathrm{mg}$ of the samples, dissolved in $0.8 \mathrm{~mL}$ of heavy water $\left(\mathrm{D}_{2} \mathrm{O}\right)$.

\section{Size-Exclusion Chromatography (SEC) of Copolymer/Drug Conjugate}

As detailed in our previous study [23], average molecular weight distribution of the copolymer and modified product was measured by a Viscotek size exclusion chromatography (SEC) system (Houston, USA) with $660 \mathrm{~nm}$ refractive index and $670 \mathrm{~nm}$ right-angle light scattering (RALS) apparatus. Polystyrene standard was used for quadruple detector array calibration. Powdered samples were prepared in tetrahydrofuran (THF) and eluted (1.0 $\mathrm{mL} / \mathrm{min}$ ) at room temperature. OmniSEC 4.1 software program (Viscotek Cooperation, Houston USA) was used for obtained data analysis [24,25].

\section{Cytotoxicity of MAVA Copolymer and MAVA/MF Conjugate}

Cultivation of L929 mouse fibroblast cell lines: Detailed information about this method can be obtained from our previous work [35]: L929 (ATCC cell line, NCTC clone 929) mouse connective tissue fibroblast cell line, was cultured in DMEM, in the presence of fetal calf serum $(10 \%)$ and L-glutamine $(2 \mathrm{mM} / \mathrm{ml})$ without adding antibiotics to the cell culture medium. Cultivation was performed for the cultures in the humidified incubator at $37{ }^{\circ} \mathrm{C}$ and $5 \% \mathrm{CO}_{2}$, until complete cell monolayer integration ( 7 days).

Cell proliferation assay: As we clearly explained in our previous study [35], the cell proliferation assay mainly measures the number of living cells by dividing the tetrazolium salts to formazan by cellular enzymes in presence of the WST-1 labeling reagent. This method is based on a simple and well known kit protocol [35]. Briefly, after the user's kit protocol, quantification was performed by a scanning multi-well spectrophotometer (wavelength $450 \mathrm{~nm}$ ) according to the absorbance measurement of the dye solution. Seeded cells in 96-well microtiter plates were adjusted to $1 \times 10^{5}$ cells $/ \mathrm{mL}$ in a main volume of 100 $\mu \mathrm{L}$ per well. For the cytotoxic analysis of the MAVA; MF, and MAVA/MF 500, 250, 125, 62.5, 31.2 , and $15.6 \mu \mathrm{g} / \mathrm{mL}$ concentrations were applied. Incubation was performed for $24 \mathrm{~h}$ at $37^{\circ} \mathrm{C}$ in a humidified atmosphere with $5 \% \quad \mathrm{CO}_{2}$. After the incubation period, WST-1 labeling reagent was gently supplemented to the medium as $1: 1$ volume ratio $(10 \mu \mathrm{l})$ in each well. The absorbance values, using the control group comparison, were recorded at $450 \mathrm{~nm}$ using a Thermo Scientific microplate photometer (Multiskan FC, USA). Ten $\mu$ l of WST1 labeling reagent/one hundred $\mu \mathrm{l}$ of medium was also added to one well (selected blank position) on ELISA reader for background control purpose. After the start of the tetrazolium reaction $(2 \mathrm{~h})$ absorbance was recorded and experiments were carried out in six replicates for reliable results. Percentage viability was calculated according to user's manual and following Eq. (1) the optical density (OD) of the test products was compared with that of the negative control.

Cell Viability $(\%)=[(\mathrm{OD} 450 \quad($ Sample $) / O D 450$ $($ Negative control) $) \times 100]$

\section{Statistical analysis}

Optical density (OD) results, with six replicates, were presented as mean \pm SD. Statistical ANOVA (one-way analysis of variance) was performed with 95\% confidence levels for multiple comparisons. Furthermore, Student's $t$-test was also used for two group comparisons. 
G. Karakus et al.: Design, synthesis, structural characterization and cell cytotoxicity of a new derivative ...

RESULTS AND DISCUSSION

\section{FTIR Analysis}

Unmodified copolymer (Figure 3a) had the main anhydride containing polymer structure [26-31]: symmetric and asymmetric carbonyl $(\mathrm{C}=\mathrm{O})$ stretches of MA at $1855.48 \mathrm{~cm}^{-1}$, and $1780.59 \mathrm{~cm}^{-1}$, respectively $[26,27]$. Frequency vibrations of $\mathrm{C}-\mathrm{O}-$ $\mathrm{C}$ bond on anhydride ring were found at 1025.21 $\mathrm{cm}^{-1}$ and $934.96 \mathrm{~cm}^{-1}$ [28]. Another carbonyl $(\mathrm{C}=\mathrm{O})$ symmetric and asymmetric stretches of VA was at $1715.67 \mathrm{~cm}^{-1}$ [29], $\mathrm{CH}_{3}$ and $\mathrm{CH}_{2}$ belong to VA stretching frequencies at $1373.80 \mathrm{~cm}^{-1}$ and 1430.38 $\mathrm{cm}^{-1}$, respectively $[30,31]$, characteristic acetyl ($\mathrm{COCH}_{3}$ ) group stretching frequencies of VA were at $1095.30 \mathrm{~cm}^{-1}$ [26], C-C vibration at $603.84 \mathrm{~cm}^{-1}$, $\mathrm{C}-\mathrm{O}-\mathrm{C}$ stretching at $1214.91 \mathrm{~cm}^{-1}$, and $\mathrm{C}-\mathrm{H}$ group stretching vibration at $2943.19 \mathrm{~cm}^{-1}[32,33]$.

Characteristic copolymer repeating units disappeared almost completely in MAVA/MF as a sign of the success of the ring-opening reaction (Figure $3 \mathrm{~b}$ ). It also means that a high percentage of the repeated anhydride rings were modified after copolymer hydrolysis and nucleophilic amino ($\mathrm{NH}_{2}$ ) group attack of MF [34]. Modification/conjugation of anhydride ring generally accompanied by functional group formation, for example, results in carboxylic group with amide or ester structure [34]. In this study conjugation reaction was verified with formation of a carboxylic acid and amide functionality. Characteristic - $\mathrm{CH}$ stretching vibration of VA along with the copolymer backbone stretching vibrations at $1403 \mathrm{~cm}^{-1}$ [35], CO-O-C ester groups or C-C of VA located at $1226.97 \mathrm{~cm}^{-1}$, and a $\mathrm{CH}_{2}$ deformation at $1585 \mathrm{~cm}^{-1}$ [36]. Peak observed at $1755 \mathrm{~cm}^{-1}$ could be assigned to $-\mathrm{COOH}$ carboxylic acid units [32]. $1394.31 \mathrm{~cm}^{-1}$ could be associated with MF because the same peak was also observed on pure MF spectrum. Peak $1066.28 \mathrm{~cm}^{-1}$ belongs to $\mathrm{PO}_{4}{ }^{3-}$ antisymmetric stretching frequency [37-43], $117267 \mathrm{~cm}^{-1}$ and $1052.22 \mathrm{~cm}^{-1}$ were also assigned to $\mathrm{PO}_{4}{ }^{3-}$ antisymmetric stretching movement [44]. According to literature, Breitinger and co-workers emphasized that the deceptively simple strong vibrations centered at $1059 \mathrm{~cm}^{-1}$ including minimum four components of inorganic phosphate
$\left(\mathrm{PO}_{4}\right)$, were produced by removal of the threefold degeneracy of $\mathrm{PO}_{4}$ and activation of the general mode of phosphate $\left(\mathrm{PO}_{4}\right)$ [44]. Furthermore, 629.77 $\mathrm{cm}^{-1}$ was detected in the spectrum, because a series of infrared bands such as $620 \mathrm{~cm}^{-1}, 643 \mathrm{~cm}^{-1}$ and $673 \mathrm{~cm}^{-1}$ were assigned to out of vibrations of plane bending vibrational mode of the phosphate anion $\left(\mathrm{PO}_{4}{ }^{3-}\right)$ and peak $892 \mathrm{~cm}^{-1}$ was associated with water vibrational modes [44]. Because the vibrational frequencies around $1577 \mathrm{~cm}^{-1}$ could be an overtone/combination band, the observed peak $1585.82 \mathrm{~cm}^{-1}$ was also considered as overtone and combination [44].

In addition to multiple phosphate vibrational modes detection, observed peaks were assigned to $\mathrm{PO}_{4}{ }^{3-}, \mathrm{OH}$, and $\mathrm{H}_{2} \mathrm{O}$ stretching and bending modes. Conjugate, MAVA/MF, consists of main polymeric backbone linked by $\mathrm{PO}_{4}$ groups of miltefosine through an amide bond. As a result, at the molecular level there are phosphate units which are not equivalent in the conjugate structure. Peak located at $3662.75 \mathrm{~cm}^{-1}$ could be attributed to both primary amide and also carboxylic acid groups related to the most evident vibrational peak for conjugation reaction.

As reported previously by Das and co-workers, Figure $3 \mathrm{c}$ presents the IR spectra of the pure miltefosine. Peak assignments for the characteristics of MF: at $1471 \mathrm{~cm}^{-1}$ (bending vibrations for $-\mathrm{CH}_{2}$ ) and $1242 \mathrm{~cm}^{-1}$ (asymmetric stretching vibrations for $-\mathrm{PO}_{2}$ ) and overlap of 1078 $\mathrm{cm}^{-1}$ (symmetric stretching vibrations for $-\mathrm{PO}_{2}^{-}$) and $1064 \mathrm{~cm}^{-1}$ (stretching vibrations for $\mathrm{P}-\mathrm{O}-\mathrm{C}$ ) were detected in the solid state, crystalline form, of the drug MF [45].

\section{NMR Analysis}

${ }^{1} H$-NMR Analysis: ${ }^{1} \mathrm{H}-\mathrm{NMR}$ spectrum of the copolymer (Figure 4a) showed the characteristic peaks as follows: chemical shift belongs to protons of anhydride rings at $3-4 \mathrm{ppm},-\mathrm{CH}_{2}$ protons on VA approximately at $\sim 2.3-2.7 \mathrm{ppm},-\mathrm{CH}$ fragment (bound directly to oxygen) produced a multiplet peak at around $\sim 4.9-5.3 \mathrm{ppm}[46,47]$, and all of the $-\mathrm{CH}_{3}$ protons, adjacent to $\mathrm{C}=\mathrm{O}$ (carbonyl group), located at $\sim 1.7-2.0 \mathrm{ppm}[48-50]$. 


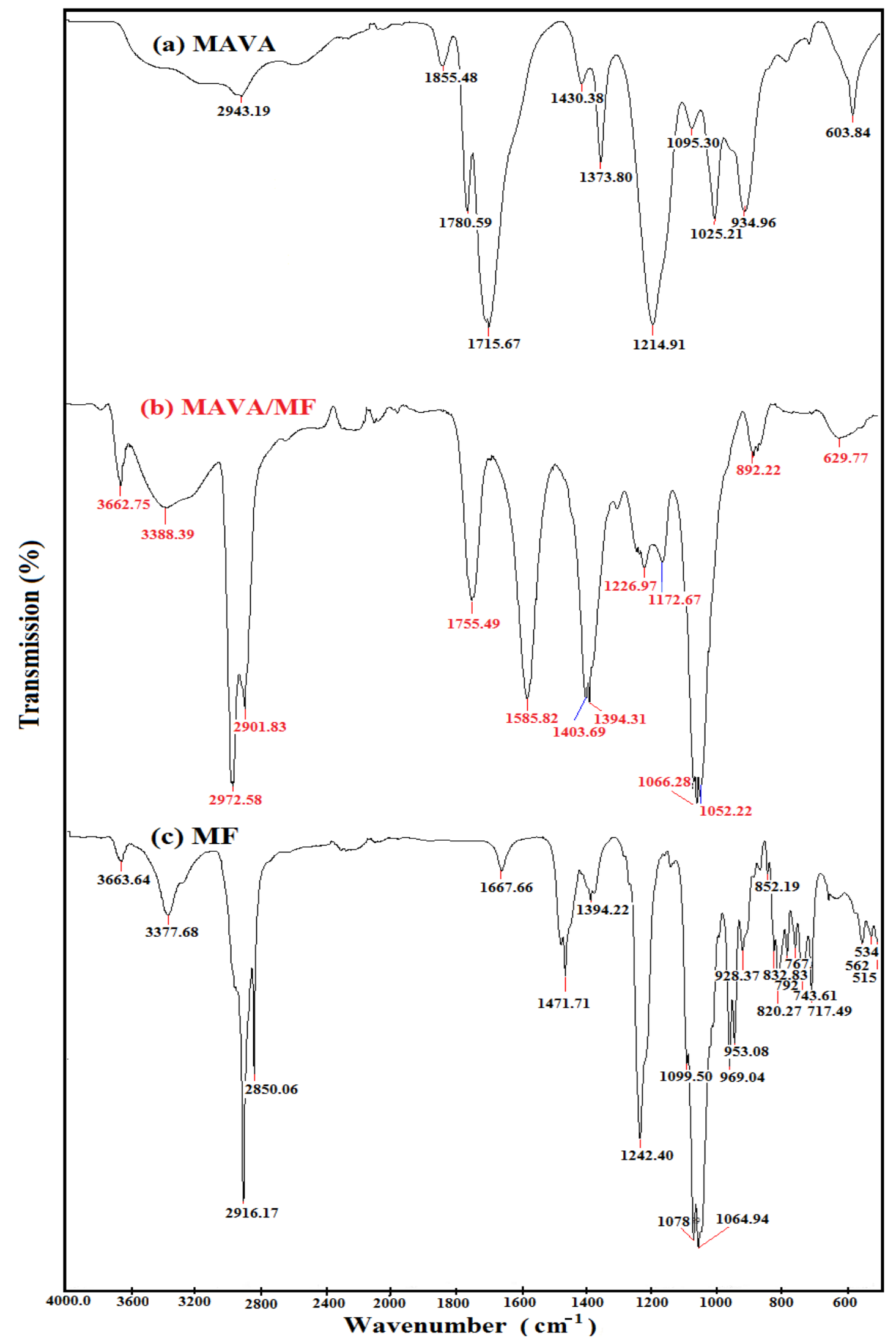

Figure 3. FTIR spectra of the copolymer, conjugation product, and MF agent. (a) MAVA, (b) MAVA/MF, and (c) MF 
G. Karakus et al.: Design, synthesis, structural characterization and cell cytotoxicity of a new derivative ...<smiles>CC1C(=O)OC(=O)C1CC1CCC(C)(C)O1</smiles>

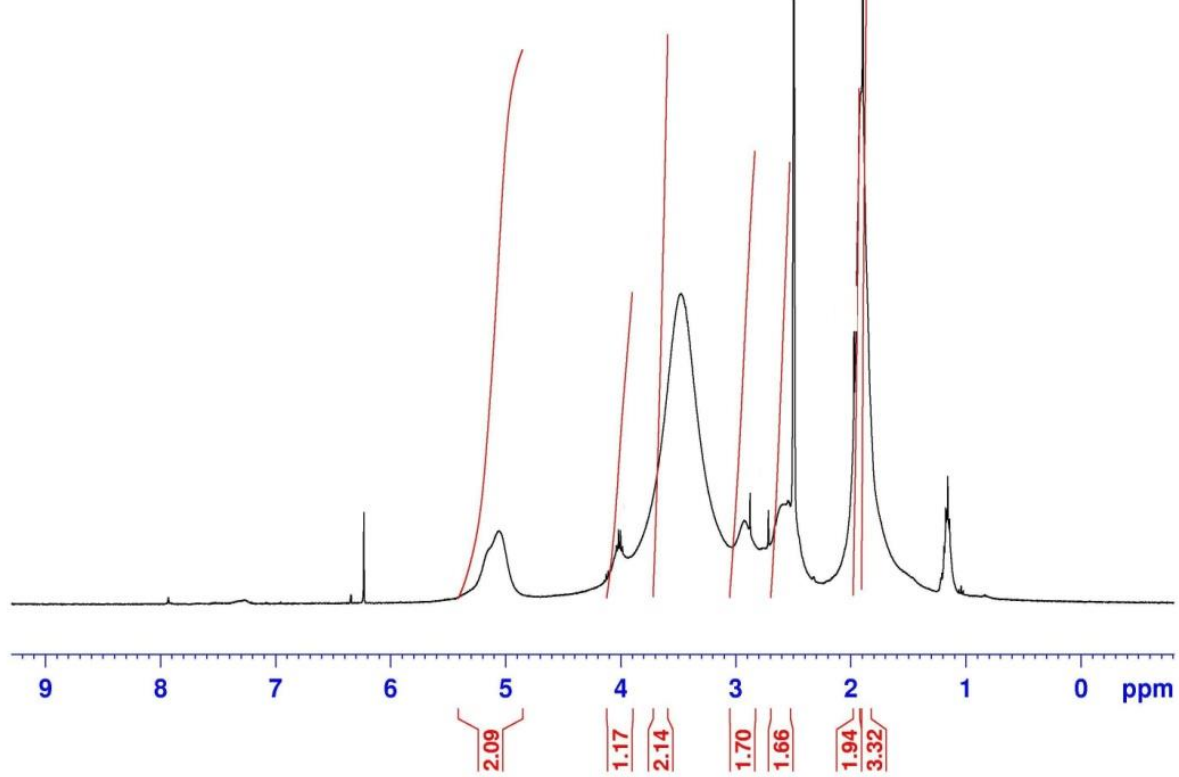

(a)

MAVA / MF

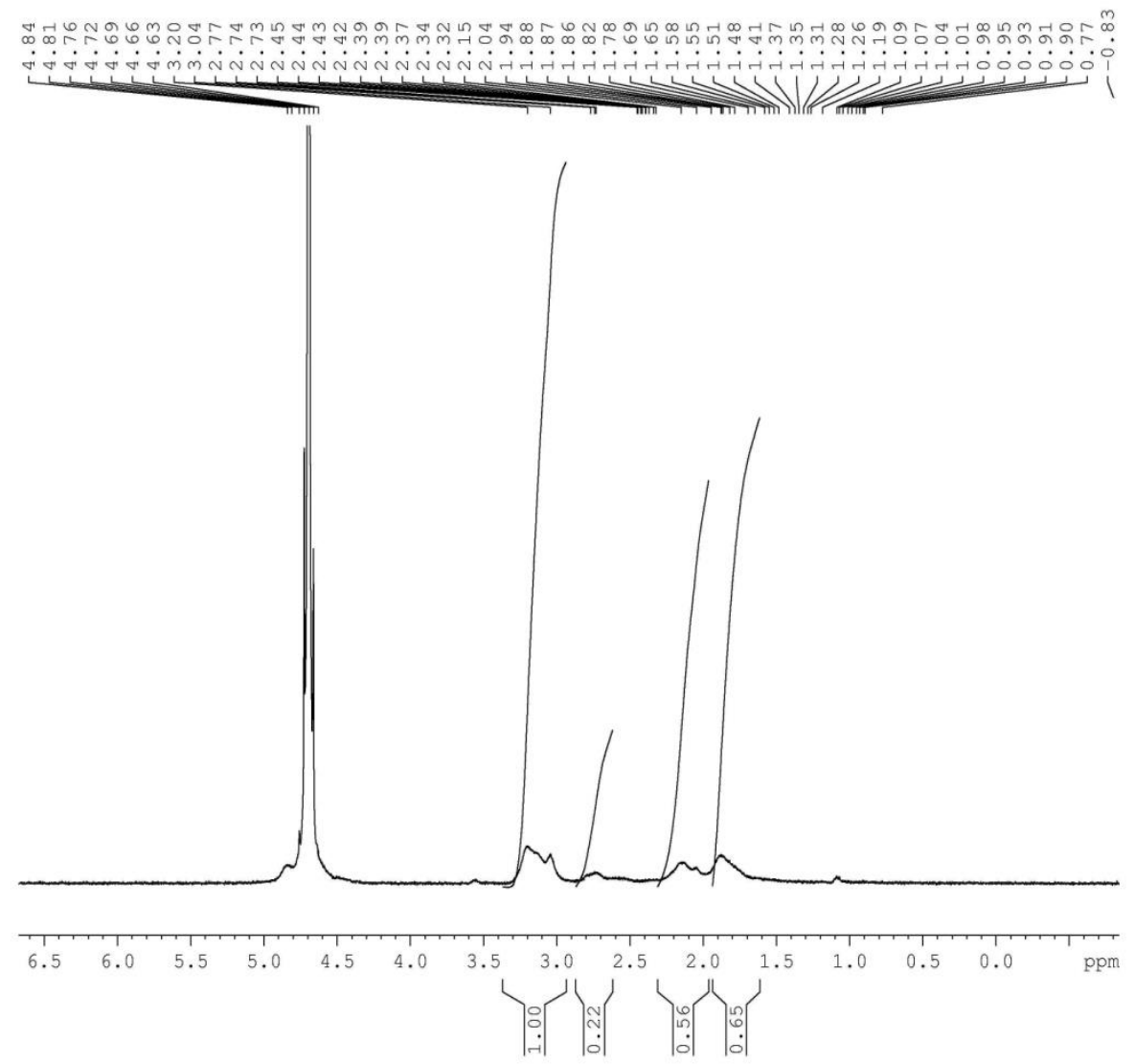

(b) 
G. Karakus et al.: Design, synthesis, structural characterization and cell cytotoxicity of a new derivative ... MF

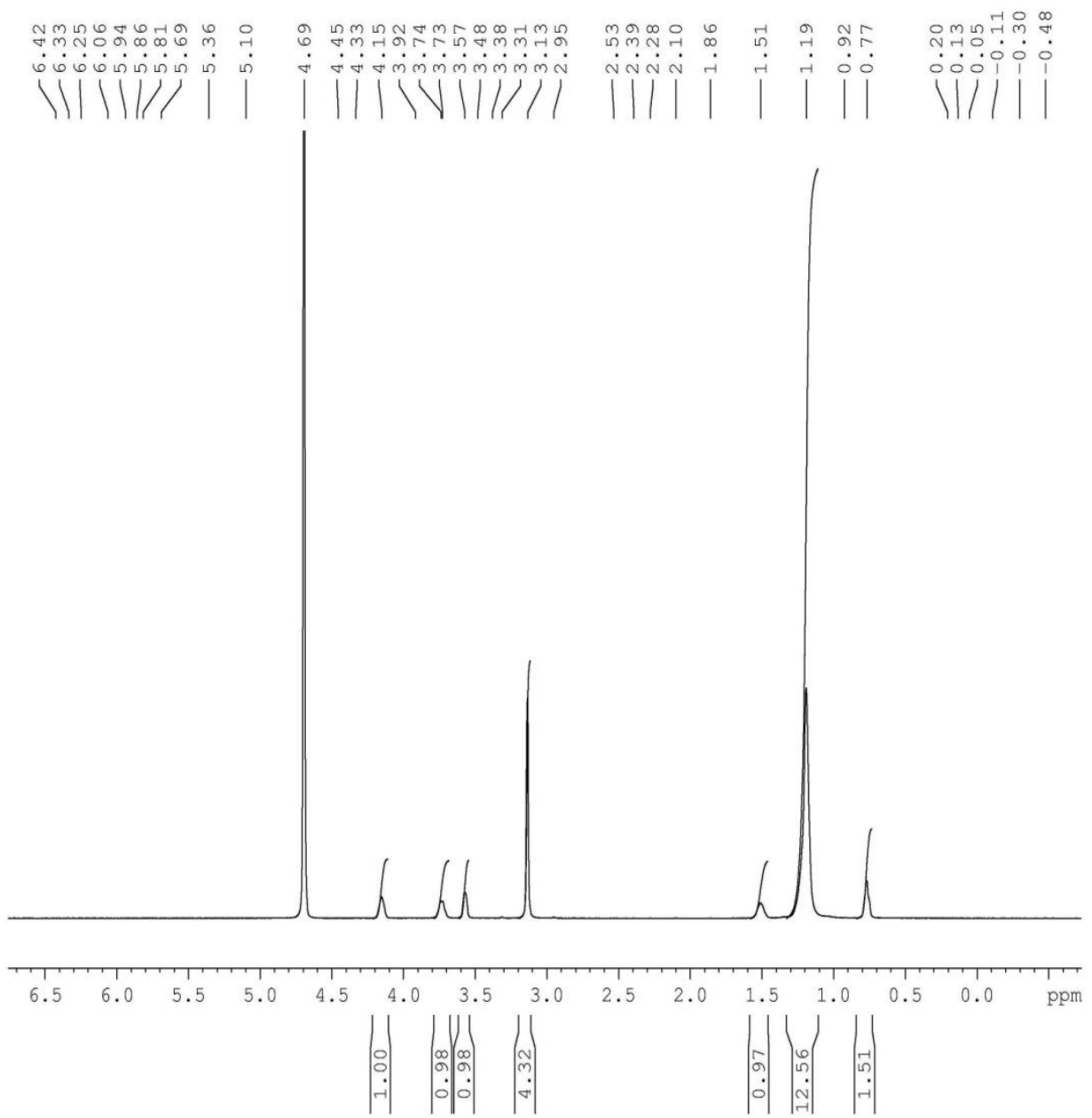

Figure 4. ${ }^{1} \mathrm{H}-\mathrm{NMR}$ spectra of the copolymer, conjugation product, and MF agent.

(c) (a) MAVA, (b) MAVA/MF, and (c) MF.

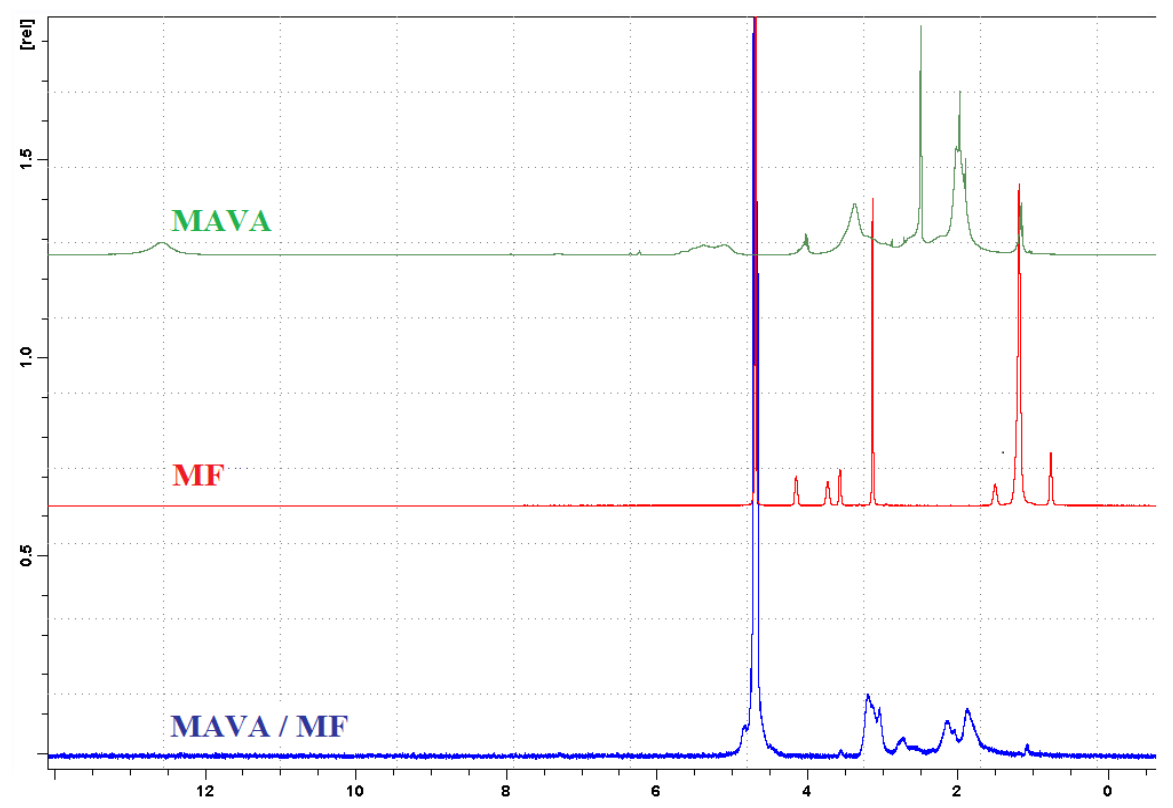

Figure 5. Overlapped ${ }^{1} \mathrm{H}-\mathrm{NMR}$ spectra of the three components MAVA, MF and MAVA/MF. 
G. Karakus et al.: Design, synthesis, structural characterization and cell cytotoxicity of a new derivative ...

MAVA / MF

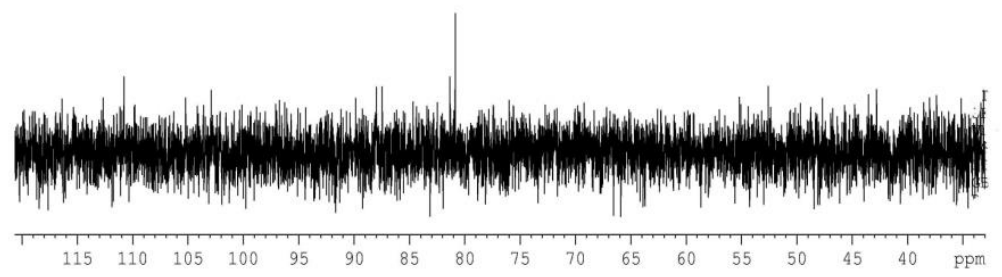

(a)

MF

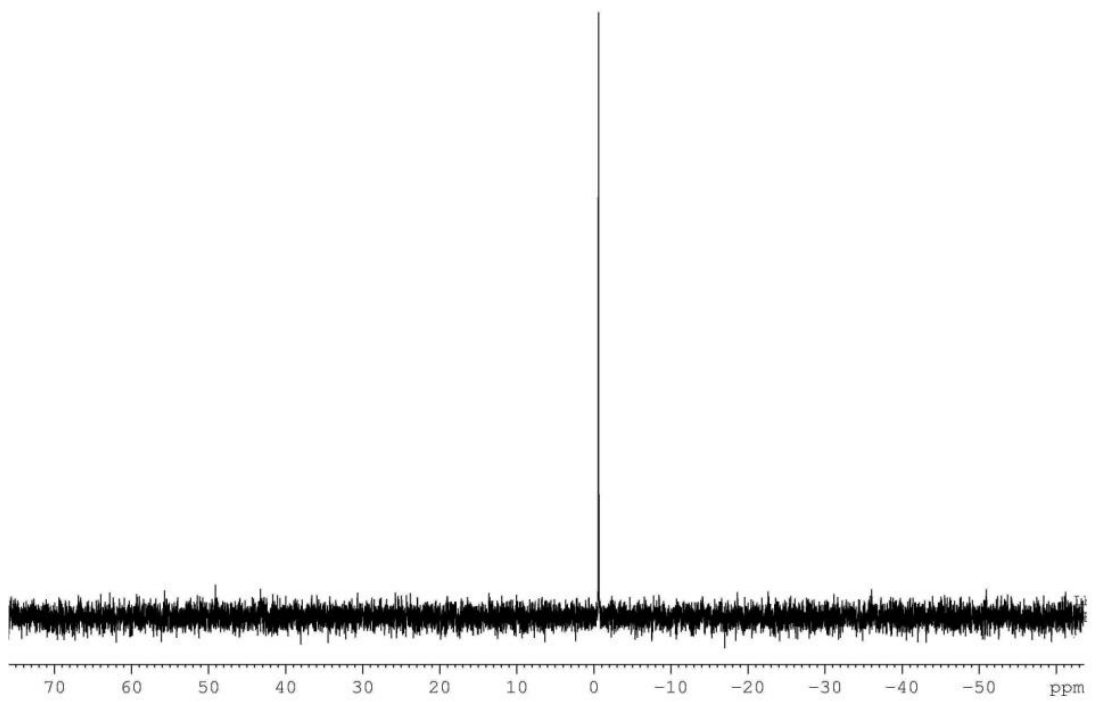

(b)

Figure 6. ${ }^{31} \mathrm{P}-\mathrm{NMR}$ spectra of the conjugation product and MF agent.(a) MAVA/MF and (b) MF.

MAVA/MF conjugate (Figure $4 \mathrm{~b}$ ) peaks are as follows: aliphatic $-\mathrm{CH}_{3}$ peaks of both copolymer backbone and end group of MF appeared at 2.77 ppm. It is taken into account that possible intramolecular hydrogen bonds with direct $-\mathrm{NH}$ and $-\mathrm{OH}$ fragments could not produce completely integrated peaks. Because the $-\mathrm{NH}$ peaks of amide linkage, evidence for the success of the chemical conjugation reaction, were masked/overlapped by the organic solvent peak at around 5.0-8.5 ppm, therefore, they were not detected. However, peaks observed at $4.84 \mathrm{ppm}$ were assigned to amide linkage considering amide were overlapped by the solvent in the 4.63-4.84 ppm range with a slight 
G. Karakus et al.: Design, synthesis, structural characterization and cell cytotoxicity of a new derivative...

difference in chemical shift. $\mathrm{D}_{2} \mathrm{O}$ solvent peaks were observed at $4.76 \mathrm{ppm}$ [51] and $4.81 \mathrm{ppm}$ (TSP). $-\mathrm{CH}_{2}$ protons intensity on VA and MF appeared at 1.31-1.37 ppm. Peak range 2-3 ppm was attributed to the $\mathrm{RNH}-\mathrm{CH}_{2}-(\mathrm{C}$ is attached directly to $\mathrm{N}$ ) as a marker of conjugation reaction, because the copolymer does not include any $\mathrm{N}$ atom. Due to the most important evidence of chemical modification of the copolymer with MF, $1.19,1.26$ and $1.28 \mathrm{ppm}$ could be assigned to characteristic $\mathrm{P}-\mathrm{O}-\mathrm{CH}_{2}$ - linkage resulting from $\mathrm{MF}$ (Figure 4c) on the conjugate [52]. As can be clearly seen from the overlapped spectrum (Figure 5), both copolymer and MF chemical shifts were overlapped at around $1-5 \mathrm{ppm}$ range. The most evident peak in the ${ }^{1} \mathrm{H}-\mathrm{NMR}$ for the conjugation and also ring opening reaction of anhydride ring, shows the presence of phosphorus chemical shifts in the newly synthesized conjugate. All results proved that amide linkages and phosphorus on MAVA/MF were confirmed by ${ }^{1} \mathrm{H}-\mathrm{NMR}$ and FTIR spectra. However, this conjugation reaction should be supported by a more detailed analysis like ${ }^{31} \mathrm{P}-$ NMR analysis.

${ }^{31}$ P-NMR Analysis: Phosphorus-31 nuclear magnetic resonance ( $\left.{ }^{31} \mathrm{P}-\mathrm{NMR}\right)$ is theoretically the same as ${ }^{1} \mathrm{H}-\mathrm{NMR}$ spectroscopy. The ${ }^{31} \mathrm{P}$ nucleus is useful in NMR spectroscopy due to its relatively high gyromagnetic ratio. Moreover, ${ }^{31} \mathrm{P}$ has a $100 \%$ natural isotopic abundance property. Similar to the ${ }^{1} \mathrm{H}$ nucleus, the ${ }^{31} \mathrm{P}$ nucleus has a nuclear spin of $\pm 1 / 2$ which makes spectra relatively easy and fast to interpret. As the phosphorus nucleus is coupled to a hydrogen nucleus bound directly to it, that is, this coupling separated by a single bond, is expected to be very high. Figure 6a shows the MAVA/MF spectrum, only one peak observed again at $80.83 \mathrm{ppm}$ for $\mathrm{PO}_{4}^{-3}$ with some phosphorus chemical shift. It can be said that conjugation reaction is verified by ${ }^{31} \mathrm{P}-\mathrm{NMR}$ due to phosphorus presence on the conjugate. Figure $6 \mathrm{~b}$ shows a poor MF spectrum, only one peak observed at $0.66 \mathrm{ppm}$ for $\mathrm{PO}_{4}^{-3}$ that is an evidence of phosphorus presence [53]. Overlapped ${ }^{31} \mathrm{P}-\mathrm{NMR}$ spectrum is also provided for the free and conjugated MF to compare both spectra (Figure 7).

It should be stressed that ${ }^{31} \mathrm{P}-\mathrm{NMR}$ is an excellent technique for studying phosphorus containing compounds, such as small organic compounds, metal coordination complexes, high molecular weight molecules, etc. Size-exclusion chromatography (SEC) was used for approximate molecular weights $(\mathrm{Mw})$ measurement of pure copolymer and conjugated copolymer. Results obtained for MAVA copolymer: $3.98 \times 10^{5} \mathrm{Da}$ and MAVA/MF conjugate: $1.39 \times 10^{6} \mathrm{Da}$ (Da: daltons) are promising. SEC results confirmed that the molecular weight of conjugated copolymer with $\mathrm{MF}$, reached almost threefold percentage, by the conjugation of MF to the copolymer backbone.

\section{Supposed Reaction Mechanism for the Copolymer and Conjugate}

Basic FTIR, ${ }^{1} \mathrm{H}-\mathrm{NMR}$ and ${ }^{31} \mathrm{P}-\mathrm{NMR}$ spectroscopic analysis and obtained highly watersoluble characteristic of the conjugated product revealed that copolymer synthesized by free-radical polymerization and conjugation mechanism was in agreement with the assumption that the MF molecule was bound to the copolymer backbone by a ring-opening reaction (Schemes 1 and 2, respectively).

\section{Average Molecular Weight Distribution of Copolymer/Drug Conjugate}

Molecular weights of the MAVA copolymer and MAVA/MF conjugate were $\sim 184$ and $\sim 548 \mathrm{gmol}^{-1}$, respectively.

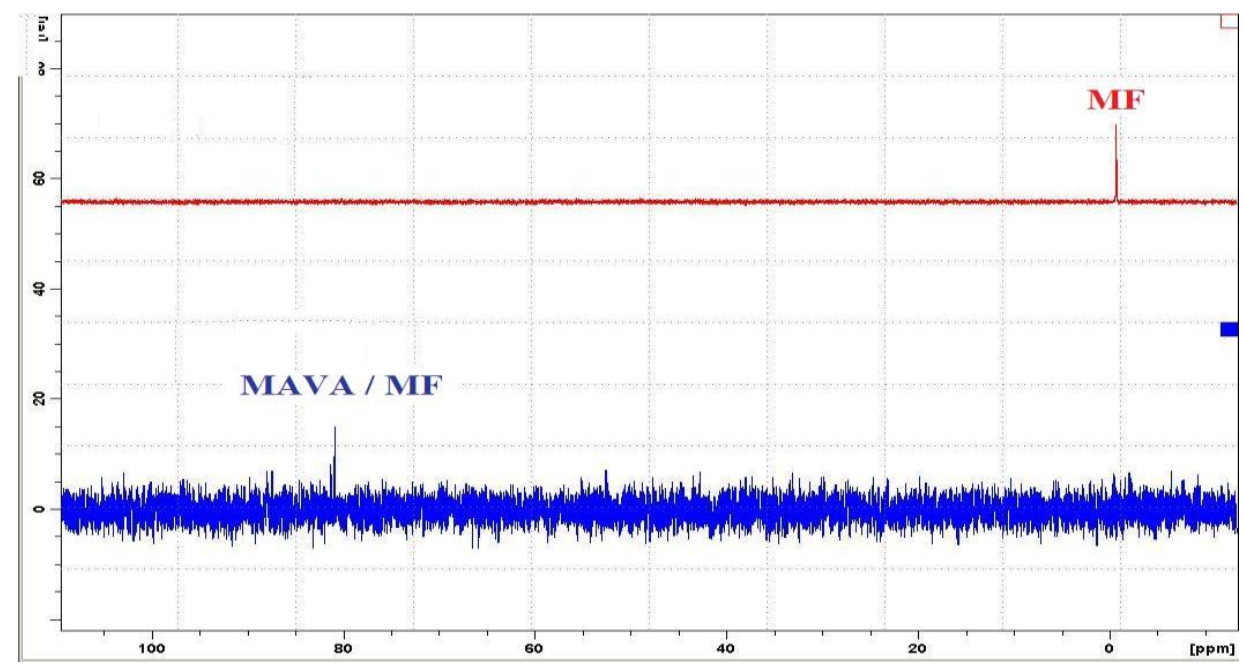

Figure 7. Overlapped ${ }^{31} \mathrm{P}-\mathrm{NMR}$ spectra of the two components MF and MAVAMF. 
G. Karakus et al.: Design, synthesis, structural characterization and cell cytotoxicity of a new derivative...

Cytotoxicity of MAVA Copolymer and MAVA/MF Conjugate

Cytotoxicity of agents: Quantitative analysis, according to WST-1 cytotoxicity test $(24 \mathrm{~h})$, were implemented for copolymer (MAVA), MF, and conjugate (MAVA/MF) (Figure 8).

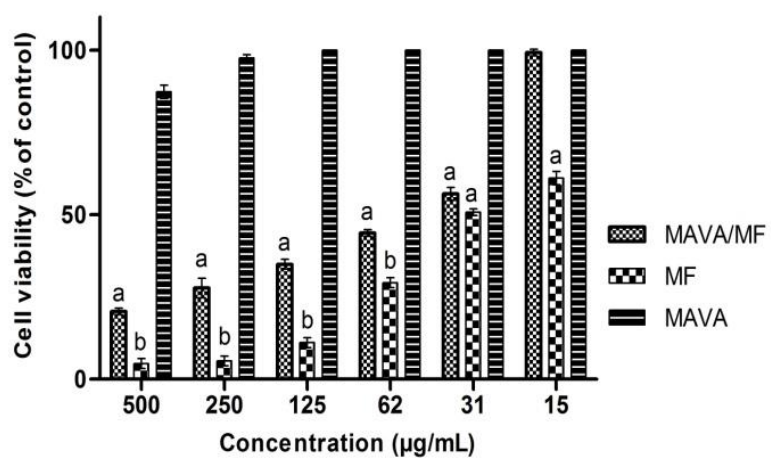

Figure 8. In vitro cytotoxic effect of the copolymer, MF, and MAVA/MF conjugate on L929 fibroblast cells lines. a $\mathrm{P}<0.05$ vs control. ${ }^{\mathrm{b}} \mathrm{P}<0.05$ vs control, MAVA/MF 500, 250, 125 and $62.5 \mathrm{mg} / \mathrm{mL}$.

The figure clearly shows that even at the highest concentration $(500 \mu \mathrm{g} / \mathrm{mL})$ of the copolymer, there was no adverse effect on living cells. The cytotoxicity results of MF and MAVAlMF 500, $250,125,62.5 \mathrm{mg} / \mathrm{mL}$ were compared with the control group for statistical evaluation, and were found significantly different $(\mathrm{P}<0.05)$ (Figure 8$)$. It could be said for the cytotoxicity as follows: MF $>$ MAVA $>$ MAVA/MF (at 500, 250, 125, 62 and $31 \mathrm{mg} / \mathrm{mL}$ ) and MF>MAVA=MAVA/MF (at 15 $\mathrm{mg} / \mathrm{mL}$ ). MAVA/MF copolymer/drug couple was successfully designed with less cytotoxicity than the free drug (MF).

\section{CONCLUSIONS}

To design a new copolymer/drug conjugate, nontoxic vinyl base copolymer (MAVA) was selected as the biocompatible drug carrier for MF. Chemical conjugation of the selected biologically active compound, MF (antileishmanial drug), was achieved by ring-opening reactions.

Potential toxicity measurement is generally used for the investigation of the viability effects of test compounds on the cells grown for a drug candidate. Synthetic polymers are considered to be promising materials for biomedical applications. Cell cytotoxicity assays of MAVA, MF, and MAVA/MF were carried out by quantitative WST1 test $(24 \mathrm{~h})$. As a promising result it was observed that MAVA did not damage cell viability even at its highest concentration $(500 \mu \mathrm{g} / \mathrm{mL})$. The cytotoxicity results of MF and MAVA/MF 500, $250,125,62.5 \mathrm{mg} / \mathrm{mL}$, by comparing with control group, were found to be statistically significantly different $(\mathrm{P}<0.05)$.

Promising results should be emphasized, considering that MF is a highly toxic drug, that MAVA/MF had no cytotoxicity and is synthesized economically in a short time.

The initial goal of this experiment was to obtain a less/non-toxic drug having high water-soluble characteristics by comparing with free MF. On the other hand, preserving the original characteristics of miltefosine in the conjugate is a crucial step for rational copolymer/drug design.

In this study, in the direction of our main goal the conjugation reaction for copolymer/drug couple was successfully carried out with better water solubility and also less cytotoxicity than the free drug. We also hope that the biological activity of this conjugate (MAVA/MF) will be higher than that of the free drug (MF) with more comprehensive biological tests to be performed.

Acknowledgement: In this study, the polymeric samples, MAVA and MAVA/MF, were synthesized in the Research Center (CUTFAM), Cumhuriyet University, Faculty of Medicine (Sivas, Turkey). FTIR and NMR analysis were carried out at the Technology Research and Developing Centre (TAUM), Erciyes University (Kayseri, Turkey). Molecular weight measurements of the samples were performed by SEC (Size-Exclusion Chromatography) analysis at Yildiz Technical University (Istanbul, Turkey).

Disclosure Statement: The authors of the manuscript solemnly declare that no scientific and/or financial conflicts of interest exist with other people or institutions.

\section{REFERENCES}

1. I. Popescu, D.M. Suflet, I.M. Pelin, G.C. Chitanu, Rev. Roum. Chim., 56, 173 (2011).

2. T. Minko, Drug. Discov. Today Tech., 2, 15 (2005).

3. G.R. Saad, R.E. Morsi, S.Z. Mohammady, M.Z. Elsabee, J. Polym. Res., 15, 115 (2008).

4. O. Atıc1-Galioglu, A. Akar, R. Rahimian, Turk. $J$. Chem., 25, 259 (2001).

5. H. Patel, D.A. Raval, D. Madamwar, S.R. Patel, Die Angewandte Makromol. Chem., 263, 25 (1998).

6. H.Y. Liu, K. Cao, Z. Li, B.G. Yao, G.H. Hu, J. Appl. Polym. Sci., 104, 2418 (2007).

7. B.L. William, R. David, Kryscio1, V.S. Brandon, A.P. Nicholas, Annu. Rev. Chem. Biomol. Eng., 1, 149 (2010).

8. H. Ringsdorf, J. Polymer. Sci., 51, 135 (1975).

9. R. Duncan, Res. Foc. Rev., 2, 441 (1999).

10. K. Hoste, K.D. Winne, E. Schacht, Int. J. Pharmaceut., 277, 119 (2004).

11. K. Greish, T. Sawa, J. Fang, T. Akaika, H. Maeda, J. Cont. Rel., 97, 219 (2004). 
G. Karakus et al.: Design, synthesis, structural characterization and cell cytotoxicity of a new derivative ...

12. H. Maeda, M. Ueda, T. Morinaga, T. Matsumoto, $J$. Med. Chem., 28, 455 (1985).

13. A. Kobayashi, T. Oda, H. Maeda, J. Bioact. Compat. Pol., 3, 319 (1988).

14. N. Ohtsuka, T. Konno, Y. Myauchi, H. Meada, Cancer, 59, 1560 (1987).

15. H. Maeda, T. Matsumoto, T. Konno, K. Iwai, M. Ueda, J. Protein Chem., 3, 181 (1984).

16. K. Greish, J. Fang, T. Inutsuka, A. Nagamitsu, H. Maeda, Clin. Pharmacokinet., 42, 1089 (2003).

17. H. Maeda, Adv. Drug Deliv. Rev., 6, 181 (1991).

18. C. Mènez, M. Buyse, M. Besnard, R. Farinotti, P.M. Loiseau, G. Barratt, Antimicrob. Agents, 50, 3793 (2006).

19. M. Bruch, D. Mäder, F. Bauers, T. Loontjens, R. Mülhaupht, J. Polym. Sci. Pol. Chem., 38, 1222 (2000).

20. G. Karakus, H.B. Zengin, Z. Akin Polat, A.F. Yenidunya, S. Aydin, Polym. Bull., 70, 1591 (2013).

21. V. Nguyen, W. Yoshida, Y. Cohen, J. Appl. Polym. Sci., 87, 300 (2003).

22. D. Fles, R. Vukovic, A.E. Kuzmic, G. Bogdanic, V. Pilizota, D. Karlovic, K. Markus, K. Wolsperger, D. Vikic-Topic, Croat. Chem. Acta, 76, 69 (2003).

23. G. Karakus, Z. Akin Polat, A. Sahin Yaglioglu, M. Karahan, A.F. Yenidunya, J. Biomat. Sci-Polym., E. 24, 1260 (2013).

24. E. Chernikova, P. Terpugova, C. Bui, B. Charleux, Polym., 44, 4101 (2003).

25. Y. Li, S. Richard-Turner, Eur. Polym. J., 46, 821 (2010)

26. C.M. Xiao, J. Tan, G.N. Xue, Express. Polym. Lett., 4, 9 (2010).

27. G.C. Chitanu, I. Popescu, A. Carpov, Rev. Roum. Chim., 51, 923 (2006).

28. M.A. Krayukhina, S.A. Kozybakova, N.A. Samoilava, V.G. Babak, S.Z. Karaeva, I.A. Yamskov, Russ. J. Appl. Chem., 80, 1145 (2007).

29. Z. Qiao, Y. Xie, M. Chen, J. Xu, Y. Zhu, Y. Qian, Chem. Phys. Lett., 321, 504 (2000).

30. V. Sunel, M. Popa, A.D. Stoican, A.A. Popa, C. Uglea, Mater. Plast., 45, 149 (2008).

31. Z.M.O. Rzayev, Int. Rev. Chem. Eng., 3, 153 (2011)

32. J. Pal, H. Singh, A.K. Ghosh, J. Appl. Polym. Sci., 92, 102 (2004).

33. J.H. Jeong, Y.S. Byoun, S.B. Ko, Y.S. Lee, J. Ind. Eng. Chem., 7, 310 (2001).

34. L. Xiao, H. Shimotani, M. L J. Ozawa, N. Dragoe, K. Saigo, K. Kitazawa, J. Polym. Sci. Pol. Chem., 37, 3632 (1999).
35. G. Karakus, Z. Akin Polat, A.F. Yenidunya, H.B. Zengin, C.B. Karakus, Polym. Int., 62, 492 (2013).

36. H. Kaplan Can, A.L. Doğan, Z.M.O. Rzaev, A.H. Uner, A. Güner, J. Appl. Polym. Sci., 96, 2352 (2005).

37. R.L. Frost, Y. Xi, R. Scholz, M.B. Fernanda, Spectrochim. Acta. A103, 143 (2013).

38. R.L. Frost, Y. Xi, R. Scholz, M.B. Fernanda, A.D.M. Luiz Filho, Spectrochim. Acta, A104, 250 (2013).

39. R.L. Frost, A. Lopez, Y. Xi, A. Granja, R. Scholz, R.M.F. Lima, Spectrochim. Acta, A114, 309 (2013).

40. R.L. Frost, Y. Xi, M. Beganovic, F.M. Belotti, R. Scholz, Spectrochim. Acta, A107, 241 (2013).

41. R.L. Frost, Y. Xi, R. Scholz, Spectrochim. Acta, A108, 244 (2013).

42. R.L. Frost, Y. Xi, R. Scholz, F.M. Belotti, Spectrochim. Acta, A105, 359 (2013).

43. R.L. Frost, Y. Xi, R. Scholz, F.M. Belotti, M. Beganovic, Spectrochim. Acta, A110, 7 (2013).

44. R.L. Frost, R. Scholz, A. Lòpez, Y. Xi, Spectrochim. Acta, Part A: Molecular and Biomolecular Spectroscopy 124, 243 (2014).

45. S. Dos, W. Khan, S. Mohsin, N. Kumar, Polym. Adv. Technol., 22, 172 (2011).

46. S. Hwang, C.H. Lee, I.S. Ahn, J. Ind. Eng. Chem., 14, 487 (2008).

47. E. Lee, B.H. Moon, Y. Park, S. Hong, S. Lee, Y. Lee, Y. Lim, Bull. Korean Chem. Soc., 29, 507 (2008).

48. D. Jacquemin, E.A. Perpete, I. Ciofini, J. Chem. Theory Comput., 6, 2071 (2010).

49. K.J. Yoon, J.H. Woo, Y.S. Seo, Fiber Polym., 4, 182 (2003).

50. G. Nemtoi, C. Beldie, C. Tircolea, I. Popa, I. Cretescu, I. Humelnicu, D. Humelnicu, Eur. Polym., J., 37, 729 (2001)

51. H.E. Gottlieb, V. Kotlyar, A. Nudelman, J. Org. Chem., 62, 7512 (1997).

52. B. Basnar, M. Litschauer, S. Abermann, E. Bertagnolli, G. Strasser, M-A. Neouze, Electronic Supplementary Information. Layer-by-layer assembly of titania nanoparticles based ionic networks, Chemical Communications, 47, 361 (2011).

53. H. Kaur, K. Seifert, G.E. Hawkes, G.S. Coumbarides, J. Alvar, S.L. Croft, Am. J. Trop. Med. Hyg., 92 (Suppl. 6), 31 (2015). 


\title{
Optimization of $\beta$-carotene solubility in pressurized hot water using a dynamic method and factorial methodology
}

\author{
N. Ebrahimi, A. Haghighi Asl*, P. Mottahedin \\ Extraction Research Group, Faculty of Chemical, Gas and Petroleum Engineering, Semnan University, Semnan, \\ Iran
}

Received, February 20, 2018; Revised May 28, 2019

In this experimental investigation, the solubility of $\beta$-carotene in pressurized hot water was measured at temperatures in the range of $(70-130){ }^{\circ} \mathrm{C}$ and the pressure of 20 bar by using a dynamic method. The influence of different variables, including temperature and flow rate in the range of $(0.15-0.64) \mathrm{ml} / \mathrm{min}$, on the solubility of $\beta$ carotene was investigated. Furthermore, the optimization of these parameters was done through the experimental design by using factorial methodology. The results of the experiments showed that the temperature was the main parameter affecting the solubility of $\beta$-carotene in pressurized hot water. The best conditions for solubility of $\beta$-carotene in pressurized hot water were the temperature of $85{ }^{\circ} \mathrm{C}$ and a flow rate of $0.64 \mathrm{ml} / \mathrm{min}$. $\beta$-carotene's solubility data in pressurized hot water were correlated with the dielectric constant values of the solvent mixture.

Keywords: Solubility, $\beta$-carotene, Pressurized hot water, Factorial methodology, Correlation, Dielectric constant

\section{INTRODUCTION}

$\beta$-carotene is a main subgroup of carotenoids. It can be found in vegetables, for example, pumpkin [1]. Many of the carotenoid compounds can be extracted from deep color vegetables and fruits because they are rich sources of phenolic compounds, including flavonoids and carotenoids [2-5]. On the other hand, for the food engineering design applications, it is important to have fundamental physicochemical data, such as solute solubility in the solvents. Solubility data are used for optimization of extraction processes. $\beta$-carotene solubility in solvents such as ethane and supercritical $\mathrm{CO}_{2}$ is measured [6-8]. Many carotenoids are sensitive to environmental factors such as light, heat, and oxygen; therefore, such measurements are challenging [9].

In recent years, due to the growing interest in subcritical fluid technology, a number of novel approaches have been proposed to measure the solubility of organic components in pressurized hot water [10-20]. These methods can be basically classified into static and dynamic methods [21]. To our knowledge, pressurized hot water, defined as water between $100{ }^{\circ} \mathrm{C}$ and $374{ }^{\circ} \mathrm{C}$, is an effective solvent for both polar and non-polar compounds [22]. Water is a solvent with tunable polarity. When the temperature increases, the polarity of water decreases. Therefore, the solubility of non-polar organics increases [23].

In the static method, an extra amount of solute and a certain amount of solvent are loaded into an equilibration cell. After the equilibrium is achieved, the sample solution is provided for analysis [21]. In the dynamic method, the solvent flows through an equilibrium solubility cell continuously. The cell contains an excess amount of the solute under the experimental temperature and pressure conditions. After the system reaches the equilibrium conditions the sample solution flows through a valve or restrictor for analysis. Miller et al. [24] used the dynamic method to measure the solubility of hydrophobic organics in pressurized hot water. Also, Zhang et al. [25] used the same method to investigate the solubility of carbohydrates in pressurized hot water. Takebayashi et al. [26] used the dynamic method for measuring the solubility of terephthalic acid in pressurized hot water whose temperature was in the range of $75-275^{\circ} \mathrm{C}$. Karasek et al. [27] used the dynamic method to measure the solubility of solid polycyclic aromatic hydrocarbons in pressurized hot water at temperatures ranging from $313 \mathrm{~K}$ to the melting point. Modeling the solubility of polycyclic aromatic hydrocarbons can be useful for predicting the solubility data in pressurized hot water [28]. The experimental procedure of the dynamic method is easy and quick. Also, it is difficult to measure the solubility of sensitive compounds above the boiling point of water using a static apparatus [9].

The objective of this research is to measure the solubility of $\beta$-carotene in pressurized hot water at temperatures ranging from 70 to $130{ }^{\circ} \mathrm{C}$ and a constant pressure of $20 \mathrm{bar}$. Since the solubility of $\beta$-carotene is low in water at room temperature, the

* To whom all correspondence should be sent:

E-mail: ahaghighi@semnan.ac.ir 
$N$. Ebrahimi et al.: Optimization of $\beta$-carotene solubility in pressurized hot water using a dynamic method ... pressurized hot water is used to enhance its solubility in water. The dynamic method has been used for these measurements. In this experimental investigation, the effect of temperature and flow rate on the solubility of $\beta$-carotene has been reported. Additionally, the mentioned variables were optimized through the design of experiments using factorial methodology. Also, $\beta$-carotene solubility data were correlated with the dielectric constants of the solvent.

\section{EXPERIMENTAL}

\section{Materials}

$\beta$-Carotene $(\geq 93 \%)$ was from Sigma-Aldrich. In this work, HPLC-grade water was utilized for all experiments. HPLC-grade ethyl acetate, acetonitrile, and methanol were purchased from DAEJUNG.

\section{Apparatus and methods}

The system used for measuring the solubility of $\beta$-carotene was based on a modification of the system used by Karasek et al. [29].

Solubility measurements were conducted with a dynamic flow type system [30]. This apparatus is schematically shown in Fig. 1. The experimental system had an HPLC pump (SY-8100 series, BFRL Co., Germany) which can work with very low flow rates. The inlet of pipelines was equipped with a burette to check the flow rate of the pump. The solubility cell was located in an oven. The role of this oven was to control the temperature (up to 250 $\pm 1{ }^{\circ} \mathrm{C}$ ) by using a PID controller. The sole solvent, HPLC grade water, was maintained in the ultrasonic cleaner system for $20 \mathrm{~min}$ at a temperature of $60{ }^{\circ} \mathrm{C}$. After that, a 5-L Pyrex feed tank was filled with water and purged with nitrogen for $1 \mathrm{~h}$ to eliminate dissolved $\mathrm{O}_{2}$. In each experiment, the cell was filled with $0.1 \mathrm{~g}$ of $\beta$ carotene. To avoid the particles' flow, two stainless steel filter, two fabric filters, and a soft metal filter were used at both cell sides.

In all experiments, the pressure of 20 bar was selected because the liquid water was needed at the extraction temperatures. When the outlet valve was closed, the HPLC pump was turned on to obtain the specified pressure. Subsequently, the pump was turned off and by using the oven the system reached the specified temperature. At this time, the outlet valve was opened and the HPLC pump started to work with the specified rate in which the pressure was kept constant at 20 bar. At the end of the pipeline, a fused-silica capillary tube (i.d. $75 \mu \mathrm{m}$ ) was located inside the oven. Due to the usage of the capillary tube, the pressure was kept at the desired capillary tube in the oven, to minimize the measurement errors. When each run finished, the samples are analyzed using HPLC.

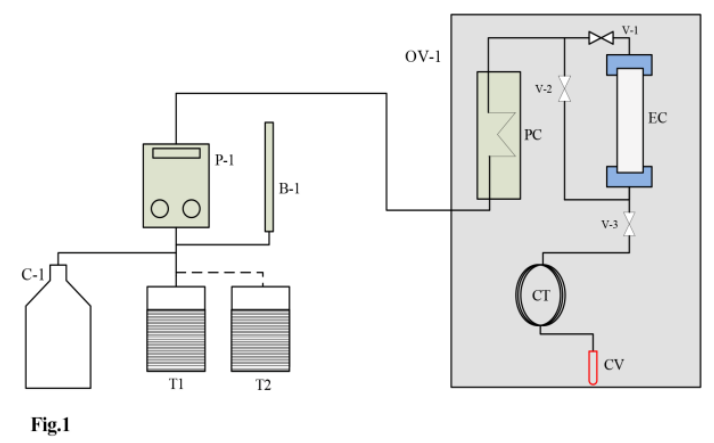

Fig. 1. Schematic diagram of the solubility measurement system, B-1: burette, C-1: nitrogen cylinder, EC: cell, PC: preheating coil, OV-1: oven, P-1: HPLC pump, T1: water tank, T2: solvent tank, v-1: cell inlet valve, v-3: cell outlet valve, v-2: bypass valve, CT: capillary tube, CV: sample collection vial.

The experimental system was equipped with an HPLC pump (SY-8100 series, BFRL Co., Germany) which can work with very low flow rates. The inlet of pipelines was connected with a burette to check the flow rate of the pump. The solubility cell was located in an oven. The role of this oven was to control the temperature (up to 250 $\pm 1{ }^{\circ} \mathrm{C}$ ) by using a PID controller. The sole solvent, HPLC-grade water, was maintained in a ultrasonic cleaner system for $20 \mathrm{~min}$ at a temperature of $60^{\circ} \mathrm{C}$. After that, the 5-L Pyrex feed tank was filled with water and purged with nitrogen for $1 \mathrm{~h}$ to eliminate dissolved $\mathrm{O}_{2}$. In each experiment, the cell was filled with $0.1 \mathrm{~g}$ of $\beta$-carotene. To avoid the particles' flow, two stainless steel filters, two fabric filters, and a soft metal filter were used at both cell sides.

In all experiments, the pressure of 20 bar was selected because liquid water was needed at the extraction temperatures. When the outlet valve was closed, the HPLC pump was turned on to obtain the specified pressure. Subsequently, the pump was turned off and by using the oven the system reached the specified temperature. At this time, the outlet valve was opened and the HPLC pump started to work with the specified rate in which the pressure was kept constant at 20 bar. At the end of the pipeline, a fused-silica capillary tube (i.d. $75 \mu \mathrm{m}$ ) was located inside the oven. Due to the usage of the capillary tube, the pressure was kept at the desired value. Samples were collected after the capillary tube. When each run finished, the samples were analyzed by HPLC.

\section{$\beta$-Carotene quantification analysis}

The calibration of HPLC was conducted by 
$N$. Ebrahimi et al.: Optimization of $\beta$-carotene solubility in pressurized hot water using a dynamic method ... solutions containing a $\beta$-carotene stock solution in acetonitrile. The concentrations of $\beta$-carotene were 200, 100, 50, 25 and $12.5 \mathrm{ppm}$. As can be seen in Fig. 2, the calibration curve was regressed with a linear function.

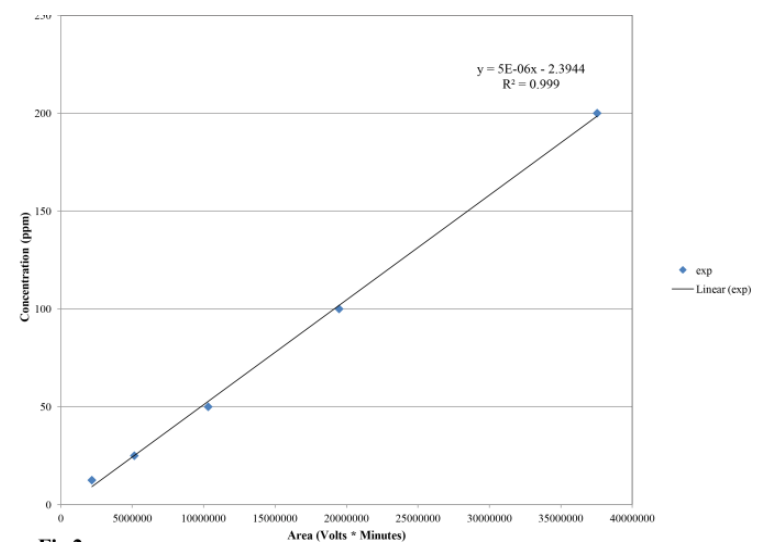

Fig. 2. Calibration curve for $\beta$-carotene

\section{Factorial design}

The experimental design of the factors affecting the solubility, including temperature and pressurized hot water flow rate, was investigated under full factorial design. The factorial design was done to reduce the cost, to design optimization, and to avoid time-consuming analysis methods [31]. The constants of the factorial equations were obtained by MINITAB 16. Each statistical analysis was done two times for each solubility process to ensure their reproducibility. Table 1 indicates the factors, ranges, and levels of independent variables in the factorial design.

Table 1. Ranges of the independent variables in the factorial design

\begin{tabular}{llcc}
\hline Factor & Type & Levels & Values \\
\hline $\mathrm{T}\left({ }^{\circ} \mathrm{C}\right)$ & Fixed & 3 & $70,85,130$ \\
$\mathrm{Q}(\mathrm{ml} / \mathrm{min})$ & Fixed & 3 & $0.15,0.42,0.64$ \\
\hline
\end{tabular}

\section{Dielectric constant model}

Table 2. Dielectric constant values for temperatures between $0{ }^{\circ} \mathrm{C}$ and $200{ }^{\circ} \mathrm{C}$ and $0 \%$ and $20 \%$ (v/v) ethanol in water solutions [32].

\begin{tabular}{cccc}
\hline $\mathrm{T}$ & \multicolumn{3}{c}{ Ethanol \% (v/v) } \\
\cline { 2 - 4 }$\left({ }^{\circ} \mathrm{C}\right)$ & 0 & 5 & 20 \\
\hline 0 & 80.37 & 77.43 & 68.66 \\
50 & 72.54 & 69.82 & 61.69 \\
100 & 65.47 & 62.95 & 55.43 \\
110 & 64.15 & 61.66 & 54.26 \\
120 & 62.84 & 60.40 & 53.11 \\
130 & 61.57 & 59.16 & 51.99 \\
140 & 60.32 & 57.95 & 50.88 \\
150 & 59.10 & 56.76 & 49.81 \\
160 & 57.90 & 55.60 & 48.75 \\
170 & 56.72 & 54.46 & 47.72 \\
180 & 55.57 & 53.34 & 46.71
\end{tabular}

$\begin{array}{llll}190 & 54.44 & 52.25 & 45.72 \\ 200 & 53.34 & 51.18 & 44.75\end{array}$

Table 2 [32] illustrates the dielectric constant values for different temperatures and different volume percentages of ethanol in water.

$\beta$-Carotene solubility data from this work and a previous work [30] can be correlated with the dielectric constant values in table 2 .

\section{RESULTS AND DISCUSSION HPLC analysis}

The HPLC analysis of $\beta$-carotene was conducted using a reversed-phase HPLC system (Knauer Company, Germany). The HPLC chromatograms of $\beta$-carotene are shown in Fig. 3. The solvents used were (A) acetonitrile:water (9:1 v/v) and (B) ethyl acetate. The solvent gradient utilized was developed as follows: $0-40 \%$ solvent B $(0-20 \mathrm{~min})$, 40-60\% solvent B (20-25 min), 60-100\% solvent B (25-25.1 min), $100 \%$ solvent B (25.1-35 $\mathrm{min})$, and $100-0 \%$ solvent $\mathrm{B}(35-35.1 \mathrm{~min})$ at a flow rate of $1.0 \mathrm{ml} \mathrm{min}{ }^{-1}$ [1]. The temperature of the column was maintained at $20^{\circ} \mathrm{C}$. The injection volume was $20 \mu \mathrm{l}$.
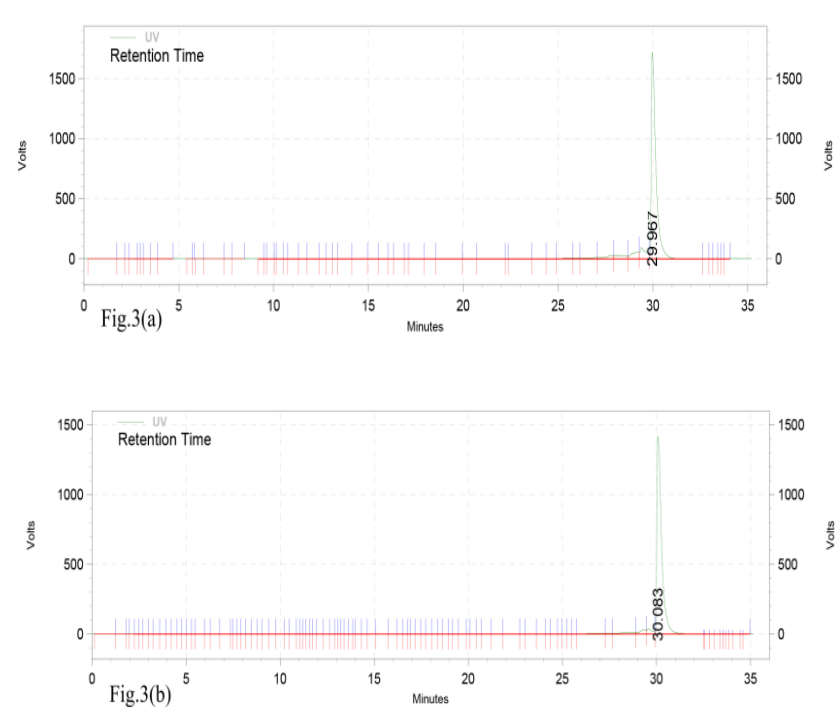

Fig. 3. HPLC chromatogram of (a) $\beta$-carotene standard and (b) $\beta$-carotene (obtained at $85^{\circ} \mathrm{C}$ )

\section{Factorial analysis}

The obtained experimental solubilities of $\beta$ carotene in pressurized hot water are presented in Table 3. Table 4 shows the importance of each parameter by considering the $\mathrm{P}$ value and contribution term. The most significant variables affecting the yield of $\beta$-carotene solubility were the linear terms of temperature and flow rate and the interaction term of temperature and flow rate $(\mathrm{P}<0.05)$. R-squared $=99.84 \%$ and R-squared $(\operatorname{adj})$ $=99.69 \%$ indicate that the experimental data are in good agreement with the predicted values. 
N. Ebrahimi et al.: Optimization of $\beta$-carotene solubility in pressurized hot water using a dynamic method ... Table 3. The result of the two factors-three levels

\begin{tabular}{cccc}
\hline Exp. & $\mathrm{T}\left({ }^{\circ} \mathrm{C}\right)$ & $\mathrm{Q}(\mathrm{ml} / \mathrm{min})$ & $\mathrm{C}(\mathrm{ppm})$ \\
\hline 1 & 70 & 0.15 & 71.456 \\
2 & 70 & 0.42 & 87.587 \\
3 & 70 & 0.64 & 105.586 \\
4 & 85 & 0.15 & 85.242 \\
5 & 85 & 0.42 & 130.004 \\
6 & 85 & 0.64 & 173.338 \\
7 & 130 & 0.15 & 6.654 \\
8 & 130 & 0.42 & 9.653 \\
9 & 130 & 0.64 & 10.878 \\
10 & 70 & 0.15 & 65.023 \\
11 & 70 & 0.42 & 89.618 \\
12 & 70 & 0.64 & 110.989 \\
13 & 85 & 0.15 & 88.949 \\
14 & 85 & 0.42 & 137.006 \\
15 & 85 & 0.64 & 180.188 \\
16 & 130 & 0.15 & 5.992 \\
17 & 130 & 0.42 & 8.154 \\
18 & 130 & 0.64 & 10.622 \\
\hline
\end{tabular}

Table 4. $\mathrm{P}$ value and contribution term

\begin{tabular}{ccc}
\hline Source & P value & Contribution \\
\hline $\mathrm{T}$ & 0.000 & 0.83 \\
$\mathrm{Q}$ & 0.000 & 0.11 \\
$\mathrm{~T}^{*} \mathrm{Q}$ & 0.000 & 0.06 \\
\hline
\end{tabular}

\section{Effect of independent variables on the solubility}

The effect of operating parameters (temperature and pressurized hot water flow rate) on the solubility of $\beta$-carotene was investigated. The plots of the response (beta carotene solubility) against the independent variables are shown in Figs. 4 and 5.

As shown in Fig.4 (a) at a constant water flow rate, an increase in temperature leads to an increase in the solubility of $\beta$-carotene up to the temperature of $85^{\circ} \mathrm{C}$. After this temperature, due to the thermal degradation of $\beta$-carotene, the solubility decreases. Thermal degradation of $\beta$-carotene is visible by the change in the color of the solution and decrease of the area under the chromatogram peak. According to Fig.4 (b) at a constant temperature, increasing the flow rate of water enhances the solubility $\beta$ carotene with a mild slope. It is because of higher superficial velocity and so higher coefficient of mass transfer. Fig.5 (a, b) shows the contour plot of the $\beta$-carotene solubility $v s$. T, Q.

\section{Dielectric constant model}

Fig. 6 shows the solubility data for $\beta$-carotene in pressurized hot water solutions against the dielectric constant values of the solvent mixture.
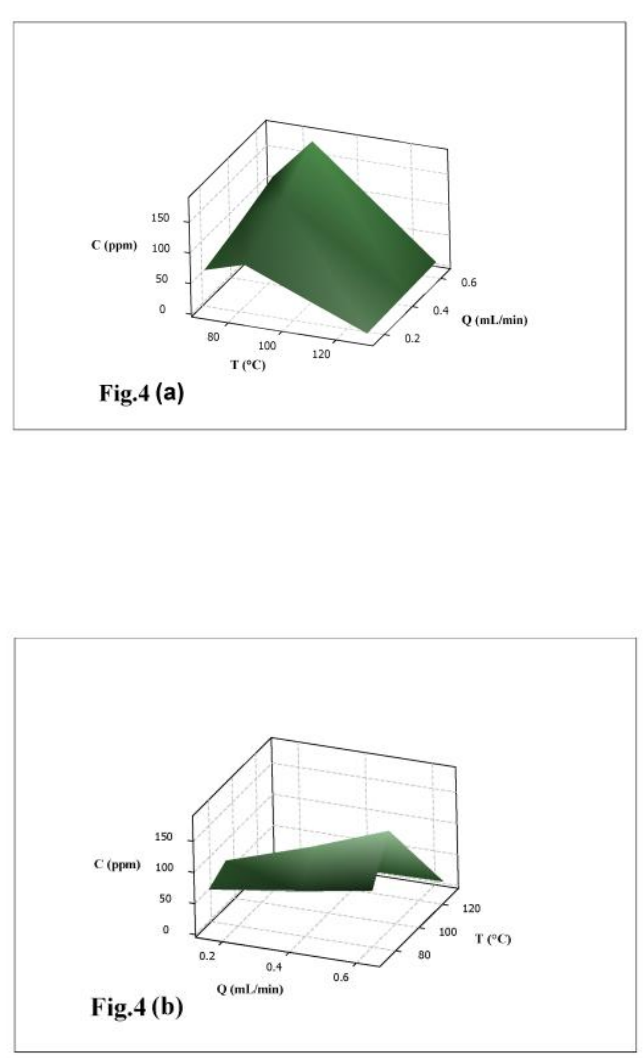

Fig. 4. (a, b) Factorial plots of $\beta$-carotene solubility as a function of temperature and flow rate at a pressure of $20 \mathrm{bar}$.
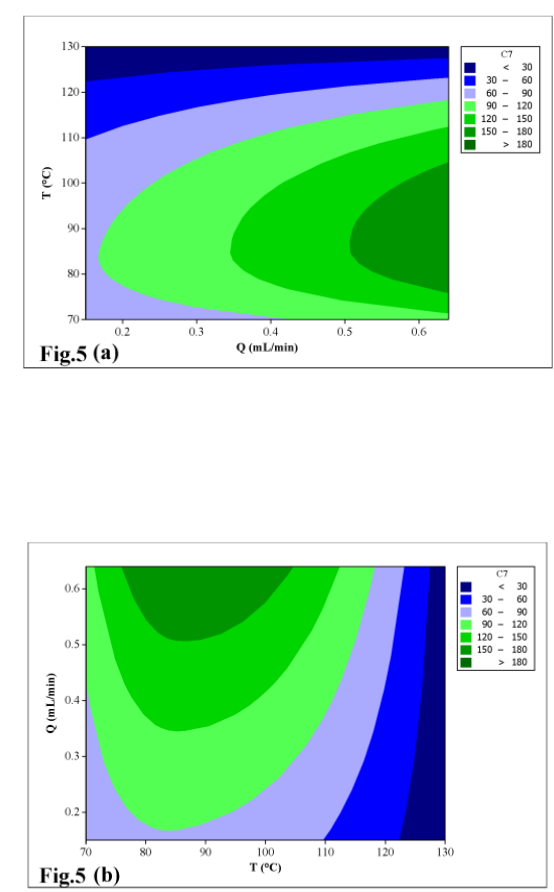

Fig. 5. (a, b) Contour plots of $\beta$-carotene solubility 
$N$. Ebrahimi et al.: Optimization of $\beta$-carotene solubility in pressurized hot water using a dynamic method ... vs. T, Q

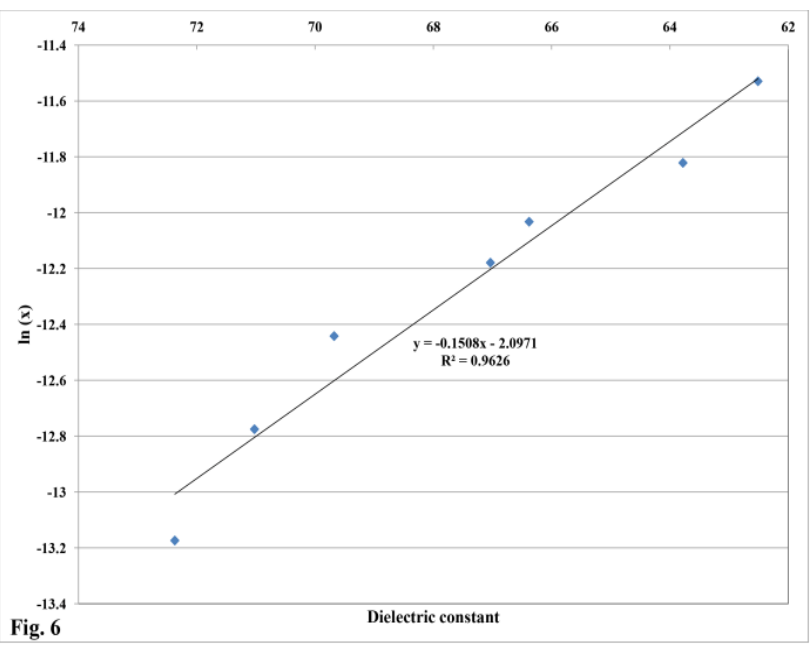

Fig. 6. Solubility of $\beta$-carotene in pressurized hot water solutions vs. dielectric constant values.

As can be seen in Fig. 6, the following equation stands for the solubility of $\beta$-carotene in pressurized hot water solution as a function of dielectric constant:

$$
\ln \mathrm{x}=\mathrm{Ad}+\mathrm{B}
$$

where $\mathrm{x}$ is the solubility and $\mathrm{d}$ is the dielectric constant. The constants $\mathrm{A}$ and $\mathrm{B}$ for $\beta$-carotene are as follows: $\mathrm{A}=-0.1508$ and $\mathrm{B}=-2.0971$.

The validity and goodness of the model were checked by the coefficient of determination $\left(\mathrm{R}^{2}\right)$ value. $R^{2}=0.9626$ indicates acceptable model validation.

\section{CONCLUSIONS}

The experimental solubility measurement of $\beta$ carotene in pressurized hot water was carried out in the temperature range of $(70-130){ }^{\circ} \mathrm{C}$ and water flow rate of $0.15-0.64 \mathrm{ml} / \mathrm{min}$. The dynamic approach was used to investigate the solubility of $\beta$ carotene in pressurized hot water. The operating conditions were optimized by using factorial methodology. The optimal conditions for solubility of $\beta$-carotene were the temperature of $85^{\circ} \mathrm{C}$ and the flow rate of $0.64 \mathrm{ml} / \mathrm{min}$. Also, the experimental solubility data of $\beta$-carotene were correlated with the dielectric constants of pressurized hot water solutions with $\mathrm{R}^{2}=0.9626$.

\section{REFERENCES}

1. S. Norshazila, J. Irwandi, R. Othman, H. H. Yumi Zuhanis, Int. Food Research J., 19, 531 (2012).

2. J. Y. Qian, D. Liu, A. G. Huang, Food Chem., 87, 283 (2004).

3. A. Sass-Kiss, J. Kiss, P. Milotay, M. M. Kerek, M. Toth-Markus, Food Research Int., 38, 1023 (2005).

4. A. Trappey, H. A. Bawadi, R. R. Bansode, J. N. Losso, Food Chem., 91, 665 (2005).
5. E. Cieślik, A. Gręda, W. Adamus, Food Chem., 94 135 (2006).

6. R.L. Mendes, B.P. Nobre, J.P. Coelho, A.F. Palavra, J. Supercrit. Fluids, 16, 99, (1999)

7. B.N. Hansen, A.H. Harvey, J.A.P. Coelho, A.M.F Palavra, and Th.J. Bruno, J. Chem. Eng. Data, 46, 1054, (2001).

8. K. Sakaki, J. Chem. Eng. Data, 37, 249 (1992).

9. K. Srinivas, J. W. King, L. R. Howard, \& J. K. Monrad, J. Food Eng., 100, 208 (2010).

10. W.H. Teoh, R. Mammucari, S.A.B. Vieira de Melo, N.R. Foster, Ind. Eng. Chem. Res., 52, 5806 (2013).

11. E. Yabalak, Ö. Görmez, B. Gözmen, A. M. Gizir, Int. J. Ind. Chem., 6, 23 (2015).

12. P. Karásek, B. Hohnová, J. Planeta, and M. Roth, J. Chem. Eng. Data, 55, 2866 (2010).

13. P. Karásek, B. Hohnová, J. Planeta, L. Št'avíková, M. Roth, Chemosphere, 90, 2035 (2013).

14. A. G. Carr, R. Mammucari, N. R. Foster, Ind. Eng. Chem. Res. 49, 3403 (2010).

15. A. G. Carr, R. Mammucari, N. R. Foster, Ind. Eng. Chem. Res. 49, 9385 (2010).

16. P. Karásek, J. Planeta, M. Roth, J. Chem. Eng. Data, 53, 160 (2008).

17. P. Karásek, J. Planeta, M. Roth, J. Chem. Eng. Data, 53, 816 (2008).

18. K. Srinivas, J. W. King, L. R. Howard, J. K. Monrad, J. Chem. Eng. Data, 55, 3101 (2010).

19. P. Huang, R. Yang, T. Qiu, X. Fan, J. Supercrit. Fluids, 81, 221 (2013).

20. W.H. Teoh, S.A.B. Vieira de Melo, R. Mammucari, N. R. Foster, Ind. Eng. Chem. Res., 53, 10238 (2014).

21. K. Bei, C. Zhang, J. Wang, K. Li, J. Lyu, J. Zhao, J. Chen, I. M. Chou, Z. Pan, Fluid Phase Equil., 425, 93 (2016).

22. P. Mottahedin, A. Haghighi Asl, M. Khajenoori, $J$. Food Proces. Preserv., 41, e13095 (2017).

23. A. G. Carr, R. Mammucari, N. R. Foster, Chem. Eng. J., 172, 1 (2011).

24. D. J. Miller, S. B. Hawthorne, J. Chem. Eng. Data, 45, 78 (2000).

25. D. Zhang, F. Montanés, K. Srinivas, T. Fornari, E. Ibánez, J. W. King, Ind. Eng. Chem. Research, 49, 6691 (2010).

26. Y. Takebayashi, K. Sue, S. Yoda, Y. Hakuta, T. Furuya, J. Chem. Eng. Data, 57, 1810 (2012).

27. P. Karásek, J. Planeta, M. Roth, J. Chromat. A, 1140, 195 (2007).

28. P. Mottahedin, A. Haghighi Asl, M. N. Lotfollahi, J. Solution Chem., 46, 2191 (2017).

29. P. Karásek, J. Planeta, M. Roth, J. Chem. Eng. Data, 51, 616 (2006).

30. P. Mottahedin, A. Haghighi Asl, \& M. N. Lotfollahi, J. Mol. Liquids, 237, 257 (2017).

31. O. Anurukvorakun, Mahidol University J. Pharma. Sci, 40, 7 (2013).

32. A. G. Carr, A. Branch, R. Mammucari, N. R. Foster, J. Supercrit. Fluids, 55, 37 (2010). 


\title{
Modified graphite electrodes as potential cathodic electrocatalysts for microbial electrolysis cells
}

\author{
E. Chorbadzhiyska ${ }^{1,2^{*}}$, I. Bardarov ${ }^{1,2}$, Y. Hubenova ${ }^{3,4}$, M. Mitov ${ }^{1,2}$ \\ ${ }^{1}$ Department of Chemistry, South-West University, Blagoevgrad, Bulgaria \\ ${ }^{2}$ Innovative Centre for Eco Energy Technologies, South-West University "Neofit Rilski", Blagoevgrad, Bulgaria \\ ${ }^{3}$ Department of Biochemistry and Microbiology, Plovdiv University, Plovdiv, Bulgaria \\ ${ }^{4}$ Department of Electrocatalysis and Electrocrystallization, Institute of Electrochemistry and Energy Systems \\ "Academician Evgeni Budevski”, Bulgarian Academy of Sciences (IEES-BAS), Sofia, Bulgaria
}

Received May 13, 2019; Accepted May 28, 2019

Microbial electrolysis cell (MEC) is an ecologically clean and innovative technology for hydrogen production. The development of cost-effective cathodes with high catalytic activity for hydrogen evolution reaction (HER) in nearneutral electrolytes is the most critical challenge for the practical application of MEC technology. In this study, graphite electrodes, functionalized with non-noble metal oxides, were produced and after electrochemical pre-treatment investigated as potential cathodes for MEC. The morphology of the developed materials was analyzed by scanning electron microscopy (SEM). Their electrochemical performance in neutral phosphate buffer solution (PBS) was explored by means of linear sweep voltammetry (LSV) and chronoamperometry (CA). The results from both methods show that all modified electrodes exhibit higher electrocatalytic activity towards HER than that of bare graphite, which is a prerequisite for further evaluation of these materials as cathodes in real MEC.

Keywords: modified graphite electrodes, hydrogen production, microbial electrolysis cell

\section{INTRODUCTION}

Hydrogen production is becoming increasingly important in view of using hydrogen in fuel cells. However, most of the production of hydrogen so far comes from the combustion of fossil fuels and water electrolysis, which have disadvantages like usage of non-renewable sources and high energy input.

Microbial electrolysis cell (MEC) is an ecologically clean, renewable and innovative technology for hydrogen production. MECs produce hydrogen mainly from waste biomass assisted by exoelectrogenic bacterial strains [1-4]. By adding a small electrical input (a minimum of $0.14 \mathrm{~V}$ compared to $1.23 \mathrm{~V}$ needed for water electrolysis) $[5,6]$ hydrogen can be evolved on the cathode. To date, carbon-based materials such as carbon cloth and carbon paper are applied in most MEC cathodes due to their stability in a microbial inoculum mixture, high conductivity and high specific surface area. However, they possess relatively low electrocatalytic activity for hydrogen evolution reaction (HER).

Crucial for the efficiency of MEC bio-hydrogen production is the choice of a proper cathodic catalyst [7]. Up to now, cathodes containing platinum have been mostly used in MECs $[8,9]$. The high cost and poisoning of $\mathrm{Pt}$ by different chemicals (e.g. sulfides) have driven researchers to investigate non-precious metal catalysts as an alternative. There is a gap in the knowledge about the catalytic activity of such materials towards hydrogen evolution under neutral medium and ambient temperature conditions, appropriate for microbial growth in MEC [10]. Therefore, the development of cost-effective cathodes for nearneutral $\mathrm{pH}$ and ambient temperature conditions is the most critical challenge for the practical application of MEC technology.

Transition metal-based catalysts are promising candidates in this respect, taking into consideration that the kinetics of the overall process in MEC is usually controlled by the relatively slow extracellular electron transfer from the biocatalyst (exoelectrogenic bacteria) to the anode. Depending on the precursors, different methods for preparation of supported metal catalysts are applied [11]. In the cases when metal oxides are used as precursors, they are usually transformed in dispersed metal catalysts by thermal treatment in hydrogen flow or reduction by a chemical reagent such as formaldehyde or hydrazine.

In this study we demonstrate a possibility for preparation of electrodes with good catalytic activity for HER in a neutral electrolyte by functionalization of graphite with non-precious metal oxides $\left(\mathrm{Fe}_{2} \mathrm{O}_{3}, \mathrm{Fe}_{3} \mathrm{O}_{4}, \mathrm{Mn}_{3} \mathrm{O}_{4}, \mathrm{TiO}_{\mathrm{x}}\right)$ and

\footnotetext{
* To whom all correspondence should be sent:

E-mail: elli_e1@swu.bg
} 
E. Chorbadzhiyska et al.: Modified graphite electrodes as potential cathodic electrocatalysts ...

subsequent electrochemical reduction. The morphology of the developed materials was characterized by means of scanning electron microscopy (SEM). Their electrocatalytic activity for HER in neutral phosphate buffer solution (PBS) was examined by means of linear sweep voltammetry (LSV) and chronoamperometry (CA).

\section{MATERIALS AND METHODS}

\section{Production and characterization of graphite-based electrodes}

Electrodes with geometric area of $1 \mathrm{~cm}^{2}$ were made using a procedure closely resembling the method used for producing synthetic graphite. The ingredients used were $50 \%$ finely powdered spectroscopic graphite, $30 \%$ carbon pitch with a softening point of $110{ }^{\circ} \mathrm{C}$ (Koppers Inc., USA) and $20 \%$ metal oxide $\left(\mathrm{Fe}_{2} \mathrm{O}_{3}, \mathrm{Fe}_{3} \mathrm{O}_{4}, \mathrm{Mn}_{3} \mathrm{O}_{4}\right.$ or $\left.\mathrm{TiO}_{\mathrm{x}}\right)$ as a catalyst precursor. The mixture was first homogenized in a rotary ball mill for 1 hour, then pressed at $250 \mathrm{MPa}$ in a stainless steel form and sintered for 30 minutes at $150{ }^{\circ} \mathrm{C}$. The electrode preforms were than pyrolyzed in argon at atmospheric pressure with a heating rate of $5{ }^{\circ} \mathrm{C} / \mathrm{min}$ and maintained at $800{ }^{\circ} \mathrm{C}$ for 1 hour. Titanium wires (1.6 $\mathrm{mm}$ thick) were screwed in the electrode samples and served as current collectors. The produced electrodes are further denoted as graphite, $\mathrm{Fe}_{2} \mathrm{O}_{3} /$ graphite, $\mathrm{Fe}_{3} \mathrm{O}_{4} /$ graphite, $\mathrm{Mn}_{3} \mathrm{O}_{4} /$ graphite and $\mathrm{TiO}_{\mathrm{x}} /$ graphite.

Before electrocatalytic tests, the as-prepared electrodes were pre-treated electrochemically in $100 \mathrm{mM}$ phosphate buffered saline (PBS, $\mathrm{pH}$ 7.4) by sweeping the potential with a scan rate of 1 $\mathrm{mV} / \mathrm{s}$ from 0 to $-1.2 \mathrm{~V}(v s$. $\mathrm{Ag} / \mathrm{AgCl}, 3 \mathrm{M} \mathrm{KCl})$ in order to reduce the incorporated metal oxide precursors.

The morphology of the developed materials was analyzed by scanning electron microscopy (SEM) using JEOL 6300.

\section{Electrocatalytic activity and corrosion behavior of the developed electrodes in PBS}

Investigations of the electrocatalytic activity and corrosion resistance of the developed electrodes were carried out in a three-electrode cell with 50 $\mathrm{cm}^{3}$ electrolyte volume. The tested sample was connected as a working electrode and a platinum wire was used as a counter electrode. All potentials were measured against $\mathrm{Ag} / \mathrm{AgCl}(3 \mathrm{M} \mathrm{KCl})$ reference electrode. The electrochemical studies were performed by using PalmSens 3 potentiostat/galvanostat, equipped with MUX8 multiplexer.
Linear sweep voltammetry (LSV) from 0 to -1.2 $\mathrm{V}$ (vs. $\mathrm{Ag} / \mathrm{AgCl})$ was performed to evaluate the electrocatalytic activity of the studied materials towards HER in neutral (PBS) solution. The potential was swept with a scan rate of $1 \mathrm{mV} / \mathrm{s}$. LSV tests were carried out in duplicate and the second scan was used for analysis of performance based on the voltage needed to initiate hydrogen production, $\mathrm{V}_{\mathrm{e}}$, and the slope in the voltammogram, $\mathrm{V}_{\mathrm{h}}$, reflecting the current (resp. hydrogen) production rate at an applied voltage. The linear region in the LSVs was analyzed by linear regression to obtain the value of $\mathrm{V}_{\mathrm{e}}$ and the slope $\mathrm{V}_{\mathrm{h}}$.

Chronoamperometric measurements were also carried out at $-1.0 \mathrm{~V}$ (vs. $\mathrm{Ag} / \mathrm{AgCl}$ ) for $10 \mathrm{~min}$ and the responding current was monitored. The quantity of the produced hydrogen was calculated by integration of the areas under the chronoamperometric curves, applying the Faraday's law.

The corrosion stability of the newly produced electrodes in used neutral electrolyte was examined by anodic polarization with a scan rate of $1 \mathrm{mV} / \mathrm{s}$ from -0.6 to $+0.2 \mathrm{~V}$ ( $v s$. $\mathrm{Ag} / \mathrm{AgCl}$ ). The corrosion tests with each electrode were performed before and after cathodic pre-treatment. The obtained data are presented as Tafel plots, from which the values of the corresponding corrosion potentials, $E_{\text {corr., }}$, and corrosion currents, $I_{\text {corr. }}$, are estimated.

\section{RESULTS AND DISCUSSION}

After preparation of electrodes, their morphology was analyzed by scanning electron microscopy. The obtained micrographs are presented in Figure 1. The SEM images reveal that the produced materials consist of big conglomerates, in which particles of the metallic catalyst precursors are incorporated. In all cases, the distribution of these particles is not uniform, having different forms and size.

As a result of the electrochemical pre-treatment, the incorporated metal oxide precursors undergo reduction, which is obviously seen from the voltammograms obtained during the performed procedure (Fig. 2). The cathodic peak obtained with non-modified graphite could be assigned to reduction of impurities (e.g. organic compounds) on the electrode surface. Having in mind that the produced materials possess bulk 3D-structure without macropores, most probably only the particles on the electrode surface, exposed to the electrolyte, are partially reduced as a result of the applied electrochemical procedure. Nevertheless, this leads to a change in the corrosion behavior illustrated on Fig. 3 and summarized in Table 1. 
E. Chorbadzhiyska et al.: Modified graphite electrodes as potential cathodic electrocatalysts ...

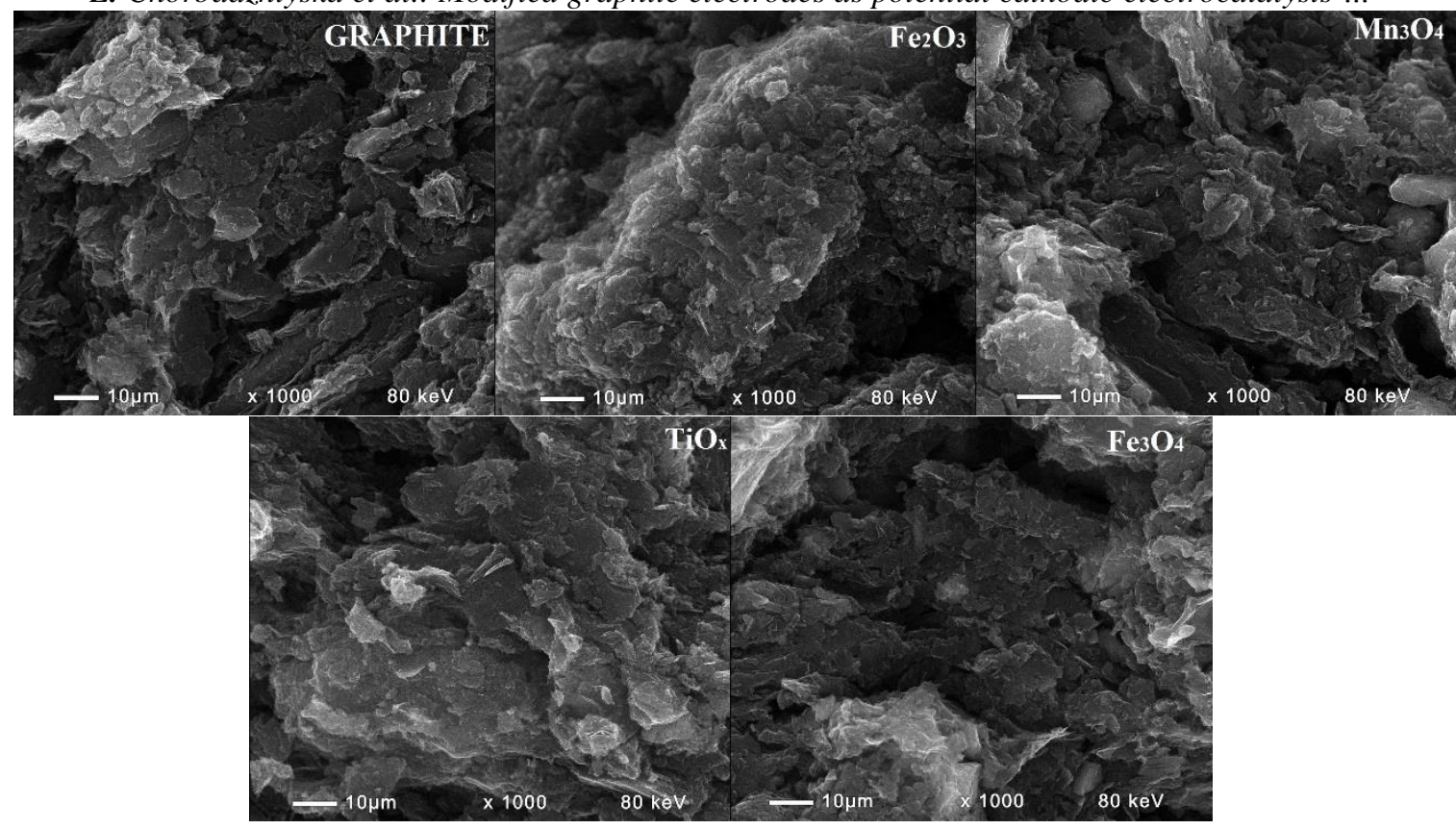

Fig. 1. SEM images of produced electrode materials

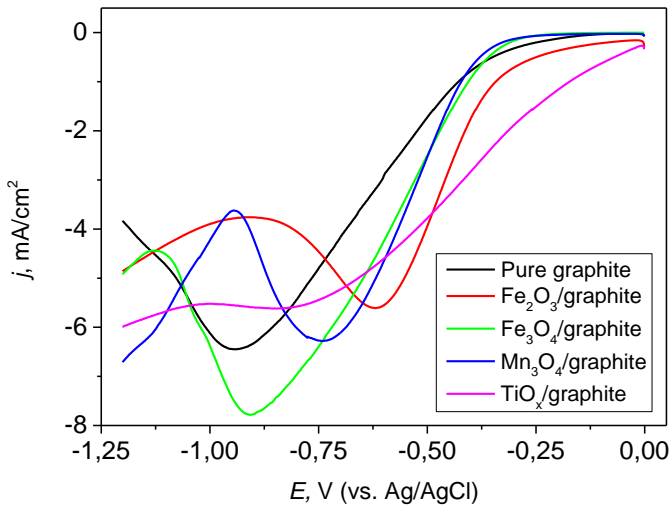

Fig. 2. Linear voltammograms obtained during cathodic pre-treatment of investigated electrodes.

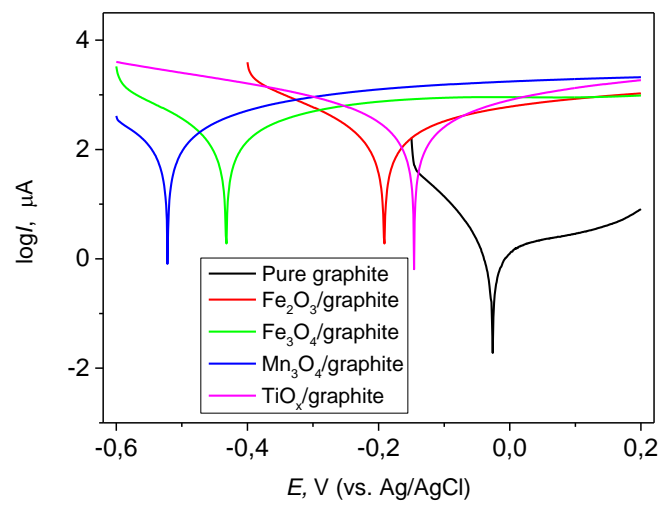

Fig. 3. Tafel plots of modified and non-modified graphite electrodes after cathodic pre-treatment.

Table 1. Values of the corrosion potential, $E_{\text {corr. }}$, and corrosion current density, $j_{\text {corr }}$ derived from Tafel plots, obtained with the investigated materials before and after cathodic pre-treatment.

\begin{tabular}{|c|c|c|c|c|}
\hline \multirow{2}{*}{ Material } & \multicolumn{2}{|c|}{ Before cathodic pre-treatment } & \multicolumn{2}{c|}{ After cathodic pre-treatment } \\
\cline { 2 - 5 } & $\mathrm{E}_{\text {corr }}, \mathrm{V}$ & $\mathrm{j}_{\text {corr }}, \mu \mathrm{A} . \mathrm{cm}^{-2}$ & $\mathrm{E}_{\text {corr }}, \mathrm{V}$ & $\mathrm{j}_{\text {corr }}, \mu \mathrm{A} . \mathrm{cm}^{-2}$ \\
\hline Graphite & +0.015 & 0.04 & -0.026 & 1.10 \\
\hline $\mathrm{Fe}_{2} \mathrm{O}_{3} /$ graphite & +0.043 & 9.77 & -0.190 & 143.55 \\
\hline $\mathrm{Fe}_{3} \mathrm{O}_{4} /$ graphite & -0.002 & 0.87 & -0.429 & 140.93 \\
\hline $\mathrm{Mn}_{3} \mathrm{O}_{4} /$ graphite & +0.002 & 10.27 & -0.522 & 70.96 \\
\hline $\mathrm{TiO}_{\mathrm{x}} /$ graphite & -0.070 & 21.42 & -0.145 & 149.97 \\
\hline
\end{tabular}

The inhomogeneity of the modified electrodes due to incorporation of metal oxides in the graphite matrix results in their lower corrosion resistance in comparison with the bare graphite electrodes. The surface reduction of the metal oxides as a result of the cathodic pre-treatment is confirmed by the shift of the corrosion potentials in negative direction, as

well as by the increase in corrosion currents, indicating existence of much more particles in lower oxidation state. In both cases (before and after pre-treatment), the electrodes modified with 
E. Chorbadzhiyska et al.: Modified graphite electrodes as potential cathodic electrocatalysts ... $\mathrm{TiO}_{\mathrm{x}}$ exhibit the highest corrosion rate, resp. the

Table 2. Values of the minimum voltage needed to initiate substantial current $\mathrm{V}_{\mathrm{e}}$ and hydrogen production rate $\mathrm{V}_{\mathrm{h}}$, derived from linear voltammograms obtained with the investigated materials

\begin{tabular}{|c|c|c|}
\hline Electrode & $\mathrm{V}_{\mathrm{e}}, \mathrm{mV}(\mathrm{vs}$. & $\mathrm{V}_{\mathrm{h}}, \mathrm{mA} / \mathrm{V}$ \\
\hline Graphite & -0.65 & 2.65 \\
\hline $\mathrm{Fe}_{2} \mathrm{O}_{3} /$ graphite & -0.42 & 3.00 \\
\hline $\mathrm{Fe}_{3} \mathrm{O}_{4} /$ graphite & -0.48 & 4.58 \\
\hline $\mathrm{Mn}_{3} \mathrm{O}_{4} /$ graphite & -0.40 & 5.44 \\
\hline $\mathrm{TiO}_{\mathrm{x}} /$ graphite & -0.44 & 4.98 \\
\hline
\end{tabular}

After pre-treatment, the electrodes were tested in respect to electrocatalytic activity towards HER by means of LSV and CA.

The lack of cathodic peaks on the linear voltammograms (Fig. 4) indicates that no other reduction reaction except HER takes place on the produced electrodes after their initial pre-treatment. The values of $V_{e}$ and $V_{h}$ (Table 2), derived from the LVs, show that the applied modification decreases the HER overpotential and at the same time enhances the hydrogen production rate. The minimum potental $\left(\mathrm{V}_{\mathrm{e}}\right)$ needed to initiate substantial current obtained with bare graphite is $0.65 \mathrm{~V}$, while for all modified electrodes it ranges between $-0.40 \mathrm{~V}$ and $-0.48 \mathrm{~V}$ (vs. $\mathrm{Ag} / \mathrm{AgCl})$. As seen from the data, the highest $\mathrm{V}_{\mathrm{h}}$ and the lowest $\mathrm{V}_{\mathrm{e}}$ values, revealing the best catalytic performance [12], are achieved with $\mathrm{Mn}_{3} \mathrm{O}_{4}$ /graphite electrode.

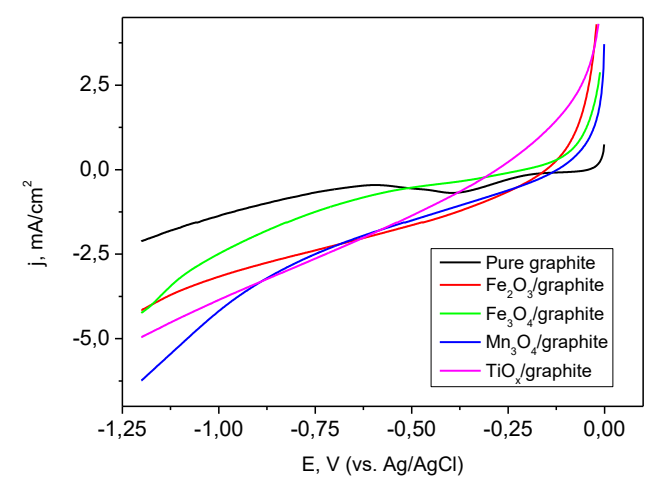

Fig. 4. Linear voltammograms obtained with modified electrodes in neutral PBS; scan rate $1 \mathrm{mV} / \mathrm{s}$.

The results from the chronoamperometric measurements confirm those obtained by LSV. The steady-state currents (Fig. 5a) and the corresponding hydrogen production rates (Fig. $5 b$ ) achieved with all modified materials are 2 to 4 times higher than that obtained with bare graphite. The highest hydrogen production rate of $1.6 \mathrm{ml} . \mathrm{cm}^{-}$ ${ }^{2} \cdot \mathrm{h}^{-1}$ is achieved with $\mathrm{Mn}_{3} \mathrm{O}_{4}$-modified electrode. The rest of the studied electrodes, except lowest corrosion resistance.

$\mathrm{Fe}_{2} \mathrm{O}_{3}$ /graphite and $\mathrm{Fe}_{3} \mathrm{O}_{4} /$ graphite, arrange in the same order determined by LSV tests. The achieved values of hydrogen production rate with the modified electrodes in this study are comparable with those obtained with other supported non-noble metal catalysts [13] and even with Pd-Au [14], examined at similar experimental conditions.
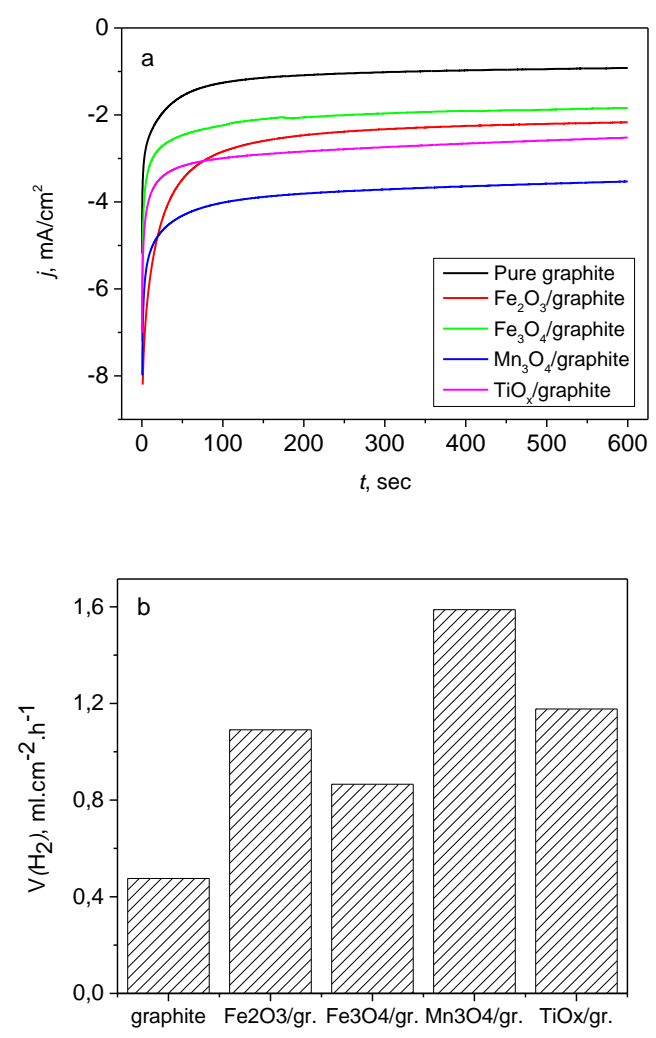

Fig. 5. a) Chronoamperograms obtained at $-1.0 \mathrm{~V}$ (vs. $\mathrm{Ag} / \mathrm{AgCl}$ ) with the investigated electrodes; b) Hydrogen evolution rate estimated from the chronoamperograms.

\section{CONCLUSIONS}

The results from this study show that costeffective electrodes with high electrocatalytic activity for HER in neutral electrolyte can be produced from non-precious metal oxides precursors mixed with graphite, and subsequent electrochemical reduction. From all tested graphite electrodes modified with $\mathrm{Fe}_{2} \mathrm{O}_{3}, \mathrm{Fe}_{3} \mathrm{O}_{4}, \mathrm{Mn}_{3} \mathrm{O}_{4}$ or $\mathrm{TiO}_{\mathrm{x}}$, the highest hydrogen production rate was achieved with the $\mathrm{Mn}_{3} \mathrm{O}_{4}$ /graphite. Further studies, aiming at practical application of this type of electrodes as cathodes in MECs, are going to be performed.

Acknowledgements: This study was funded by the National Science Fund of Bulgaria through Contract DM 09/3/2016 and partially supported by National Science Program Eplus (Contract D01214/2018). The authors are grateful to 
E. Chorbadzhiyska et al.: Modified graphite electrodes as potential cathodic electrocatalysts ...

Koppers International B.V. for providing free samples of their Carbon Pitch.

\section{REFERENCES}

1. H. Liu, S. Grot, B. Logan, Environ. Sci. Technol., 39, 4317 (2005).

2. R. Rozendal, H. Hamelers, G. Euverink, S. Metz, C. Buisman, Int. J. Hydrogen Energy., 31, 1632 (2006).

3. B. Logan, D. Call, S. Cheng, H. Hamelers, T. Sleutels, A. Jermiasse, et al., Environ. Sci. Technol., 42, 8630 (2008).

4. B. Logan, Microbial fuel cells, New York: Wiley; 2007.

5. J. Ditzig, H. Liu, B. Logan, Int. J. Hydrogen Energy, 32, 2296 (2007).

6. A. Kadier, Y. Simayi, M. Kalil, P. Abdeshahian, A. Hamid, Renew. Energy, 71, 466 (2014).
7. B. Conway, B. Tilak, Electrochim. Acta, 47, 357 (2002).

8. R. Rozendal, H. Hamelers, K. Rabaey, J. Keller, Trends Biotechnol., 26, 450 (2008).

9. B. Tartakovski, M. Manuel, H. Wang, S. Guiot, Int. J. Hydrogen Energy, 34, 672 (2009).

10. C., Santoro, F., Soavi, C., Arbizzani, A., Serov, S., Kabir, K., Carpenter, O., Bretschger, P., Atanassov, Electrochim. Acta, 220, 672 (2016).

11. F. Pinna, Catalysis Today, 41, 129 (1998).

12. Y. Zhang, M. Merrill, B. Logan, Int. J. Hydrogen Energy, 35, 12020 (2010).

13. M. Mitov, E. Chorbadzhiyska, L. Nalbandian, Y. Hubenova, J. Power Sources, 356, 467 (2017).

14. E Chorbadzhiyska, M Mitov, L Nalbandian, Y Hubenova, Int. J. Hydrogen Energy, 40, 7329 (2015). 


\title{
Friedel-Crafts acylation of 2-methoxynaphthalene with acetic anhydride catalyzed by phosphotungstic acid in ionic liquid
}

\author{
Y. Guo, J. Sun, F. Guo, Yu. He, P. Chen* \\ School of Chemistry and Materials Science, Liaoning Shihua University, Dandong Road 1, Fushun 113001, China
}

Received, November 12, 2016; Revised, February 23, 2018

The Friedel-Crafts acylation of 2-methoxynaphthalene (2-MN) with acetic anhydride (AA) was carried out in the ionic liquid (IL) butylpyridinium tetrafluoroborate $\left([\mathrm{BPy}] \mathrm{BF}_{4}\right)$ using phosphotungstic acid $\left(\mathrm{H}_{3} \mathrm{PW}_{12} \mathrm{O}_{40}\right)$ as the catalyst. The $\left[\mathrm{BPy}_{\mathrm{BF}}\right.$-mediated 2-MN acylation displays good conversion and selectivity towards 1-acyl-2methoxynaphthalene (1-AC-2-MN), with 70.4\% conversion of 2-MN and $96.4 \%$ selectivity to $1-\mathrm{AC}-2-\mathrm{MN}$ obtained under the optimal conditions. Owing to the rearrangement of 1-AC-2-MN, 6-acyl-2-methoxynaphthalene (6-AC-2-MN) can be detected after $1 \mathrm{~h}$ of reaction time, with the highest $6-\mathrm{AC}-2-\mathrm{MN}$ yield of $11.3 \%$ obtained under the examined reaction conditions. The system can be recycled and reused at least 6 times without significant loss of activity, indicating the good stability of the $\mathrm{H}_{3} \mathrm{PW}_{12} \mathrm{O}_{40} /[\mathrm{BPy}] \mathrm{BF}_{4}$ catalytic system.

Keywords: Friedel-Crafts acylation, Ionic liquid, Heteropolyacid, 2-Methoxynaphthalene

\section{INTRODUCTION}

Aromatic ketones are important intermediates in fine chemical, pharmaceutical and agrochemical industrial processes [1,2]. Friedel-Crafts acylation is one of the most effective methods for preparing aromatic ketones. A typical example is the acylation of 2-MN, which produces the important synthetic intermediates 1-AC-2-MN and 6-AC-2$\mathrm{MN}$ [3-5]. Current industrial processes for 2-MN acylation use acyl halides or anhydrides as acylating reagents over stoichiometric amounts of Lewis acids (such as $\mathrm{AlCl}_{3}$ ) or proton acids as catalysts [6]. However, these catalysts generate many problems as they are polluting, corrosive, and the catalysts are not recyclable during the work-up $[7,8]$. Due to the environmental restrictions, replacement of conventional catalytic methods is desirable. An alternative would be the application of heterogeneous catalysts instead of Lewis or proton acid catalysts, since they are more environmentally friendly, recyclable and reusable, and some of them also show good shape-selective properties in the acylation of 2-MN [9-13]. Accordingly, the acylation of 2-MN with various solid catalysts including BEA [9-13], HY [14,15], beta [16-21], MCM-41 [22,23], ITQ-7 [24,25], HMS [26], Nafion/silica composites [27,28], etc., has been extensively studied in the past decades. However, they are in general less active towards 2$\mathrm{MN}$ acylation as compared to Lewis or proton acid catalysts (for example, $\mathrm{AlCl}_{3}$ ).

Heteropolyacids (HPA), being a type of strong acid catalysts, have attracted much attention both in the academic research field and industrial applications in recent years [29,30]. Among the various Keggin type - HPA, the phosphotungstic acid $\mathrm{H}_{3} \mathrm{PW}_{12} \mathrm{O}_{40}$ has been found to be effective towards many organic reactions such as oxidation [31-38], esterification [39-44], Friedel-Crafts alkylation [45-48] and acylation [49-54]. Owing to its high stability and solid precipitation property, it is normally recyclable and reusable in the homogeneous catalytic system, and is also less corrosive as compared to Lewis or proton acids. Despite its excellent catalytic performance has been recognized, the acylation of 2-MN using $\mathrm{H}_{3} \mathrm{PW}_{12} \mathrm{O}_{40}$ as catalyst has not yet been fully examined.

Theoretically, acylation of 2-MN generally occurs at the kinetically controlled 1-position, forming 1-AC-2-MN as the major product. The reaction also has the trend to form another thermodynamically stable 6-position product 6-AC$2-\mathrm{MN}$ with the extension of reaction time at higher temperatures or over shape-selective zeolite catalysts (for example, HBEA [9-13]. Chlorobenzene has been found as a preferable solvent, since it can result in high acylation yield [9-13]. Considering green catalysis, the application of ionic liquids as non-volatile solvents is also attractive. Ionic liquids have many unique chemical and physical properties such as negligible volatility, good thermal stability and excellent solubility of both inorganic and organic compounds in them [55]. Many ionic liquids have been successfully applied as solvents for the Friedel-Crafts acylation reactions [56-63]. Nevertheless, to the best of our knowledge, the acylation of 2-MN using the heteropolyacid $\mathrm{H}_{3} \mathrm{PW}_{12} \mathrm{O}_{40}$ as catalyst and ionic liquid as solvent have never been reported in the literature. In this work, the Friedel-Crafts acylation of 2-MN with acetic anhydride using

\footnotetext{
* To whom all correspondence should be sent. (C) 2019 Bulgarian Academy of Sciences, Union of Chemists in Bulgaria EEmail: chenping-fs@163.com
} 
Y. Guo et al.: Friedel-Crafts acylation of 2-methoxynaphthalene with acetic anhydride catalyzed by phosphotungstic... phosphotungstic acid $\mathrm{H}_{3} \mathrm{PW}_{12} \mathrm{O}_{40}$ as renewable catalyst and the ionic liquid $\left[\mathrm{BP}_{\mathrm{y}}\right] \mathrm{BF}_{4}$ as an efficient and non-volatile solvent is reported as a result of our efforts.

\section{EXPERIMENTAL}

All chemicals were purchased from the Sinopharm Chemical Reagent Co. Ltd (China) or Acros and used without further purification. $\left[\mathrm{BP}_{\mathrm{y}}\right] \mathrm{BF}_{4}$ was synthesized according to the literature procedures [64-66]. ${ }^{1} \mathrm{H}$ NMR spectra were recorded on a Bruker Avance DPX-400 spectrometer. IR spectra were recorded on a Perkin Elmer Frontier FT-IR spectrometer. Gas chromatography (GC) and GC-MS were performed on an Agilent 7890A-5975C instrument equipped with an SE-30 capillary column $(0.25 \mathrm{~mm} \times 30 \mathrm{~m} \times$ $0.10 \mu \mathrm{m})$, a FID and a MS detector.

\section{Typical procedure for Friedel-Crafts acylation of $2-M N$}

$3.16 \mathrm{~g}$ (20 mmol) of 2-MN, $3.06 \mathrm{~g} \mathrm{(30} \mathrm{mmol)} \mathrm{of}$ $\mathrm{AA}$ and $10 \mathrm{~mL}$ of $\left[\mathrm{BP}_{\mathrm{y}}\right] \mathrm{BF}_{4}$ were added into a three-neck round flask. A certain amount of $\mathrm{H}_{3} \mathrm{PW}_{12} \mathrm{O}_{40}$ catalyst was then added to start the reaction. The reaction temperature was gradually increased to $120{ }^{\circ} \mathrm{C}$. After the reaction, the mixture was cooled down to room temperature and extracted with ethyl acetate three times $(3 \times 10 \mathrm{~mL})$. The recycled IL containing the catalyst $\mathrm{H}_{3} \mathrm{PW}_{12} \mathrm{O}_{40}$ was further dried at $80{ }^{\circ} \mathrm{C}$ under vacuum, then used directly for the next catalytic run. The organic layer containing the unreacted substrates and products was then diluted, and subjected to GC analysis. GC analysis was performed under the following conditions: initial temperature: $90{ }^{\circ} \mathrm{C}$ for 1 $\mathrm{min} \rightarrow$ ramp rate: $20{ }^{\circ} \mathrm{C} / \mathrm{min} \rightarrow$ final temperature: $260{ }^{\circ} \mathrm{C}$ for $10 \mathrm{~min}$. Injector/detector temperatures: $260{ }^{\circ} \mathrm{C} / 260{ }^{\circ} \mathrm{C}$. Nitrogen was used as carrier gas. The formation of possible products was confirmed by comparison of the retention time with that of authentic samples. Quantitative data were analyzed using a N 2000 Chemical work station software with the GC instrument. The conversion and yield were calculated from calibration curves $\left(\mathrm{r}^{2}>0.999\right)$ recorded prior to the reaction using naphthalene as the internal standard. The pure product of 1-AC-2$\mathrm{MN}$ was isolated by flash chromatography on silica

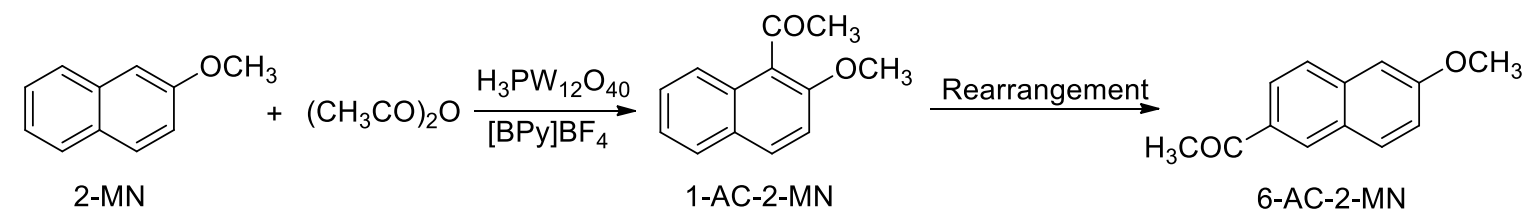

Scheme 1. Friedel-Crafts acylation of 2-MN. 
Y. Guo et al.: Friedel-Crafts acylation of 2-methoxynaphthalene with acetic anhydride catalyzed by phosphotungstic...

The reactions were carried out using different solvents, temperatures, amount of catalyst and molar ratios of the substrates. In general, 1-AC-2$\mathrm{MN}$ is observed as the major product within $1 \mathrm{~h}$ of the reaction according to GC analysis, whereas the thermodynamically stable 6-position acylated product 6-AC-2-MN can be detected after $1 \mathrm{~h}$ at higher temperatures $\left(>100^{\circ} \mathrm{C}\right)$.

\section{Influence of solvents on the acylation of 2-MN}

The reactions were carried out in $\left[\mathrm{BP}_{\mathrm{y}}\right] \mathrm{BF}_{4}$, chlorobenzene and under solvent-free condition. The data on the conversion of 2-MN and selectivity towards 1-AC-2-MN are shown in Figs. 1 and 2. When the ionic liquid $\left[\mathrm{BP}_{\mathrm{y}}\right] \mathrm{BF}_{4}$ is used as solvent, the reaction shows higher conversion than that in chlorobenzene or solvent-free condition (Fig. 1). The conversion of 2-MN gradually increases with the increase in reaction time, and finally reaches about $60.3 \%$ after $6 \mathrm{~h}$ and does not significantly increase afterwards. With respect to the product selectivity, the reactions show the highest 1-AC-2$\mathrm{MN}$ yield at $1 \mathrm{~h}$ in all examined conditions. [BPy $] \mathrm{BF}_{4}$-mediated reaction provides the best selectivity towards 1-AC-2-MN. The selectivity is almost $100 \%$ at $1 \mathrm{~h}$ and above $95 \%$ after $10 \mathrm{~h}$. The decrease in selectivity can be explained by the rearrangement of 1-AC-2-MN to 6-AC-2-MN. In the case of the reactions in chlorobenzene and solventfree condition the selectivities are $93.2 \%$ and $86.5 \%$ after $1 \mathrm{~h}$, and decrease gradually to $87.3 \%$ for chlorobenzene and $80.6 \%$ for solvent-free condition after $10 \mathrm{~h}$. The calculated yields of 6-AC$2-\mathrm{MN}$ are $3.1 \%$ in $[\mathrm{BPy}] \mathrm{BF}_{4}, 5.5 \%$ in chlorobenzene and $8.6 \%$ for solvent free conditions after $10 \mathrm{~h}$.

\section{Influence of temperature on the acylation of 2-MN}

Table 1 exhibits the data for the acylation of 2$\mathrm{MN}$ in the presence of $\left[\mathrm{BP}_{\mathrm{y}}\right] \mathrm{BF}_{4}$ at different temperatures. It can be observed that the reaction temperature significantly affects the conversion of 2-MN. The conversion of 2-MN increased rapidly with the temperature increase. All examined reactions show nearly $100 \%$ selectivity towards 1 AC-2-MN below $100^{\circ} \mathrm{C}$. The highest conversion of $2-\mathrm{MN}(66.1 \%)$ is achieved at $120{ }^{\circ} \mathrm{C}$ with 1 -AC-2$\mathrm{MN}$ selectivity of $98.3 \%$. Continued increase in the temperature leads to a decrease of 1-AC-2-MN yield but an increase of 6-AC-2-MN yield, which might be due to the acceleration of the isomerization reaction rate with the temperature increase. Accordingly, the selectivity of 1-AC-2$\mathrm{MN}$ is decreased at higher temperatures [9-13]. Nevertheless, the conversion is lower at $140{ }^{\circ} \mathrm{C}$ than that at $120{ }^{\circ} \mathrm{C}$. This might be due to the deacylation of 1-AC-2-MN at a higher temperature $\left(140{ }^{\circ} \mathrm{C}\right)$ during the isomerization process, which has also been frequently reported in the literature [9-28]. Moreover, the reaction solution becomes dark at higher temperatures $\left(>140{ }^{\circ} \mathrm{C}\right)$, which might be due to the decomposition of the ionic liquid $[\mathrm{BPy}] \mathrm{BF}_{4}$ or of the catalyst. The reaction shows $64.7 \%$ of 1-AC-2-MN as the highest yield with $98.3 \%$ selectivity at $120^{\circ} \mathrm{C}$, whereas the calculated 6-AC-2-MN yields reaches $6.8 \%$ at $140{ }^{\circ} \mathrm{C}$ and $8.3 \%$ at $160^{\circ} \mathrm{C}$.

\section{Influence of mole ratios of 2-MN and $A A$}

Table 2 shows the acylation data for different mole ratios of 2-MN and AA. It can be observed that only $55.3 \% 2-\mathrm{MN}$ conversion can be obtained with an equal equivalent of AA applied. While increasing the molar ratio of 2-MN and AA up to $1: 1.5$, the conversion reaches $66.1 \%$ with a selectivity of $98.3 \%$. Further increasing of the amount of AA leads to the decrease of both conversion and selectivity. This indicates that excessive amount of AA might also increase the activity of deacylation and acetyl migration reactions. Accordingly, the favorable molar ratio of 2-MN and AA is 1:1.5 for the formation of 1-AC-2MN.

\section{Influence of catalyst amount on the acylation of 2- $M N$}

Different amounts of catalyst were applied at a mole ratio of 2-MN : AA equal to $1: 1.5$ at $120^{\circ} \mathrm{C}$ in $[\mathrm{BPy}] \mathrm{BF}_{4}$. In general, the higher the catalyst amount applied, the higher is the conversion of 2MN (Table 3). The highest conversion of $70.4 \%$ is achieved with $0.2 \mathrm{~mol} \%$ of the catalyst applied. The conversion is not significantly increased with further increase in catalyst amount. In contrast, the selectivity slightly decreases with the increase in catalyst amount. This is also not a surprise since a high catalytic amount may also accelerate the deacylation and rearrangement reactions.

Based on the experiments performed above, we can conclude that the $\mathrm{H}_{3} \mathrm{PW}_{12} \mathrm{O}_{40} /[\mathrm{BPy}] \mathrm{BF}_{4}$ catalytic system shows a good activity towards acylation of 2-MN, with the formation of 1-AC-2$\mathrm{MN}$ as the major product and 6-AC-2-MN as a byproduct. The optimal reaction conditions for the formation of 1-AC-2-MN are: n2-MN: $\mathrm{nAA}=1: 1.5$, with $0.2 \mathrm{~mol} \%$ of $\mathrm{H}_{3} \mathrm{PW}_{12} \mathrm{O}_{40}$ catalyst applied at $120^{\circ} \mathrm{C}$ for $6 \mathrm{~h}$ in $[\mathrm{BPy}] \mathrm{BF}_{4}$ solvent. 
Y. Guo et al.: Friedel-Crafts acylation of 2-methoxynaphthalene with acetic anhydride catalyzed by phosphotungstic...

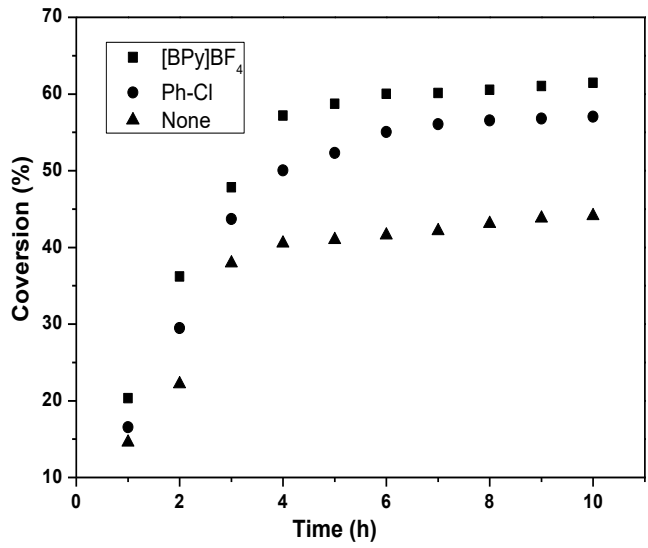

Fig. 1. Time-dependent conversion of $2-\mathrm{MN}$ in the presence of ionic liquid $[\mathrm{BPy}] \mathrm{BF}_{4}$, chlorobenzene or under solvent free conditions. Reaction conditions: 2-MN (3.16 g, $20 \mathrm{mmol})$, n2-MN:nAA =1:1.5, $\mathrm{H}_{3} \mathrm{PW}_{12} \mathrm{O}_{40}(0.06 \mathrm{~g}, 0.02 \mathrm{mmol})$, solvent $10 \mathrm{~mL}$ at $100{ }^{\circ} \mathrm{C}$

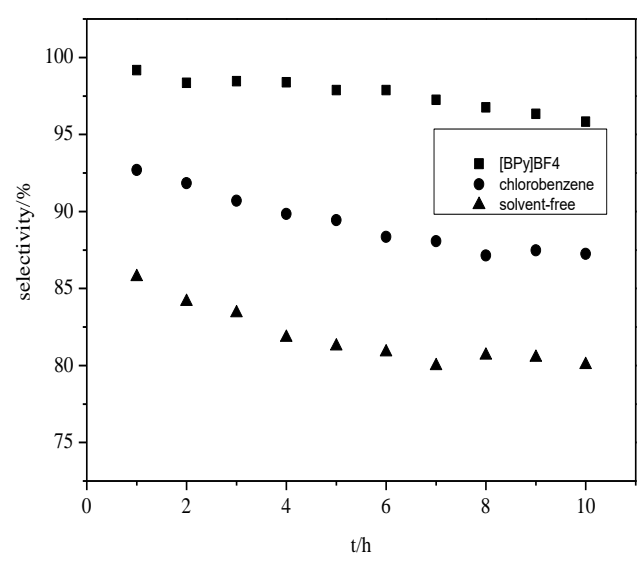

Fig. 2. Time-dependent selectivity towards 1-AC-2$\mathrm{MN}$ in the presence of ionic liquid $\left[\mathrm{BPy}^{\mathrm{B}} \mathrm{BF}_{4}\right.$, chlorobenzene or under solvent free conditions. Reaction conditions: 2-MN (3.16 g, $20 \mathrm{mmol})$, n2$\mathrm{MN}: \mathrm{nAA}=1: 1.5, \quad \mathrm{H}_{3} \mathrm{PW}_{12} \mathrm{O}_{40}(0.06 \mathrm{~g}, \quad 0.02 \mathrm{mmol})$, solvent $10 \mathrm{~mL}$ at $100^{\circ} \mathrm{C}$

Table 1. Influence of temperature on the acylation of 2-MN.

\begin{tabular}{|c|c|c|c|c|c|c|}
\hline \multirow{2}{*}{ Entry $^{\mathrm{a}}$} & \multirow{2}{*}{$\begin{array}{c}\text { Temperature } \\
\left({ }^{\circ} \mathrm{C}\right)\end{array}$} & \multirow{2}{*}{$\begin{array}{c}\text { Conversion } \\
(\%)\end{array}$} & \multicolumn{2}{|c|}{ Selectivity (\%) } & \multicolumn{2}{|c|}{ Yield (\%) } \\
\hline & & & 1-AC-2-MN & 6-AC-2-MN & 1-AC-2-MN & 6-AC-2-MN \\
\hline 1 & 60 & 30.6 & 99.9 & 0.3 & 31.2 & 0.2 \\
\hline 2 & 80 & 43.2 & 99.8 & 0.2 & 43.1 & 0.2 \\
\hline 3 & 100 & 60.1 & 98.9 & 0.1 & 60.4 & trace \\
\hline 4 & 120 & 66.1 & 98.3 & 0.9 & 64.7 & trace \\
\hline 5 & 140 & 56.6 & 84.4 & 12.8 & 48.2 & 6.8 \\
\hline 6 & 170 & 52.3 & 80.6 & 15.4 & 42.1 & 8.3 \\
\hline
\end{tabular}

${ }^{\mathrm{a}}$ Reaction conditions: 2-MN (3.16 g, $\left.20 \mathrm{mmol}\right), \mathrm{n} 2-\mathrm{MN}$ : $\mathrm{nAA}=1: 1.5, \mathrm{H}_{3} \mathrm{PW}_{12} \mathrm{O}_{40}(0.06 \mathrm{~g}, 0.02 \mathrm{mmol}),[\mathrm{BPy}] \mathrm{BF}_{4}(10$ $\mathrm{mL}$ ) for $6 \mathrm{~h}$.

Table 2. Influence of molar ratios between 2-MN and AA

\begin{tabular}{|c|c|c|c|c|c|c|}
\hline \multirow{2}{*}{ Entry $^{\mathrm{a}}$} & \multirow{2}{*}{$\begin{array}{c}\text { 2-MN: } \\
\text { AA }\end{array}$} & \multirow{2}{*}{ Conversion $(\%)$} & \multicolumn{2}{|c|}{ Selectivity (\%) } & \multicolumn{2}{|c|}{ Yield $(\%)$} \\
\hline & & & 1-AC-2-MN & 6-AC-2-MN & 1-AC-2-MN & 6-AC-2-MN \\
\hline 1 & $1: 1$ & 55.3 & 97.8 & 1.1 & 54.1 & 0.7 \\
\hline 2 & $1: 1.3$ & 60.6 & 97.3 & 1.8 & 59.2 & 0.9 \\
\hline 3 & $1: 1.5$ & 66.1 & 98.3 & 1.7 & 65.0 & 1.1 \\
\hline 4 & $1: 1.7$ & 60.6 & 87.2 & 11.7 & 53.2 & 7.3 \\
\hline 5 & $1: 2$ & 56.1 & 68.6 & 20.1 & 39.3 & 11.3 \\
\hline 6 & 1.2 .5 & 55.3 & 67.8 & 20.3 & 37.4 & 11.4 \\
\hline
\end{tabular}

\footnotetext{
${ }^{\mathrm{a}}$ Reaction conditions: 2-MN (20 mmol), $\mathrm{H}_{3} \mathrm{PW}_{12} \mathrm{O}_{40}(0.06 \mathrm{~g}, 0.02 \mathrm{mmol}),[\mathrm{BPy}] \mathrm{BF}_{4}(10 \mathrm{~mL})$ at $120^{\circ} \mathrm{C}$ for $6 \mathrm{~h}$.
} 
Y. Guo et al.: Friedel-Crafts acylation of 2-methoxynaphthalene with acetic anhydride catalyzed by phosphotungstic...

Table 3. Influence of catalyst amount on the acylation of 2-MN

\begin{tabular}{ccccc}
\hline Entry $^{\mathrm{a}}$ & $\begin{array}{c}\text { Catalyst amount } \\
(\text { mol \%) }\end{array}$ & $\begin{array}{c}\text { Conversion } \\
(\%)\end{array}$ & $\begin{array}{c}\text { Selectivity } \\
(\%)\end{array}$ & $\begin{array}{c}\text { Yield } \\
(\%)\end{array}$ \\
\hline 1 & 0.03 & 45.5 & 99.9 & 45.7 \\
2 & 0.06 & 52.4 & 99.9 & 52.1 \\
3 & 0.10 & 66.2 & 97.8 & 65.2 \\
4 & 0.20 & 70.4 & 96.4 & 67.4 \\
5 & 0.25 & 70.2 & 91.1 & 64.1 \\
$6^{\mathrm{b}}$ & 0.30 & 70.1 & 85.4 & 60.3 \\
\hline
\end{tabular}

${ }^{a}$ Reaction conditions: 2-MN (20 mmol), n2-MN: $\mathrm{nAA}=1: 1.5, \mathrm{H}_{3} \mathrm{PW}_{12} \mathrm{O}_{40}$ as catalyst, $[\mathrm{BPy}] \mathrm{BF}_{4}(10 \mathrm{~mL})$ at $120{ }^{\circ} \mathrm{C}$ for $6 \mathrm{~h}$; ${ }^{\mathrm{b}} \mathrm{The}$ reaction was performed at $140{ }^{\circ} \mathrm{C}$ for $10 \mathrm{~h}$.

\section{Recycling and reuse of catalyst and ionic liquid}

After the acylation reaction, the products and unreacted substrates were extracted by ethyl acetate. The resulting ionic liquid solution containing the catalyst $\mathrm{H}_{3} \mathrm{PW}_{12} \mathrm{O}_{40}$ was further dried at $80^{\circ} \mathrm{C}$ under vacuum. Then, the mixture was used directly for the next catalytic run; no additional catalyst was added to the system. Figure 3 shows the catalytic data of the recycled $\mathrm{H}_{3} \mathrm{PW}_{12} \mathrm{O}_{40} /[\mathrm{BPy}] \mathrm{BF}_{4}$ system for acylation of 2$\mathrm{MN}$. The data (Fig. 3) indicate that the catalytic system can be recovered and reused at least 6 runs without significant loss of activity, with the overall 2 -MN conversion higher than $60.2 \%$, indicating a good stability of the catalytic system. However, the conversion starts to decrease after 6 runs. It was observed that the color of the recycled $\mathrm{H}_{3} \mathrm{PW}_{12} \mathrm{O}_{40} /[\mathrm{BPy}] \mathrm{BF}_{4}$ solution gradually turned from colorless to brownish with the increase in recycling times, which indicates the decomposition of ionic liquid or catalyst.

\section{Acylation of other aromatic compounds}

In order to further evaluate the catalytic performance of $\mathrm{H}_{3} \mathrm{PW}_{12} \mathrm{O}_{40} /[\mathrm{BPy}] \mathrm{BF}_{4}$ system, the acylation of other aromatic compounds was also examined in our studies. The mono-substituted benzene derivatives including anisole, toluene and $p$-xylene were selected as substrates for acylation reactions in our studies. The catalytic data are summarized in Table 4.

Despite the catalytic system shows good conversion of 2-MN, it seems not active towards benzene ring-derived aromatic compounds. All the examined reactions result in only low to medium product yield. It is known that the aromaticity of naphthalene ring is weaker than that of the benzene ring, thus the electrophilic substitution of naphthalene derivatives is easier than that of benzene-derived aromatic compounds. Accordingly, 2-MN shows higher activity than anisole, toluene and $p$-xylene towards the acylation reactions. As to different functional group(s)substituted benzenes, since the electron-donating ability of methoxyl group is stronger than that of methyl group, anisole shows higher activity than toluene for acylations while in the case of $p$-xylene, the activity is lower than that of toluene. This might be due to the steric effect of the two methyl groups at para-positions, which makes the acylation hard to occur. Moreover, the electrophilic substitution is known to be more active at para-position than at ortho-position of the phenyl ring, which may be also responsible for the low activity of $p$-xylene.

\section{CONCLUSIONS}

The acylation of 2-MN was carried out for the first time with acetic anhydride in $[\mathrm{BPy}] \mathrm{BF}_{4}$ ionic liquid using phosphotungstic acid as catalyst, resulting in the formation of kinetically stable 1AC-2-MN as main product and thermodynamically stable 6-AC-2-MN as byproduct. A conversion of $70.4 \%$ with $96.4 \%$ of 1 -AC-2-MN selectivity can be obtained under the conditions of n2-MN:nAA $=1: 1.5,0.2 \mathrm{~mol} \% \mathrm{H}_{3} \mathrm{PW}_{12} \mathrm{O}_{40}$ catalyst applied at $120{ }^{\circ} \mathrm{C}$ for $6 \mathrm{~h}$ in $[\mathrm{BPy}] \mathrm{BF}_{4}$ solvent. The recycled ionic liquid and catalyst mixture can be reused at least 6 times without significant loss of activity, indicating a good stability of the $\mathrm{H}_{3} \mathrm{PW}_{12} \mathrm{O}_{40}$ l $[\mathrm{BPy}] \mathrm{BF}_{4}$ catalytic system. 
Y. Guo et al.: Friedel-Crafts acylation of 2-methoxynaphthalene with acetic anhydride catalyzed by phosphotungstic...

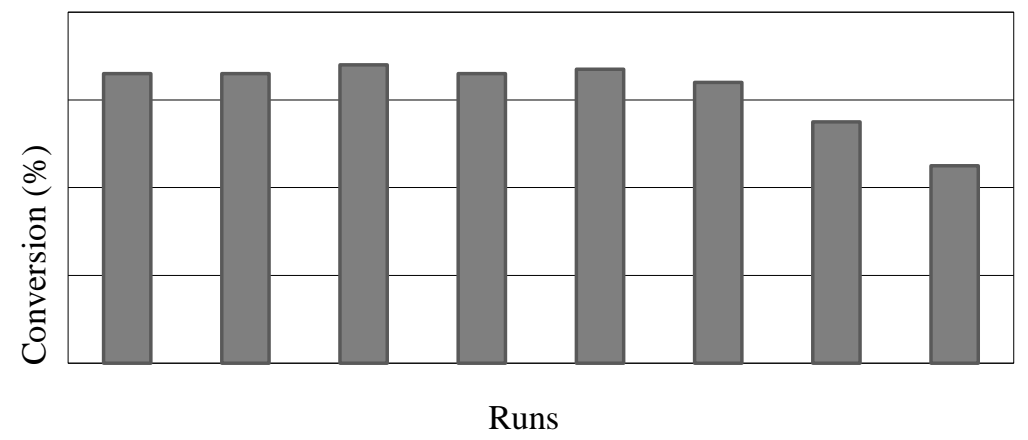

Fig. 3. Reuse of the catalytic system for acylation of 2-MN. Reaction conditions: 2-MN (3.16 g, $20 \mathrm{mmol})$, n2-MN: $\mathrm{nAA}=1: 1.5, \mathrm{H}_{3} \mathrm{PW}_{12} \mathrm{O}_{40}(0.06 \mathrm{~g}, 0.02 \mathrm{mmol}),[\mathrm{BPy}] \mathrm{BF}_{4}(10 \mathrm{~mL})$ at $120^{\circ} \mathrm{C}$ for $6 \mathrm{~h}$.

Table 4. Friedel-Crafts acylation of different aromatic compounds with AA

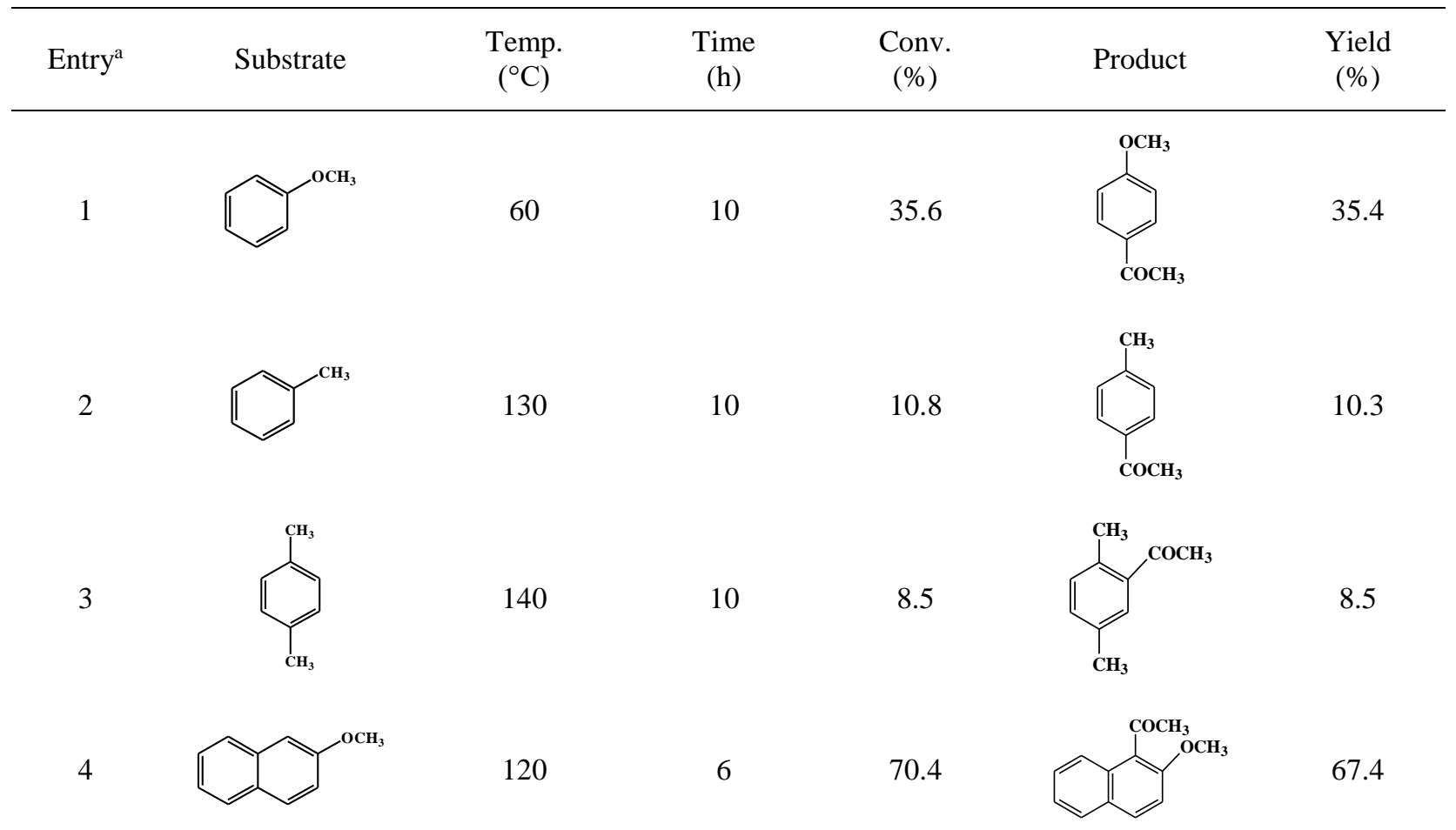

${ }^{\mathrm{a}}$ Reaction conditions: substrate $(20 \mathrm{mmol}), \mathrm{AA}(30 \mathrm{mmol}), \mathrm{H}_{3} \mathrm{PW}_{12} \mathrm{O}_{40}(0.12 \mathrm{~g}, 0.04 \mathrm{mmol}),[\mathrm{BPy}] \mathrm{BF} 4(10 \mathrm{~mL})$.

Acknowledgement: We thanks the National Science Foundation of China (21101085), Natural Science Foundation of Liaoning Province (2015020196, 20180540085), Fushun Science \& Technology Program (FSKJHT 201423), and Liaoning Excellent Talents Program in University (LJQ2012031) for the financial supports.

\section{REFERENCES}

1. H. G. Frank, J. W. Stadelhofer, Industrial aromatic chemistry, Chapter 5: Production and uses of benzene derivatives, Springer, Berlin, 1988, p. 137.

2. A. Baiker, H. U. Blaser, in: G. Ertl, H. Knözinger, J. Weitkamp (eds.), Handbook of Heterogeneous Catalysis, vol. 5, Wiley-VCH, Weinheim, 1997, p. 2422.

3. K. T. Wan, M. E. Davis. J. Catal., 152, 25 (1995).
4. M. E. Davis, Micropor. Mesopor. Mat., 4, 173 (1998).

5. S. Pivsa-Art, K. Okuro, M. Miura, S. Murata, M. Nomura, J. Chem. Soc. Perkin. Trans. 1, 1703 (1994).

6. P. J. Harrington, E. Lodewijk, Org. Process. Res. Dev., 1, 72 (1994).

7. R. A. Sheldon, H. van Bekkum, in: Fine Chemicals through heterogeneous Catalysis, R.A. Sheldon and H. van Bekkum (eds.), Wiley-VCH, Weinheim, 2001.

8. K. Tanabe, W. E. Hölderich, Appl. Catal. A-Gen., 181, 399 (1999).

9. A. Berreghis, P. Ayrault, E. Fromentin, M. Guisnet, Catal. Lett., 68, 121 (2000).

10. E. Fromentin, J M. Coustard, M. Guisnet, J. Catal., 190, 433 (2000).

11. E. Fromentin, J. Coustard, M. Guisnet, J. Mol. 
Y. Guo et al.: Friedel-Crafts acylation of 2-methoxynaphthalene with acetic anhydride catalyzed by phosphotungstic ... Catal. A-Chem., 159, 377 (2000).

12. H. Heinichen, W. Hölderich, J. Catal., 185, 408 (1999).

13. M. Casagrande, L. Storaro, M. Lenarda, R. Ganzerla, Appl. Catal. A-Gen., 201, 263 (2000).

14. P. Meric, A. Finiels, P. Moreau, J. Mol. Catal. AChem., 189, 251 (2002).

15. G. Harvey, G. Mader, Collect. Czech. Chem. Commun., 57, 862 (1992).

16. P. Andy, J. Garcia-Martinez, G. Lee, H. Gonzalez, C. Jones, M. Davis, J. Catal., 192, 215 (2000).

17. D. Das, S. Cheng, Appl. Catal. A-Gen., 201, 159 (2000).

18. S. Kim, K Lee, J. Lee, Y. Kim, K. Yoon. J. Mol. Catal. A-Chem., 152, 33 (2000).

19. L. Červený, K. Mikulcová, J. Čejka, Appl. Catal. AGen., 223, 65 (2002).

20. P. Moreau, A. Finiels, P. Meric, F. Fajula, Catal. Lett., 85, 199 (2003).

21. B. Yuan, Z. Li, Y. Liu, S. Zhang, J. Mol. Catal. AChem., 280, 210 (2008).

22. E. Gunnewegh, S. Gopie, H. Bekkum, J. Mol. Catal. A. Chem., 106, 151 (1996).

23. M. Selvaraj, K. Lee, K. Yoo, T. Lee, Micropor. Mesopor. Mat., 81, 343 (2005).

24. P. Botella, A. Corma, M. Navarro, F. Rey, G. Sastre, J. Catal., 217, 406 (2003).

25. P. Botella, A. Corma, G. Sastre, J. Catal., 197, 81 (2001).

26. P. Chen, W. Wang, Y. Zhai, J. Porous Mat., 21, 441 (2014).

27. H. Schuster, W. Hölderich, Appl. Catal. A-Gen., 350, 1 (2008).

28. A. Pârvulescu, B. Gagea, V. Pârvulescu, D. Vos, P. Jacobs, Appl. Catal. A-Gen., 306159 (2006).

29. D. Katsoulis, Chem. Rev., 98, 359 (1998).

30. A. Dolbecq, E. Dumas, C. Mayer, P. Mialane, Chem. Rev., 110, 6009 (2010).

31. K. Kamata, K. Yonehara, Y. Nakagawa, K. Uehara, N. Mizuno, Nature Chem., 2, 478 (2010).

32. C. Isabel, J. Gamelas, M. Balula, J. Mol. Catal. AChem., 262, 41 (2007).

33. S. Zhao, J. Xu, M. Wei, Y. Song, Green Chem., 13, 384 (2011).

34. J. Sousa, I. Santos, Catal. Commun., 12, 459 (2011).

35. N. Mizuno, K. Yamaguchi, K. Kamata, Catal. Surv. Asia, 15, 68 (2011).

36. P. Liu, C. Wang, C. Li, J. Catal., 262, 159 (2009).

37. Y. Wang, J. Wang. D. Zhu, M. Zhang, Green Chem., 13, 1636 (2011).

38. L. Ghamati, O. Walter, U. Amold, Eur. J. Inorg. Chem., 17, 2756 (2011).

39. Y. Yamamoto, S. Hatanaka, K. Tsuji, Appl. Catal. A-Gen., 344, 55 (2008).

40. E. Caliman, A. José, C. Sílvia, Micropor. Mesopor. Mat., 132, 103 (2010).

41. L. Wee, S. Bajpe, N. Janssens, Chem. Comm., 46 8186 (2010).

42. Y. Leng, J. Wang, D. Zhu, Angew. Chem. Int. Ed., 48168 (2009).

43. A. Alsalme, E. Kozhevnikova, I. Kozhevnikov, Appl. Catal. A-Gen., 349, 170 (2008).

44. G. Sunita, B. Devassy, A. Vinu, Catal. Comm., 9 696 (2008).

45. C. Kumar, P. Prasad, N. Lingaiah, Appl. Catal. AGen., 384, 101 (2010).

46. J. Satam, R. Jayaram, Catal. Comm., 9, 1937 (2008).

47. C. Kumar, K. Rao, P. Prasad, N. Lingaiah, J. Mol. Catal. A. Chem., 337, 17 (2011).

48. G. Kamalakar, K. Komura, Y. Kubota, Y. Sugi, J. Chem. Technol. Biot., 81, 981 (2006).

49. G. Sartori, R. Maggi, Chem. Rev., 106, 1077 (2006).

50. I. Kozhevnikov, Chem. Rev., 98, 171 (1998).

51. I. Kozhevnikov, Appl. Catal. A-Gen., 256, 3 (2003).

52. C. Castro, A. Corma, J. Primo, J. Mol. Catal. AChem., 177, 273 (2002).

53. J. Kaur, I. Kozhevnikov, Chem. Commun., 21, 2508 (2002).

54. J. Kaur, K. Griffin, B. Harrison, I. Kozhevnikov, J. Catal., 208, 448 (2002).

55. J. Huddleston, A. Visser, W. Reichert, H. Willauer, G. Broker, R. Rogers, Green Chem., 3, 156 (2001).

56. E. G. Derouane, G. Crehan, C. J. Dillon, D. Bethell, H. He, S. B. Derouane-Abd Hamid, J. Catal., 194, 410 (2000).

57. J. Boon, J. Levisky, J. Pflug, J. Wilkes, J. Org. Chem., 51, 480 (1986).

58. J. K. Surette, L. Green, R. D. Singer, Chem. Commun., 24, 2753 (1996).

59. G. D. Luer, D. E. Bartak, J. Org. Chem., 47, 1238 (1982).

60. D. S. Newman, R. E. Winans, R. L. McBeth, J. Electrochem. Soc., 131, 1079 (1984).

61. Y. Chauvin, A. Hirschauer, H. Olivier, J. Mol. Catal. A-Chem., 92, 155, (1994).

62. V. R Koch, L. L. Miller, R. A. Osteryoung, J. Am. Chem. Soc., 98, 5277 (1976).

63. T. Welton, Chem. Rev., 99, 2071 (1999).

64. S, Park, R. J. Kazlauskas, J. Org. Chem., 66, 8395 (2001).

65. J. Z. Yang, X. M .Lu, J. S .Gui, W. G. Xu, H. W. Li, J. Chem. Thermodyn., 37, 1250 (2005).

66. V. Singh, S. Kaur, V. Sapehiyia, J. Singh, G. L. Kad, Catal. Commun., 6, 57 (2005).

67. F. Durap, M. Aydemir, A. Baysal, D. Elma, B. Ak, Y. Turgut, Inorg. Chim. Acta, 411, 77 (2014).

68. Q. G. Deng, Z. L. Qin, Y. Yang, W. M. Song, J. Chem. Eng., 23, 384 (2015).

69. R. Ballini, L. Barboni, G. Bosica, J. Org. Chem., 65, 6261 (2000).

70. R. Srivastava, Catal. Lett., 139, 17 (2010). 


\section{Instructions about Preparation of Manuscripts}

General remarks: Manuscripts are submitted in English by e-mail The text must be typed on A4 format paper using Times New Roman font size 11, normal character spacing. The manuscript should not exceed 15 pages (about 3500 words), including photographs, tables, drawings, formulae, etc. Authors are requested to use margins of $2 \mathrm{~cm}$ on all sides

Manuscripts should be subdivided into labelled sections, e.g. Introduction, Experimental, Results and Discussion, etc. The title page comprises headline, author's names and affiliations, abstract and key words. Attention is drawn to the following:

a) The title of the manuscript should reflect concisely the purpose and findings of the work. Abbreviations, symbols, chemical formulas, references and footnotes should be avoided. If indispensable, abbreviations and formulas should be givenin parentheses immediately after the respective full form.

b) The author's first and middle name initials and family name in full should be given, followedby the address (or addresses) of the contributing laboratory (laboratories). The affiliation of the author(s) should be listed in detail by numers (no abbreviations!). The author to whom correspondence and/or inquiries should be sent should be indicated by asterisk $(*)$ with e-mail address.

The abstract should be self-explanatory and intelligible without any references to the text andcontaining not more than 250 words. It should be followed by key words (not more than six).

References should be numbered sequentially in the order, in which they are cited in the text. The numbers in the text should be enclosed in brackets [2], [5, 6], [9-12], etc., set on the text line. References are to be listed in numerical order on a separate sheet. All references are to be given in Latin letters. The names of the authors are given without inversion. Titles of journals must be abbreviated according to Chemical Abstracts and given in italics, the volume is typed in bold, the initial page is given and the year in parentheses. Attention is drawn to the following conventions:a) The names of all authors of a certainpublications should be given. The use of "et al." inthe list of references is not acceptable.b) Only the initials of the first and middle names should be given. In the manuscripts, the reference to author(s) of cited works should be made without giving initials, e.g. "Bush and Smith [7] pioneered...". If the reference carries the names of three or more authors it should be quoted as "Bush et al. [7]", if Bush is the first author, or as "Bush and co-workers [7]", if Bush is the senior author.

Footnotes should be reduced to a minimum. Each footnote should be typed double-spaced at thebottom of the page, on which its subject is first mentioned.Tables are numbered with Arabic numerals on the left-hand top. Each table should be referred to inthe text. Column headings should be as short as possible but they must define units unambiguously. The units are to be separated from the preceding symbols by a comma or brackets. Note: The following format should be used when figures, equations, etc. are referred to the text (followed by the respective numbers): Fig., Eqns., Table, Scheme.

Schemes and figures. Each manuscript should contain or be accompanied by the respective illustrative material as well as by the respective figure captions in a separate file (sheet).As far as presentation of units is concerned, SI units are to be used. However, some non-SI units are also acceptable, such as ${ }^{\circ} \mathrm{C}$, $\mathrm{ml}, 1$, etc.The author(s) name(s), the title of the manuscript, the number of drawings, photographs, diagrams, etc., should be written in black pencil on the back of the illustrative material (hard copies) inaccordance with the list enclosed. Avoid using more than 6 (12 for reviews, respectively) figures in the manuscript. Since most of the illustrative materials are to be presented as $8-\mathrm{cm}$ wide pictures, attention should be paid that all axis titles, numerals, legend(s) and texts are legible.

The authors are required to submit the text with a list of three individuals and their e-mail addresses that can be considered by the Editors as potential reviewers. Please, note that the reviewers should be outside the authors' own institution or organization. The Editorial Board of the journal is not obliged to accept these proposals.

The authors are asked to submit the final text (after the manuscript has been accepted for publication) in electronic form by e-mail. The main text, list of references, tables and figure captions should be saved in separate files (as *.rtf or *.doc) with clearly identifiable file names. It is essential that the name and version of the word-processing program and the format of the text files is clearly indicated. It is recommended that the pictures are presented in *.tif, *.jpg, *.cdr or *.bmp format. 
The equations are written using "Equation Editor" and chemical reaction schemes are written using ISIS Draw or ChemDraw programme.

\section{EXAMPLES FOR PRESENTATION OF REFERENCES}

\section{REFERENCES}

1. D. S. Newsome, Catal. Rev.-Sci. Eng., 21, 275 (1980).

2. C.-H. Lin, C.-Y. Hsu, J. Chem. Soc. Chem. Commun., 1479 (1992).

3. R. G. Parr, W. Yang, Density Functional Theory of Atoms and Molecules, Oxford Univ. Press, New York, 1989.

4. V. Ponec, G. C. Bond, Catalysis by Metals and Alloys (Stud. Surf. Sci. Catal., vol. 95), Elsevier, Amsterdam, 1995.

5. G. Kadinov, S. Todorova, A. Palazov, in: New Frontiers in Catalysis (Proc. 10th Int. Congr. Catal., Budapest,(1992), L. Guczi, F. Solymosi, P. Tetenyi (eds.), Akademiai Kiado, Budapest, 1993, Part C, p. 2817. 6. G. L. C. Maire, F. Garin, in: Catalysis. Science and Technology, J. R. Anderson, M. Boudart (eds), vol. 6, SpringerVerlag, Berlin, 1984, p. 161.

7. D. Pocknell, GB Patent 2207355 (1949).

8. G. Angelov, PhD Thesis, UCTM, Sofia, 2001, pp. 121-126.

9 JCPDS International Center for Diffraction Data, Power Diffraction File, Swarthmore, PA, 1991.

10. CA 127, 184 762q (1998).

11. P. Hou, H. Wise, J. Catal., in press.

12. M. Sinev, private communication.

13. http://www.chemweb.com/alchem/articles/1051611477211.html.

Texts with references which do not match these requirements will not be considered for publication!!! 


\section{CONTENTS}

B. Alev, S. Tunali, R. Yanardag, A. Yarat, Influence of storage time and temperature on activity of urease.

M. Sinirkaya, Effect of ultrasound on the dissolution of Mardin-Mazıdağı (Turkey) phosphate ore in dilute $\mathrm{H}_{3} \mathrm{PO}_{4}$ solution.

G.S. Seth, R. Kumar, R. Tripathi, Thermo-дiffusion effects on magnetohydrodynamic natural convection flow of a chemically reactive Brinkman type nanofluid in a porous medium.

M. Imran Khan, T. Hayat, M. Ijaz Khan, T. Yasmeen, Thermal properties and time dependent flow behavior of viscous fluid

S. Hassanpour, M.Bayat, A.Chaichi Nosrati, M.Ghorannevis, S. Hashemi, The eliminatory effects of cold argon plasma jet on aflatoxin B1 produced by different isolates of Aspergillus section nigri....

B. Parvizi, A. Khanlarkhani, Y. Palizdar, A.Farshad,Comparison of ANFIS and ANN modeling for predicting the behavior of a catalytic methane reformer.

$\ddot{O}$. Özşen1, $\ddot{O}$. Atli, $\dot{I}$. Kiran, Biotransformation of oleic acid and antimicrobial and anticancer activities of its biotransformation extracts................................................

R. Rehman, T. Tanveer, Spectrophotometric determination of significant minerals in milk samples found in Lahore (Pakistan) for ensuring food safety ...............................

S. Sönmez, Ö. Özden, The influence of pigment proportions and calendering of coated paperboards on dot gain.

P.M. Rafailov, R. Todorov, V. Marinova, D.Z. Dimitrov, M. M. Gospodinov, Optical spectroscopic study of Ru- and Rh-DOPED $\mathrm{Bi}_{12} \mathrm{TiO}_{20}$ crystals...........................

S. Mohammadi Chalanchi, A. Ebrahimi , A. Nowroozi, Theoretical insight to intermolecular hydrogen bond interactions between methyl $\mathrm{N}$-(2-pyridyl) carbamate and acetic acid: substituent effects, cooperativity and energy decomposition analysis........................

V.A. Kadnor, S. N. Shelke, Synthesis and biological evaluation of novel carbazole chalcones and corresponding 1,5-benzothiazepine derivatives

D.S. Bogdanović, D.H. Anđelković, I.S. Kostić, G.M. Kocić, T.D. Anđelković, The effects of temperature and ultrasound on the migration of di-(2-ethylhexyl) phthalate from plastic packaging into dairy products.

A.M. Vasileva, I.A. Iliev, V.S. Lozanov, M.B. Dimitrova, V.I. Mitev, I.P. Ivanov, In vitro study on the antitumor activity of Tanacetum vulgare L. extracts.

N.P. Gribacheva, G.M. Gecheva, V.M. Stefanova, Air pollution monitoring with mosses in Western Rhodopes, Bulgaria.....

S.B. Seo, Y.M. Kim, Optimization of high ultrasound-assisted extraction (INEFU) of active components from natural materials by response HPLC-PDA analysis..........................

G. Karakus, Z. Akin Polat, M. Karahan, Design, synthesis, structural characterization and cell cytotoxicity of new derivative poly( maleic anhydride-co-vinyl acetate)/miltefosine polymer/drug conjugate.

N. Ebrahimi, A. Haghighi Asl, P. Mottahedin, Optimization of $\beta$-carotene solubility in pressurized hot water using a dynamic method and factorial methodology.

E. Chorbadzhiyska, I. Bardarov, Y. Hubenova, M. Mitov, Modified graphite electrodes as potential cathodic electrocatalysts for microbial electrolysis cells.

Y. Guo, J. Sun, F. Guo, Yu. He, P. Chen, Friedel-Crafts acylation of 2-methoxynaphthalene with acetic anhydride catalyzed by phosphotungstic acid in ionic liquid........................... 